Fall 2013

\title{
2013 Miracle Yearbook
}

Cedarville University

Follow this and additional works at: https://digitalcommons.cedarville.edu/yearbooks

Part of the Higher Education Commons, Organizational Communication Commons, and the Public Relations and Advertising Commons

\section{Recommended Citation}

Cedarville University, "2013 Miracle Yearbook" (2013). Yearbooks. 94.

https://digitalcommons.cedarville.edu/yearbooks/94

This Book is brought to you for free and open access by DigitalCommons@Cedarville, a service of the Centennial Library. It has been accepted for inclusion in Yearbooks by an authorized administrator of DigitalCommons@Cedarville. For more information, please contact digitalcommons@cedarville.edu. 

TABLE OF CONTENTS

OPENING

DEDICATION

FALL

6

FACULTY \& STAFF

ORGANIZATIONS

FRESHMEN

64

ACTIVITIES

84

SOPHOMORES

WINTER

116

MINISTRIES

JUNIORS

146

SPRING

156

SENIORS

MIRACLE STAFF

INDEX 


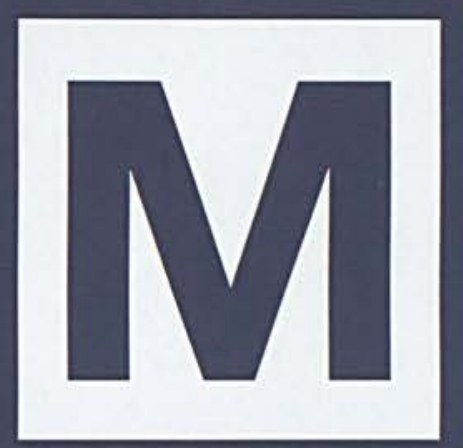

THE MIRACLE YEARBOOK

Miracle 2013 | Cedarvile University | Volume 60 | 251 N. Main Street, Cedarvile, OH 45314 


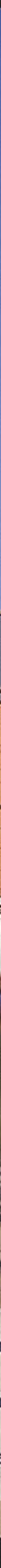





\section{DR: GLINT KOHL}

Dr. Kohl explains how sludents can integrats

their faith into theirstudies in his Digital Logic

Design class:

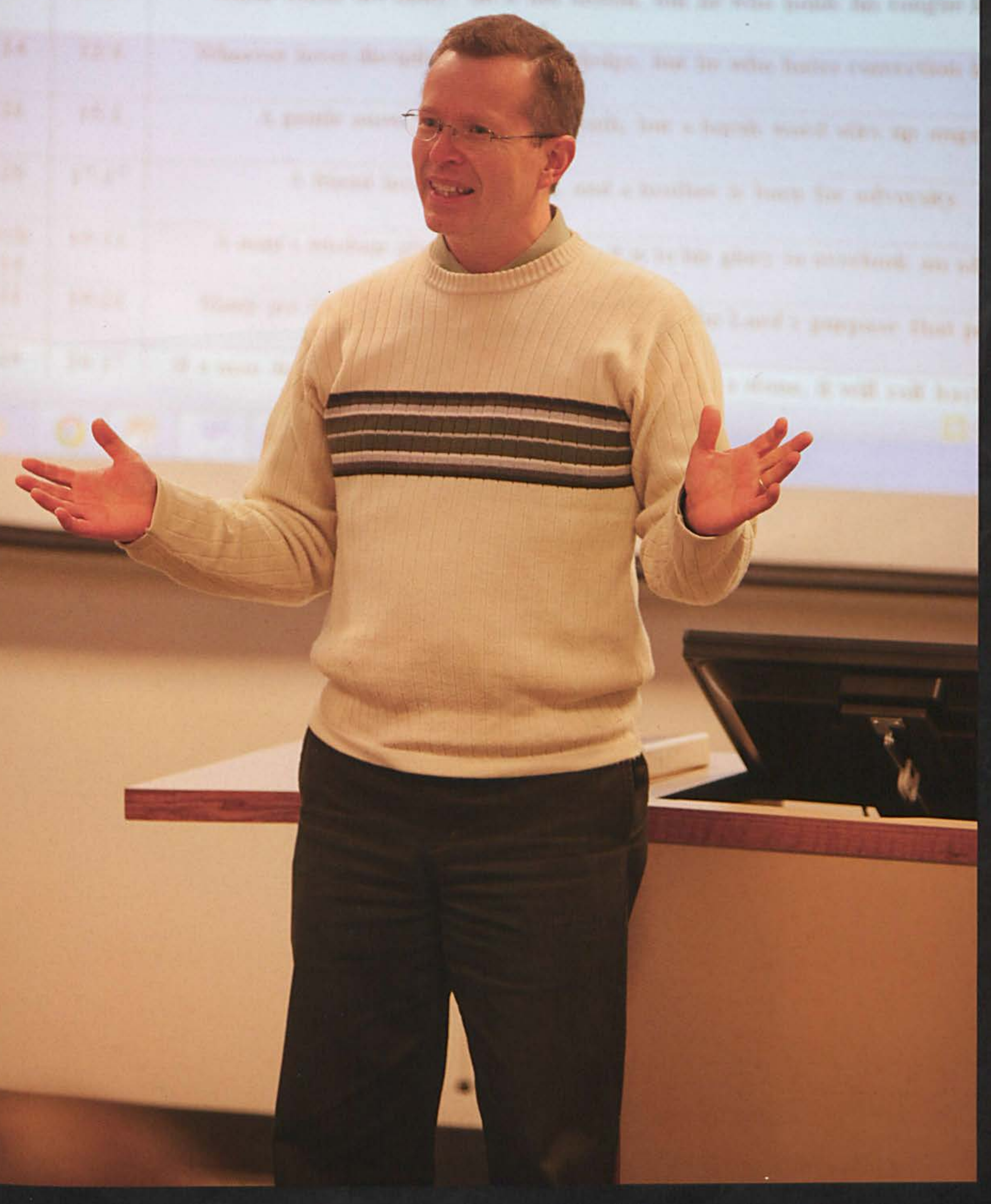




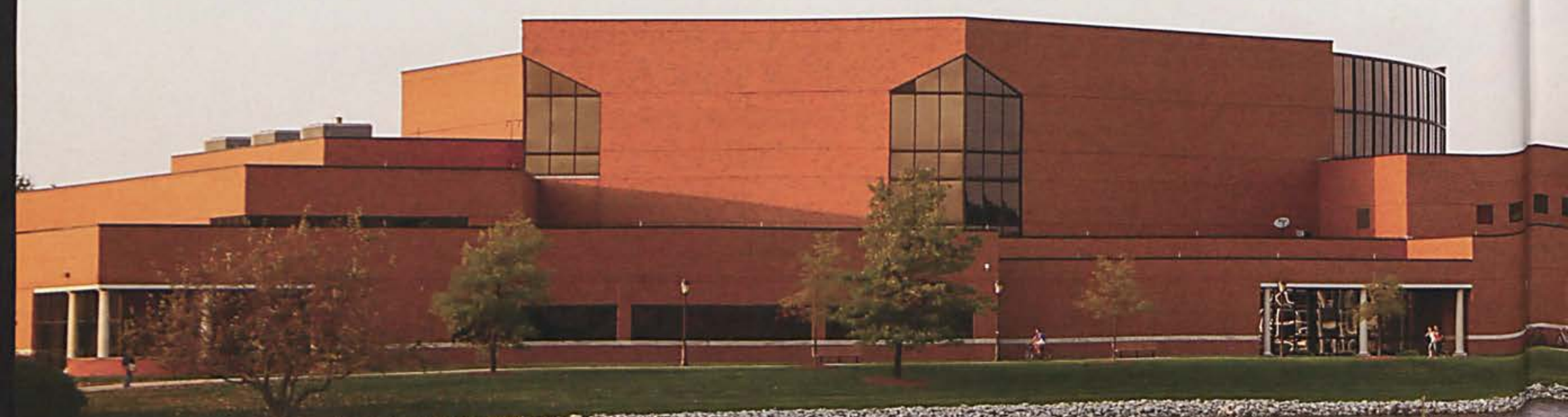

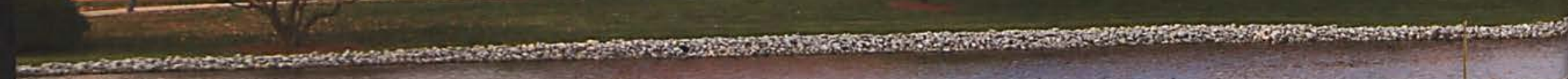

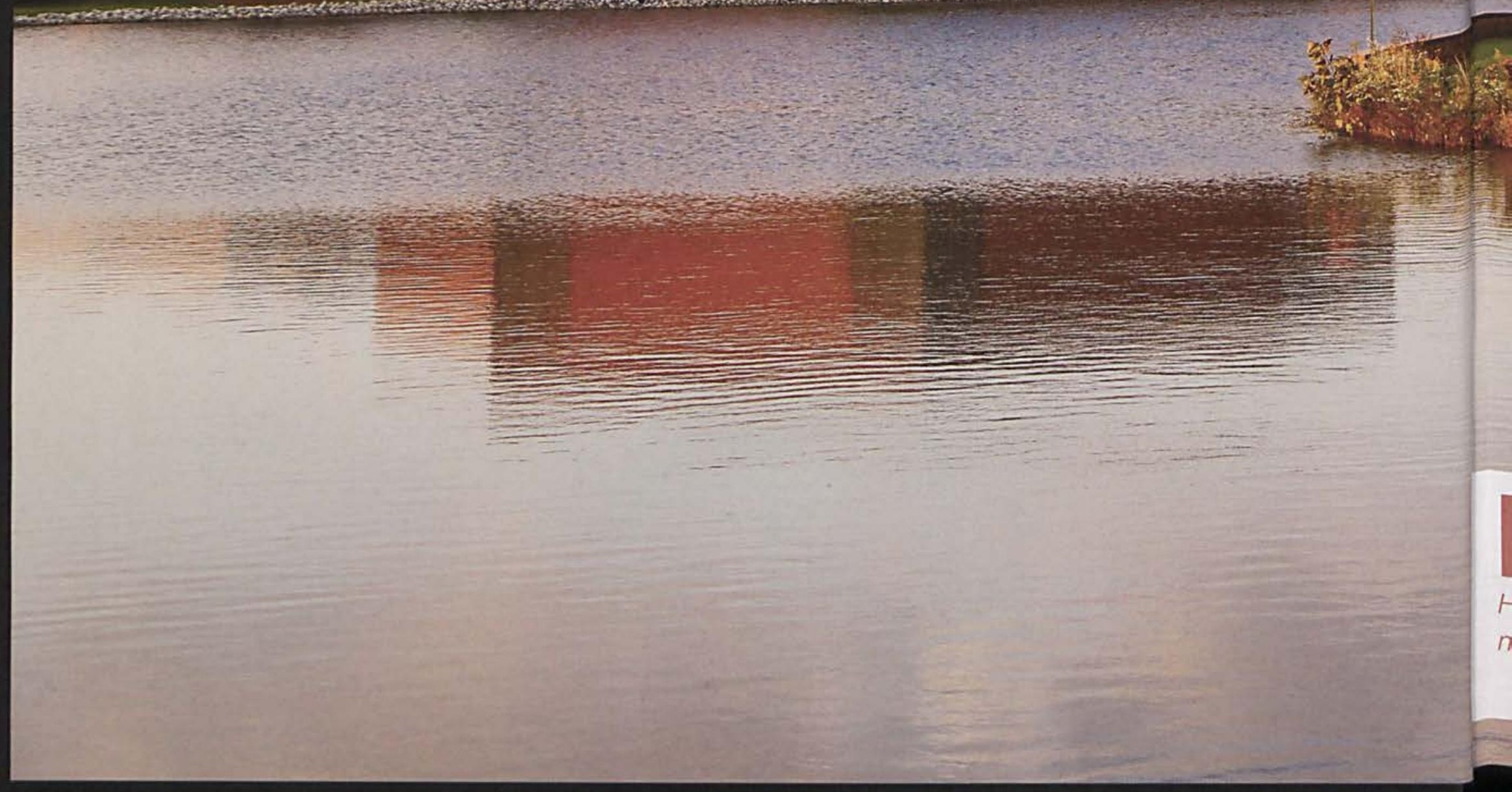




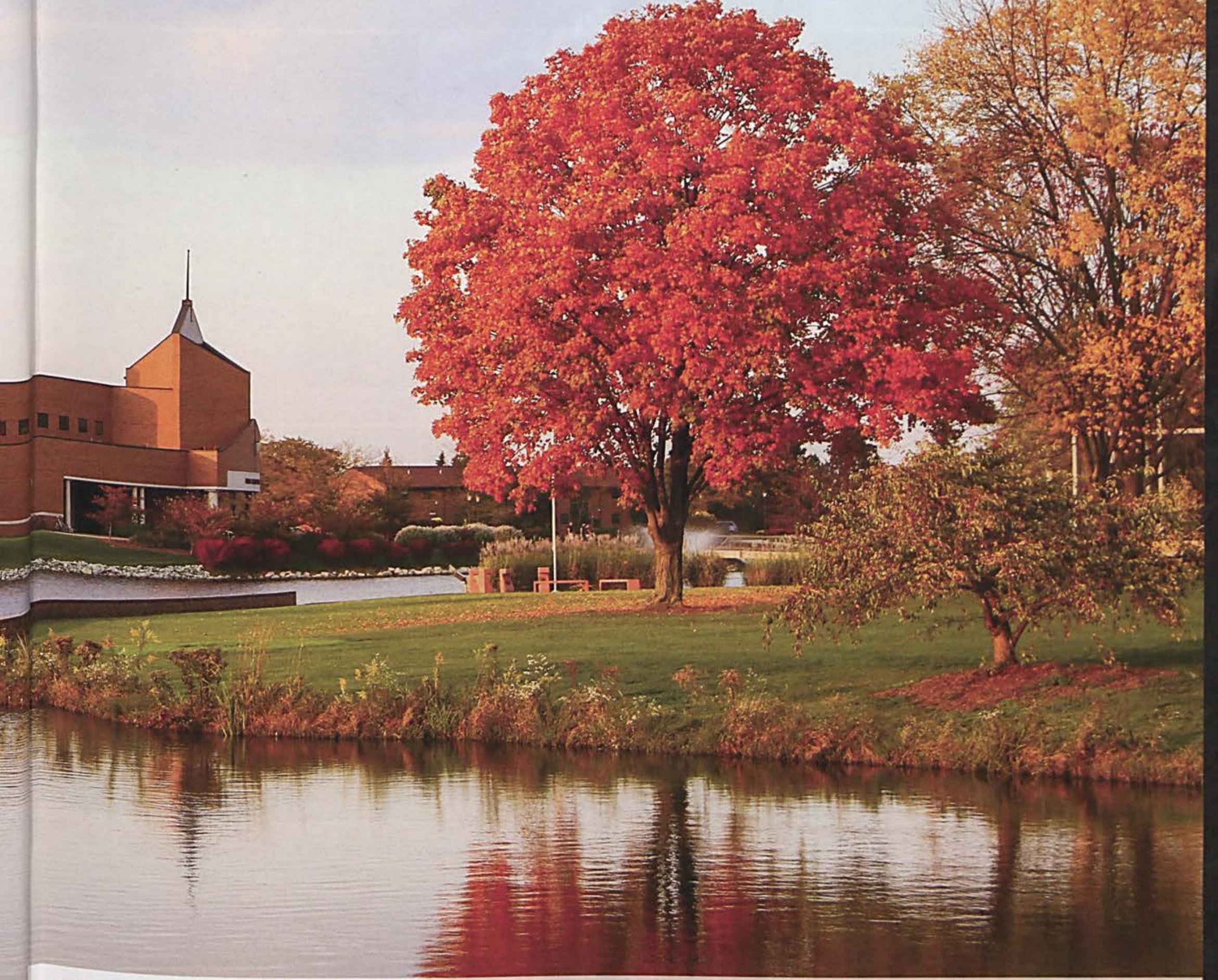

\section{FALL}

Hebrews 10:24-25 "And let us consider how to stir up one another to love and good works, not neglecting to meet together, as is the habit of some, but encouraging one another..." 


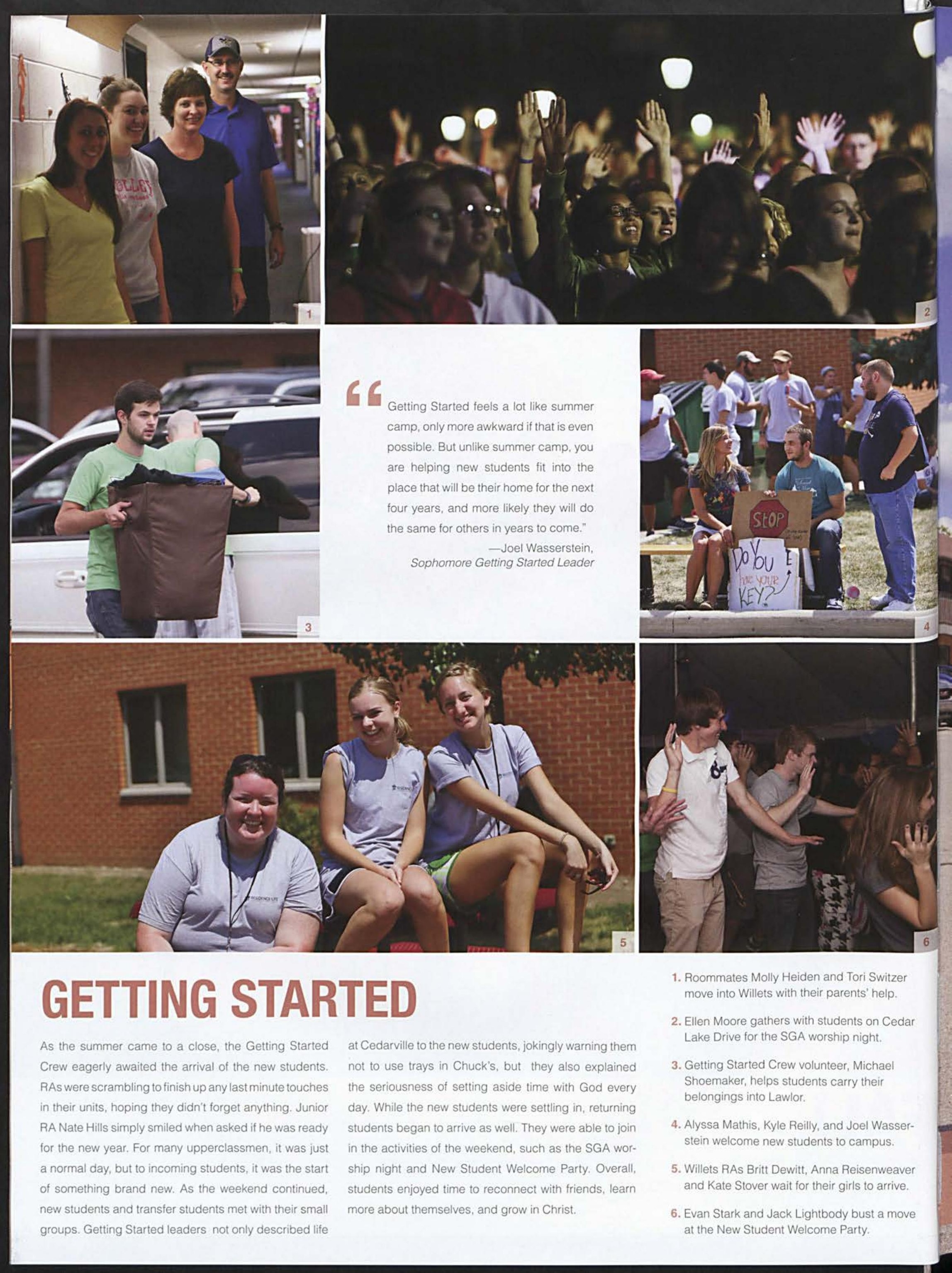




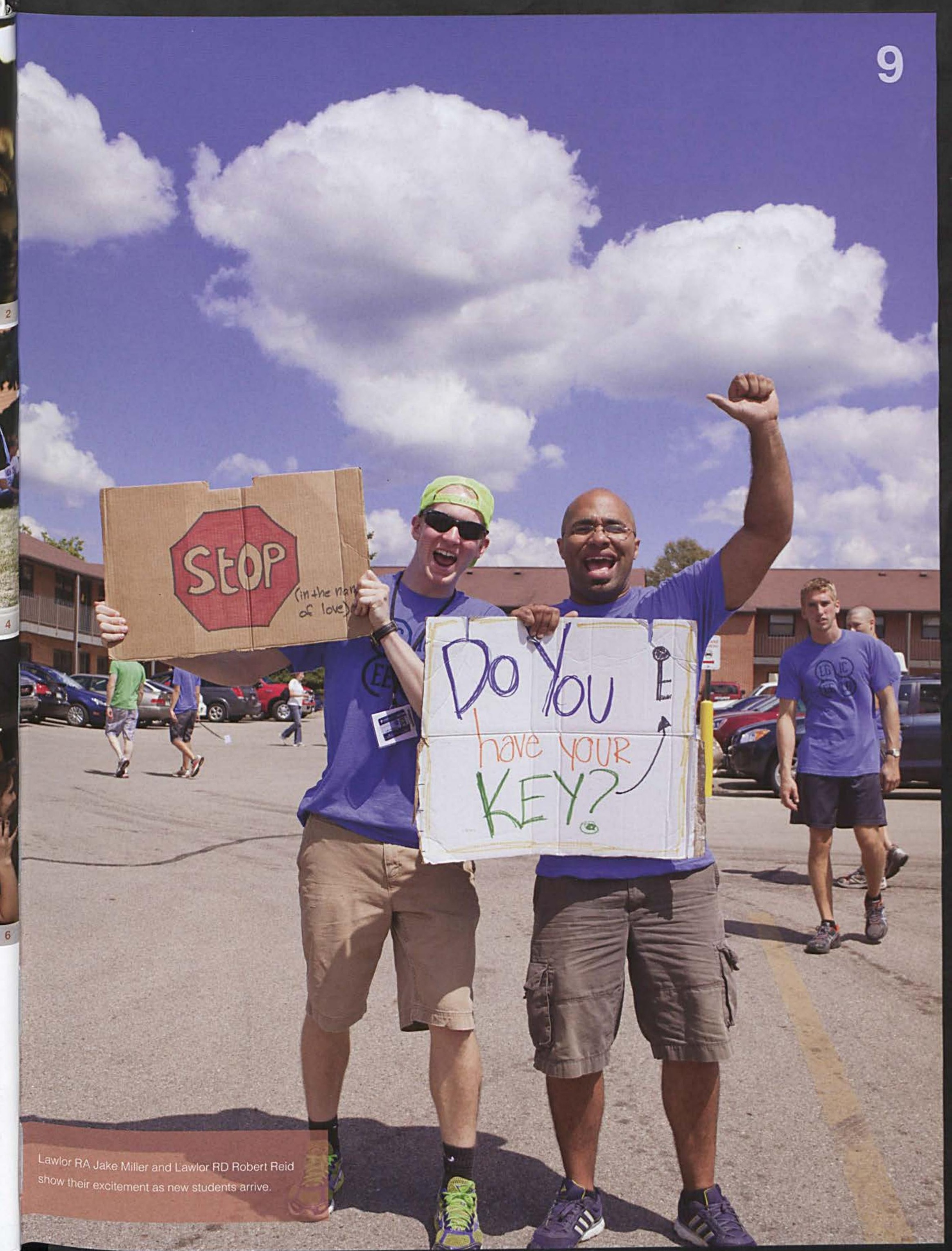




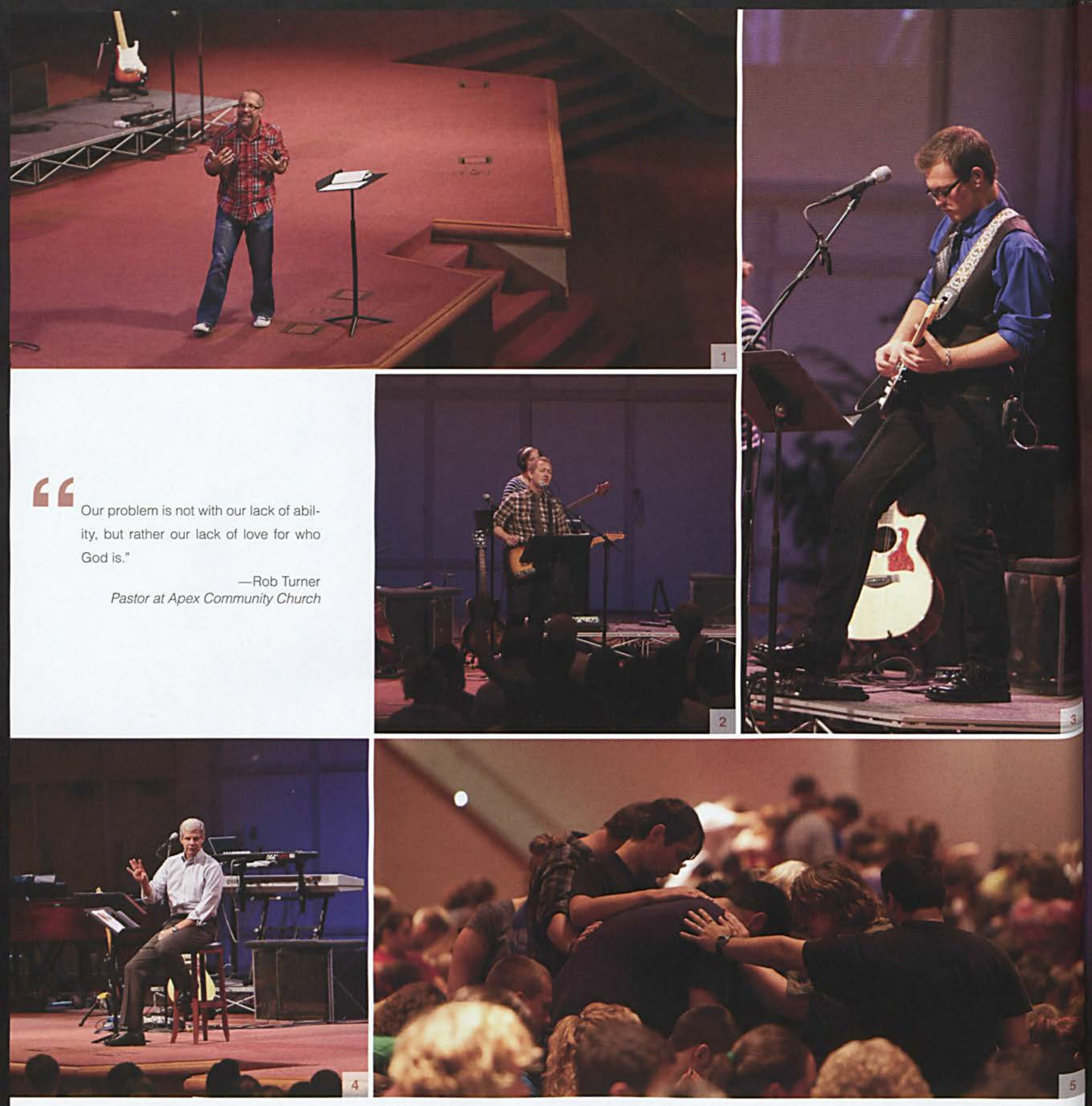

\section{FALL BIBLE CONFERENCE}

At the beginning of every year, even before classes begin, students gather together in the chapel for the Fall Bible Conference. This year, students had the privilege of hearing Pastor Rob Turner, a leader of Apex Community Church in both Xenia and Kettering. Students laughed along with Rob Turner as he told funny stories about his life. His energy and passion for God were evident, and freshmen and seniors alike were able to relate to his messages. Turner reminded students that they should never become numb to the expansion of God's fame because God is worthy to be praised in any circumstance. Patrick Anderson and the other musicians led the students in worship for this year's conference. Thousands of students lifted their voices and filled the chapel with sounds of praise. New students were awed by the chance to worship with so many other believers, and returning students were glad to be back with fellow brothers and sisters in Christ. For many, the opportunity to worship in song and in prayer encouraged a healing of heart and a renewal of mind.
1. Rob Turner challenges students to act on what they hear.

2. Patrick Anderson, assistant professor of music, begins the conference with worship.

3. Isaac Murrell introduces the song " 10,000 Reasons" to the student body.

4. Dr. Brown introduces "The Christ-centered Life" as this year's chapel theme.

5. A group of students respond to Rob's message by lifting each other up in prayer. 

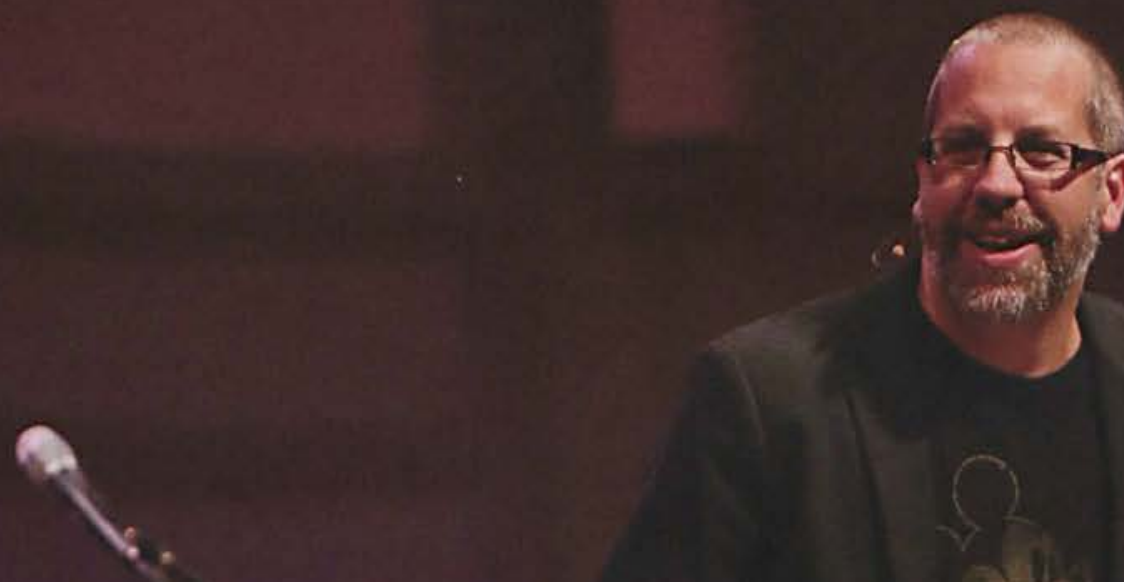

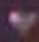
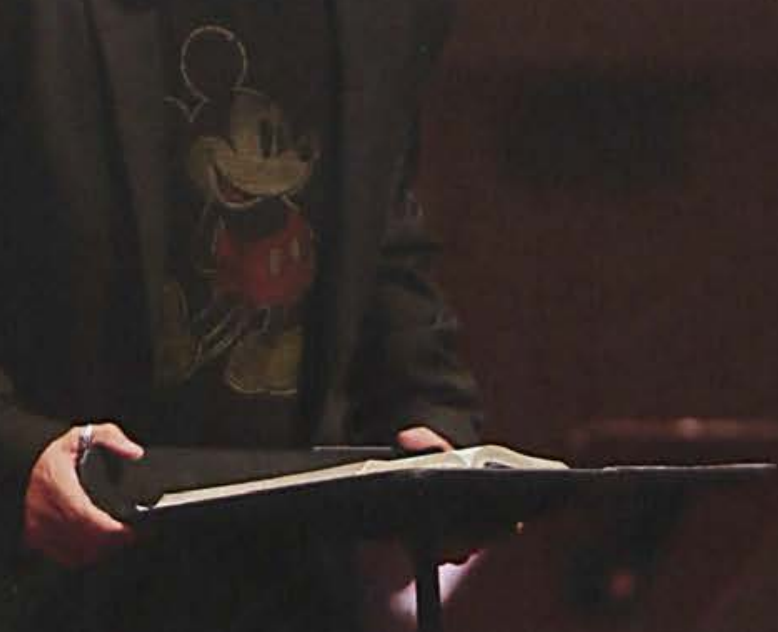

Pastor Rob Turner begins the Fall Bible Conference

with a clever and entertaining story. 

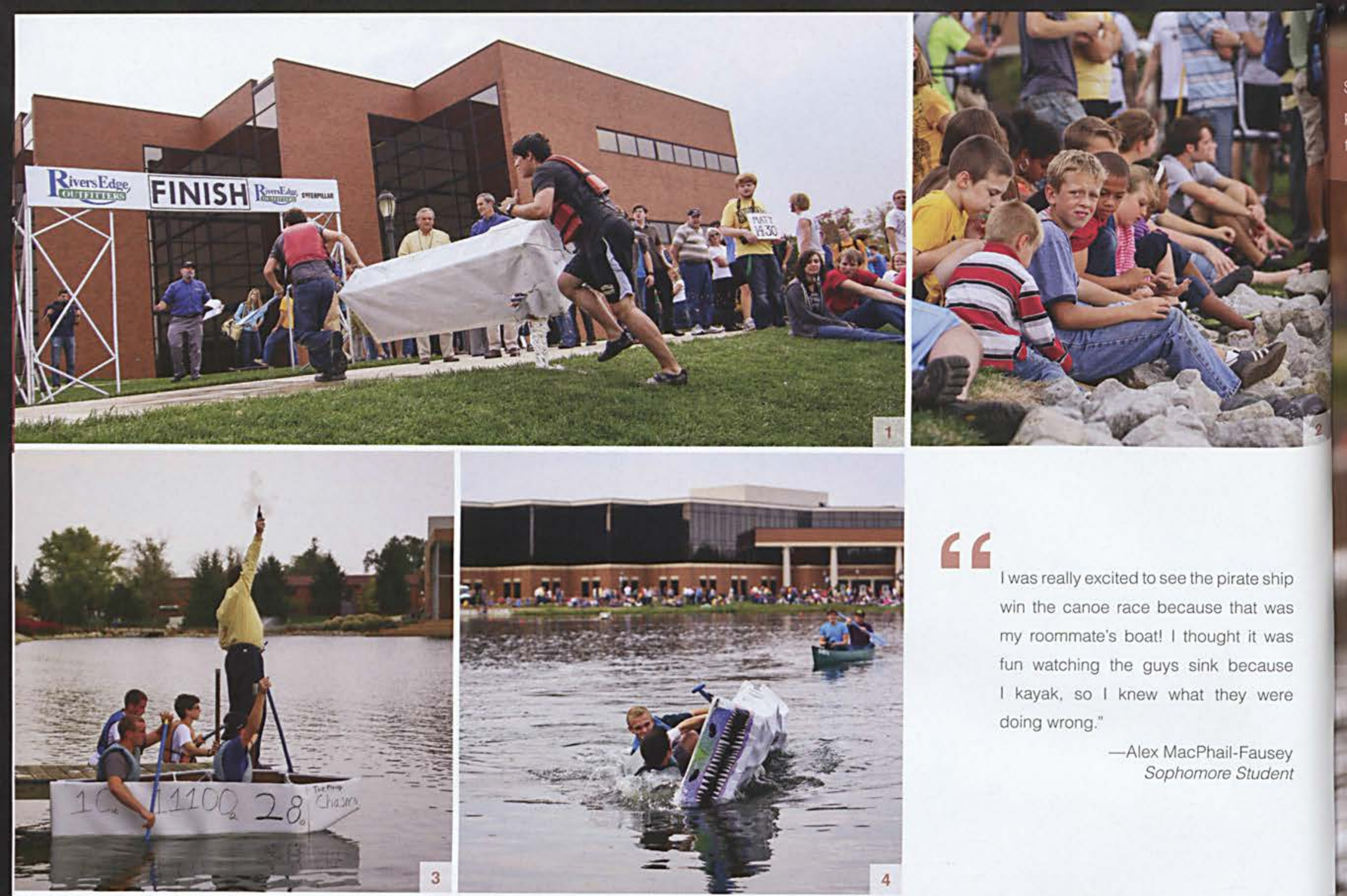

C.

I was really excited to see the pirate ship

win the canoe race because that was

my roommate's boat! I thought it was

fun watching the guys sink because

I kayak, so I knew what they were doing wrong."

-Alex MacPhail-Fausey Sophomore Student

.

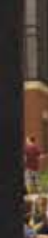

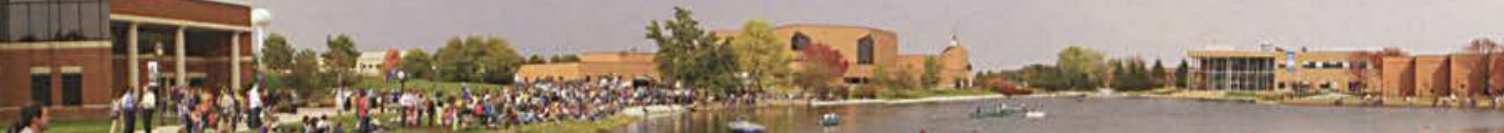
in

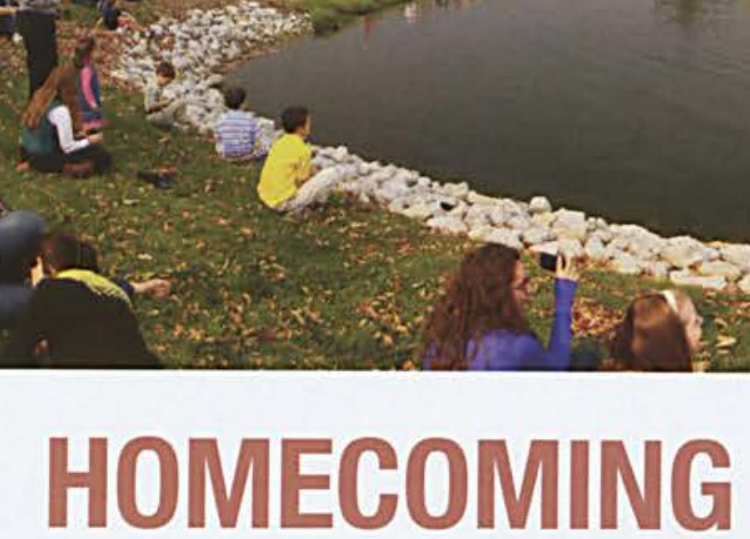

Homecoming weekend is always a great time for both students and alumni. On Friday afternoon, engineering students climbed into their hand-crafted cardboard canoes and raced across the lake. Students and visitors gathered around to watch the race and cheer on their friends. Some even hoped that the canoes would capsize so they could watch the engineering students sink into the murky waters of Cedar Lake. The engineering students hoped otherwise. As freshman Brendan McGillick patiently waited for his group to go, he said. "It better float." Unfortunately for his team, not all boats reach shore. On Saturday morning, Homecoming continued as students, children, and alumni lined the streets of Cedarville, eager to watch the parade and take part in the annual celebration, which continued with CU by the Lake. Many orgs set up tables around the lake and sold products for their fundraisers. Other homecoming activities continued throughout the night where over 600 alumni reconnected with their beloved campus.
1. Two waterlogged freshmen run up the hill in front of the ENS to the finish line of the cardboard canoe race.

2. As alumni return for Homecoming weekend, their children come too, gathering around Cedar Lake to watch the races.

3. Engineering professor Clint Kohl fires the gun, and the canoes take off.

4. Despite the fierce appearance of their canoe, these students will not win the prized Ti-89 calculator.

5. Hundreds of onlookers line the edges of the lake for the 19th Annual Canoe Race. 
Students Jameson Hale (left) and Tyler Dicks (right) paddle cautiously in the hopes that they can secure

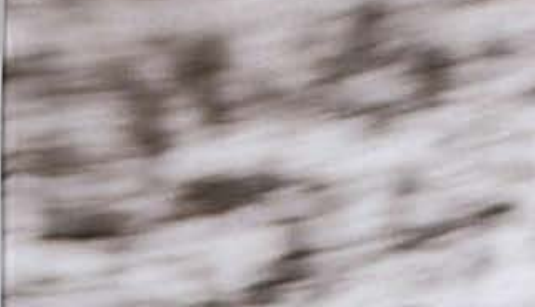

$+2-3=2$

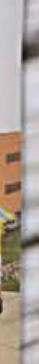

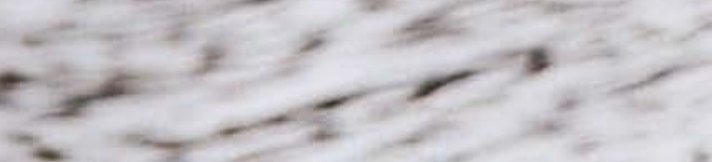$$
\text { is }
$$
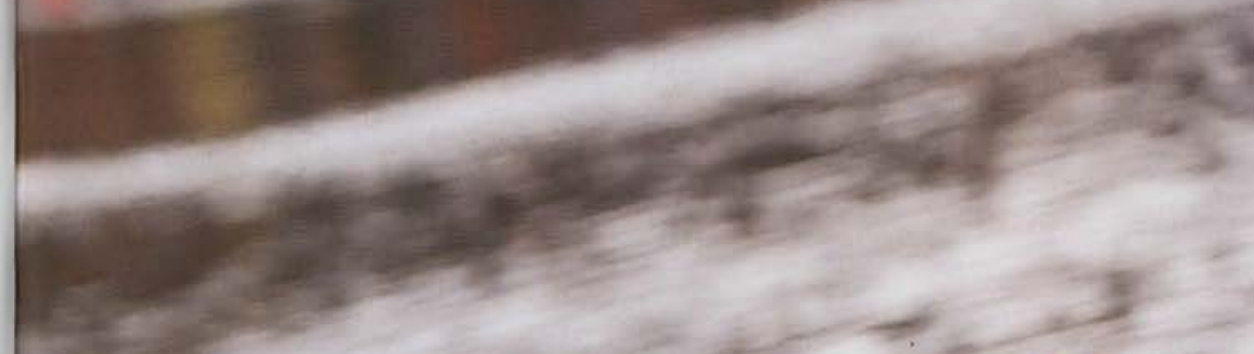

\section{$=-2$}

C.3.
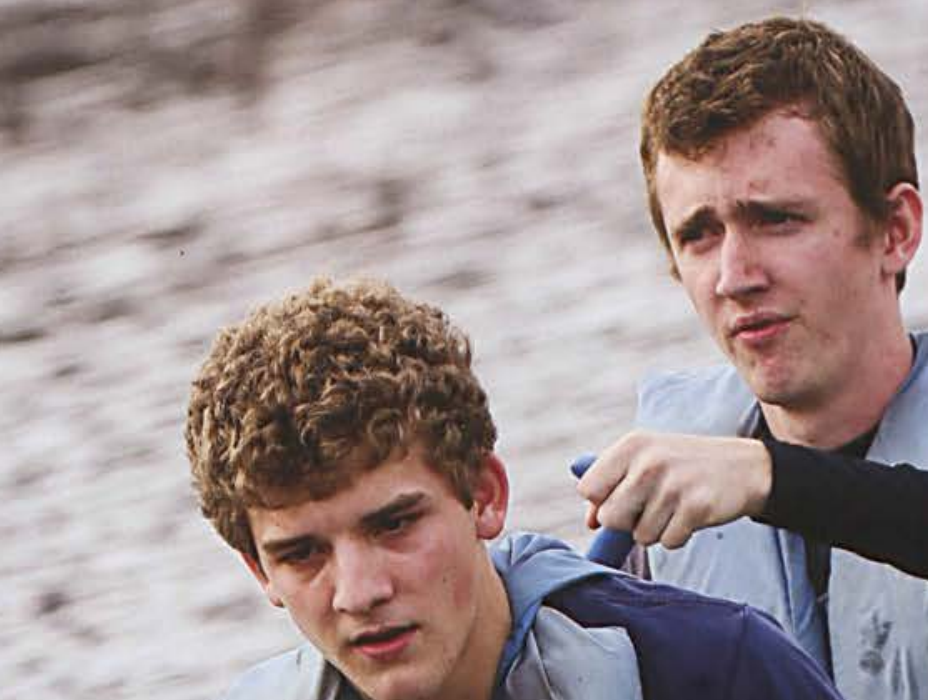

- $2=1$

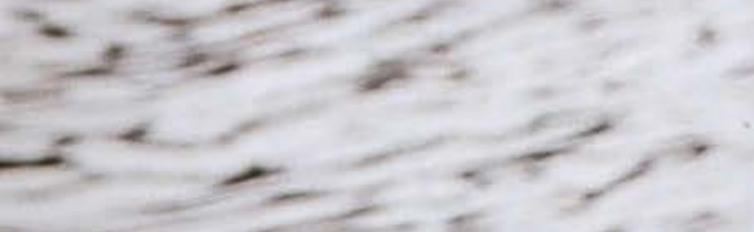

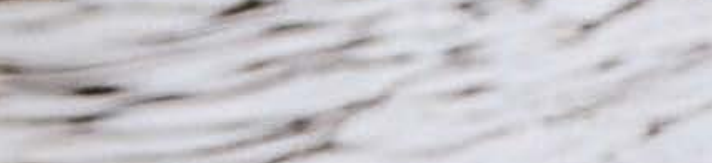

nusm

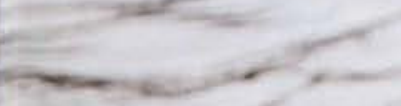

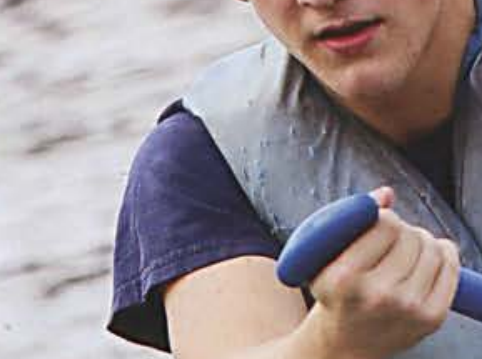

ipe 4
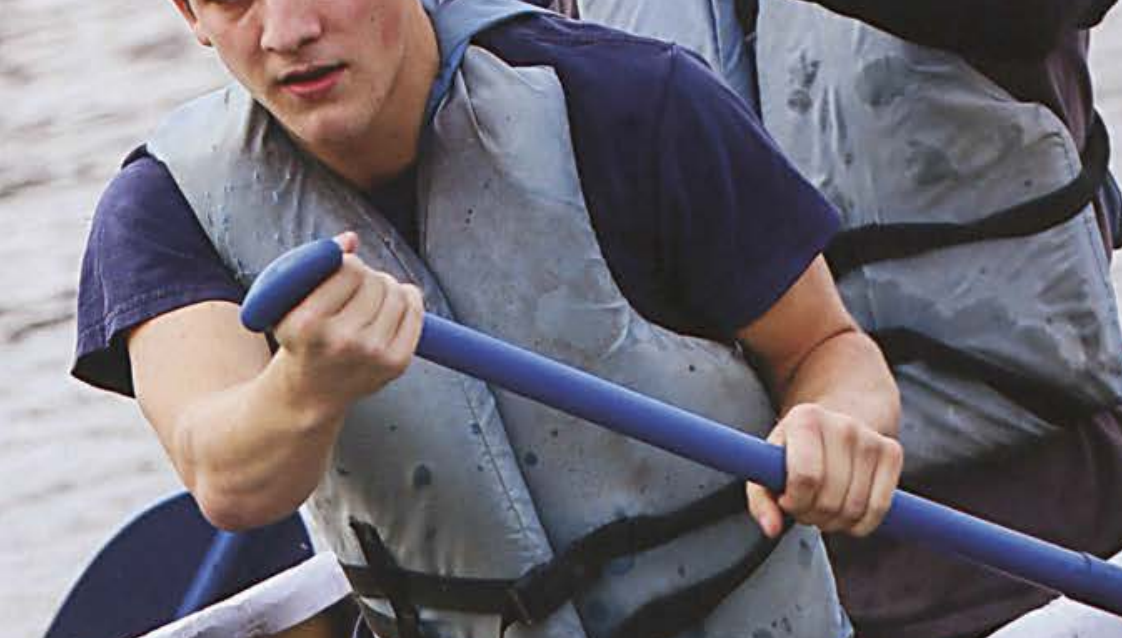

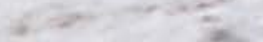

$(1-1)+9$

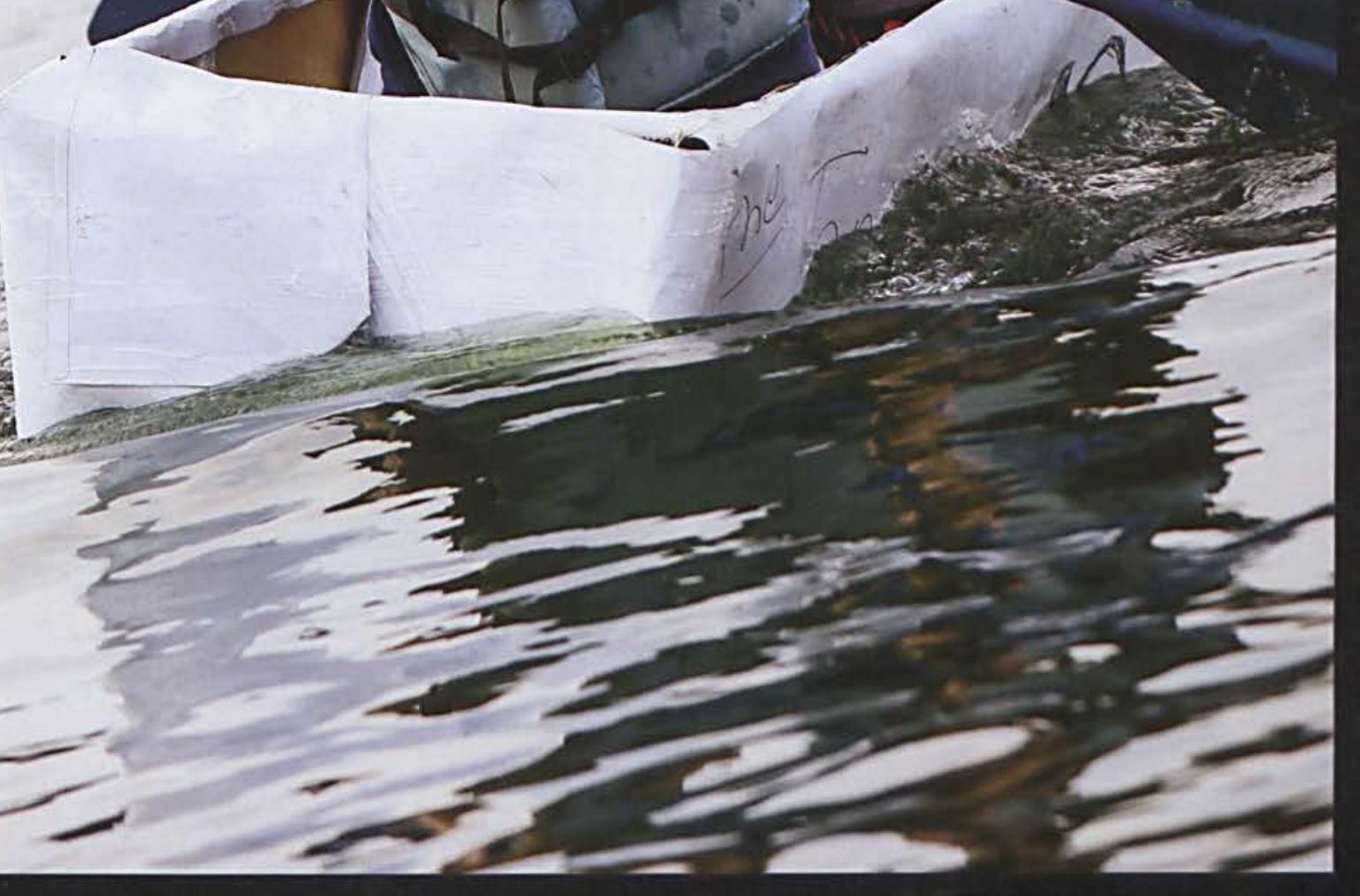




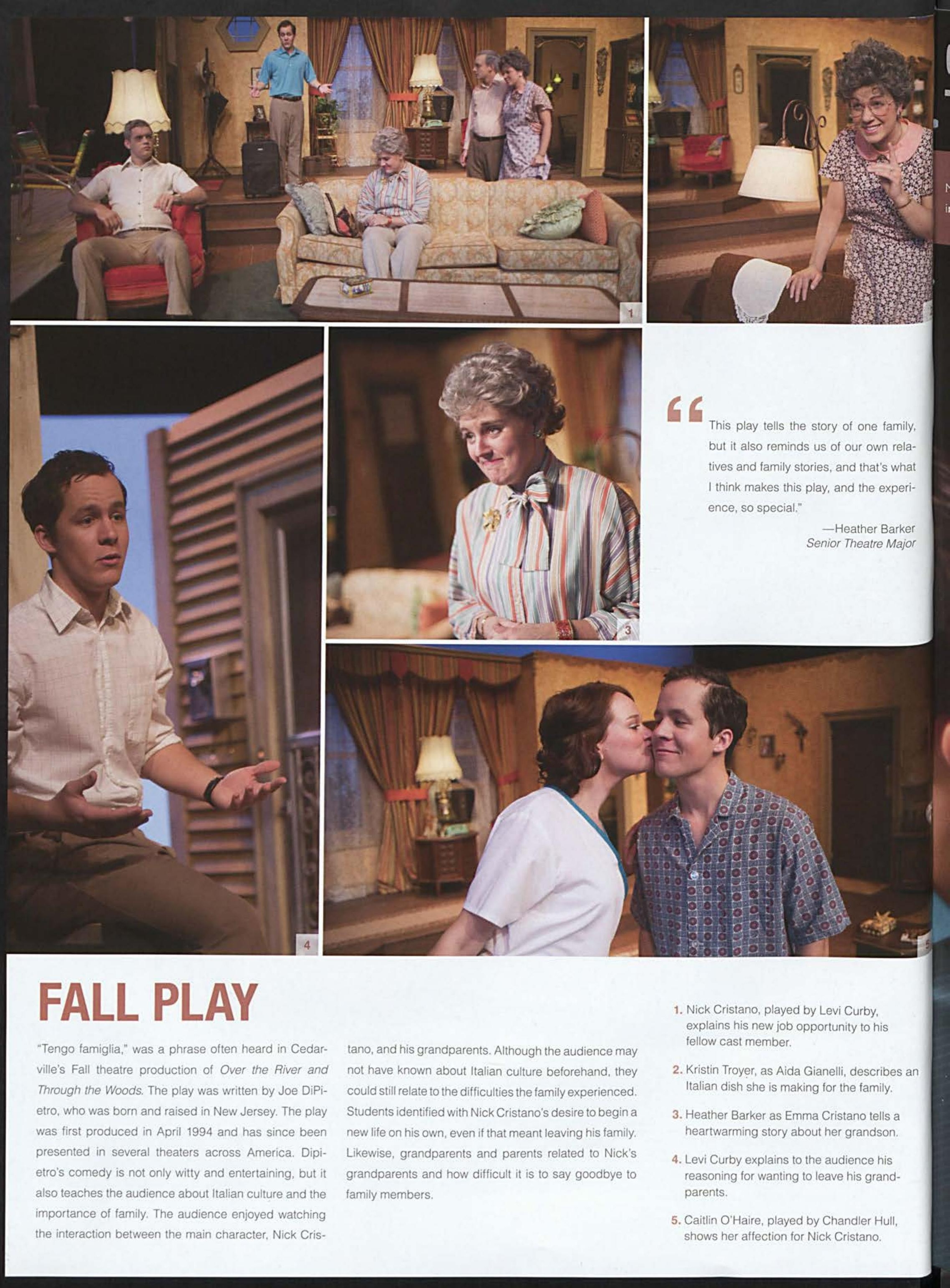




\section{OVER THE RIVER AND THROUGH FHE WOODS}

Nunzio Cristano, played by Ben Lenox. explains the importance of family to his grandson Nick. 


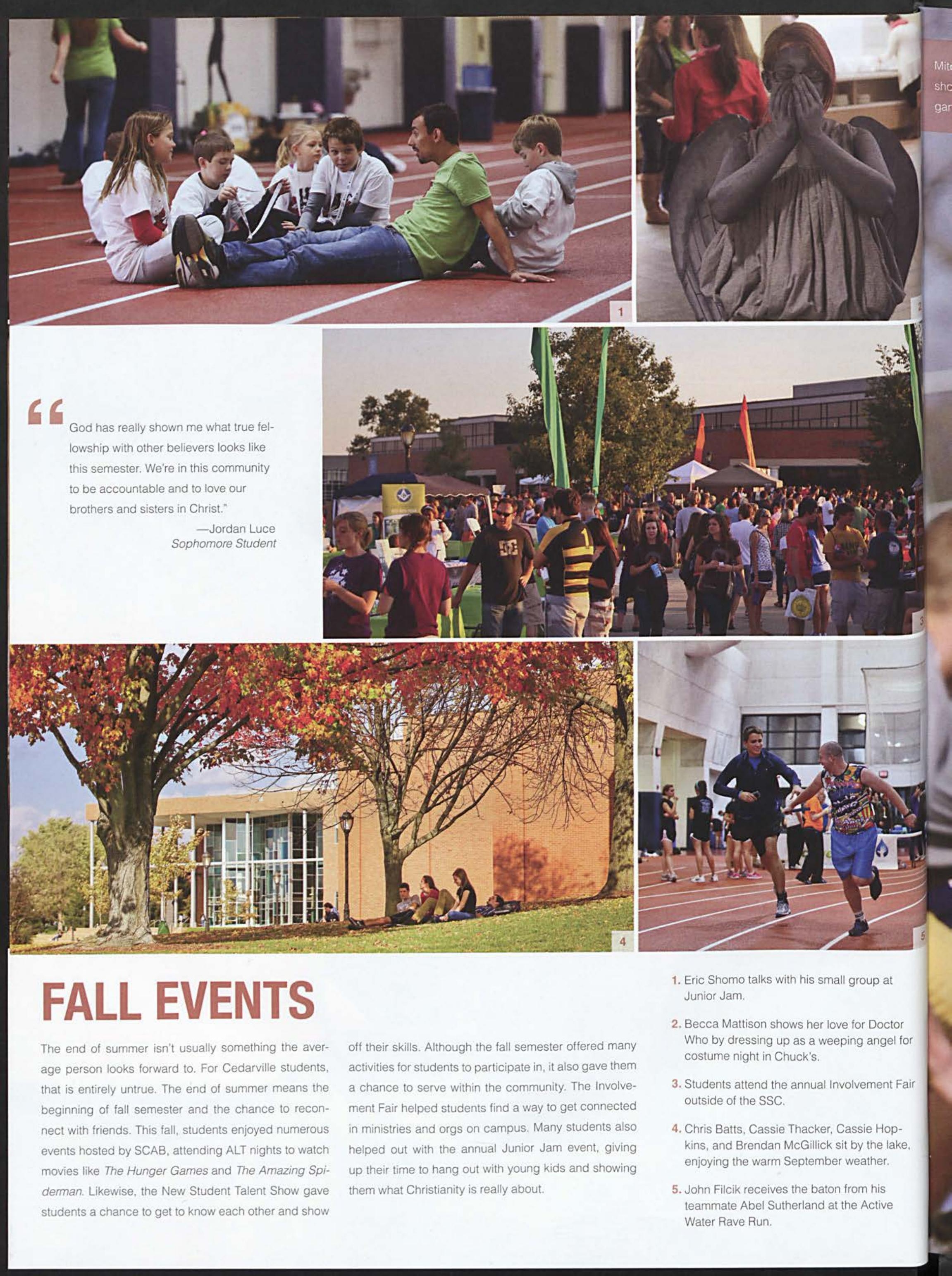




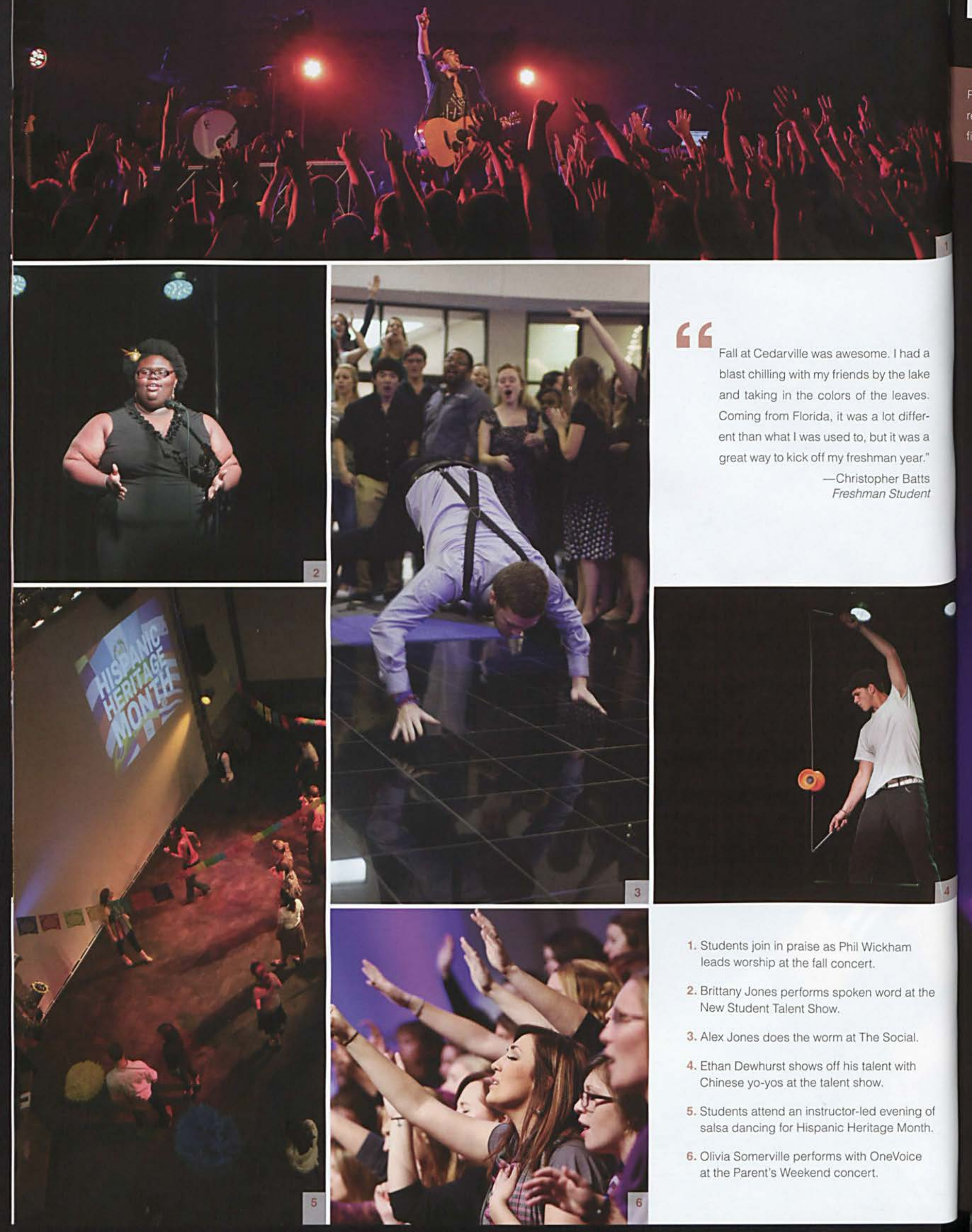




\section{FALL EVENTS}

Phil Wickham performs the song "Divine Romance",

reminding students that they can only be truly satis-

fied when they are in Christ.
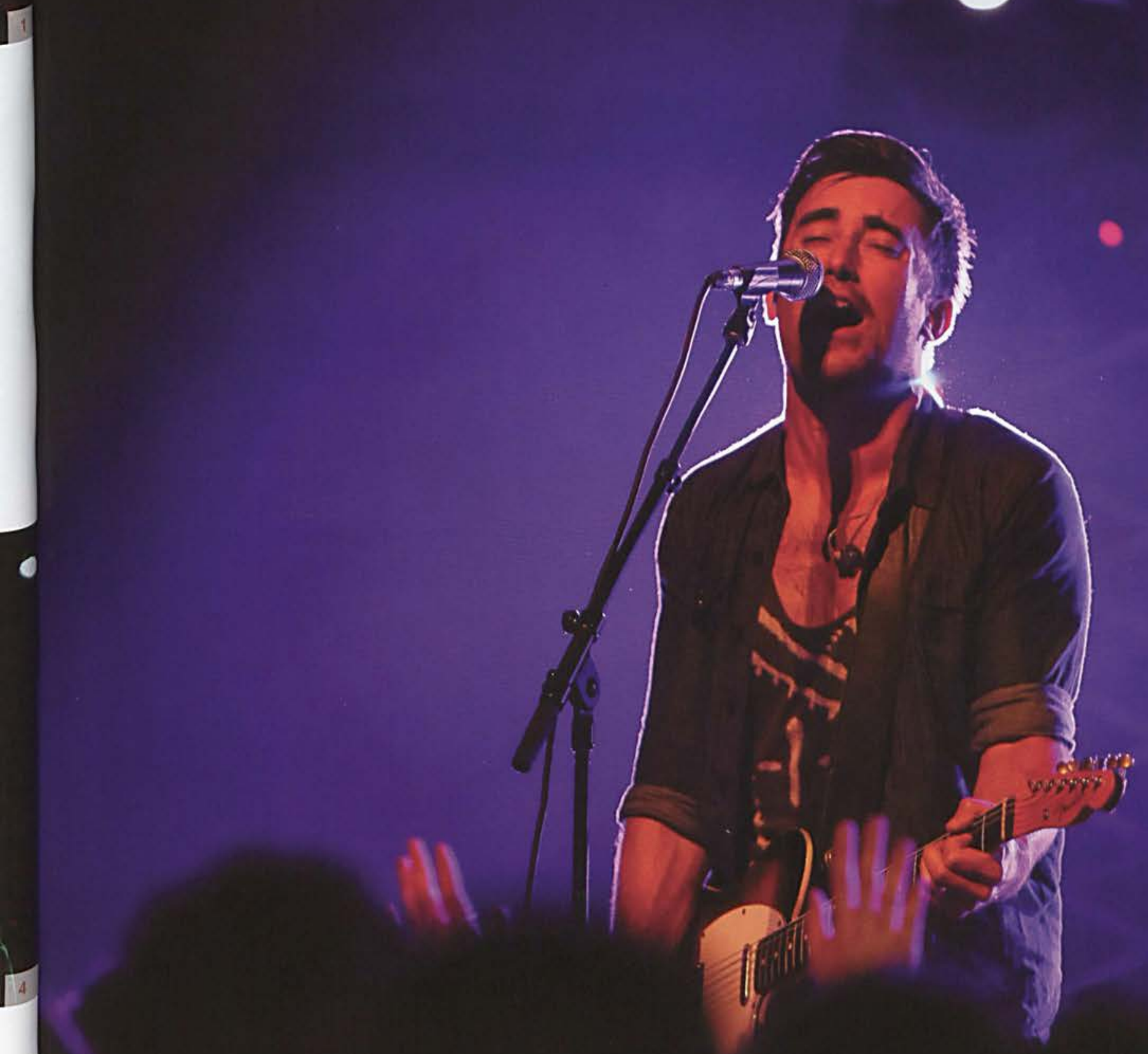


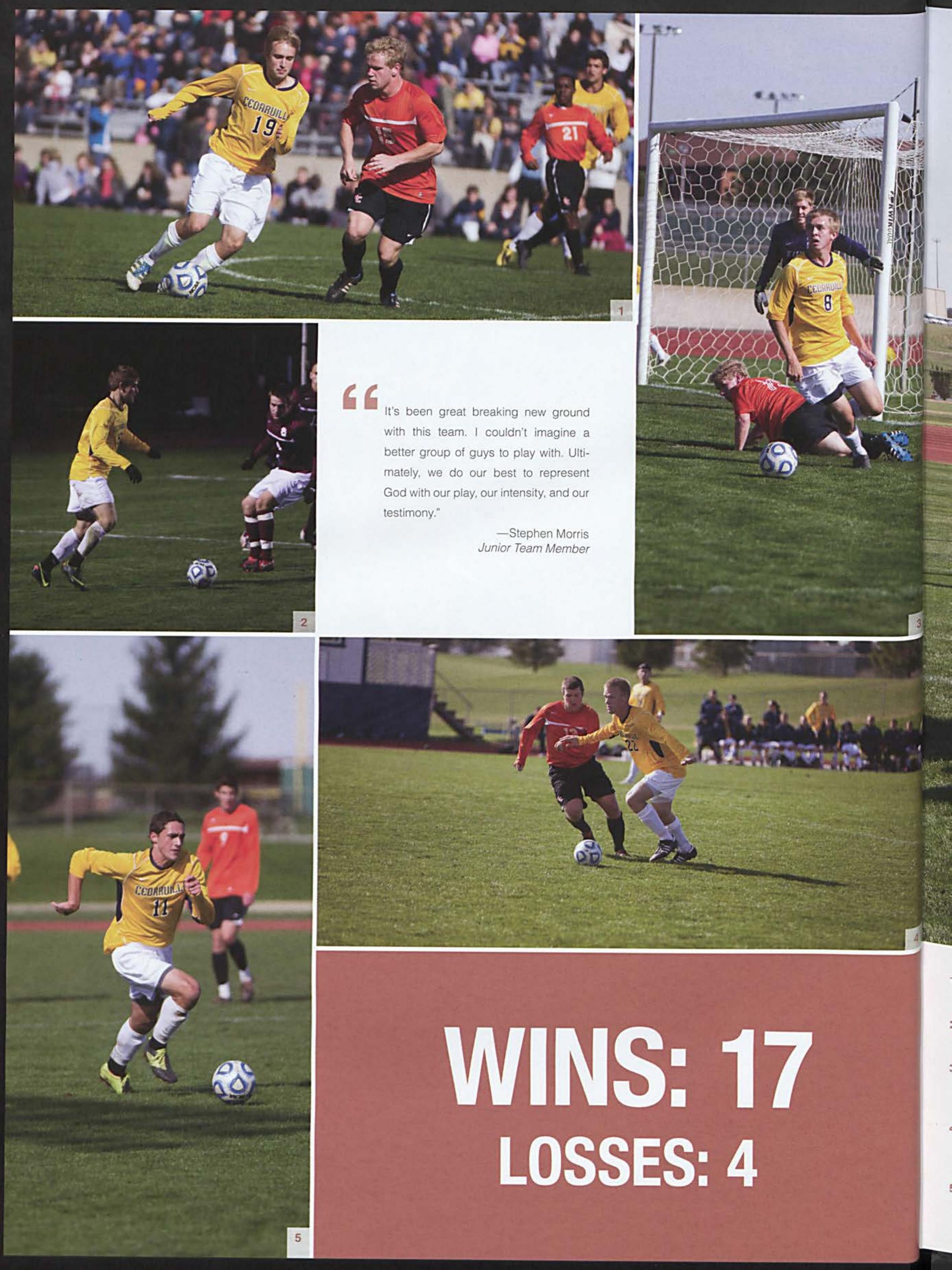




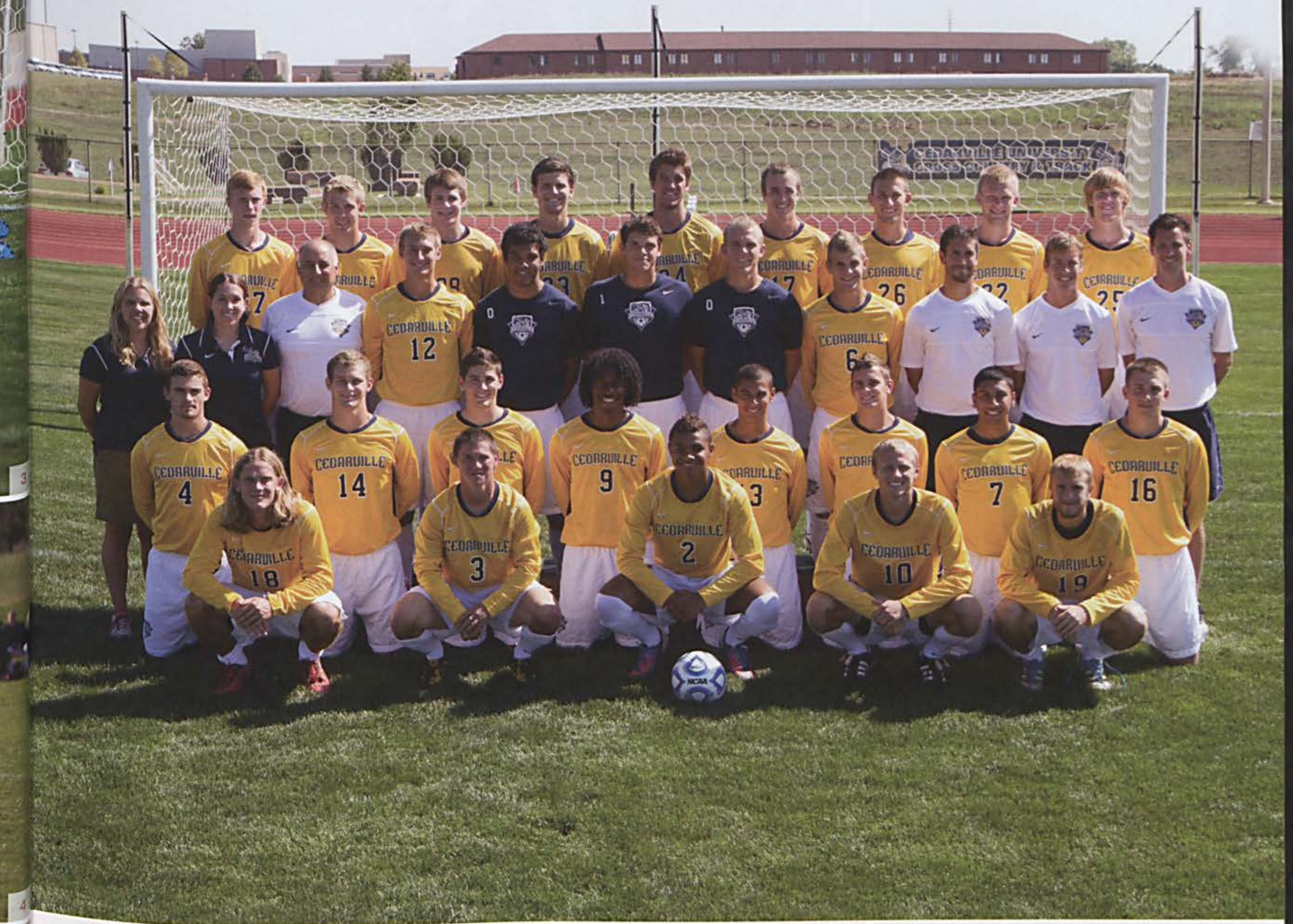

1. Daniel Rigby faces off against an opponent at the Homecoming game.

2. Forward Christian Alexander prepares to take on a player from Shepherd University,

3. Midfielder Jared Newman looks for an opportunity to score in a 5-0 victory against Georgetown College.

4. Midfielder Dillon King holds off a defender as he switches the field at Yellow Jacket Soccer Complex.

(Front row) Timmy Waller, Ryan Thurman, Zachary Harris, Jeremy Hoppe, Daniel Rigby. (Second row) Todd McKinley, Myles Greely, Connor Gilmour, Stephen Morris, Eric Hoober, Zack Gatlin, Joshua Hanif, Jon Earl. (Third row) Student Athletic Trainer Marissa Yorgey, Assistant Athletic Trainer Leyna Hebert, Assistant Coach Kevin Roper, Justin Santoro, Adam Marte, Ethan Dewhurst, Curtis Ranck, Christian Alexander, Assistant Coach Grant Knight, Assistant Coach Brett Faro, Head Coach Todd Beall. (Back row) Joe Davison, Jared Newman, Ethan Lee, Stephen Schindler, Eric Newman, Steve Ellis, Grant Kovac, Dillon King, Connor Scott. (Not pictured: Mitch Goodling and Ethan Shula)

5. Defender Ethan Shula uses his speed to carry the ball up the field. 


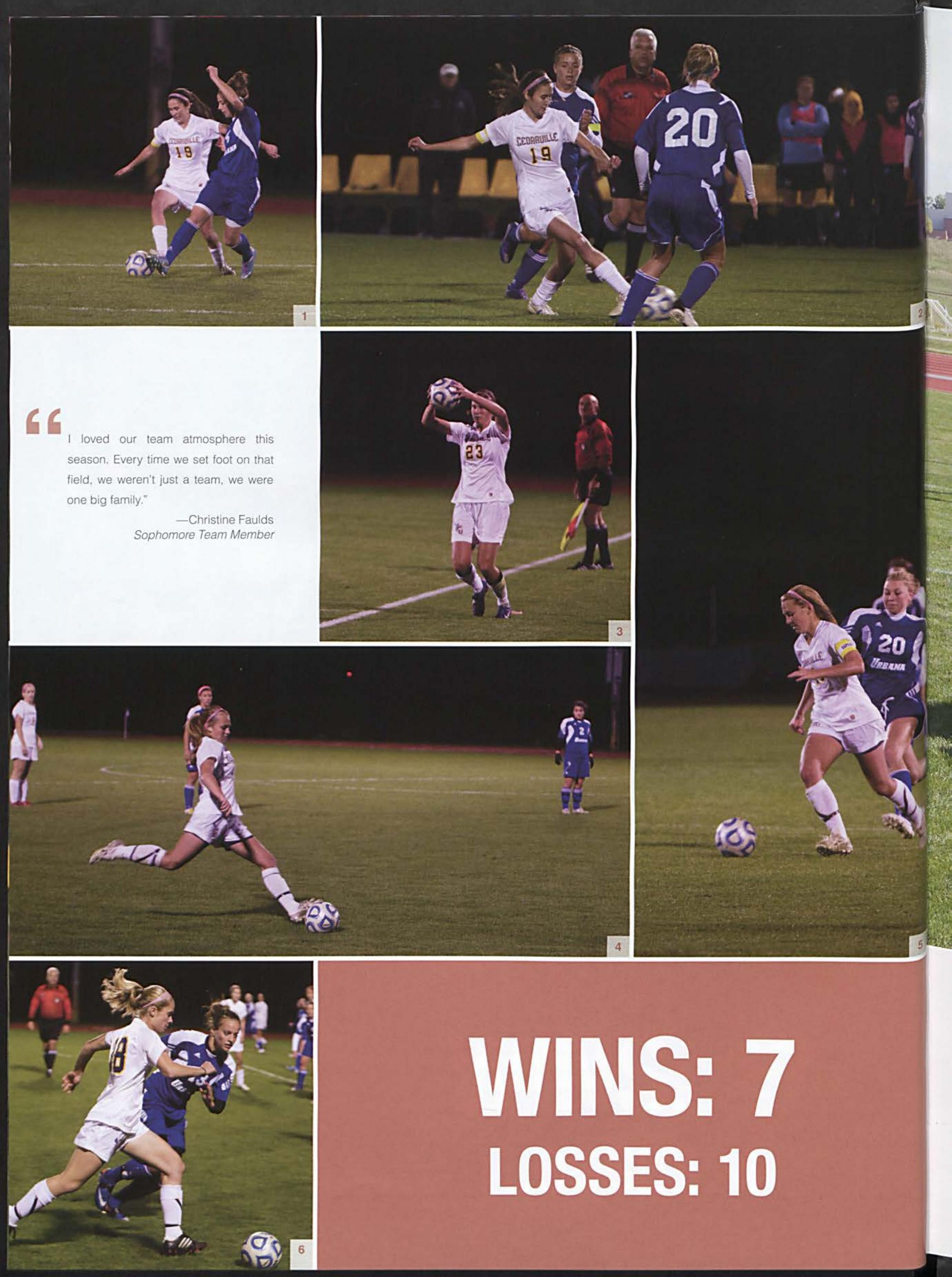



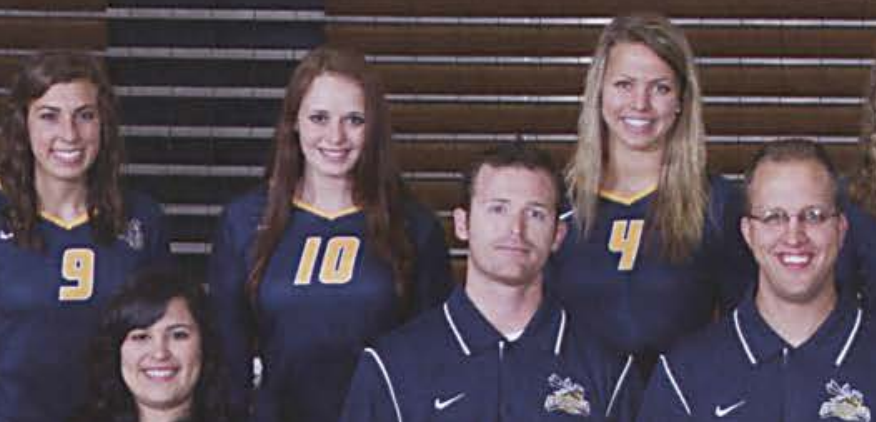

3. .

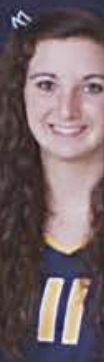

애랄.
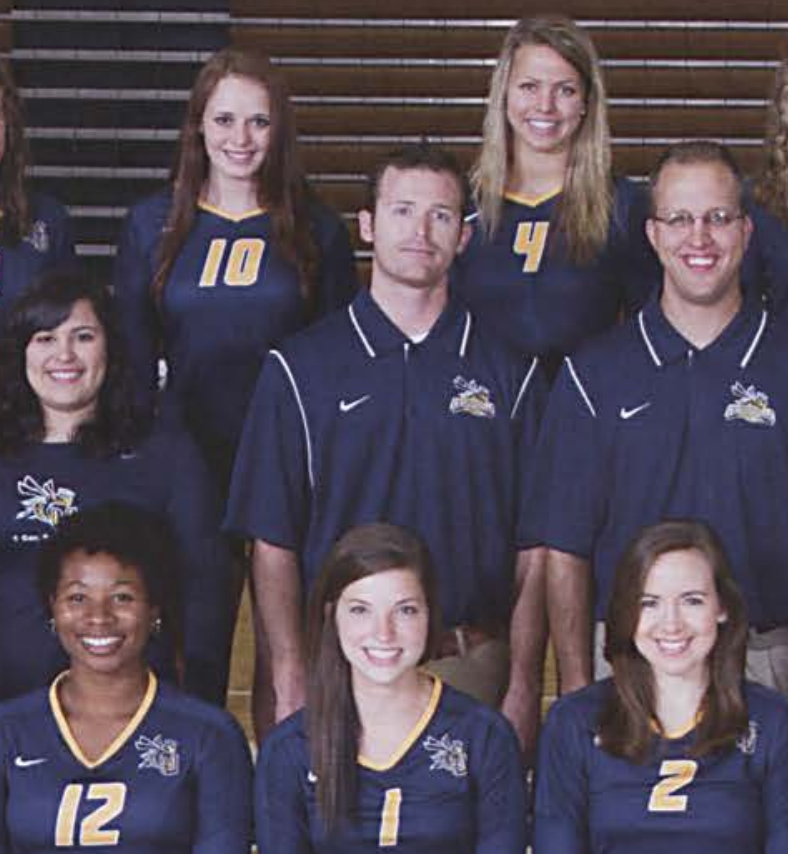

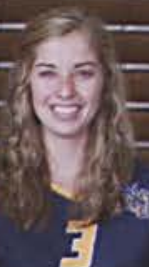

169

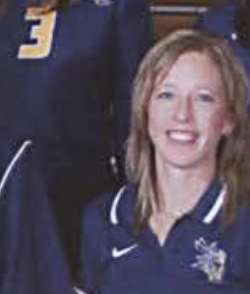

(3)

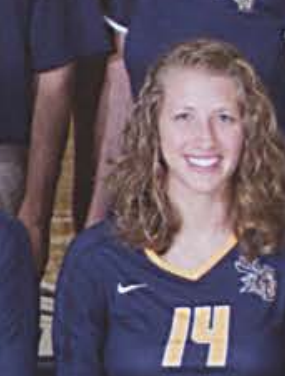

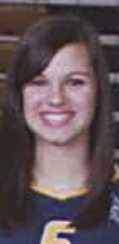

5
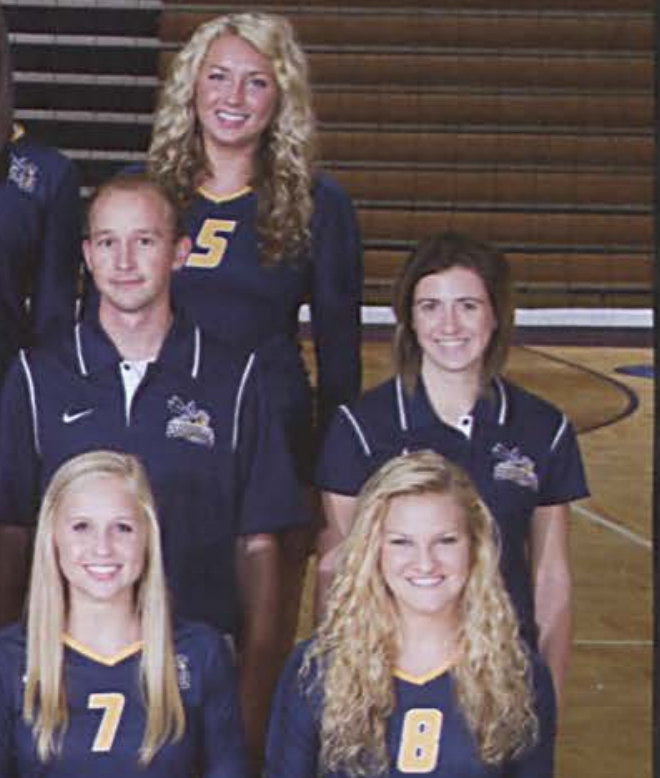

(4) $x$ 다.

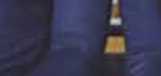<smiles>CCC1CCC(C)C1</smiles>

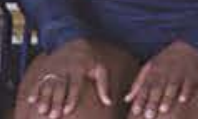
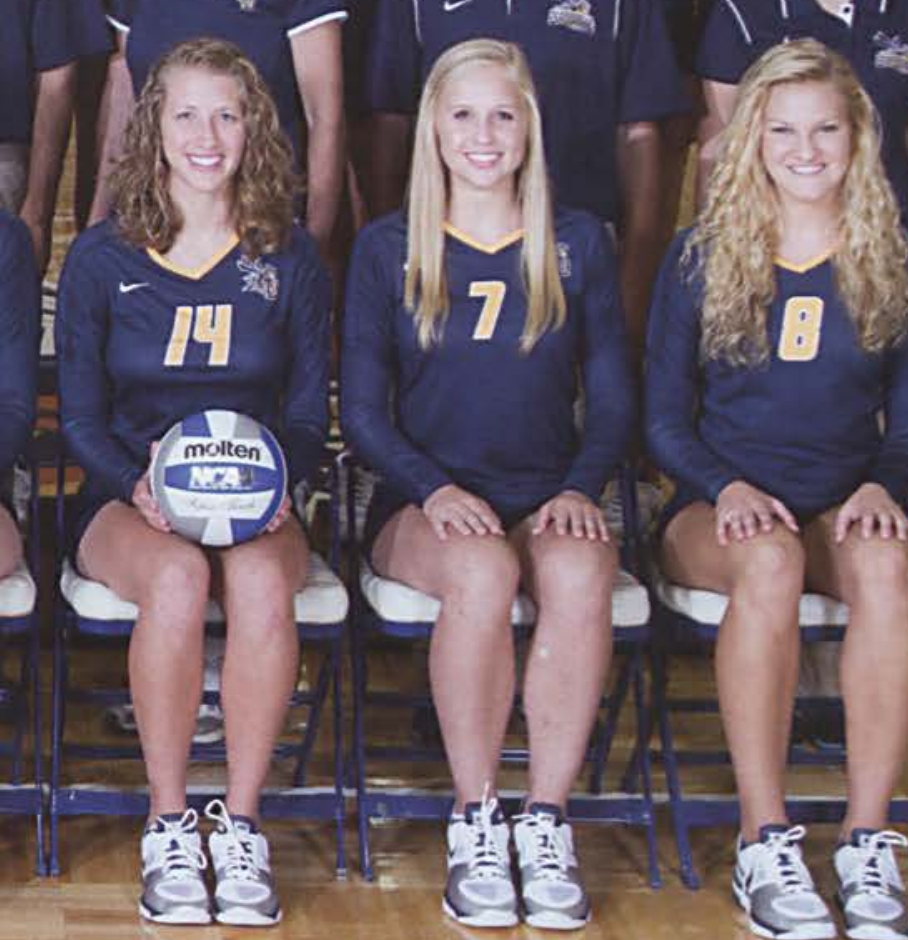

\section{(1)}




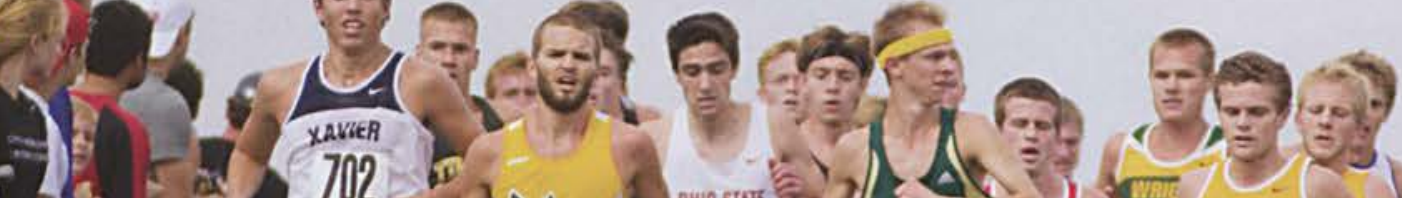

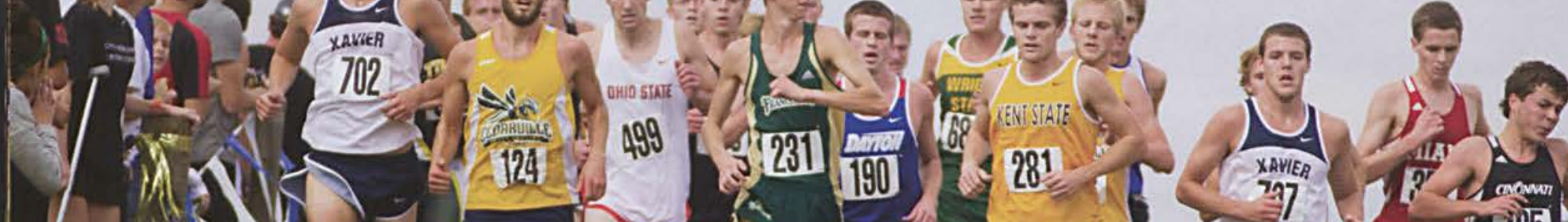

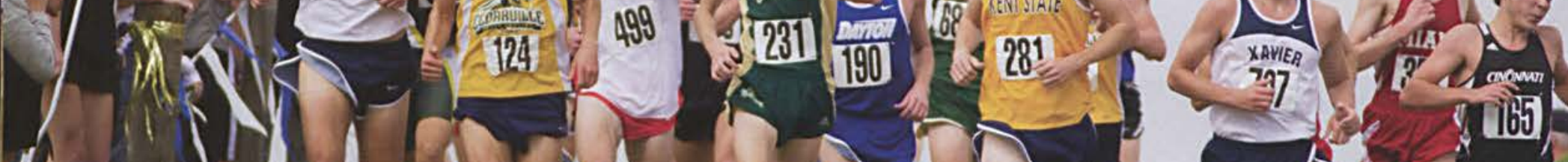

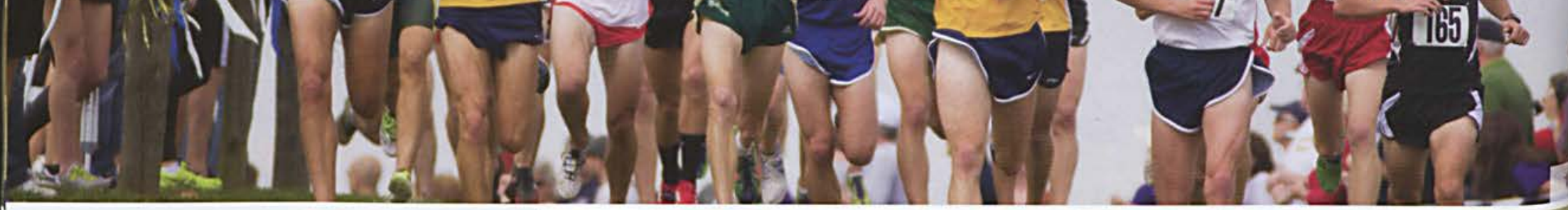

66

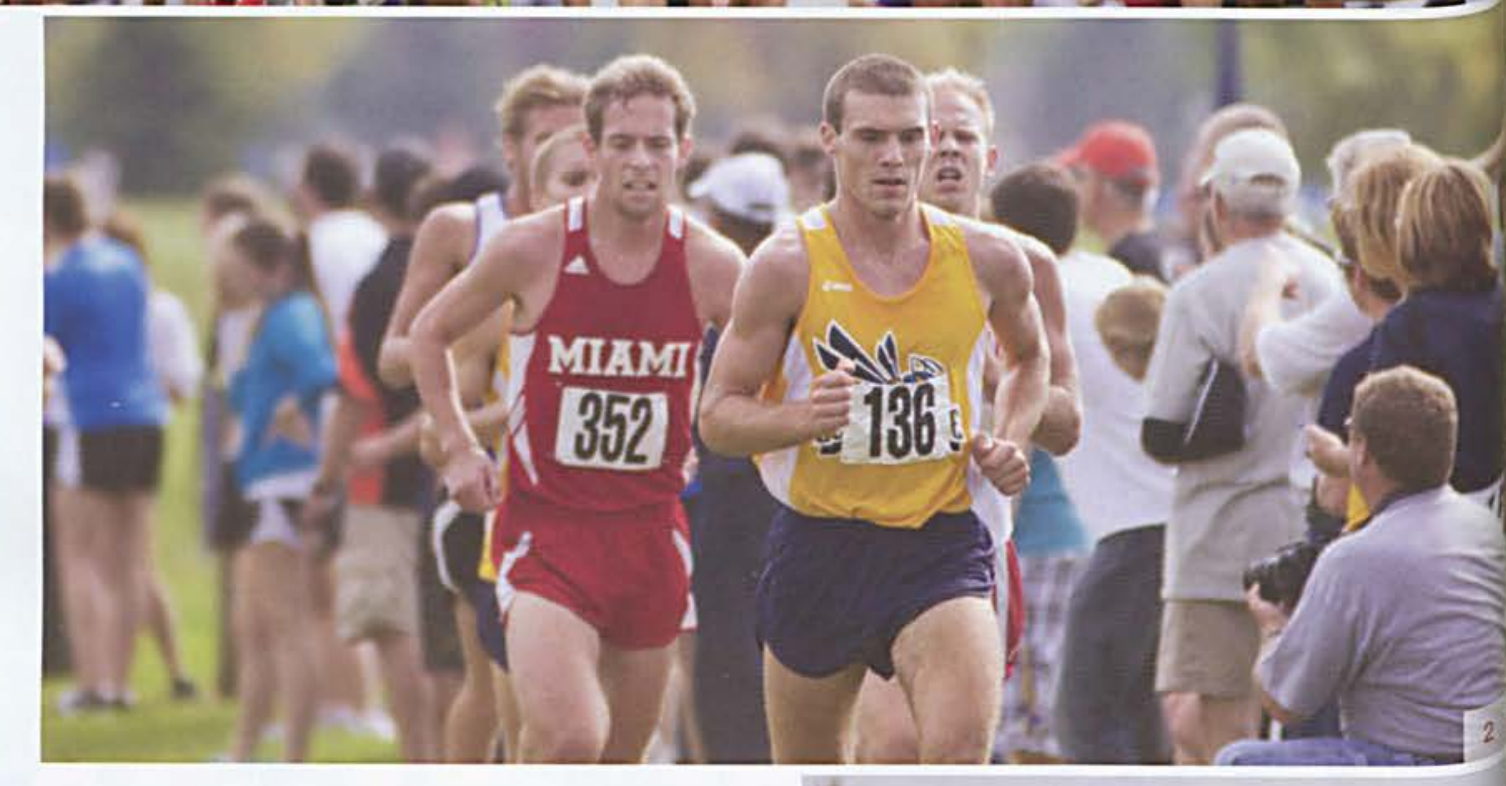

seven faster.

raderie are amazing, and it is so great

to work hard for a big goal with twenty

of your best friends. The team qualified

for nationals in Missouri this year, being

the first ever Cedarville team to make it

to nationals in the NCAA.

Senior Team Member

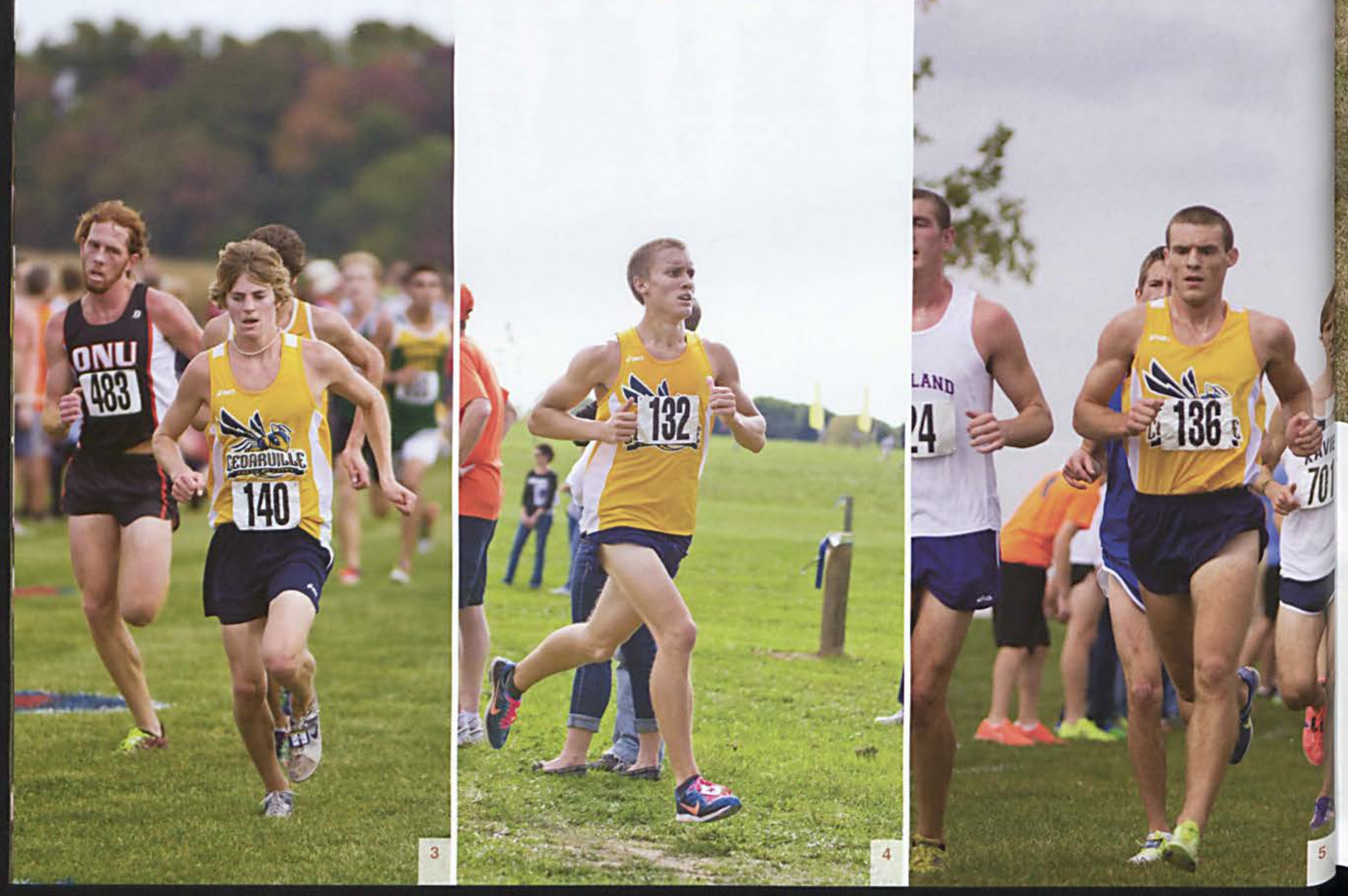




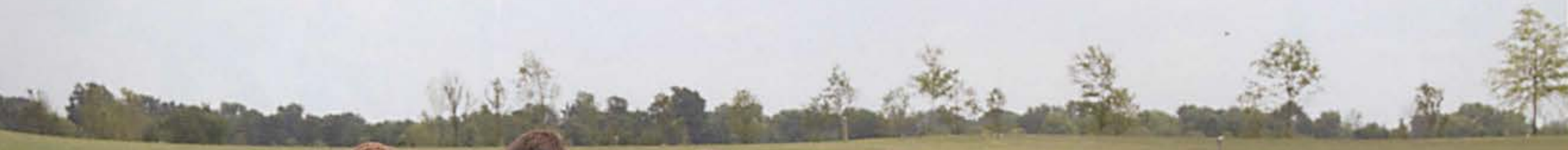

1. Amongst the pack of faces jostling for a position. Matt Brooker represents the Cedarville team.

2. Neil Klinger takes a lead and paces himself for the long distance between himself and the finish line.

3. Ben Tuttle kicks into a new gear as he tries not to let other runners overtake him.

4. Looking strong. Ryan Gustafson strides through the open field in the hopes of plac ing well for the Cedarville team.

5. With a determined face, Neil Klinger toughs it out through the long cross country haul.

\section{MEN'S CROSS COUNTRY}

(First row) Greg Johnson, Clay Watson, Jon Van Dyke, Joel Dennison, Frederick Waters. (Second row) Dylan McKevitt, Ben Tuttle, Matt Cheney, Erik Johnson, Philip Dannelly, Jacob Walter, Nathan Kilgore. (Third row) Team Chaplain Bud May, James Niemiec, Ryan Gustafson, Neil Klinger, Scott Gardner, Forrest Thayer, Head Coach Paul Orchard. (Back row) Nathaniel Burrell, Jacob Dubie, Joe Niemiec, Matt Brooker, Josiah Bragg, Sergei Brubaker. 


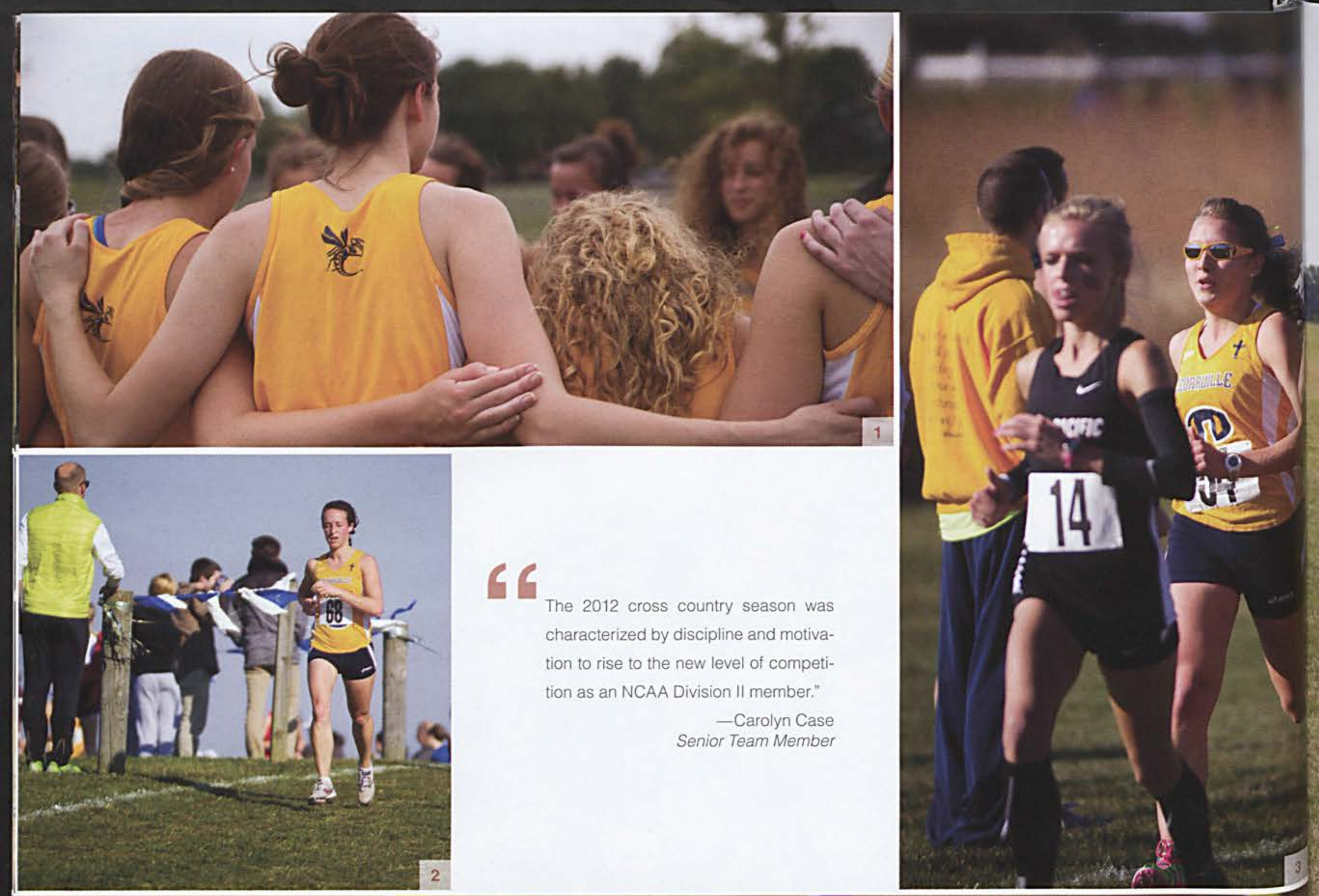

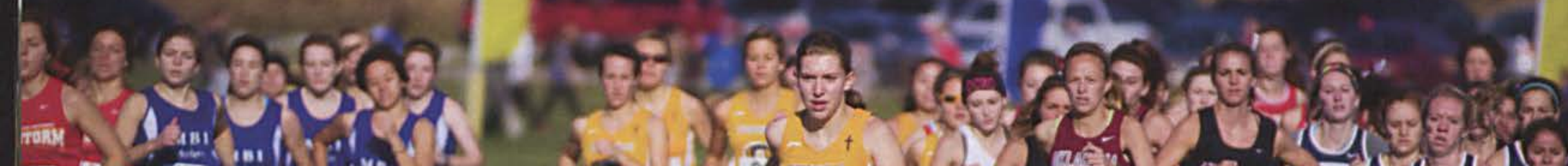
- H


12 ate 

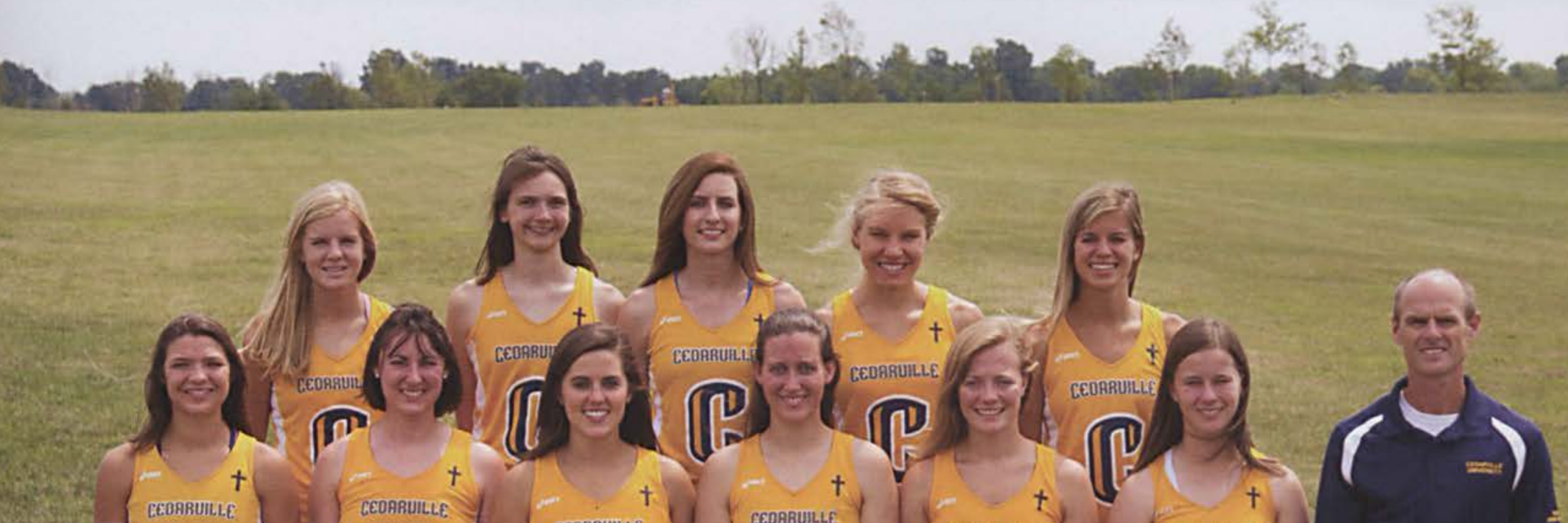

Fic $\rightarrow$ C $A$

(ึ)
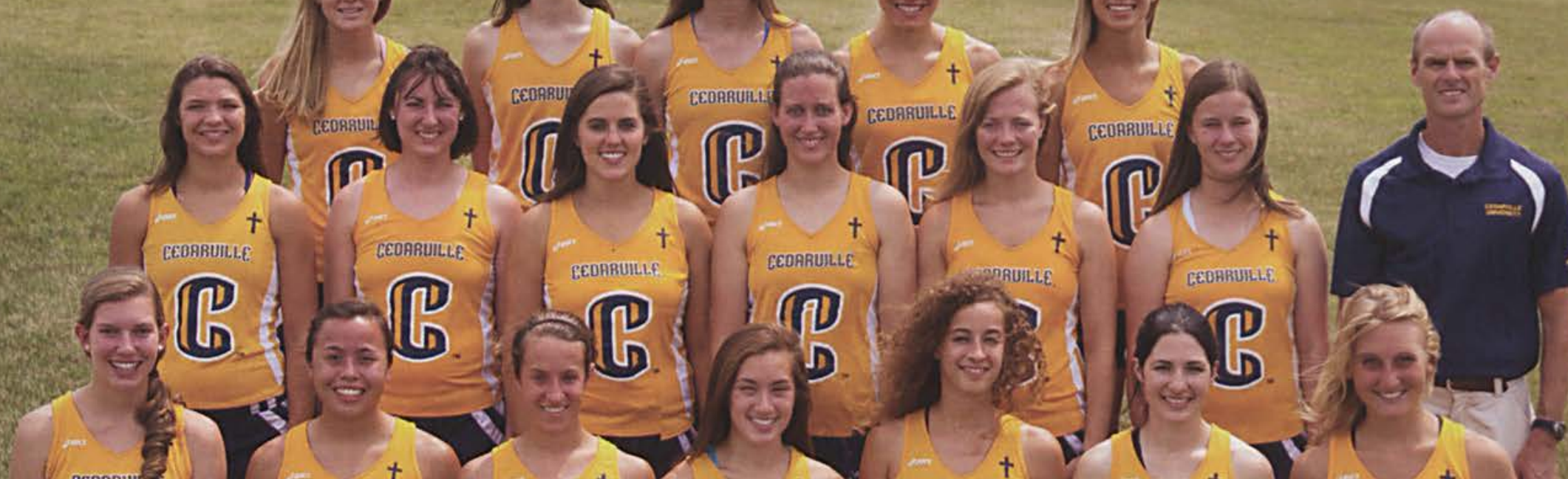

E. (x) Sill

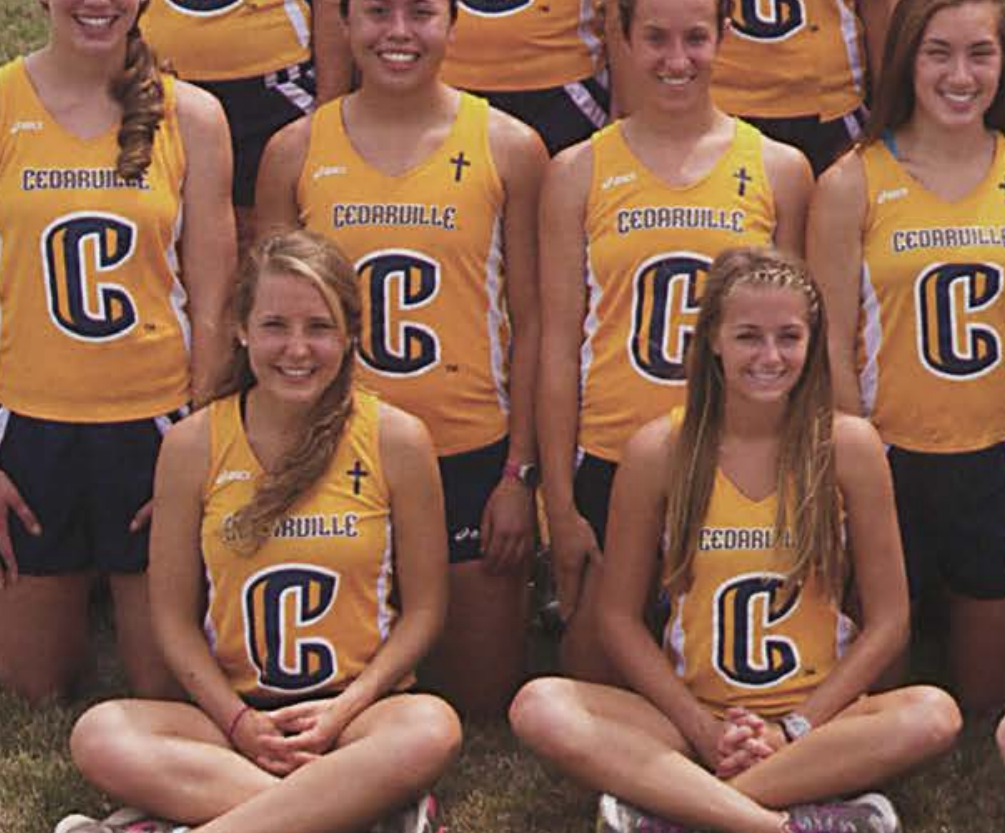

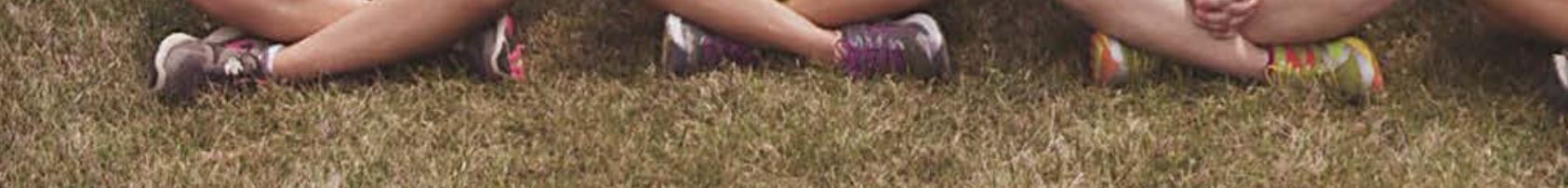

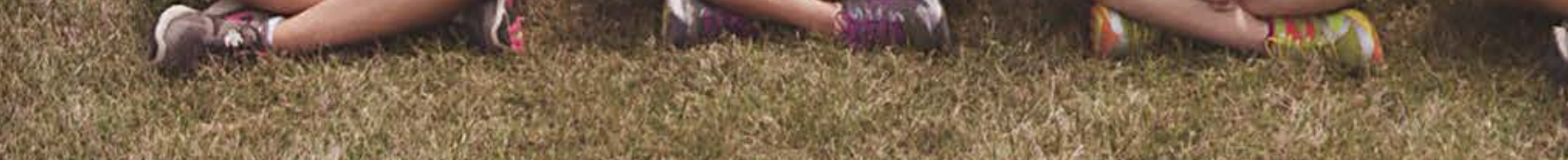

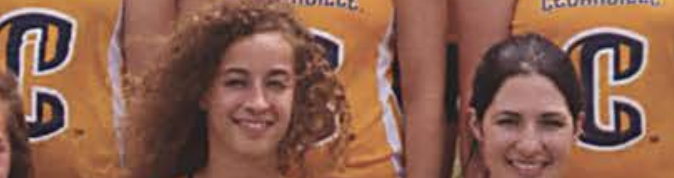
$\operatorname{lic}_{120}$ $+2(+)^{2}$
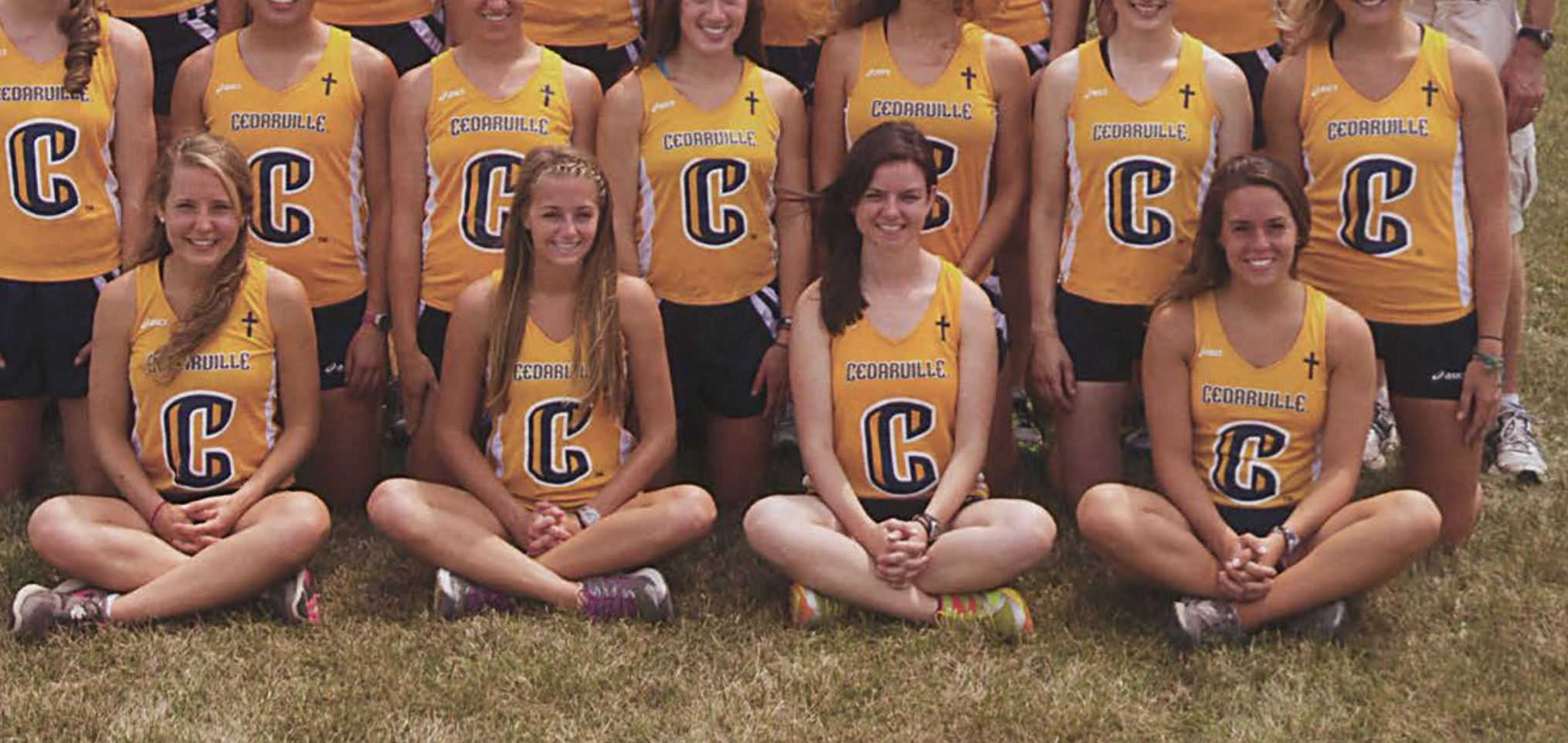

1. The team gathers together to pray before the meet begins.

2. Neola Putnam paces herself as she runs in the cool of the morning

3. Carolyn Case attempts to pass an opponent during the last leg of the race.

4. Alexandra Archambault pulls ahead at the start of the race, motivating her teammates to join her

5. As the finish line quickly approaches,

Tabitha DeHart quickens her steps.

6. Meghan Terrell steps it up at the end of the race against tough opponents.

\section{WOMEN'S CROSS COUNTRY}

(Front row) Carolyn Case, Tabby Moore, Ali Logan, Rachel McKinley. (Second row) Alexandra Archambault, Abby Wong, Neola Putnam, Kathryn Sill, Louise Van Matre, Hannah Lamos, Jasmin Banachowski. (Third row) Gina Mattes, Grace Campbell, Joanna Wheatley, Elisa Cherry, Krista Johnson, Margo Watson, Head Coach Jeff Bolender. (Back row) Melanie Redfield, Kristen Laaman, Lauren Callahan, Tabitha DeHart, Meghan Terrell. 

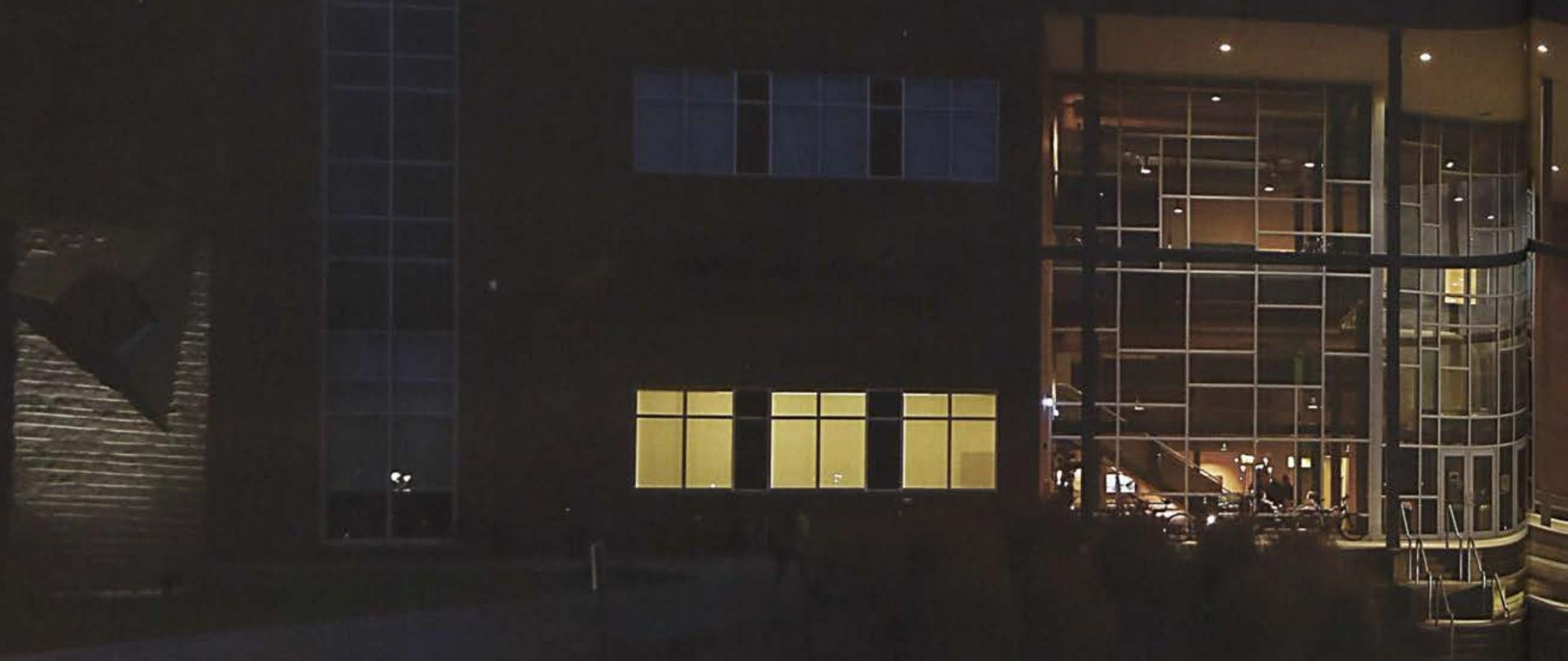

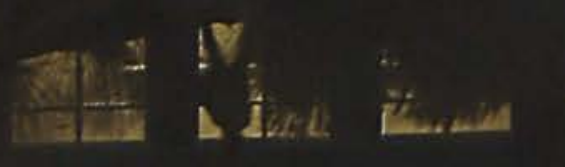

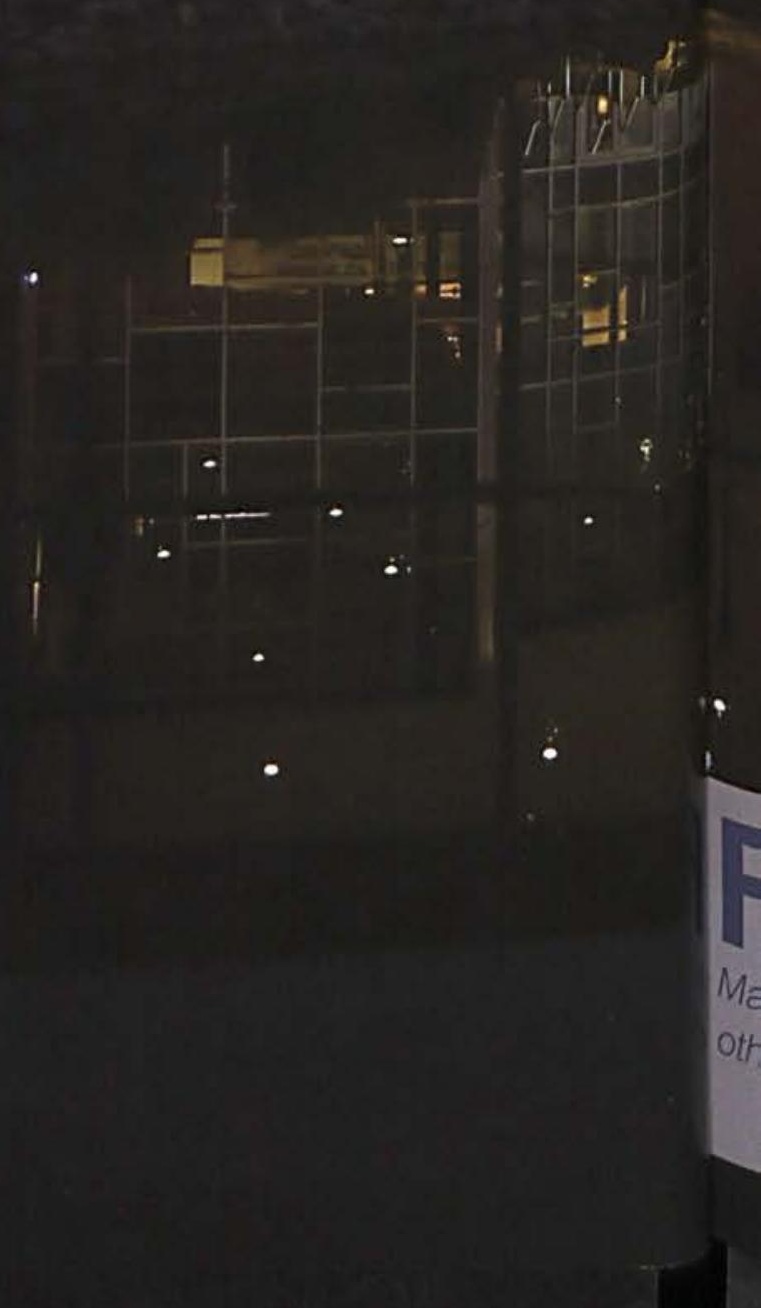




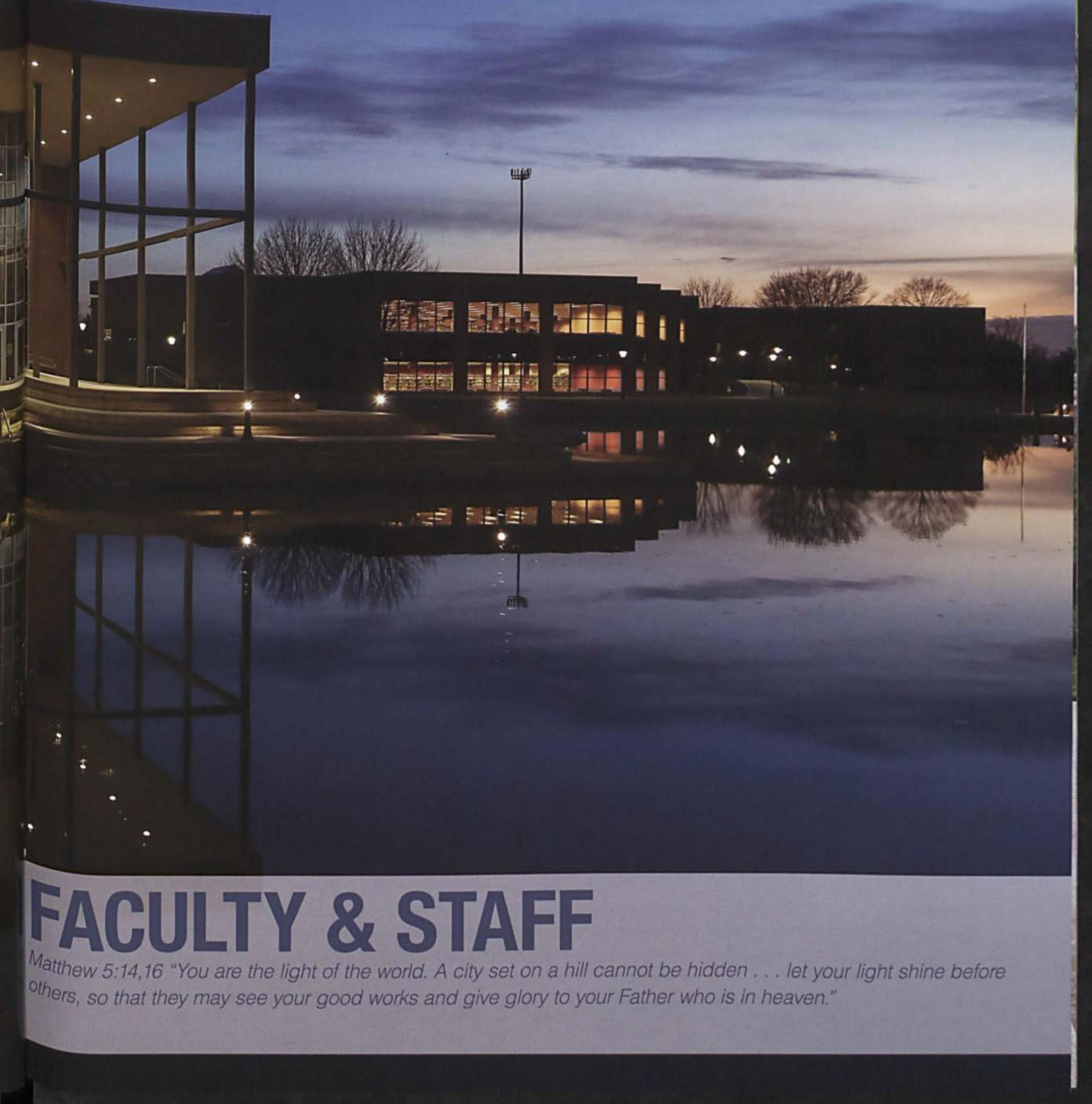




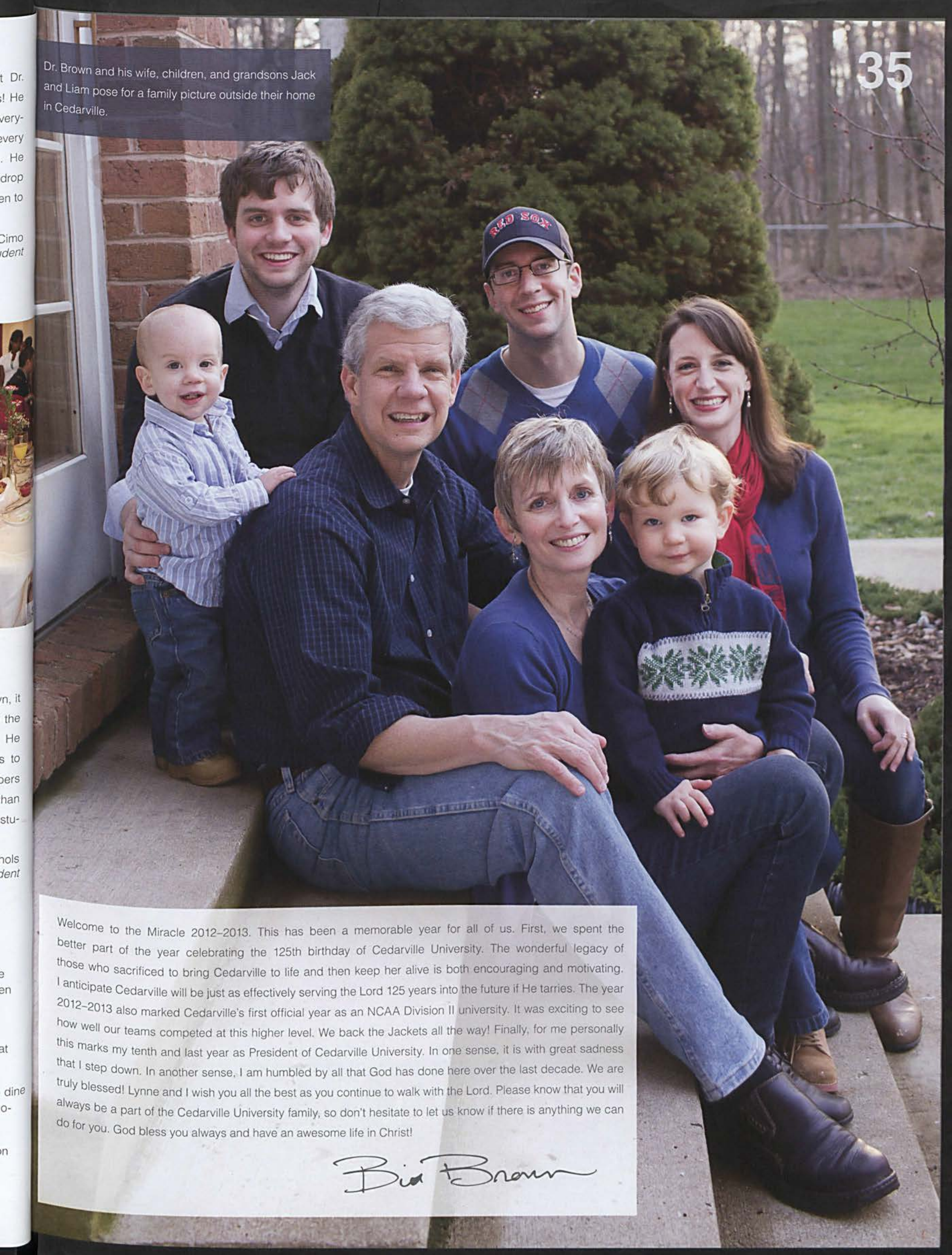




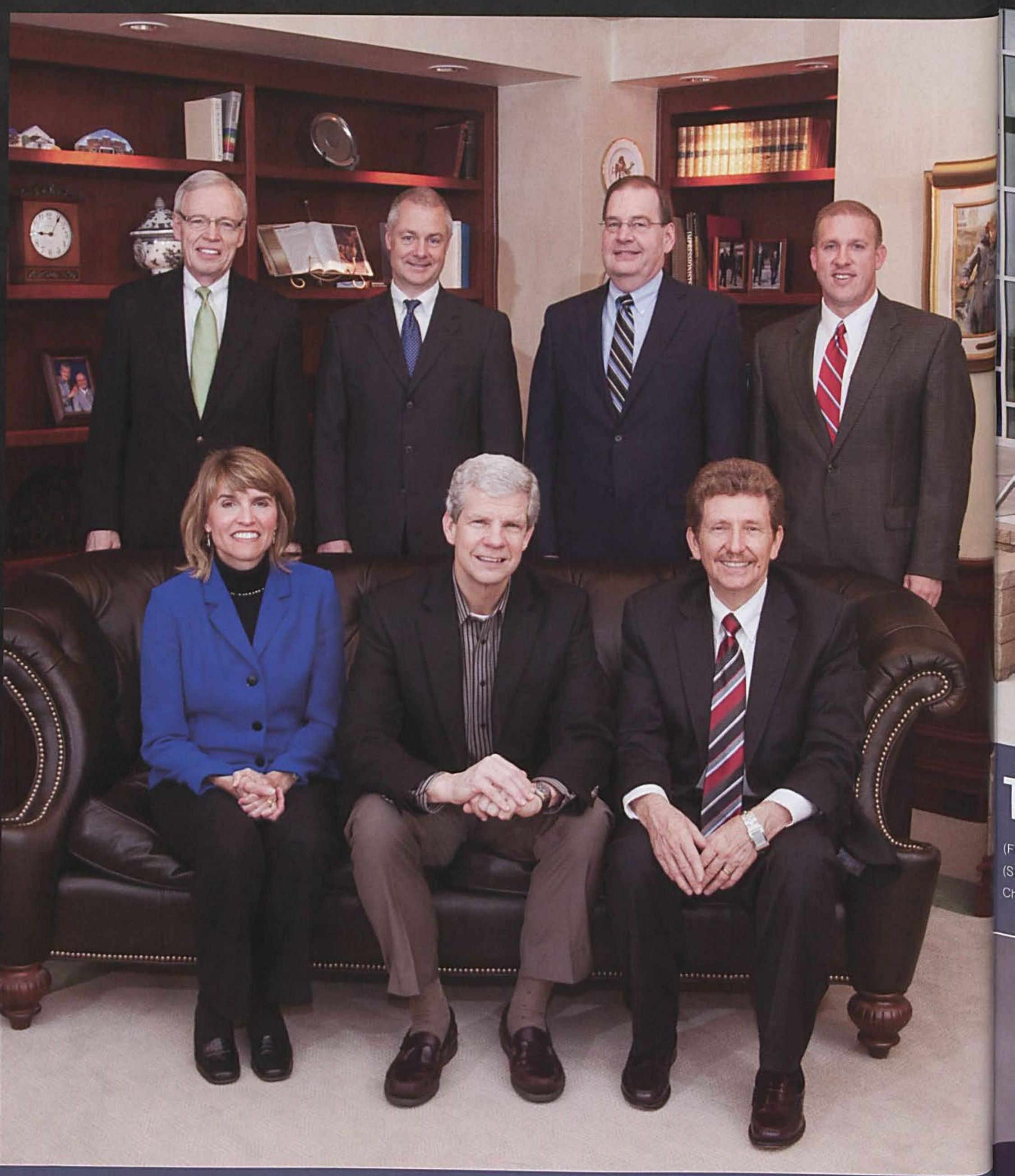

\section{ADMINISTRATIVE COUNCIL}

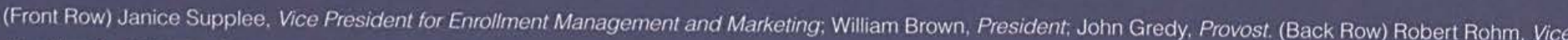

President for Christian Ministries; Carl Ruby, Vice President of Student Life; Thomas Cornman, Academic Vice President: William Bigham, Vice President for Advancemer 


\section{ART, DESIGN, AND THEATRE}

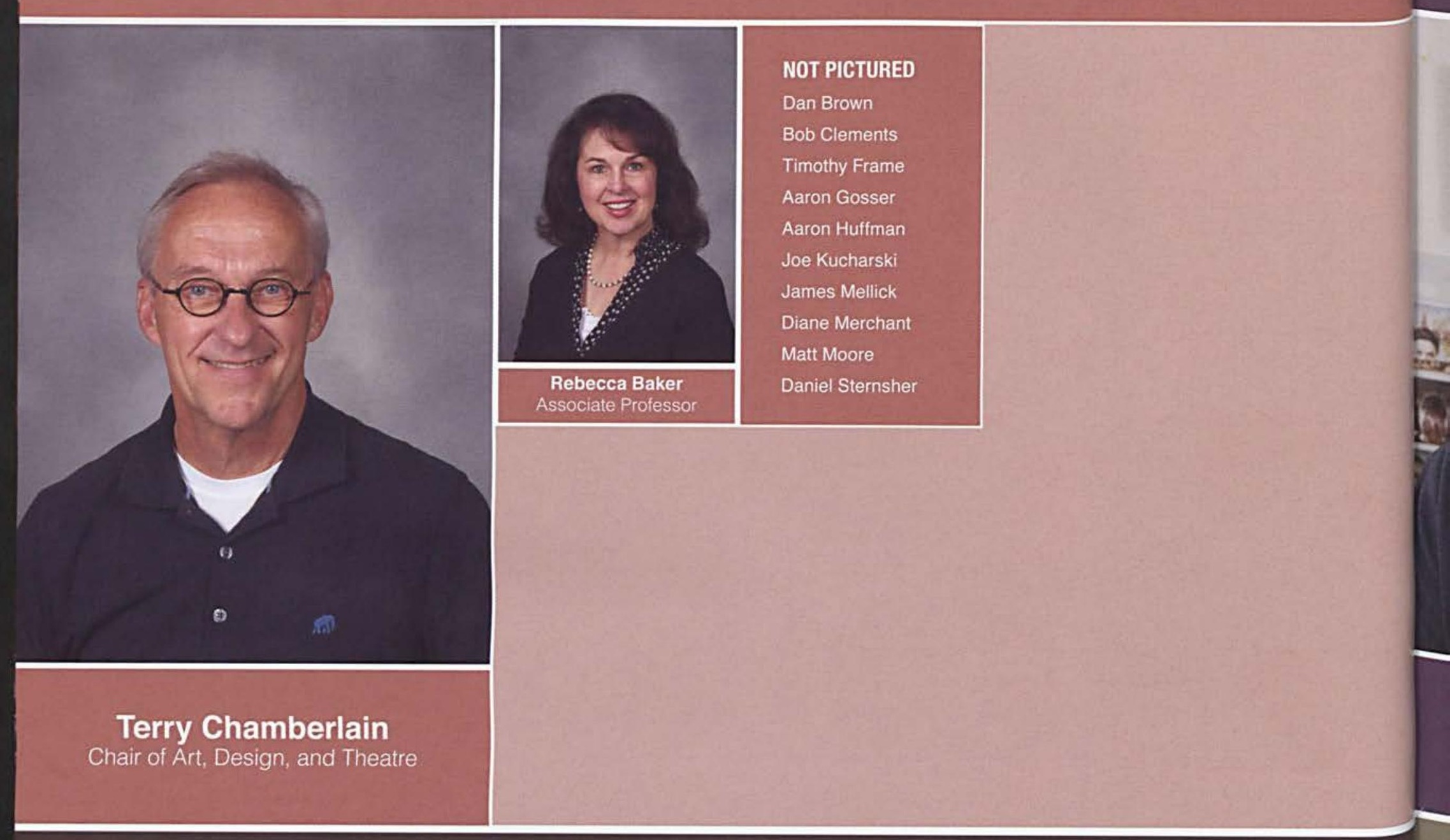

\section{BIBLICAL AND MINISTRY STUDIES}

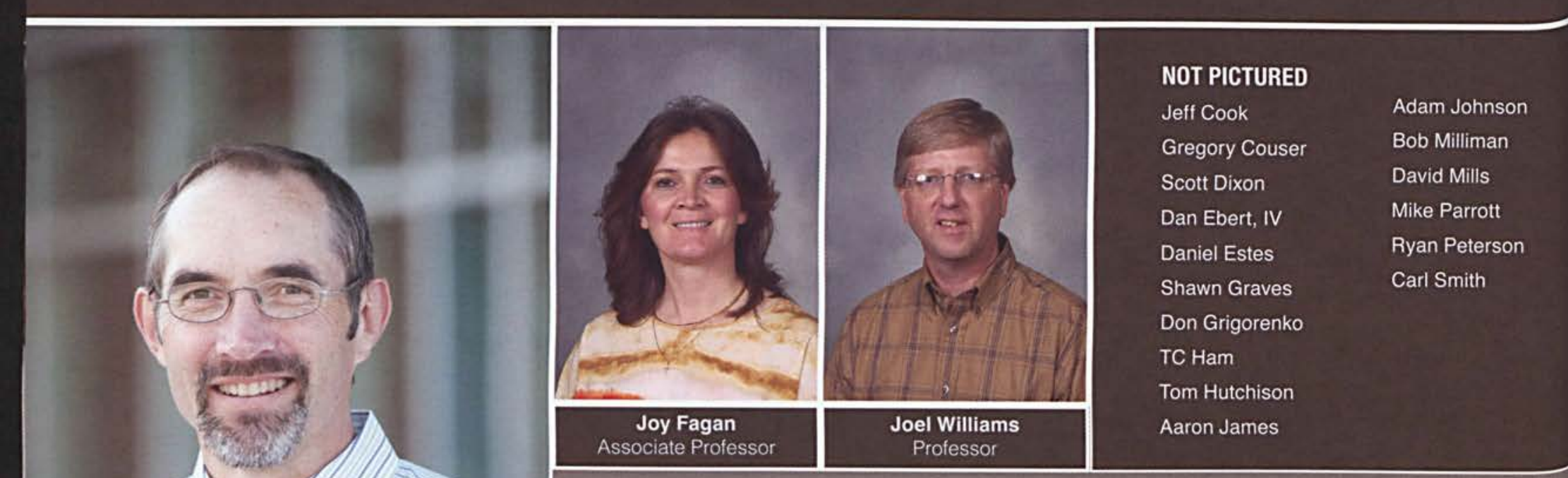

Chris Miller

Chair of Biblical and Ministry Studies 


\section{SCIENCE AND MATHEMATICS}

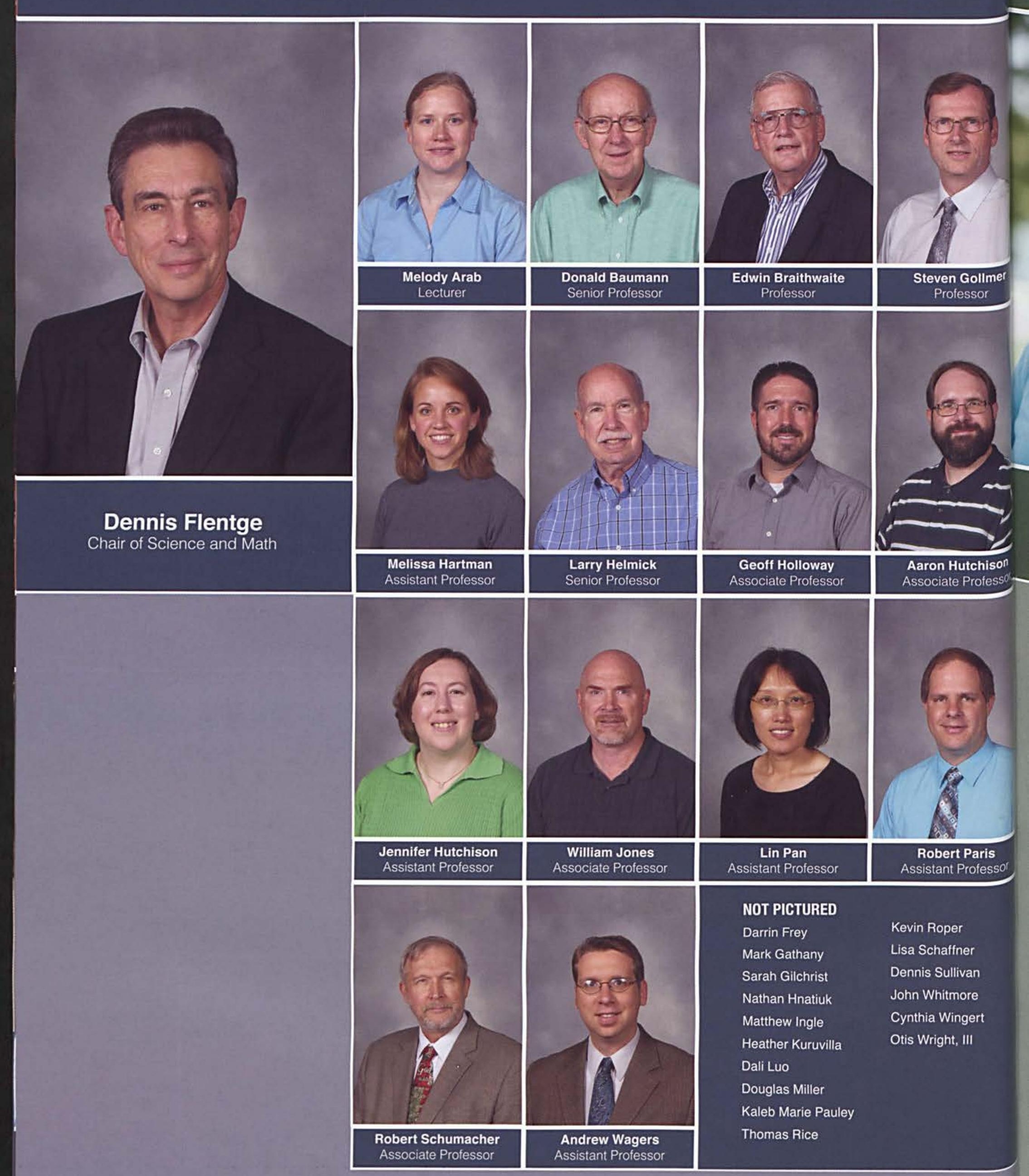




\section{KINESIOLOGY AND ALLIED HEALTH}

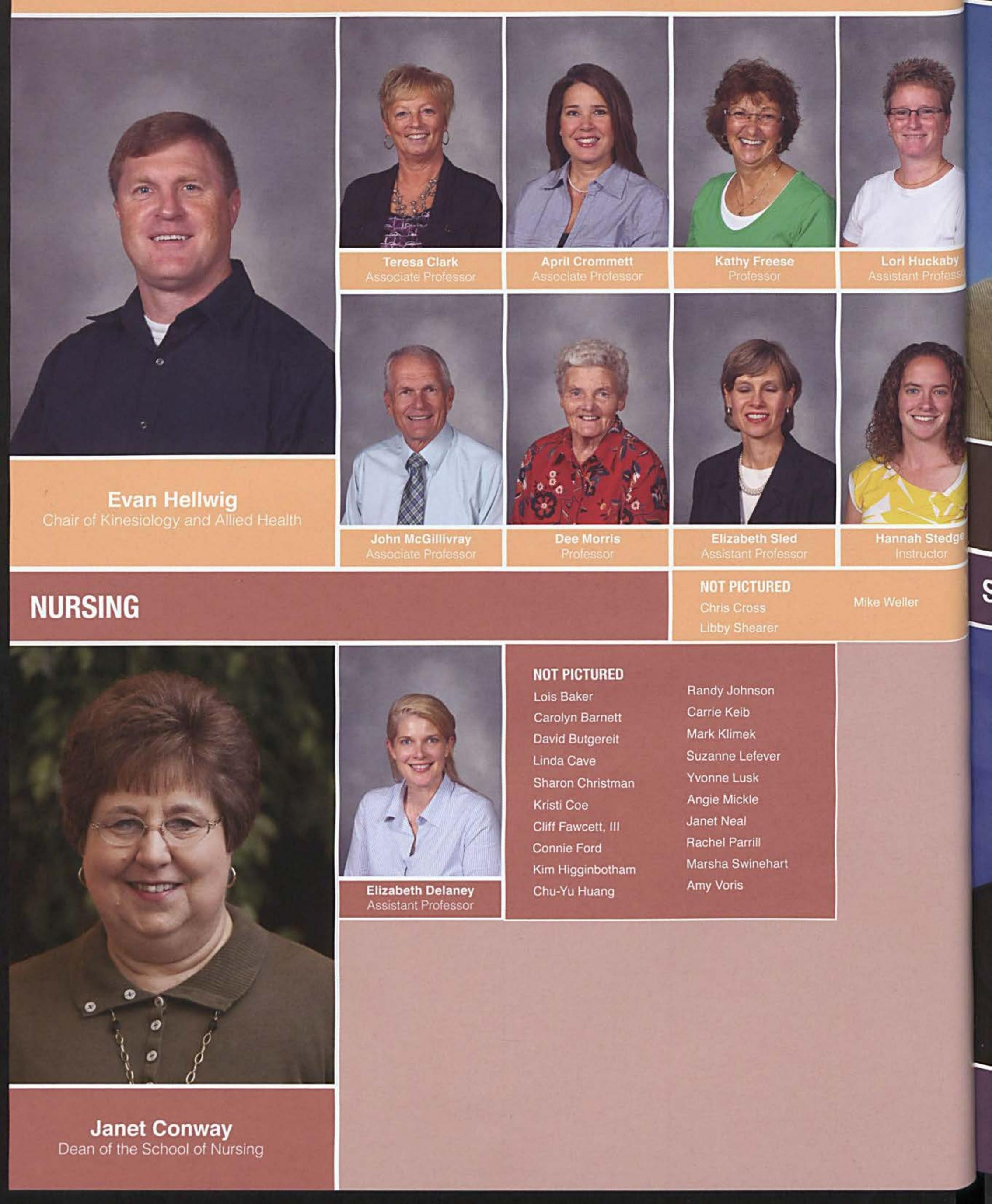




\section{PSYCHOLOGY}

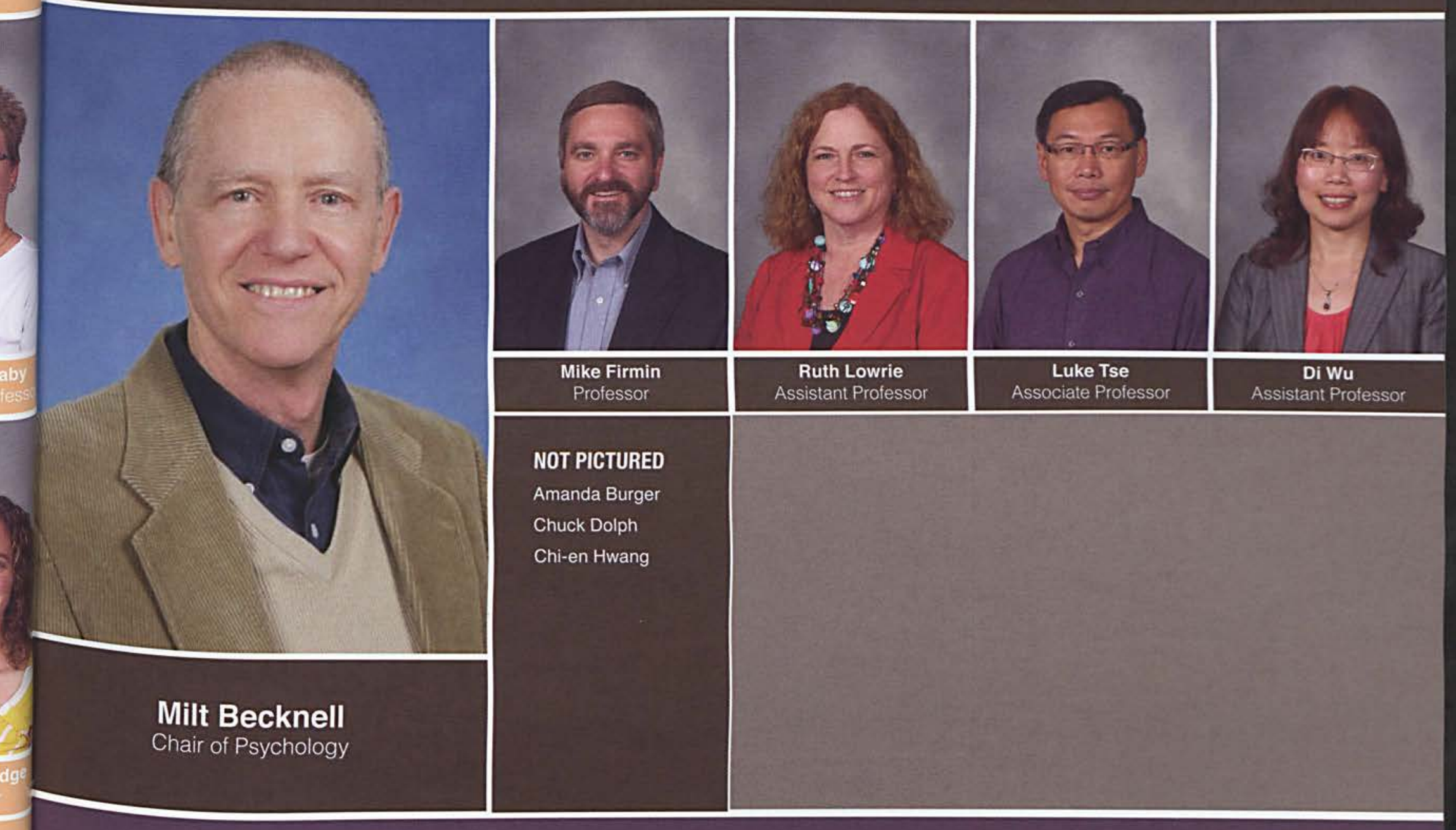

\section{SOCIAL WORK}

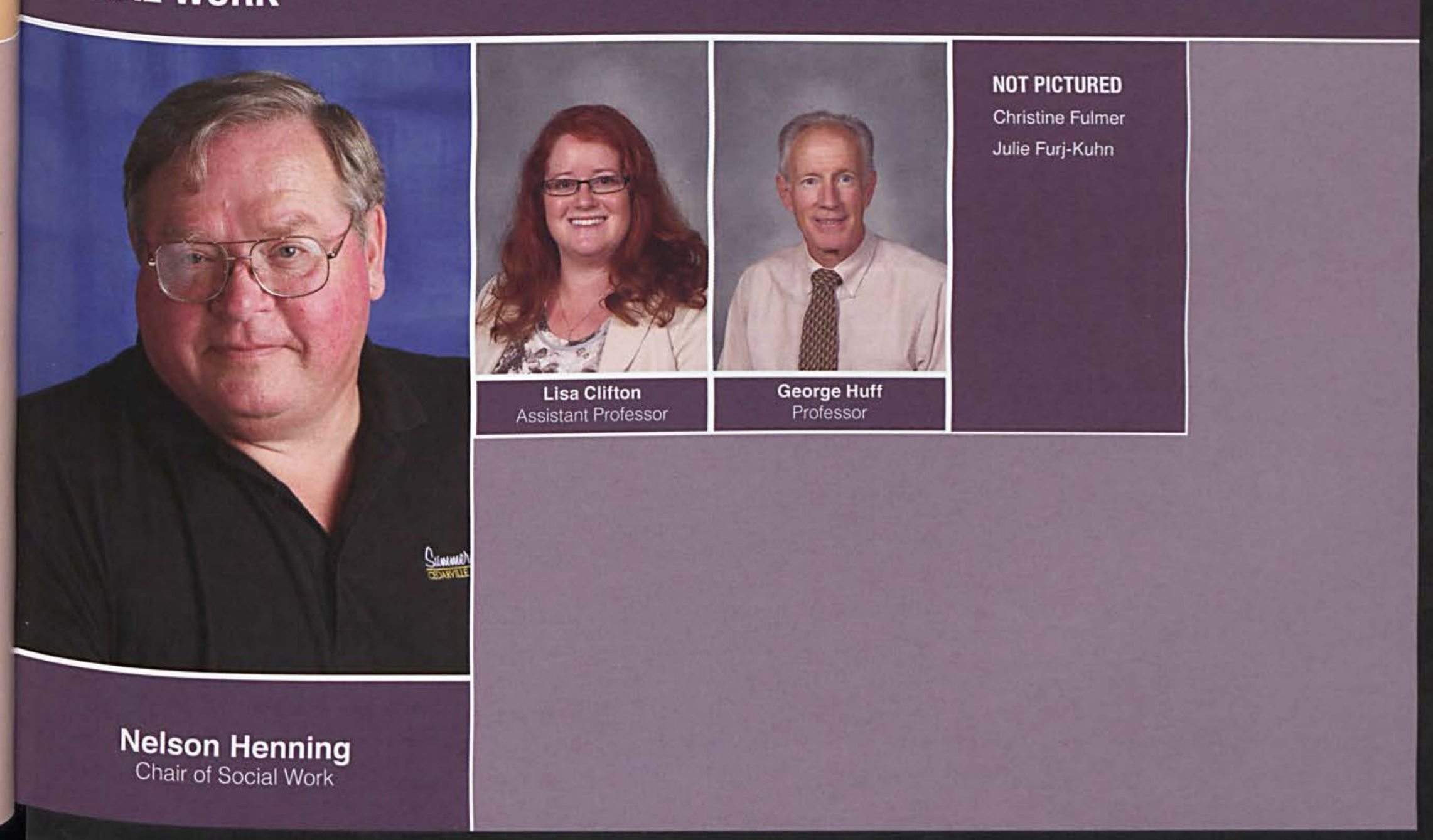




\section{SCHOOL OF BUSINESS ADMINISTRATION}

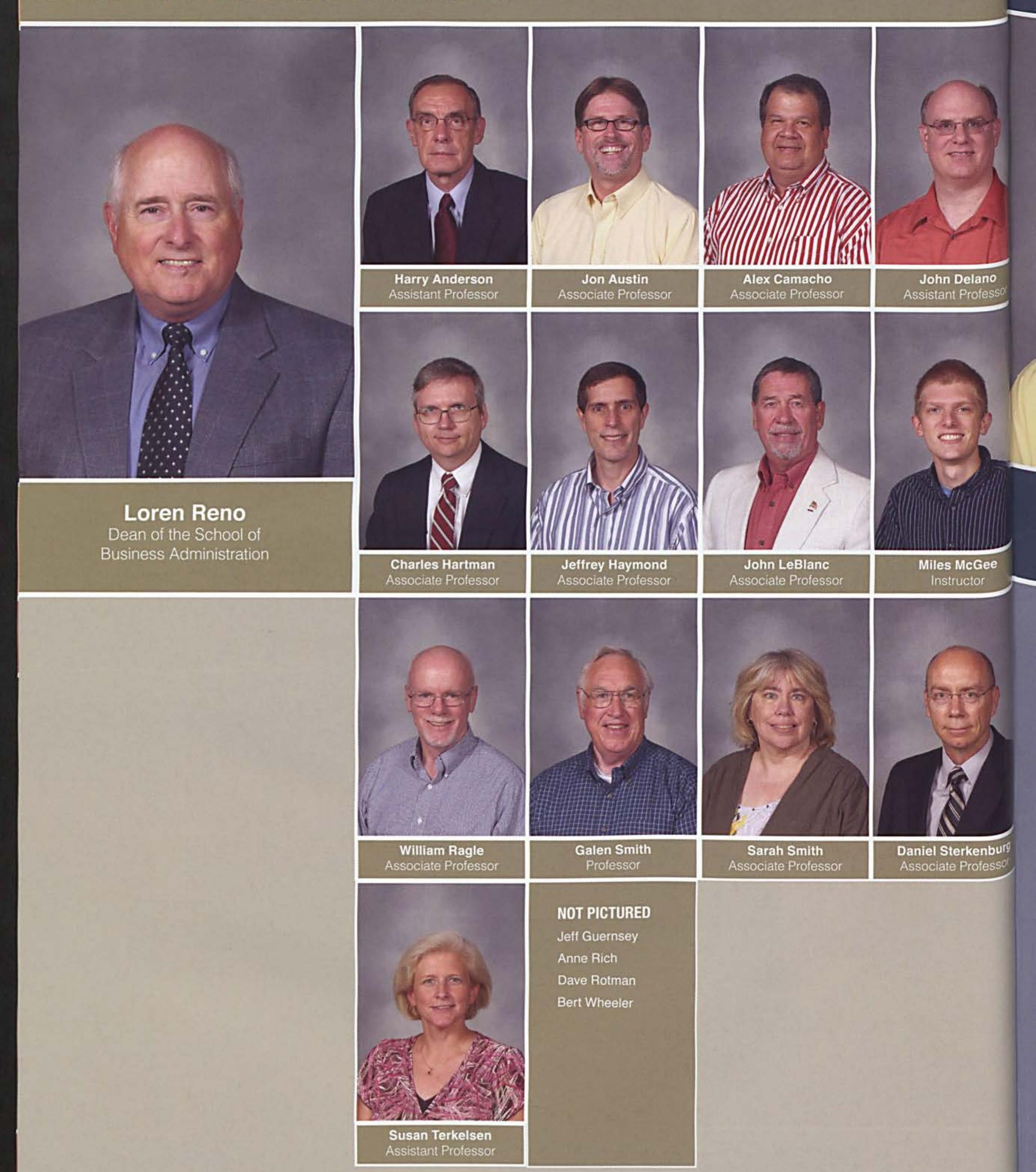




\section{ENGINEERING AND COMPUTER SCIENGE}

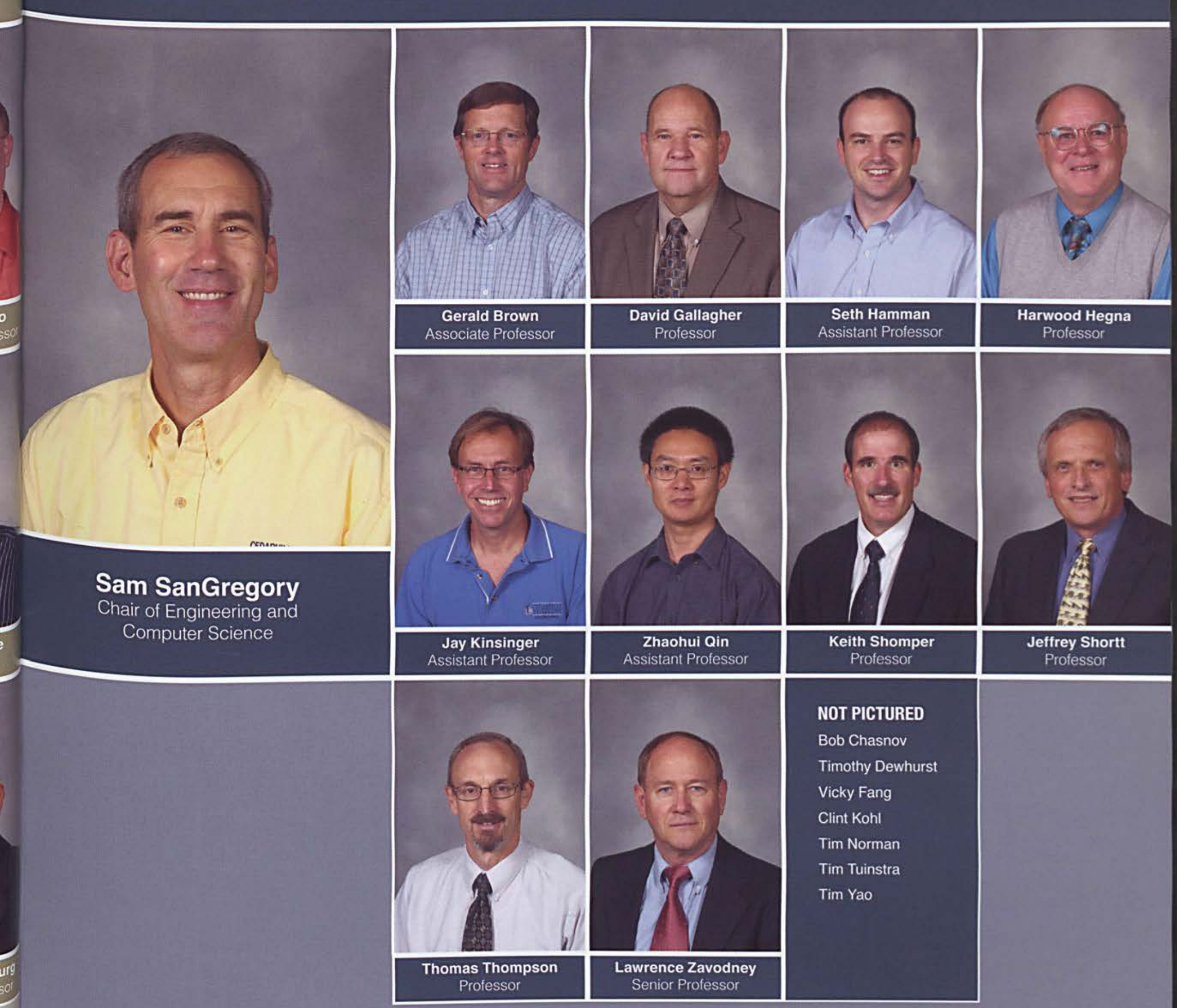




\section{FACULTY}

\section{EDUGATION}

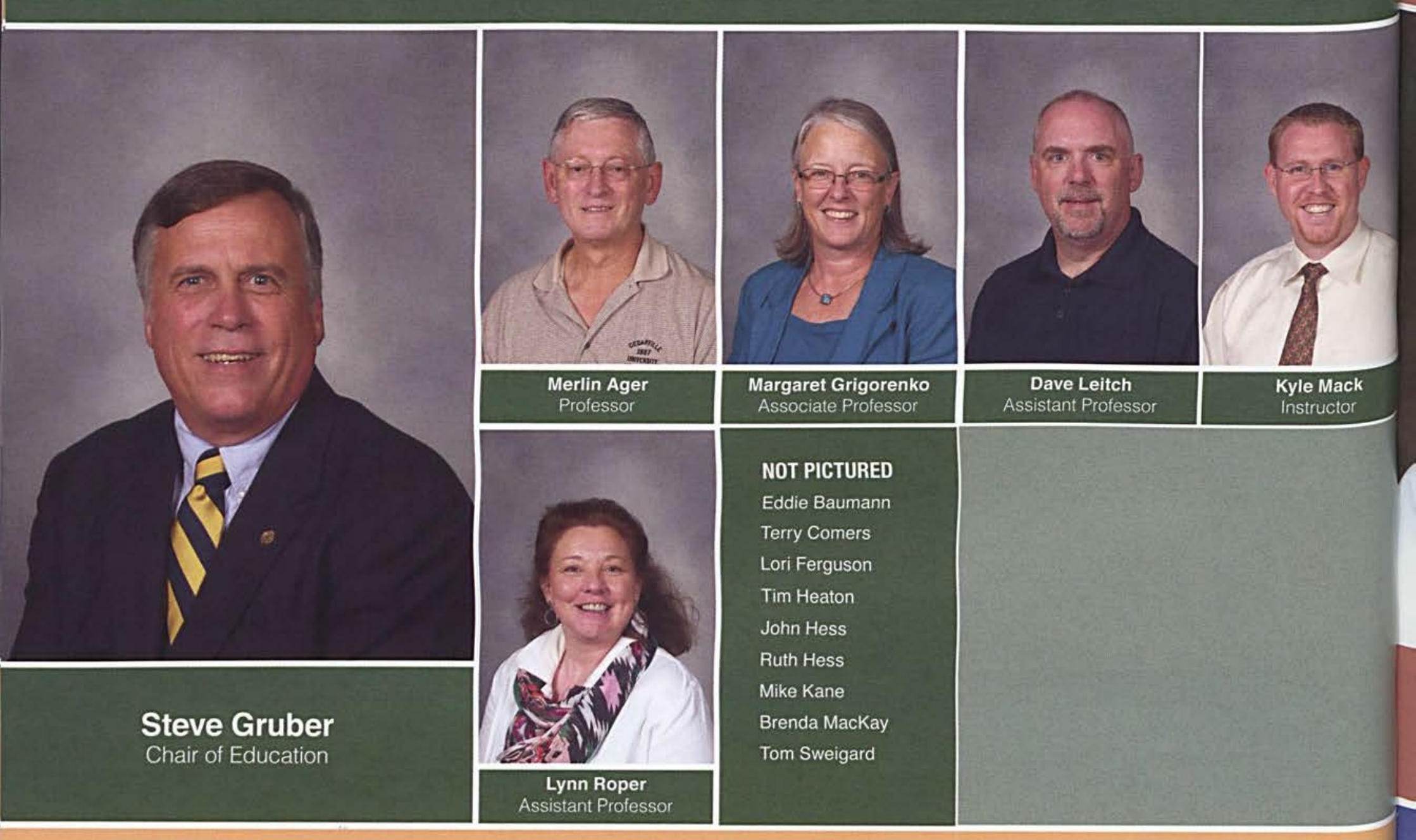

\section{MEDIA AND APPLIED GOMMUNICATIONS}

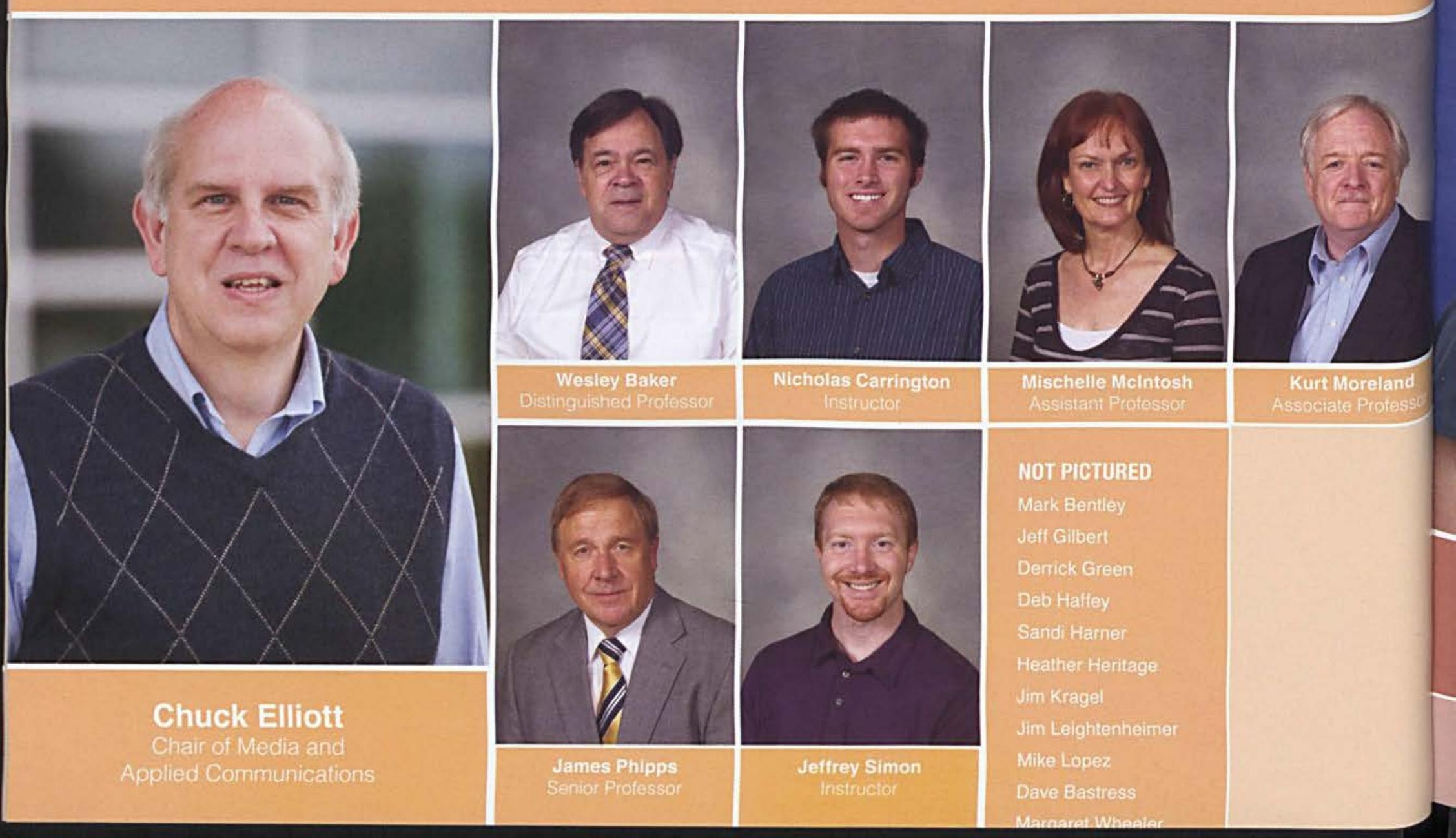




\section{LIBRARY SERVICES}

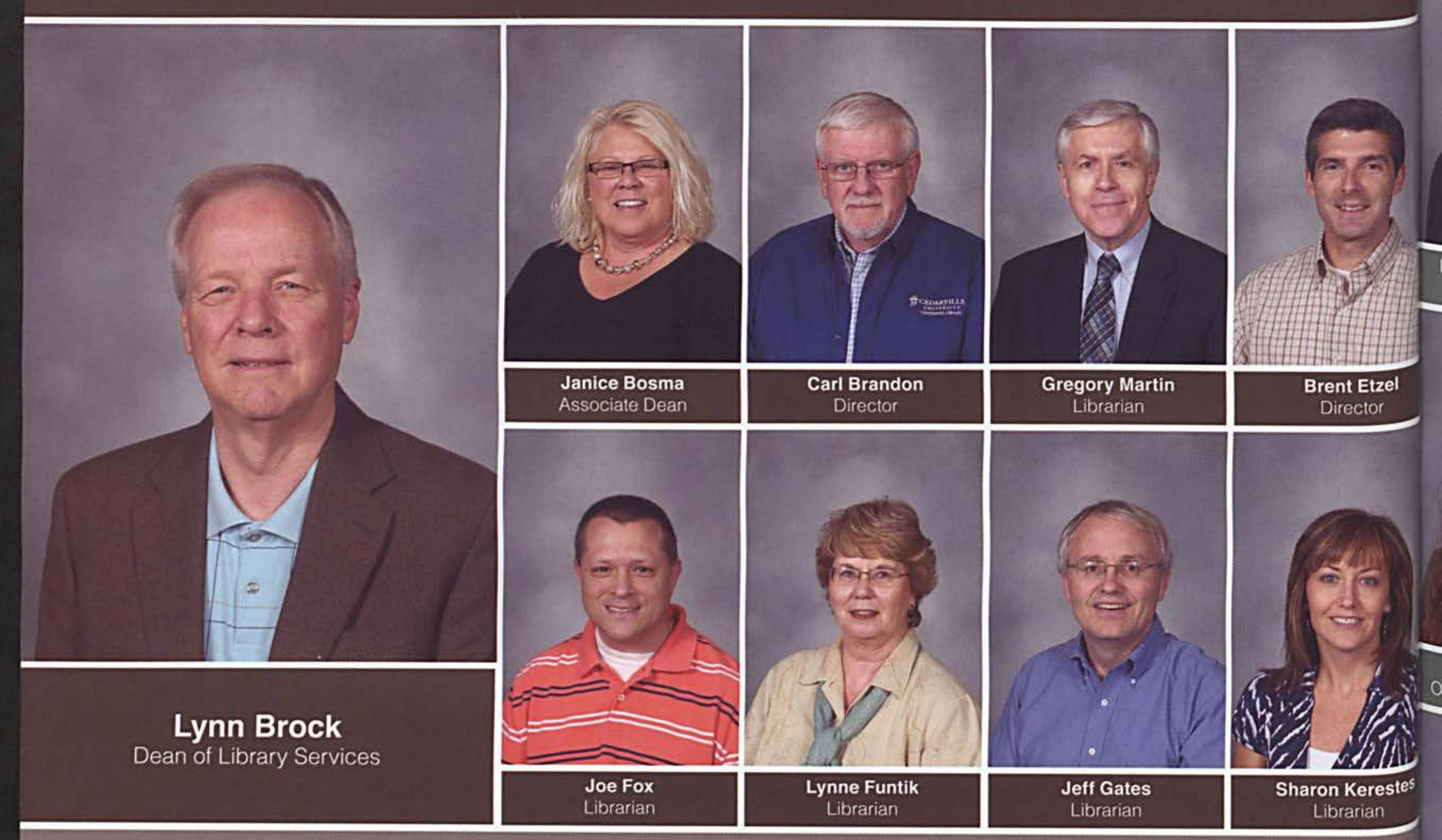





\section{STAFF}

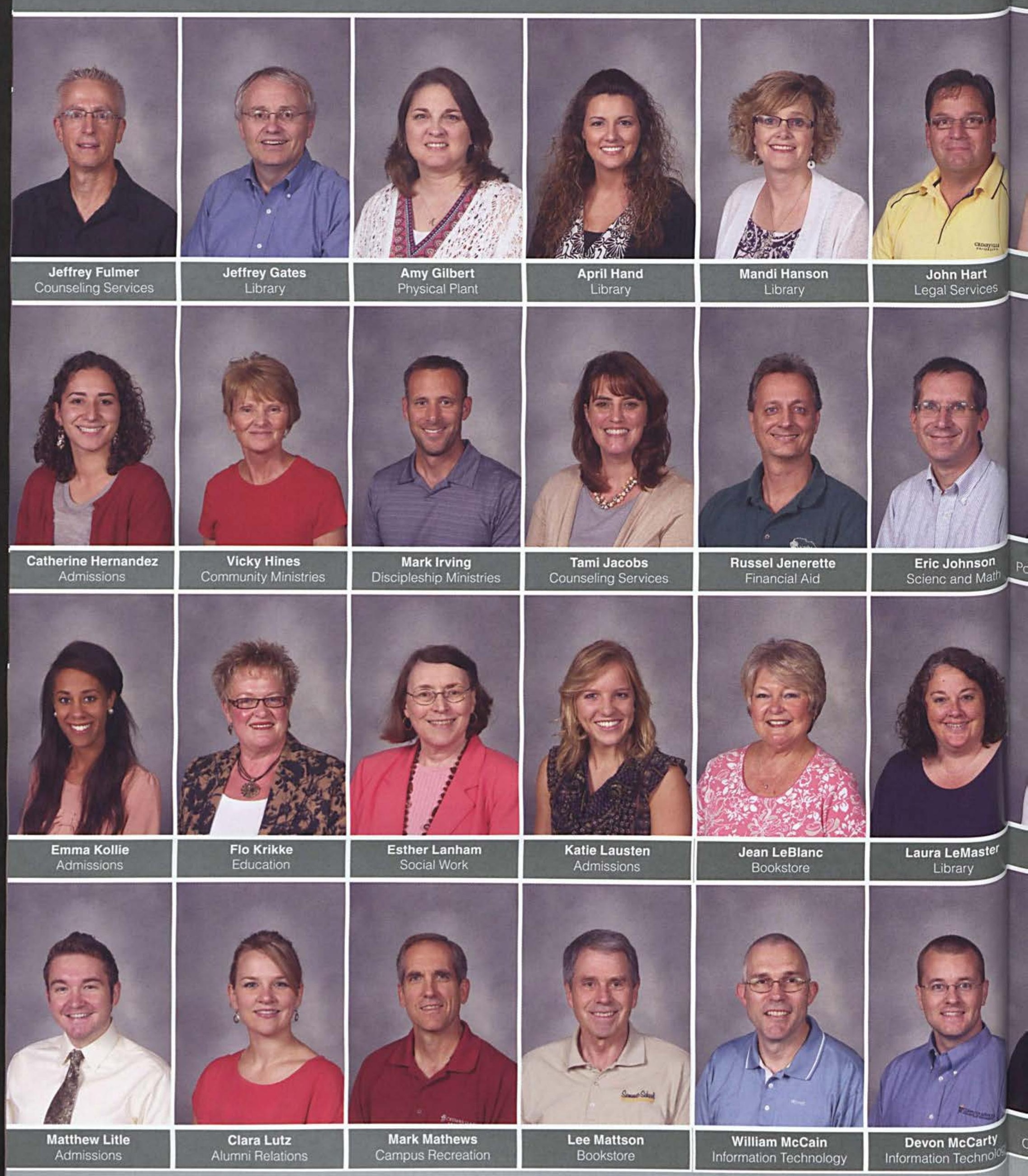




\section{STAFF}

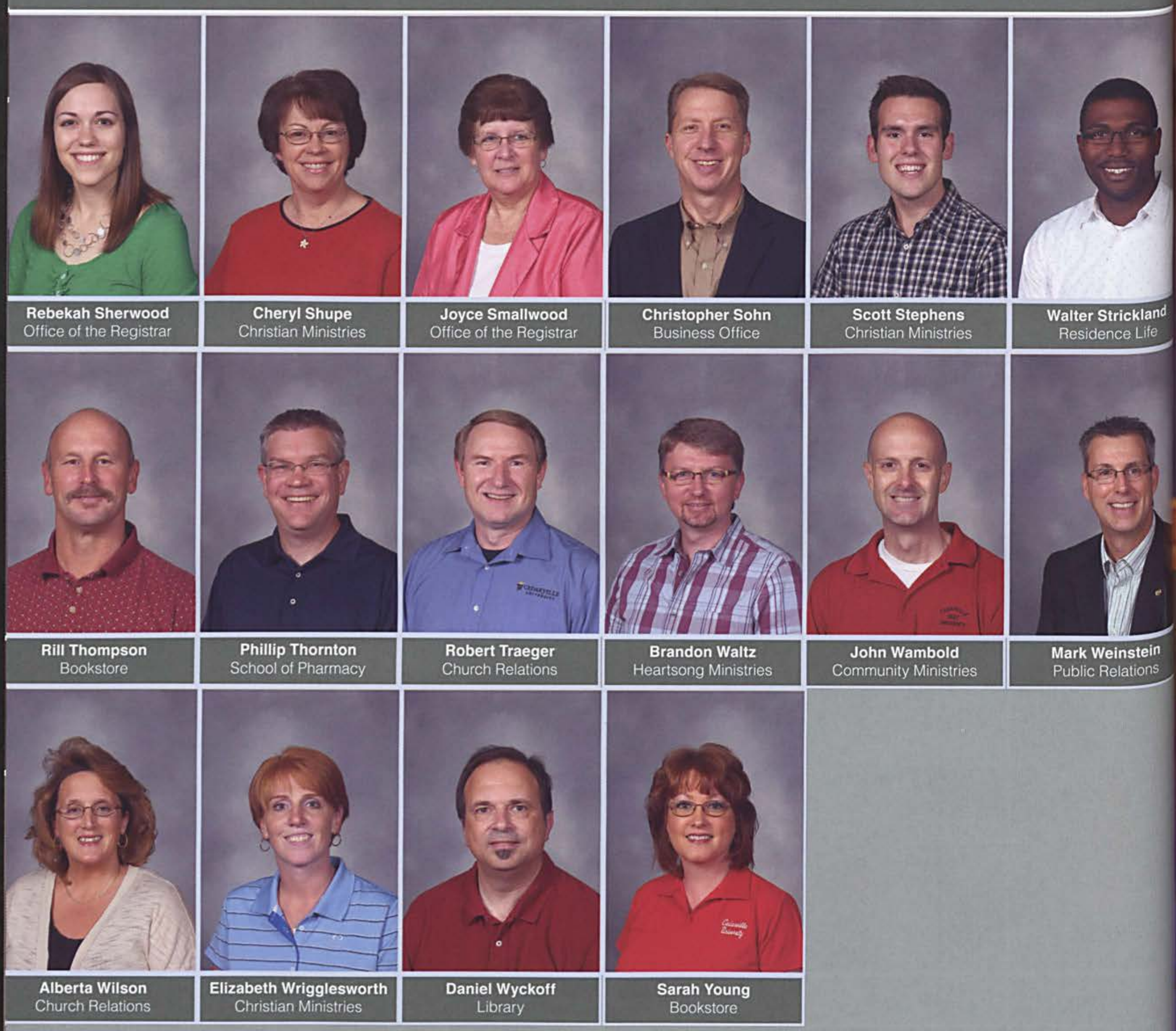




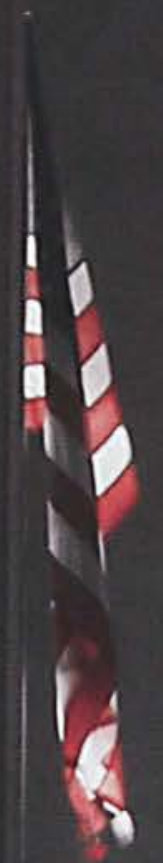

$y=0 . \cdots$

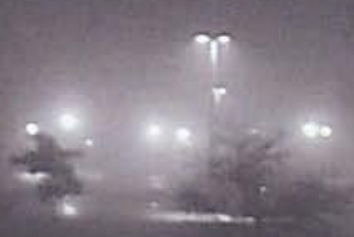





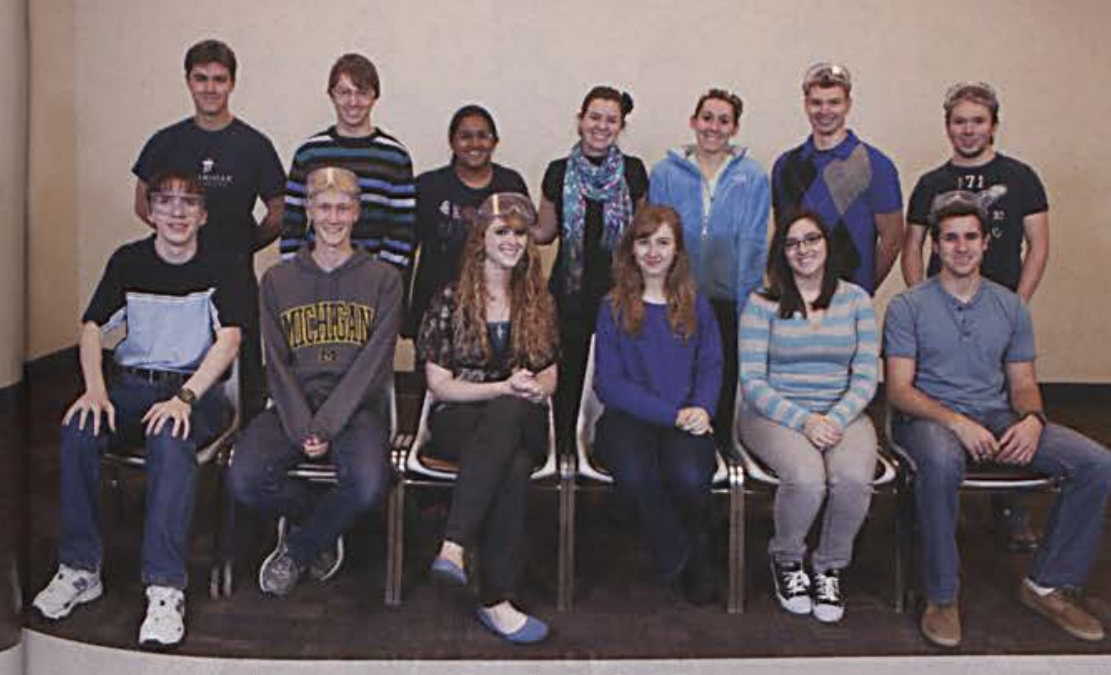
Chemistry Club

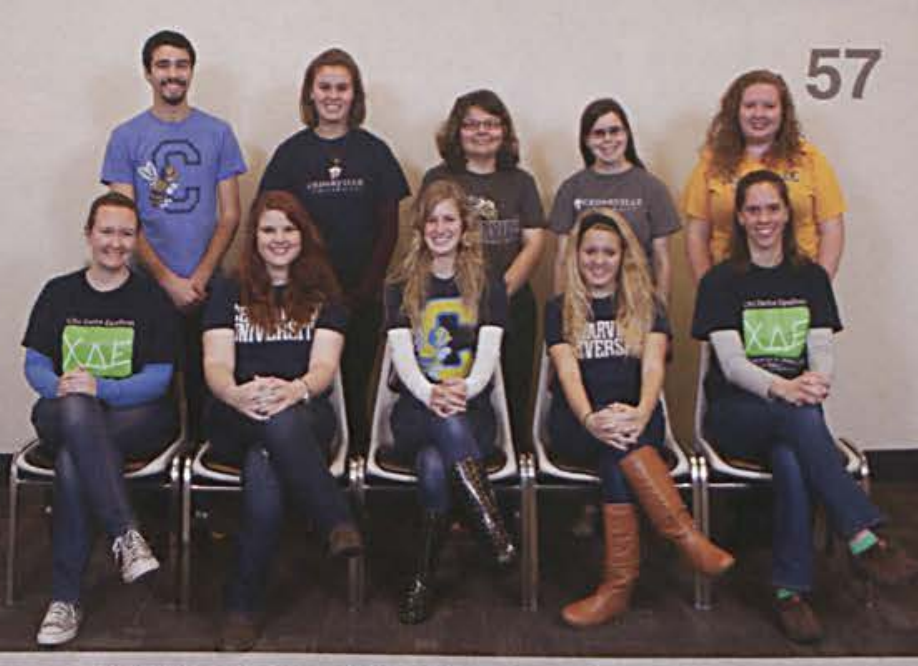

Chi Delta Epsilon (Education Organization)
00000098900

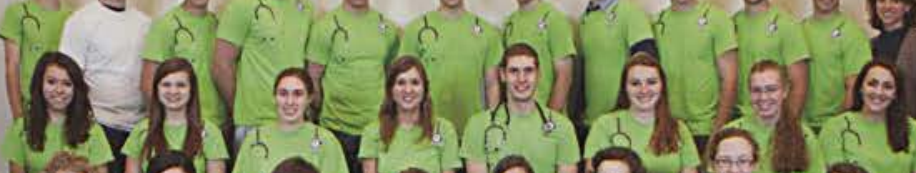

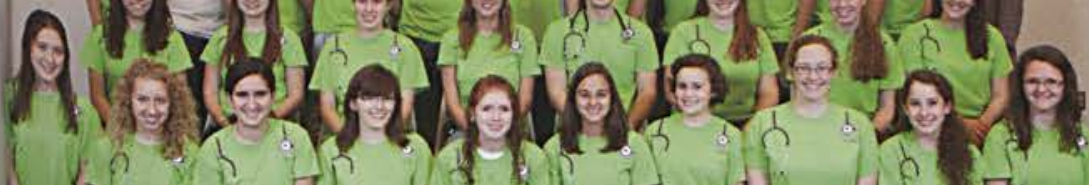

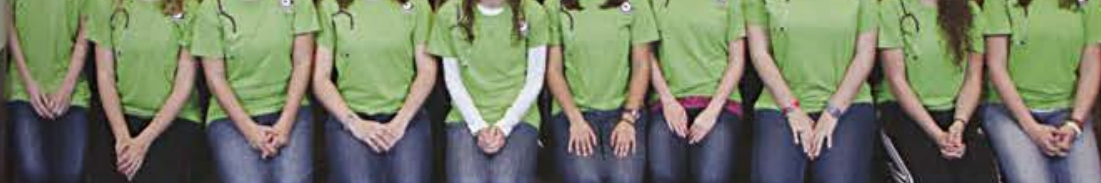

Christian Nursing Organization

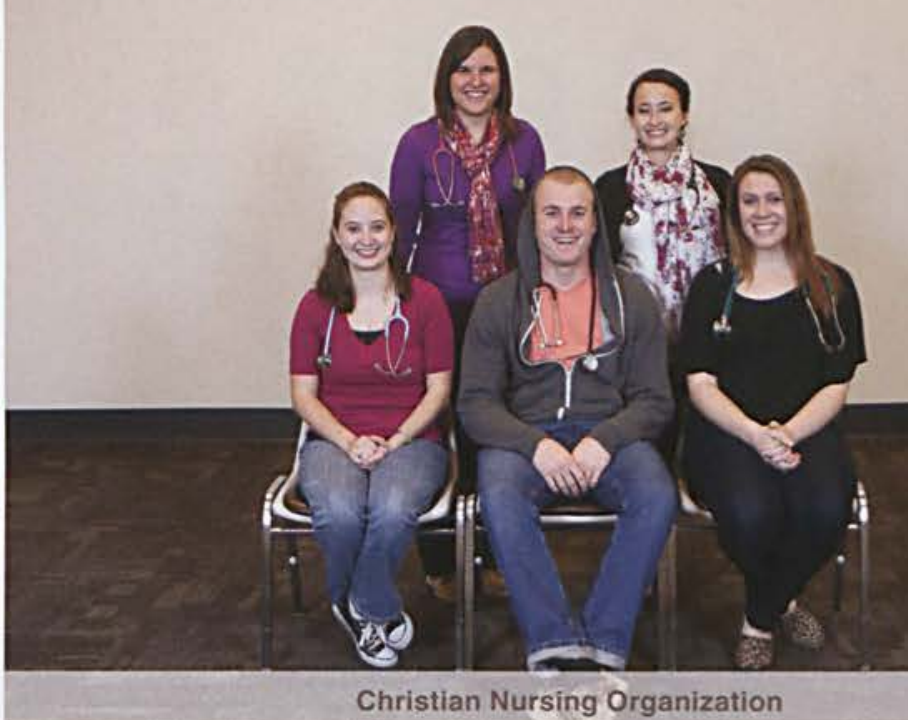

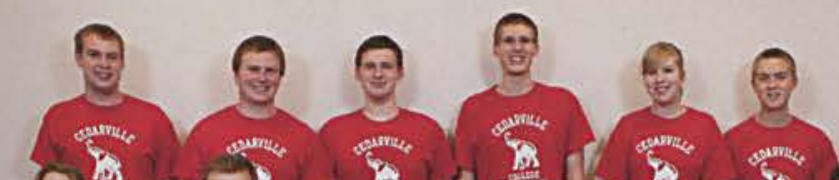

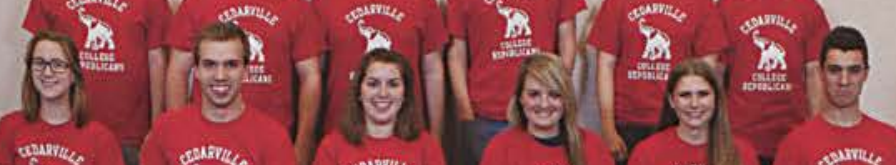
(1) xe $=$ 65 College Republicans

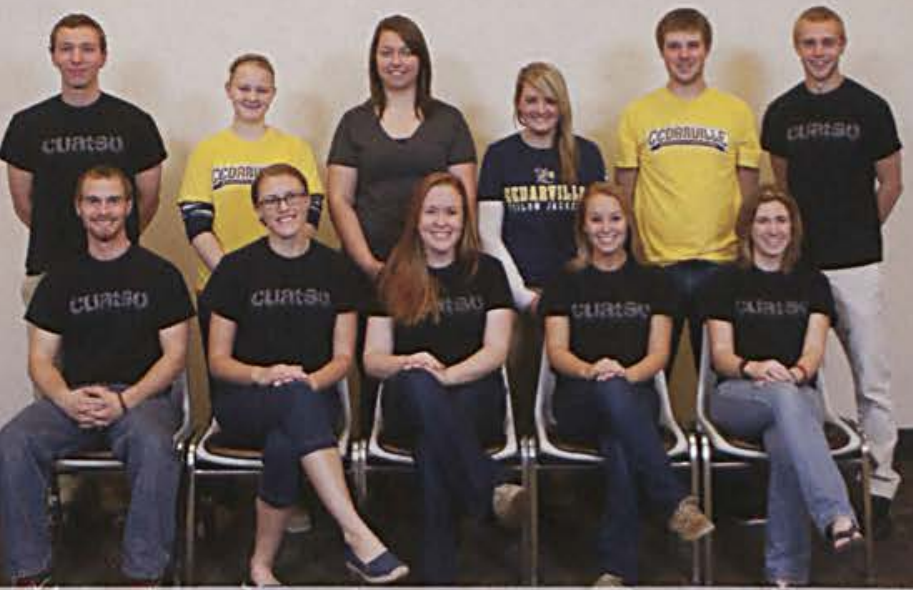

CUATSO (Athletic Training Organization)

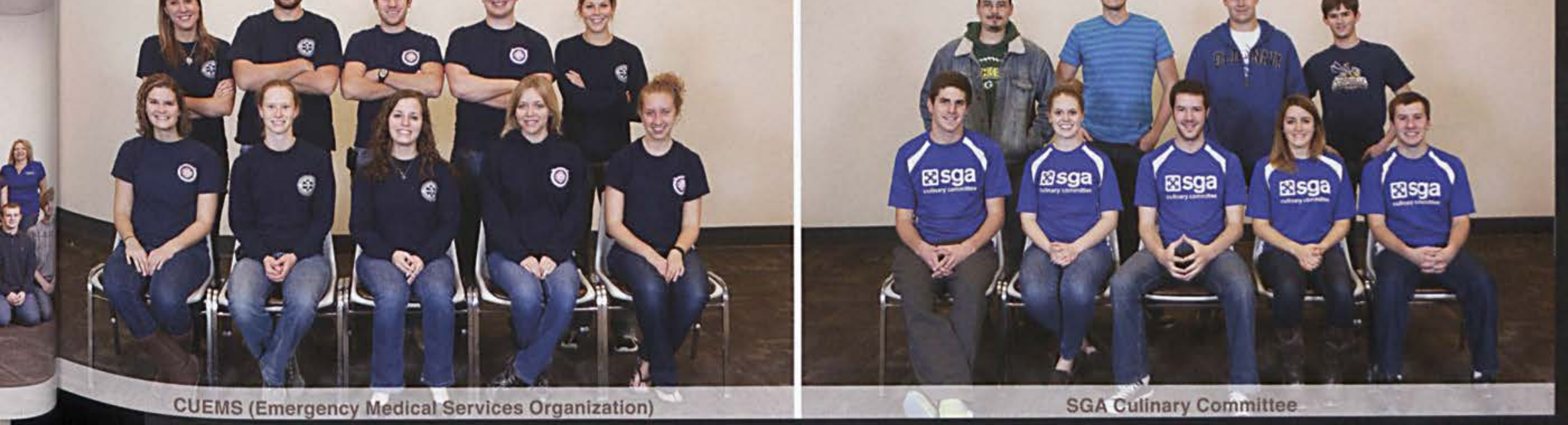


3ere $292 \%$

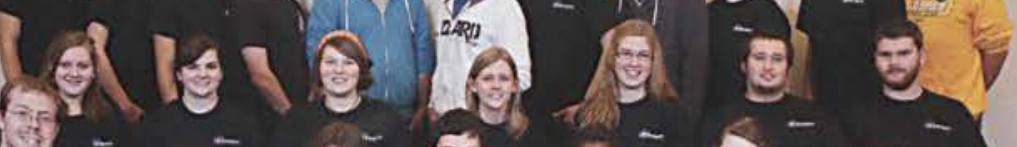

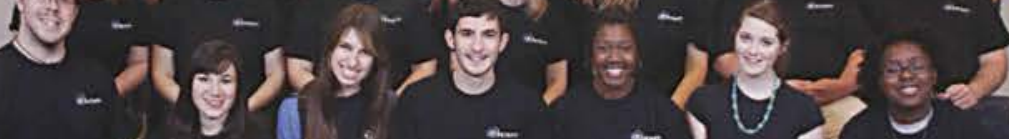

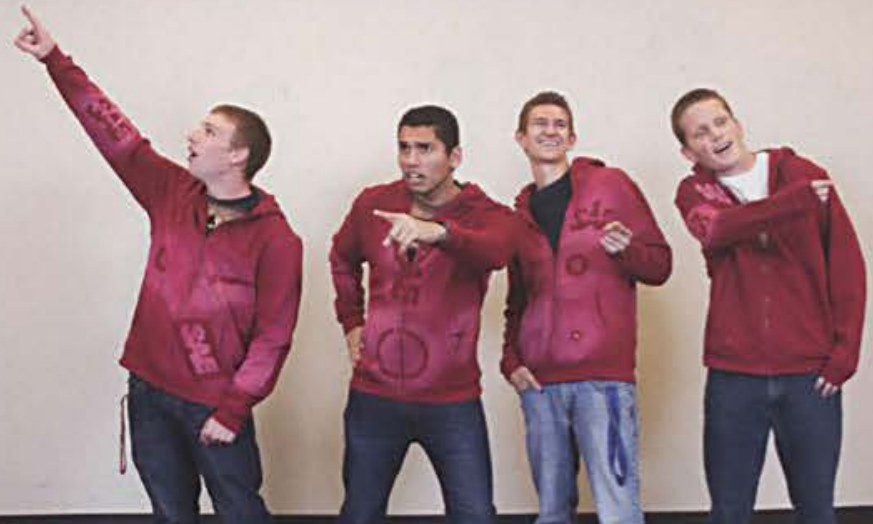

mingo axy 19 O. 1,1910 YUMU

Sanctify Ministries (Women's Purity Organization)

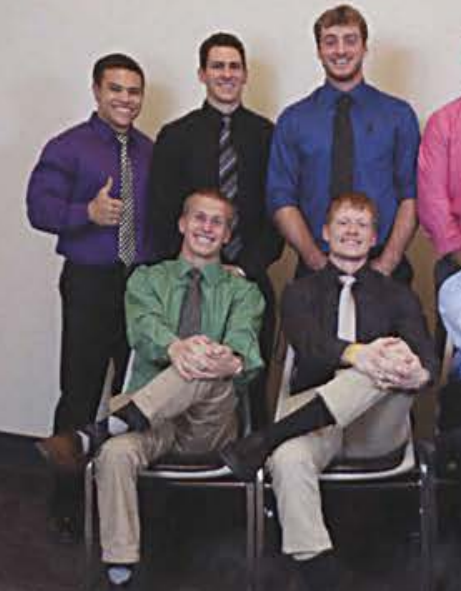

คำ

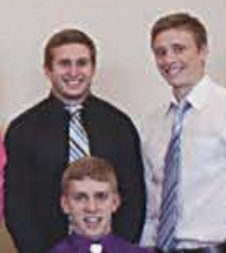

शis

1.1

$1 \leq 1$

$\rightarrow 0$

6

Sigma Phi Lambda (Men's Service Organization)

280292920 13. alle \& :

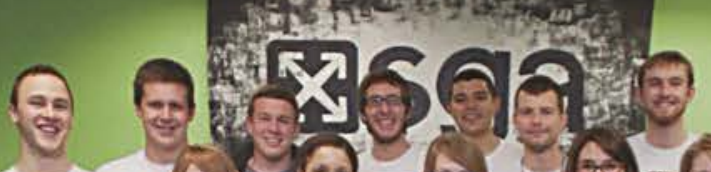

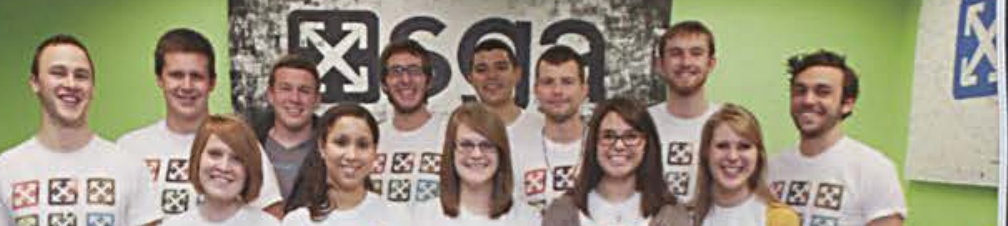
(5) IE

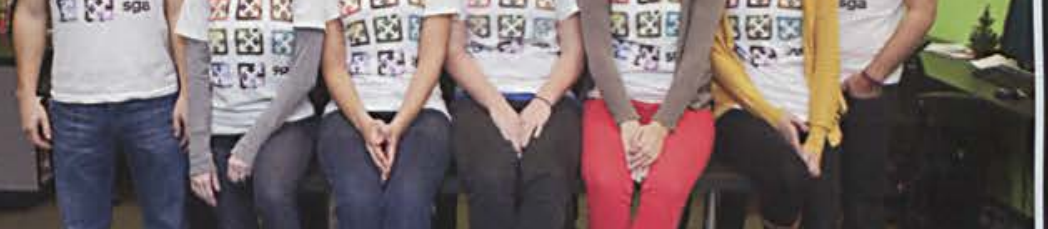

$20.4 / \operatorname{los}$

Student Government Association

Society for Women Engineers
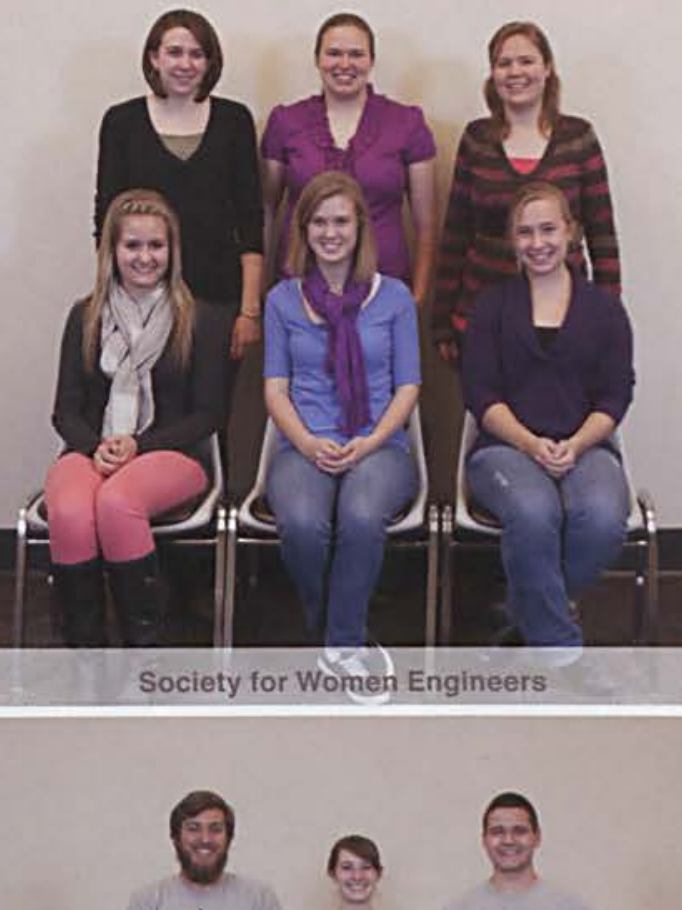
A.

요 $(20)$ a $\triangle 1$ (5) $2-3$ F, $\quad \Rightarrow$ 


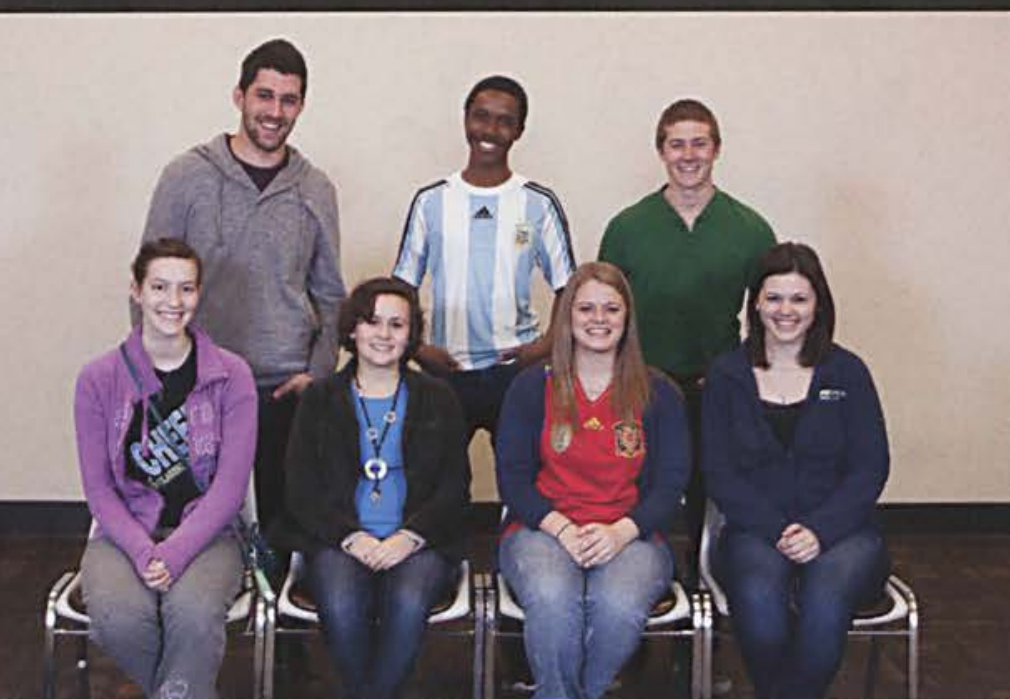

Spanish Club

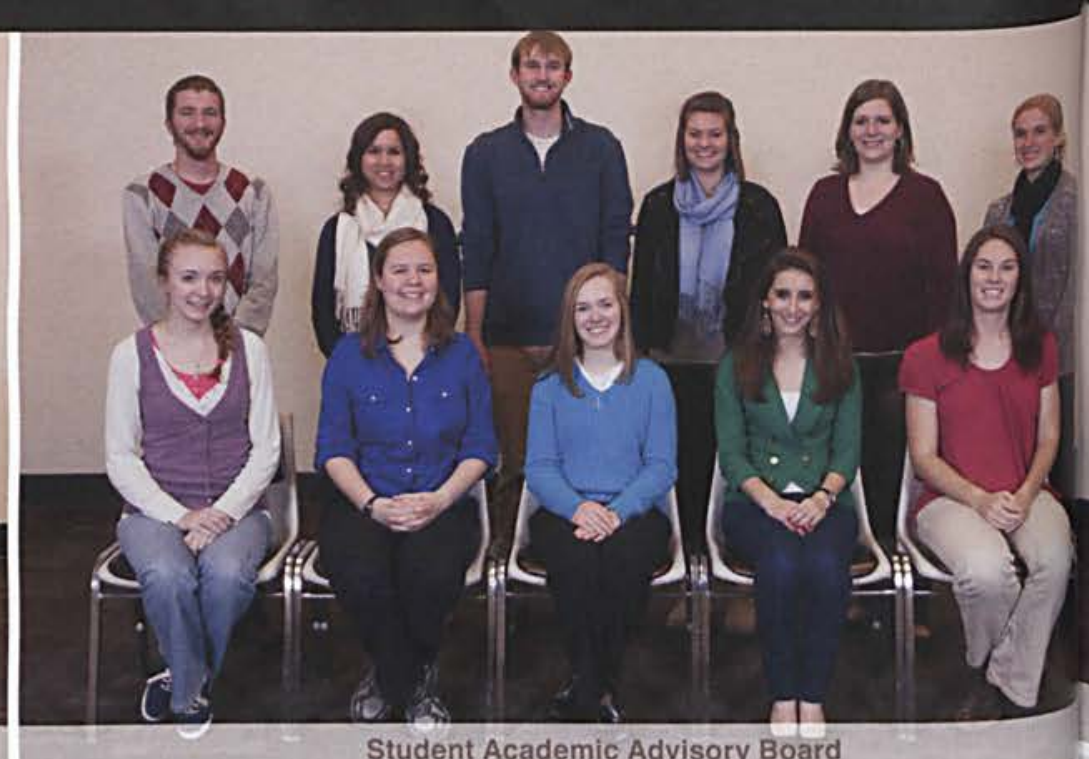

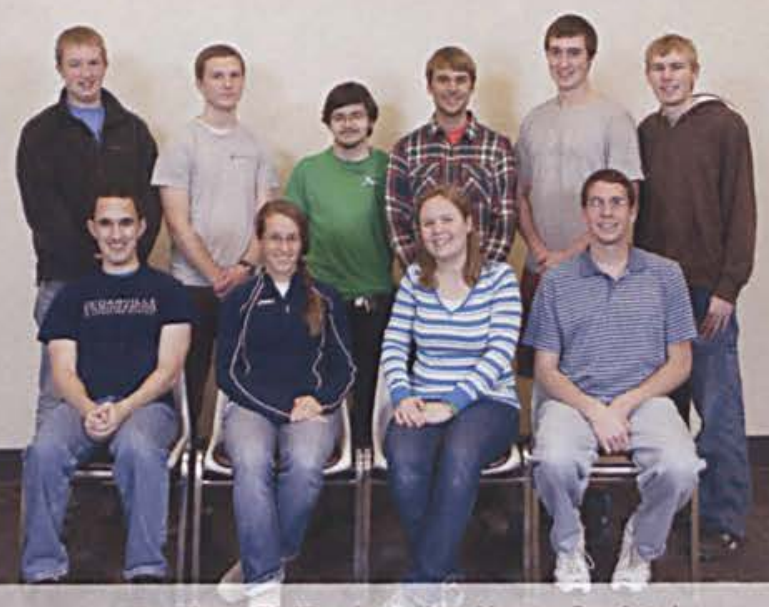

Tau Beta Pi (Engineering Honor Society)
Acenge2a2

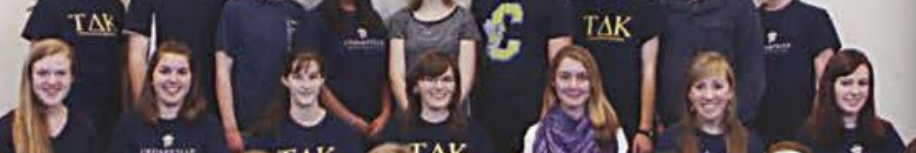

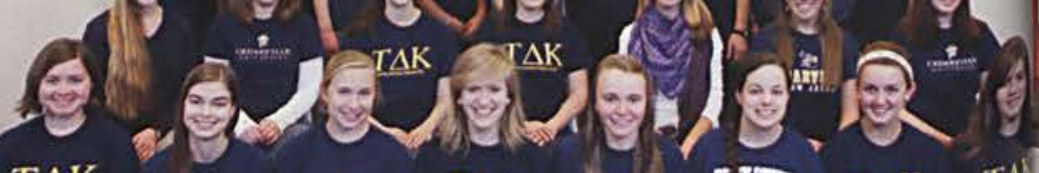
TAK

Tau Delta Kappa (Honors)

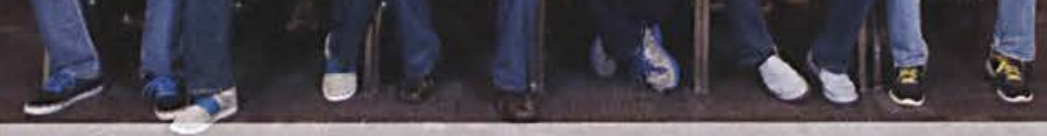

Theta Rho Epsilon (Men's Organization)

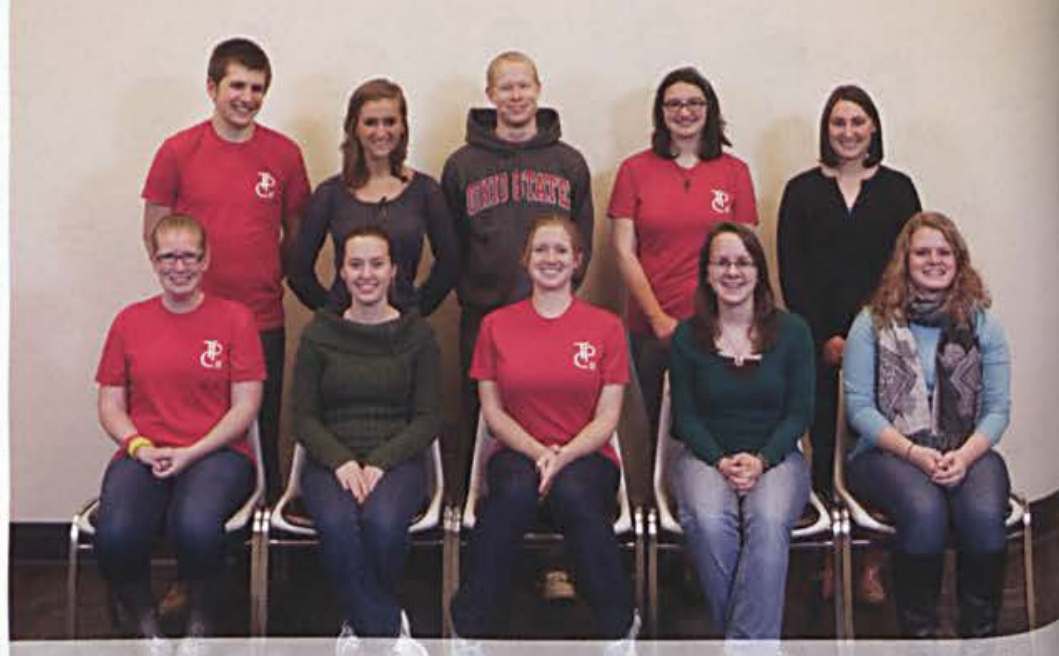

TPCu (Technical and Professional Communication Organization)

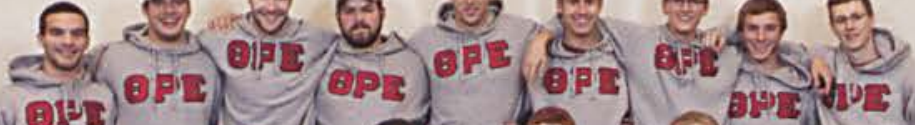

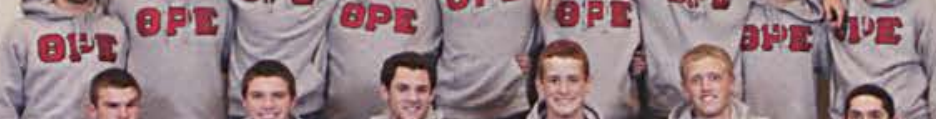
I $\Rightarrow$ क 7 물

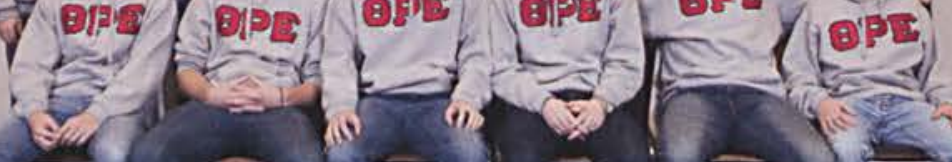
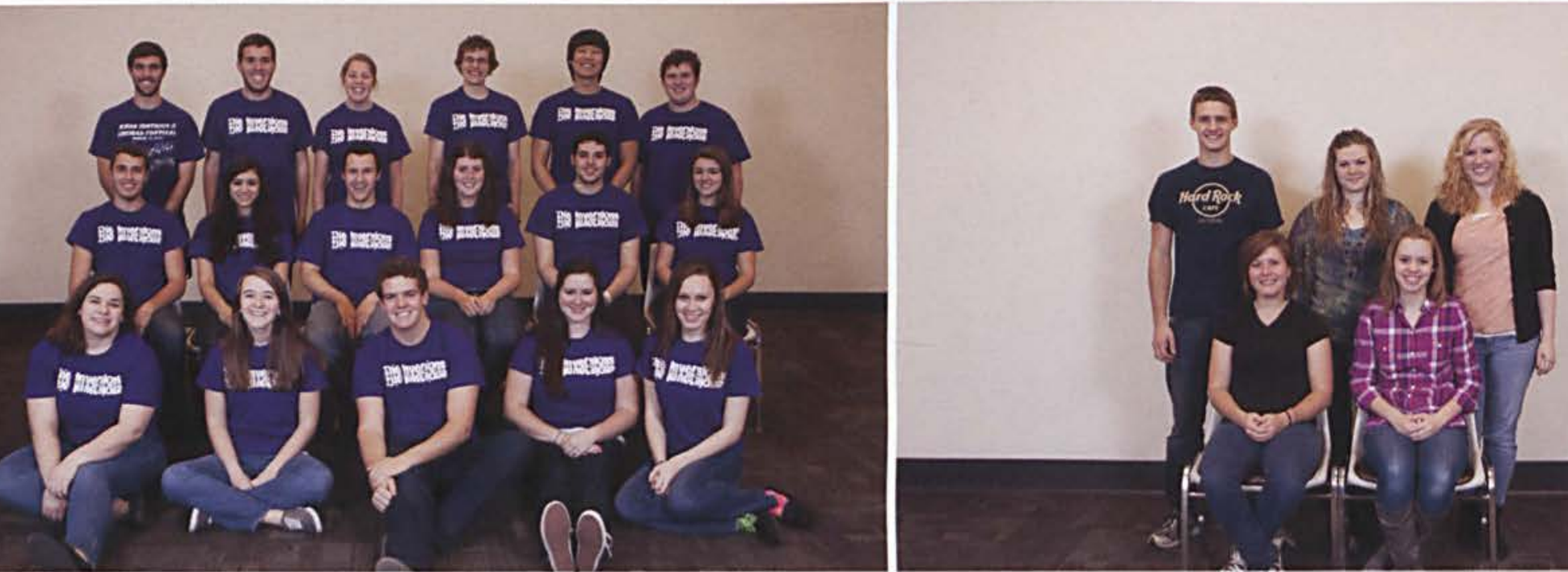

66

FRESHMEN

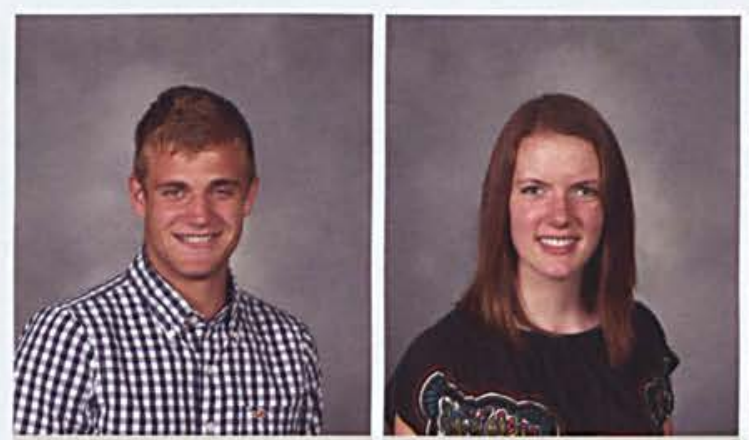

Christian Alexander

Katherine Alexander

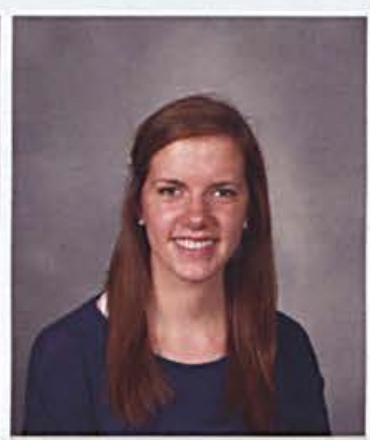

Nicole Alexander

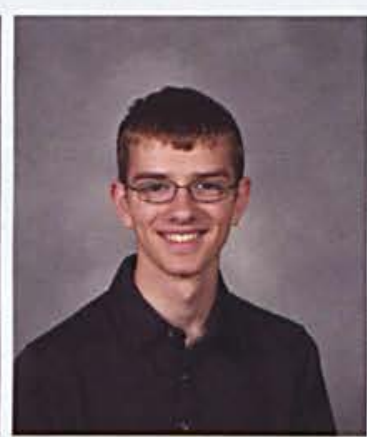

Aaron Alford

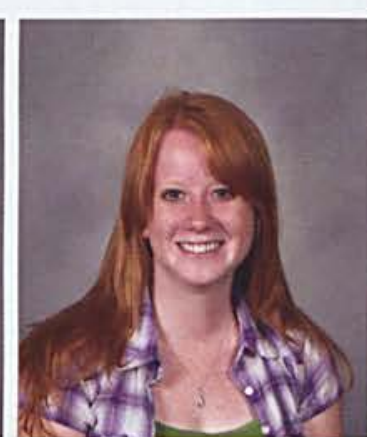

Alexis Ancona

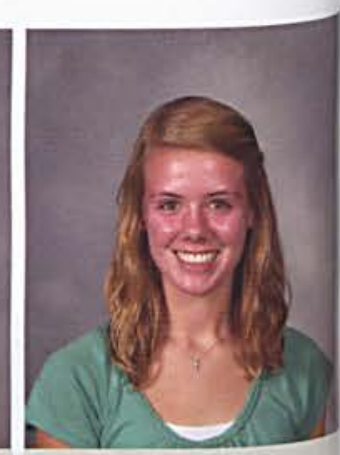

Jessica Alderink

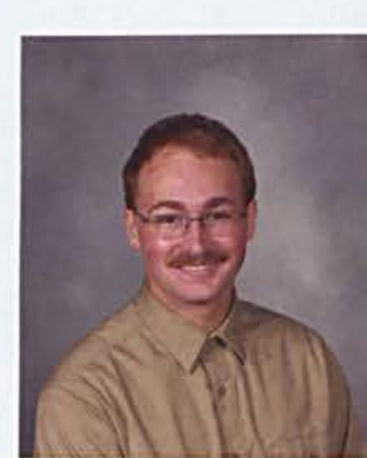

Calvin Anderson

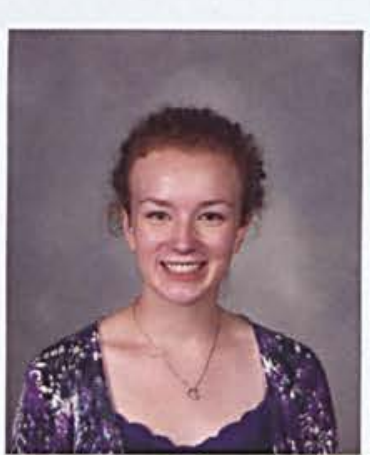

Claire Anderson

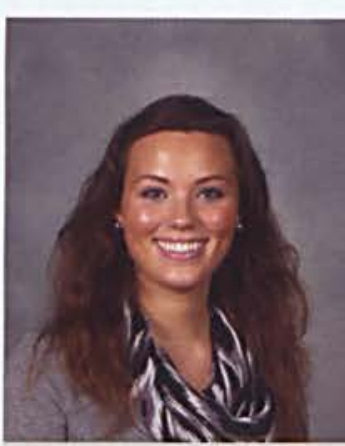

Megan Anderson

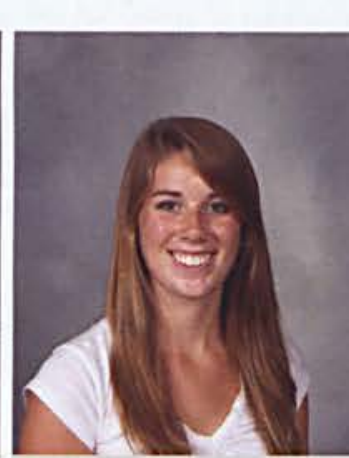

Hillary Ansiel

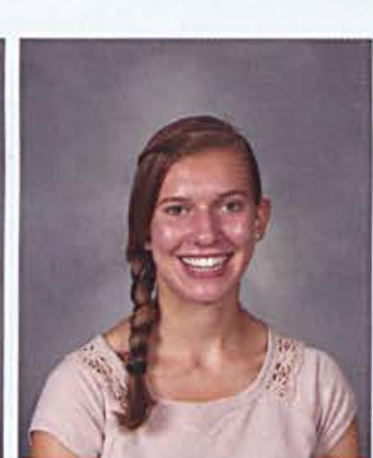

Alexandra Archambault

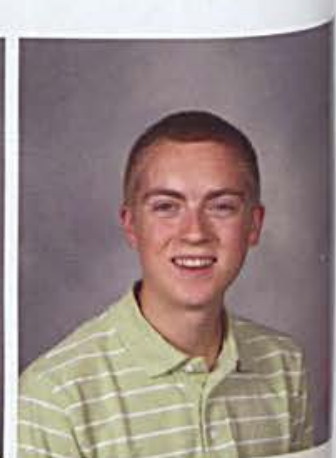

Arne Anderson

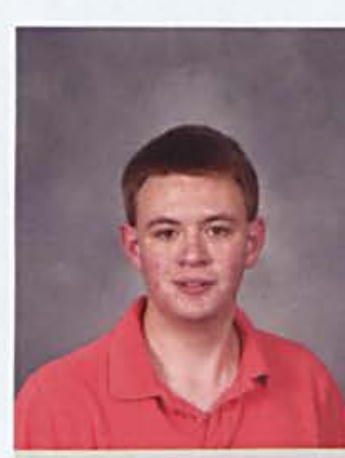

Joseph Austin

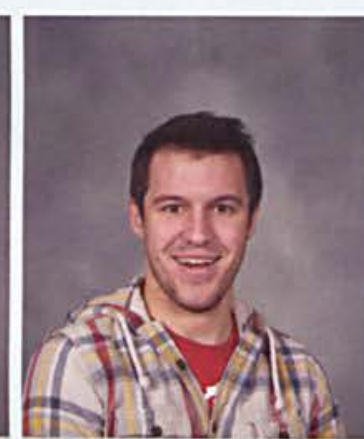

Jerry Bailey

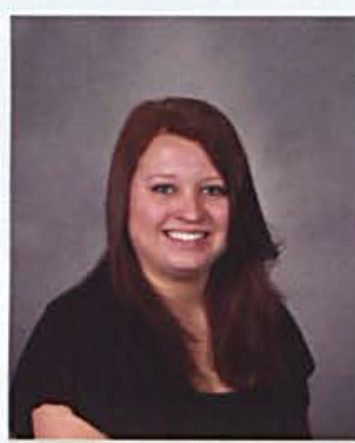

Morgan Bailey

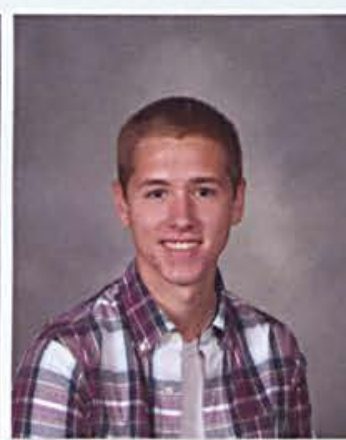

Adam Baker

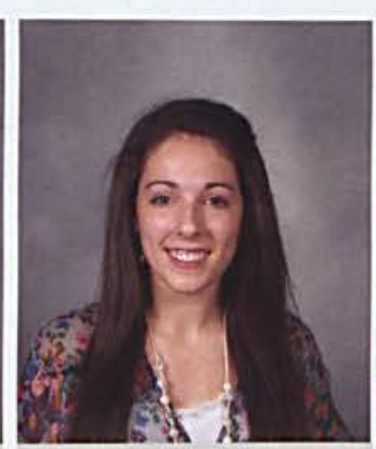

Jocelyn Baker

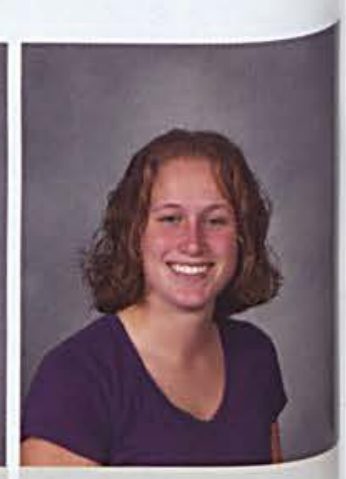

Monica Arslain
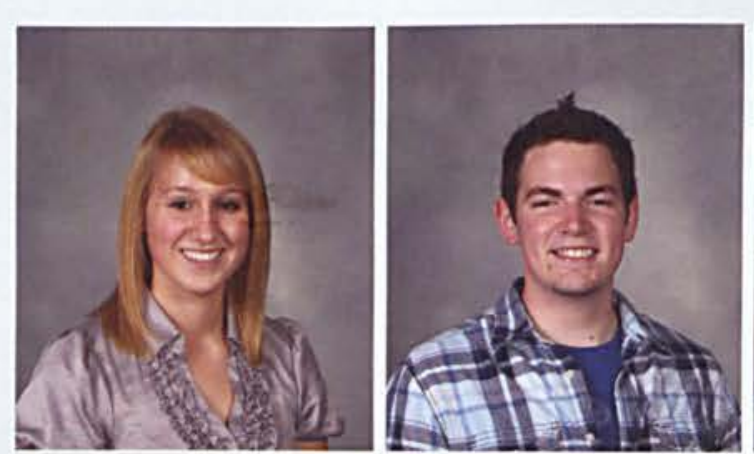

Aleska Barkoviak

Jonathan Barlow

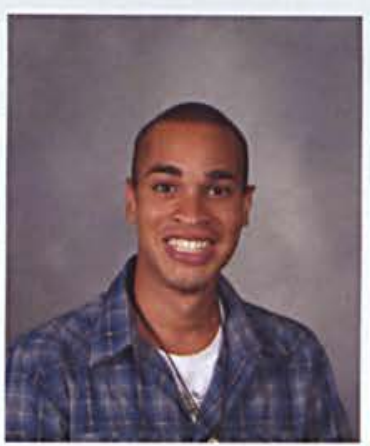

Witness Barnett

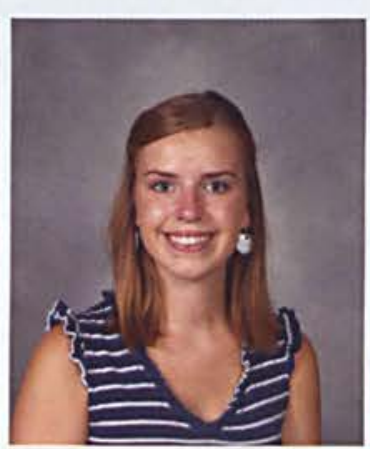

Alexis Baroni

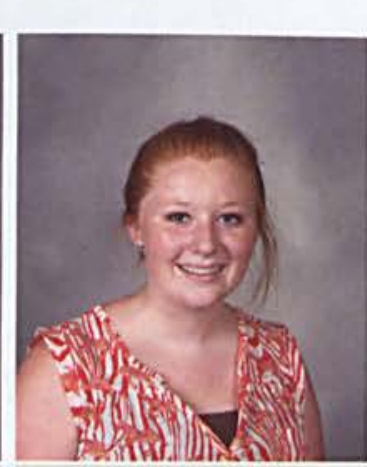

Amella Baumann

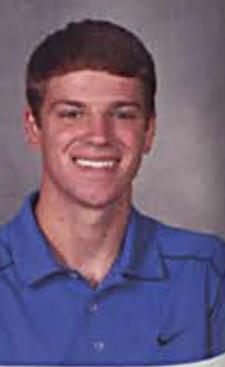

David Bancroft 


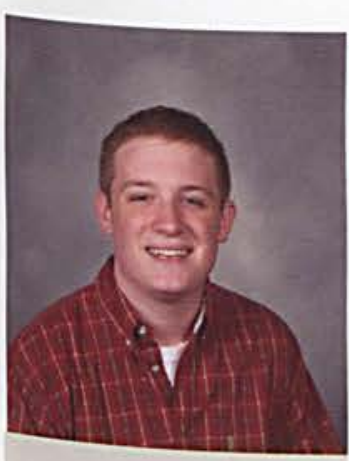

Austin Becton

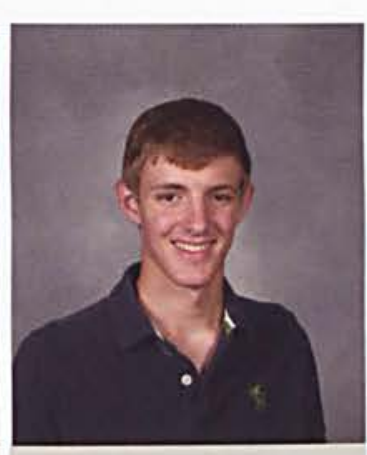

John Behnke

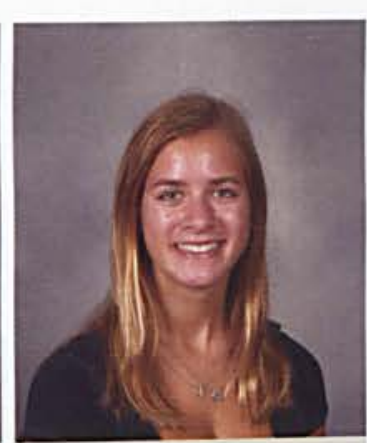

Casey Belback

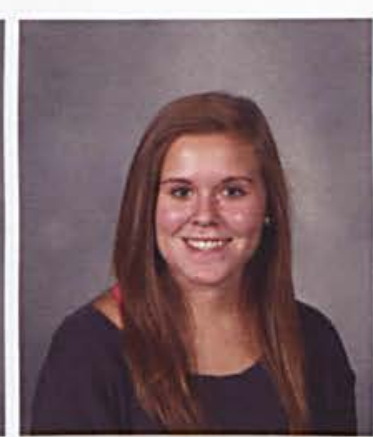

Olivia Benitez

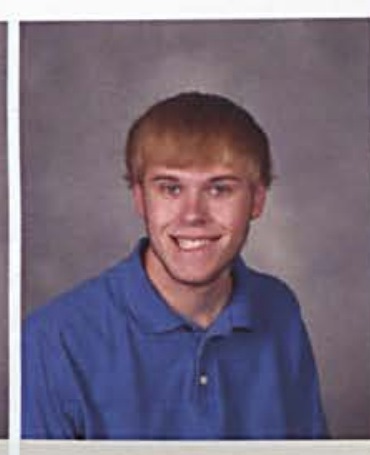

Gregory Bennett

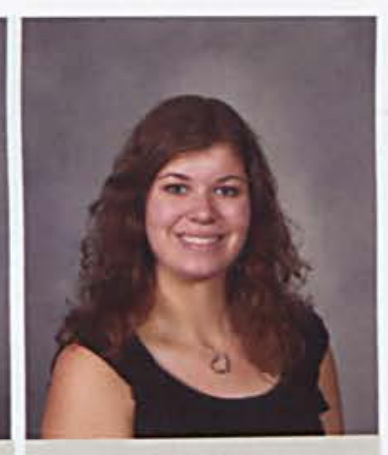

Alissa Bercaw

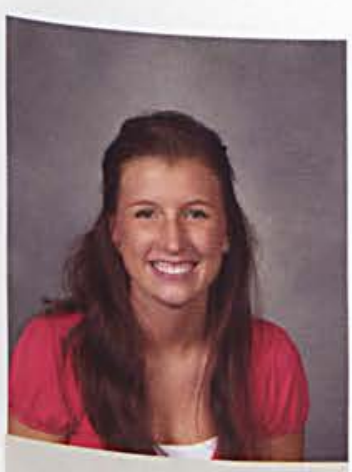

Mary Bewley

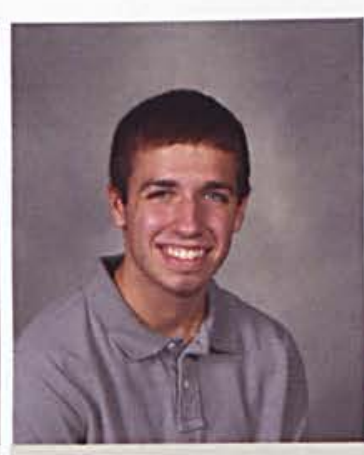

David Bieniek

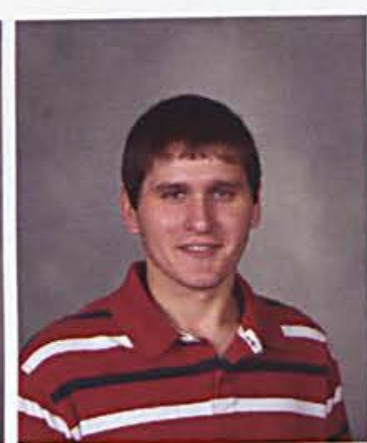

Kyle Biggs

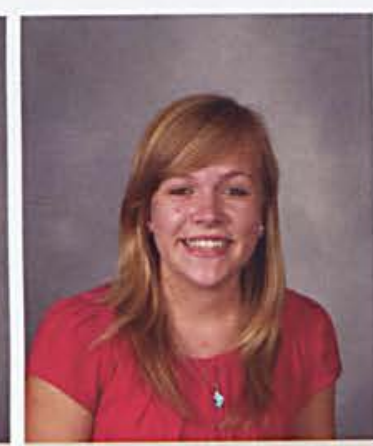

Meredith Blair

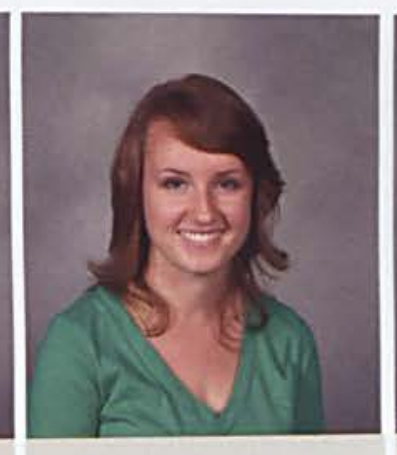

Christa Blanchard

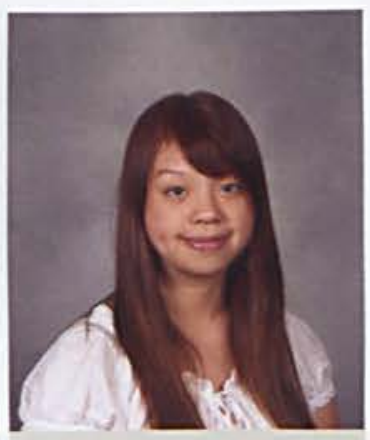

Irene Blocksom

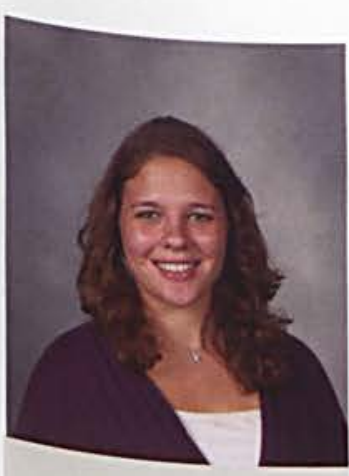

Leah Bode

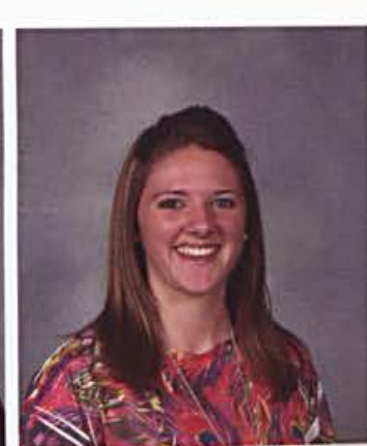

Brianna Boehning

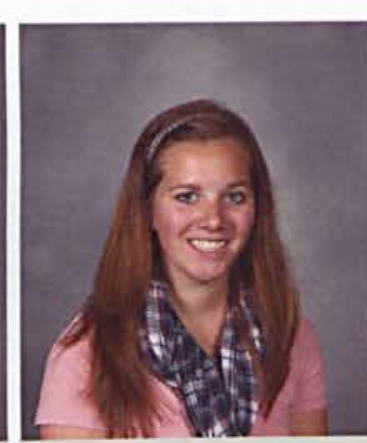

Kathleen Bond

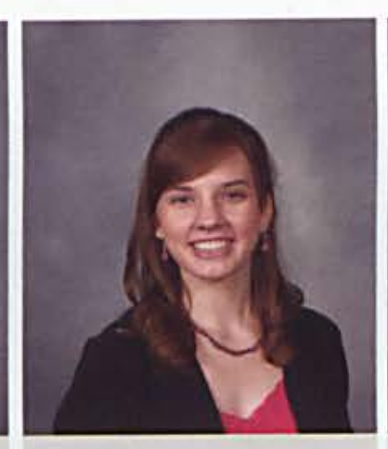

Victoria Boward

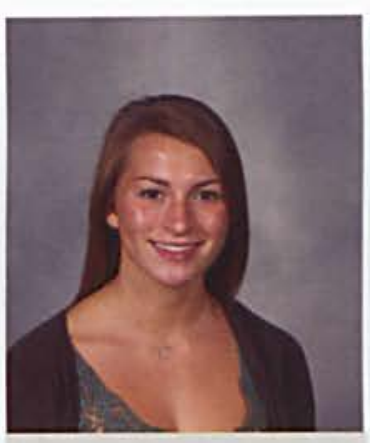

Kailey Bricker

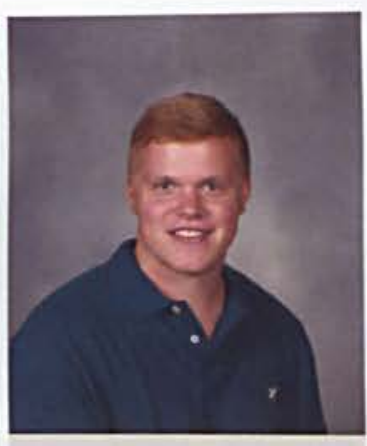

Gregory Brock

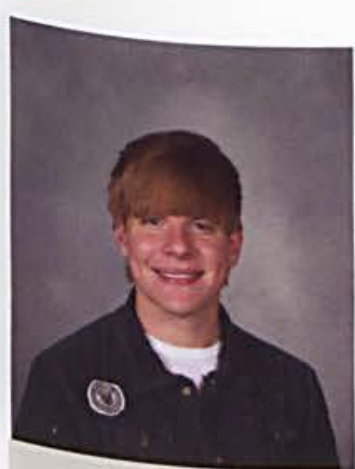

Austin Brown

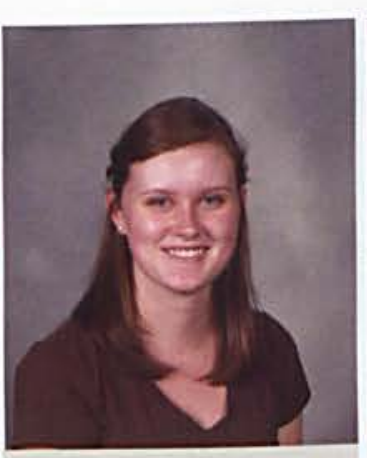

Hannah Brown

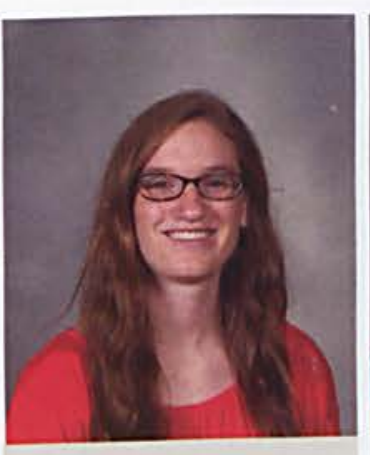

Meghan Bruckner

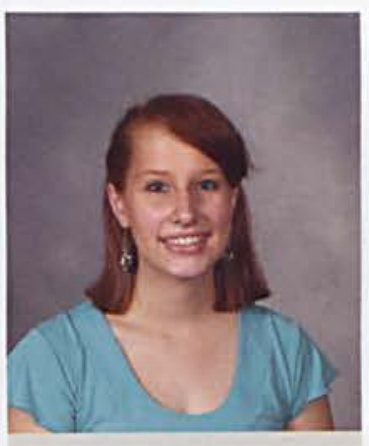

Rebecca Brumbaugh

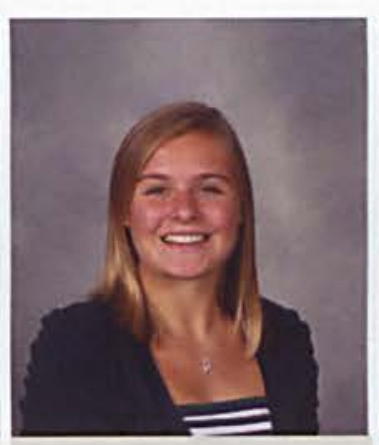

Julia Brummel

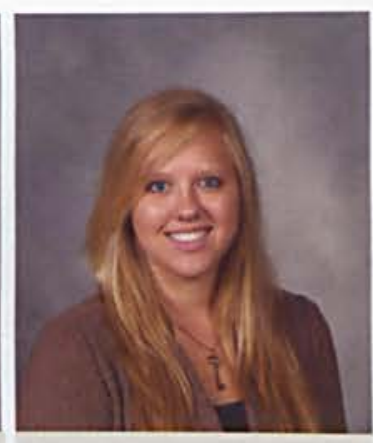

Anna Bullis
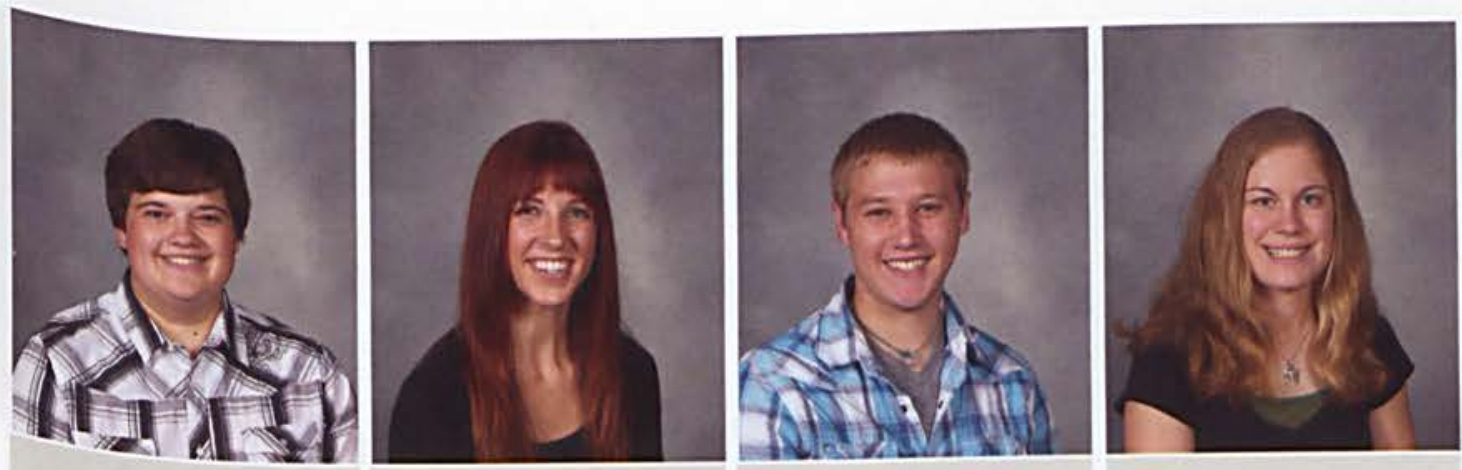

Tanya Busby

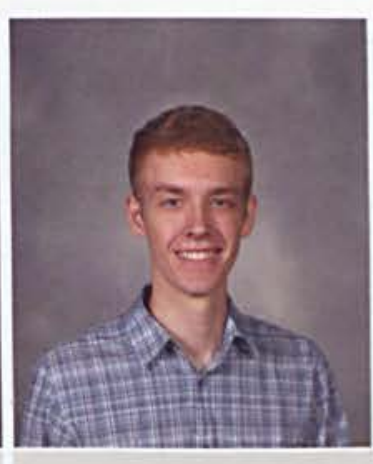

Hudson Carden

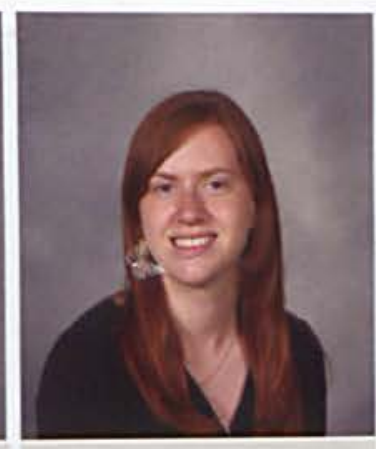

Rebecca Carpenter 
68

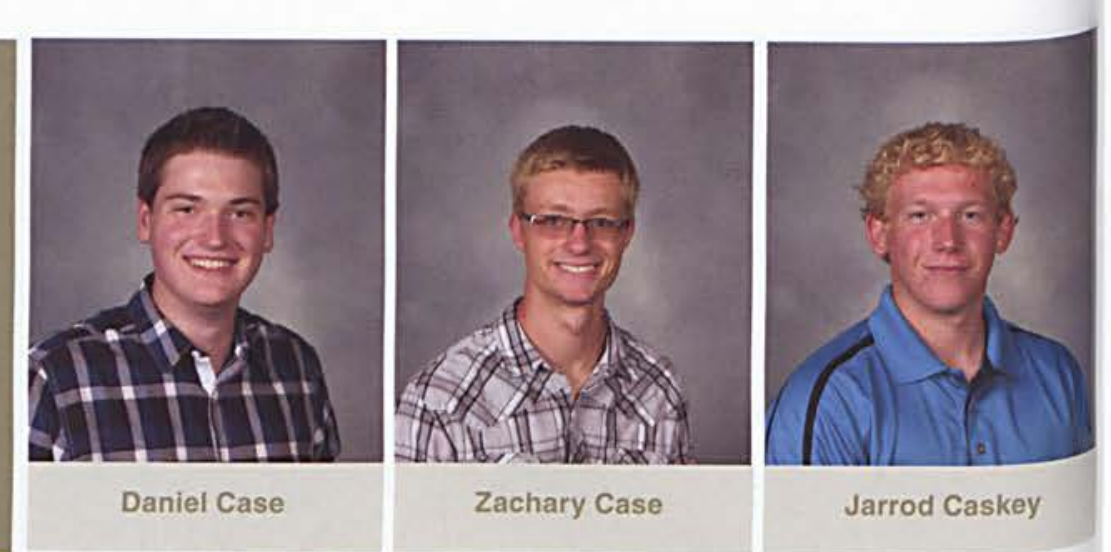

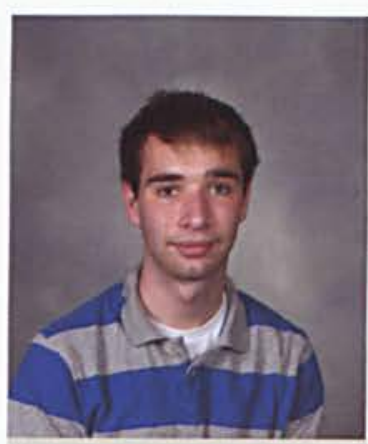

David Catanese

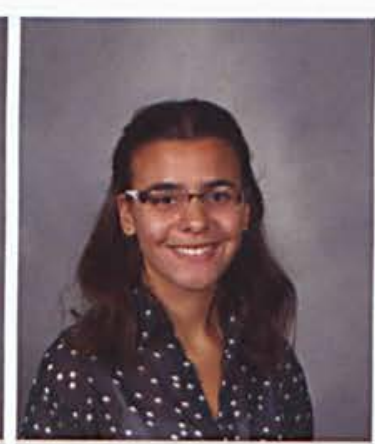

Christina Cerutti

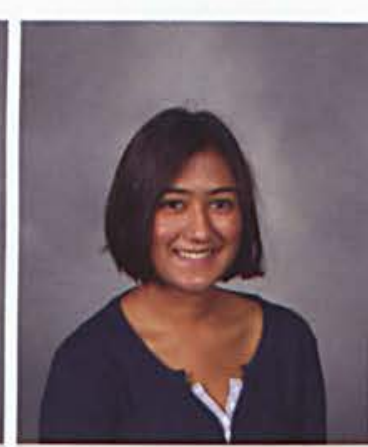

Khoisnam Chanu

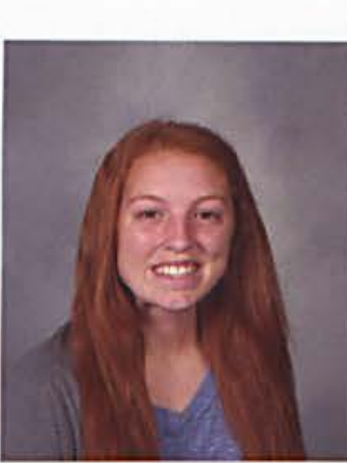

Sara Chasse

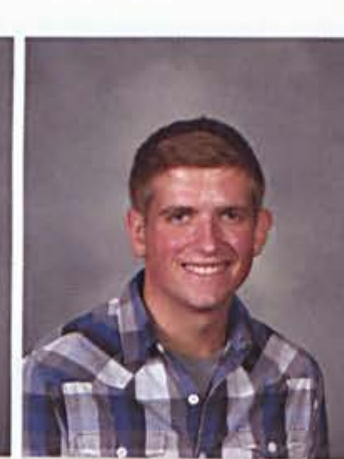

Kyle Cherry

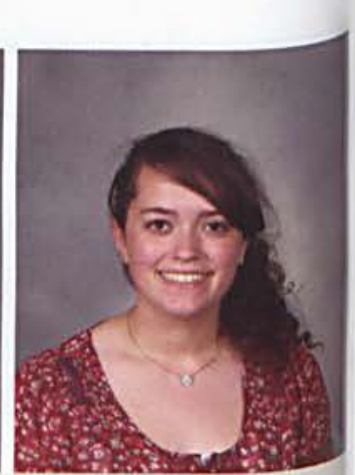

Hannah Chitty

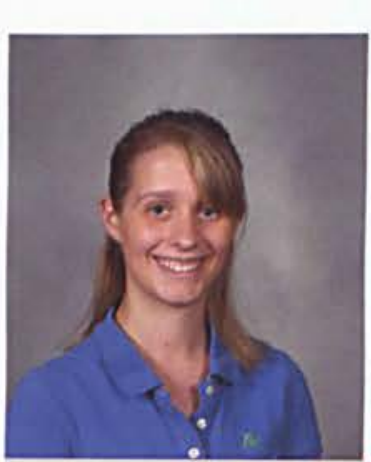

Kayleigh Clark

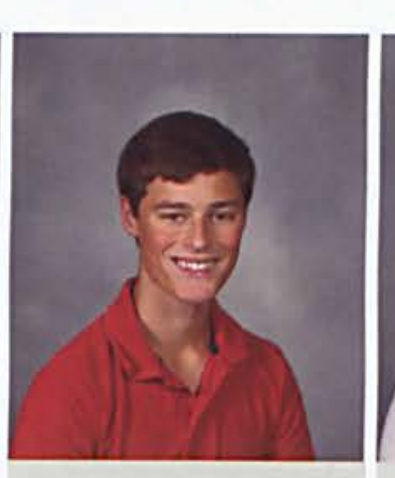

Stephen Clark

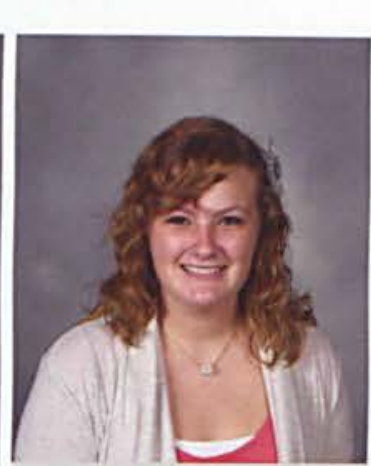

Natalie Clason

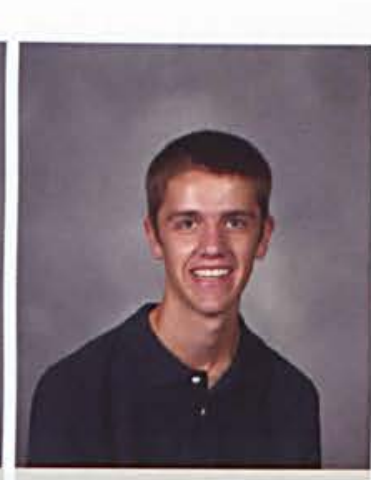

Kyle Classen

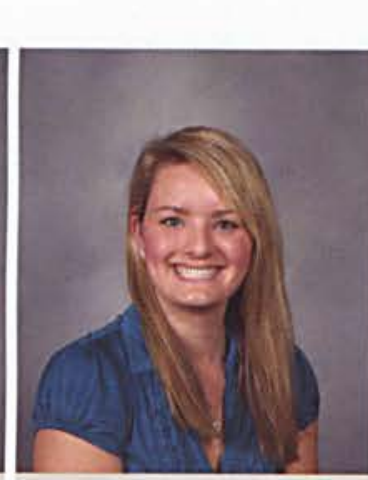

Karissa Clemens

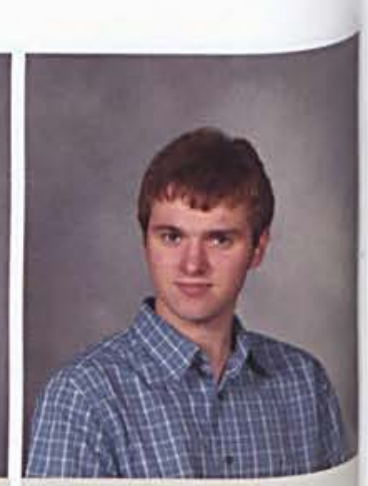

Sebastian Clifford

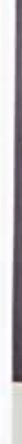

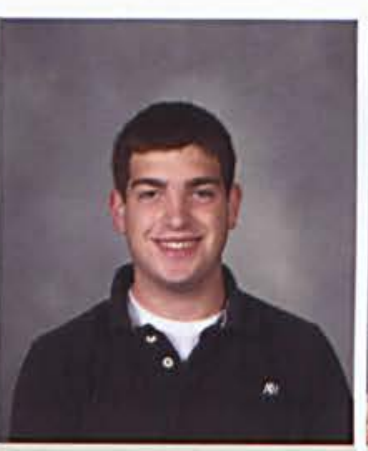

Adam Cole

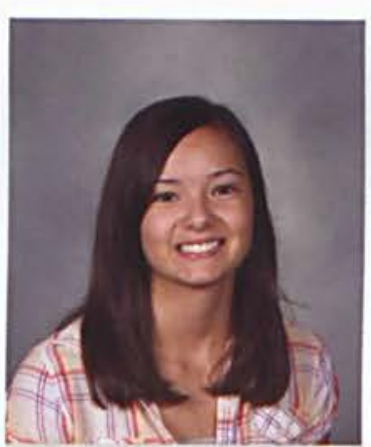

Alyssa Coleman

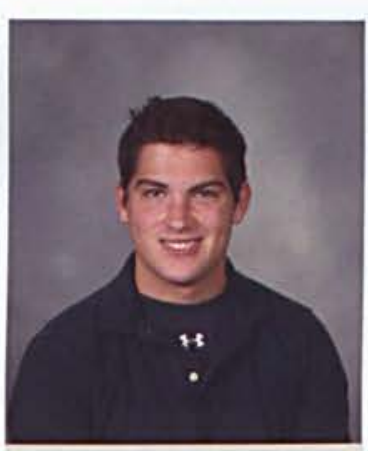

Paul Conover

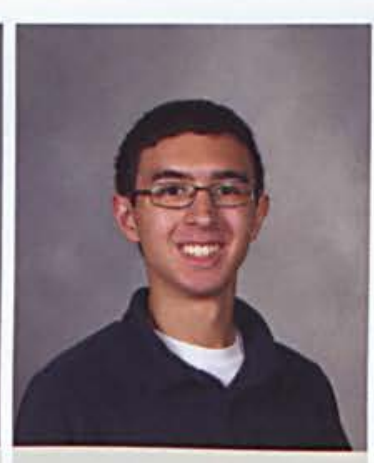

Johnathan Coraccio

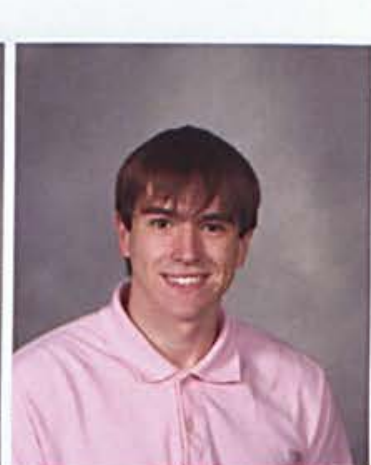

Austin Cordle

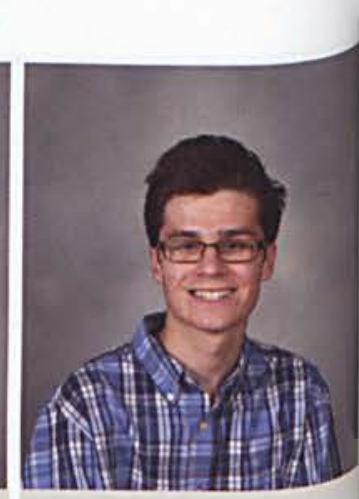

Joseph Corlito-Morell

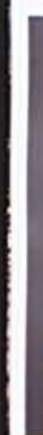
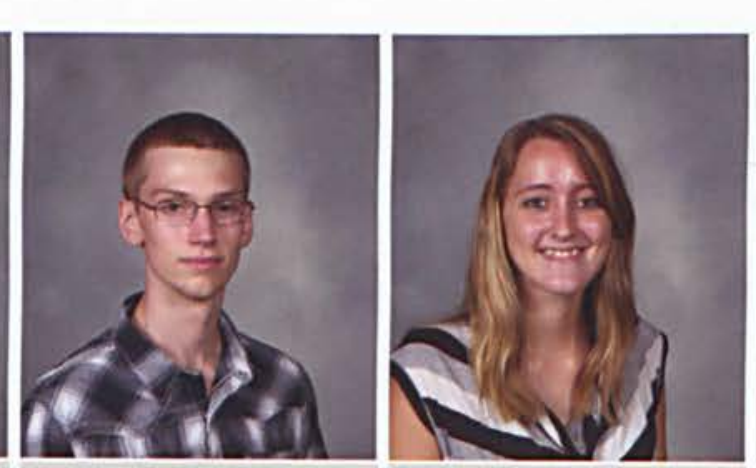

Victoria Couser

Aaron Crawley

Emily Criswell
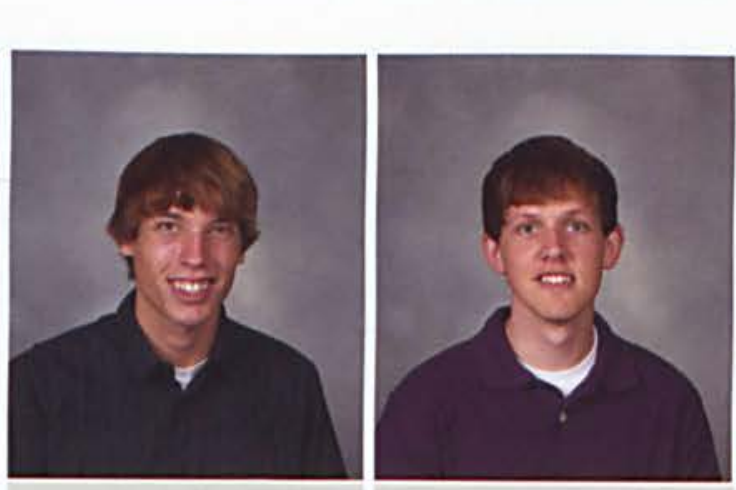

Marcus Croll

Andrew Cromwell

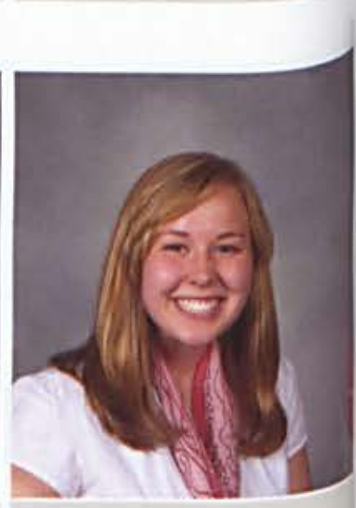

Tonl Crouch 

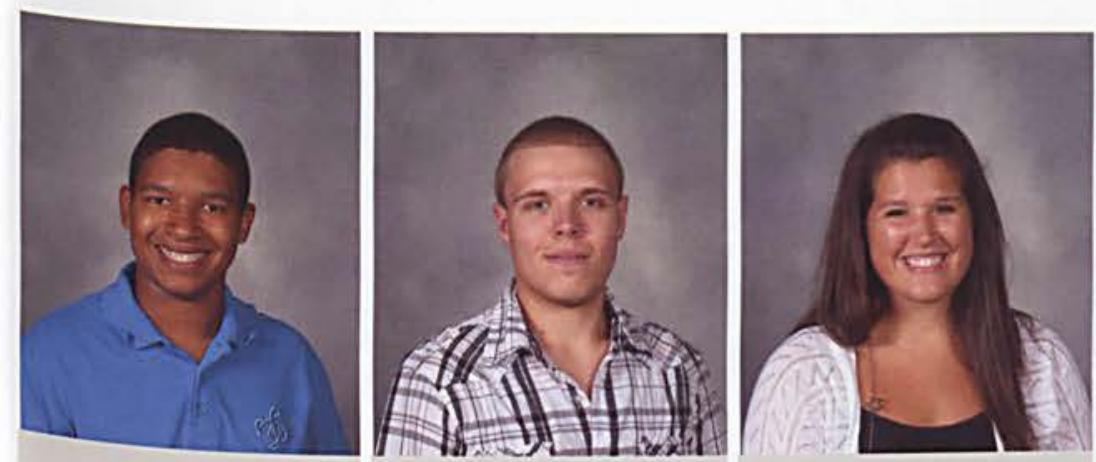

Austin Curtis

Andrew Cutting

Lindsey Cymbalak

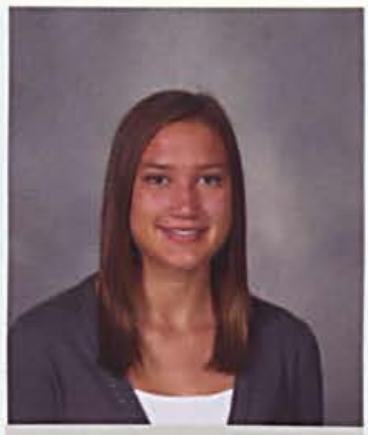

Anna Dalton

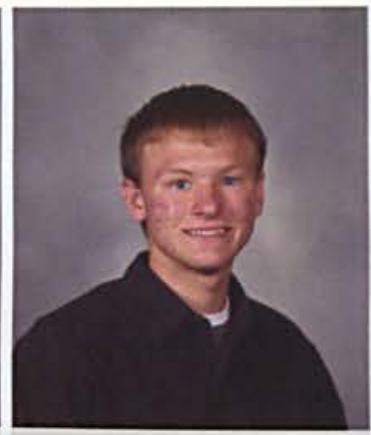

Joshua Dancer

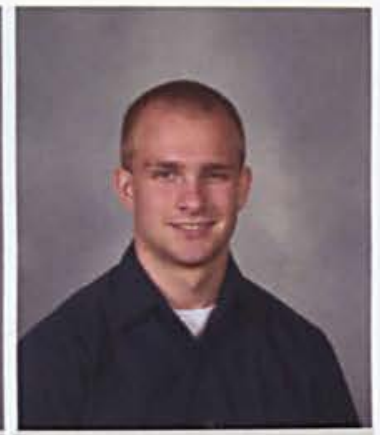

Ian Daniels

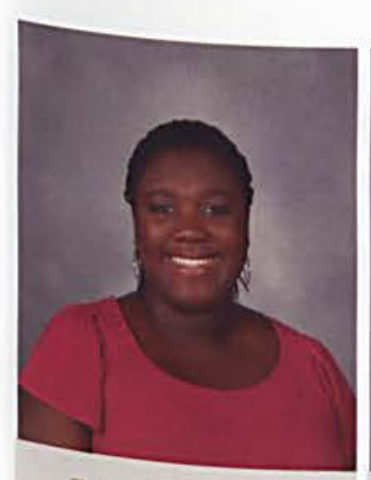

Belinda Darkwah

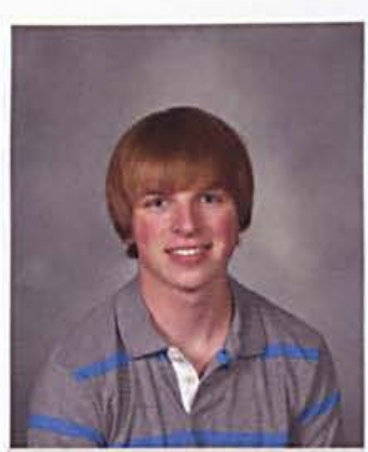

Kyle Darwin

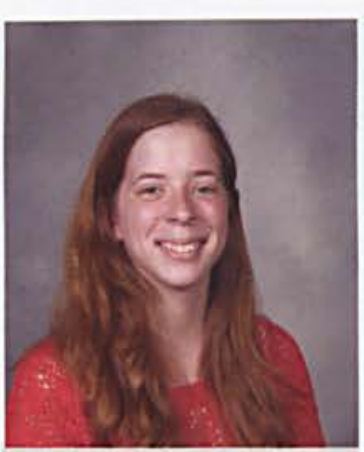

Hannah Davis

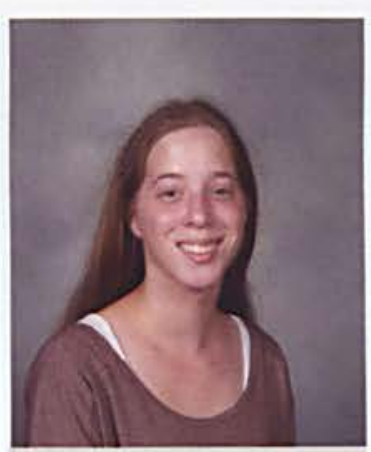

Samantha Davis

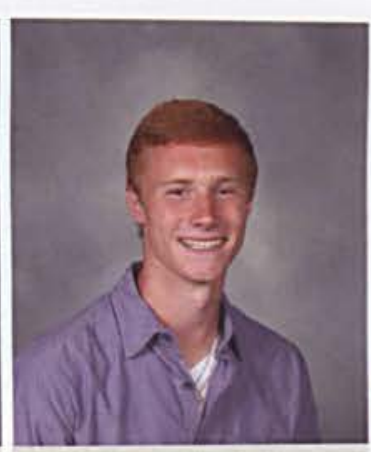

Joseph Davison

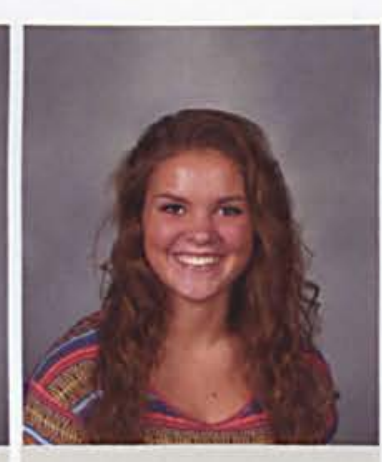

Heidi Dayton

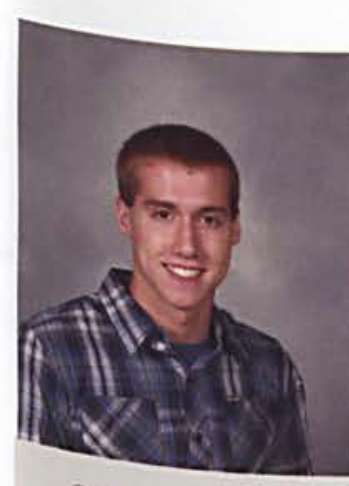

Grayson Dearing

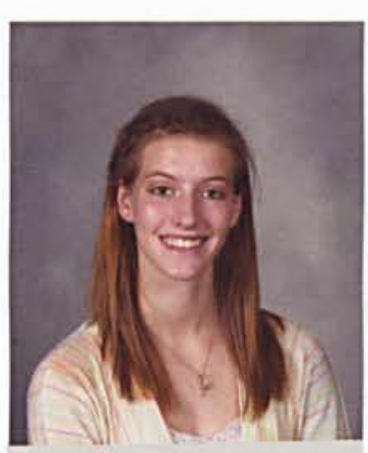

Stephanie Defore

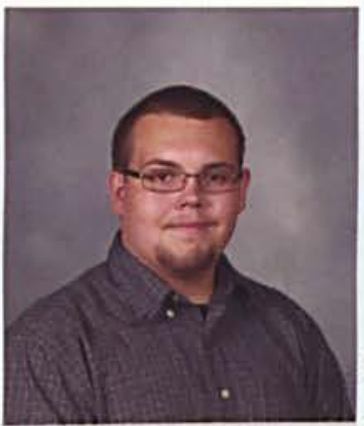

Paul DeHart

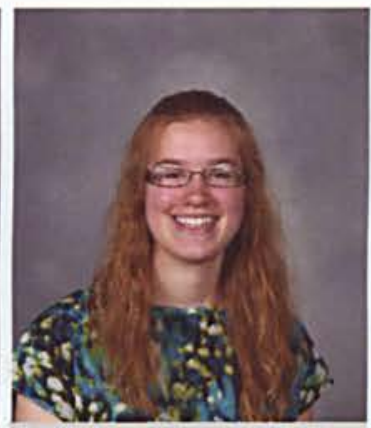

Anna Dembowski

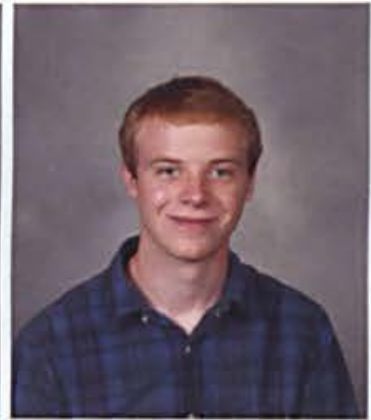

Tyler Detrick

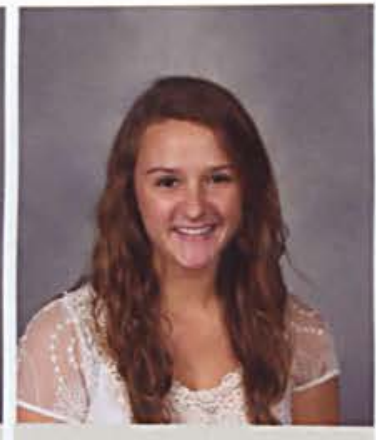

Ashley Devries

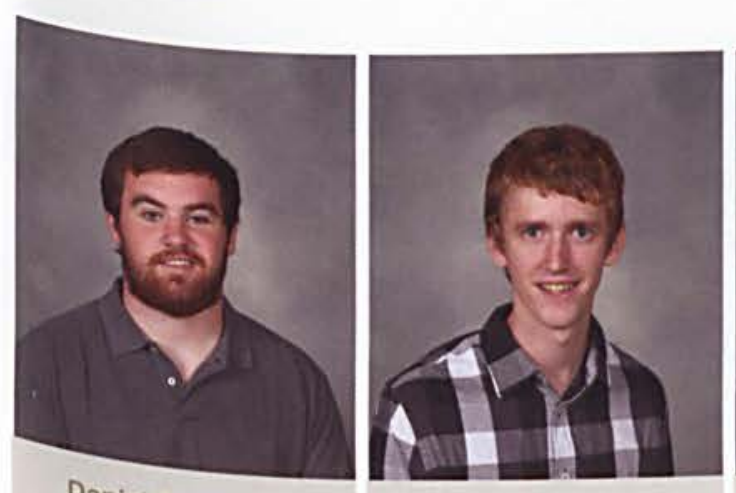

Daniel Dickerson

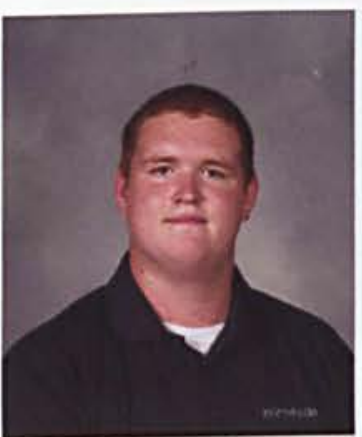

Jonathan Dodson

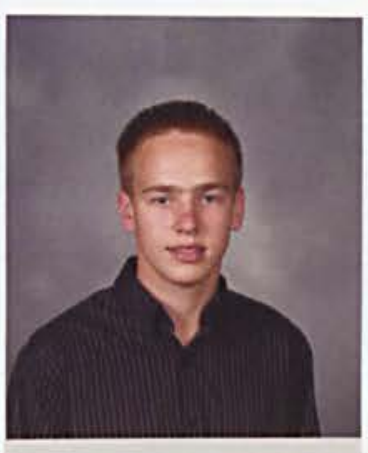

David Dombrowski

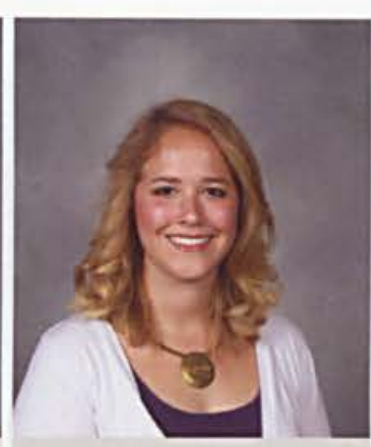

Sarah Dremann

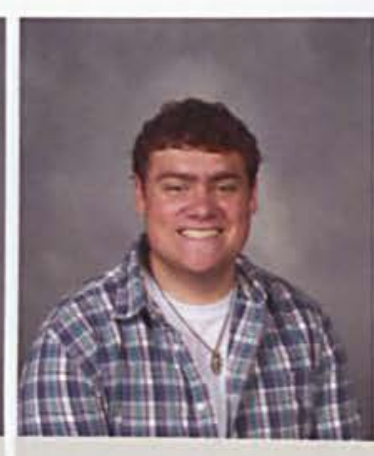

William Duncan

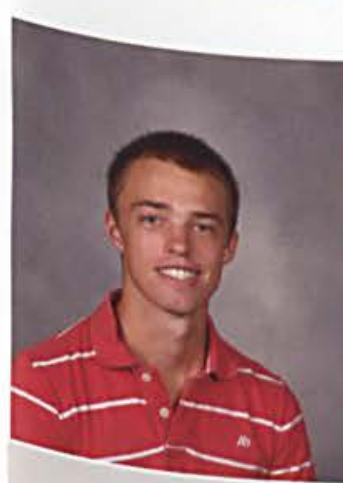

Justin Eckberg

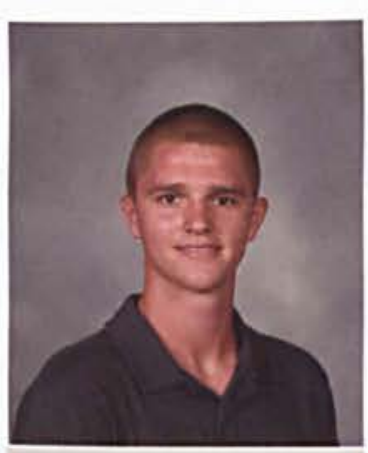

Nathan Edwards

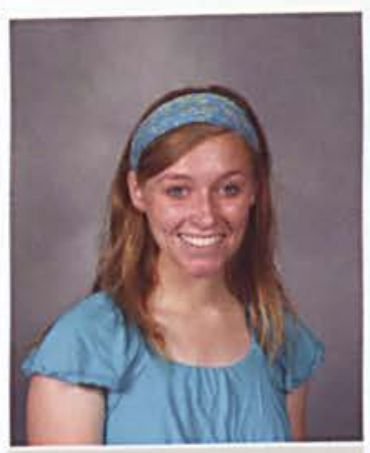

Laura Egan

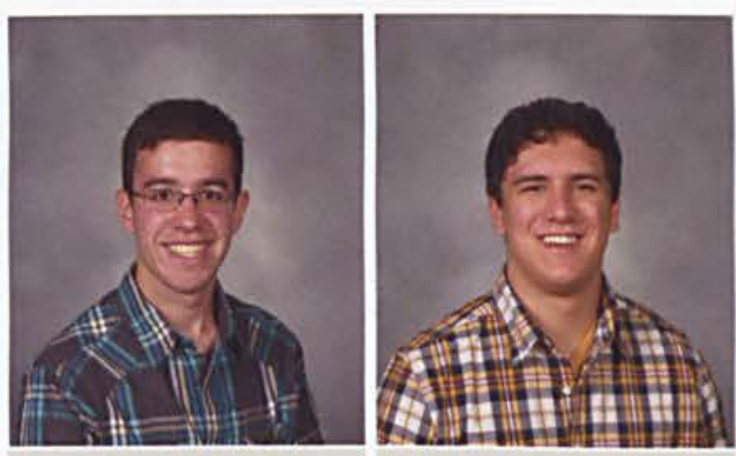

Alexander Eliopulos

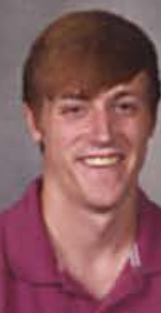

Cole Ericson 


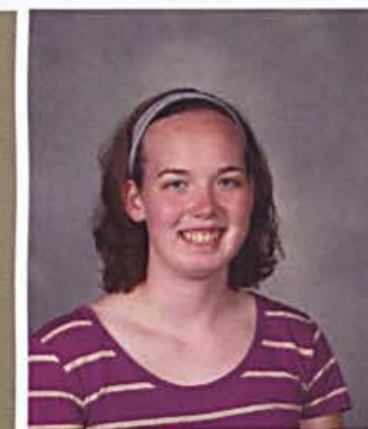

Michelle Eriksen

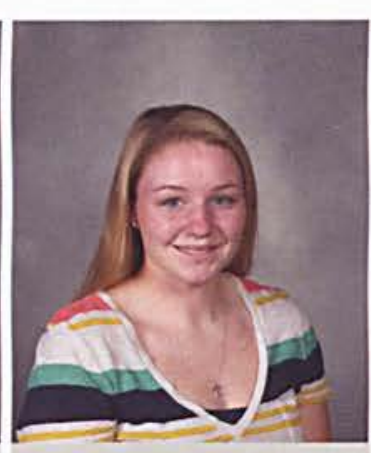

Jenna Erwin

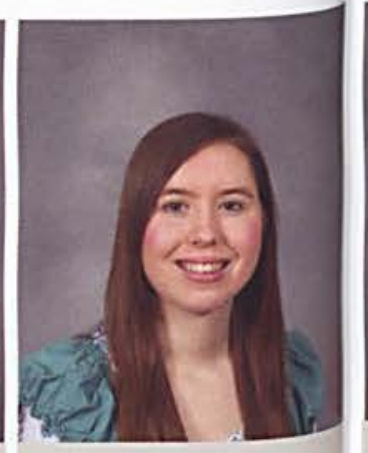

Victoria Eustace

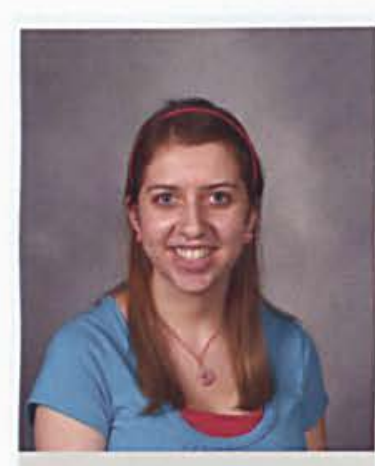

Jessica Evanoff

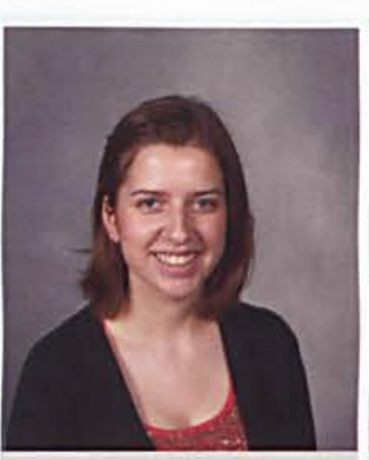

Julia Evanoff

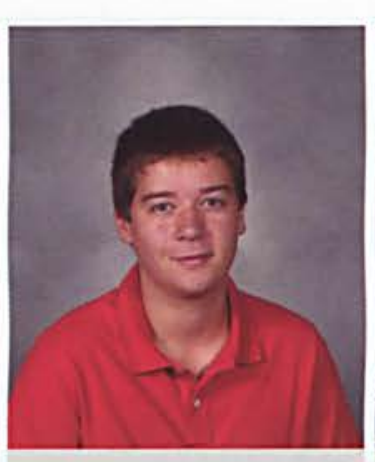

Matthew Fakhoury

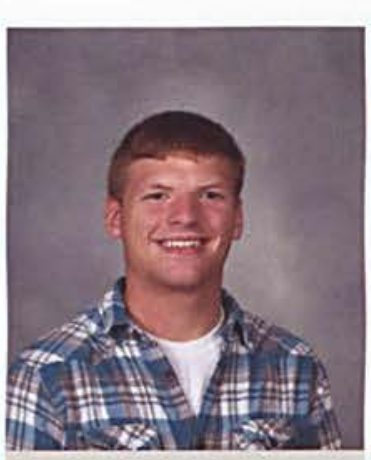

Benjamin Fawcett

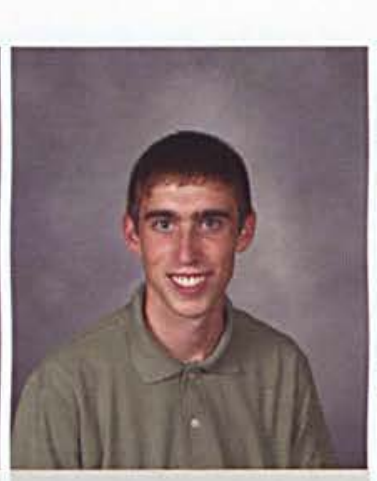

Luke Febrizio

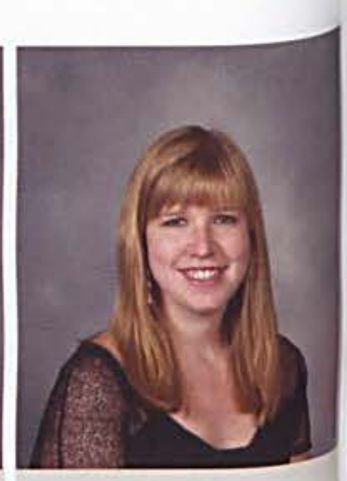

Kathryn Field

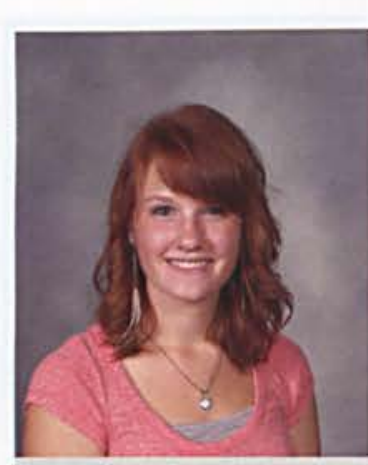

Caitlin Fillmore

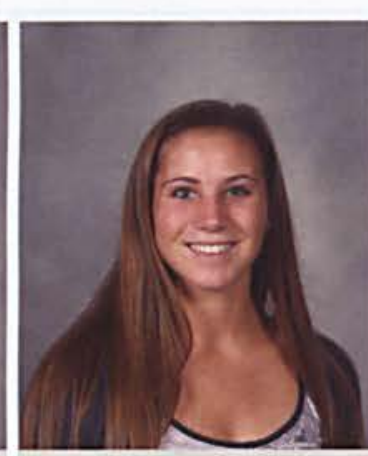

Jessica Finfrock

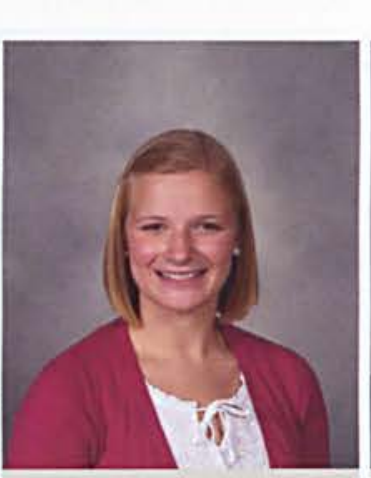

Anna Finke

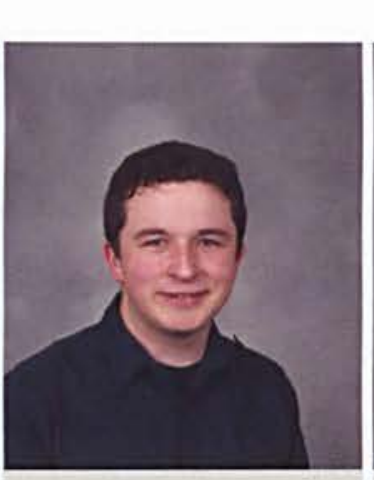

Logan Fitzgerald

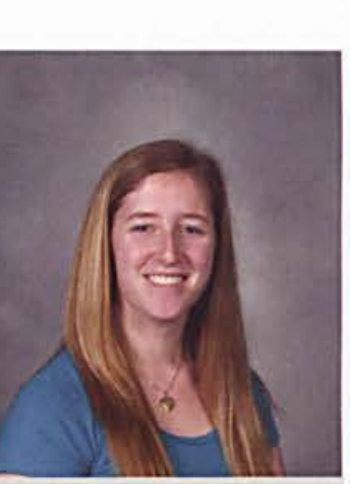

Sarah Fox

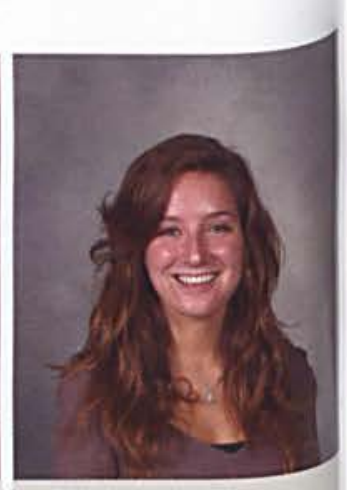

Rachel Fraga

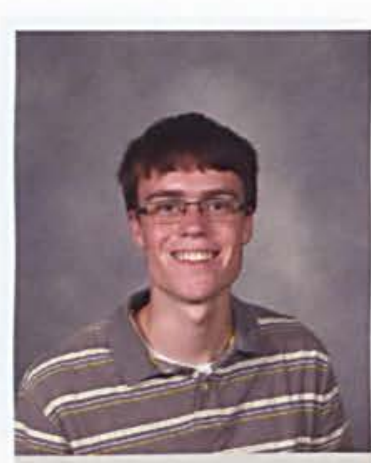

Samuel Franklin

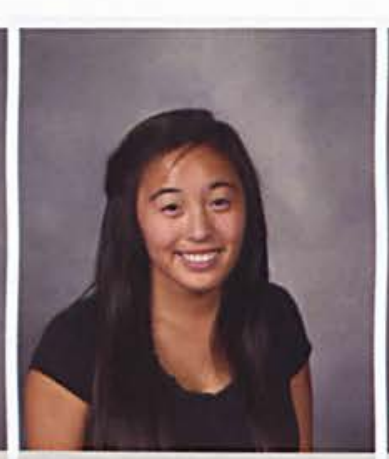

Candace Frazier

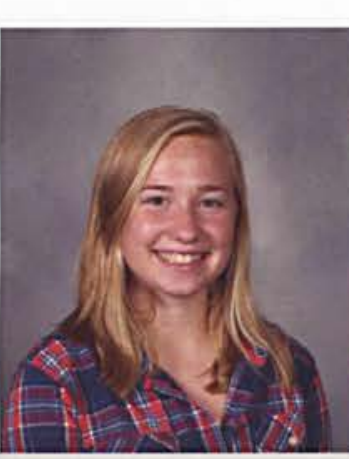

Kayla Fry

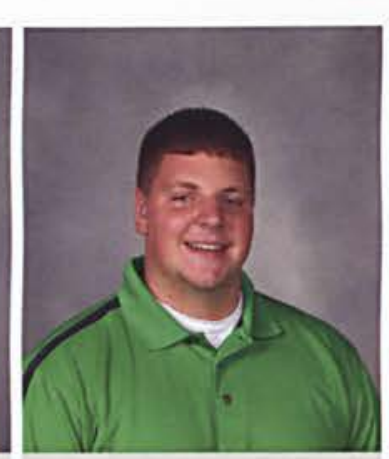

Brian Funk

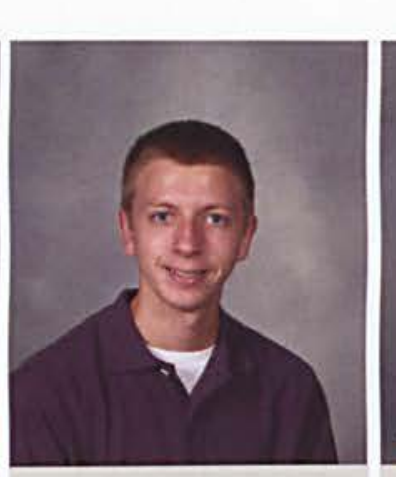

Kyle Funtik

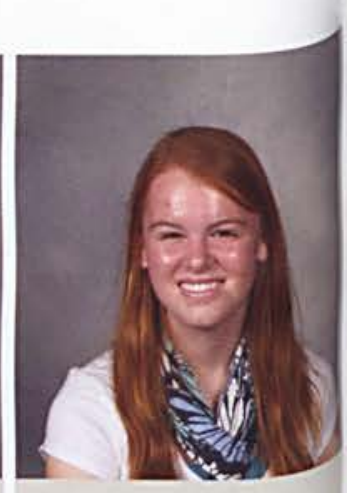

Leah Furrow

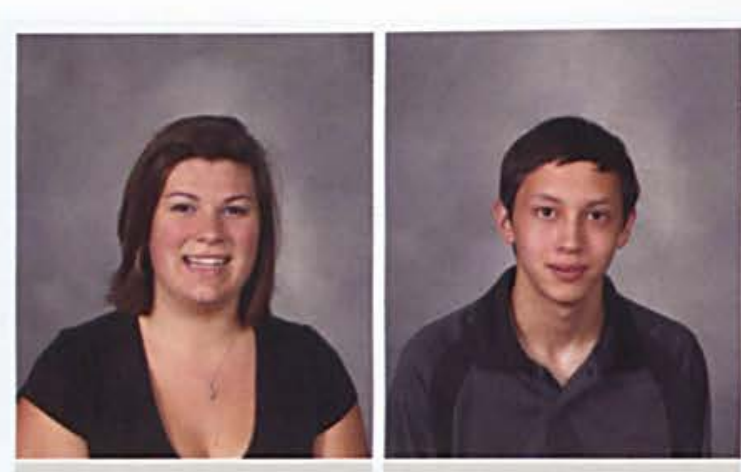

Amie Gale

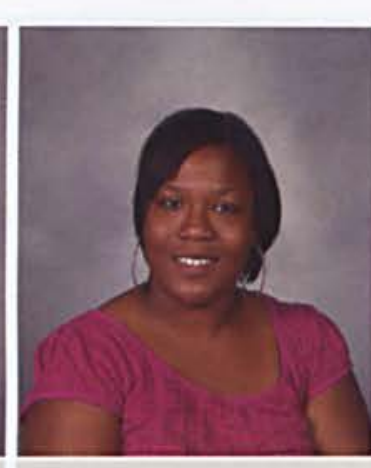

Keyana Galloway

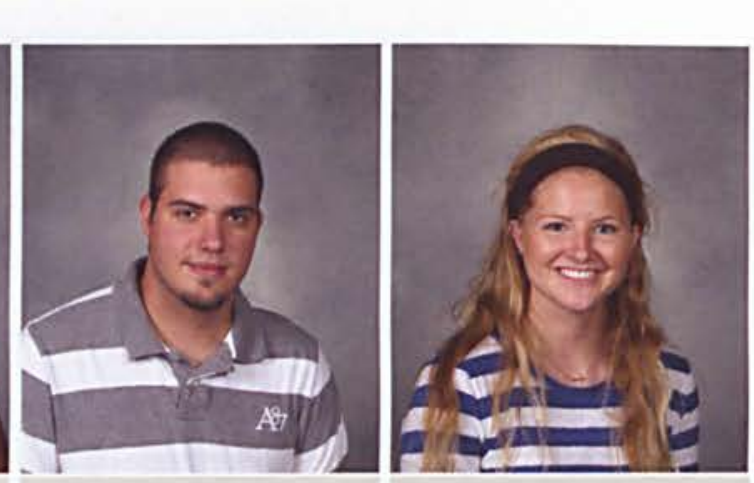

Carl Gambrell

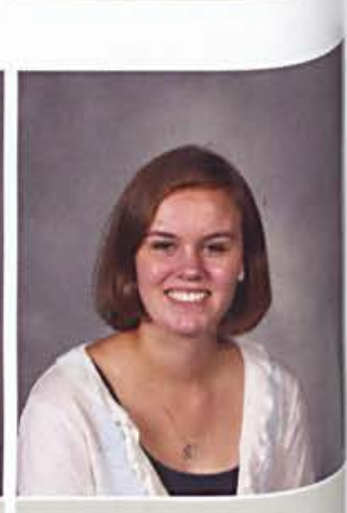

Hannah Gendron 
72 FRESHMEN

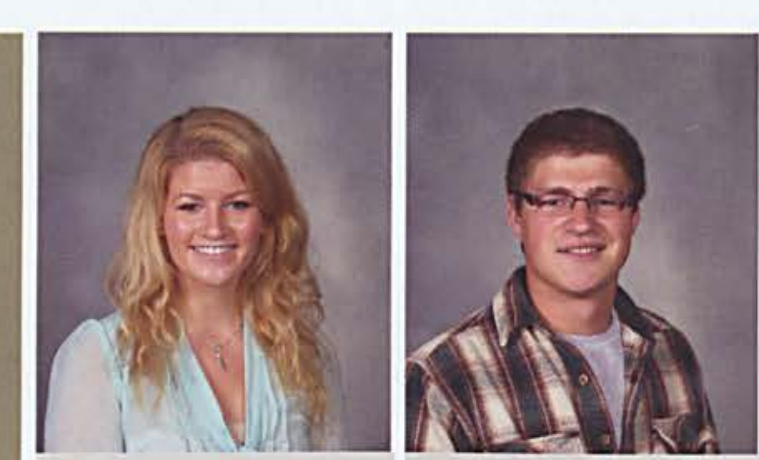

Jennifer Hefner

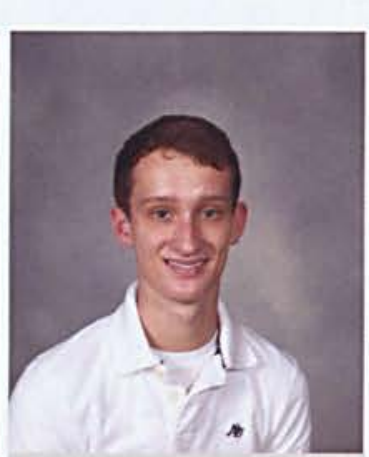

Samuel Hinckley

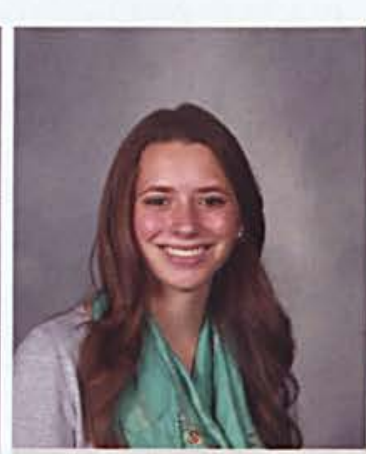

Kathryn Hinman

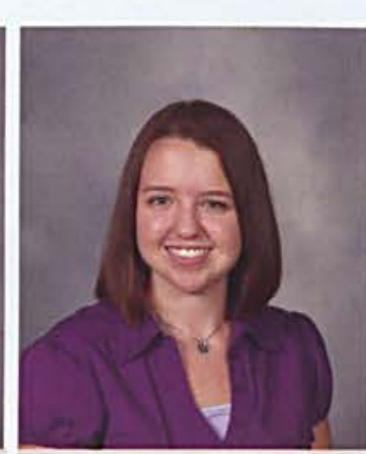

Jessica Hise
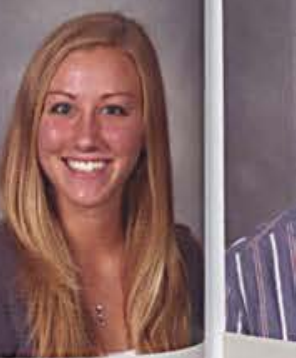

Danae Hershbergef

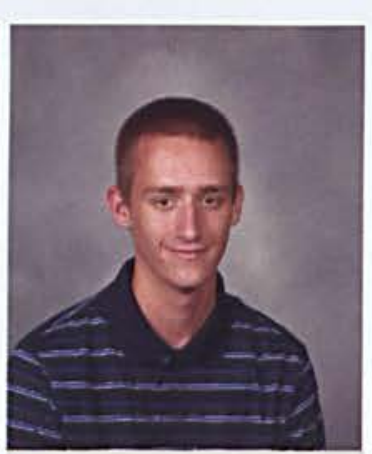

Benjamin Howder

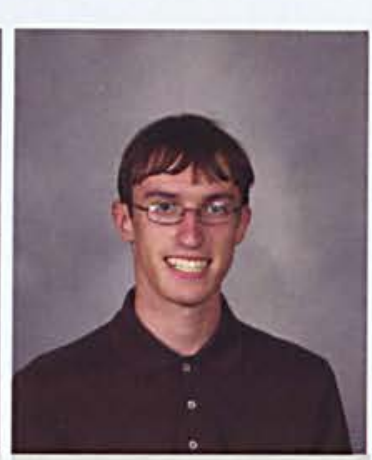

Caleb Howland

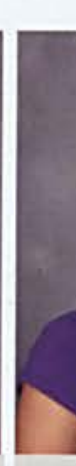

Rebecca Hughes

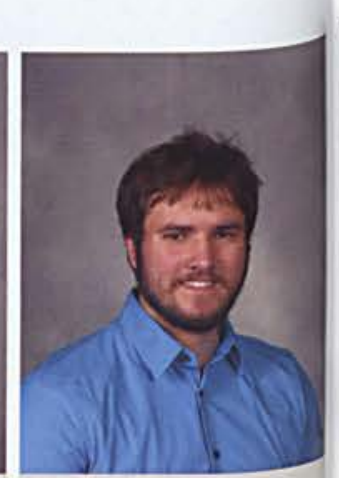

Ian Holloway

Jared Holsclaw

Grant Hooper

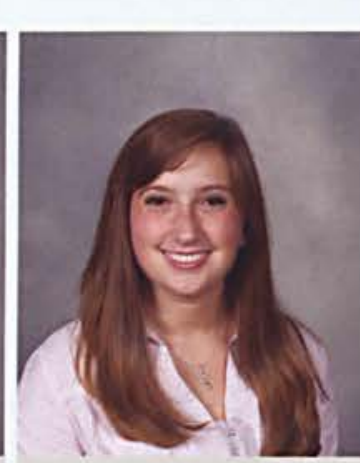

Jennifer Hutchison

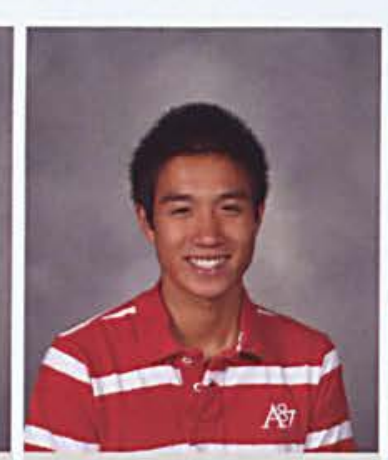

Samuel Huynh

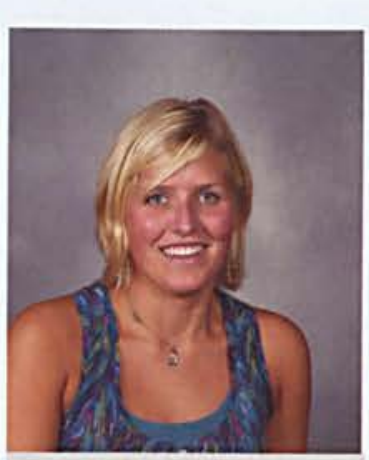

Stephanie Huyser

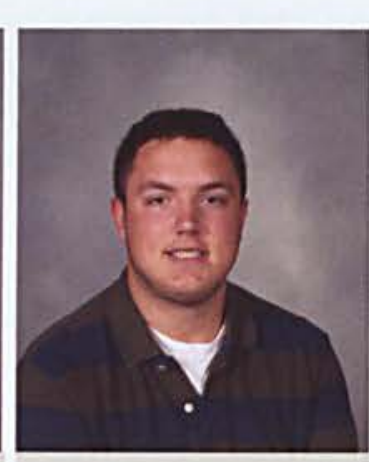

Peter Hykes

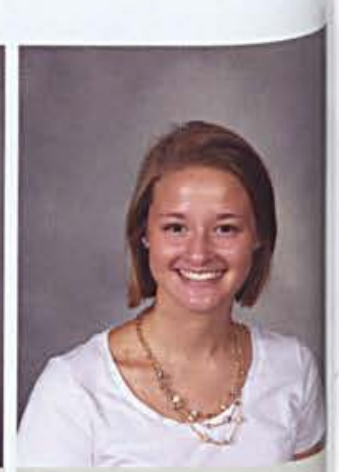

Kendra Huhta

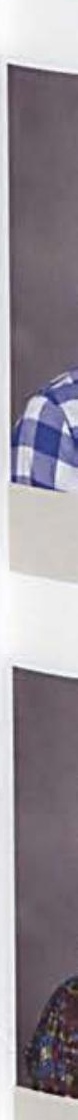

Bethany Hull

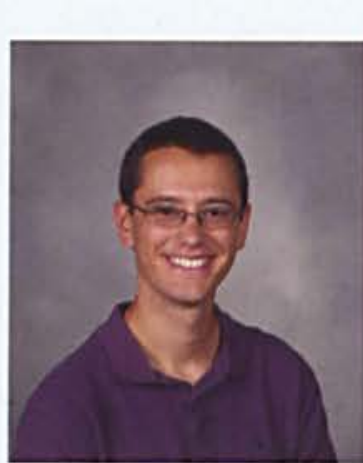

Benjamin Ingis

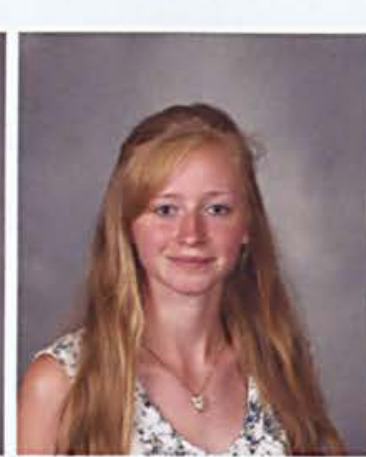

Micaila Iversen

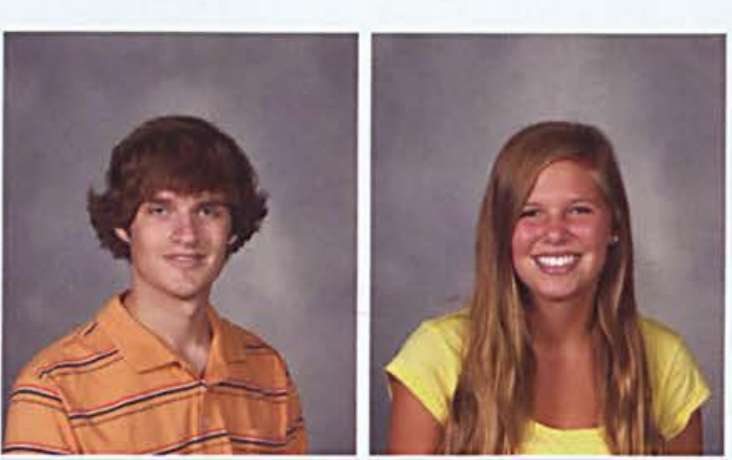

Wyatt Jackson

Alicia James

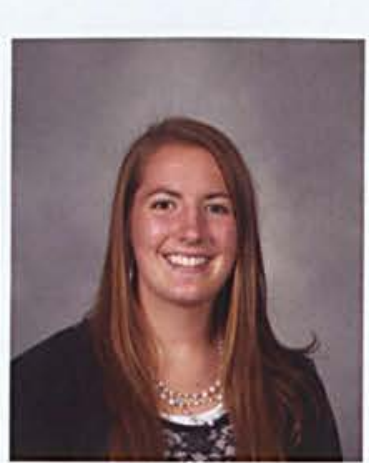

Zoe Jamison

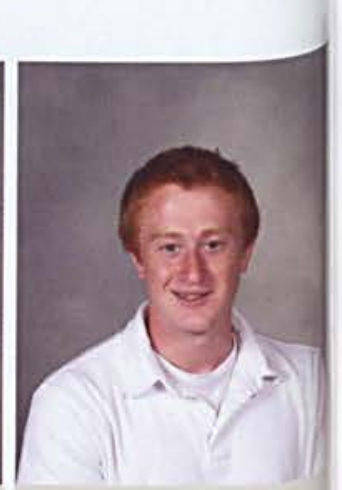

Jacob Imhof

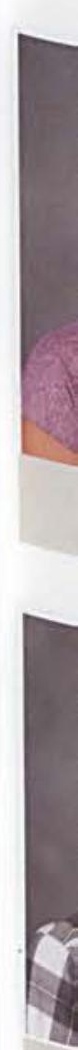

Adrian Jantzi 


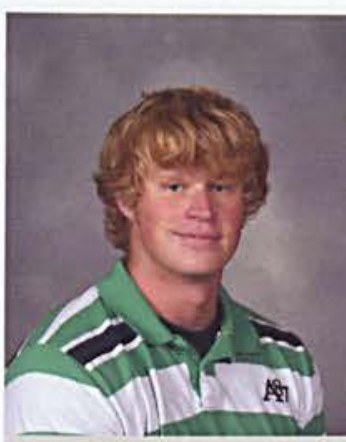

Joshua John

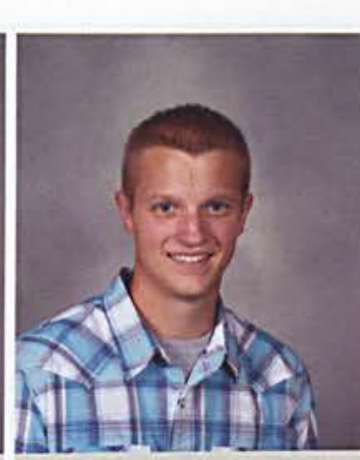

Andrew Johnson

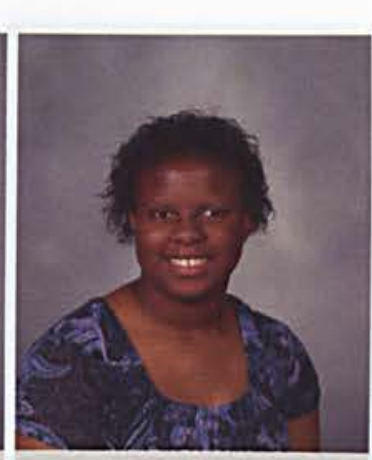

Anna Johnson

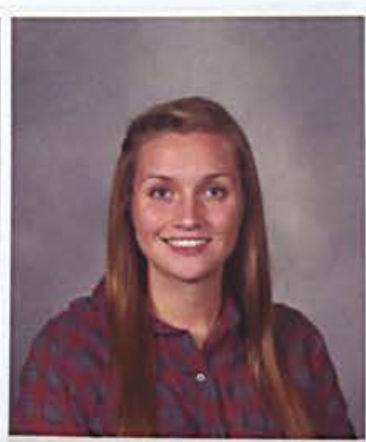

Christa Johnson

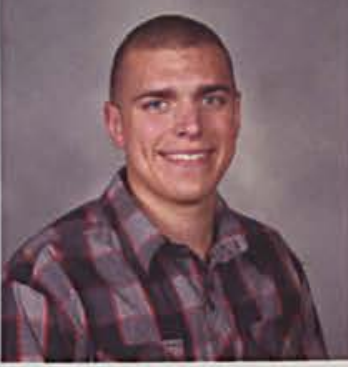

David Johnson

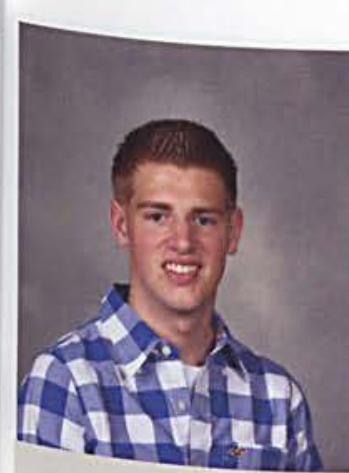

Ryan Johnson

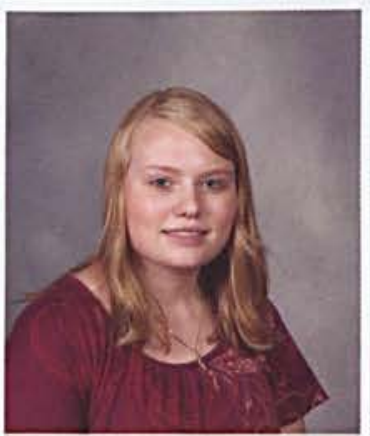

Jennifer Johnston

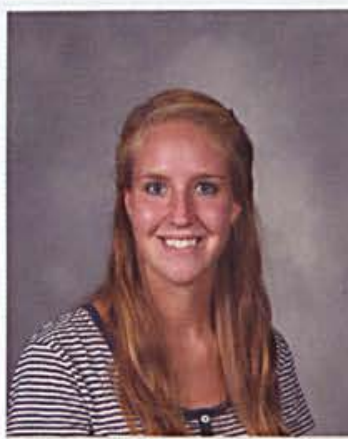

Natalie Juergemeier

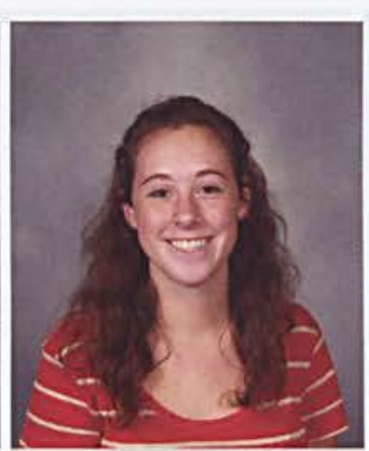

Audrey Jung

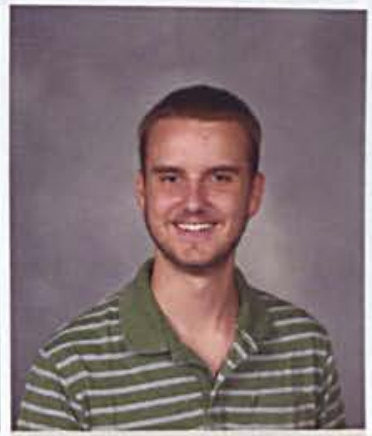

Nathan Kallman

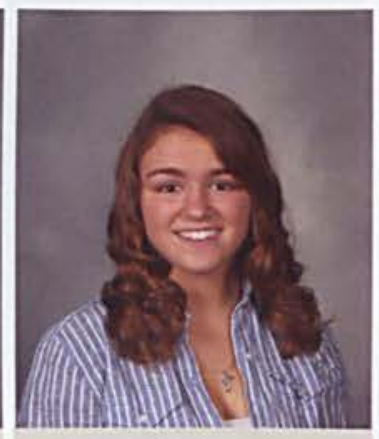

Ciara Kaufman

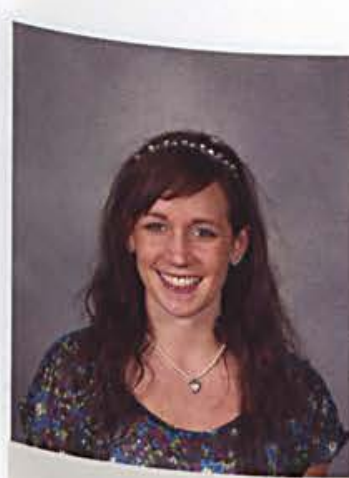

Ellery Kent

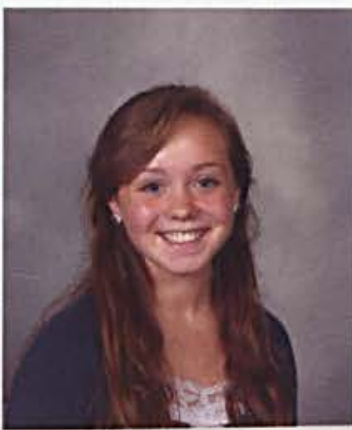

Angelica Kester

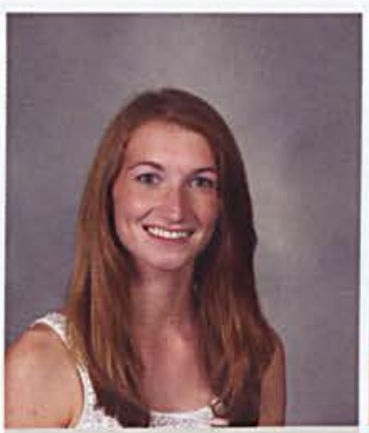

Kathleen Kidd

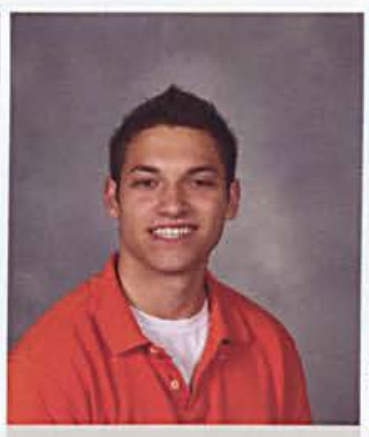

Brett King

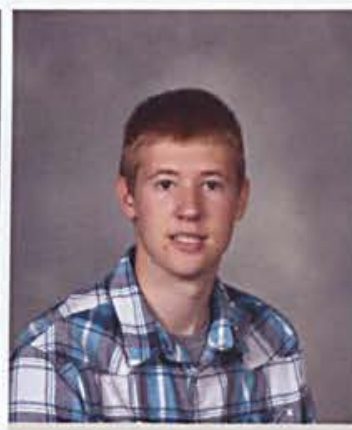

Joshua Kirschbaum

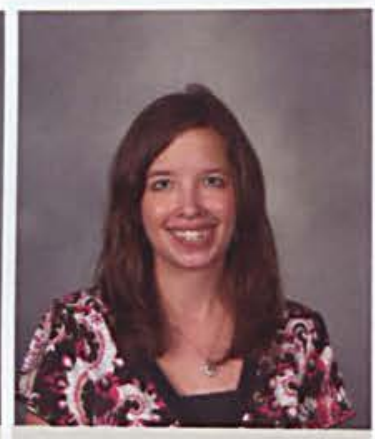

Michelle Kisha

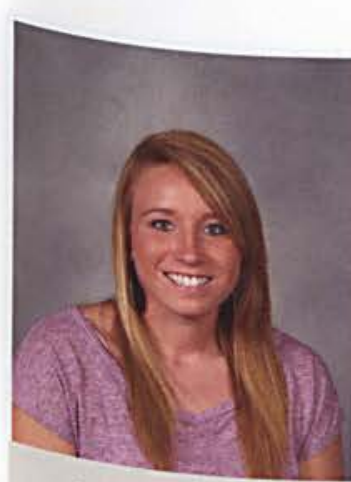

Hayley Kitchen

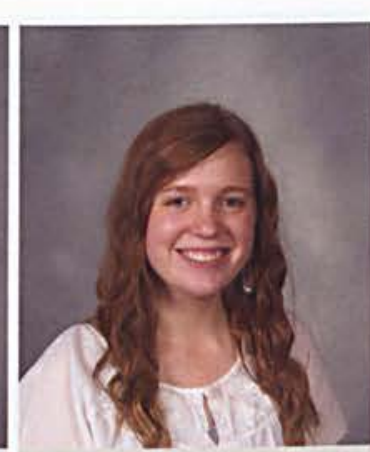

Cierra Klatt

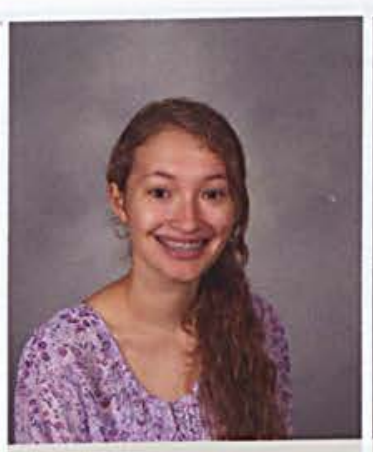

Ayana Kleckner

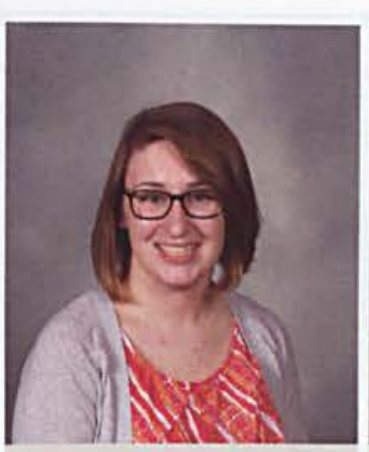

Karis Knepper

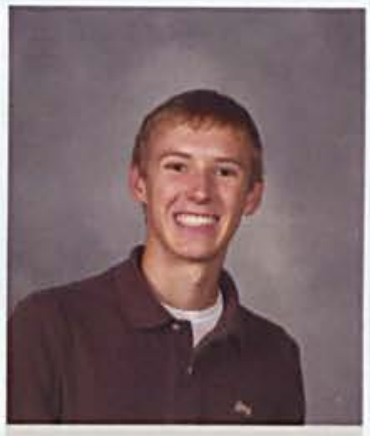

Daniel Kohl

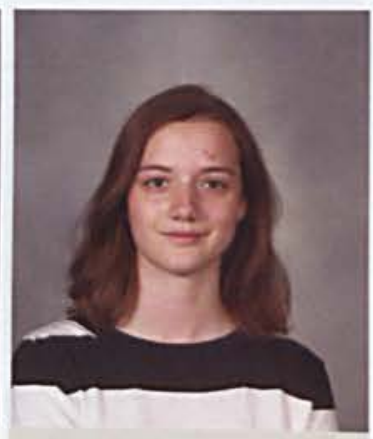

Paulene Kolota

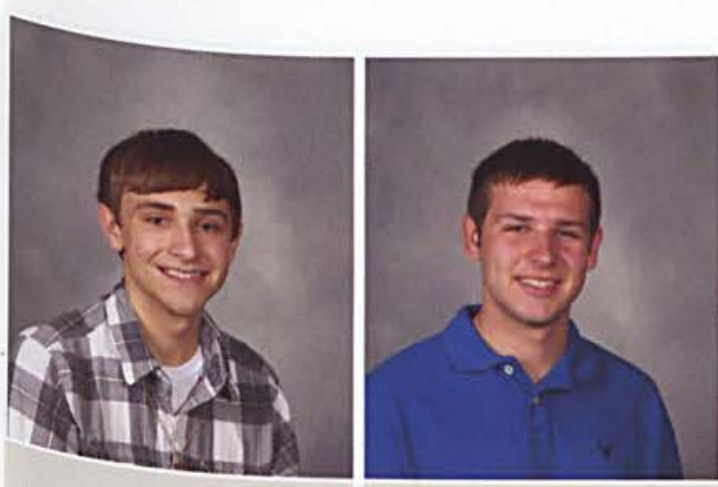

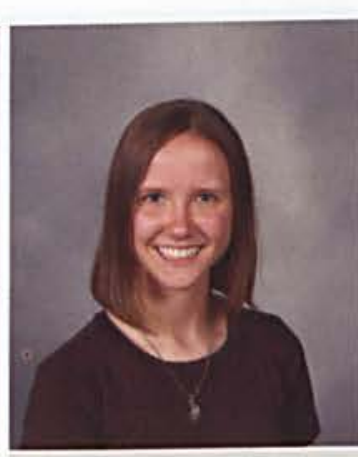

Laura Krizo

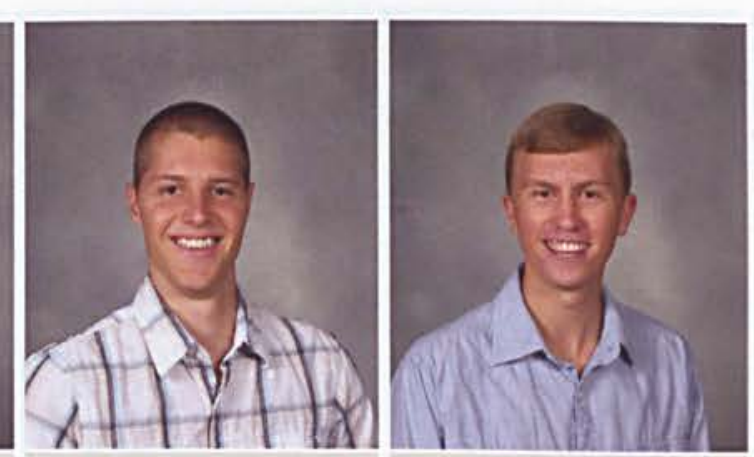

Collin Kueter

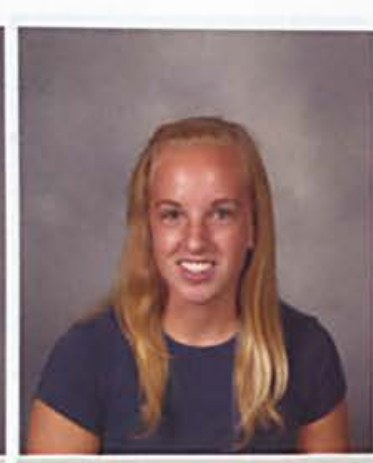

Christine Kuiken 
74

FRESHMEN

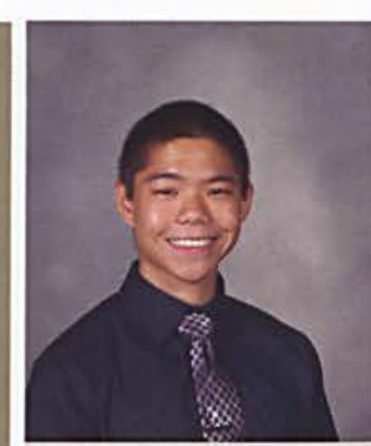

Matthew Kuo

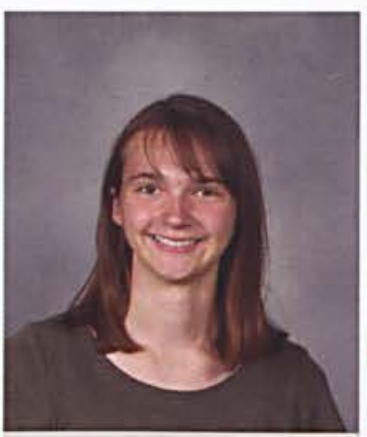

Kristen Laaman

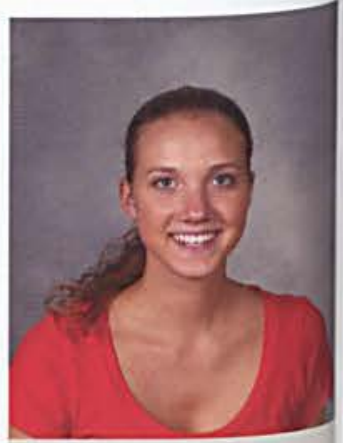

Ashley Laird
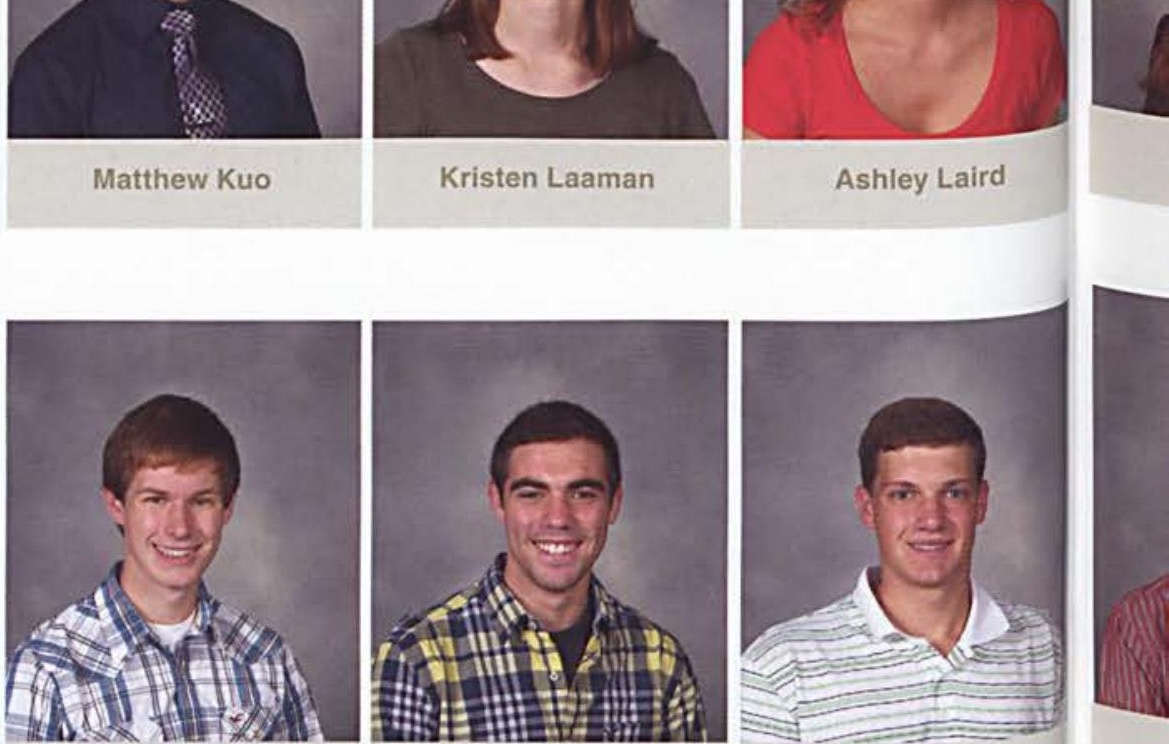

David Law

Jordan Lee

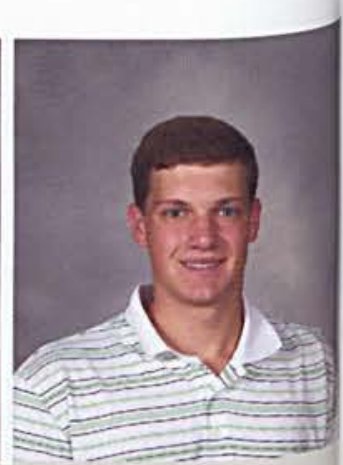

Jason Leigh

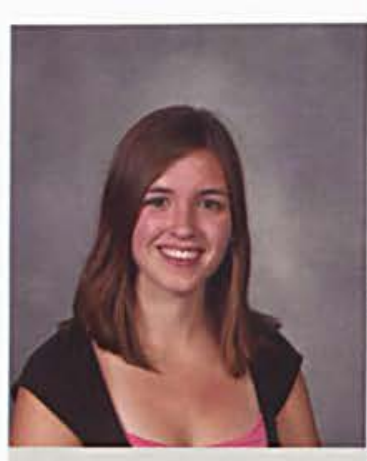

Rebecca Levergood

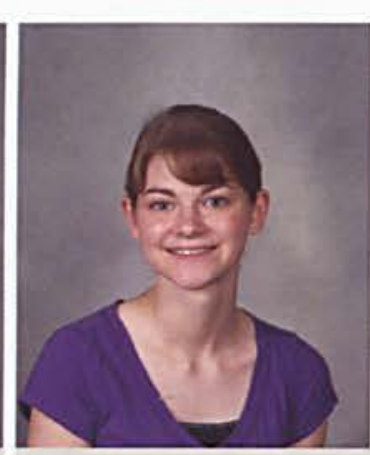

Morgan Liggett

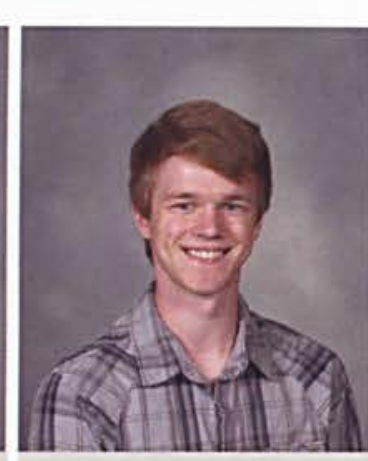

Jack Lightbody

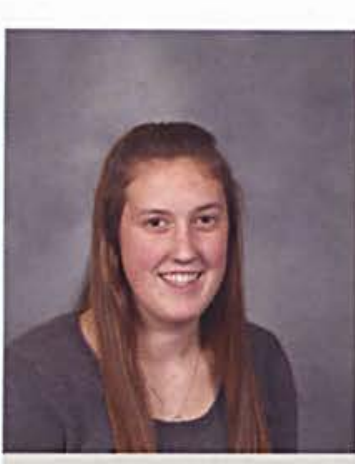

Jessica Lingaas

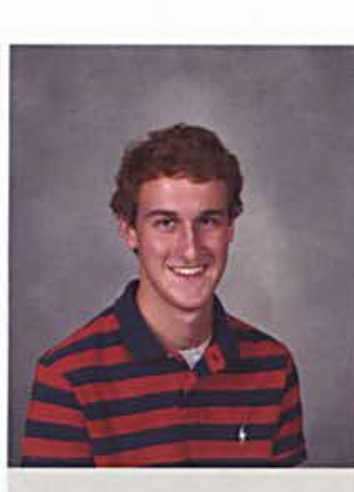

Seth Lingenfelter

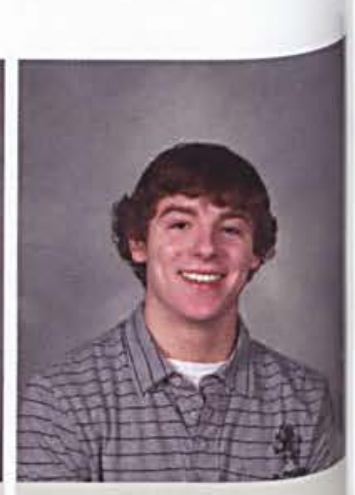

Alexander Link

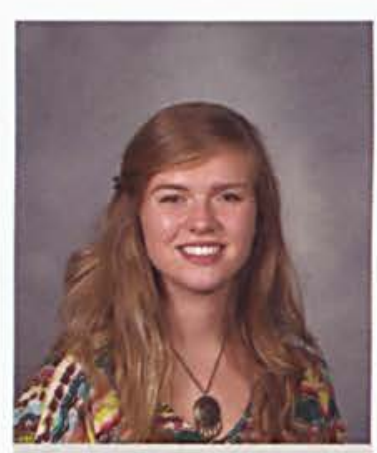

Tani Linville

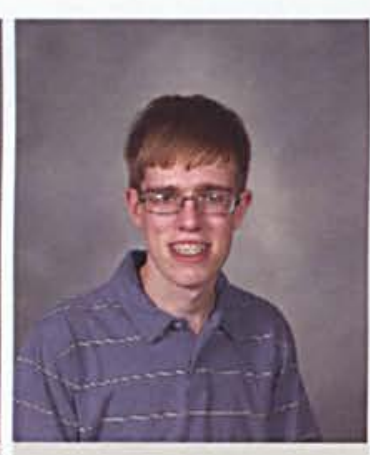

J. Little

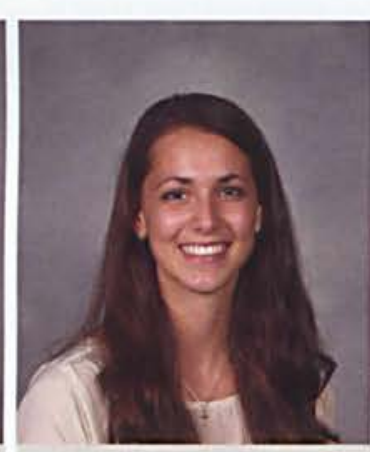

Kathryn LiVigni

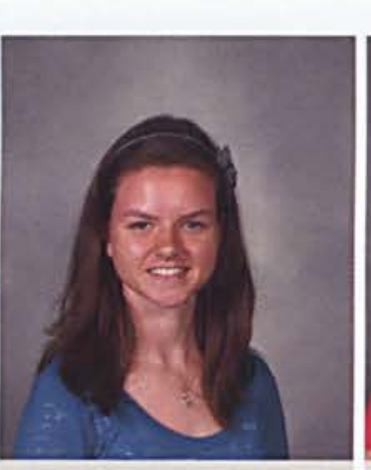

Alison Logan
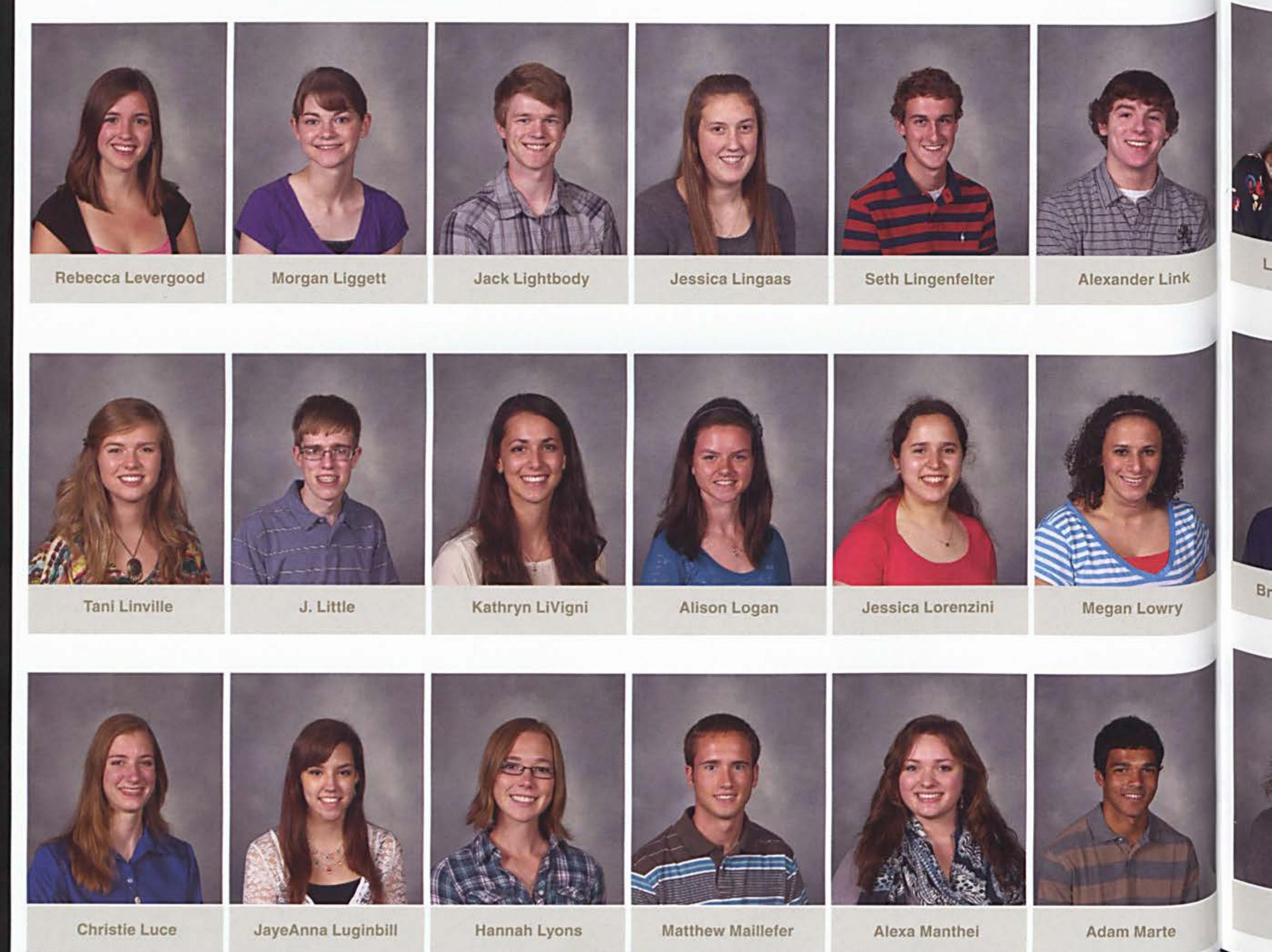


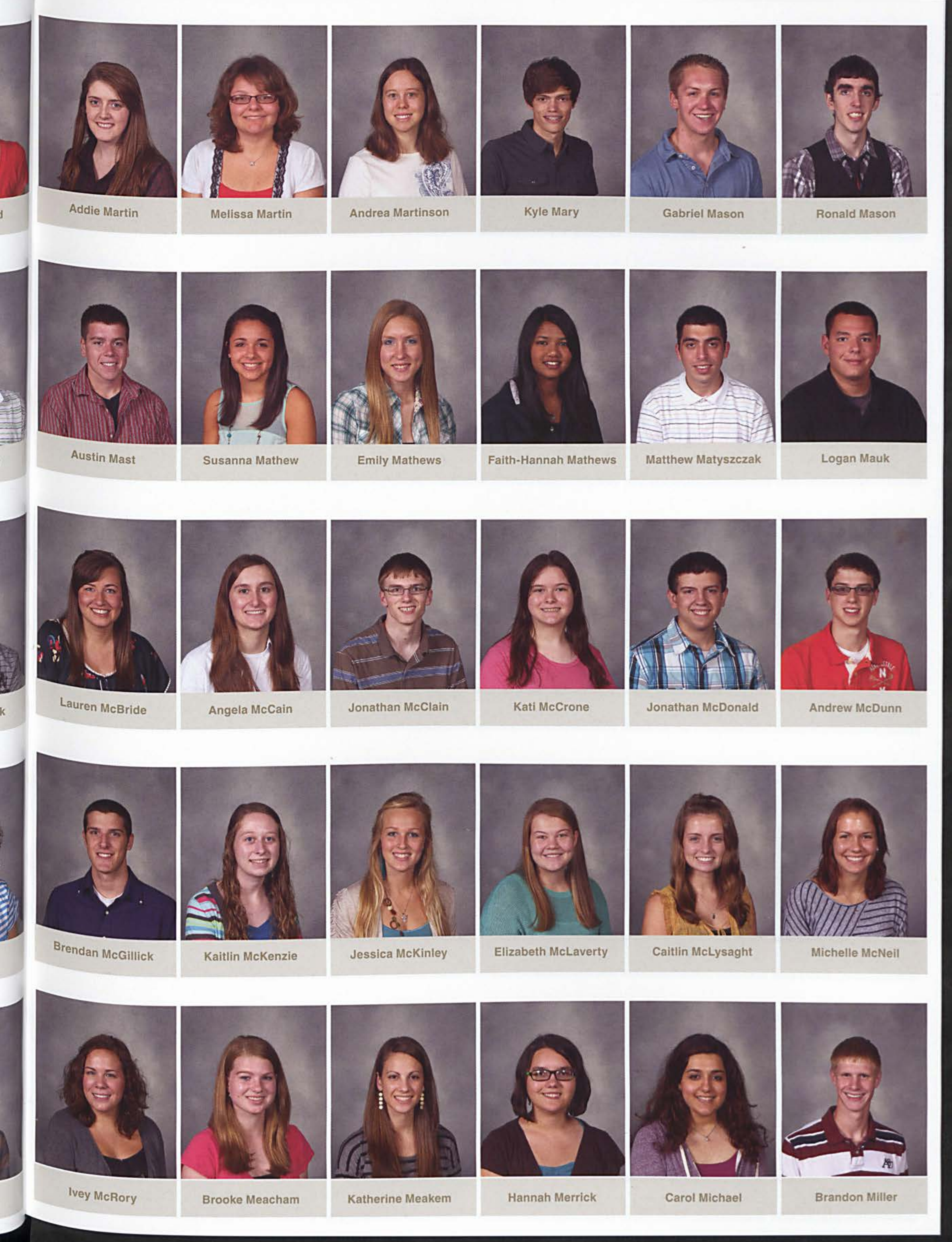


76

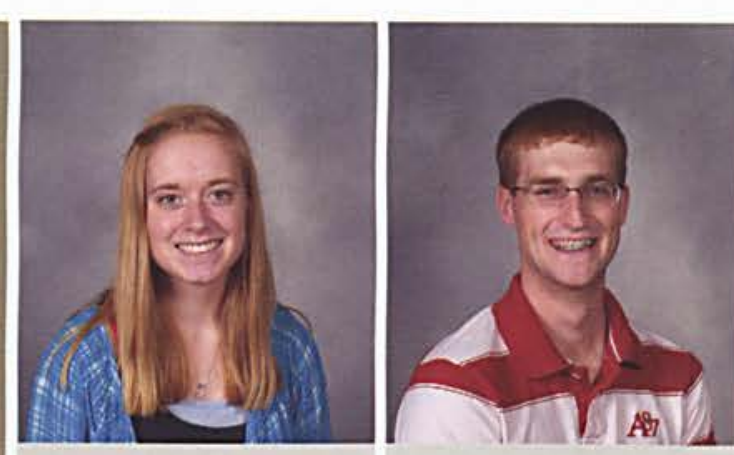

Kiyla Miller

Phillip Minich

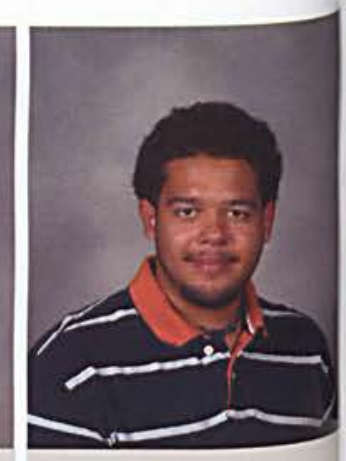

Julio Miranda

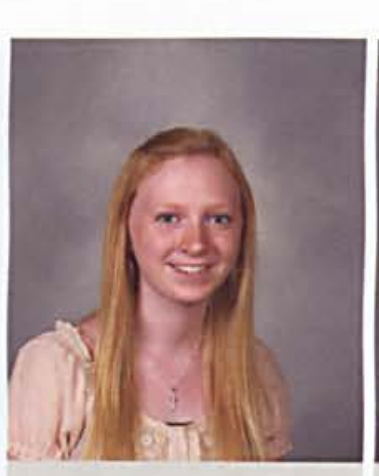

Rebekah Mitchell

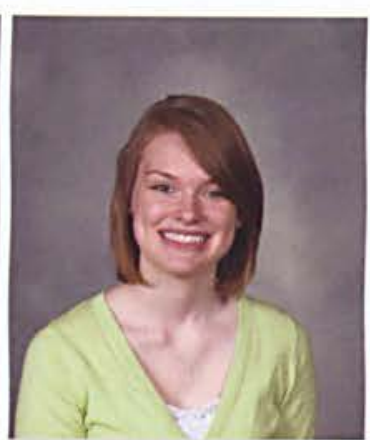

Sarah Moats

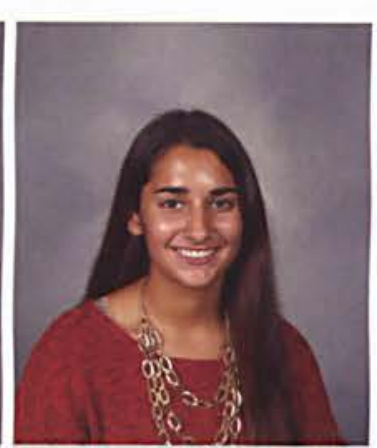

Andria Mody

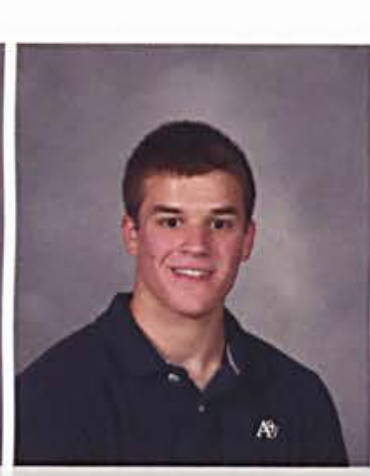

Ryan Montross

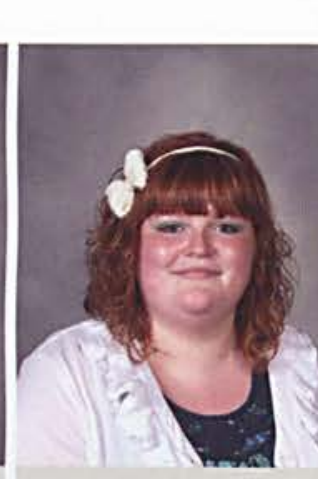

Abigail Moon

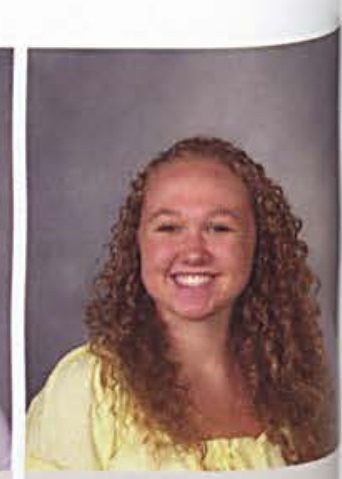

Brittnee Moore

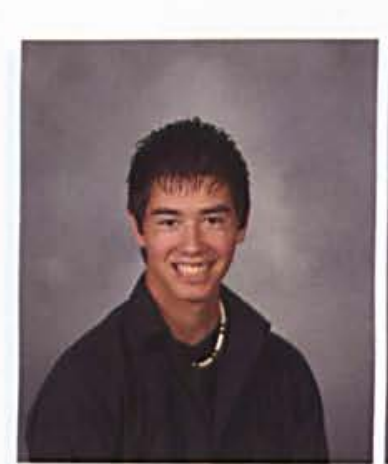

Garrett Moore

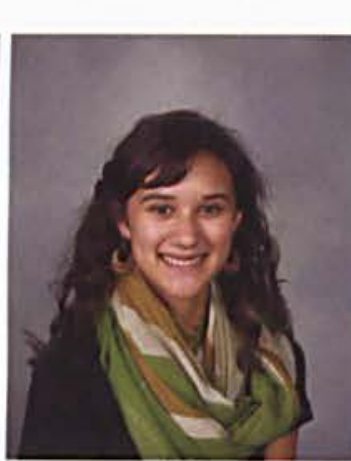

Jessica Moreno

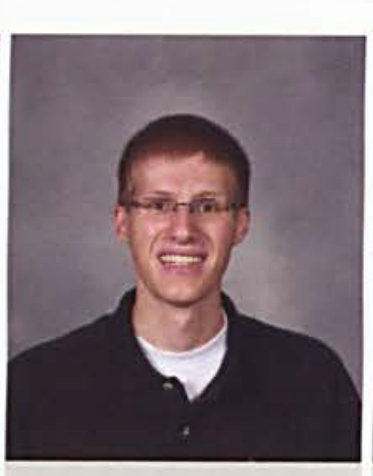

Joseph Morley

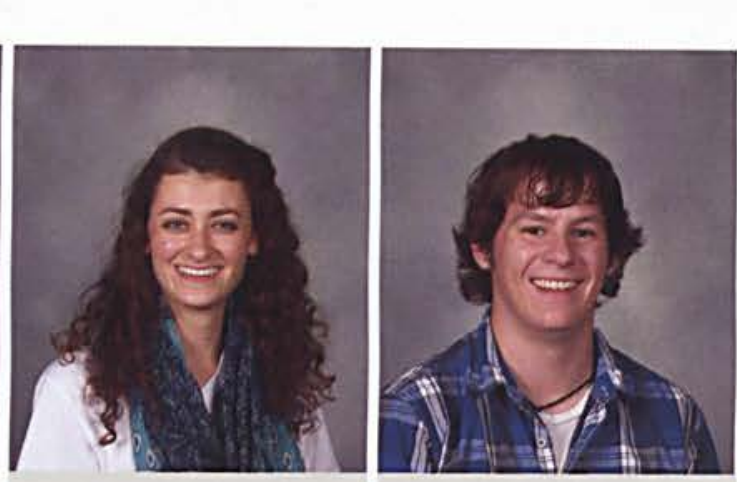

Casey Morrone

Tyler Morton

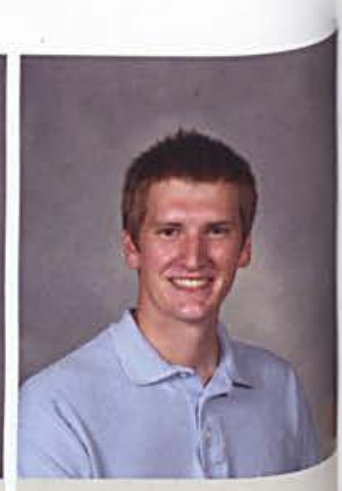

Isaac Moss

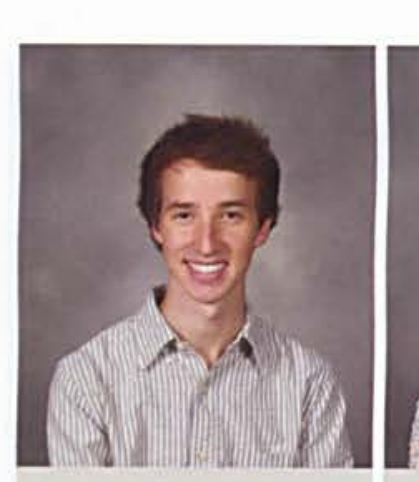

Brett Muschott

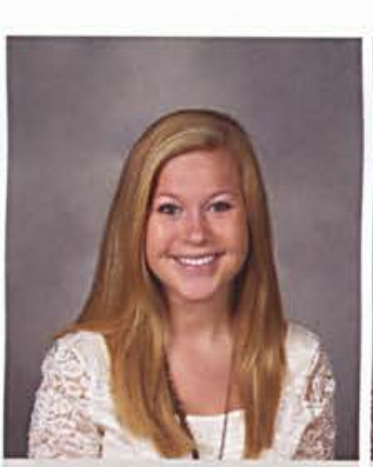

Emily Myers

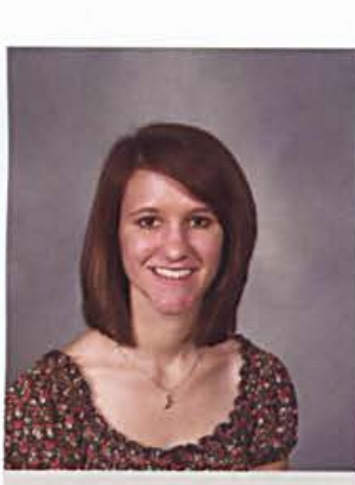

Natalie Neidig

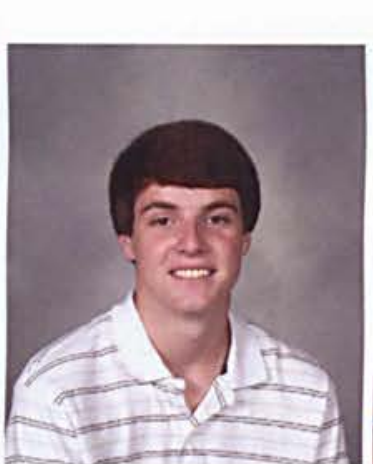

Casey Nelson

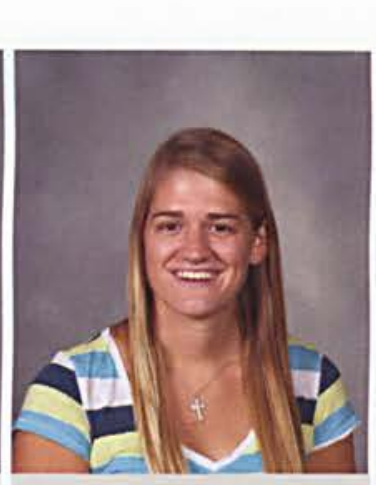

Patricia Neve

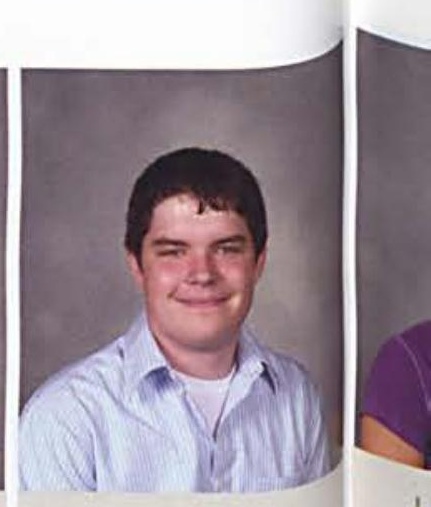

John Neville

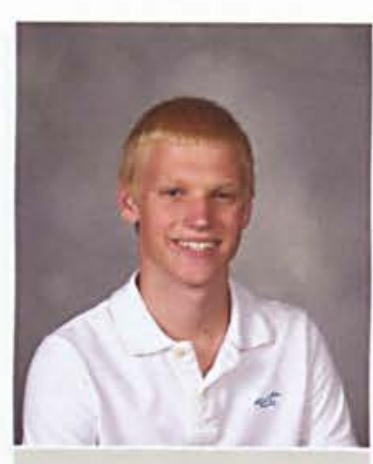

Austin Newlin

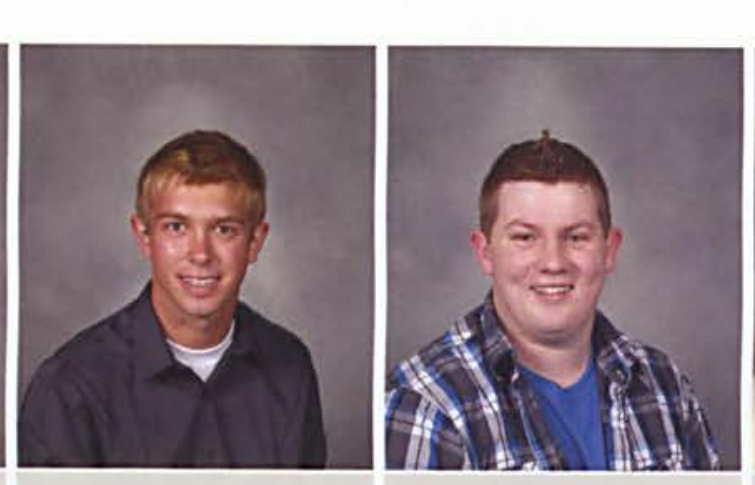

Jared Newman

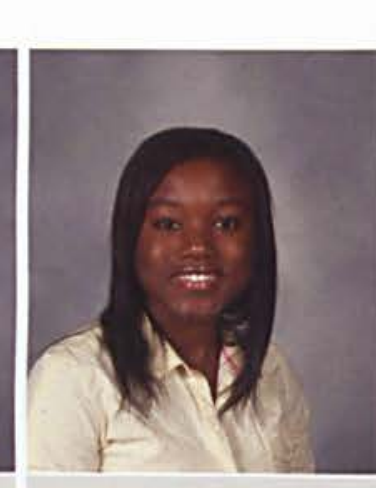

Deborah Obielodan

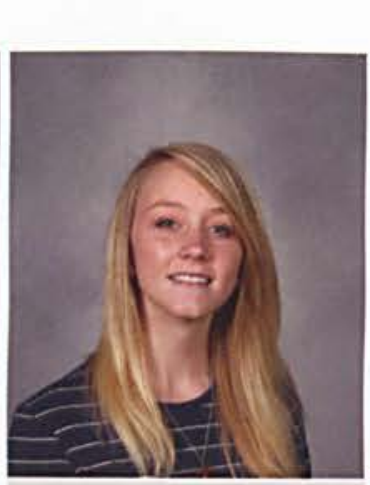

Brenna O'Brien

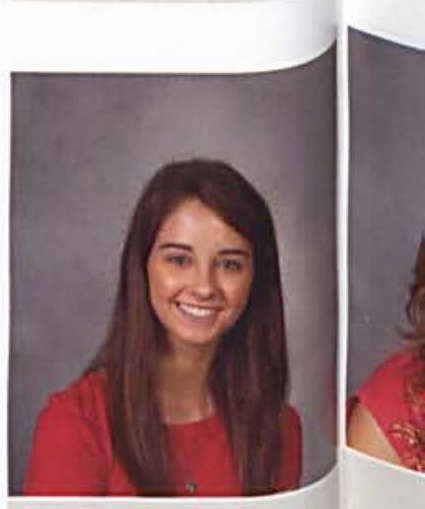

Stephanie O'Brien 


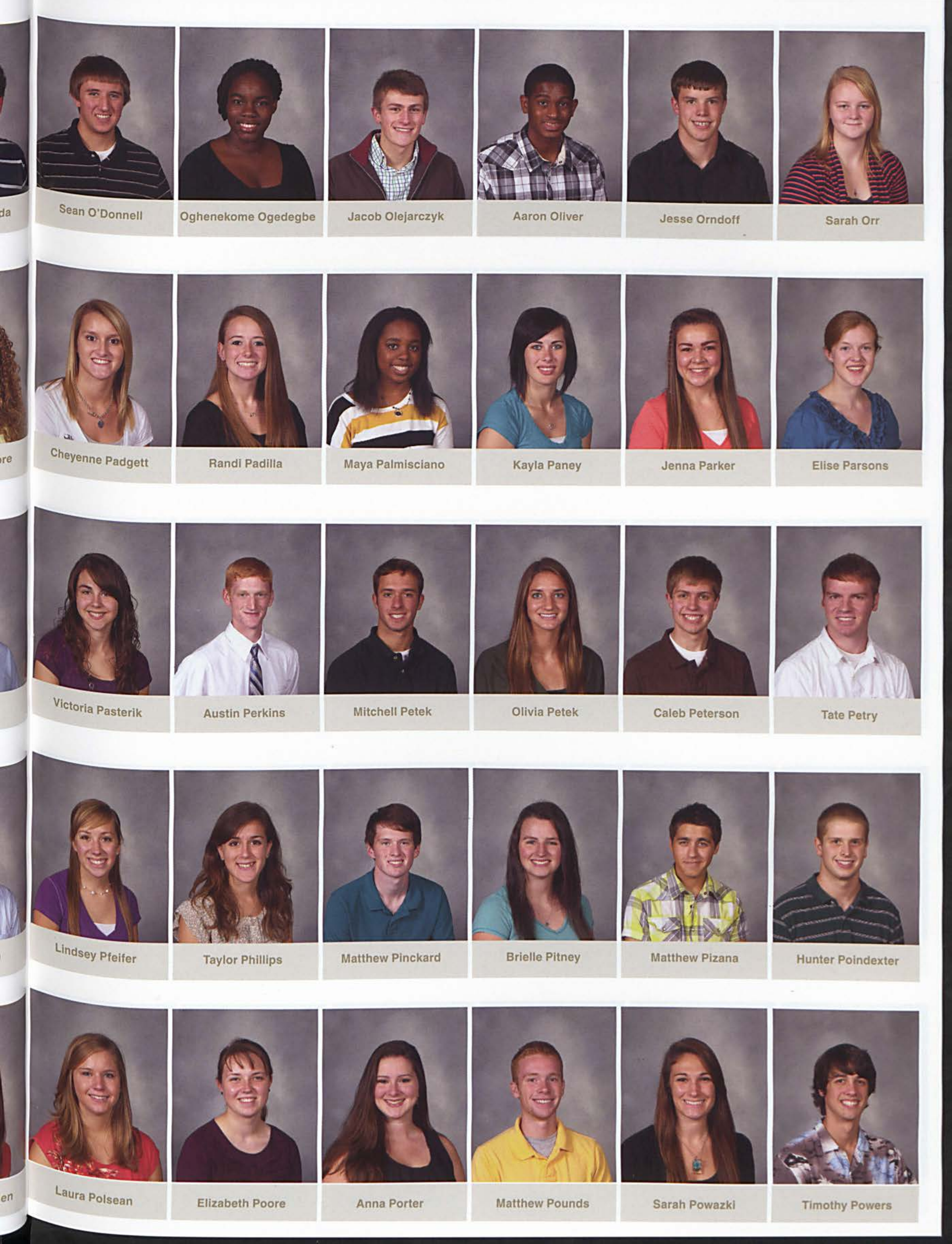


78

FRESHMEN

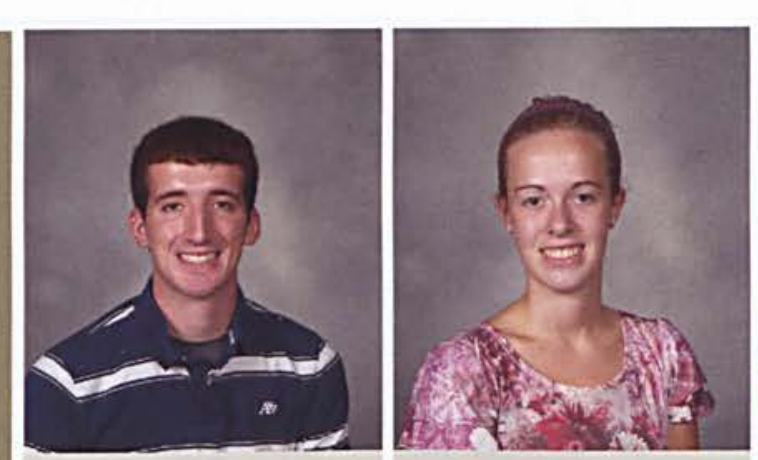

Jacob Prem

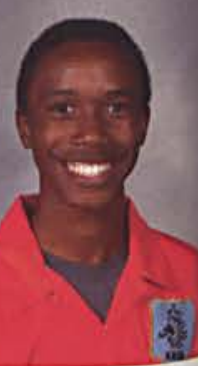

Micah Price

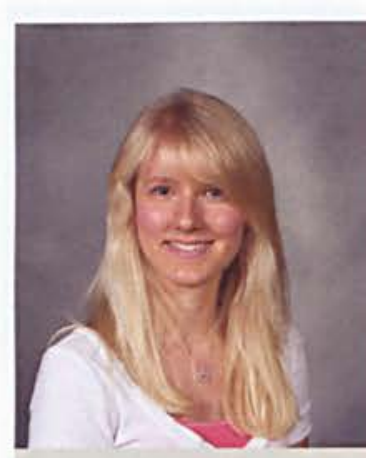

Katrina Pritchard

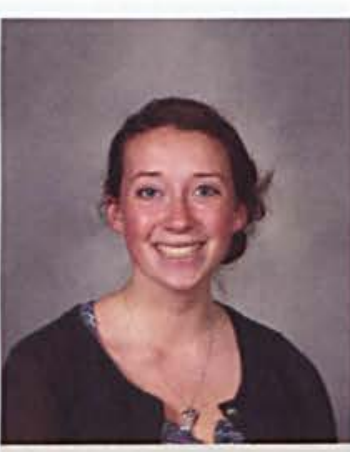

Kelley Pugh

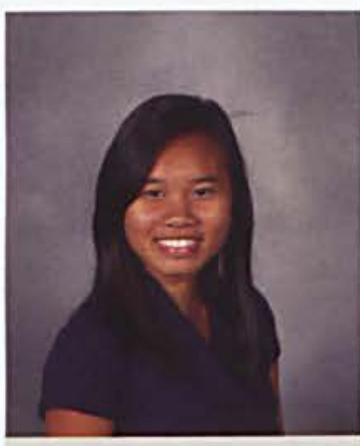

Kimberly Raisch

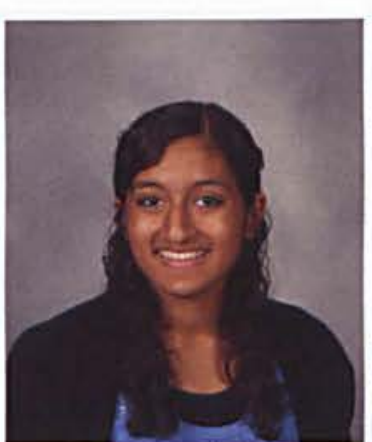

Vineeta Rao

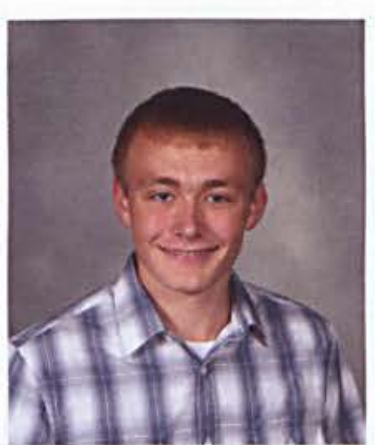

Nathaniel Raquet

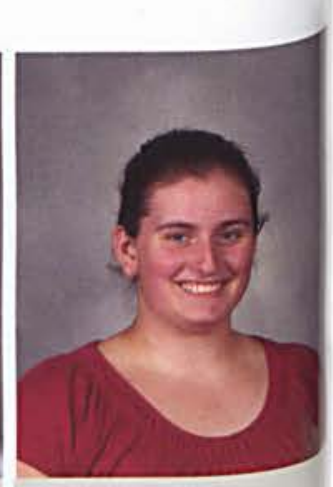

Cerianne Rea

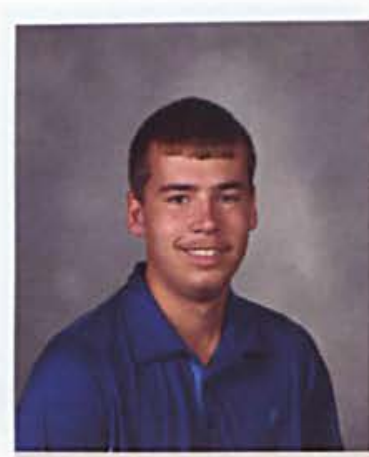

Trevor Reed

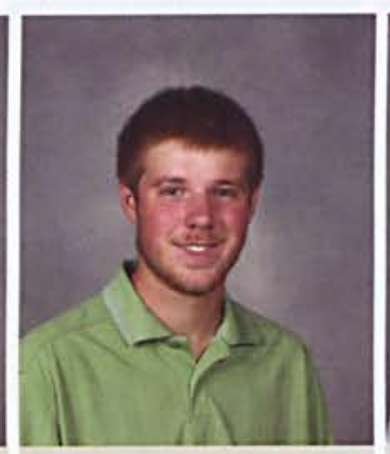

Lawrence Reeder

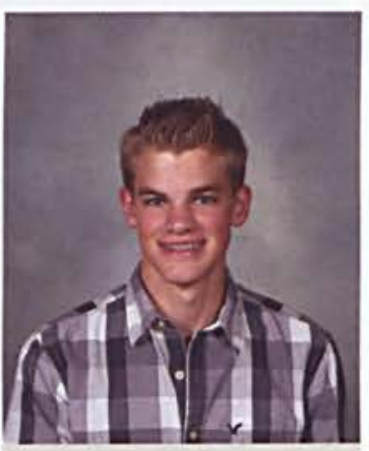

Garrison Reeves

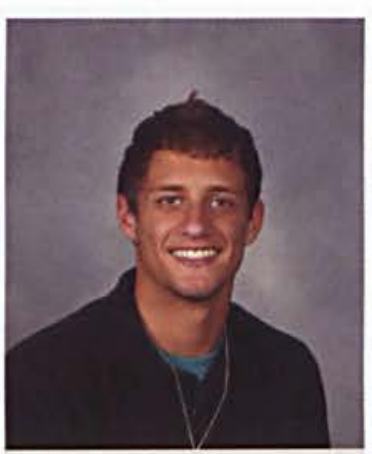

Rodrigo Reis

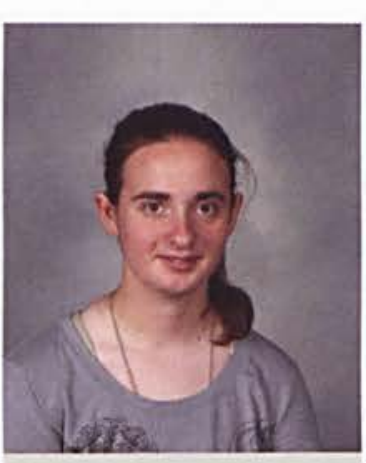

Rebekkah Reisner

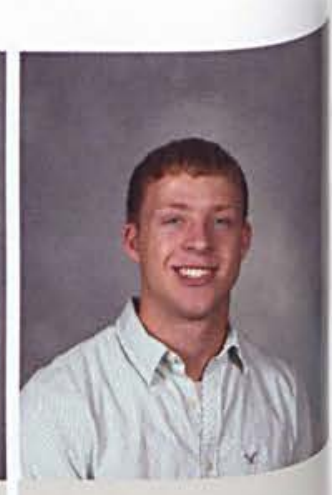

Derek Renner

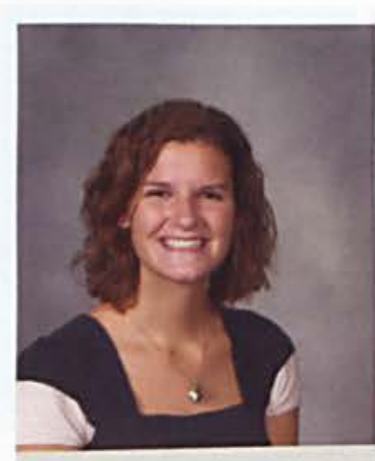

Grace Revenaugh

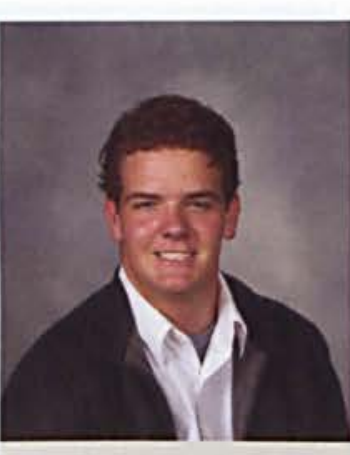

Robert Rhodes

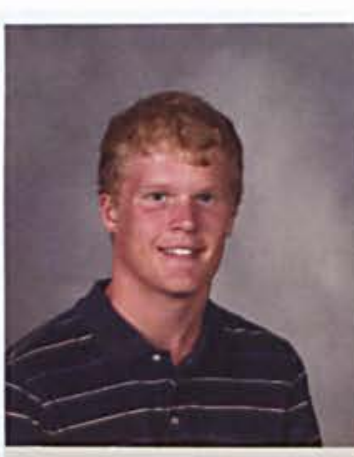

Elljah Richmond

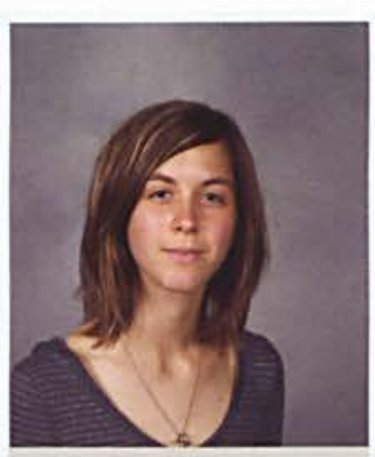

Jane Richmond

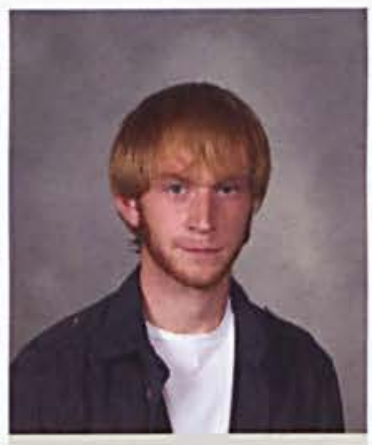

Joseph Ries

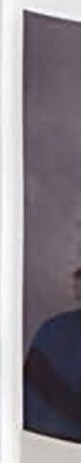

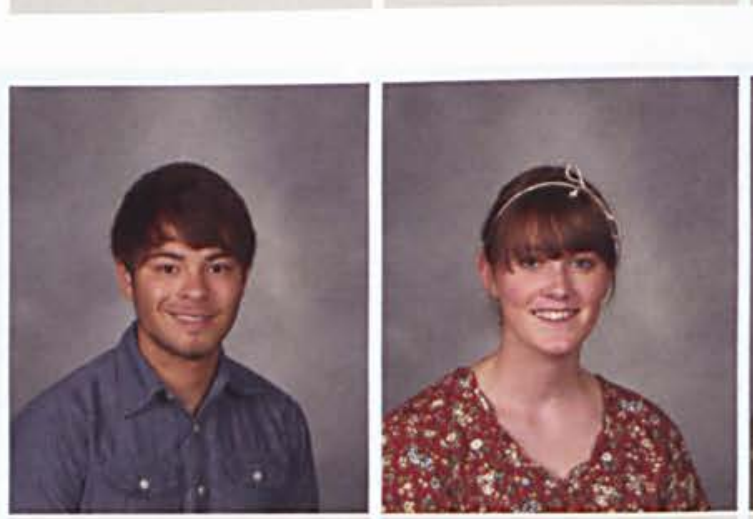

Matthew Rivera

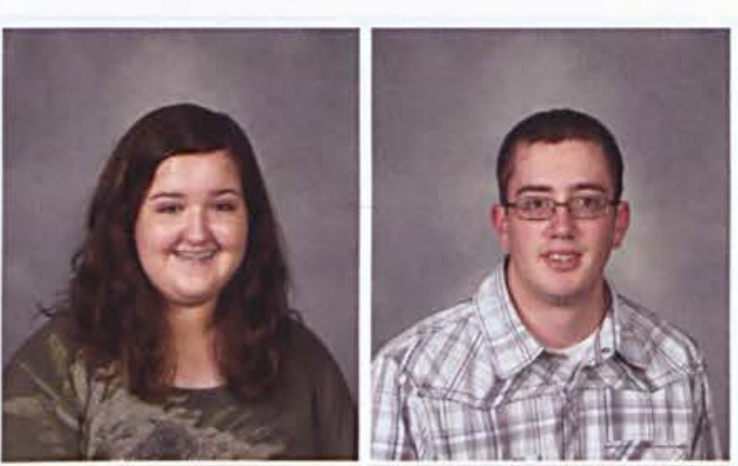

Angyla Robinson
Erik Riggleman

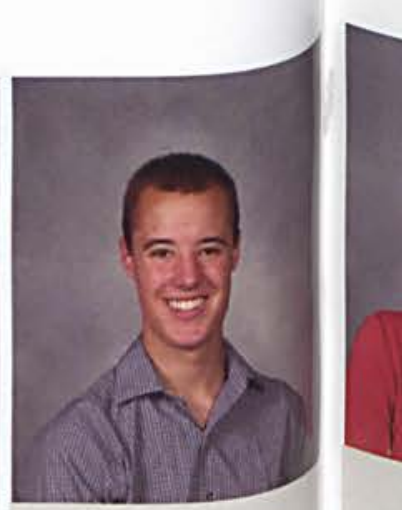

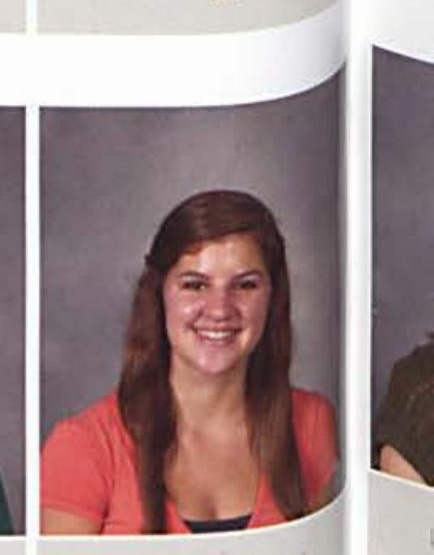

Christine Ruch 
80 FRESHMEN

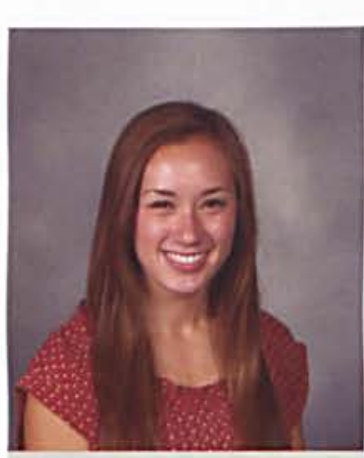

Kathryn Sill

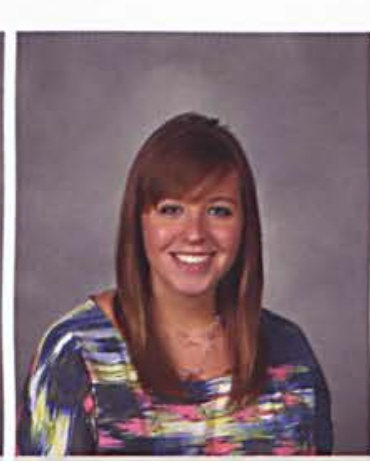

Kaitlyn Simpson

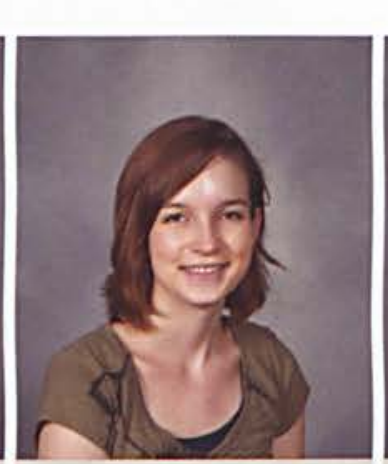

Sarah Skinner

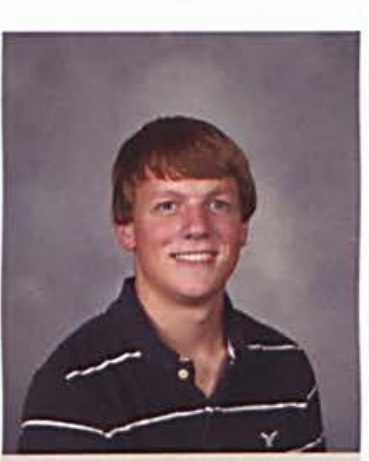

Phillip Slagh

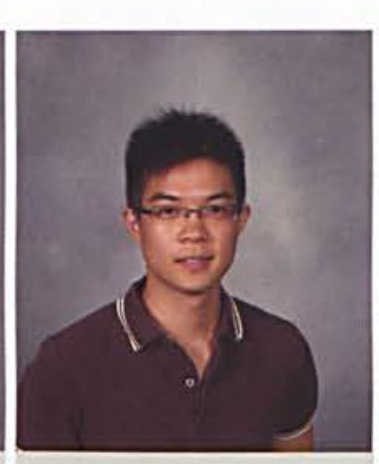

Francois Shum

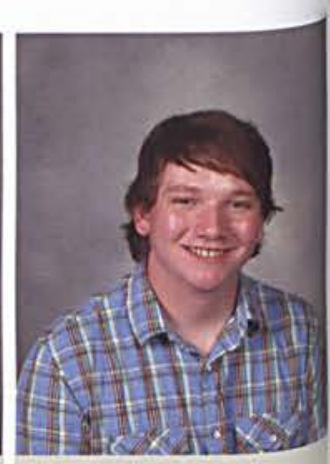

Jonathan Shumakef

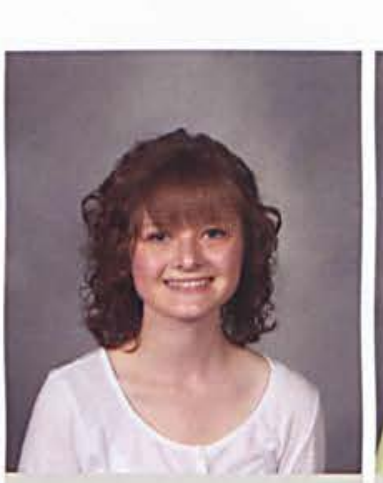

Elise Slone

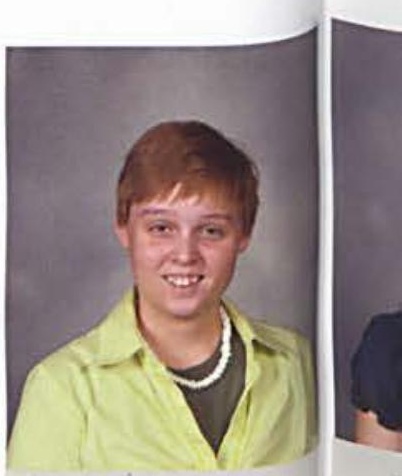

Elizabeth Slone

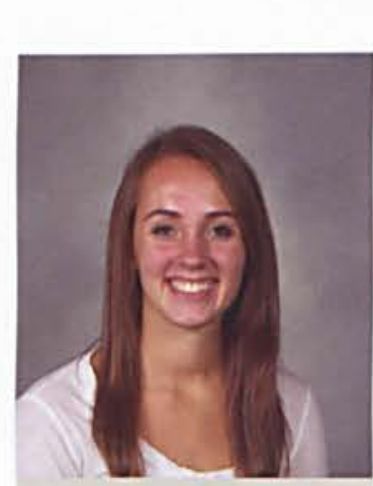

Alyssa Smith

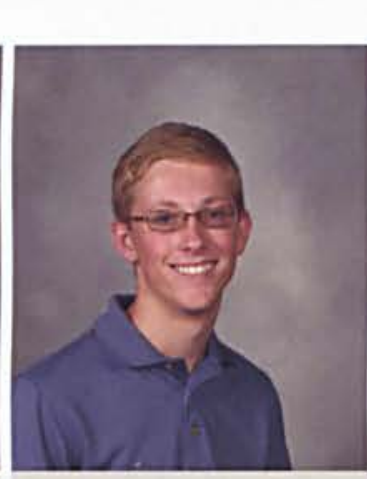

Connor Smith

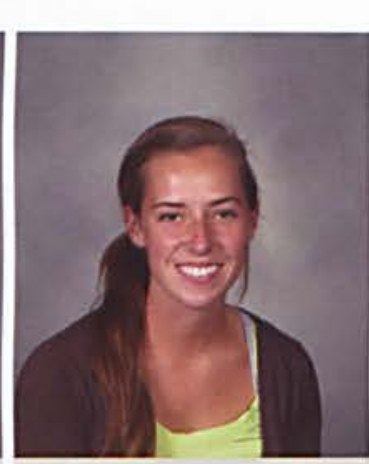

Emily Smith

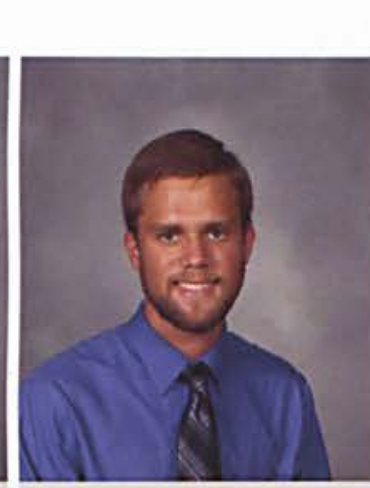

Jonathan Smith

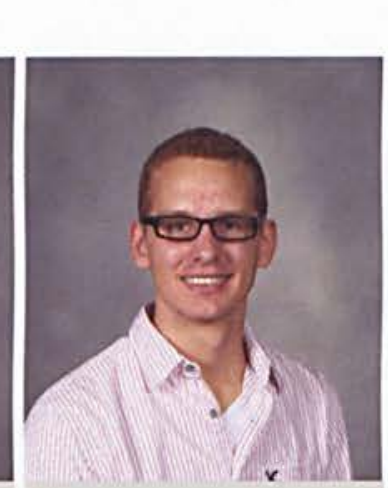

Matthew Smith

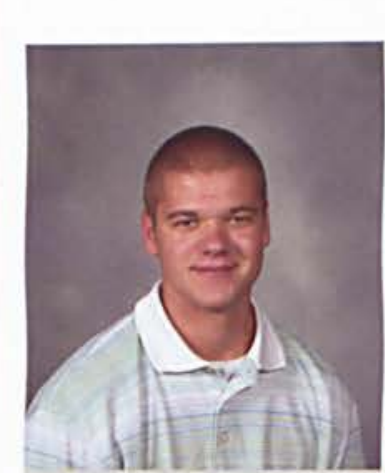

Brandon Solinger

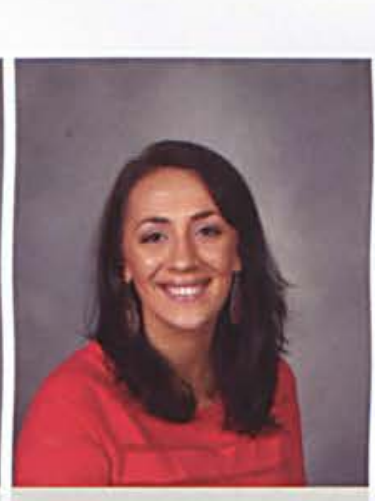

Olivia Somerville

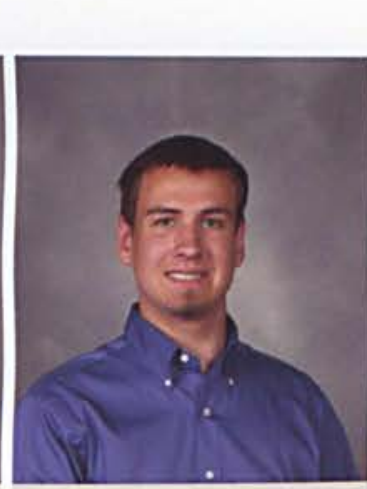

Joshua Sommers

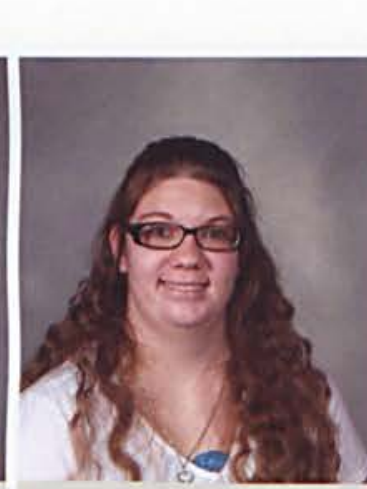

Jena Sorah

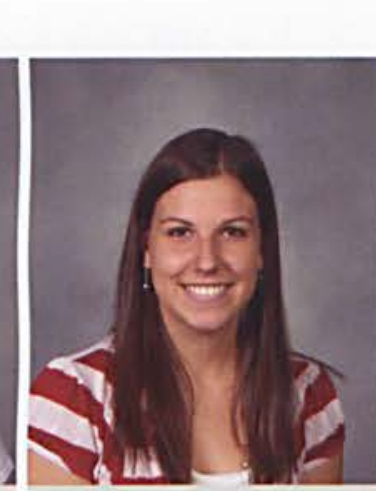

Brianna Sparks

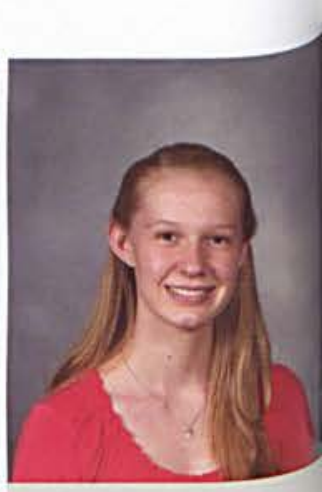

Valerie Sohn
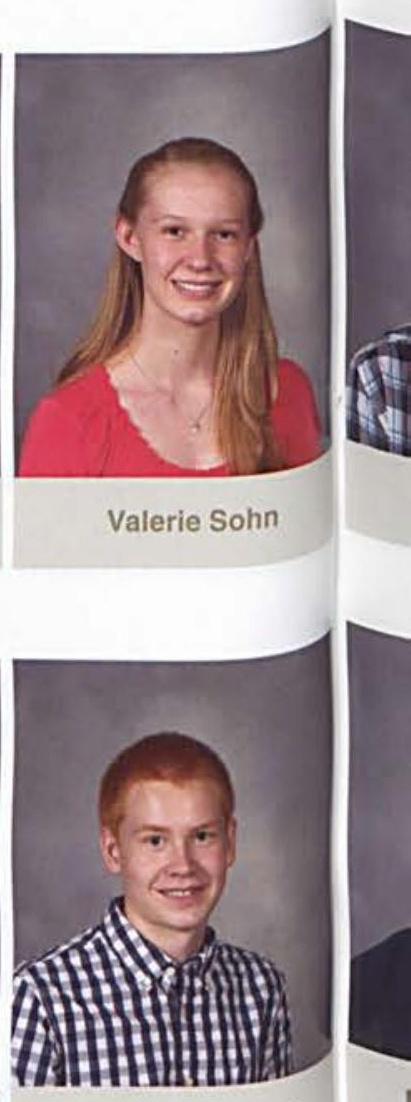

Andrew Spencer

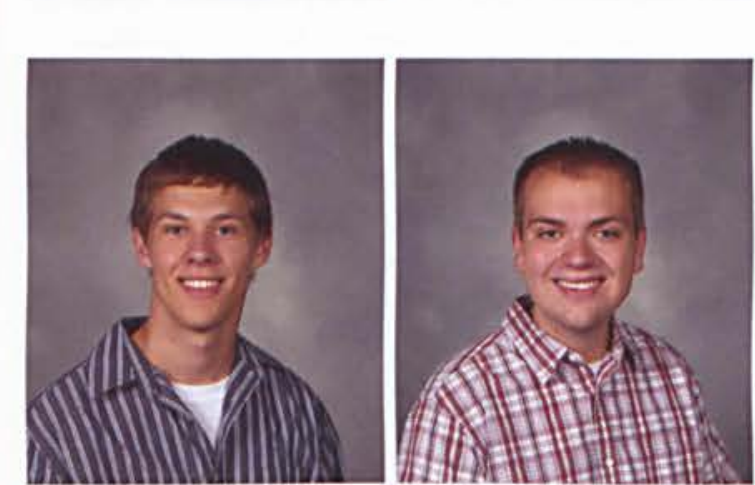

Samuel Spivey

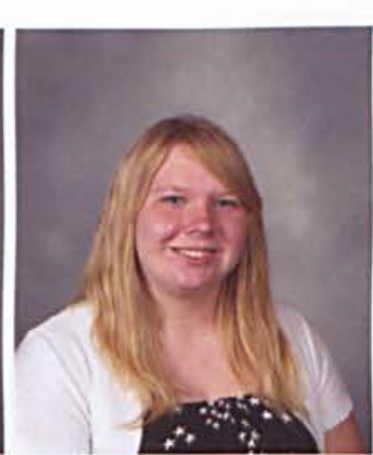

Lydia Standridge

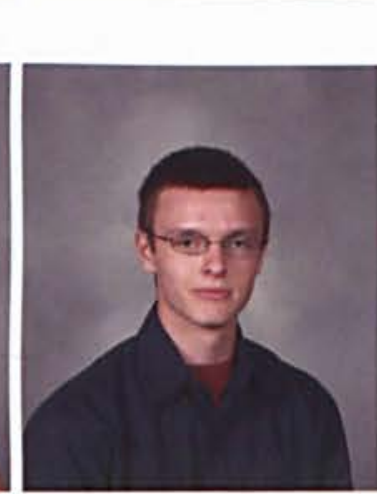

Grant Stannard

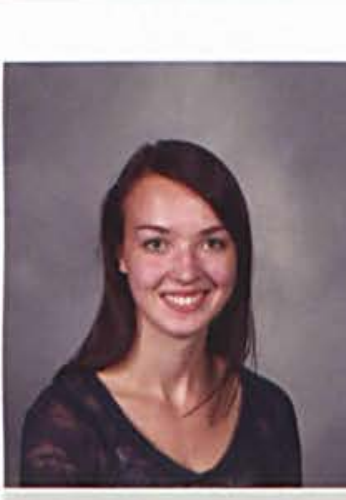

Taylor Stephens

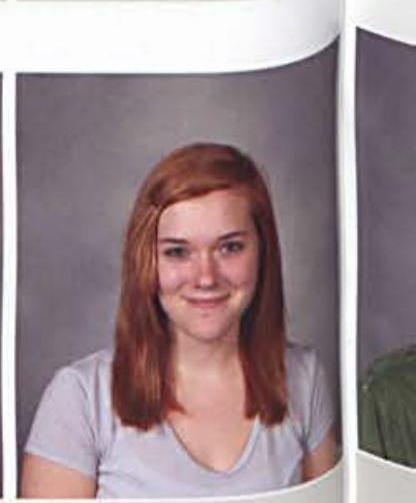

Kellee Sterling 
82 FRESHMEN

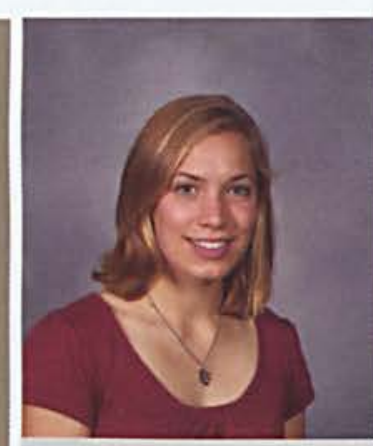

Elizabeth van der Wal

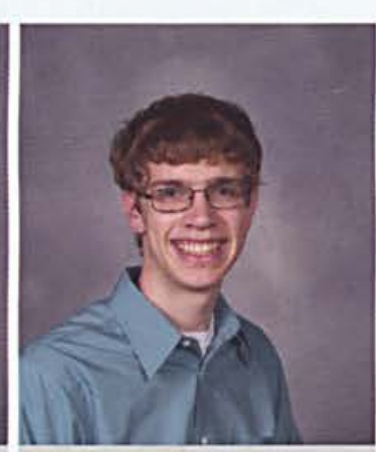

Caleb VanDyke

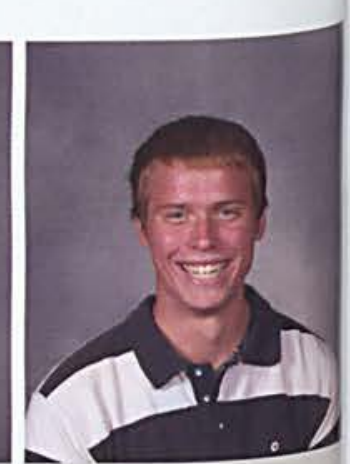

Joel Van Kampen

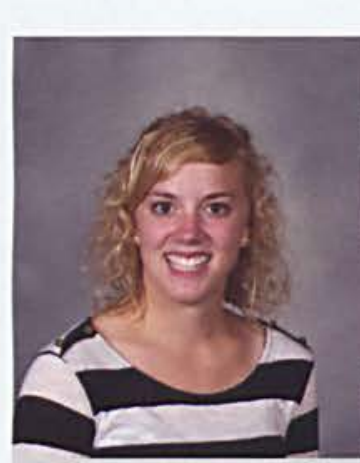

Natalie VanLith

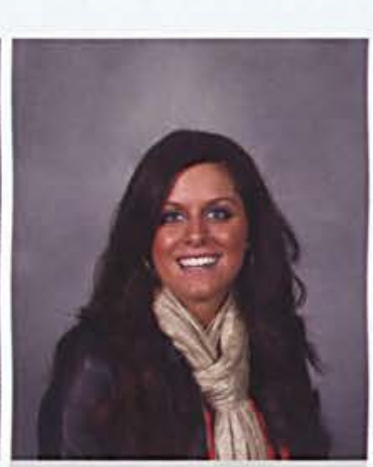

Johnna Vanstrom

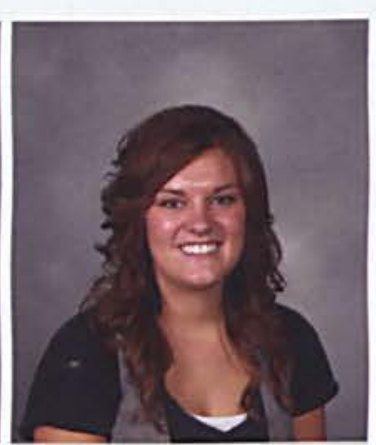

Heather Vaughn

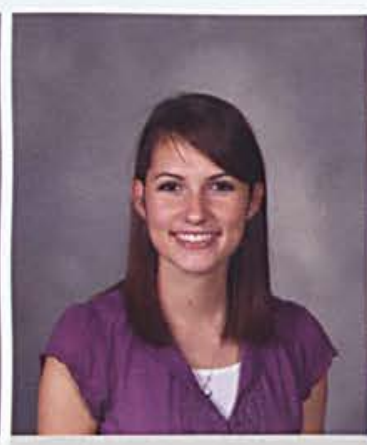

Cristina Vazquez

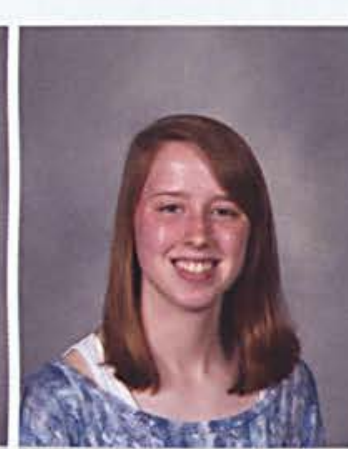

Briana Vidal

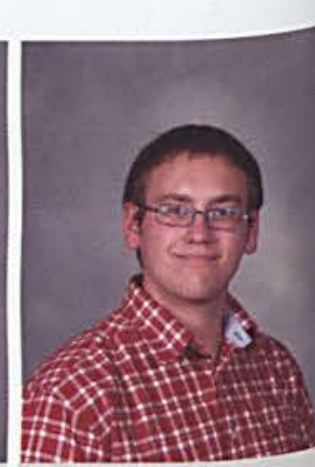

Jerome Vogt

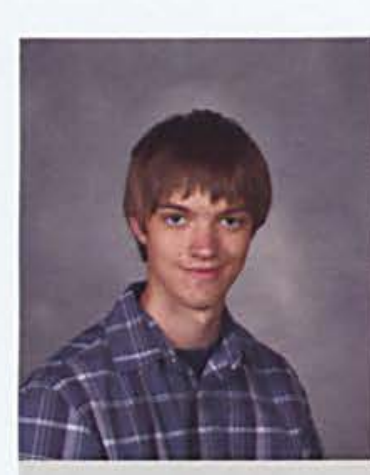

Henry Wadman

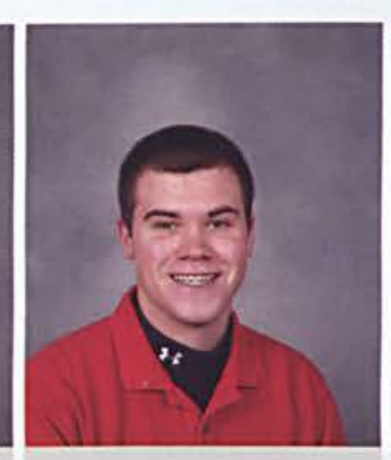

Andrew Wah

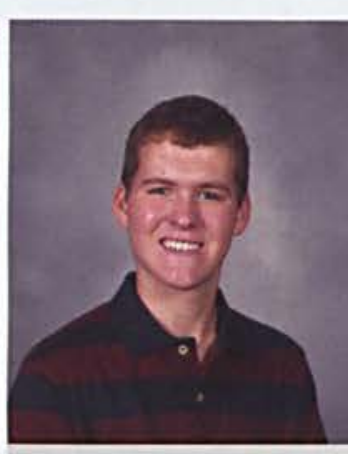

Brian Walczyk

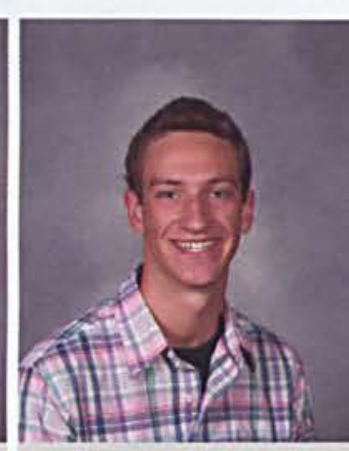

Fred Walker

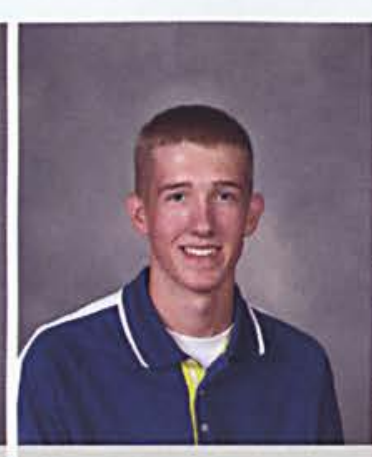

Ryan Wall

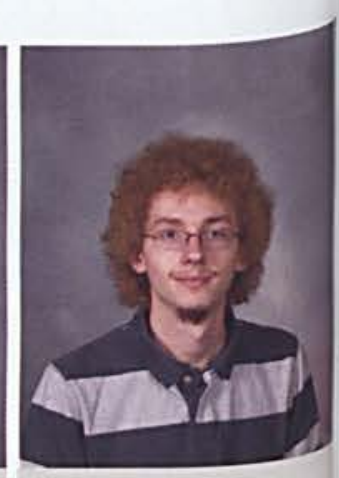

Samuel Wallace

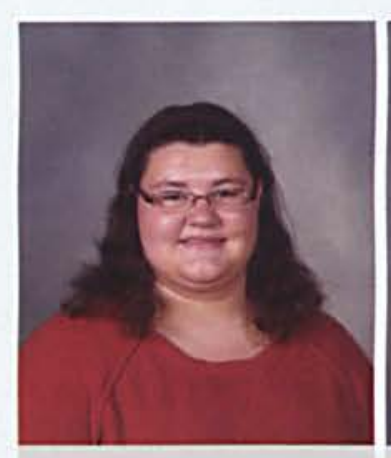

Stephanie Wallace

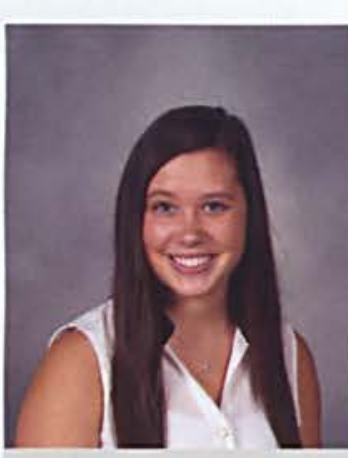

Erin Walsh

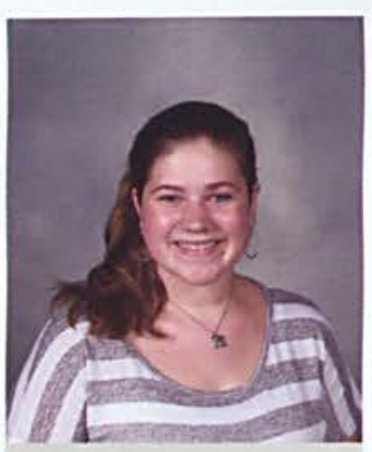

Megan Walter

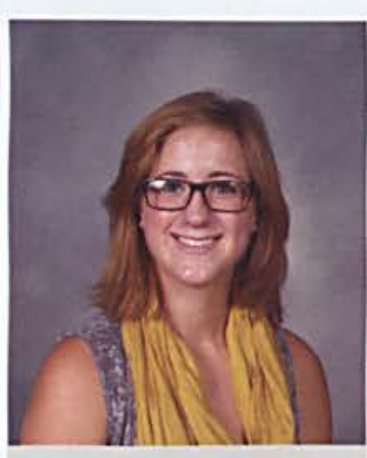

Jessica Ward

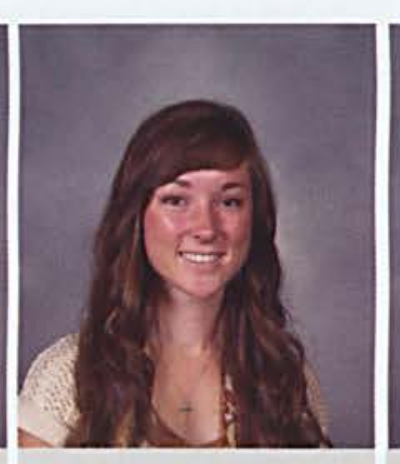

Laurel Ward
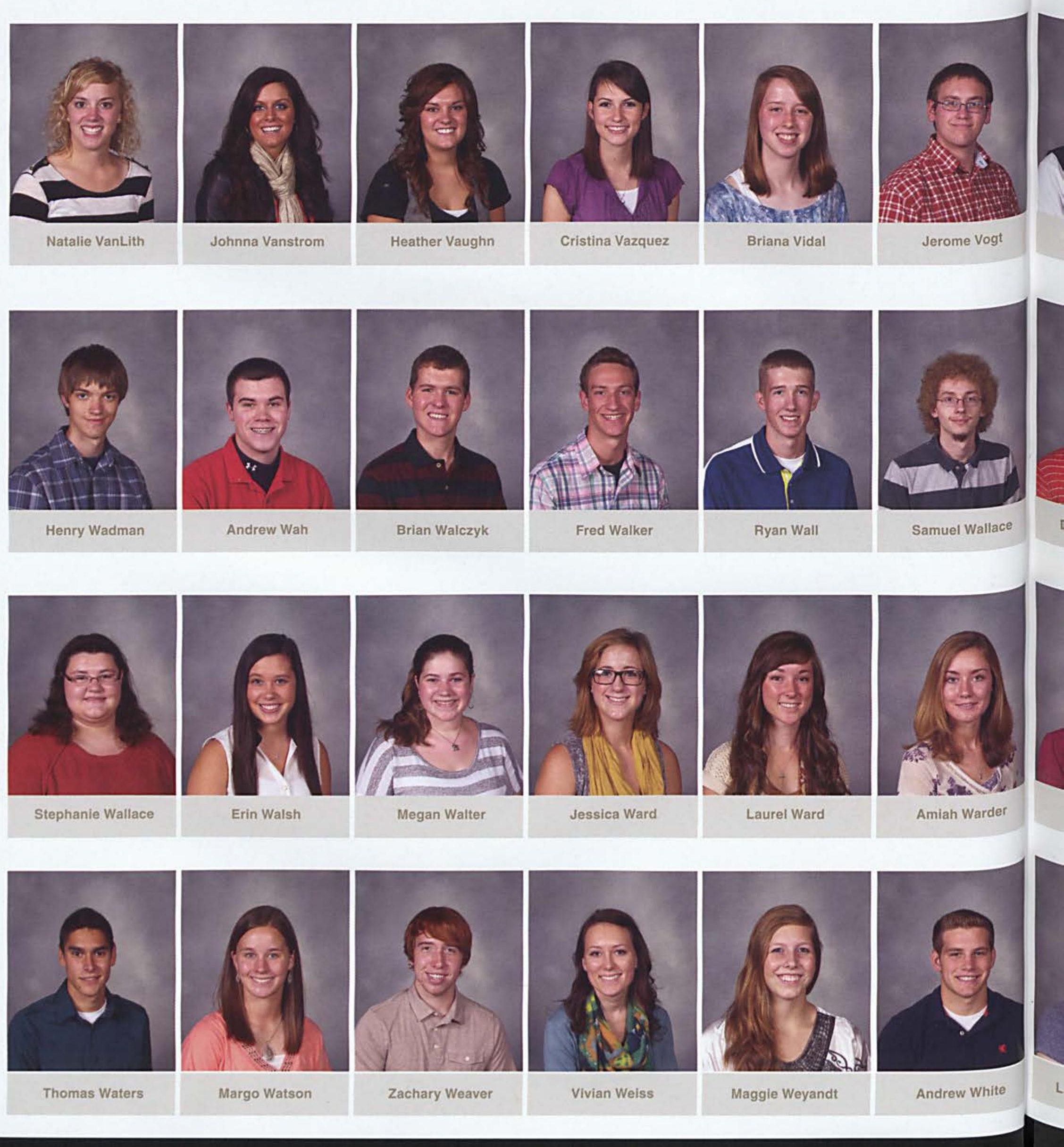


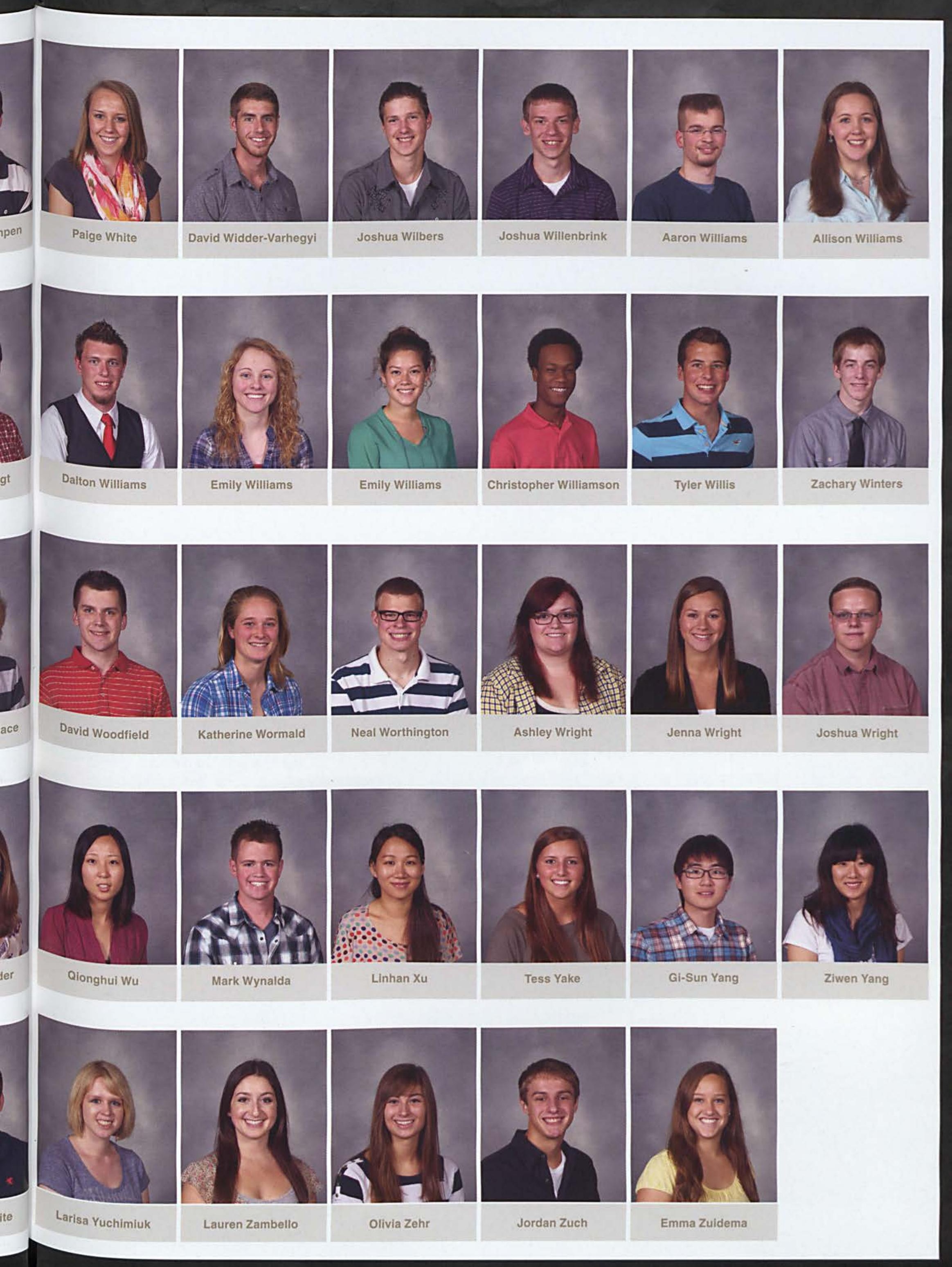




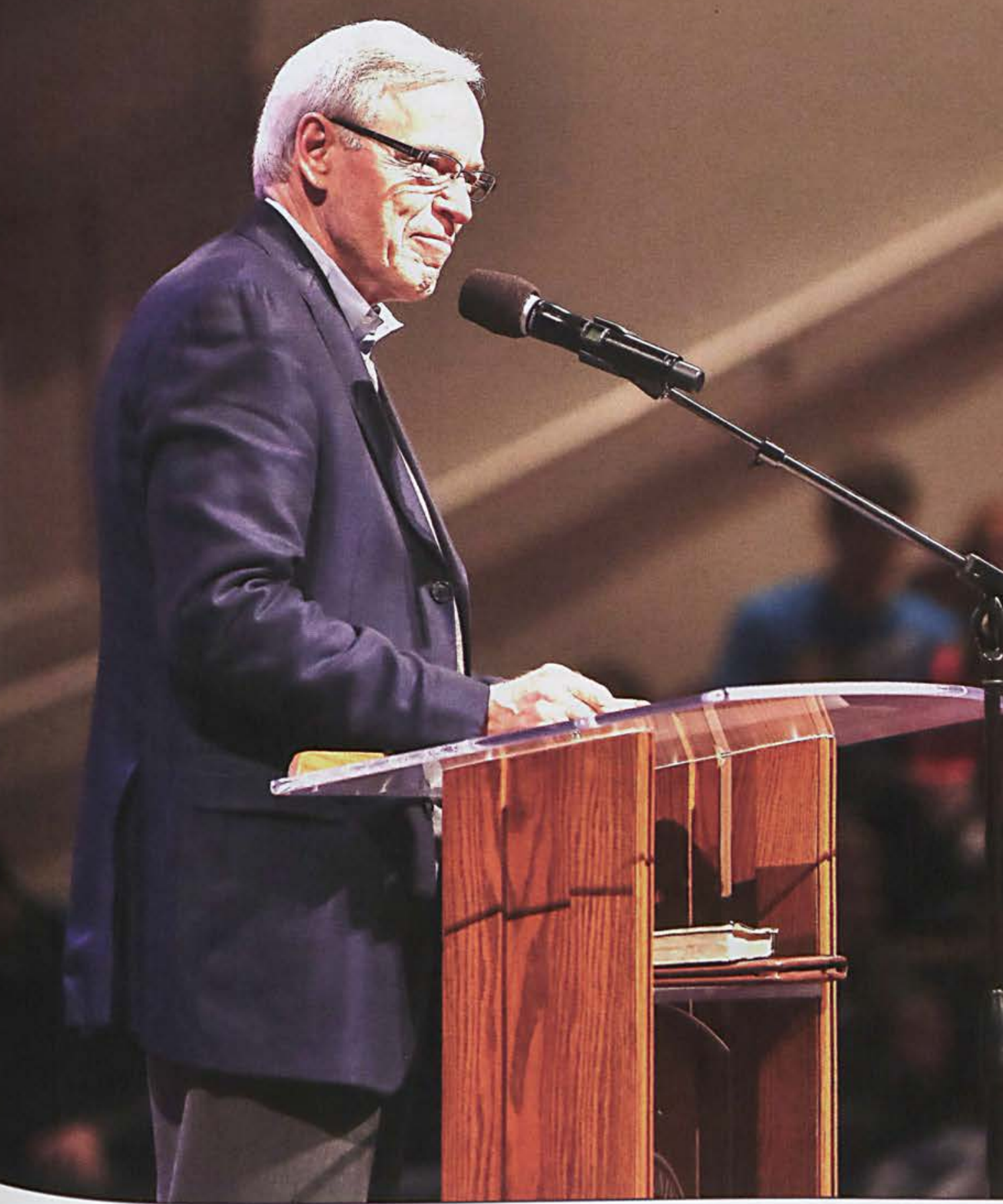

\section{CHAPEL SPEAKERS}

Good morning! Welcome to chapel!" Does that have remotely familiar ring to it? During your four years ere at Cedarville University you would have heard that approximately 596 times attendan arville Organized was stellar). Yes, your alma mater planned, The Dels from, and presented approximately 596 chaathle: Freshman the first day of Fall Bible Conference in your corw The theme for until the last chapel of your senior year

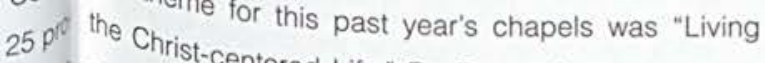
ved another, dealtered Life." Dr. Brown, in some way or ars af have heard him that most every Monday, You would ars. alone. Christian Figueredo, last year's SGA Chaplain, spoke approximately 18 times. Each chapel speaker, having been invited to speak, was informed of the emphasis for the chapel year, though not required to specifically handle the subject. I found it exciting to see how many chapels rang the bell last year (and the chapels I'm referring to would vary depending on each person's situation at the time). Chapel is core to Cedarville University. For a long time we have held chapel five days a week and required attendance for all fulltime undergraduate students. We don't plan on changing this. When students and graduates are asked what they don't want to see change at Cedarville their responses include that we should not change chapel. It is our prayer that your experience in chapel during your years here at $\mathrm{CU}$ will be an encouragement for years to come! Please come join us in chapel at 10:00 AM Monday through Friday if you are in the Cedarville area. You are always welcome

\section{Coram Deo,}

Pastor Rohm 


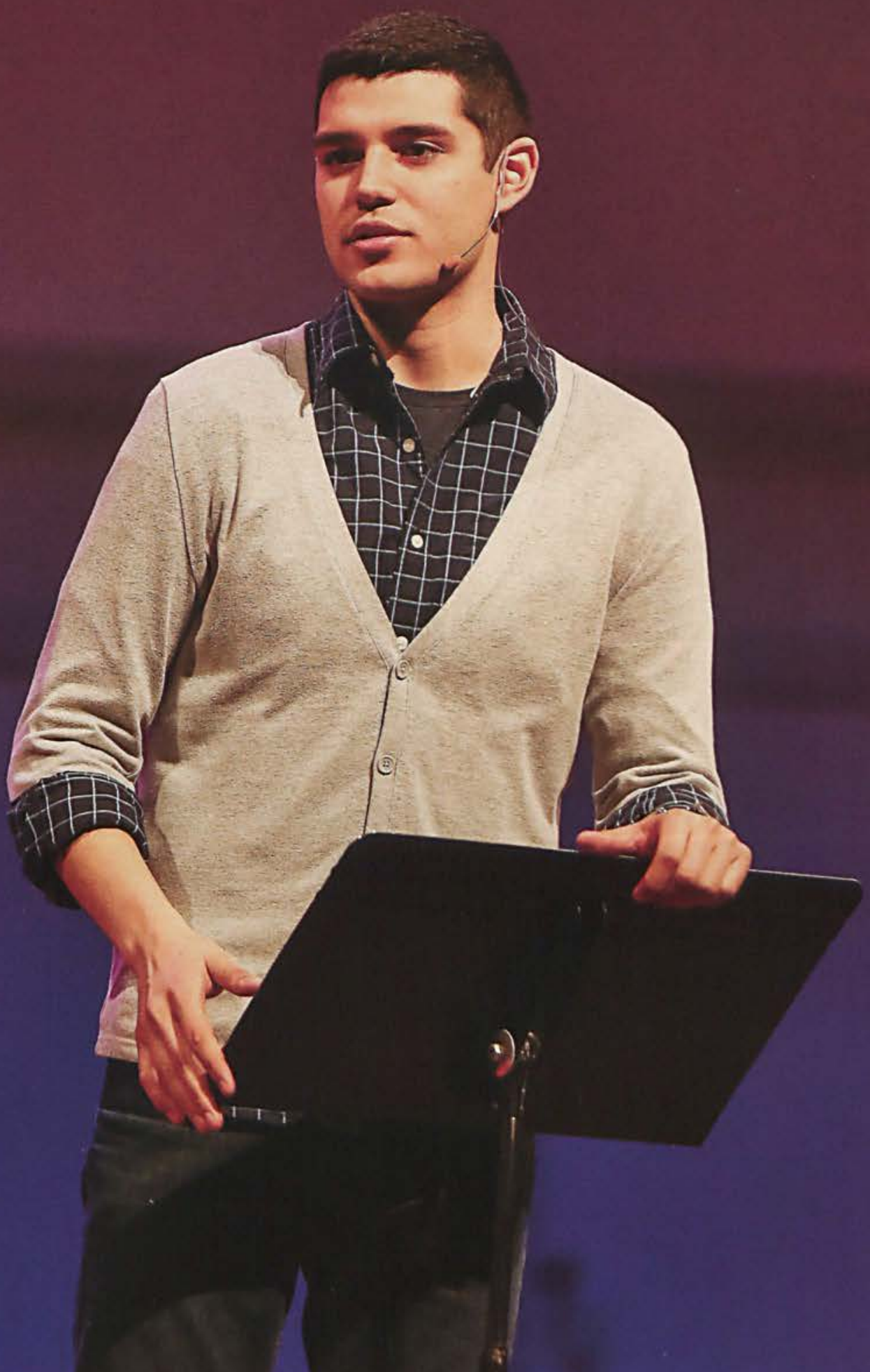

\section{SGA \& CLASS COUNCIL CHAPELS}

Do nothing out of selfish ambition or vain conceit. Rather, in humility valn conceit. looking value others above yourselves, not int SGAts of the others." (Philippians 2: 3-4) This year's anaplain, Christian Figueredo, spoke on Philippians during the 2012-2013 SGA chapels. He empha- sized the importance of having a servant's heart and being in community with other believers. Figueredo reminded students that although they're in community with believers at Cedarville, they need to continue to encourage and challenge one another. Being part of a church means investing in other people's lives and making an effort to grow as a whole and as an individual. A Christ-centered life is a life spent loving God first and foremost, and also loving others. Figueredo's bi-weekly messages helped to remind students what is truly important about the time spent at Cedarville. 


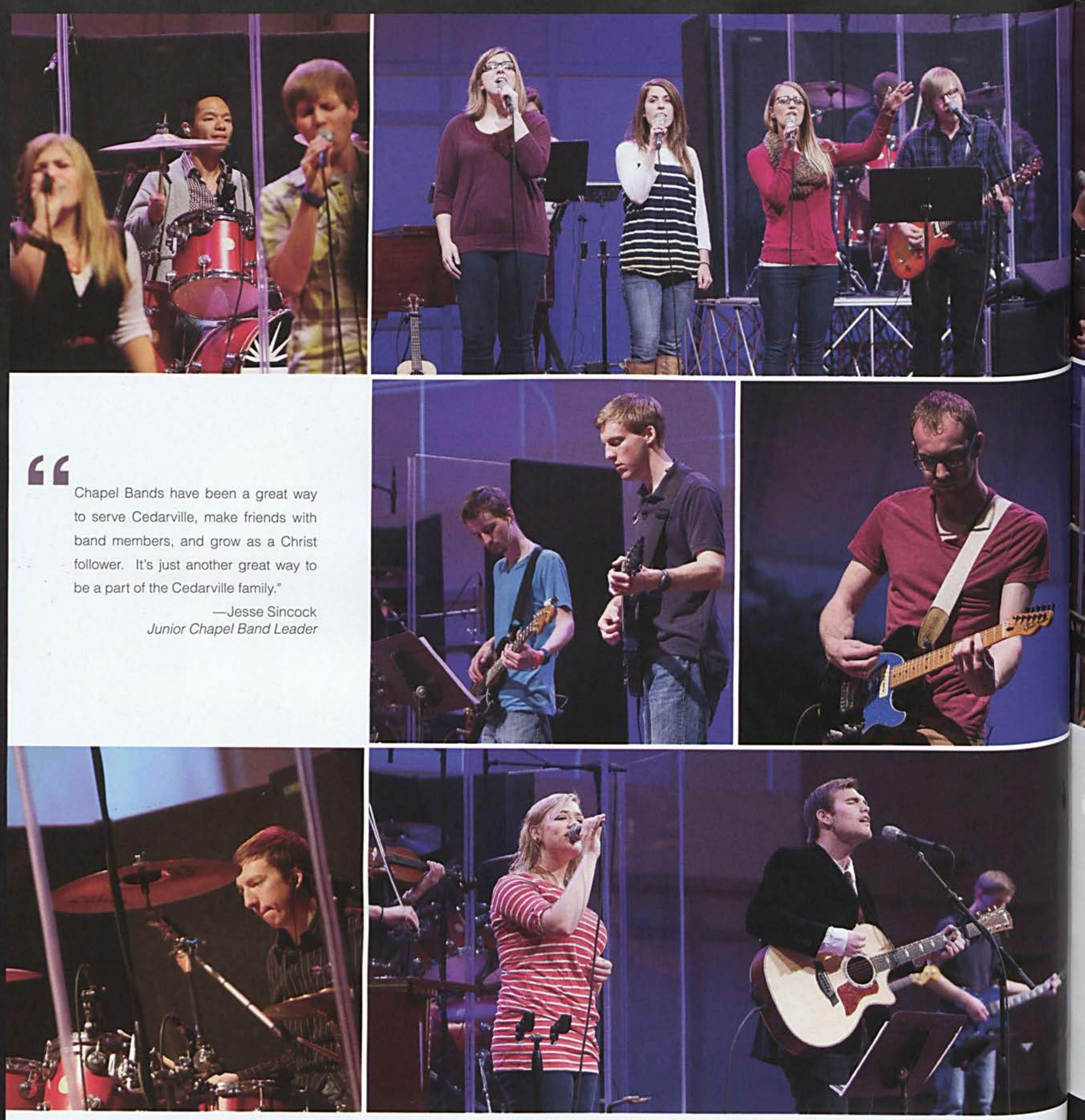

\section{CHAPEL BANDS}

Chapel is one of the most unique features of Cedarville University. At the beginning of a typical chapel service, students participate in corporate praise and worship before a speaker shares from God's word. Along with various guest bands and musical groups, there are seven student-led bands that lead worship during the course of the school year. The student-led bands rotate and lead worship around once or twice a month. In order to play in a chapel band, students audition for a chance to be part of a band at the beginning of fall semester. Student worship leaders and band members don't necessarily have to be worship majors. They are just expected to be able to commit for a whole year. Each band practices several times a month, selecting songs that will both encourage and challenge students to become more Christ-like. Sometimes speakers request a specific song to be played that will coincide with the message. Members of the chapel bands consider it a great honor to be able to lead over 3,000 people in worship to God.

\section{Chapel Band Leaders}

Sam Deloye

Sophomore Comprehensive Bible Major

Jesse Sincock

Junior Pre-seminary/Bible Major

Brian Fanelli

Junior Broadcasting and Digital Media Major

Caleb Alley

Senior Biology/Pre-med Major

Isaac Murrell

Sophomore Worship and Bible Major

Jena Baumer

Senior Worship Major

Brandon Cruise

Junior Worship Major 


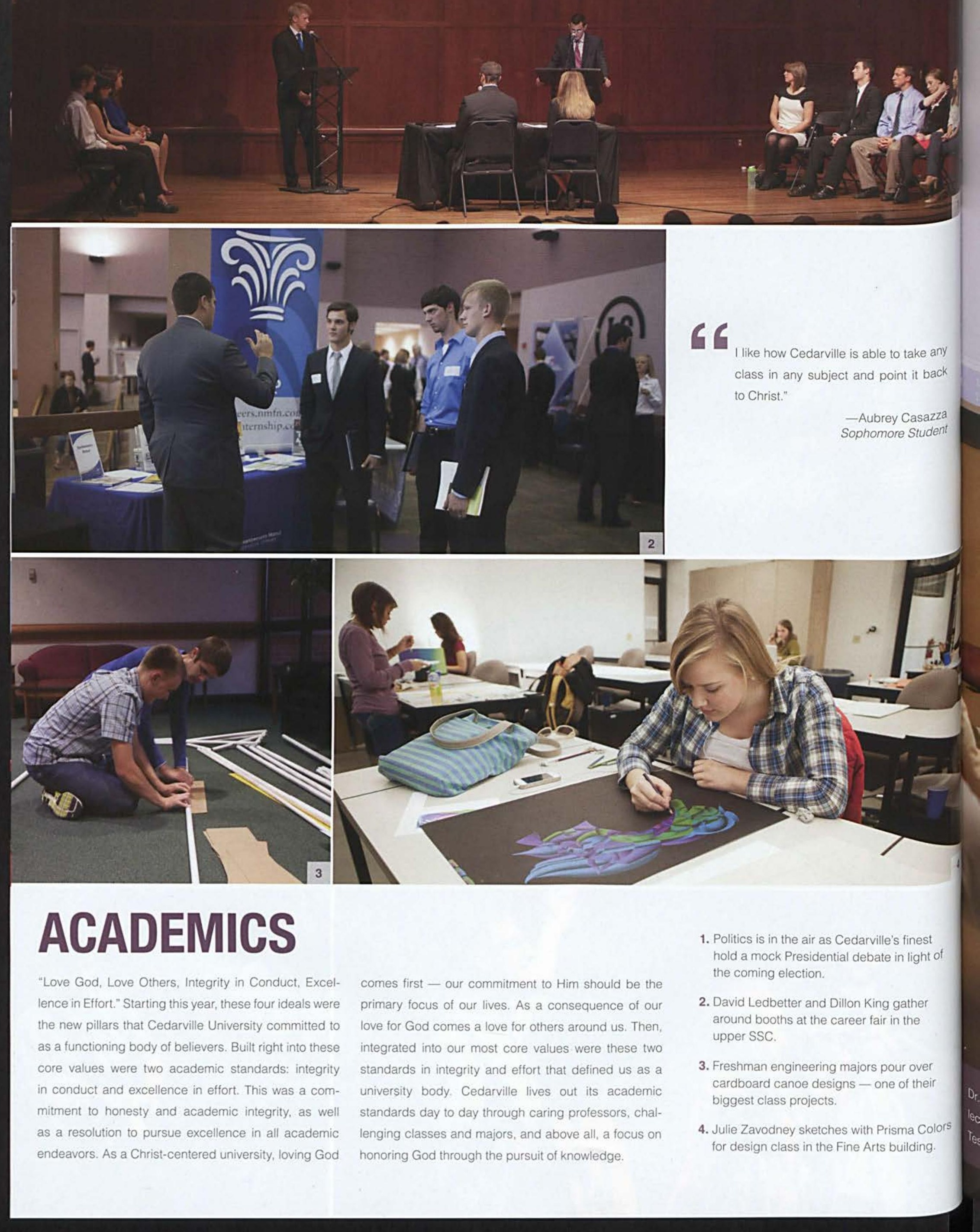




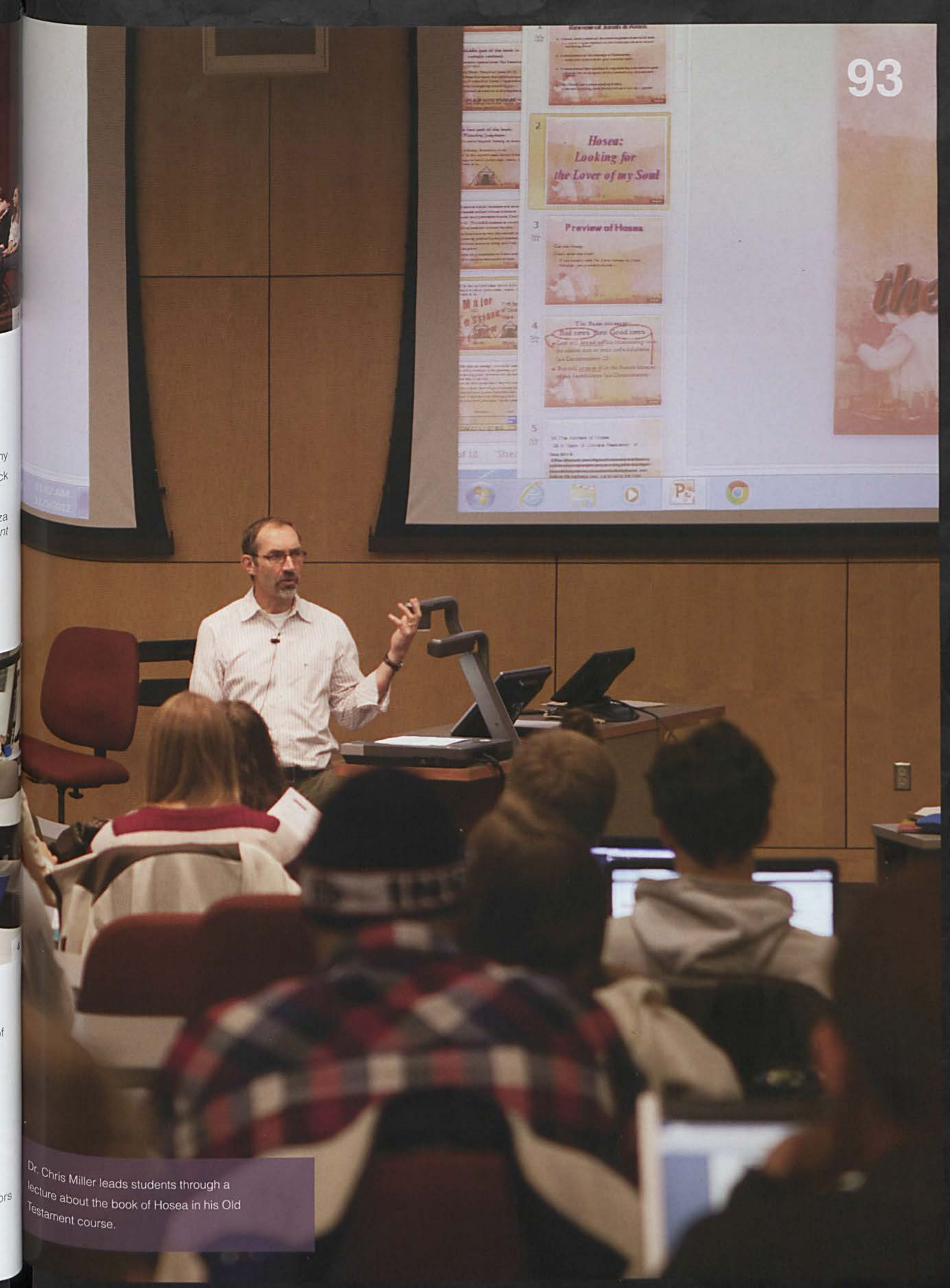




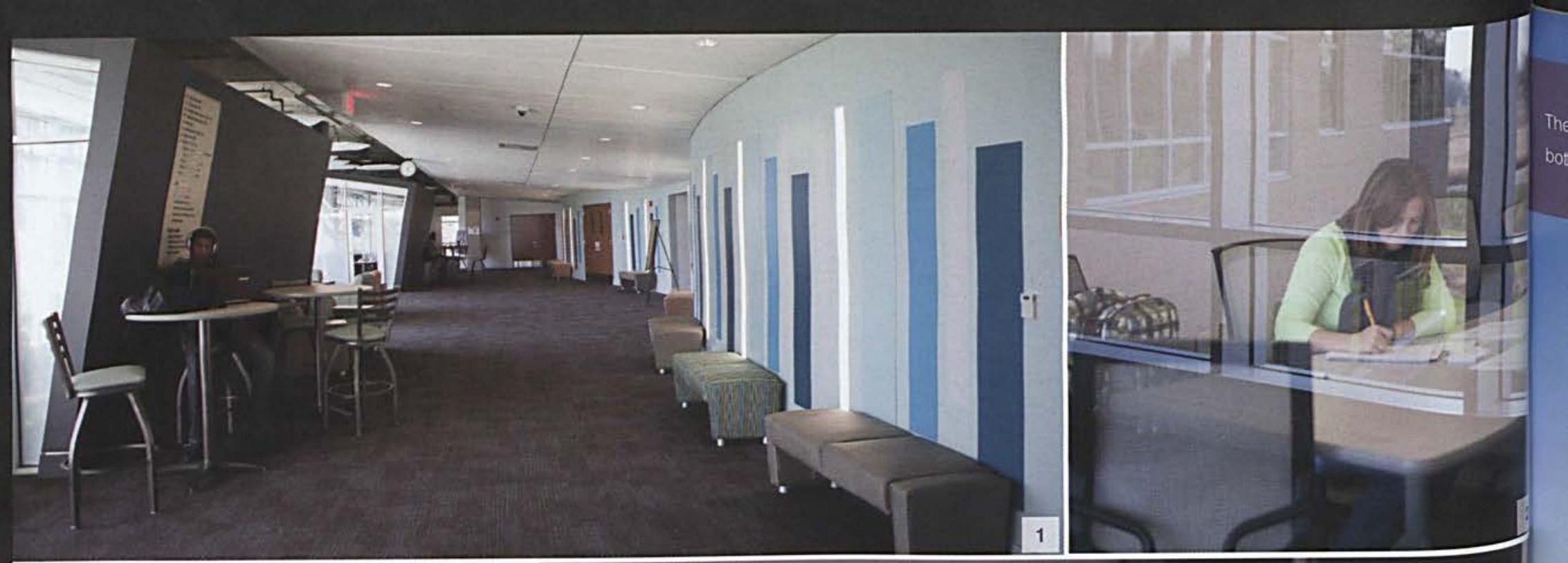

Cé

The HSC has really been such a bless-

ing. They've provided us with brand new technology and labs, and I feel like l'm going to be fully prepared for a future in nursing."

- Sarah Chalgren Sophomore Nursing Student
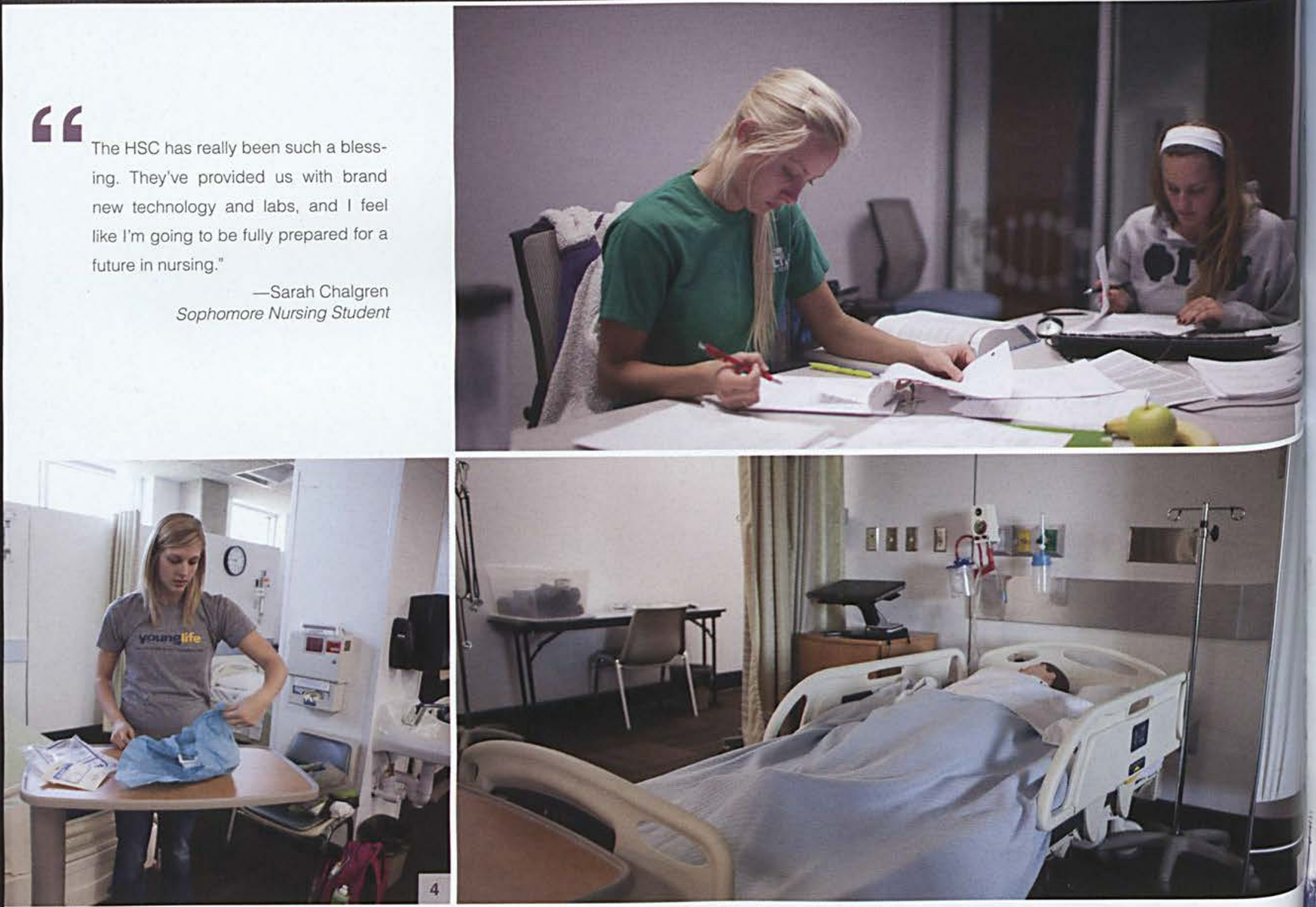

\section{HEALTH SCIENCES CENTER}

The Health Sciences Center is designed to support collaboration between the nursing and pharmacy programs. Not only does it have three floors and sixteen collaboration rooms, but it is also intentionally designed for a comfortable atmosphere with natural lighting. Each room is built with care and a well thought-out design. The design of the building supports an active learning model. This kind of learning model includes team-based learning, in which students engage with one another to achieve an overall outcome. The numerous labs within the building offer pharmacy and nursing students chances to interact with real patients and simulation manikins. The first floor simulation clinic even resembles a medical office practice and is used to train both pharmacy and nursing students. Faculty, staff, and students find the building to be very welcoming and open, allowing them to complete daily tasks with ease.
1. Students work on homework in the lobby 0 the newest building on campus, the Health Sciences Center

2. A pharmacy student works on a project fo class in one of the second floor collaboration rooms.

3. Morgan Gullion studies with a friend in a collaboration room.

4. Nursing student Emily Scheurer practices sterile technique in the teaching lab.

5. The practice lab allows students to work with simulation manikins on a daily basis. 


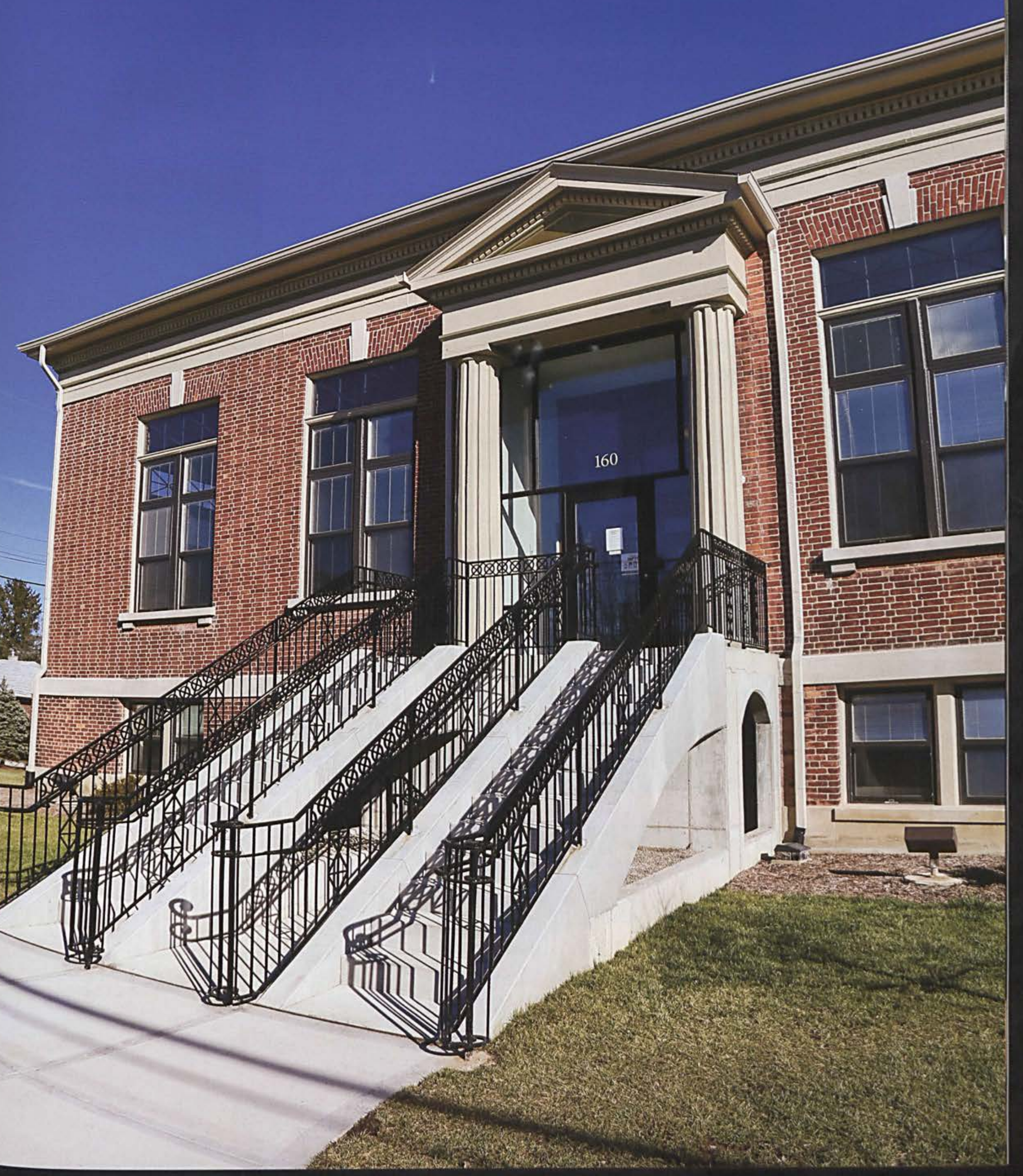




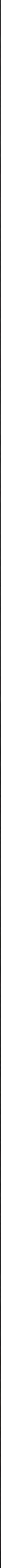





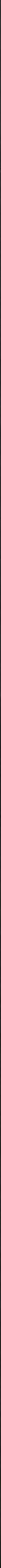





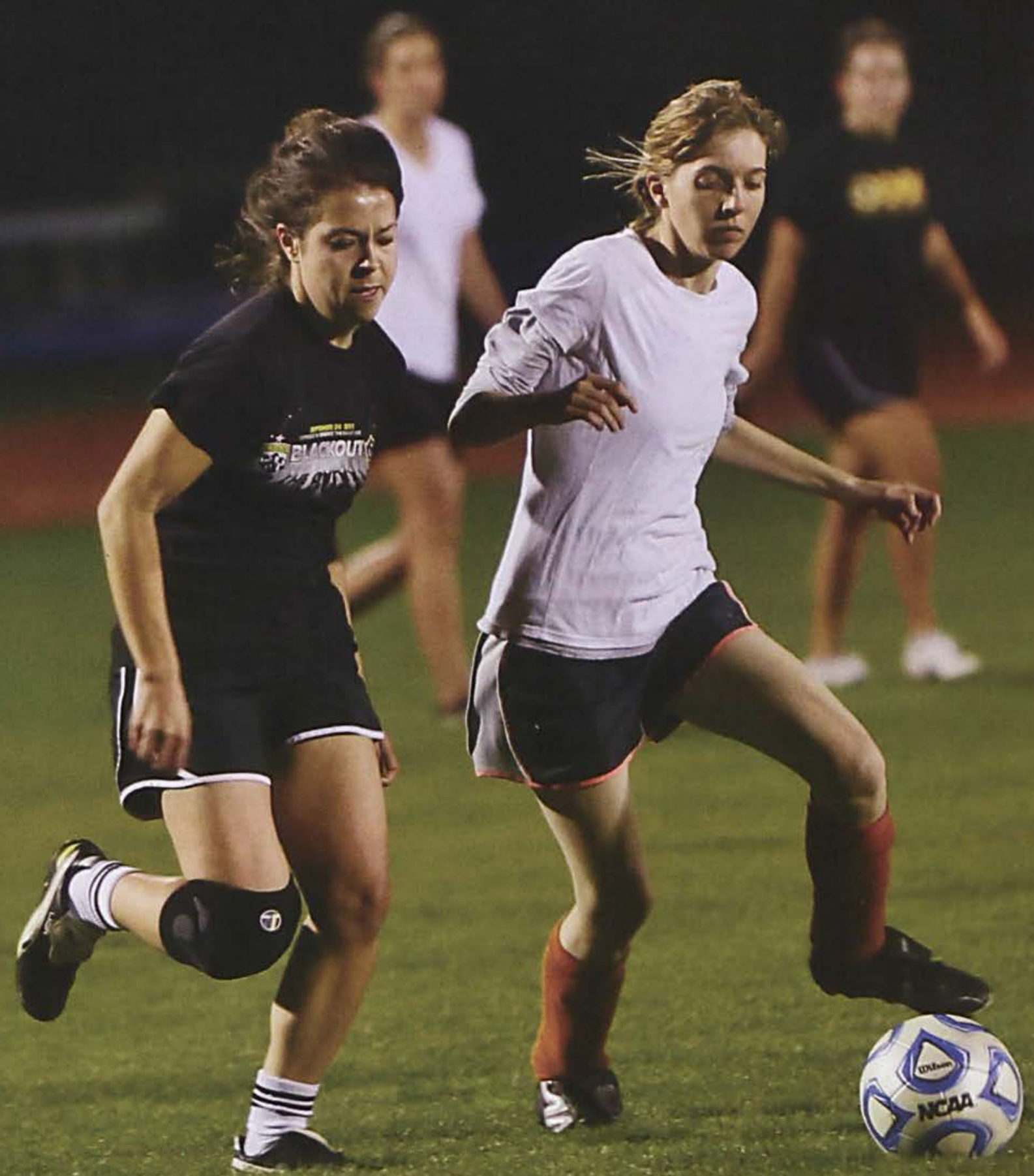

Tara Venturini (left) and Malerie Young (right) vie for

Control of the ball in intramural soccer. 


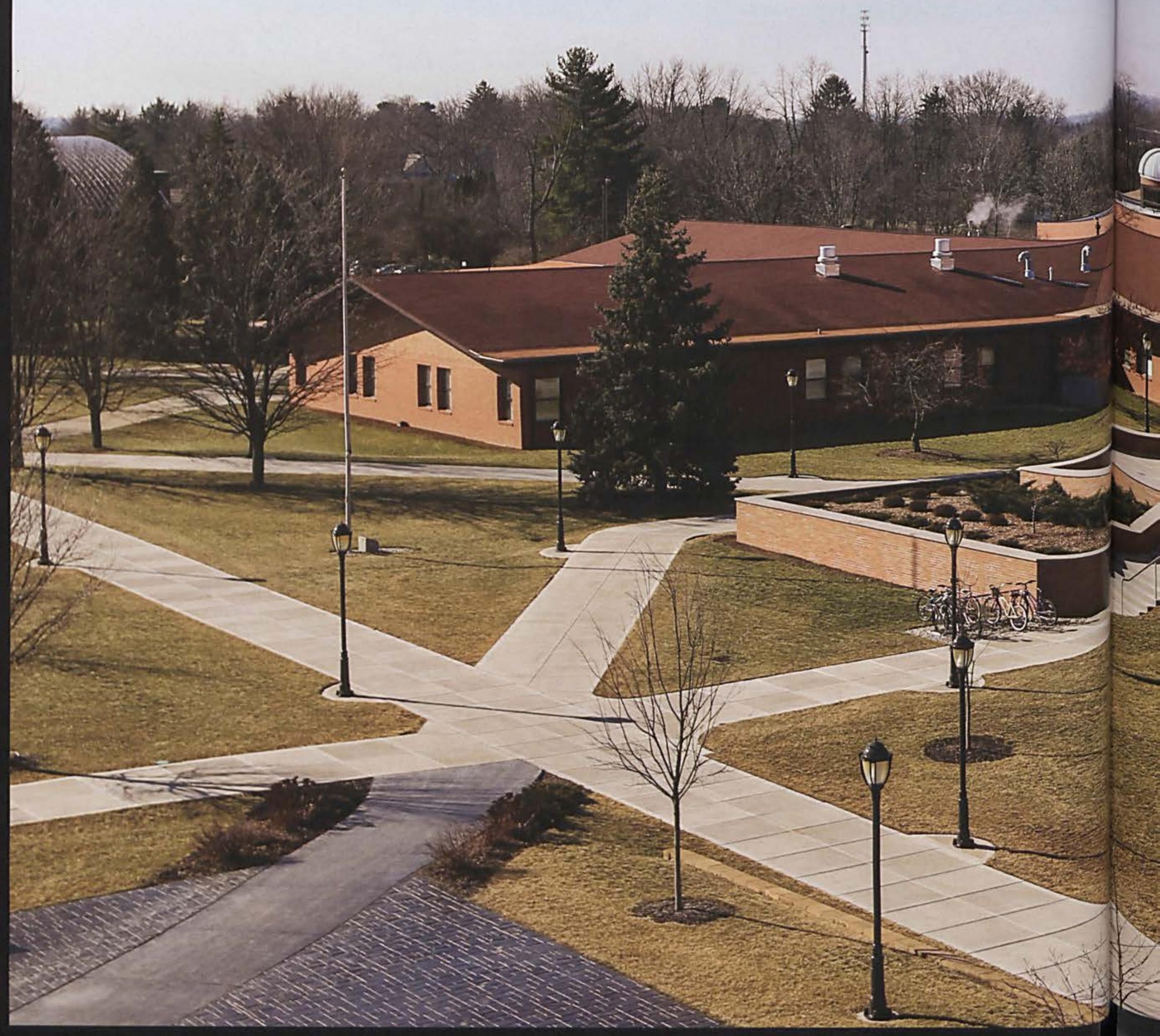




\section{SOPHOMORES}

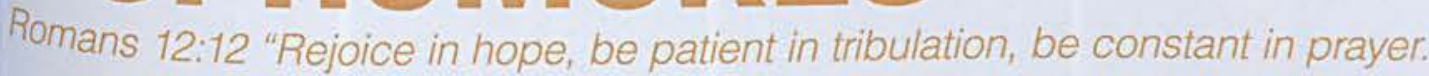

4

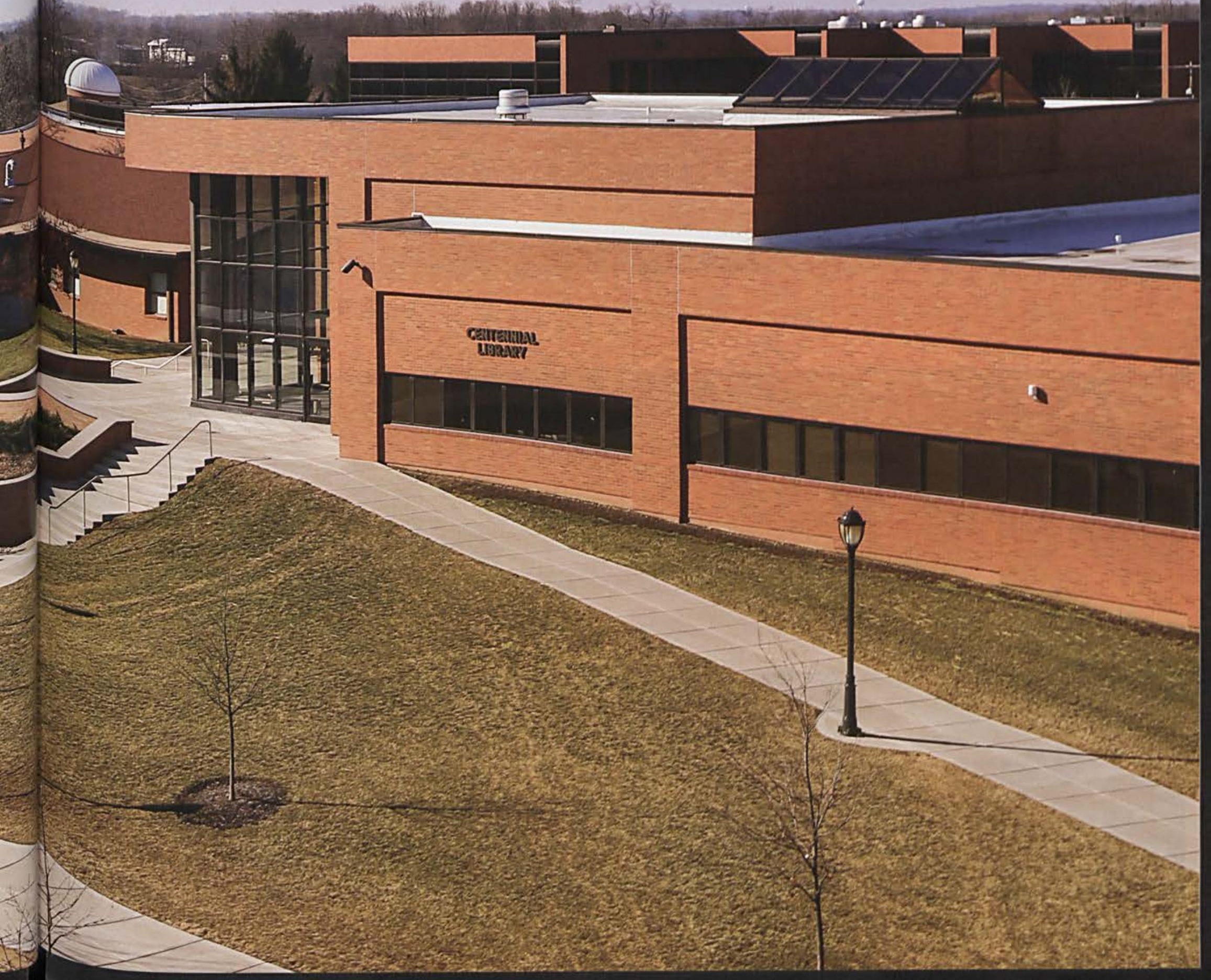



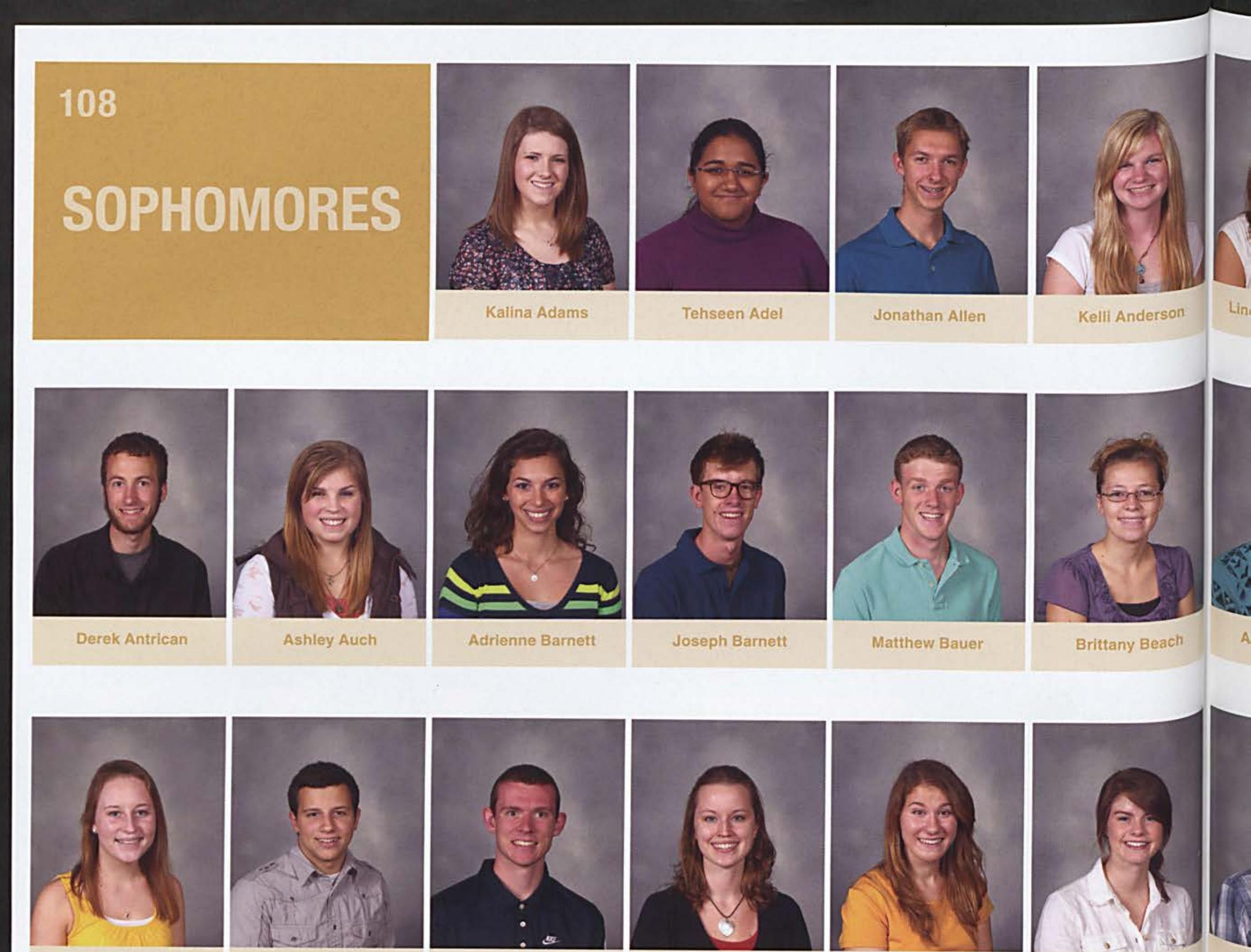

Meghan Becker

Jordan Bellanti
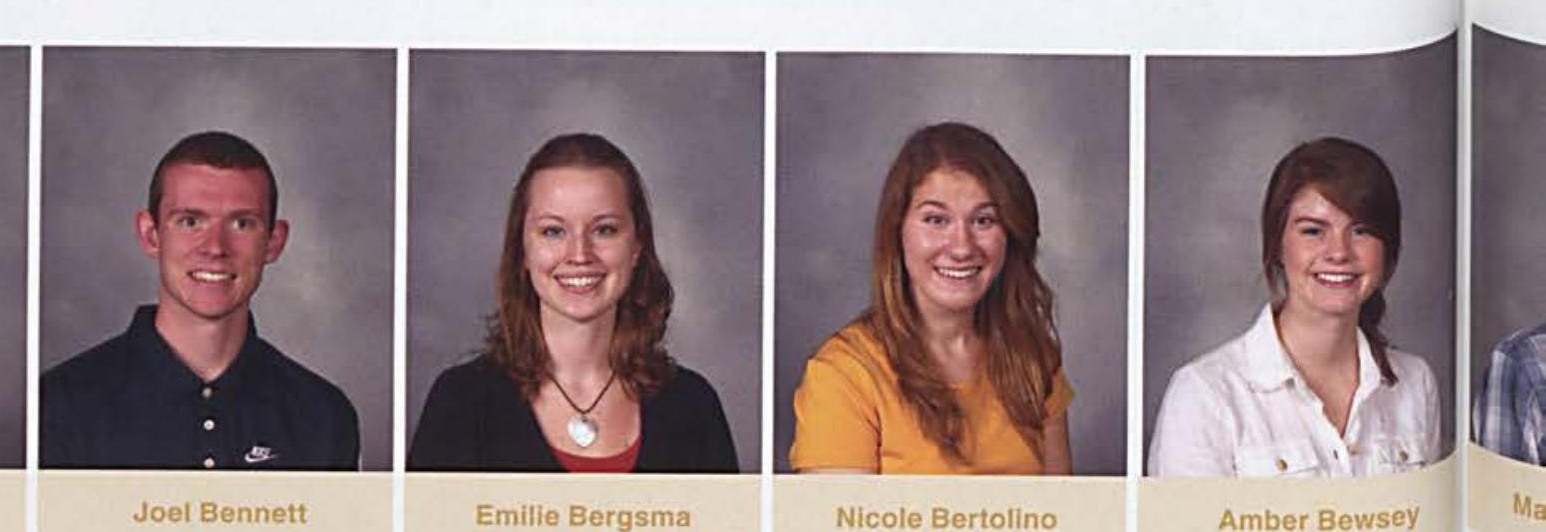

Emilie Bergsma

Nicole Bertolino

Amber Bewsey

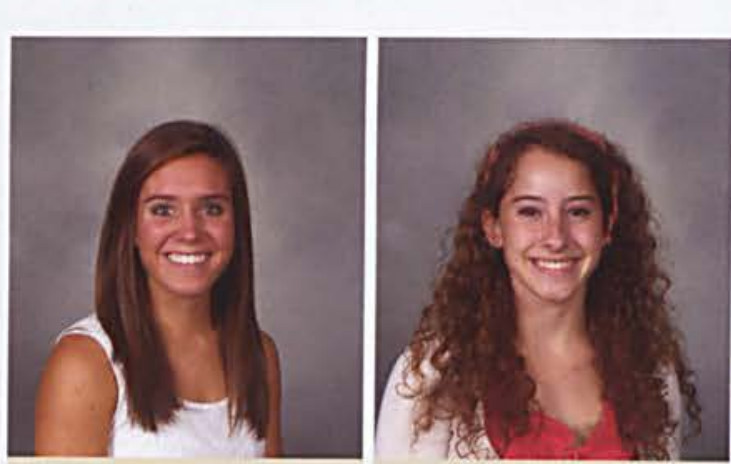

Alison Bigler

Sydney Bissonette

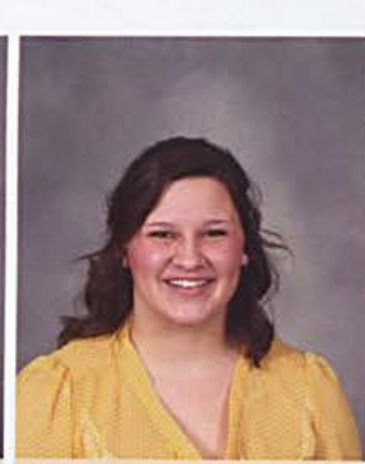

Hayley Blackburn

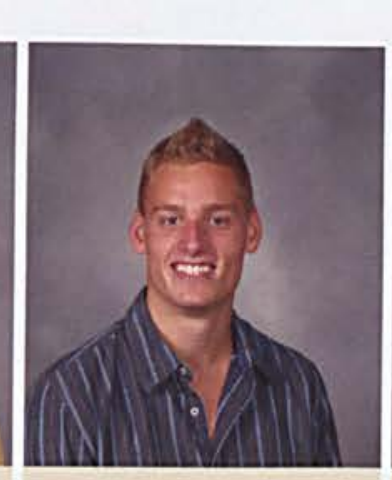

James Blackwell

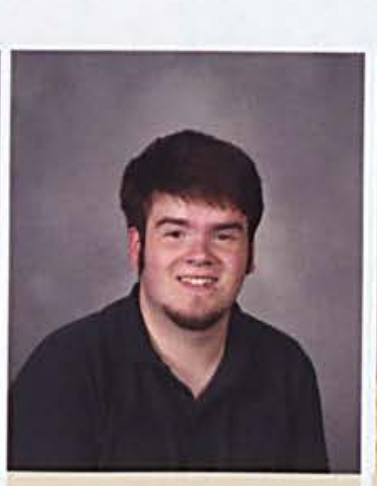

Jeremiah Bollman

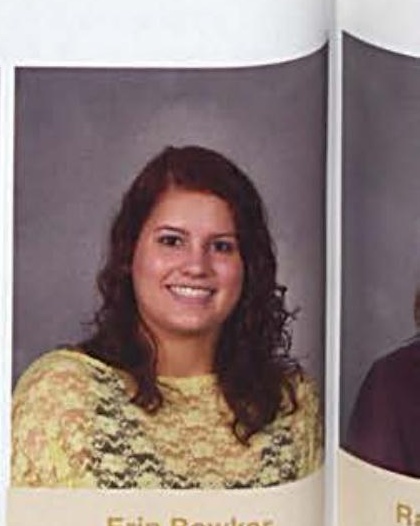

Erin Bowker
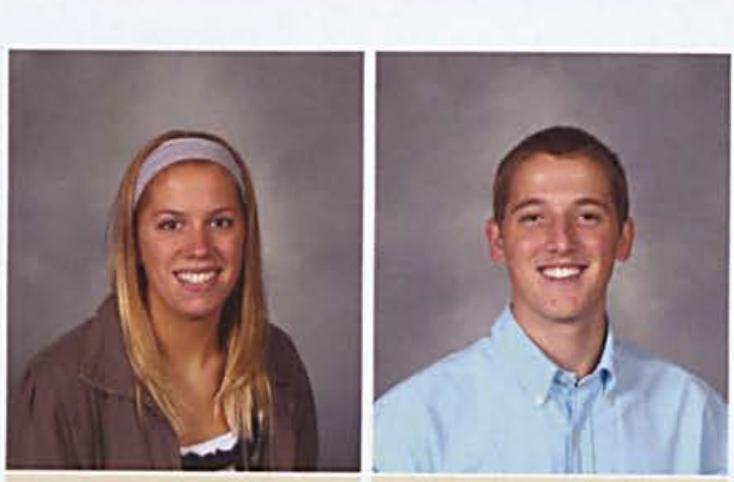

Joy Brammer

Micah Brant

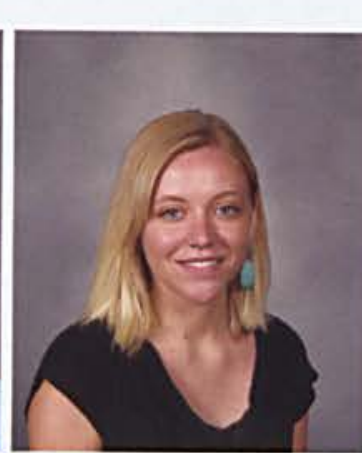

Anna Brown

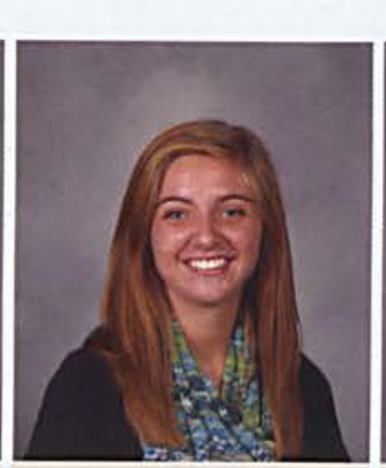

Madison Bruning

Nicole Buice

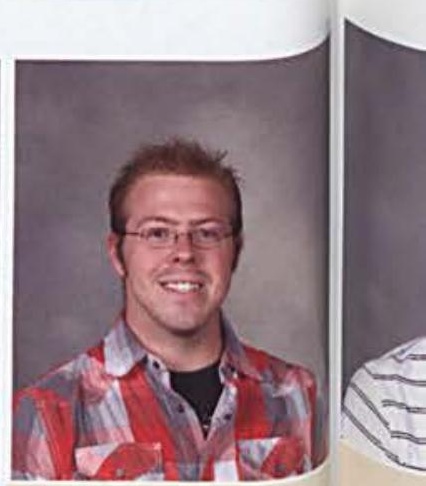

Andrew Bundy

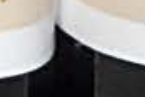




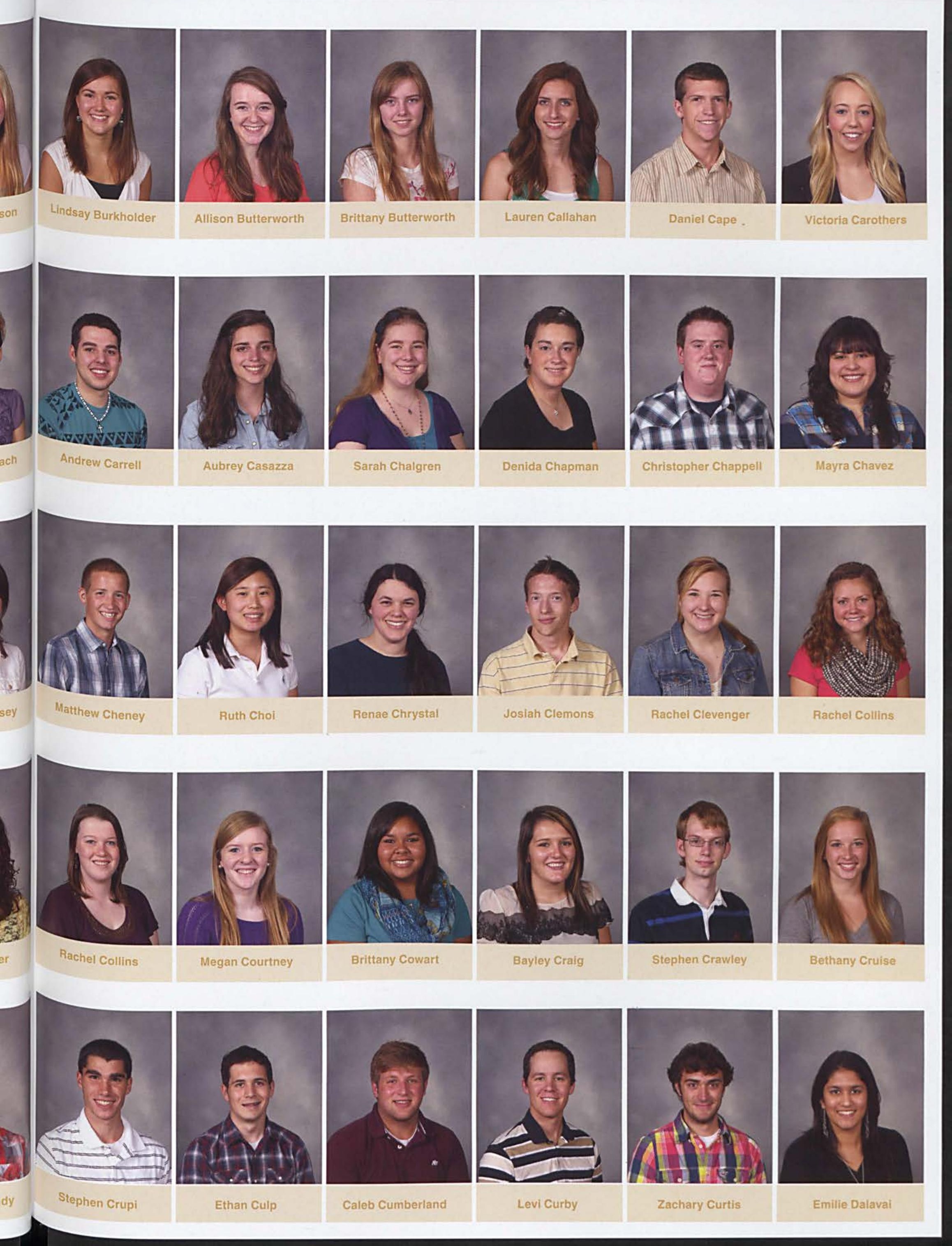




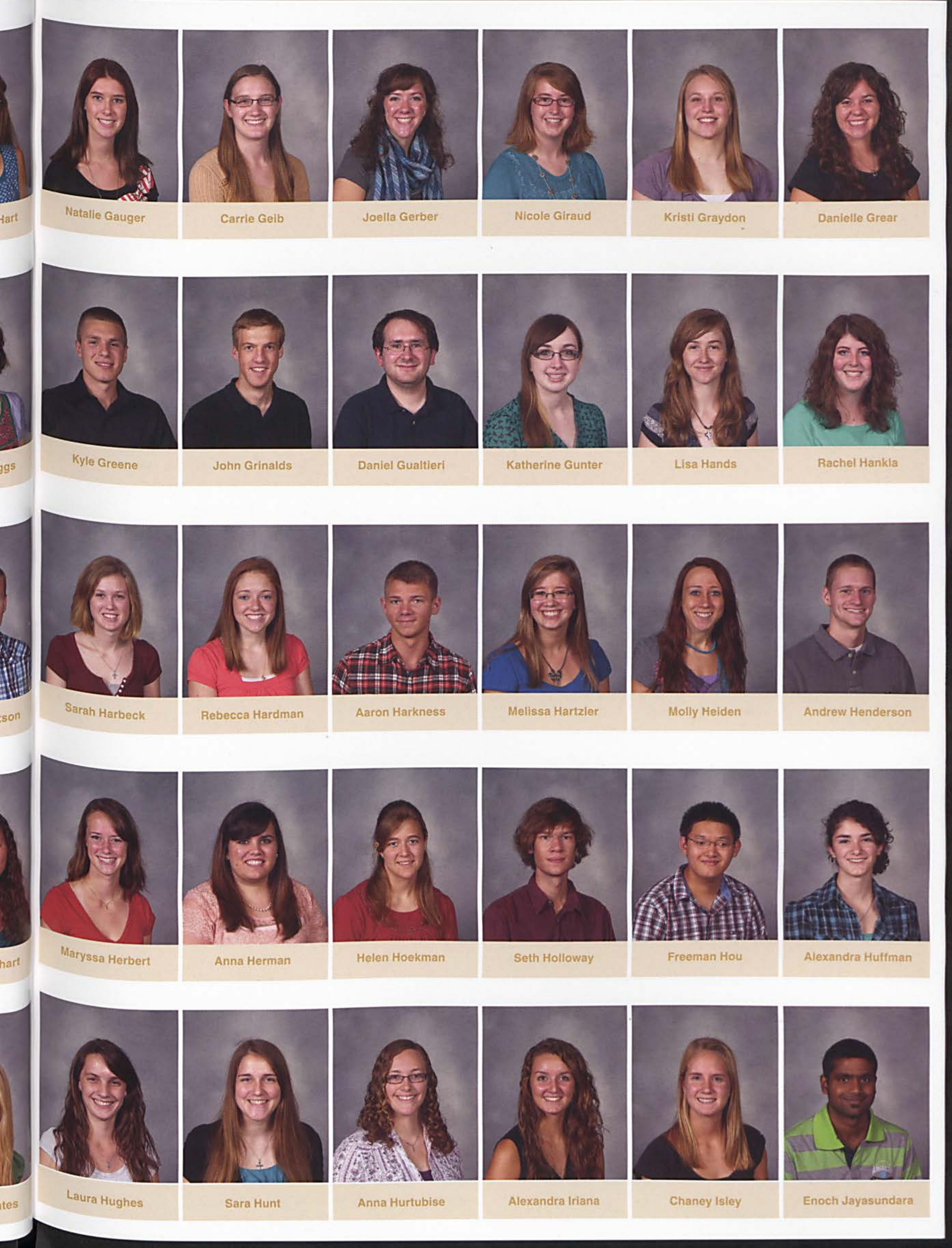




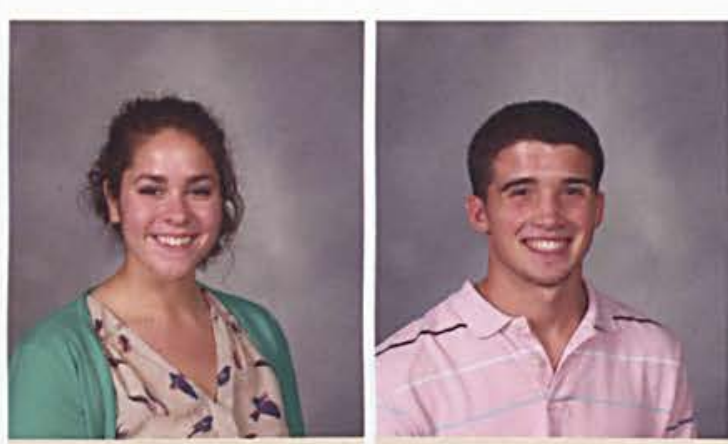

Alaina Jones

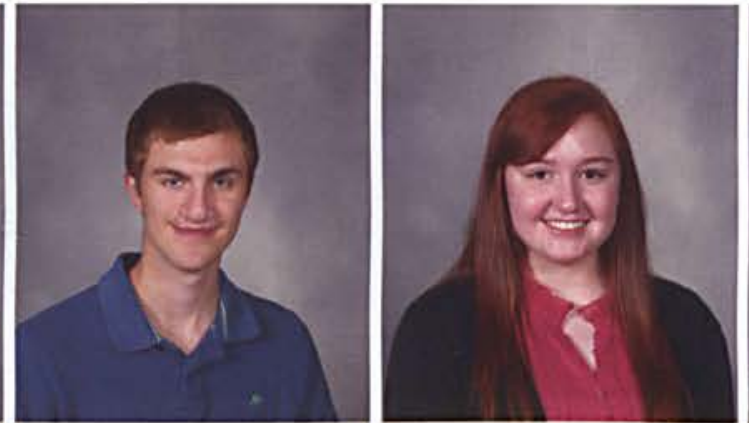

Jonathan Kearney

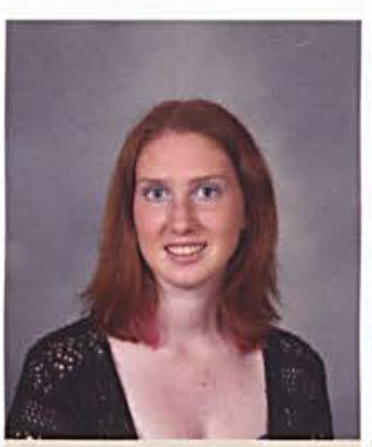

Rachel Kinnett
8

Ella Kinsinge

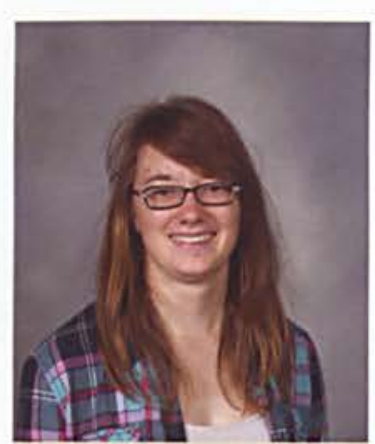

Valerie Kirk

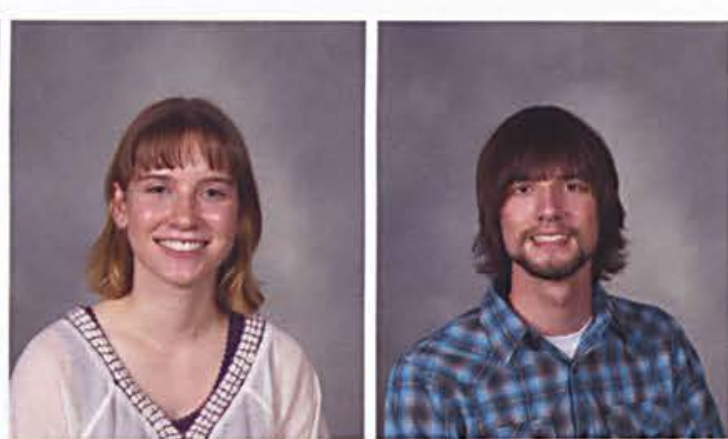

Ericka Kistler

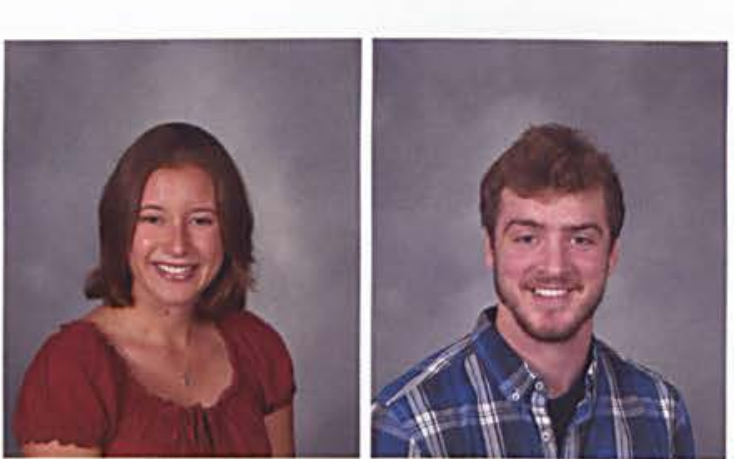

Laura Klodnicki

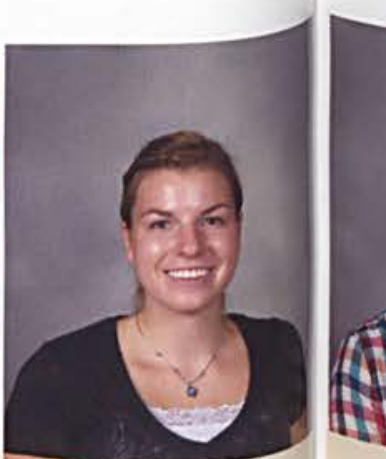

Jennifer Knepel

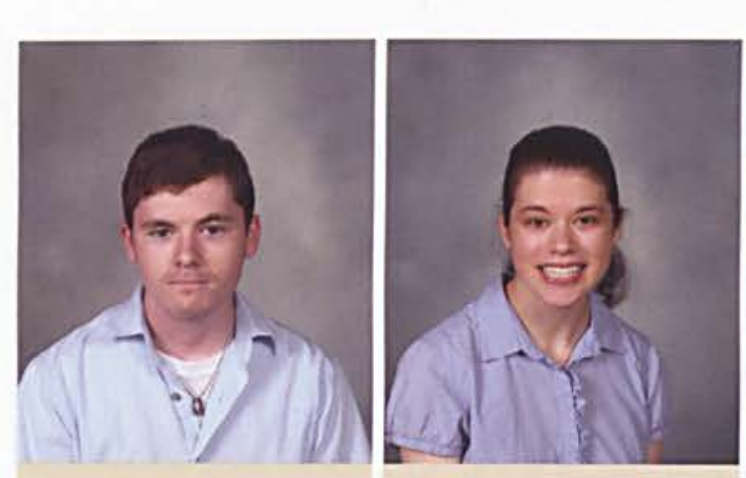

John Knowland

Meredith Knowland

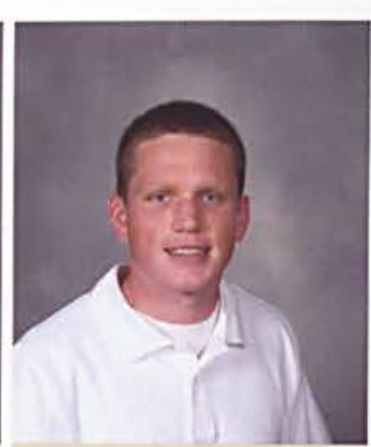

Peter Larson

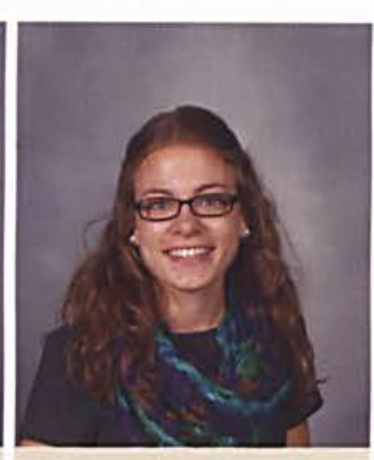

Lauren Koch

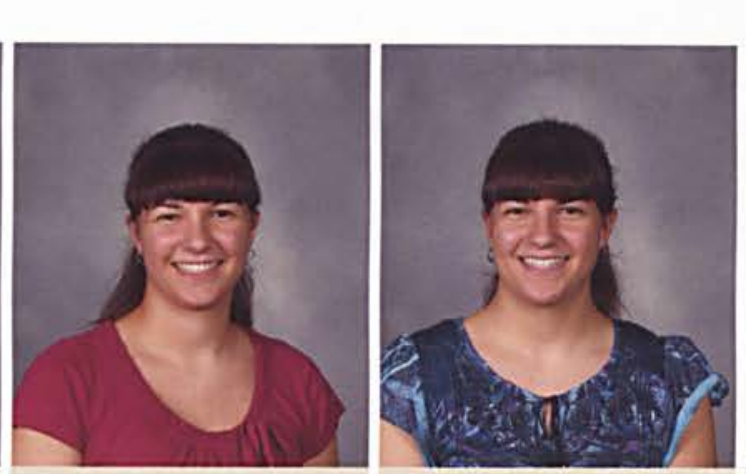

Jorian Krob

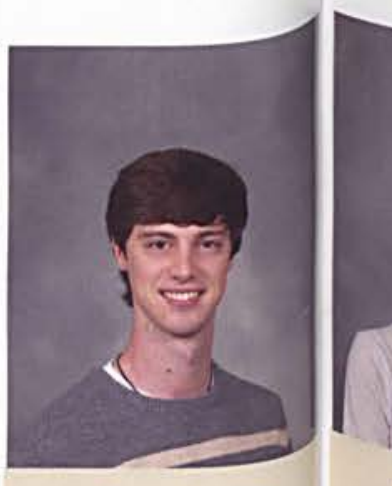

Sean Larkin

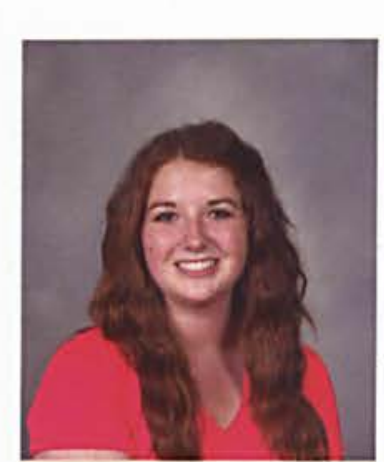

Alana Larned

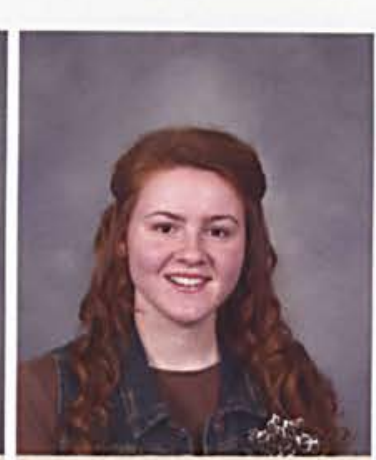

Rebecca Lawson

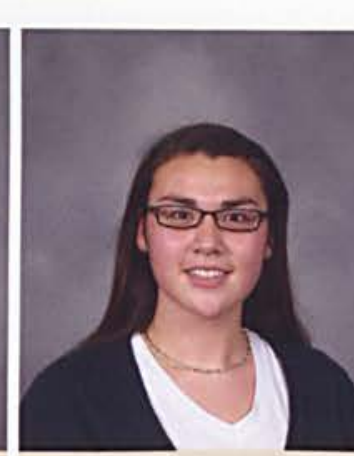

Jennifer Lee

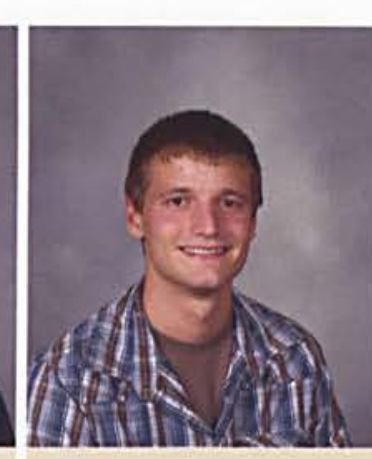

Matthew Lee

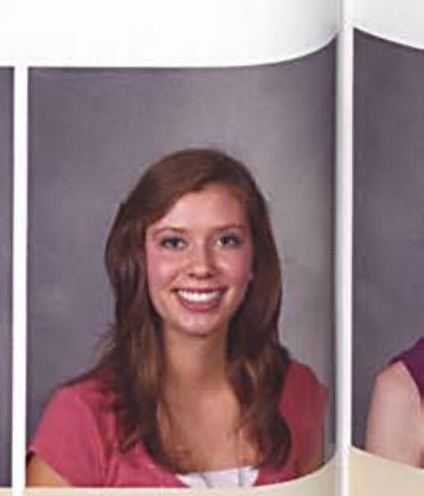

Callie Linstad

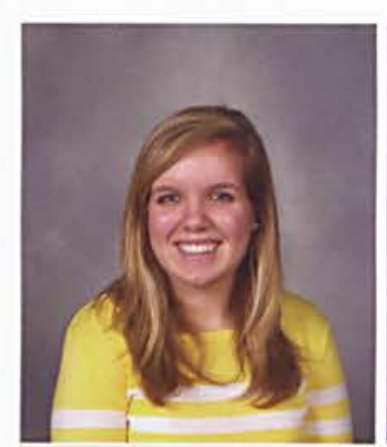

Mary Lively

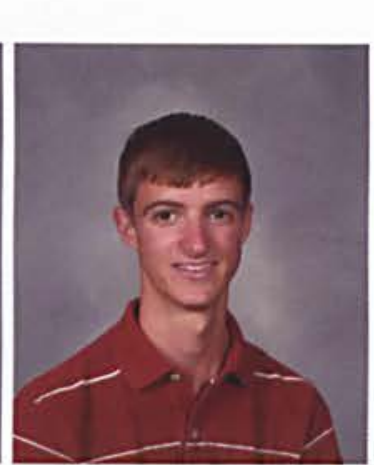

Andrew Luther

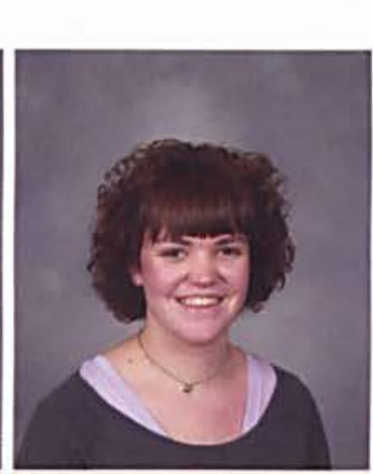

Elizabeth MacKendrick

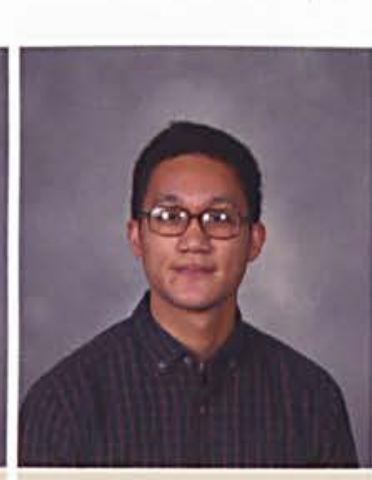

Andrew Malincon

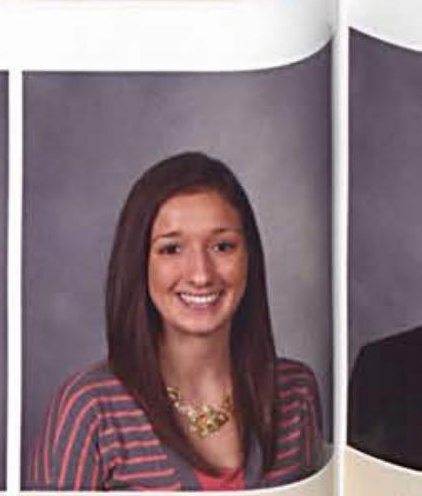

Kayla Marks 


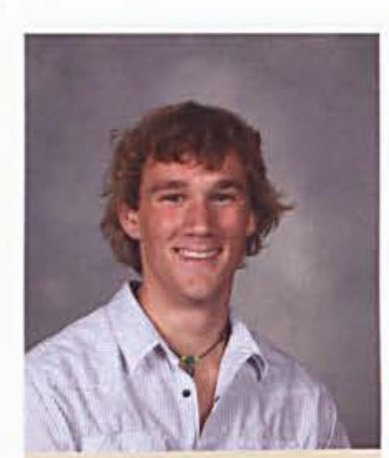

Joseph Pryor

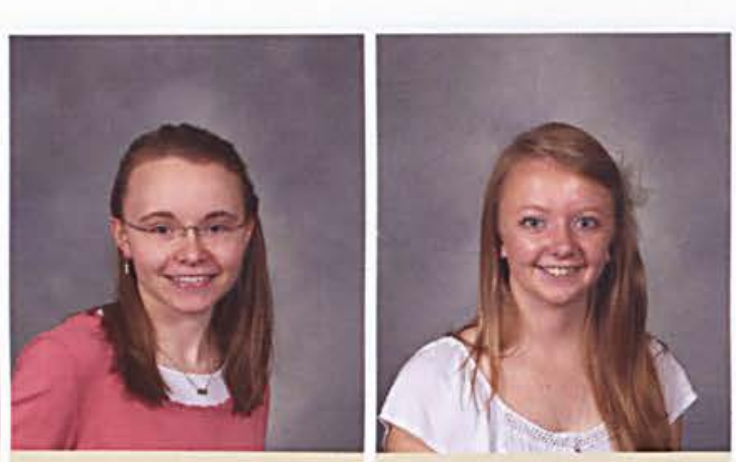

Johanna Raquet

Courtney Raymond

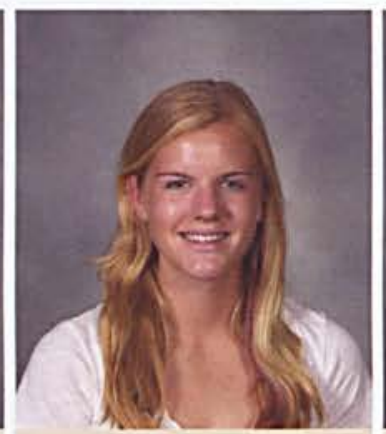

Melanie Redfield

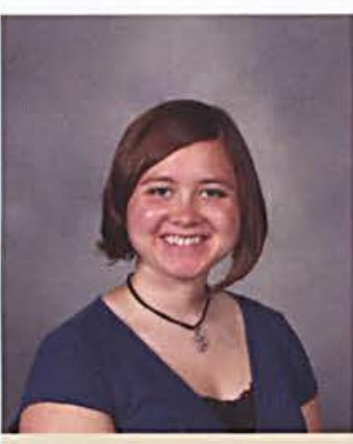

Kathryn Roberts

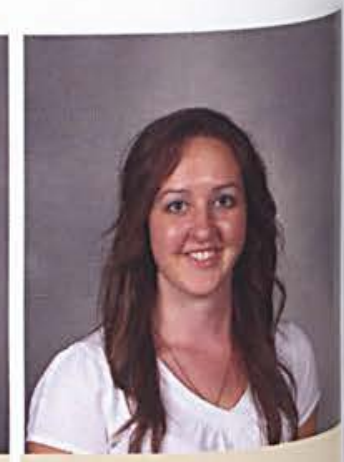

Tabitha Roberts

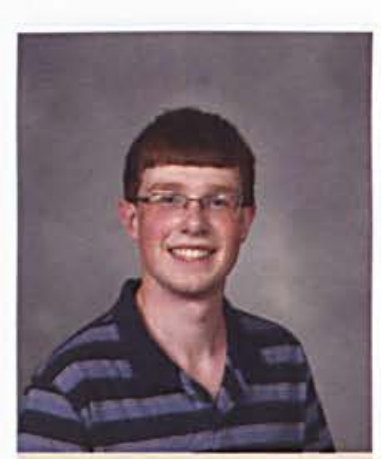

Andrew Robertson

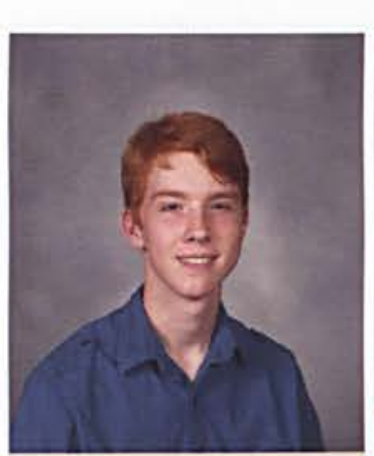

Daniel Robinson

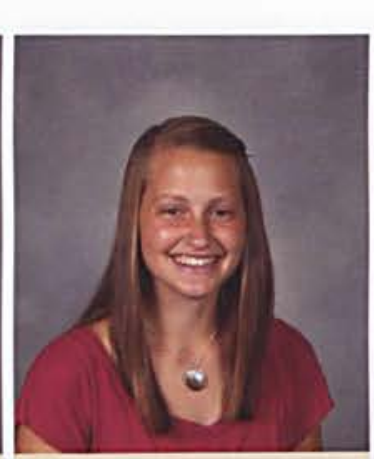

Melissa Ruhlman

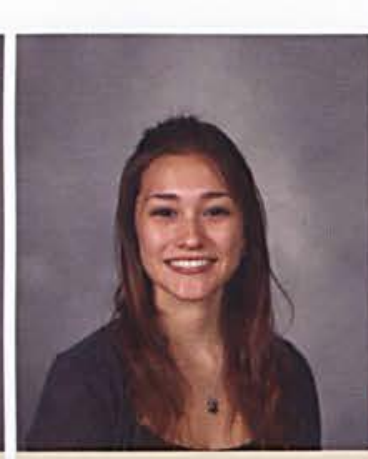

Amy Ruiz-Bueno

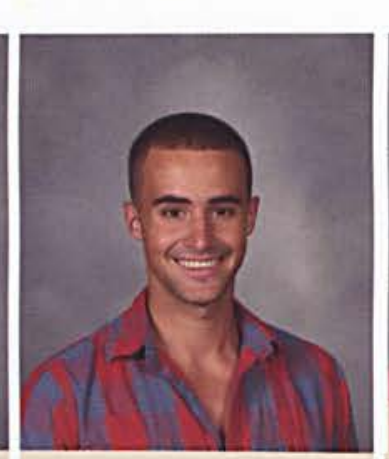

Benjamin Sanders

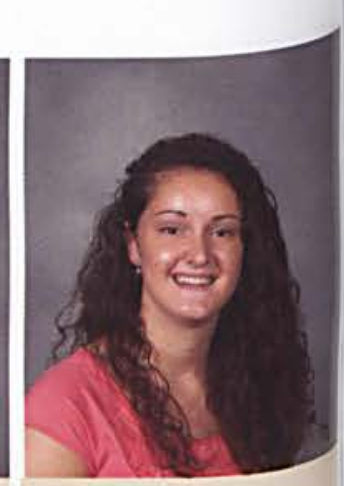

Hannah Scanlori

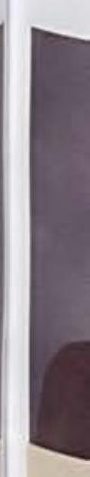

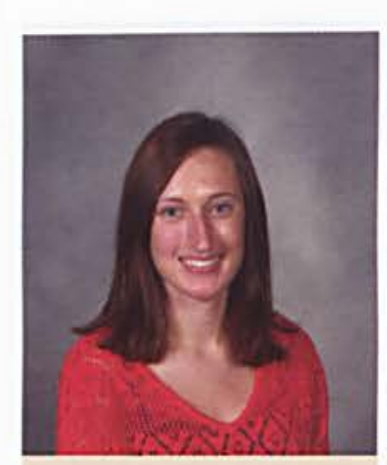

Kelly Schaffter

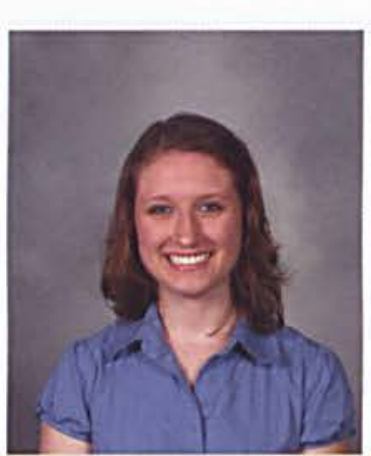

Annelise Schick

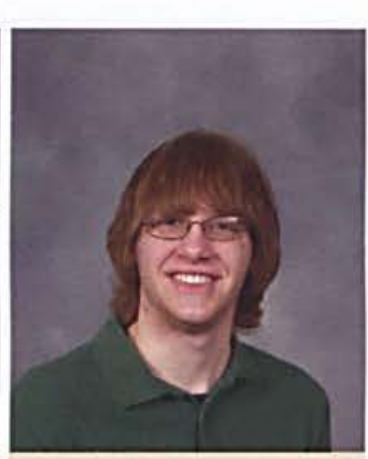

Erich Schroeder

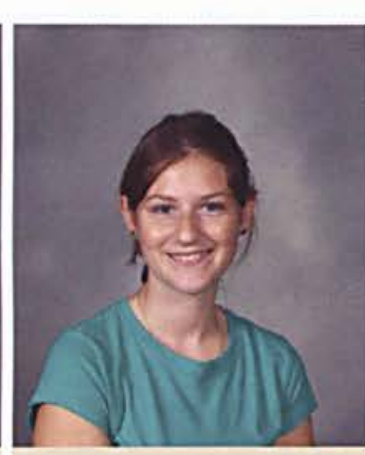

Amanda Seals

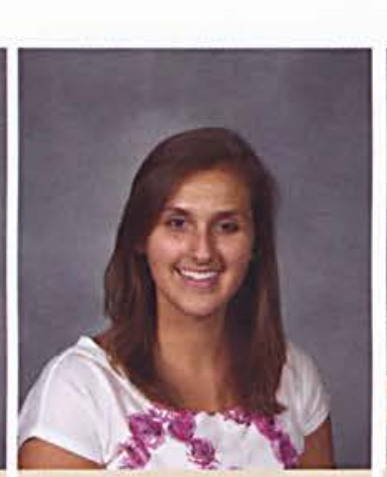

Cassandra Sensky

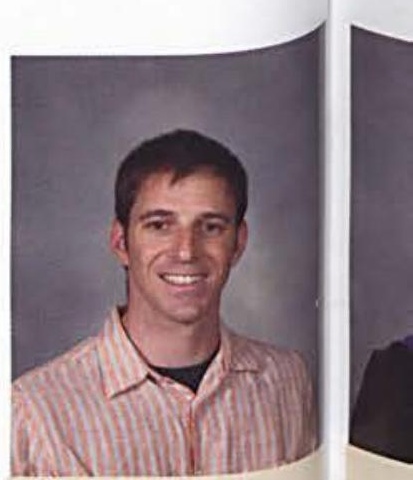

Troy Sergeant

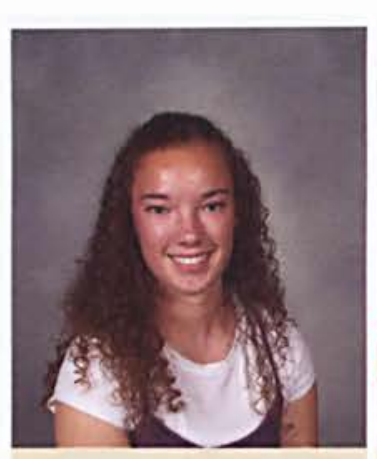

Hannah Shock

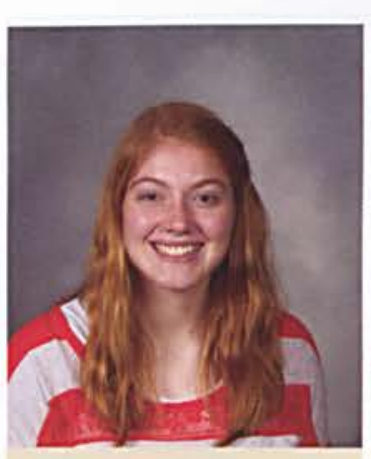

Victoria Shrum

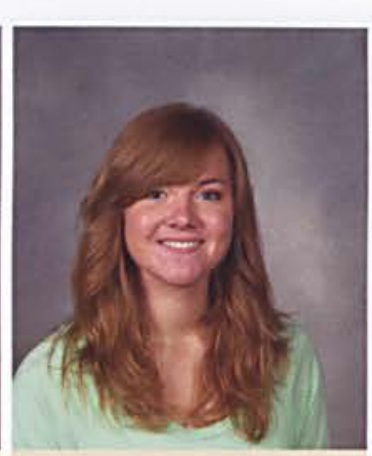

Rijah Shuck

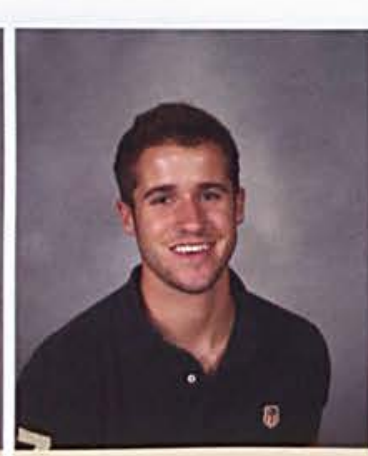

Zachary Sirois

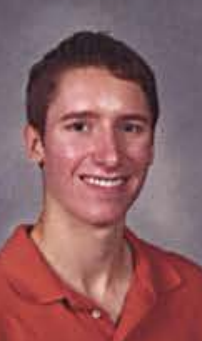

Steele Skrenta

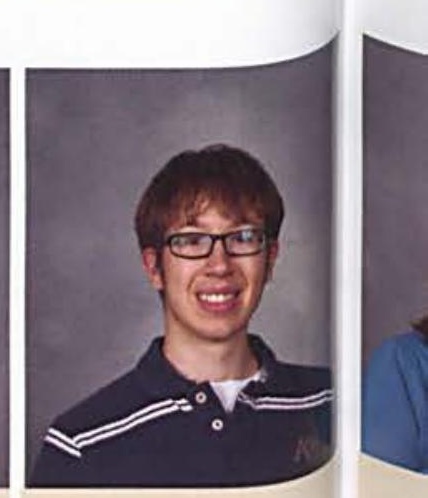

Josiah Sleppy
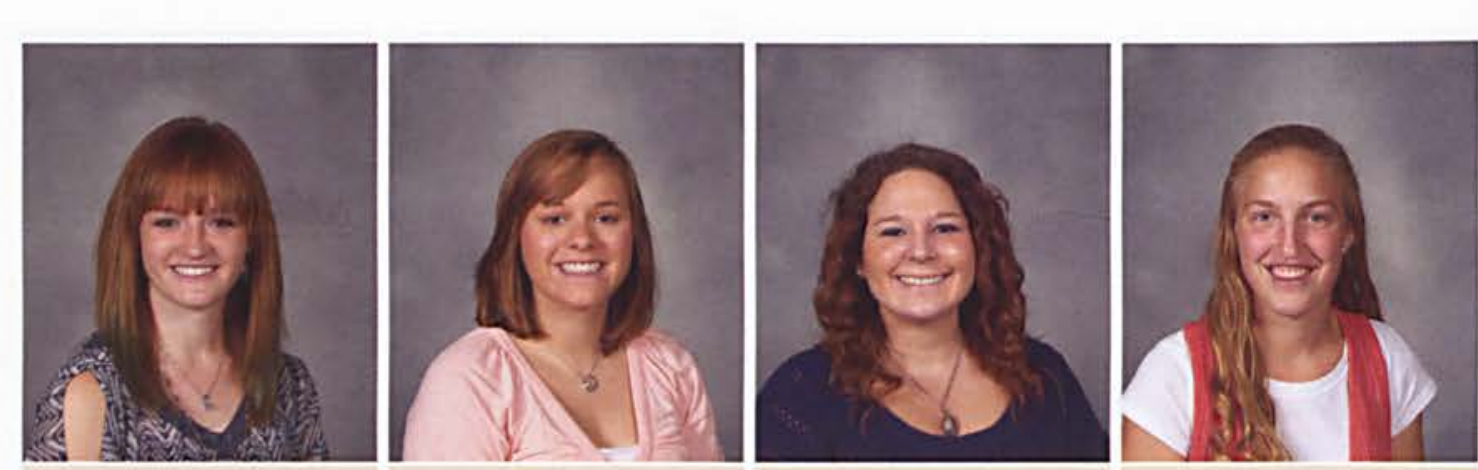

Courtnie Spain

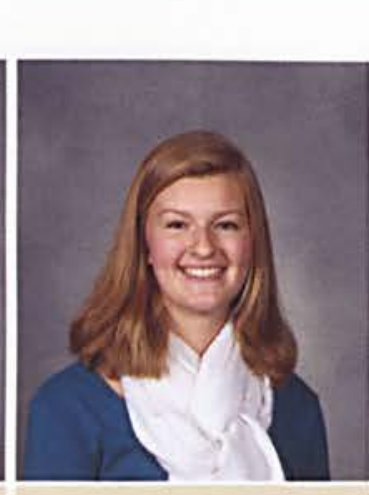

Lillian Stobbe

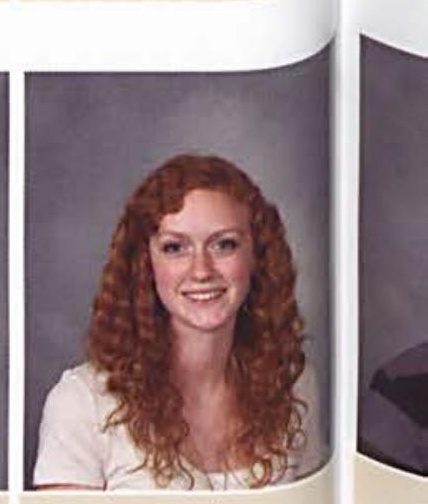

Sarah Straight 

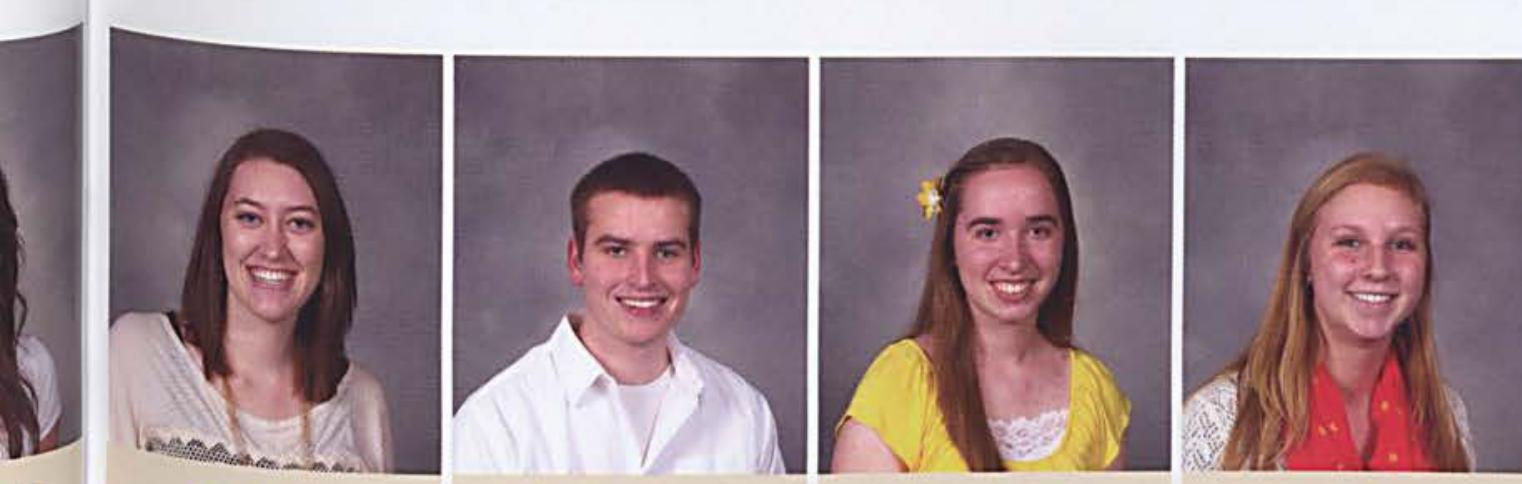

Tyler Tavierne

Rebecca Taylor

Courtney Teague

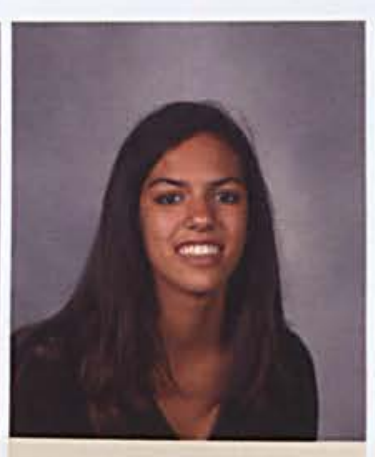

Aerial Thurman

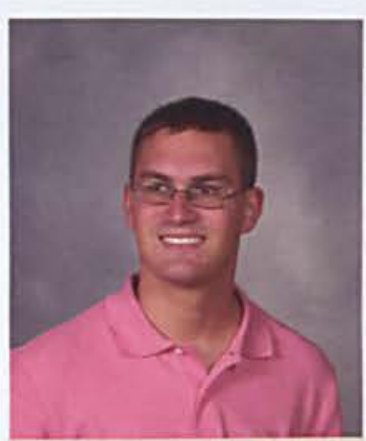

Jordan Treece

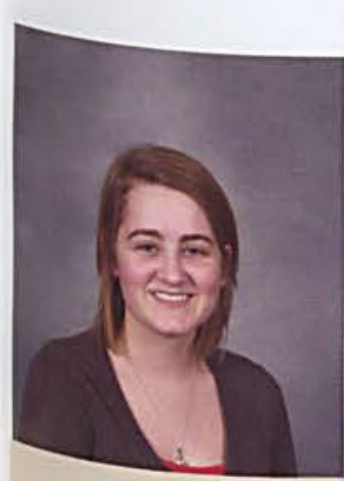

Emily Trigg

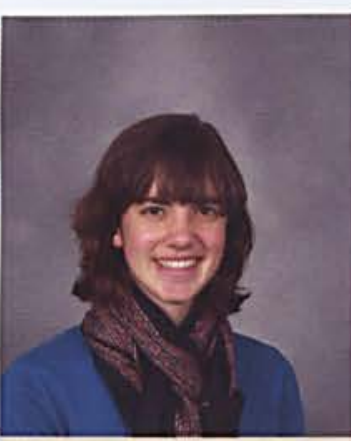

Megan Troyer

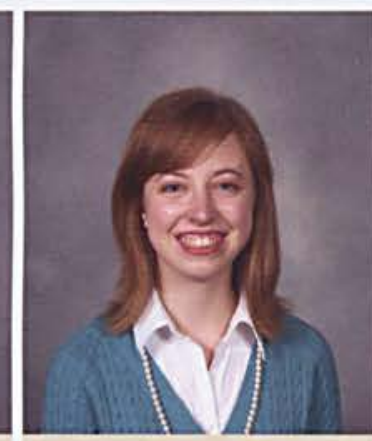

Lynley Turkelson

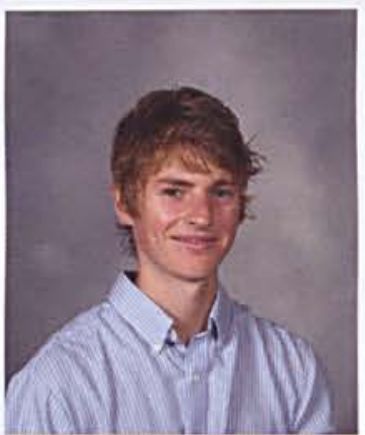

Benjamin Tuttle

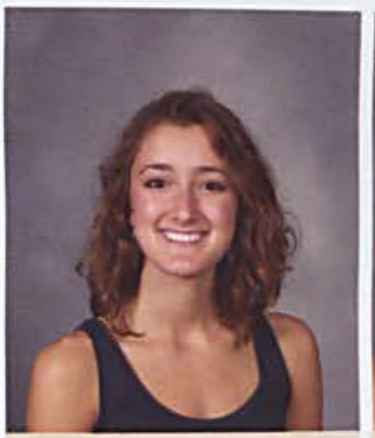

Caytlin Van Etten

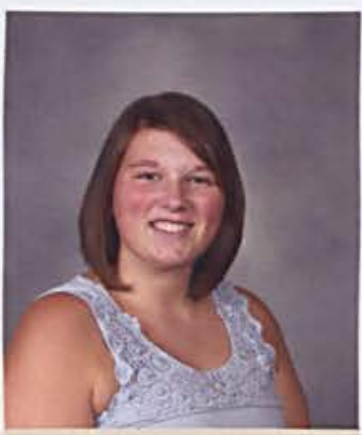

Kayla Vencill

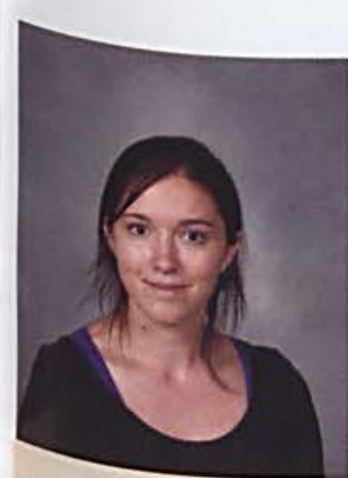

Racher Wadman

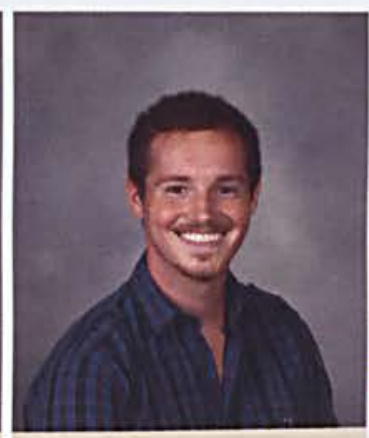

Luke Weaver

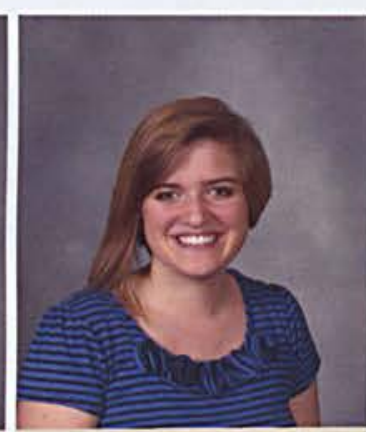

Kelsey Weir

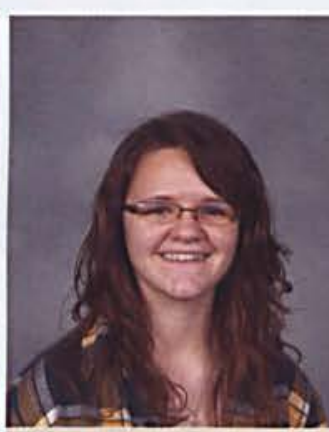

Darcy Whitney

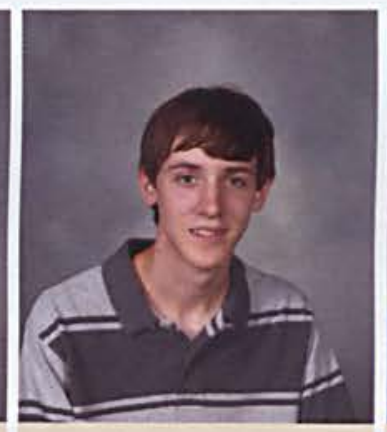

Bradley Williams

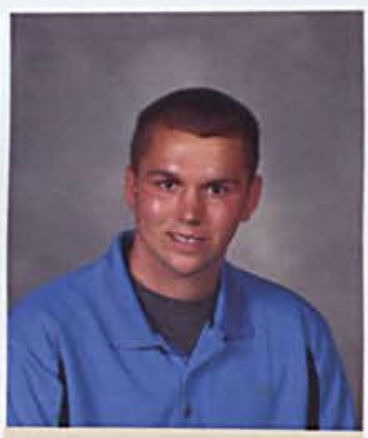

Cody Williams

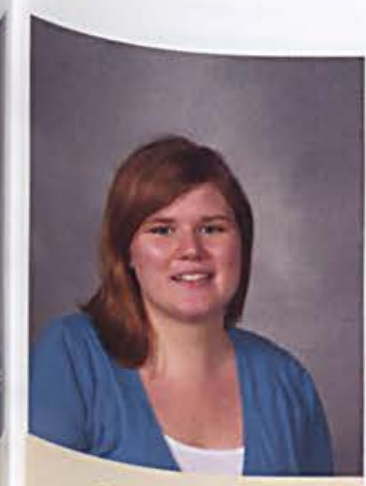

Caroline Wills

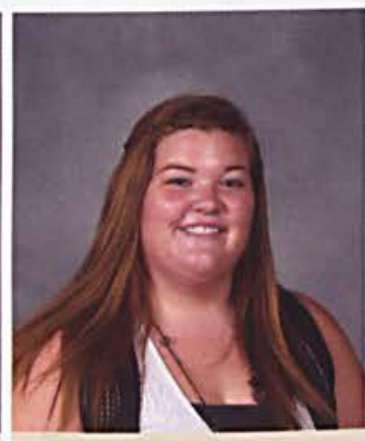

Donielle Winters

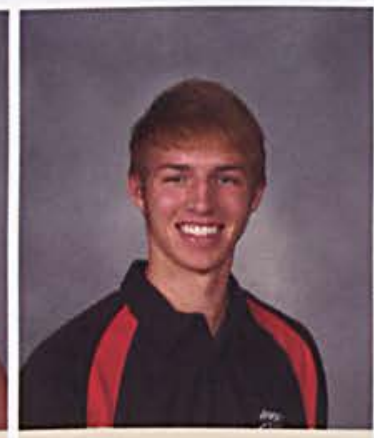

Colin Wirrig

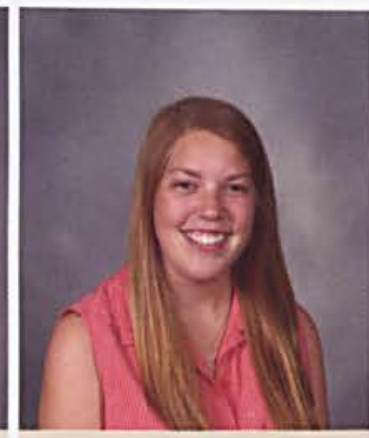

Heather Wismer

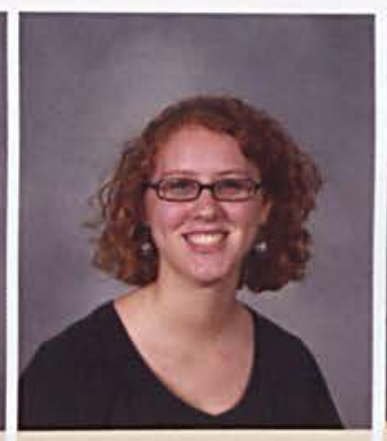

Ashley Worsham

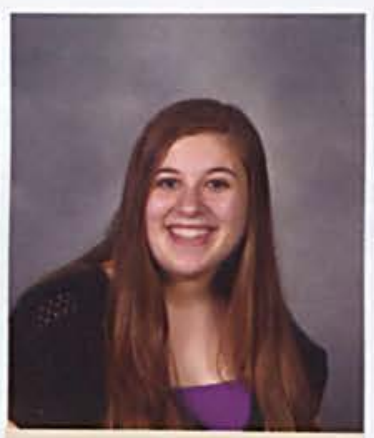

Hannah Wunderlich

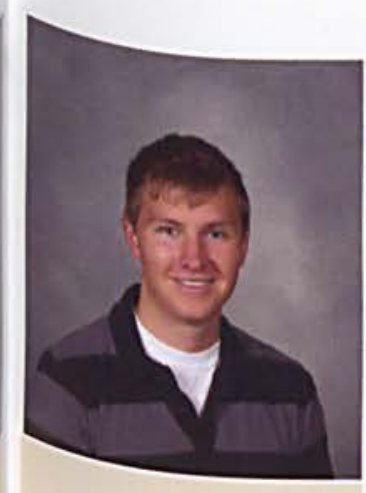

Seth Yates

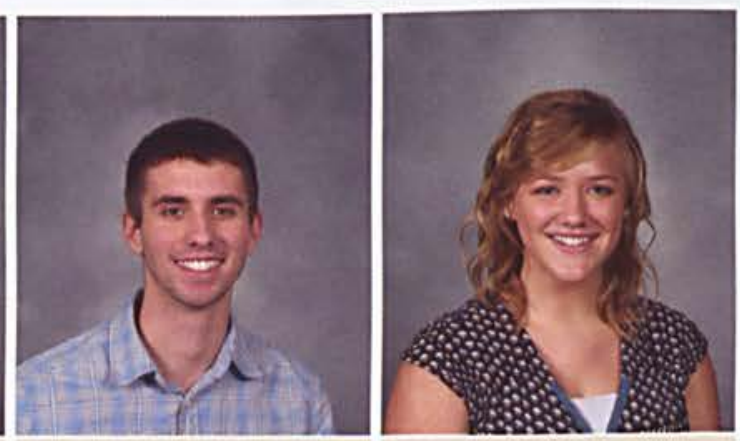

David Yoder

Julie Zavodney

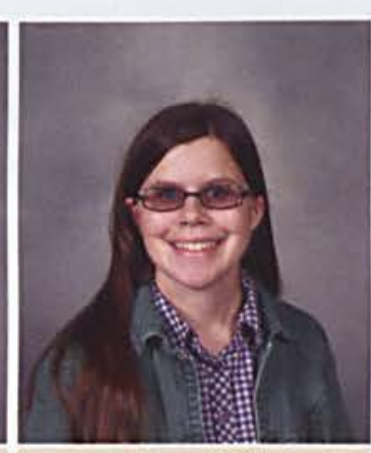

Rachel Zeiter

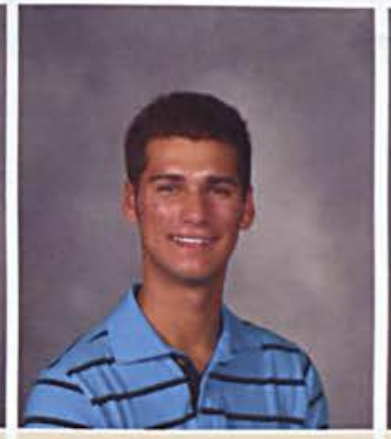

Jordan Zender

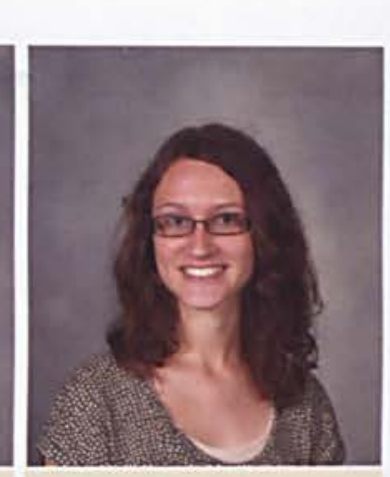




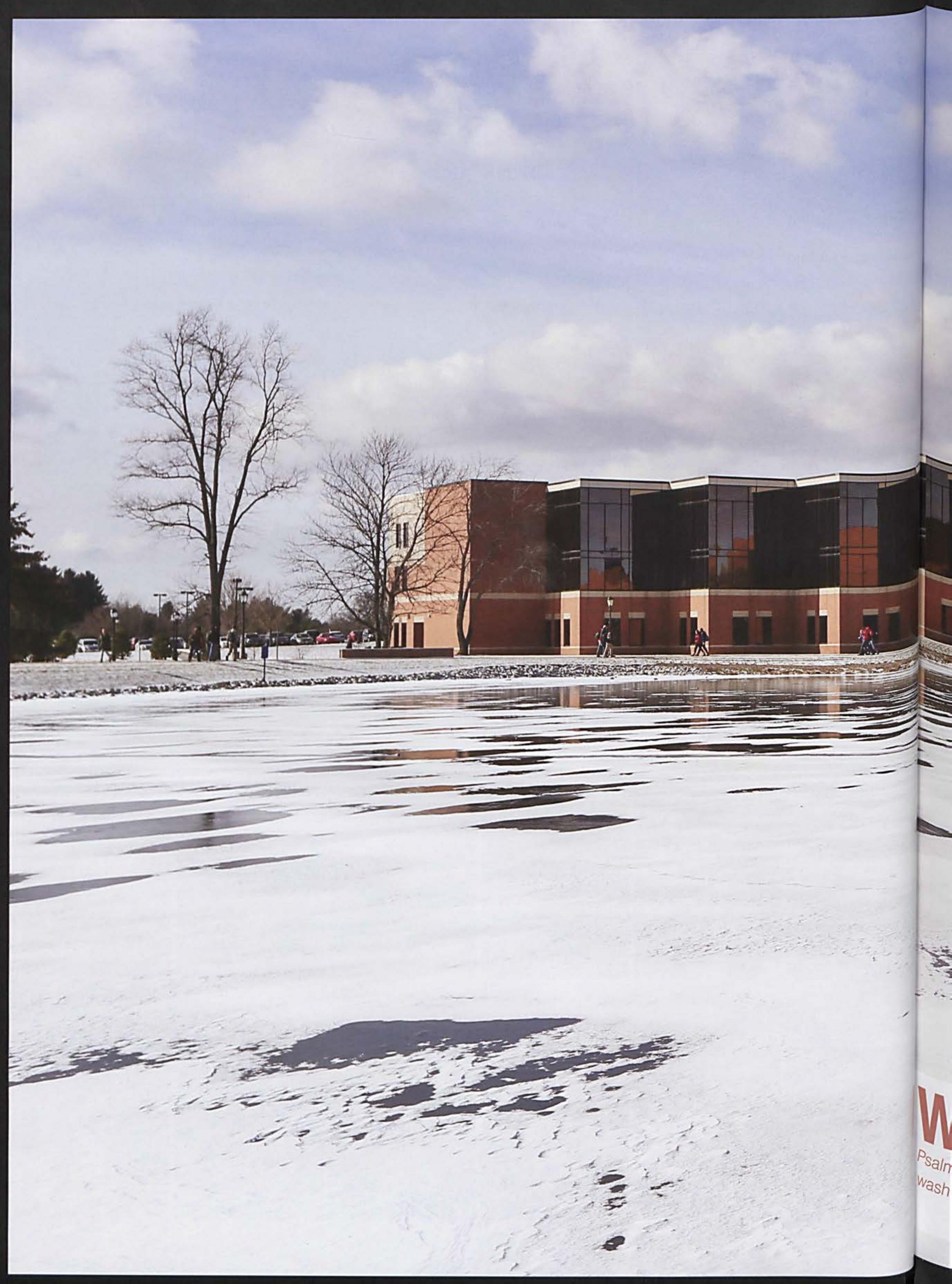




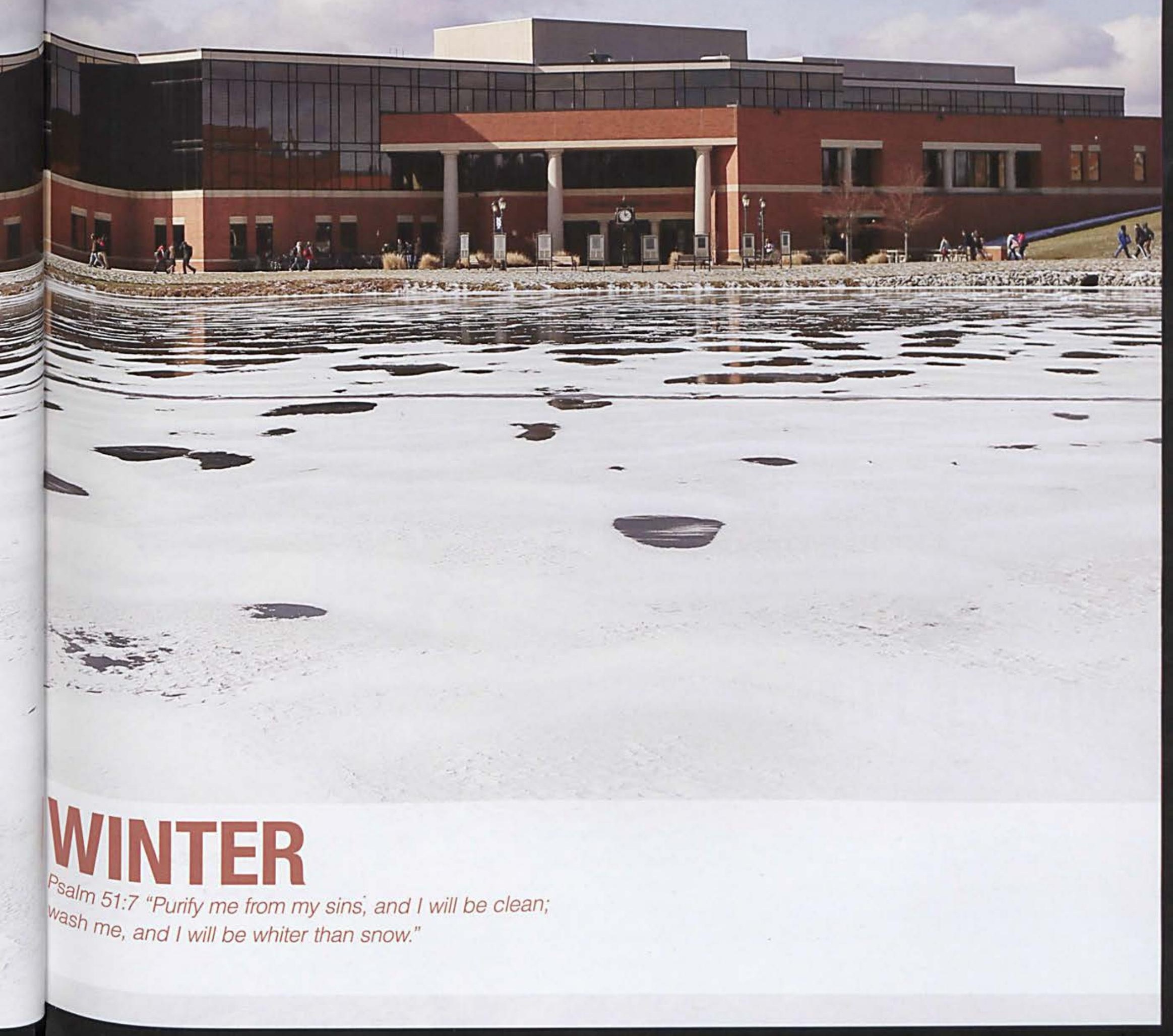




\section{JOSEPH AND THE AMAZING TECHNICOLOR DREAIMCOAT}
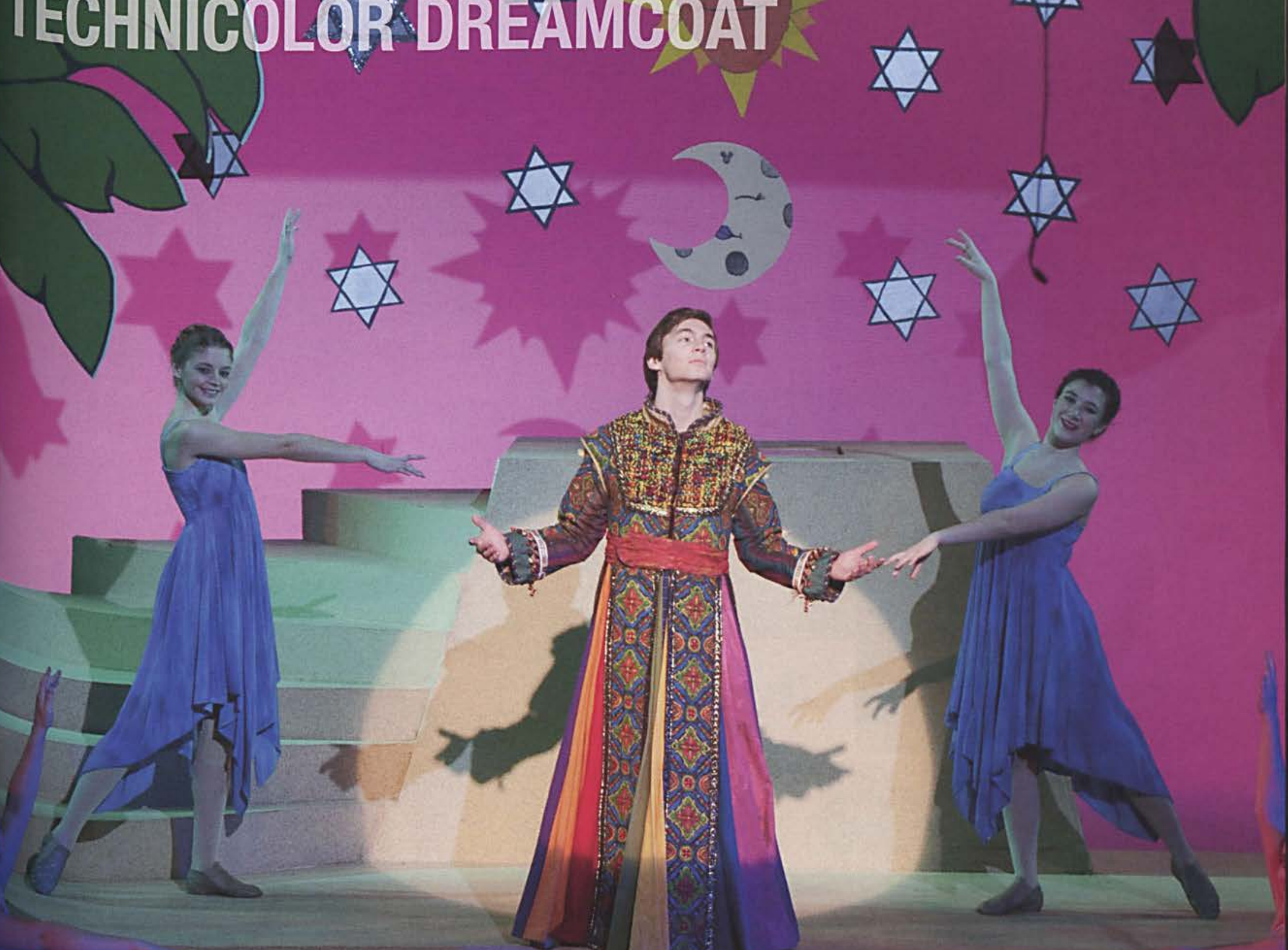


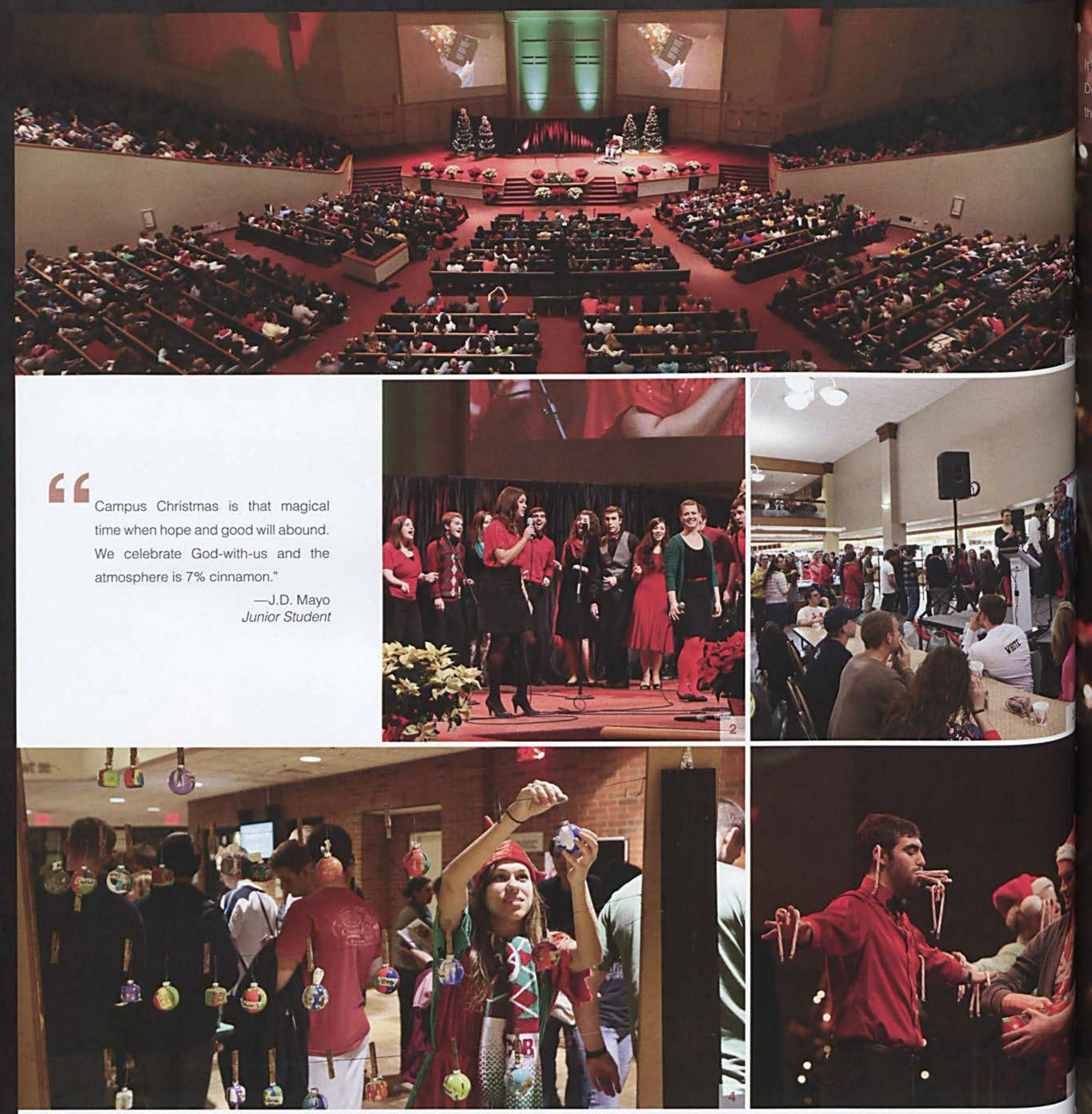

\section{CAMPUS CHRISTMAS}

Campus Christmas is perhaps the most wonderful time of the year at Cedarville. Some might say that even chicken tender night in Chuck's pales in comparison. The weekend after Christmas open dorms, SCAB hosted a variety of events. On Saturday night, students gathered in the chapel for a concert featuring the Inversions, an a capella music group from the university. Following the concert, Dr. Brown continued his tradition of reading Christmas stories to the student body. Since this was Dr. Brown's final Christmas reading, he read the students' favorite books from previous years: My Dad Ran Over a Frog and Peef the Christmas Bear. Only a few hours later, Chuck's even re-opened for a late night breakfast served by faculty and staff. Christmas karaoke filled the Chuck's air with "Sleigh Ride" and "The Christmas Song," some of this year's favorites. In the meantime, students created ornaments and decorated and ate Christmas cookies in the lower SSC. The weekend concluded with two showings of Elf in the Devries theatre.
1. Students pack the chapel for one of the biggest events on Cedarville's campus: the start of Campus Christmas.

2. Backed up by the rest of The Inversions, Freshman Ciara Kaufman and Senior Anna Zavodney belt it out for the crowd.

3. When it comes to midnight Chuck's karaoke, one voice soars above the rest-that is the voice of Karl Olson.

4. Like a good elf, SCAB member Molly Dinse helps out with the ornament-making station

5. Inversions member Jordan Lee competes in a candy cane challenge. 


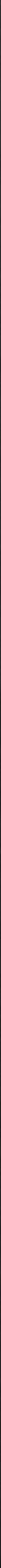




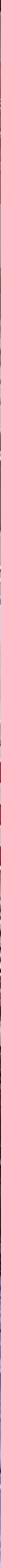

The winter semester is a time of growth and renewal into February, the campus was flooded with little sibfor students. The semester began with the Winter Blast lings. Students and their younger siblings attended a event featuring the band Gungor, run by the Student Center Activity Board (SCAB), and was soon followed by the Dream Event. In honor of Dr. Martin Luther King. Jr., the Dream Event featured a number of artistic students who performed spoken word, songs, and readings. As January came to a close, seniors were invited to attend the 100 Days Party, where they acknowledged the rapidly approaching arrival of graduation. Not long few on-campus events, including the movie Wreckit Ralph and a basketball game. Although there were many fun events throughout the semester, there were also events that brought change. In early February. Dr. Carl Ruby stepped down as Vice President for Student Life. This was a sad time for the Cedarville community, but everyone continued to remember to keep their eyes focused on Christ despite the situation.
1. Students gather in earnest prayer for the changes taking place in the university

2. Michael Gungor leads the rest of the band in a memorable show in the DMC Chapel.

3. Only 100 days left until graduation for the seniors, and they're already celebrating w their "Hundred Days" party.

4. Students in red line the path to Dr. Carl Ruby's car as the former Vice President o Student Life makes his final exit. 


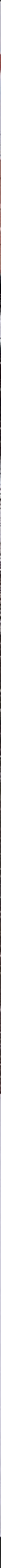




\section{WINTER EVENTS}

Cedaryille has its fair share of snow storms this year.

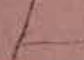
4

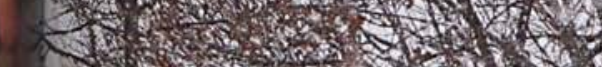

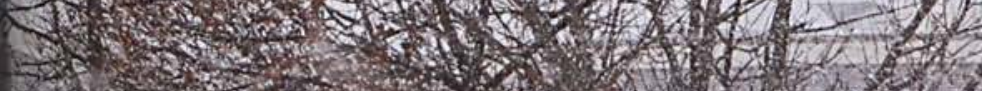
1.1.5.

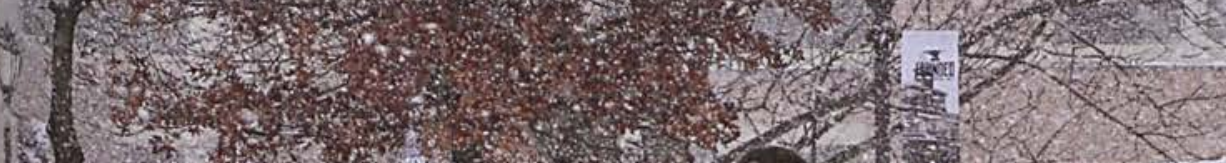

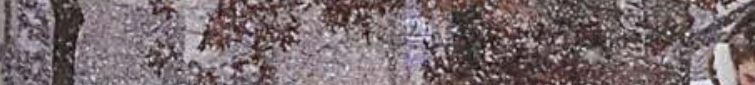

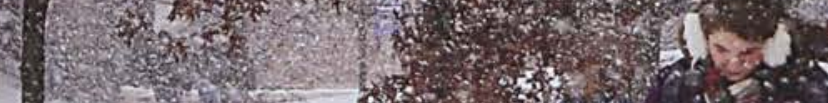
$|-| \frac{1}{4}$ ) (2)
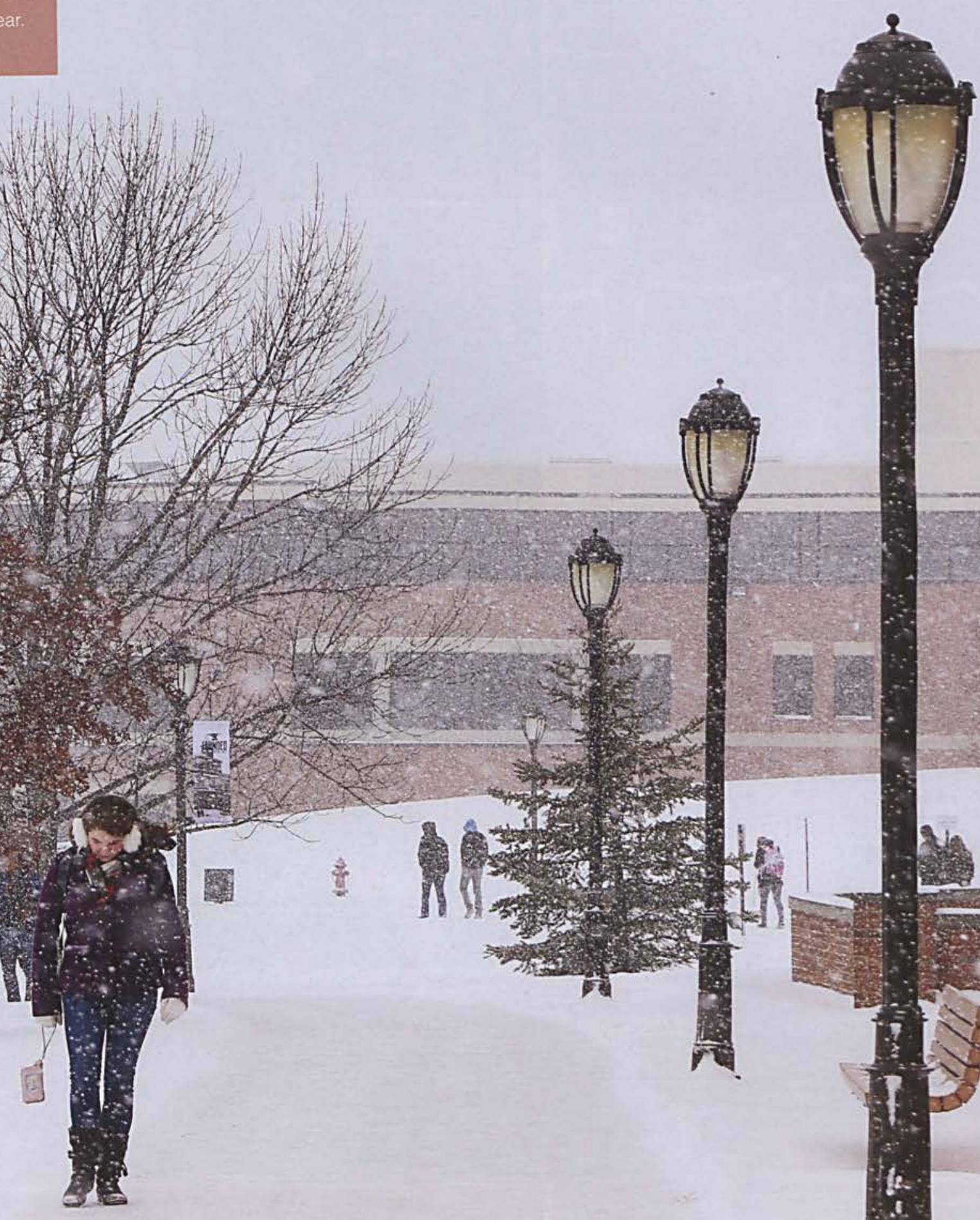

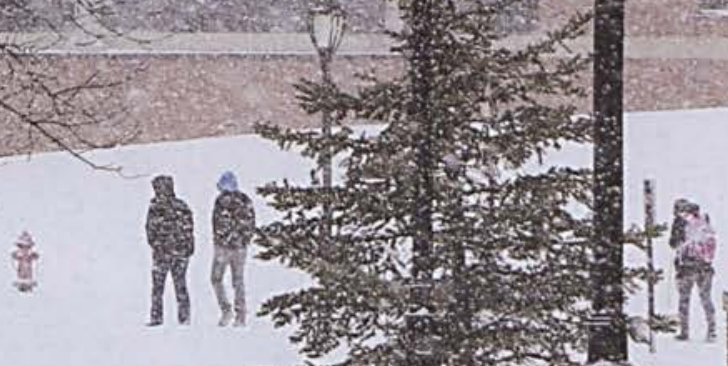

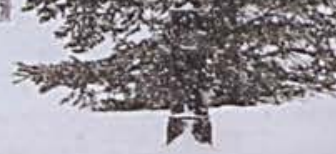

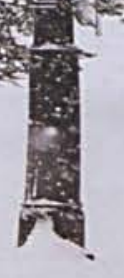

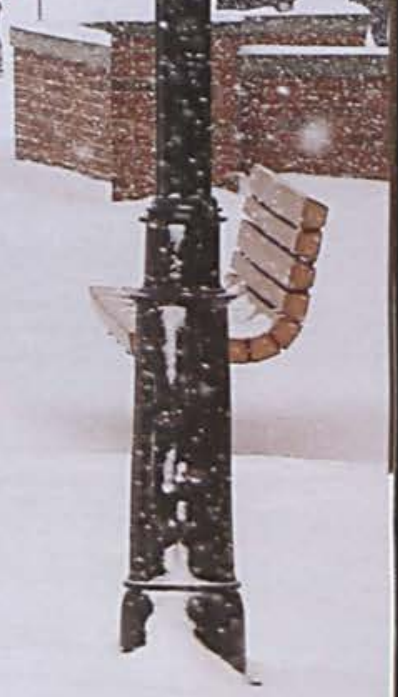




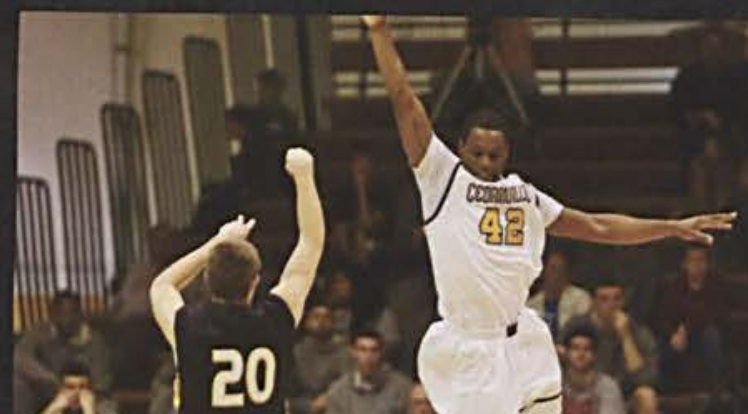

A 20 (1)

fé

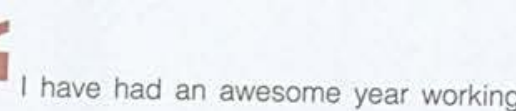

for the men's basketball team. From the

trip to Hawaii, to the daily practices, I

can honestly say I have enjoyed every

aspect of it.

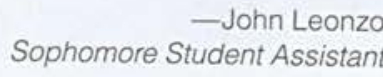
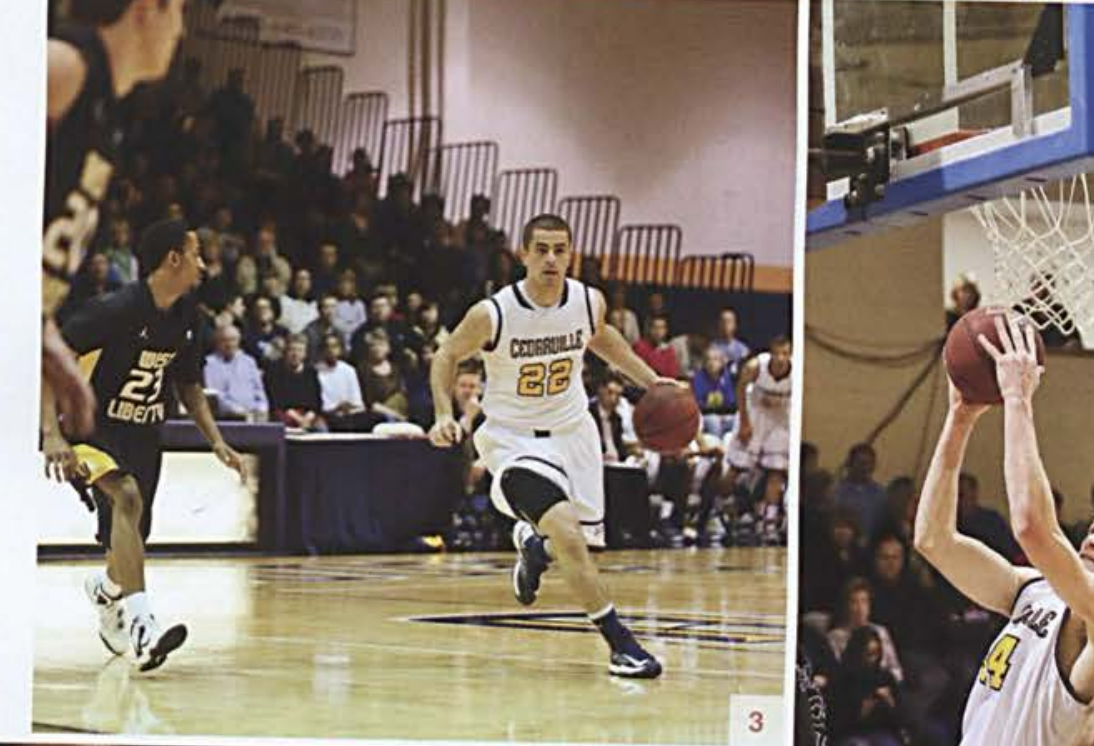

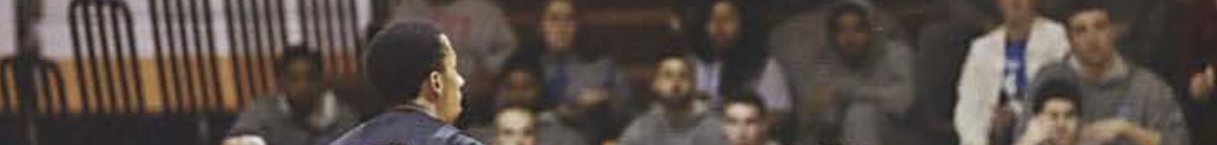

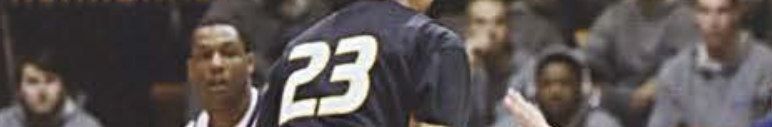

$\because=2 \pi$ ?

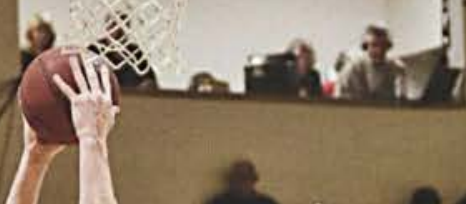

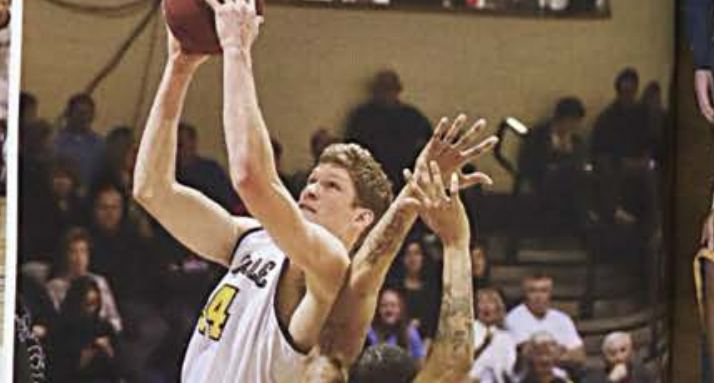

$85-9)^{2} 15$
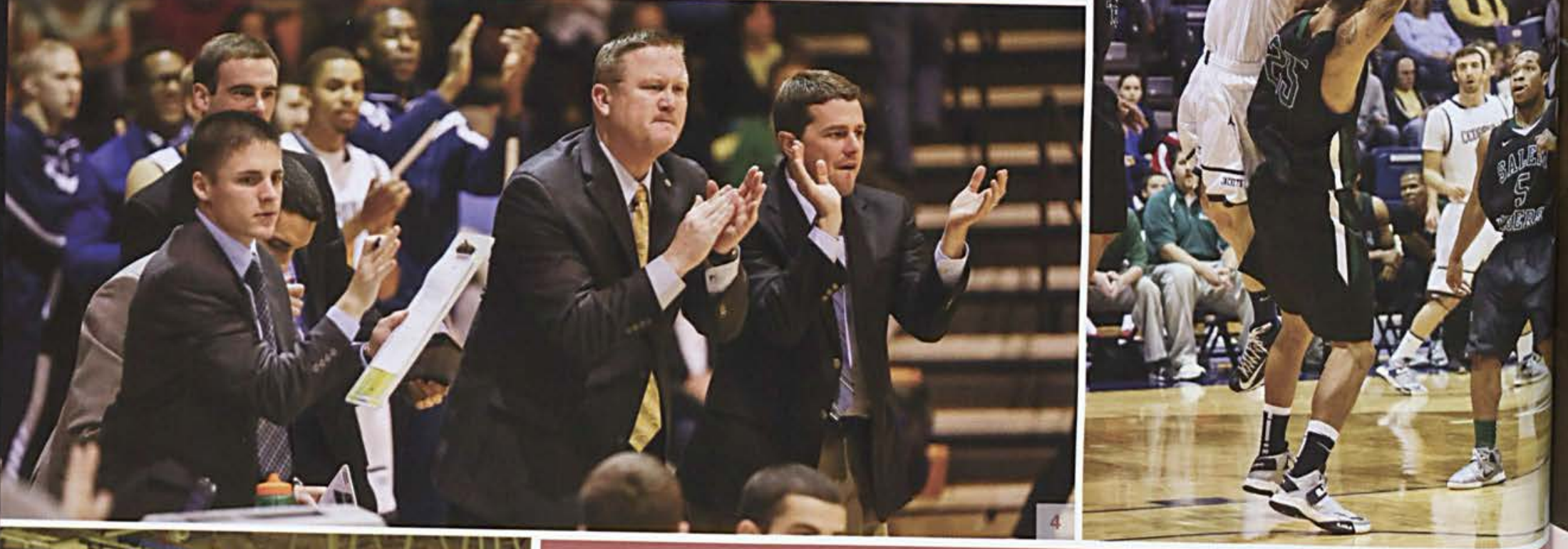

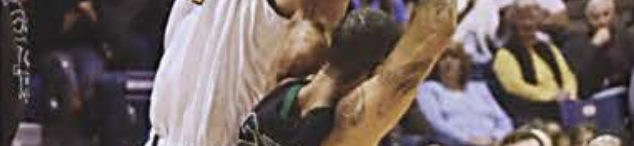

\&, 5 ,
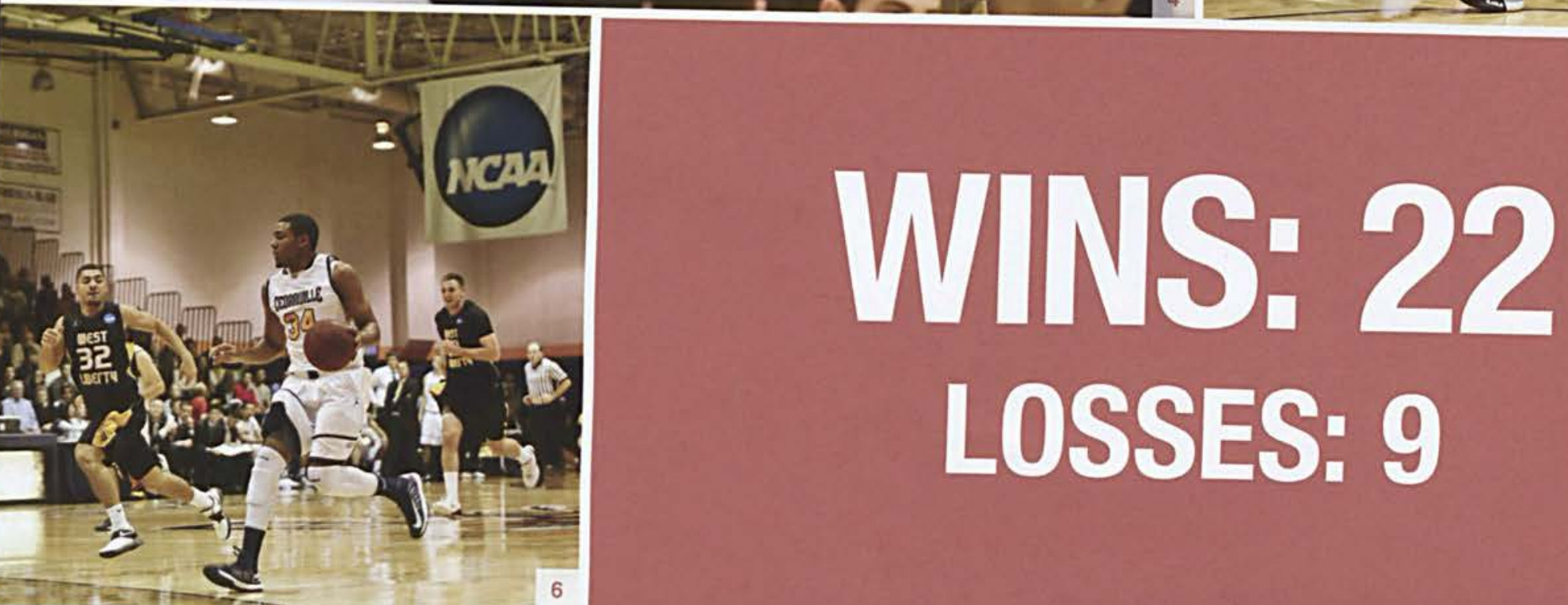


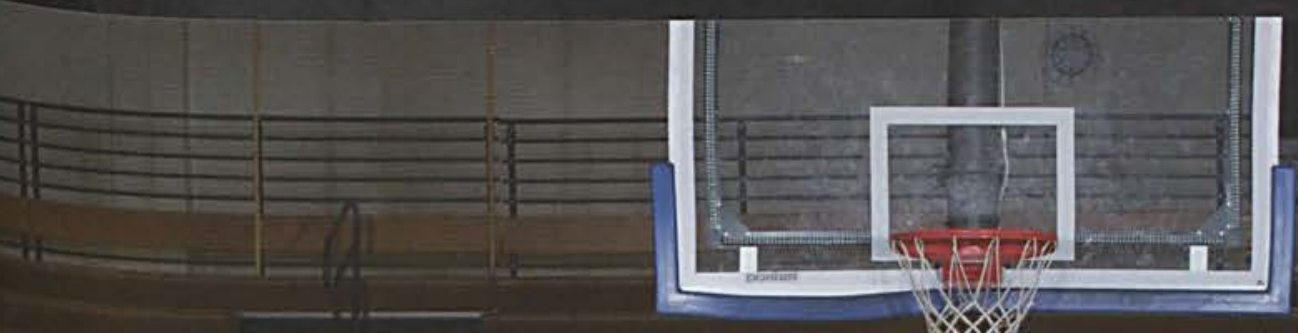

129

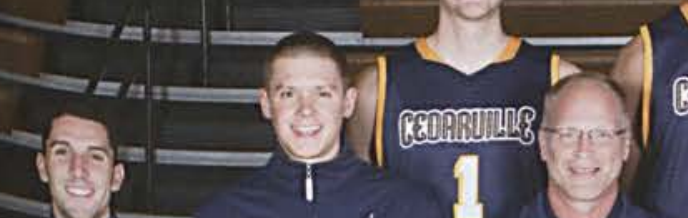

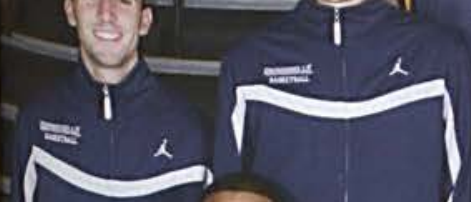

1
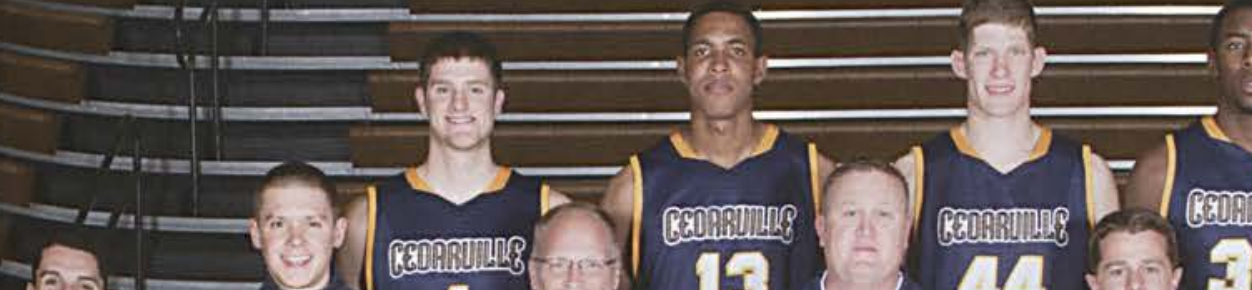

$\sqrt{=} 9$
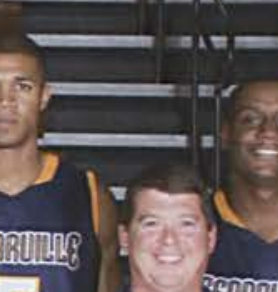

-

$=0$
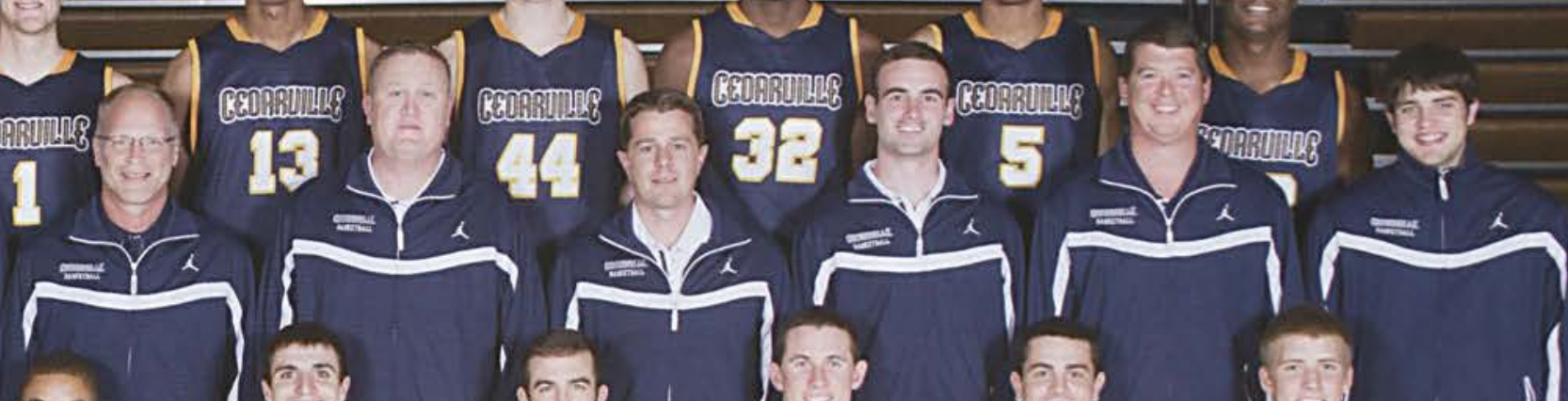

cendand

CEDPROMLR

(14. 3 - 14

$\log ^{2}, 15$

$12-\ln ^{1}=3$

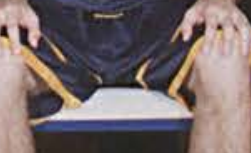

Noses
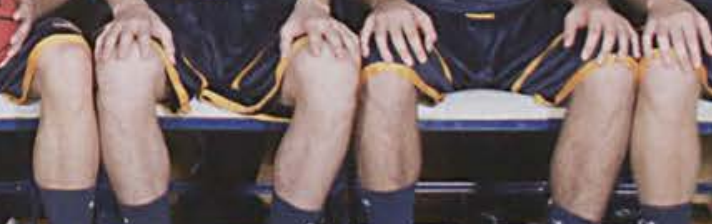

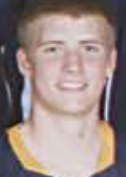

112

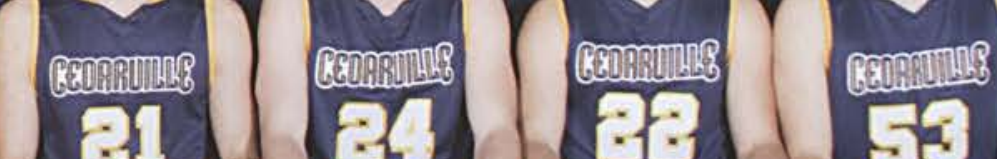

Q1 : $: 2$ : 5

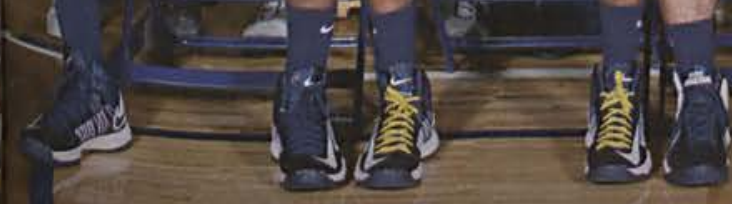

$\therefore$
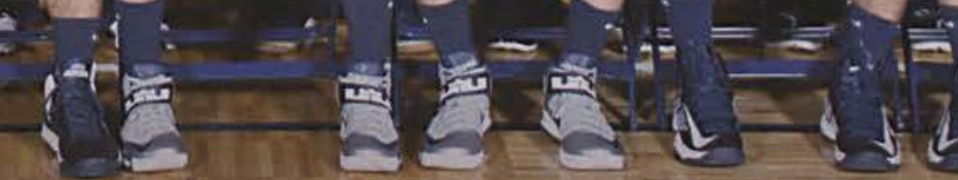

\section{MEN'S BASKETBALL}

2. Cedarville's own Daniel Hickman plays a

strong defense as the crowd looks on.

3. Dribbling the basketball, Marcus Reineke presses down the court.

4. Coaches on the bench react to a good play by the Cedarville Men's Basketball team.

5. Caleb Maxwell, a senior veteran of the

Cedarville team, leaps up to score on the

Salem Tigers.

6. Hustling down the court, Jason Cuffee

attacks the basket while the opposition tries

to keep up. 
बा $\quad 07$

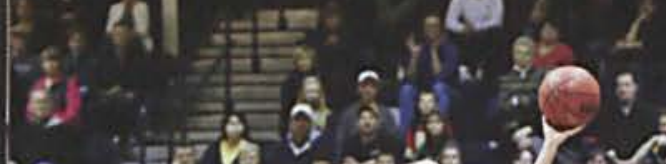

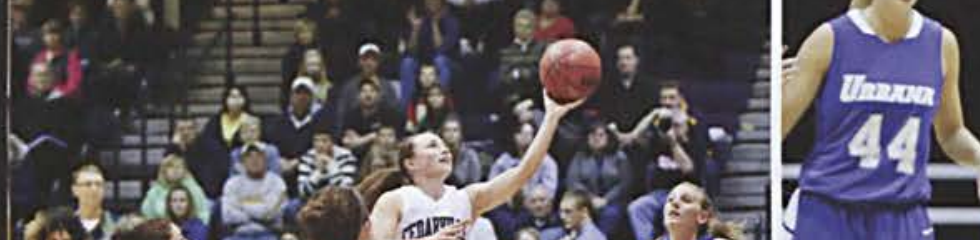
(t) 5 . 40 H: 14 , $1 x^{2} \cdot x^{-1}$

$(2+1)=$ (5) ${ }^{3}$ $4=15$ of 2012 II)

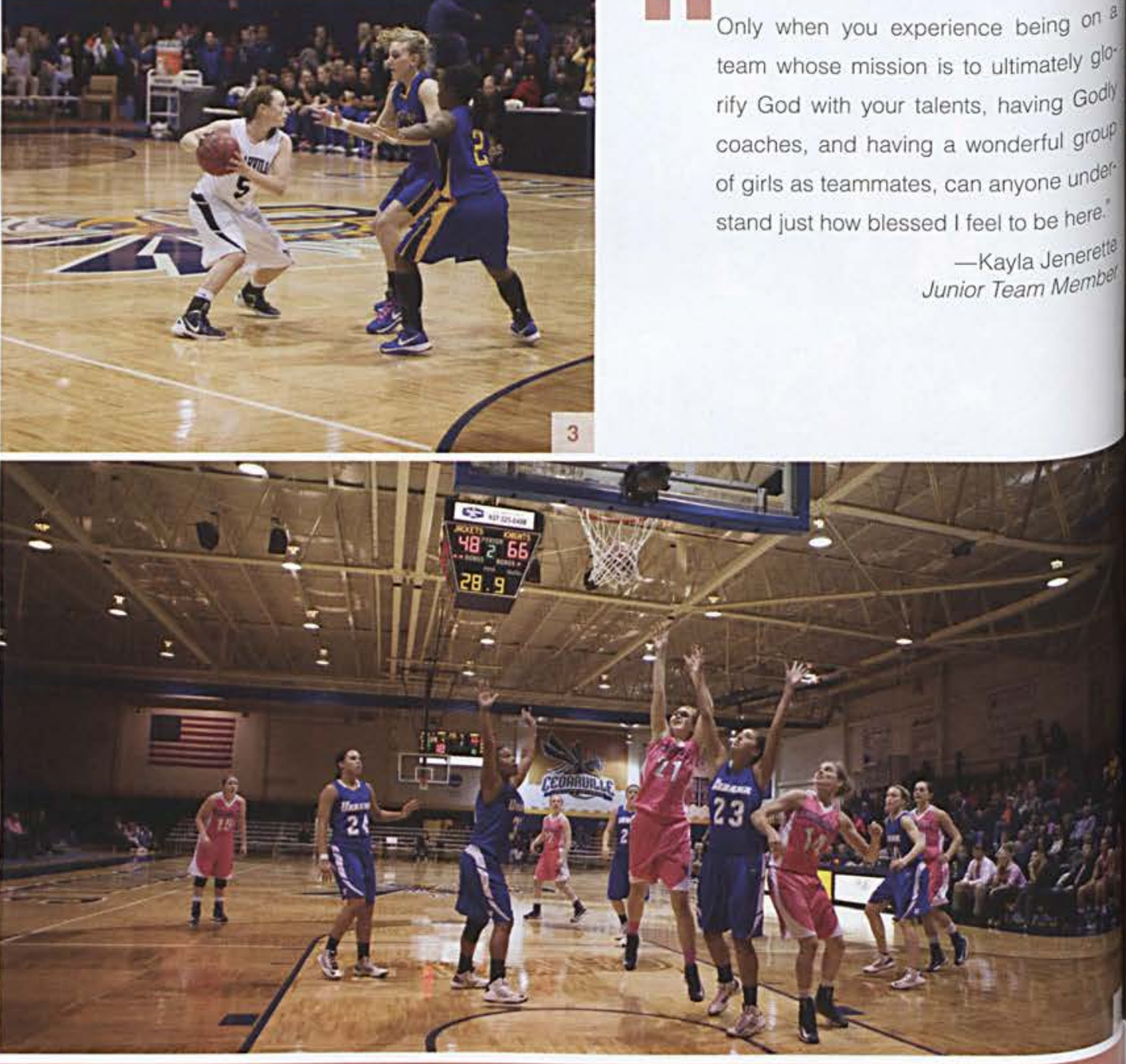

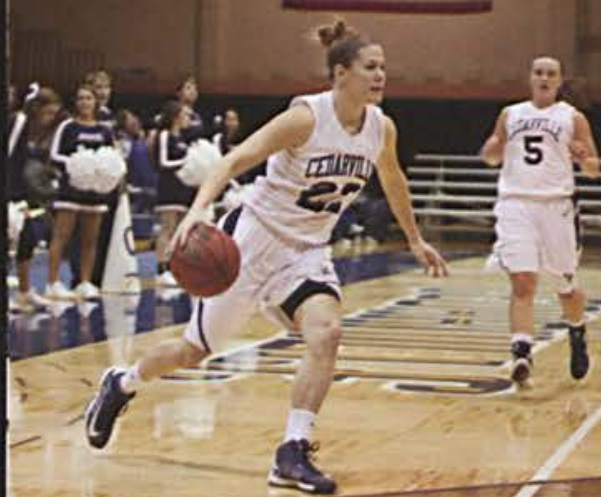

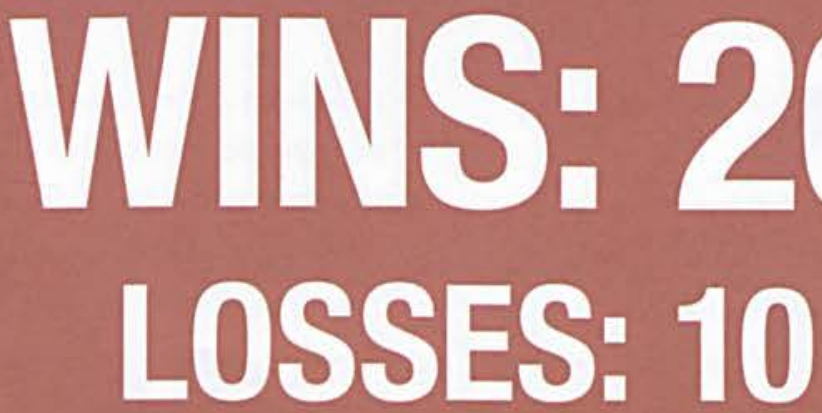




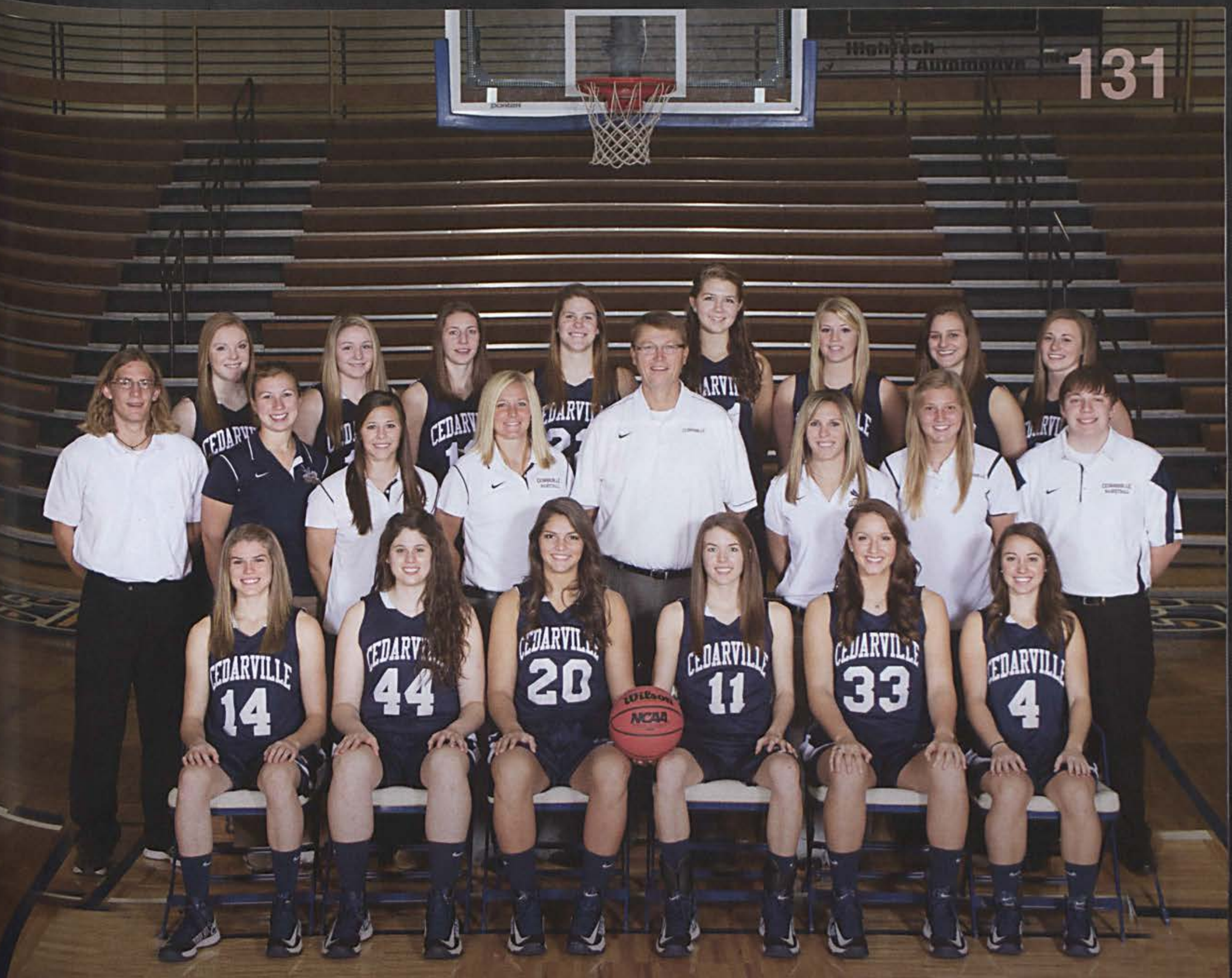

1. Raegan Ryan takes it to the basket over the heads of the Ursuline team.

2. Playing tough defense. Heidi Ansiel guards against Urbana

3. Junior Raegan Ryan looks for an open

leammate under pressure.

4. Kayla Linkous takes it up for 2 as the crowd looks on.

5. Finishing her shot strong, Ki Ketron scores while the home crowd cheers.

6. Deborah Gordon drives the ball to the hoop, looking to score for the Jackets.

\section{WOMEN'S BASKETBALL}

(Front row) Heidi Ansiel, Danielle Spiliotis, Lauryn Robinson, Becky Orchard, Deborah Chandler, Kayla Jenerette (Second row) Student Athletic Trainer Timmy Waller, Athletic Trainer Amanda Meade, Student Assistant Kenleigh Ludlow, Assistant Coach Kari Flunker, Head Coach Kirk Martin, Assistant Coach Stacie Travis, Student Assistant Payton Strawser, Student Assistant Stephen Buettell (Back row) Whitney Robinson, Kaysie Brittenham, Deborah Gordon, Ki Ketron, Kayla Linkous, Taylor Vander Plas, Raegan Ryan 


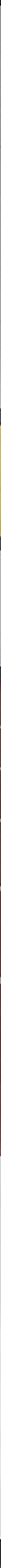





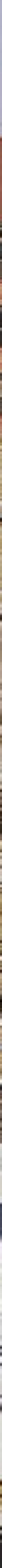




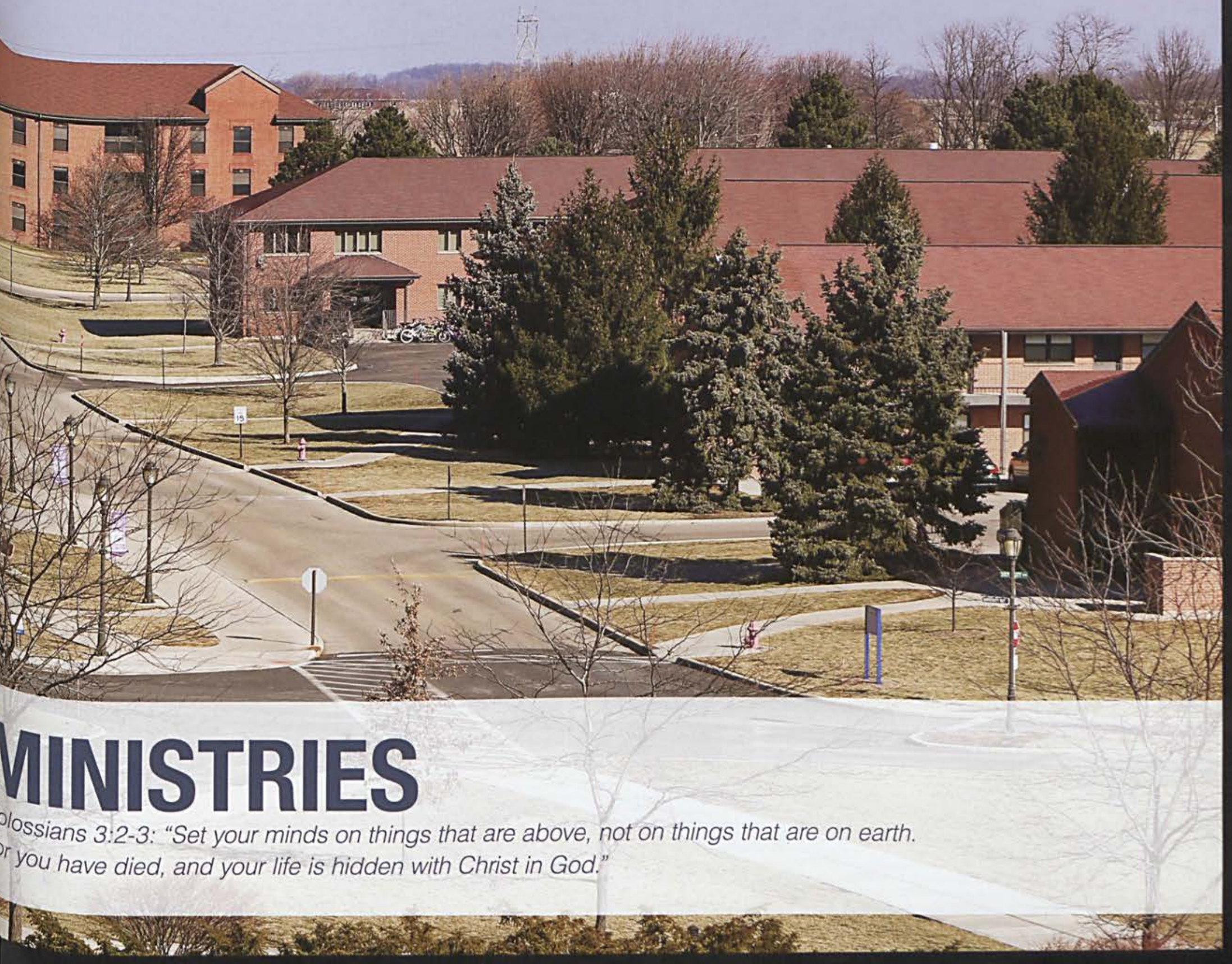




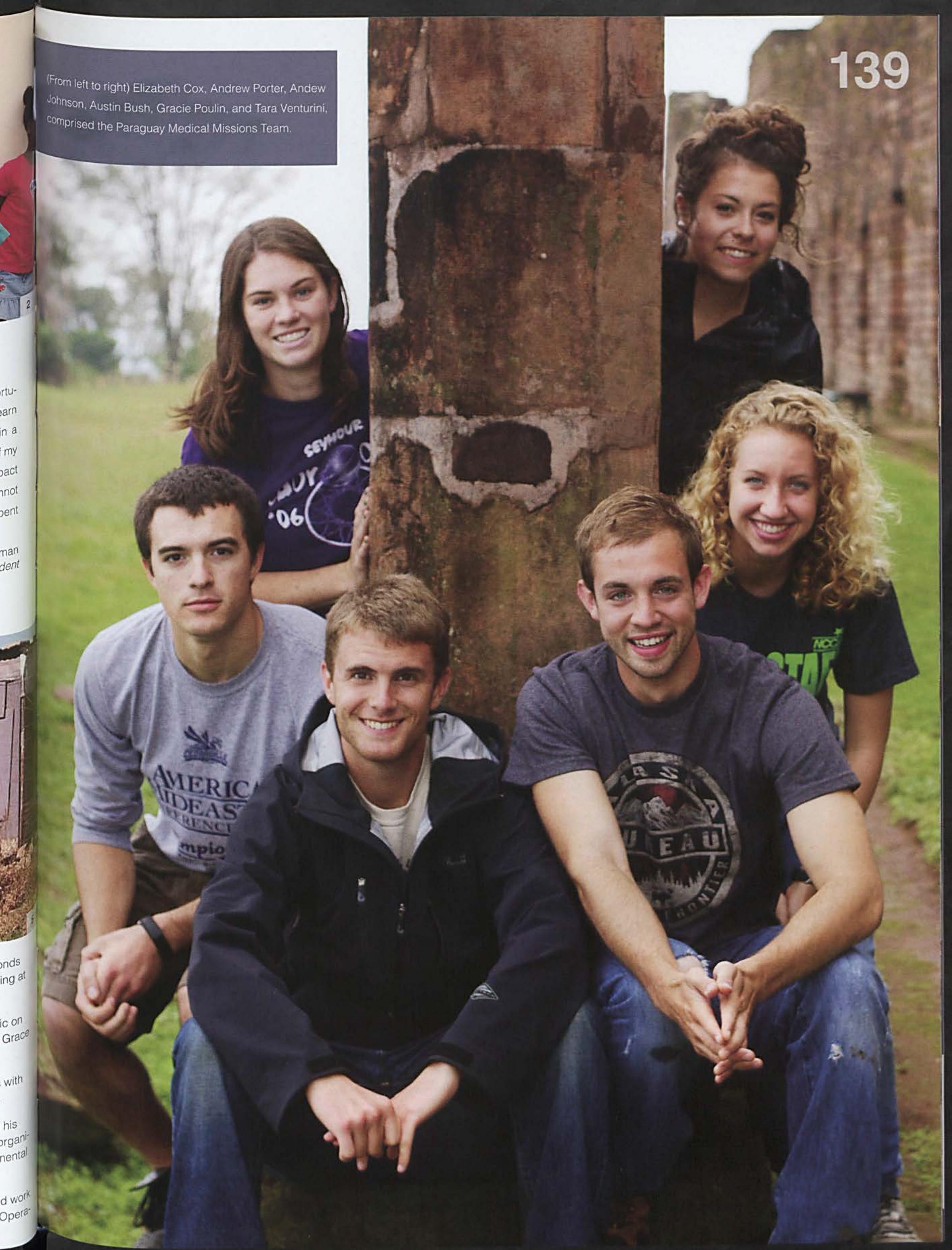




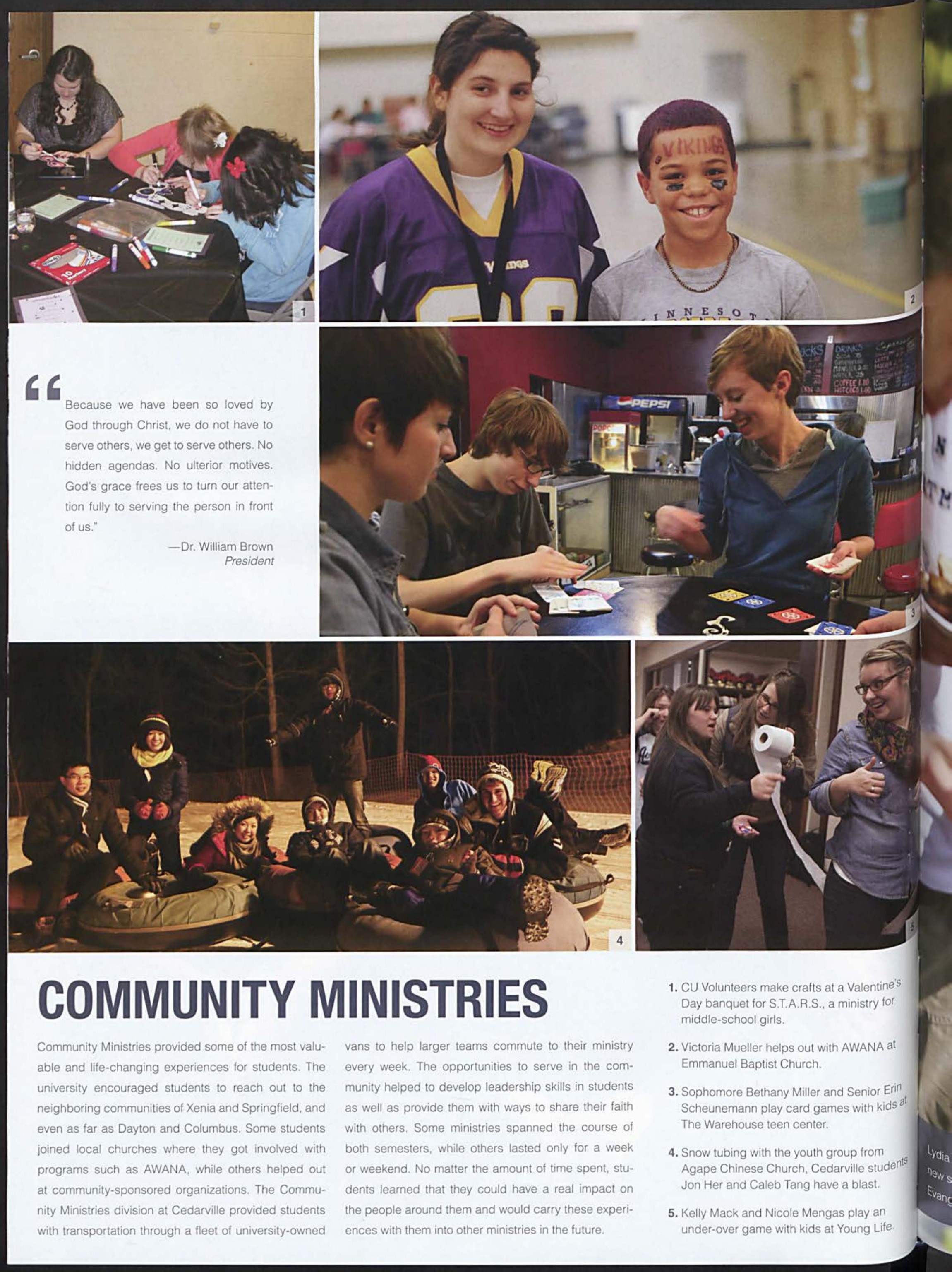





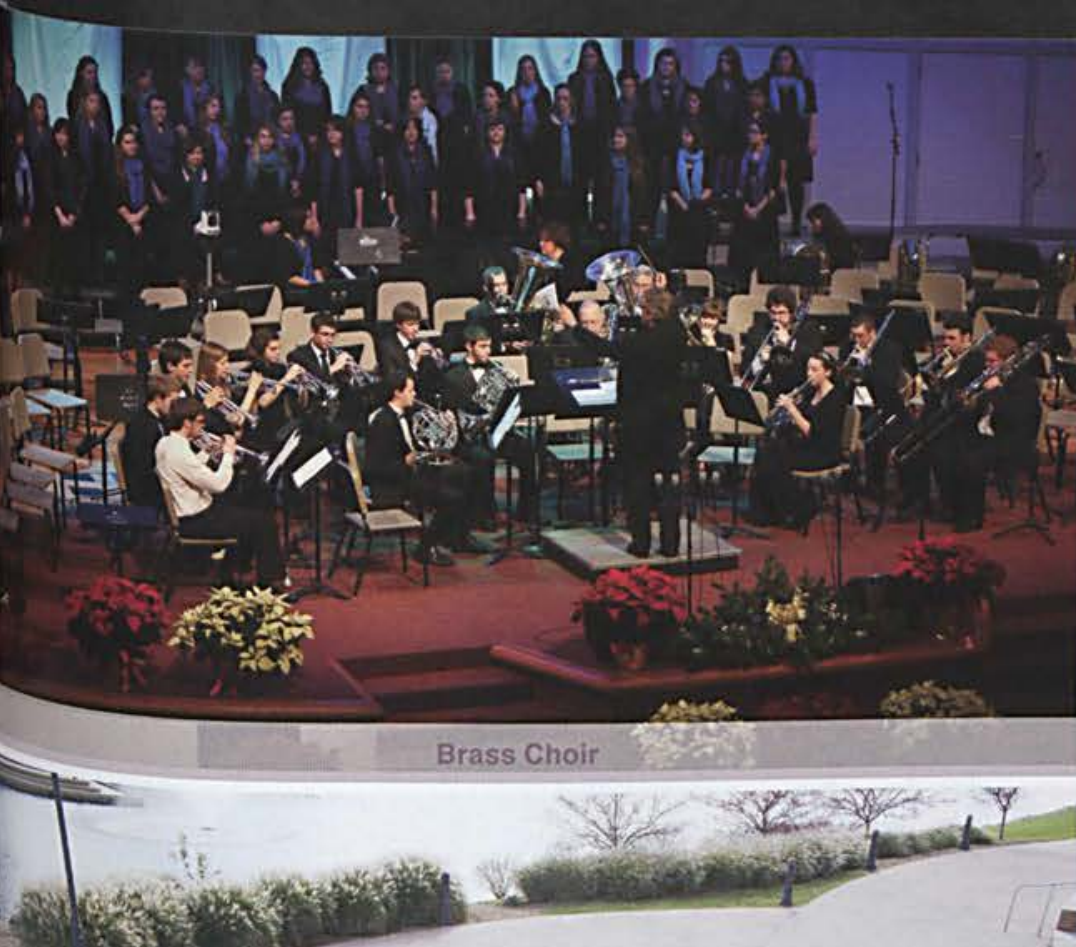

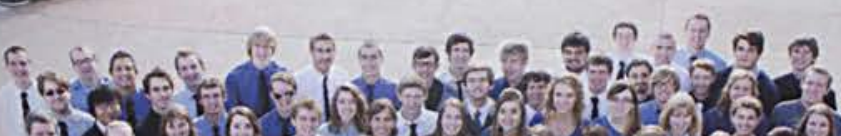

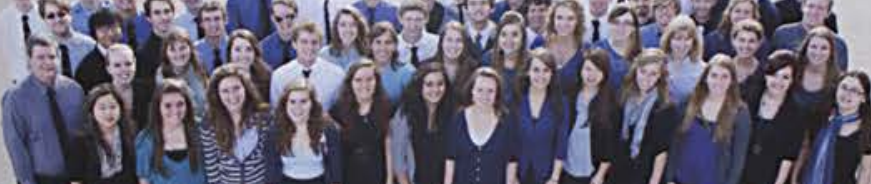

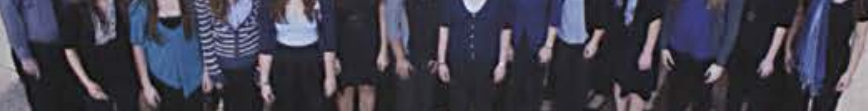

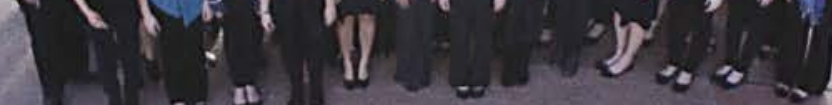

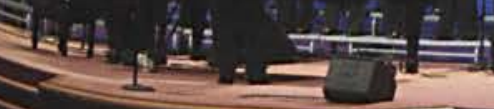

\section{Women's Choir}

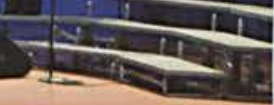

3.
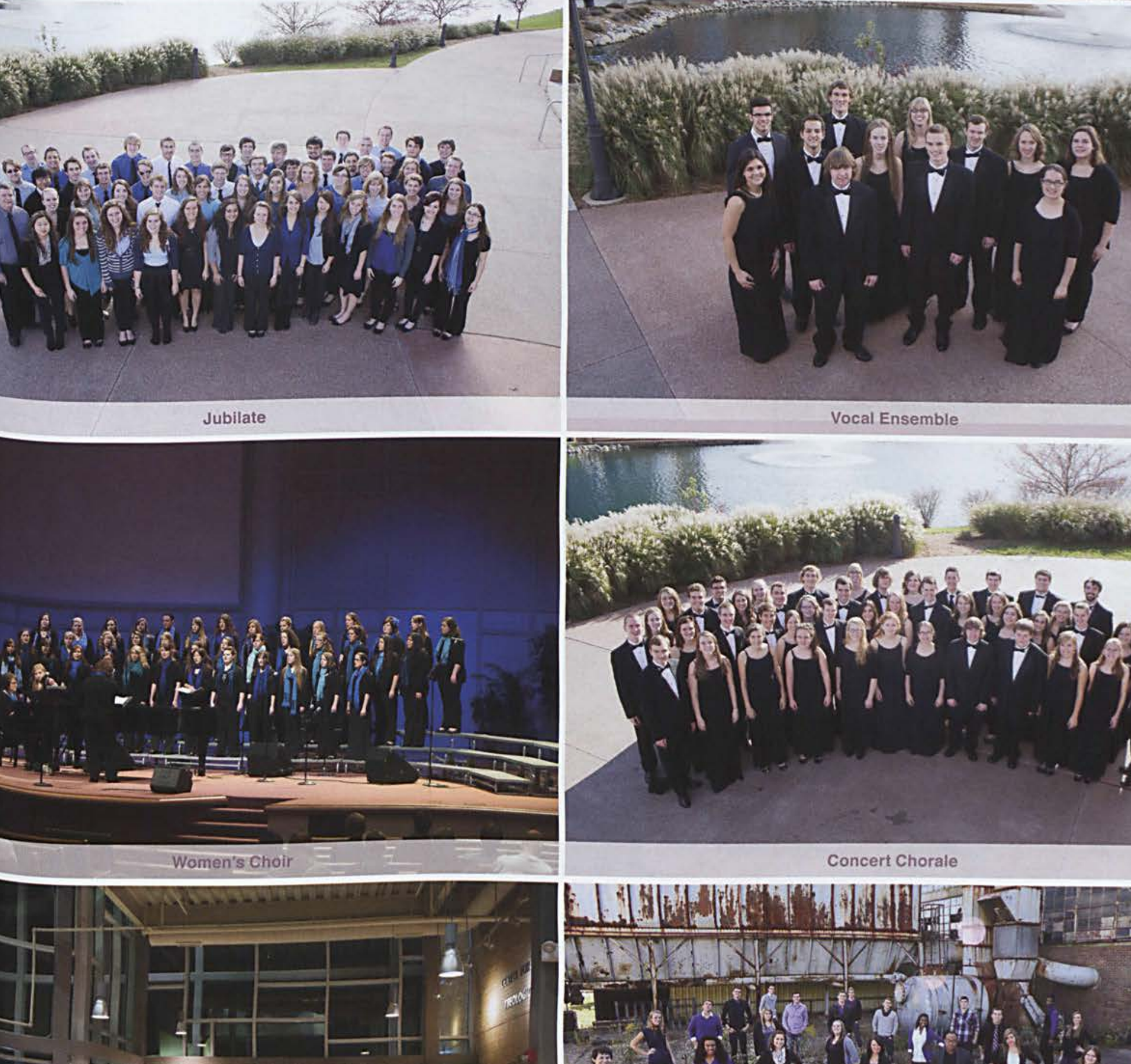

$\operatorname{ran} 4=0$ Dath 


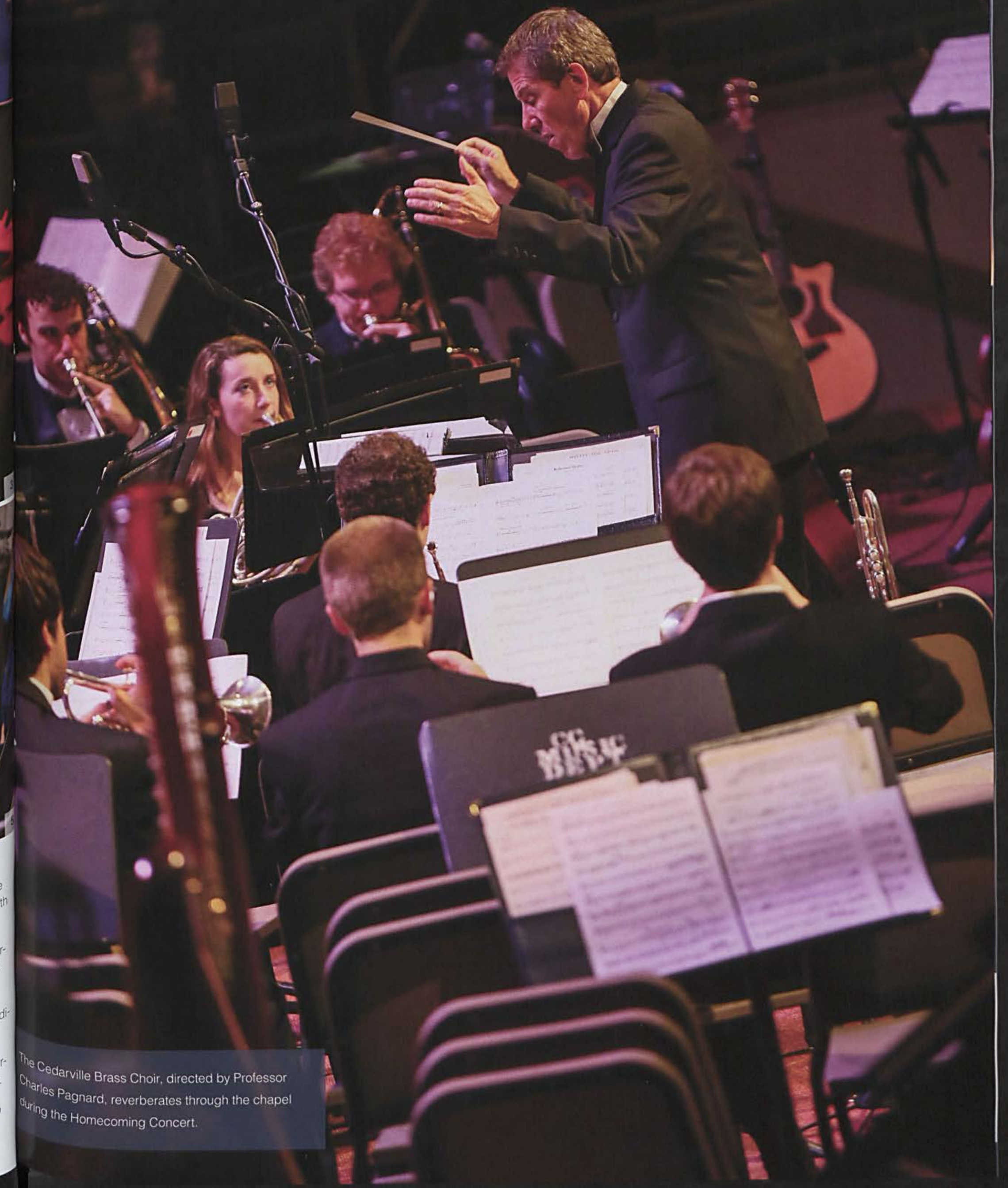




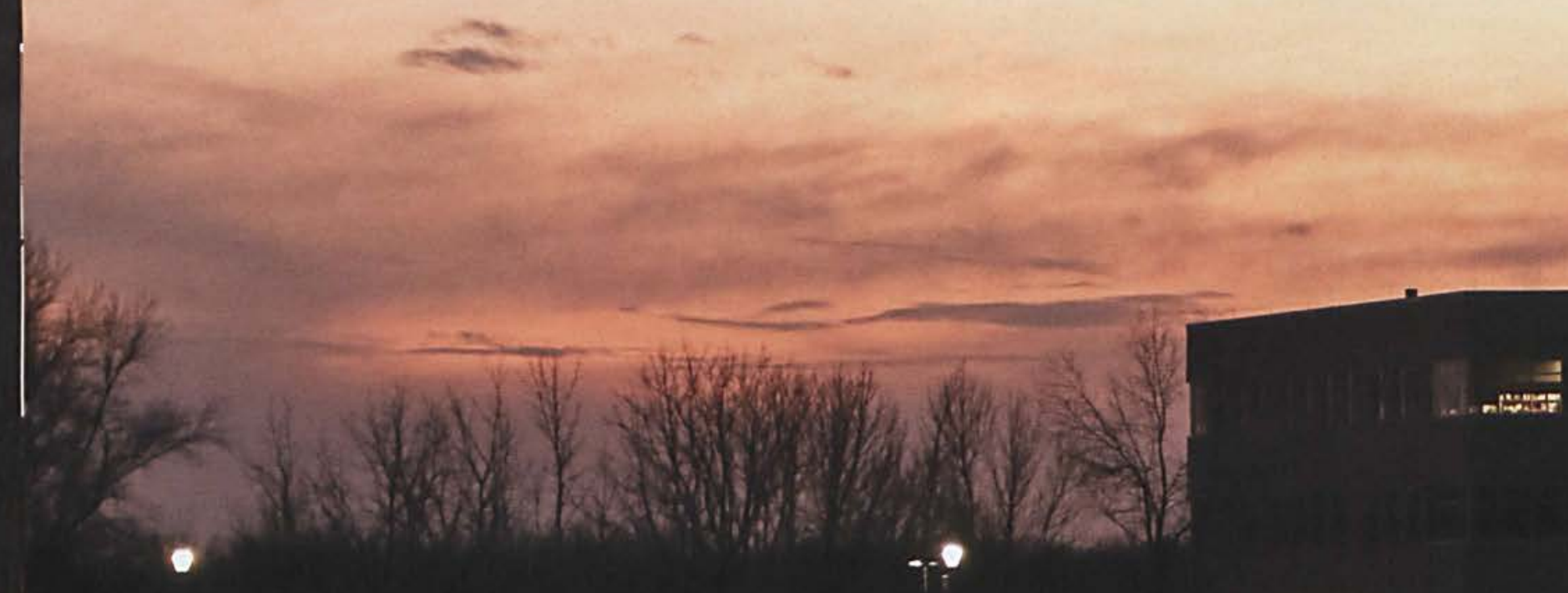

intiritivi

,

16x.

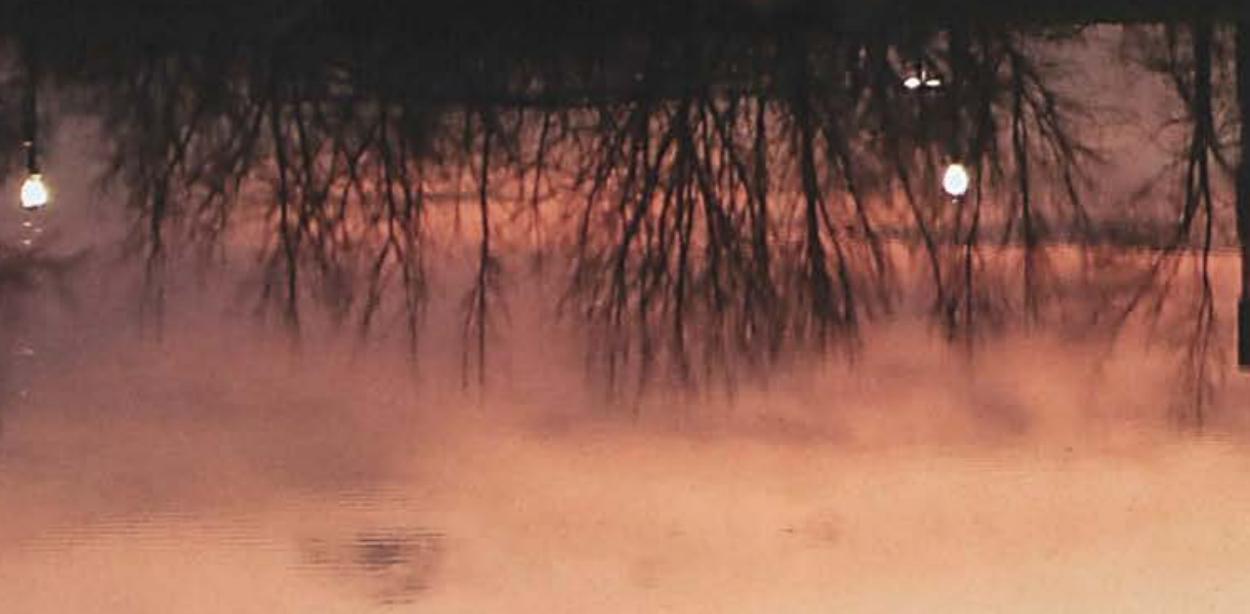

-mormaratila
E. Ifit

-

i 19

C 4 내

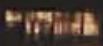


10

A

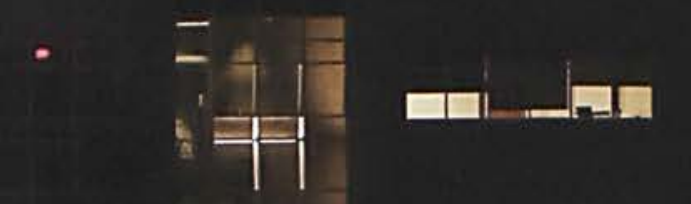

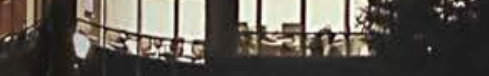

dartat

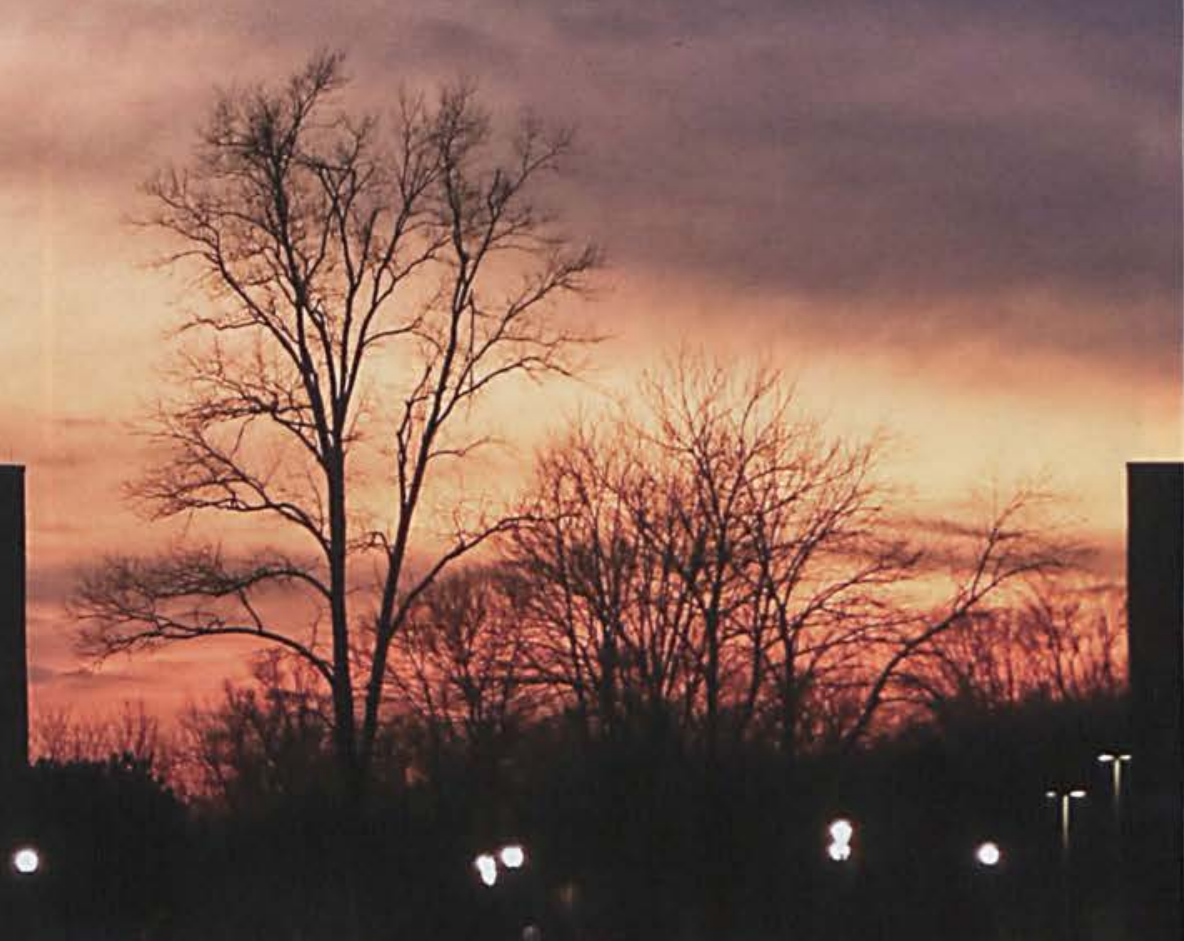

4)

"iven yese.

는

411

IIIIE * *

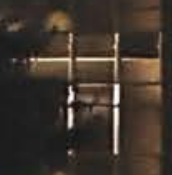

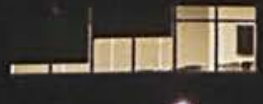

-

L1

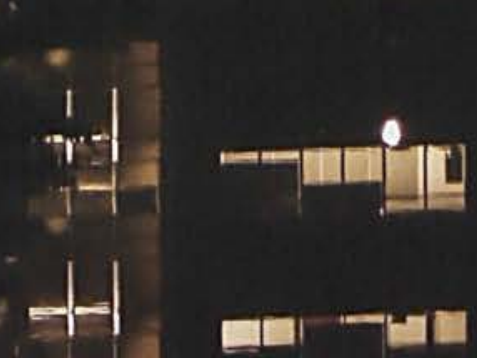

-

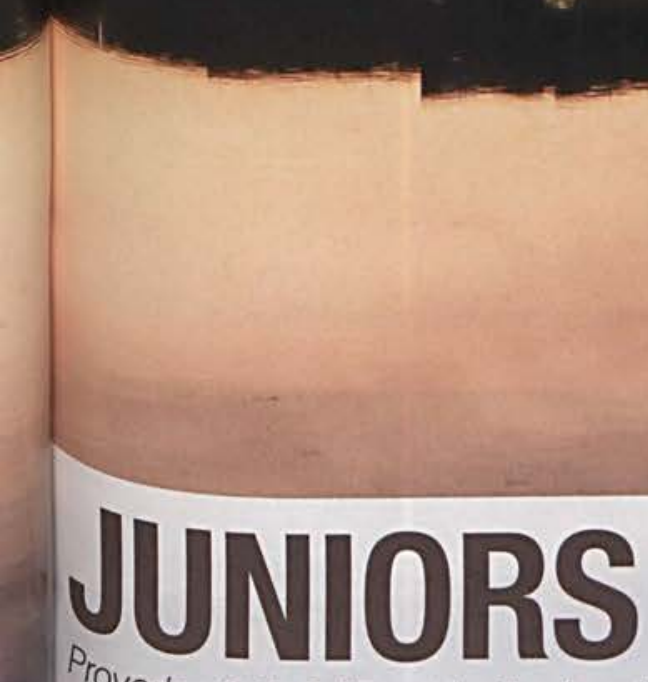

Proverbs 3:5-6 "Trust in the Lord with all your heart, and do not lean on your own

understanding. In all your ways acknowledge him, and he will make straight your paths." 
JUNIORS

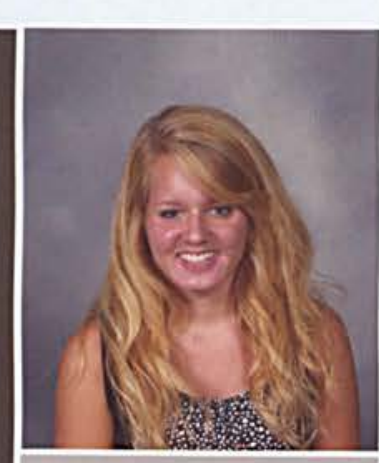

Elizabeth Acker
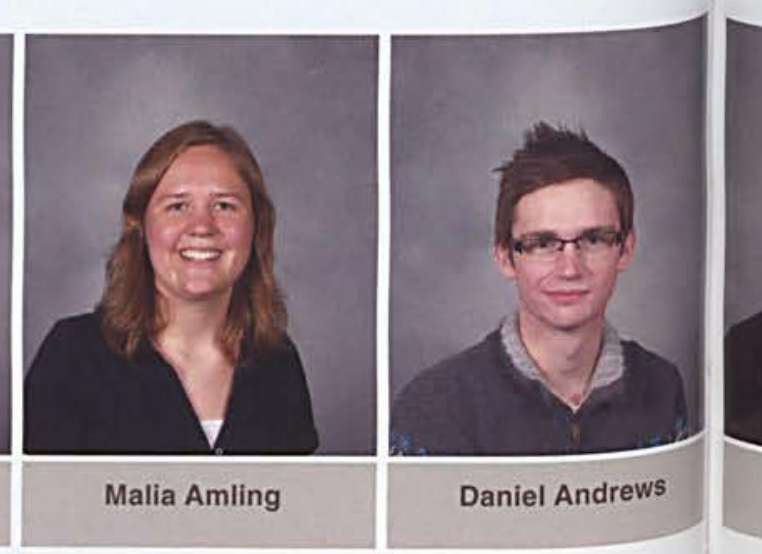

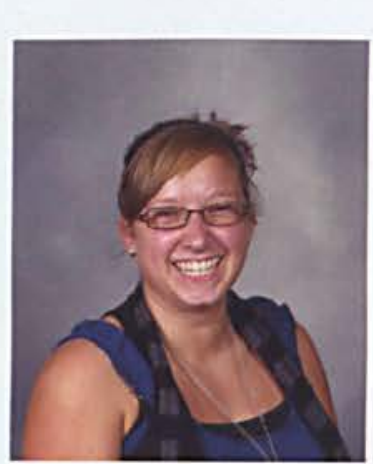

Rebekah Andrews

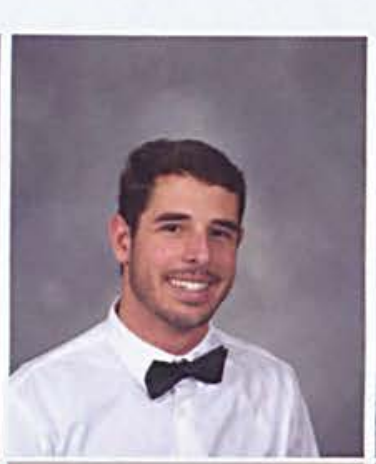

Jacob Antoun

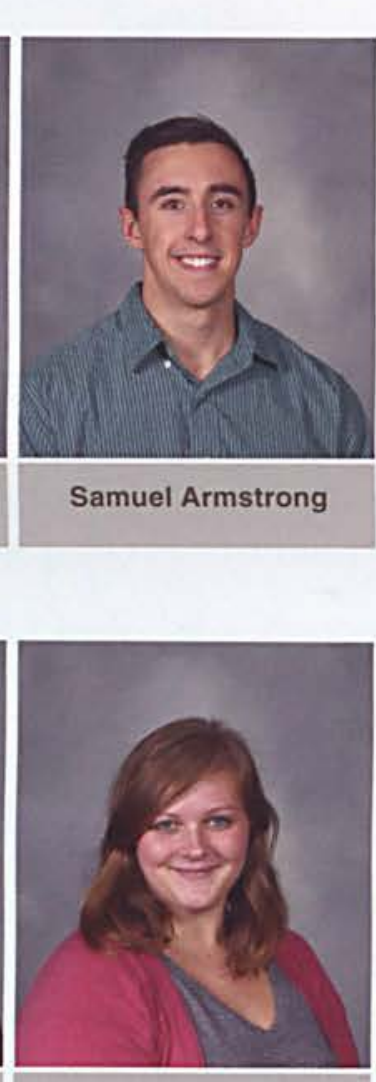

Julianna Barkas

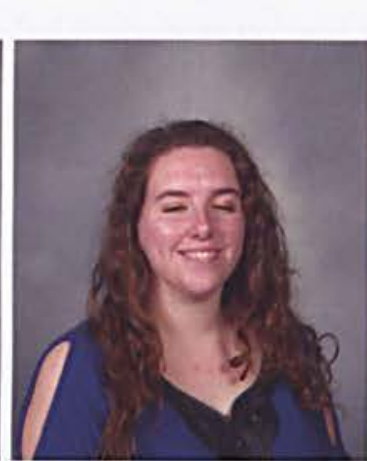

Kayla Ault

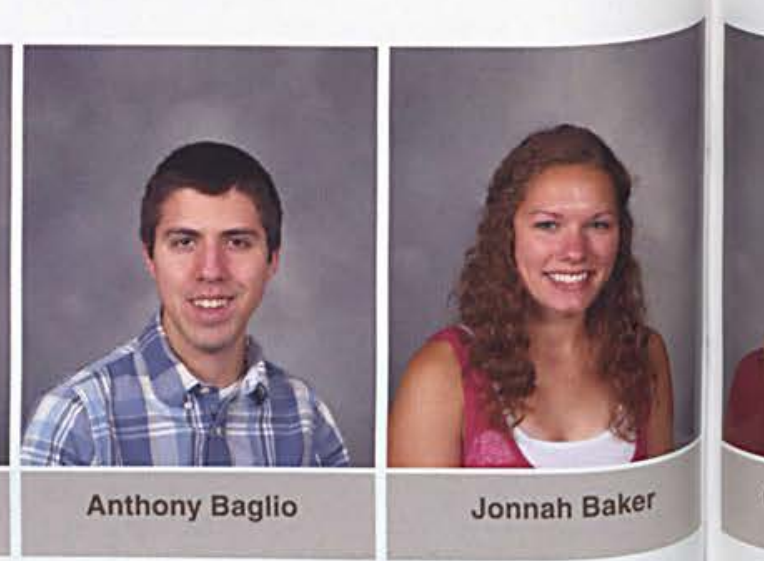

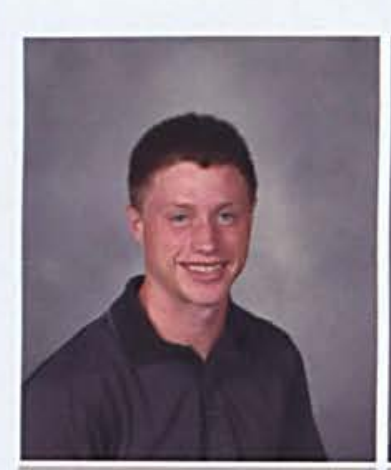

David Banz

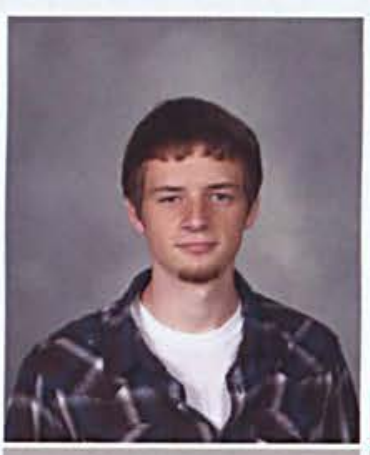

Daniel Barber

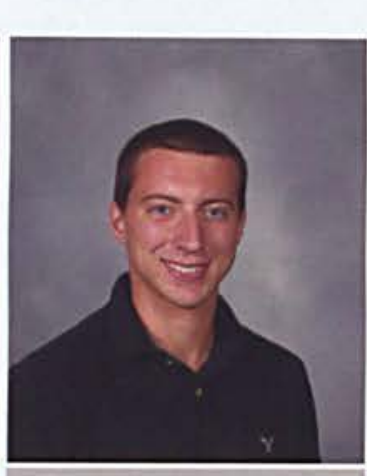

Steven Barnhart

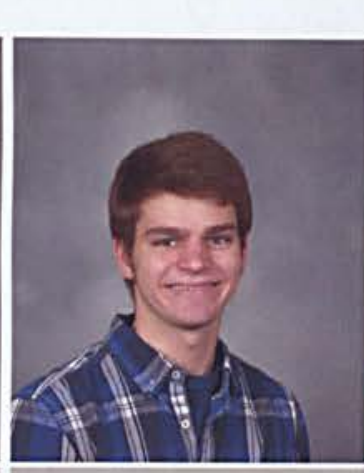

Daniel Baron
Anna Barulich

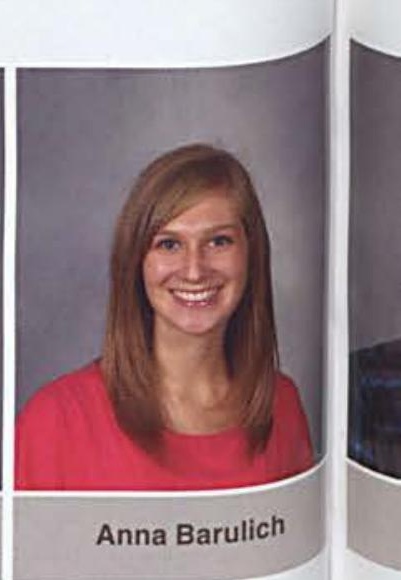

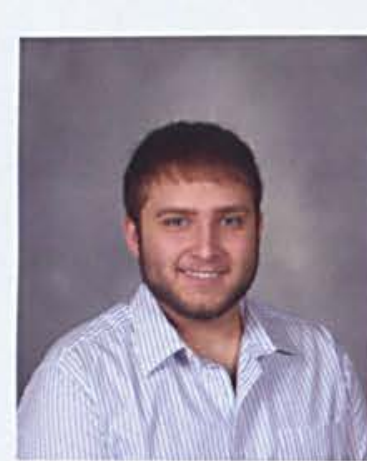

Michael Beach

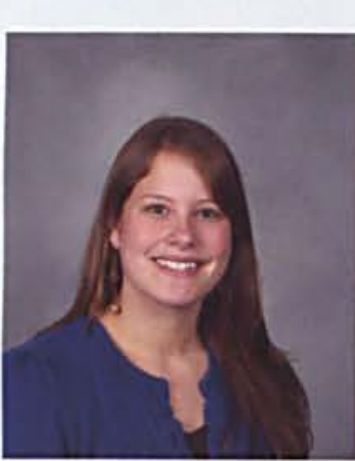

Megan Bernstein

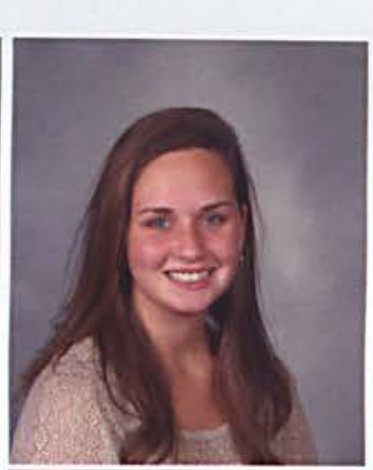

Lauren Bisbee

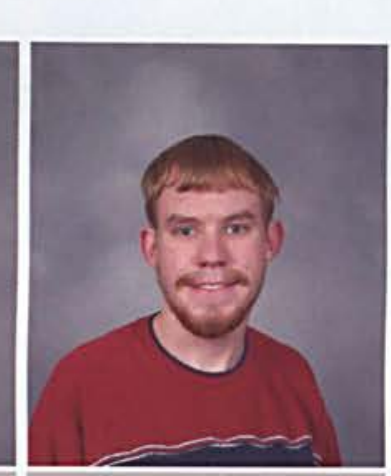

Nathaniel Bond

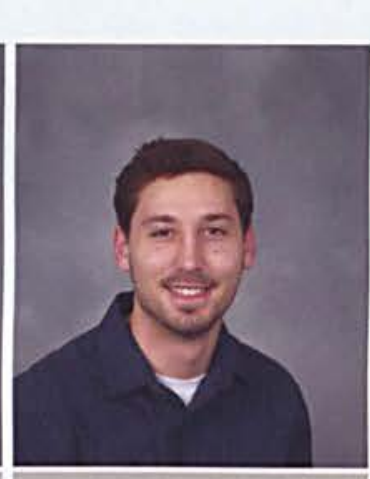

Jonathan Bonifas

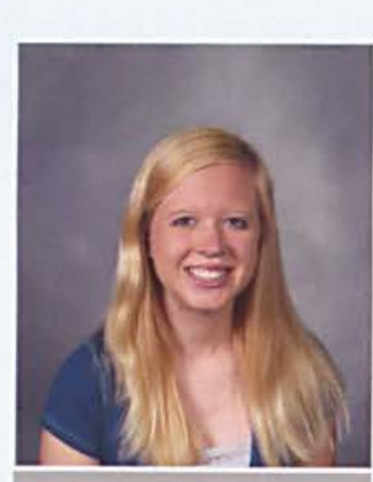

Jessica Bradshaw

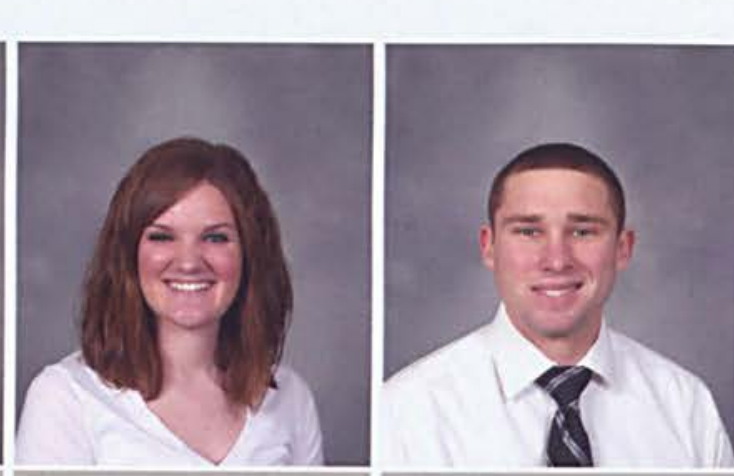

Megan Brewer
Michael Brittan

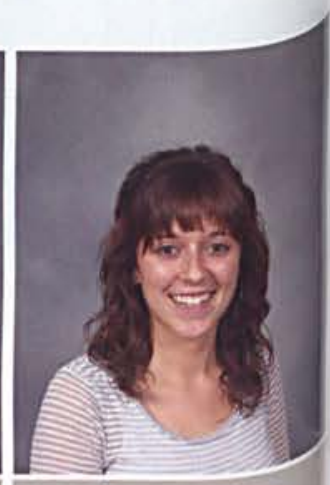

Marie Boyd 


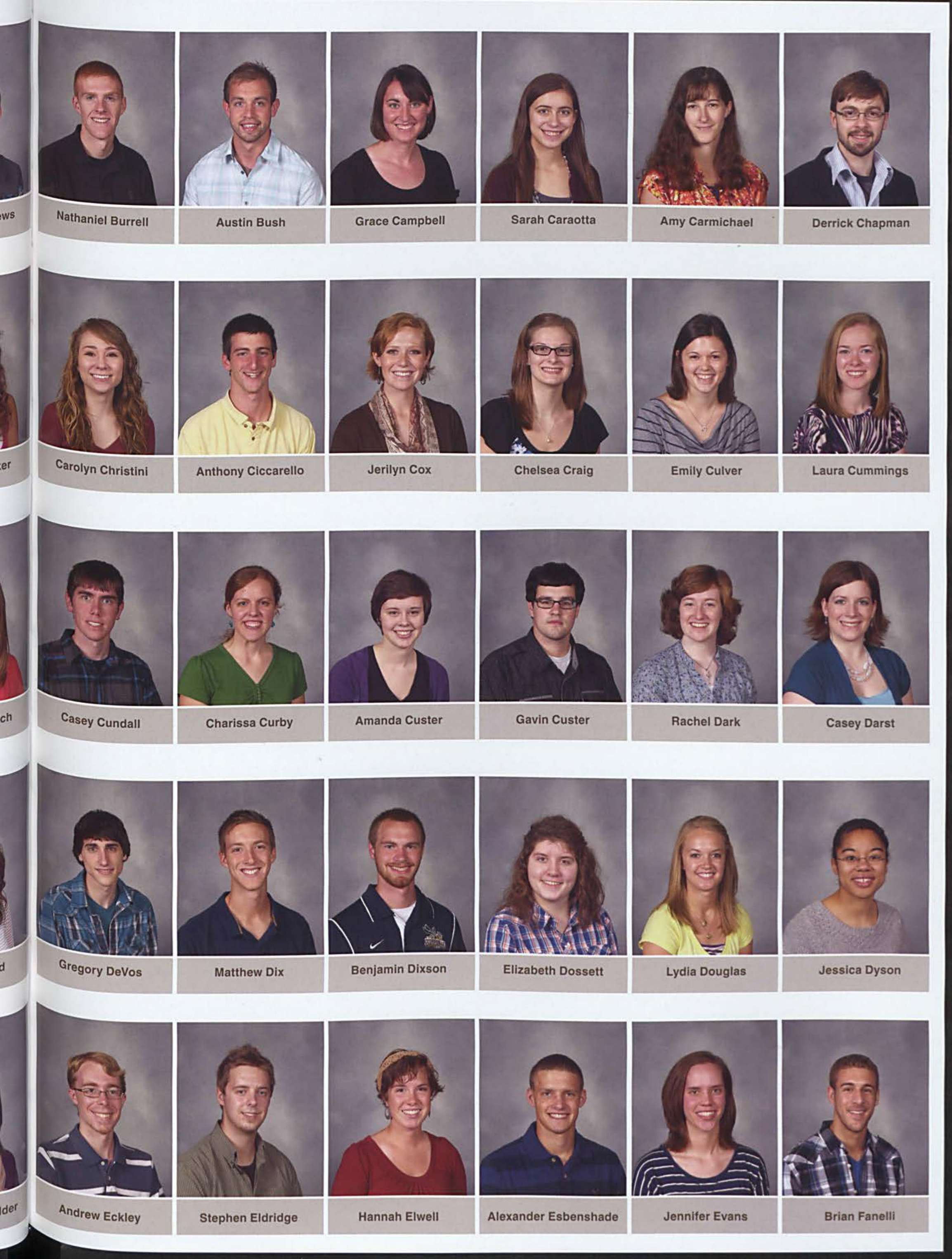


150

\section{JUNIORS}

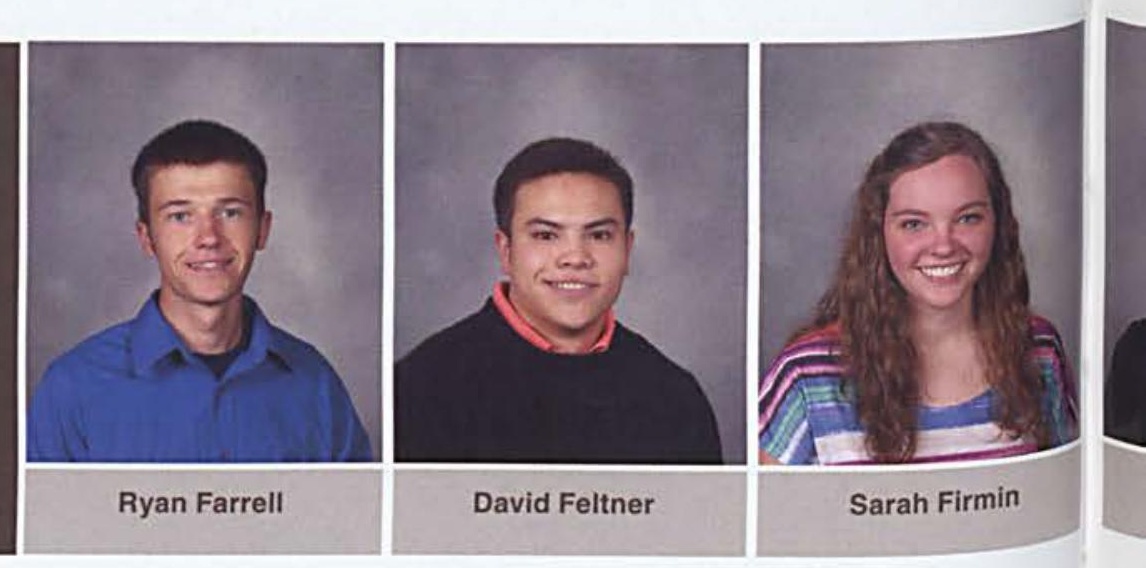

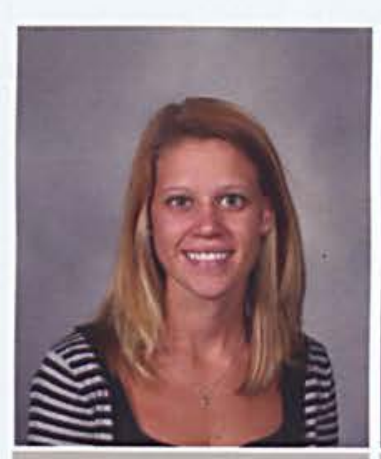

Kelsey Fladda

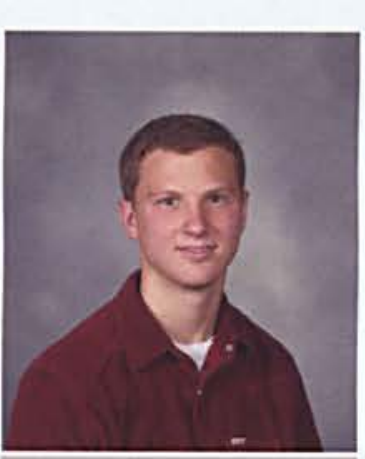

Joshua Fleming

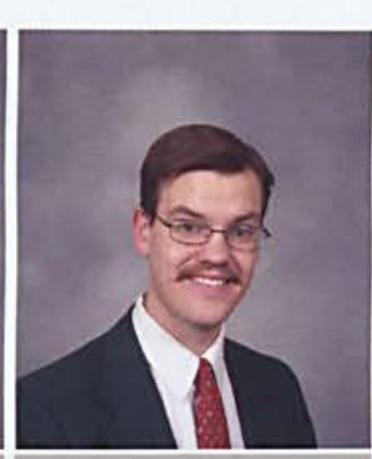

Alexander Folkerts

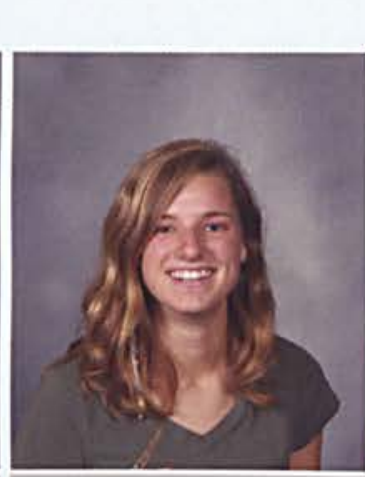

Elena Fouch

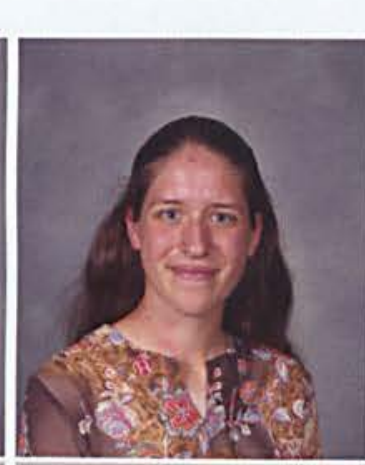

Michelle Frazer

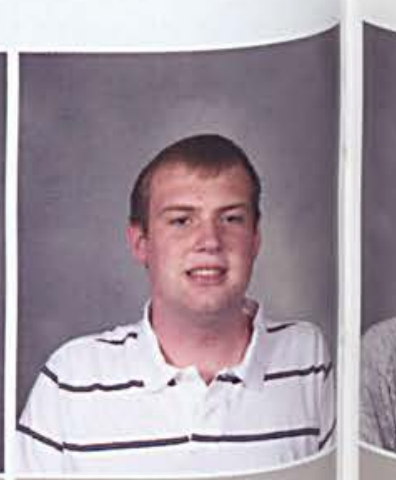

Aaron Free

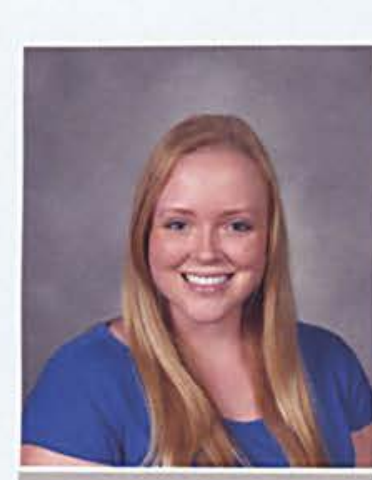

Amber Gardner

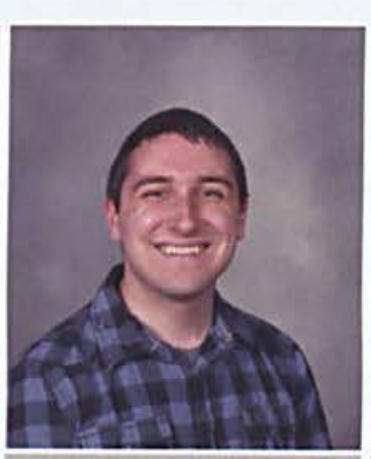

Roger Gelwicks

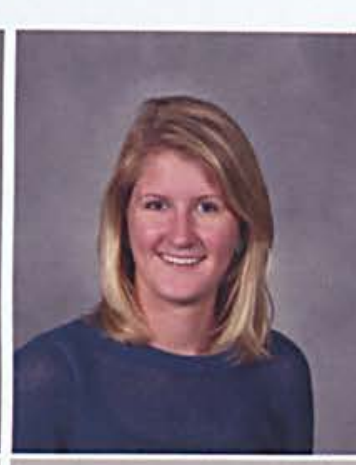

Paige Getchell
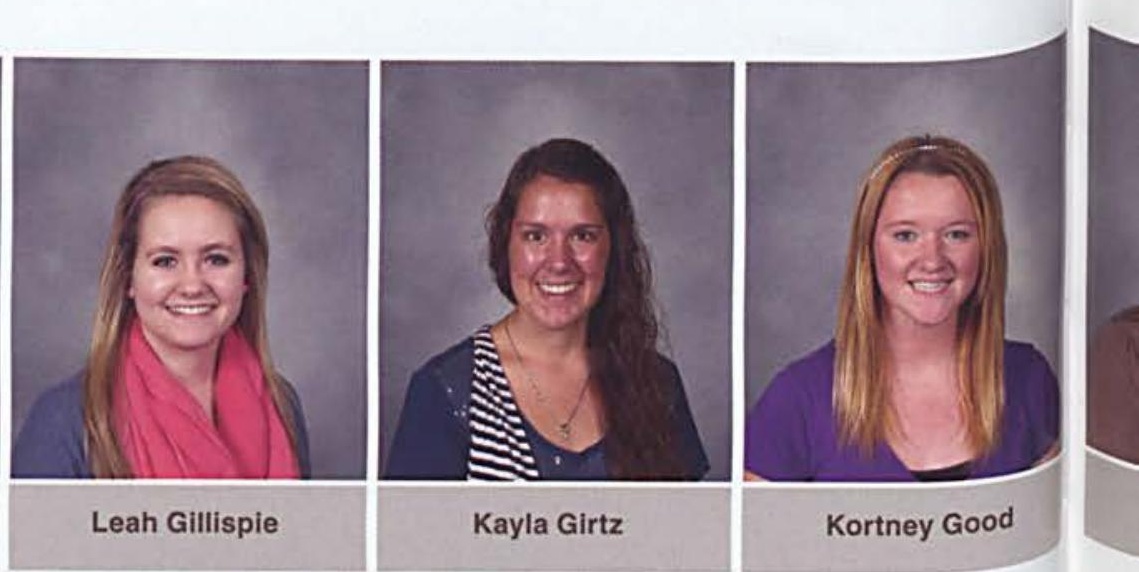

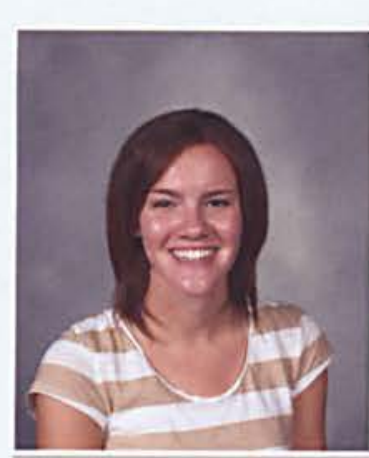

Diane Goodliffe

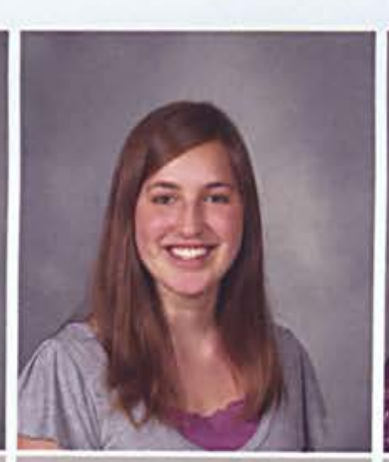

Katie Grayton

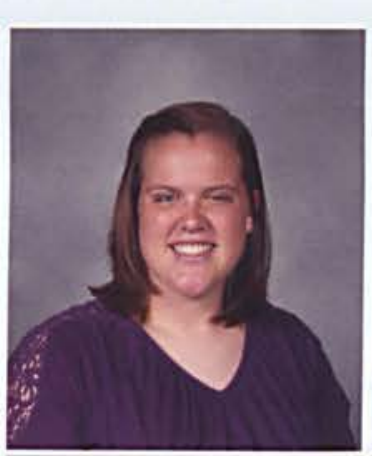

Bethany Green

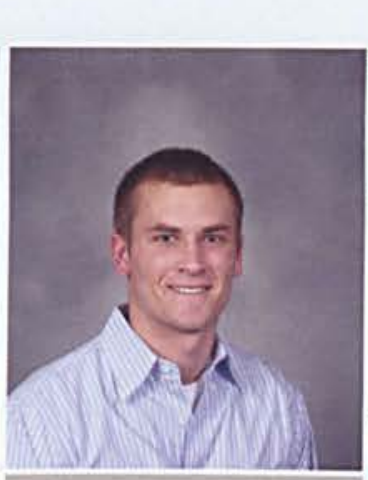

Jonathan Greenwood

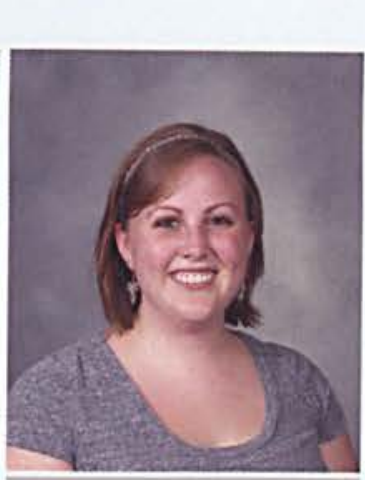

Katherine Guffey
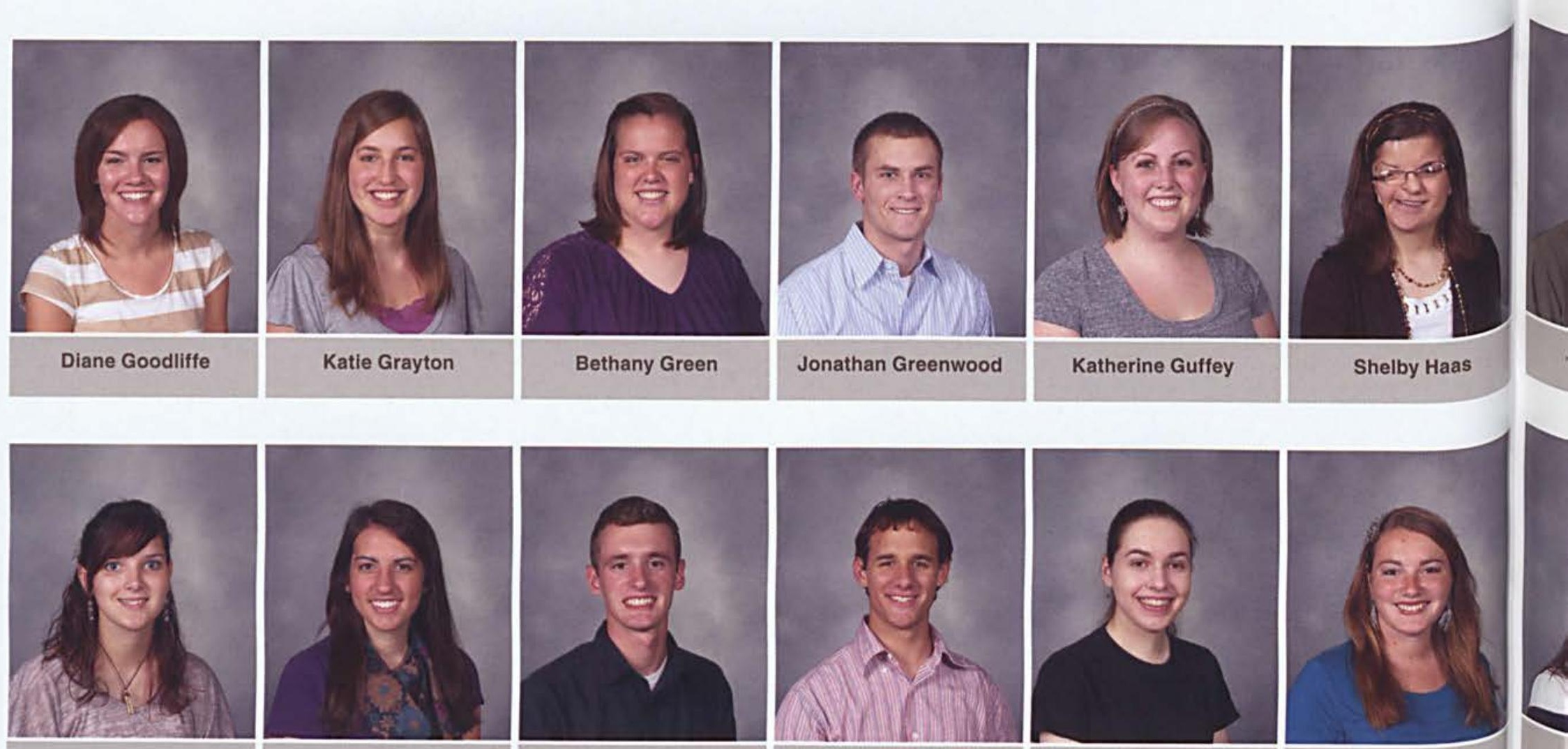

Peter Hamblen

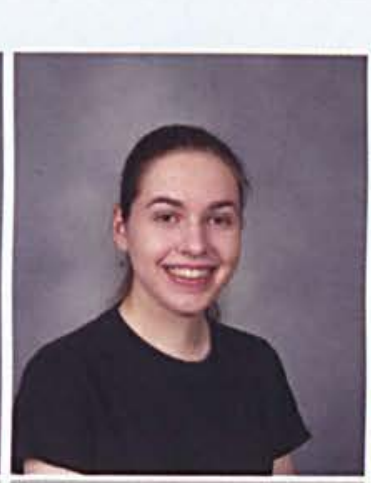

Emily Hartman

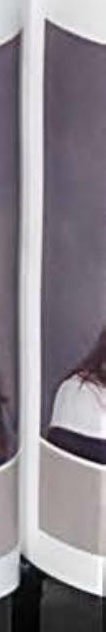




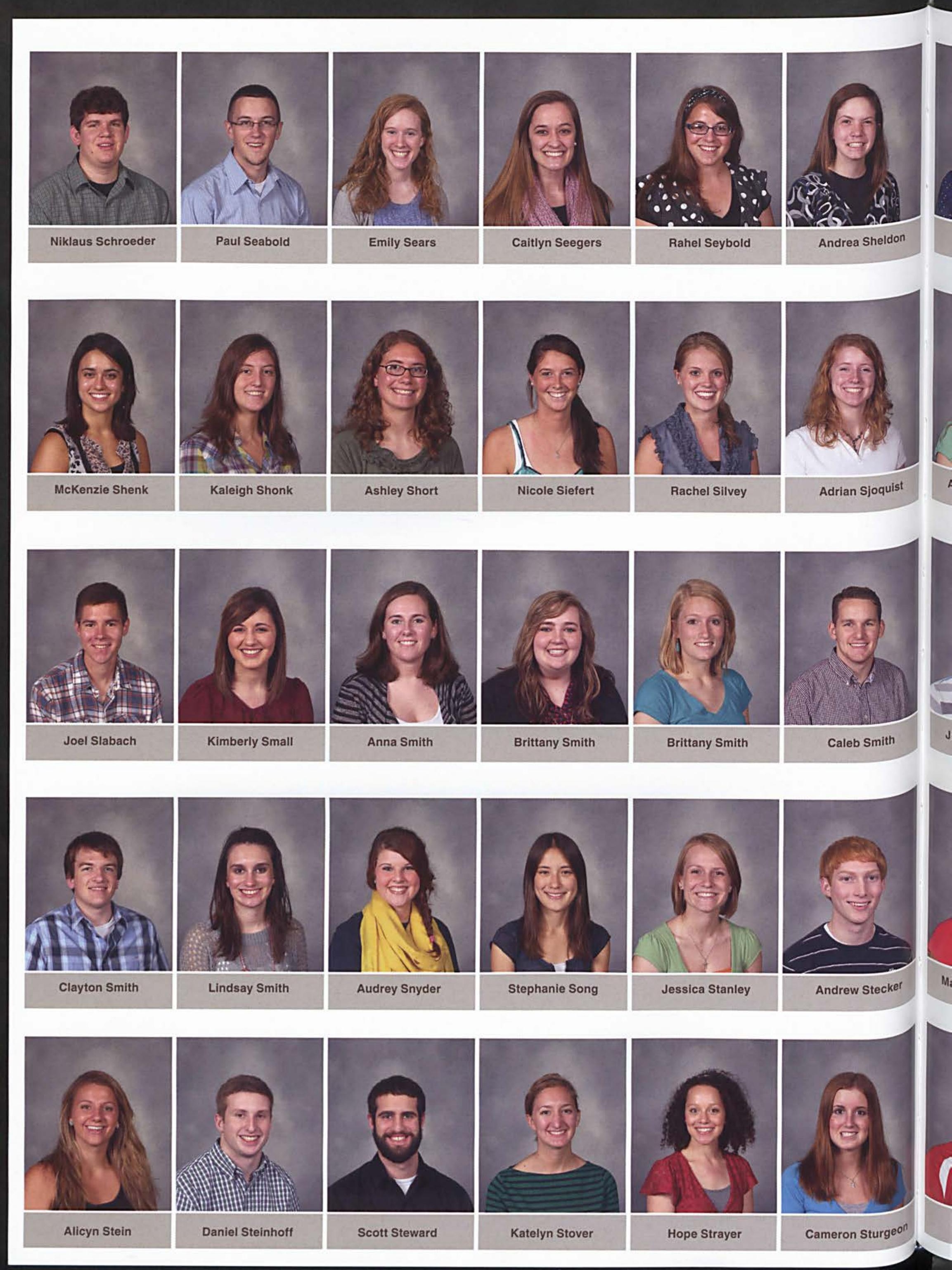




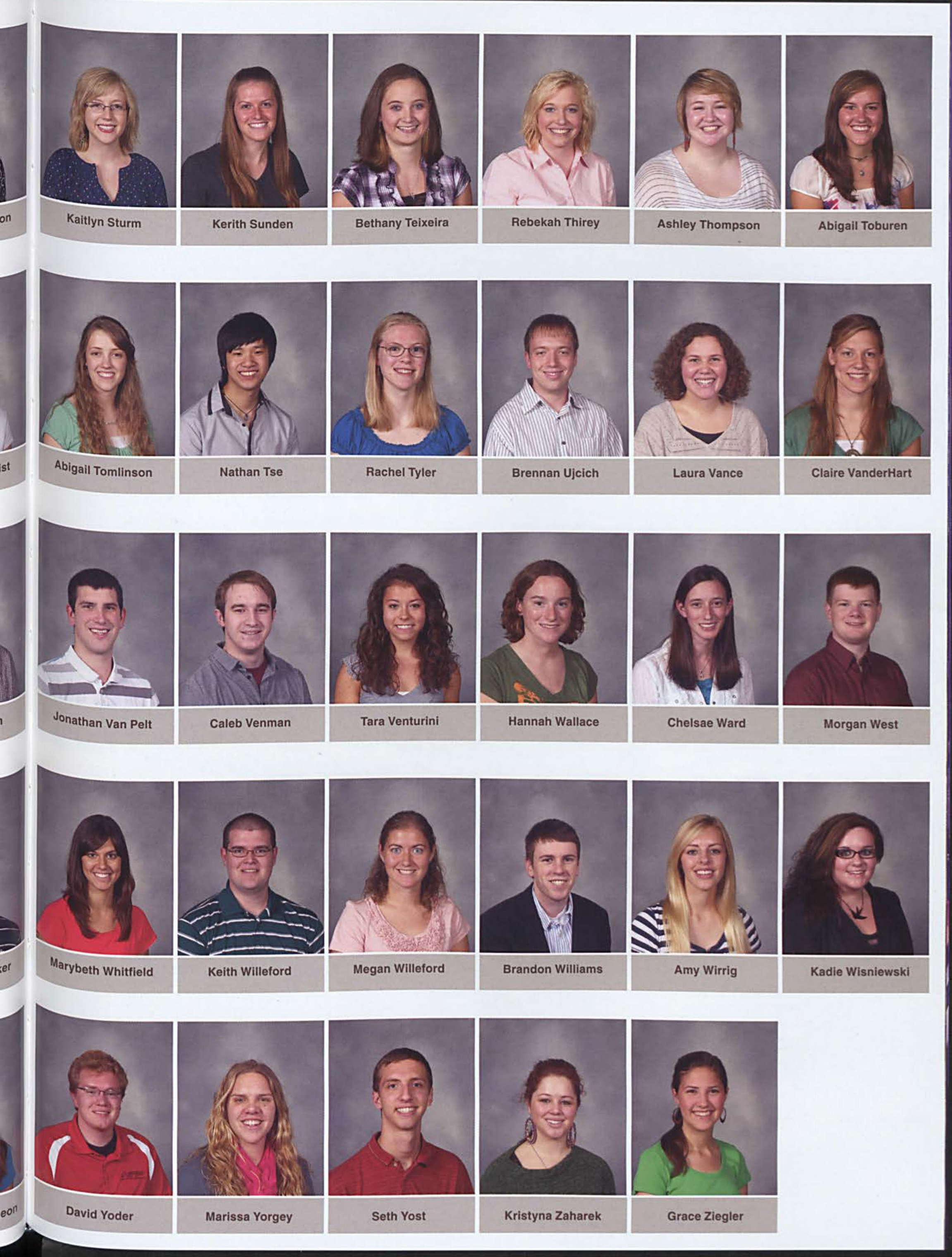




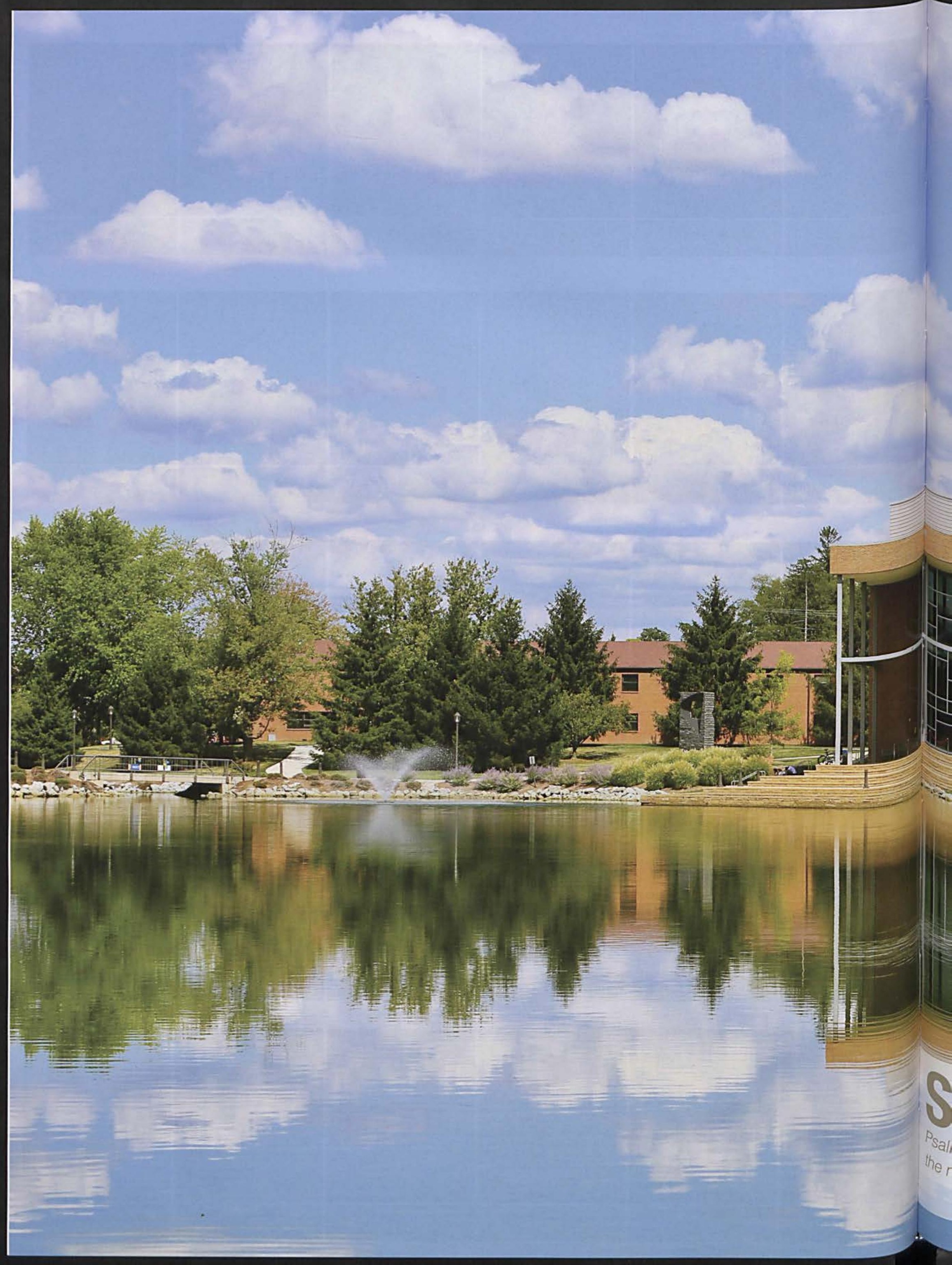




\section{FARTUFFE, OR THEIMPOSTER. \\ Nini}

Orgon tries to convince his mother that Tartuffe is Clually a scheming imposter.

\section{IIf

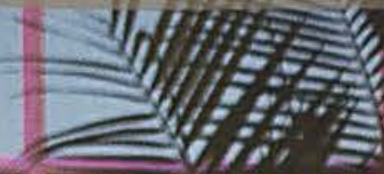

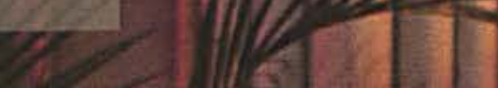

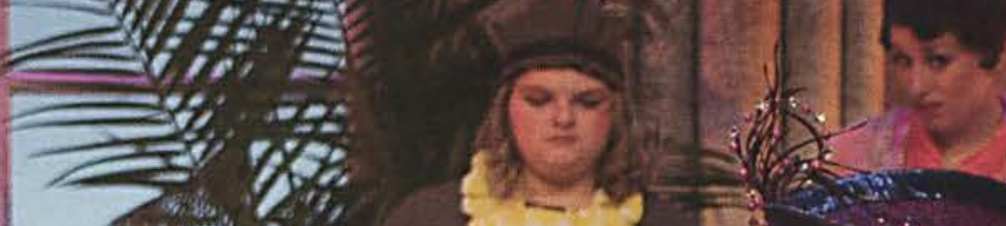

$1 \mathrm{milm}$ A

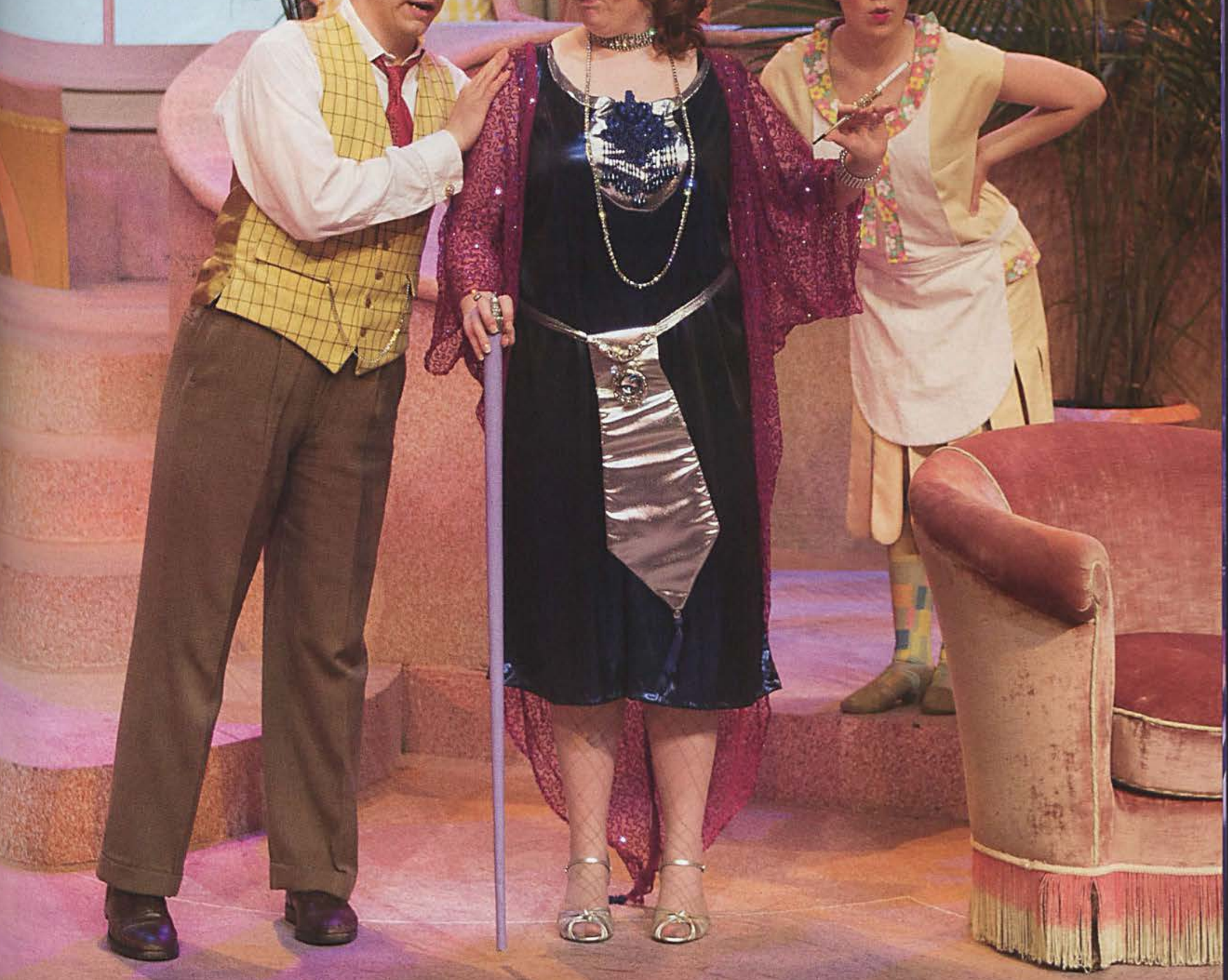




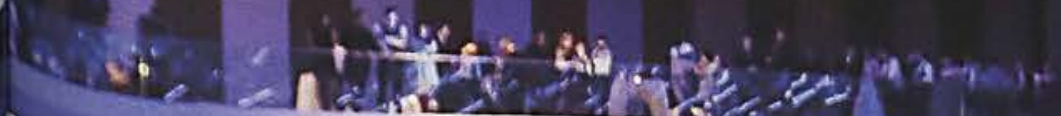

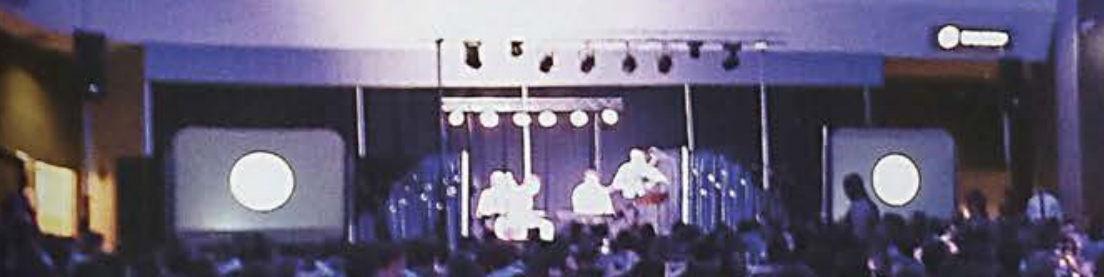

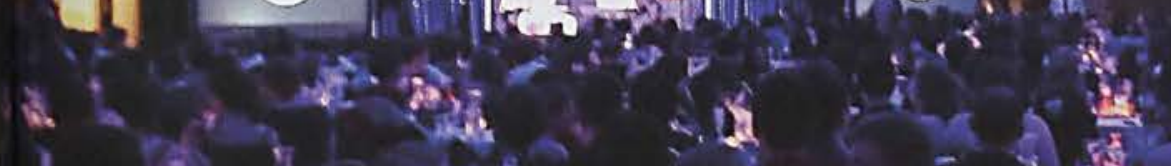

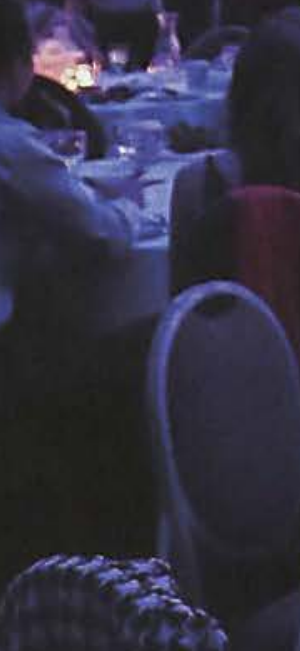

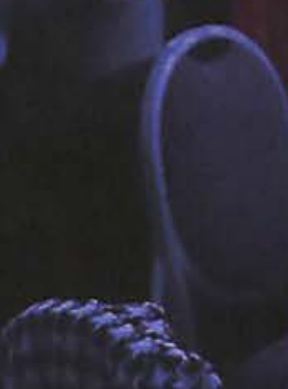

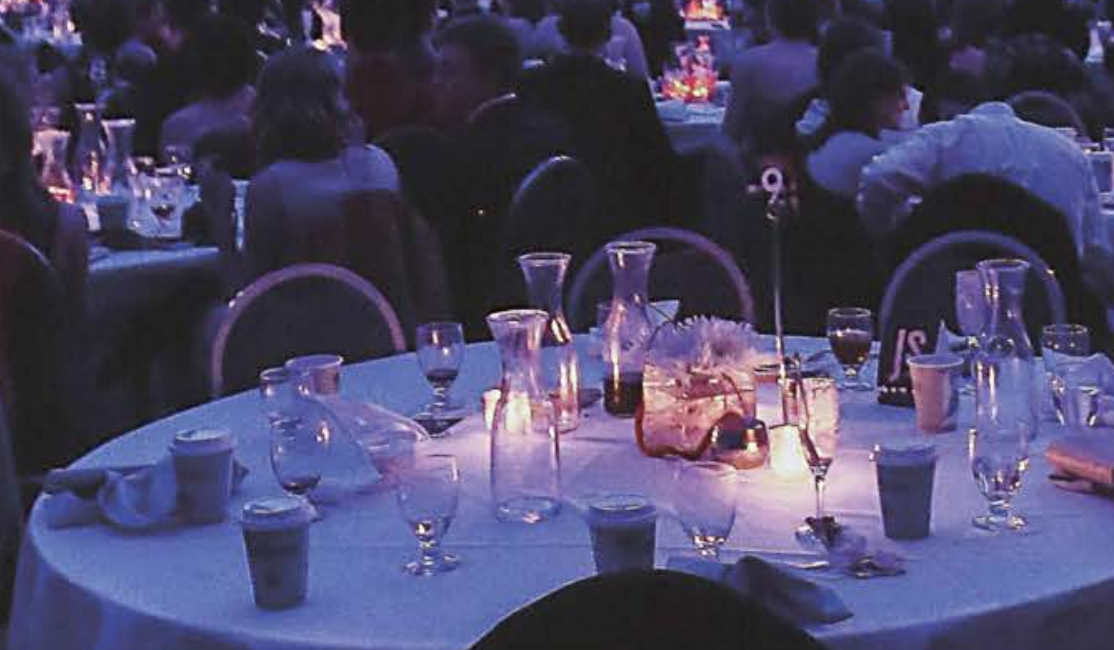

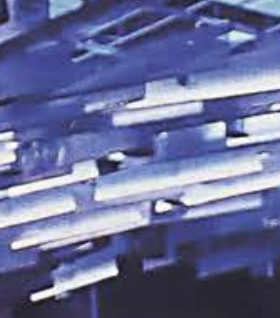

살
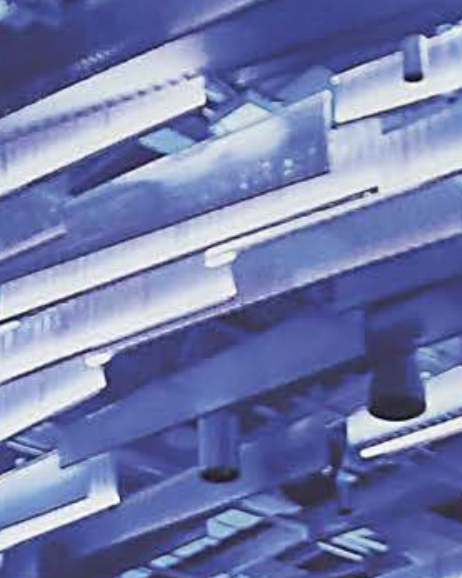

- $2+10$
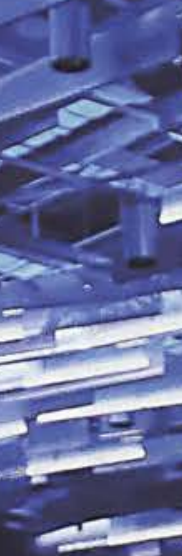

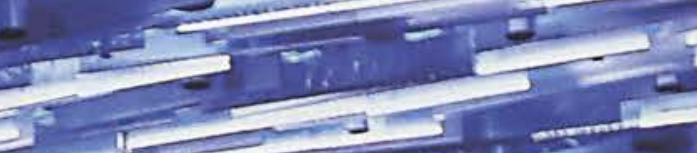

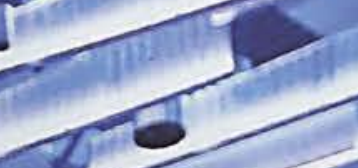
政

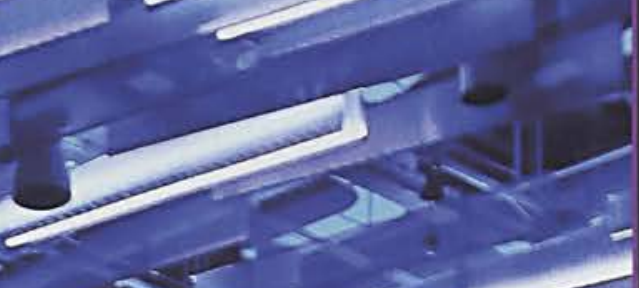
. 


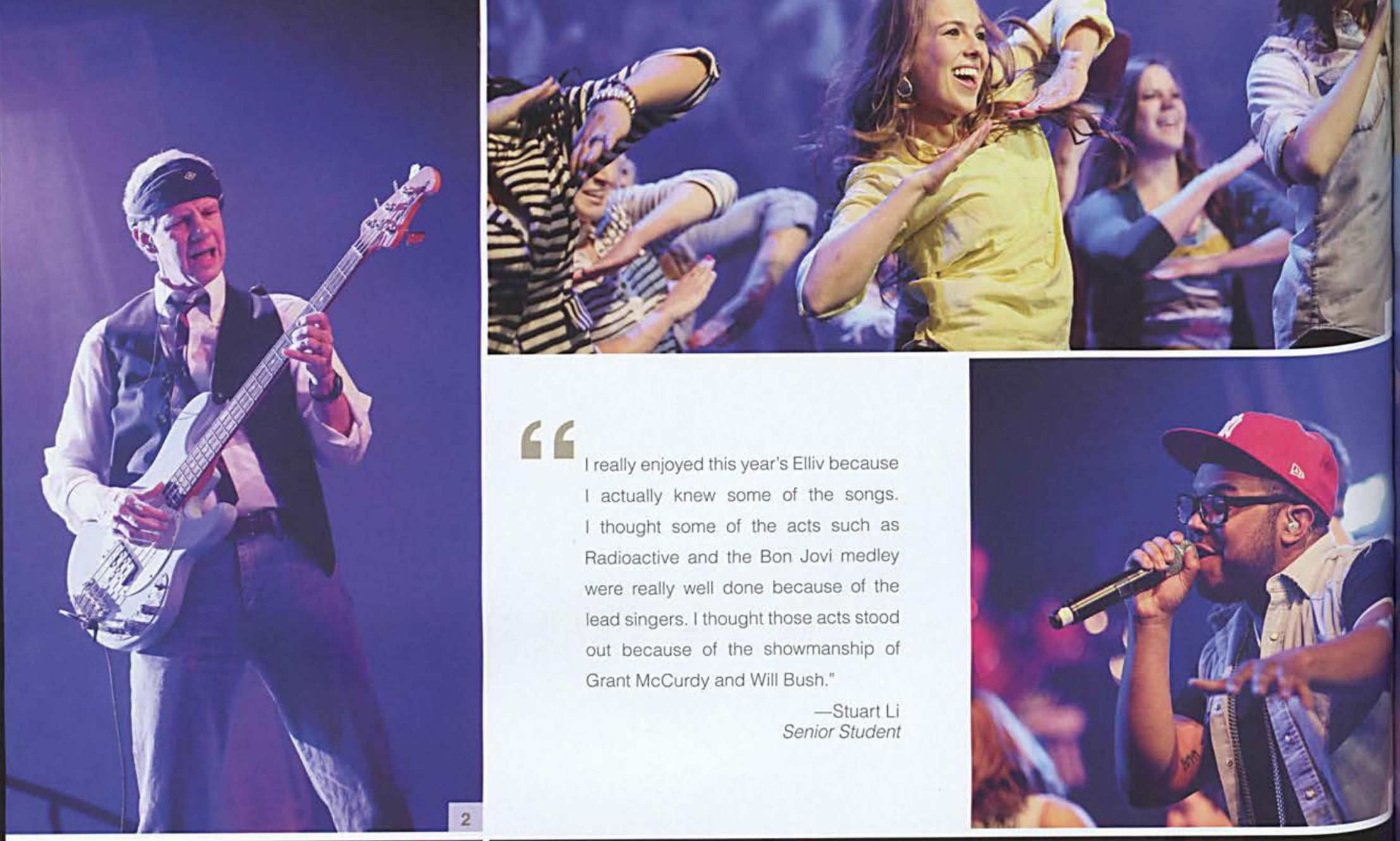

fín

I really enjoyed this year's Elliv because

I actually knew some of the songs.

I thought some of the acts such as

Radioactive and the Bon Jovi medley

were really well done because of the

lead singers. I thought those acts stood

out because of the showmanship of

Grant McCurdy and Will Bush."

-Stuart Li

Senior Student
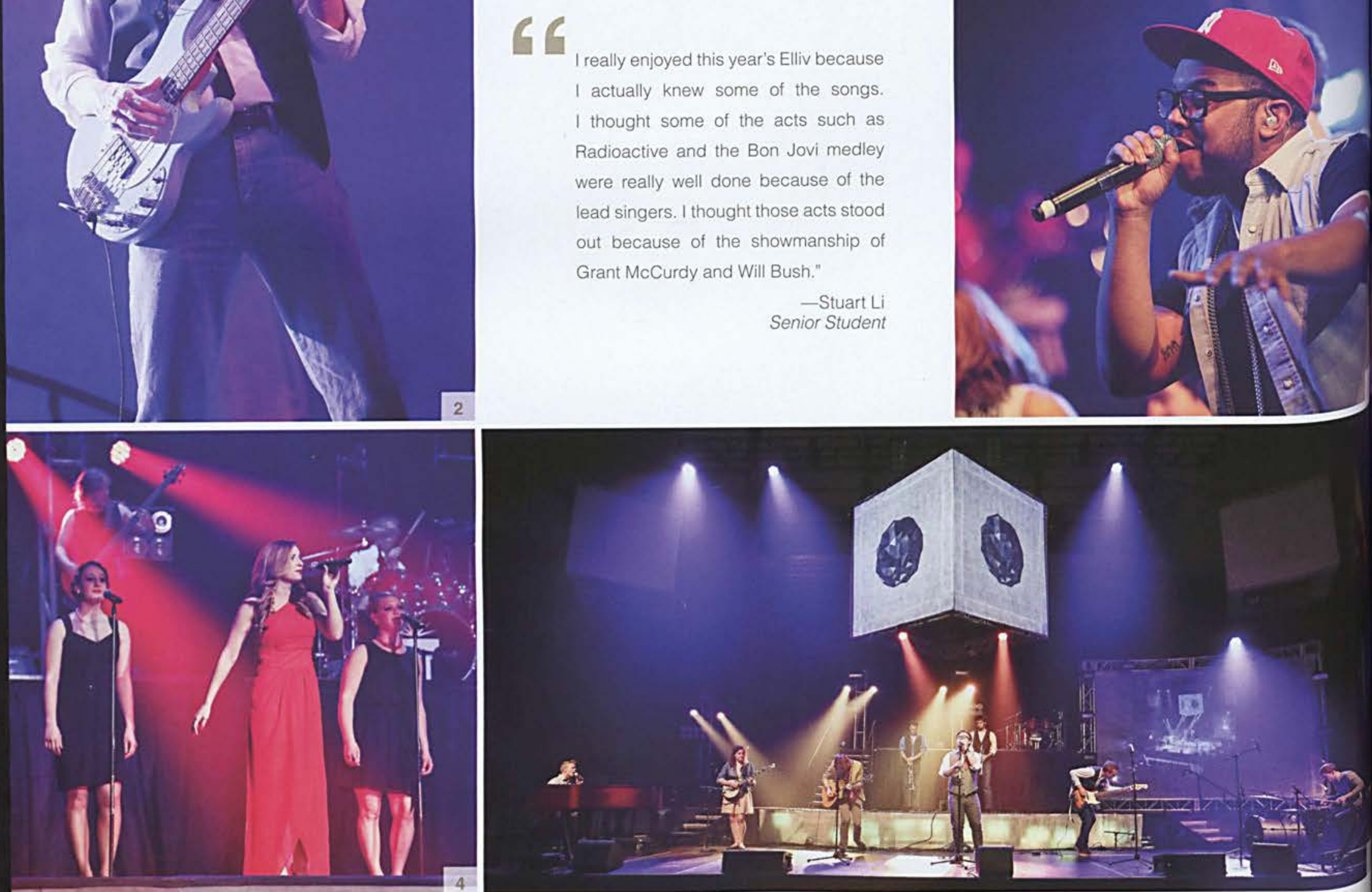

2

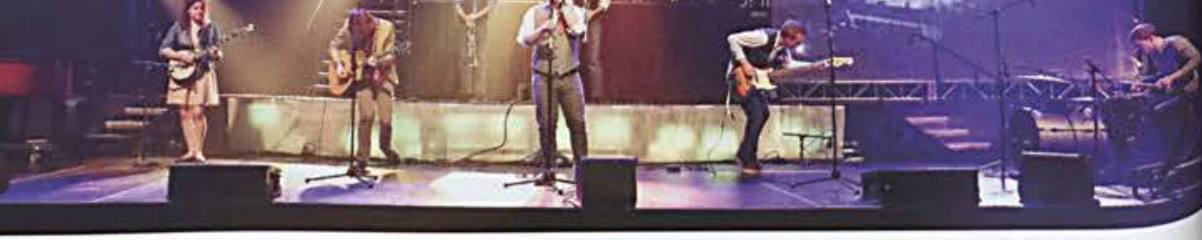

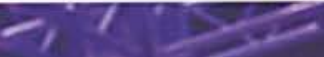
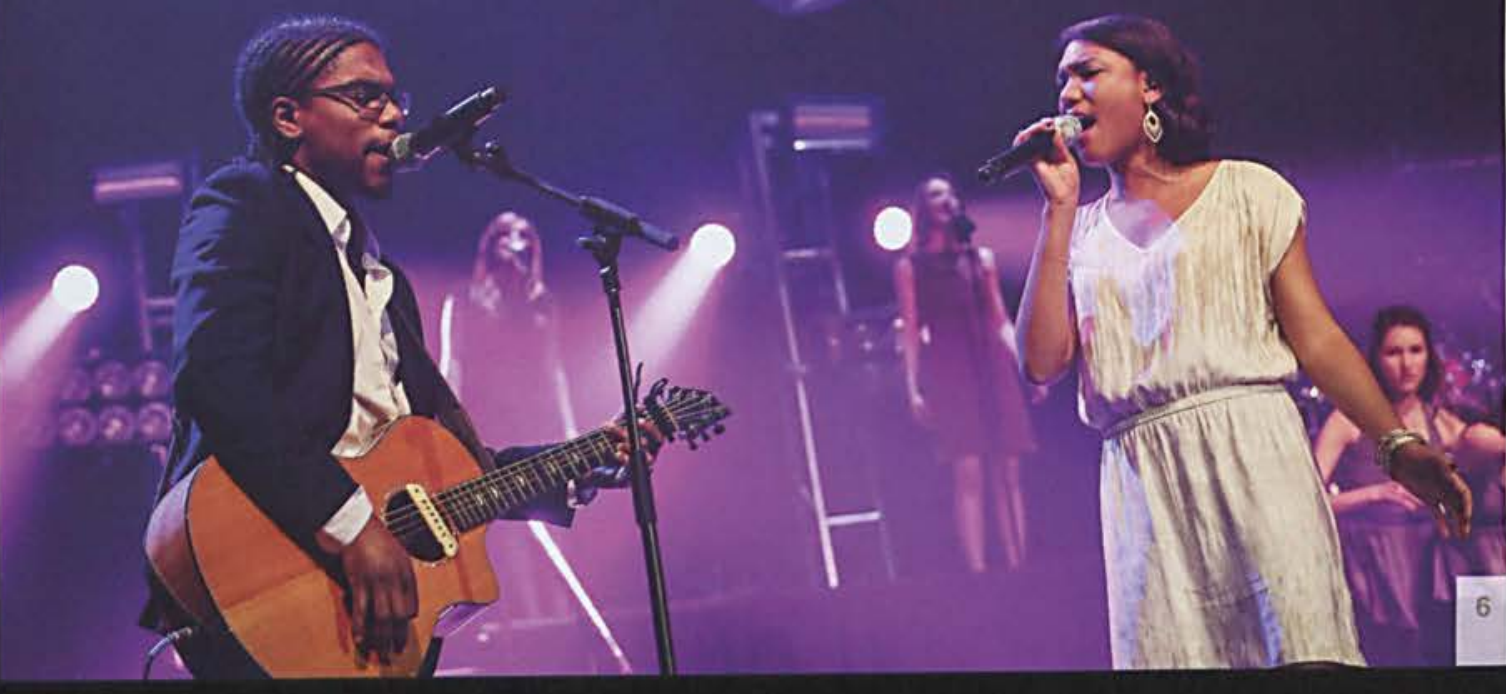

1. Students dance during "What Makes

You Beautiful" by One Direction.

2. Dr. Brown plays bass during the Bon Jovi medley.

3. Darris Sneed finishes the night with the song "Forever" by Chris Brown.

4. Abigail Butts belts out "Respect" by Aretha Franklin.

5. Eddie Liggitt performs "I Will Wait" by Mumford \& Sons.

6. Michael Pettus and Thalia Melendez sing "The Only Exception" by Paramore. 


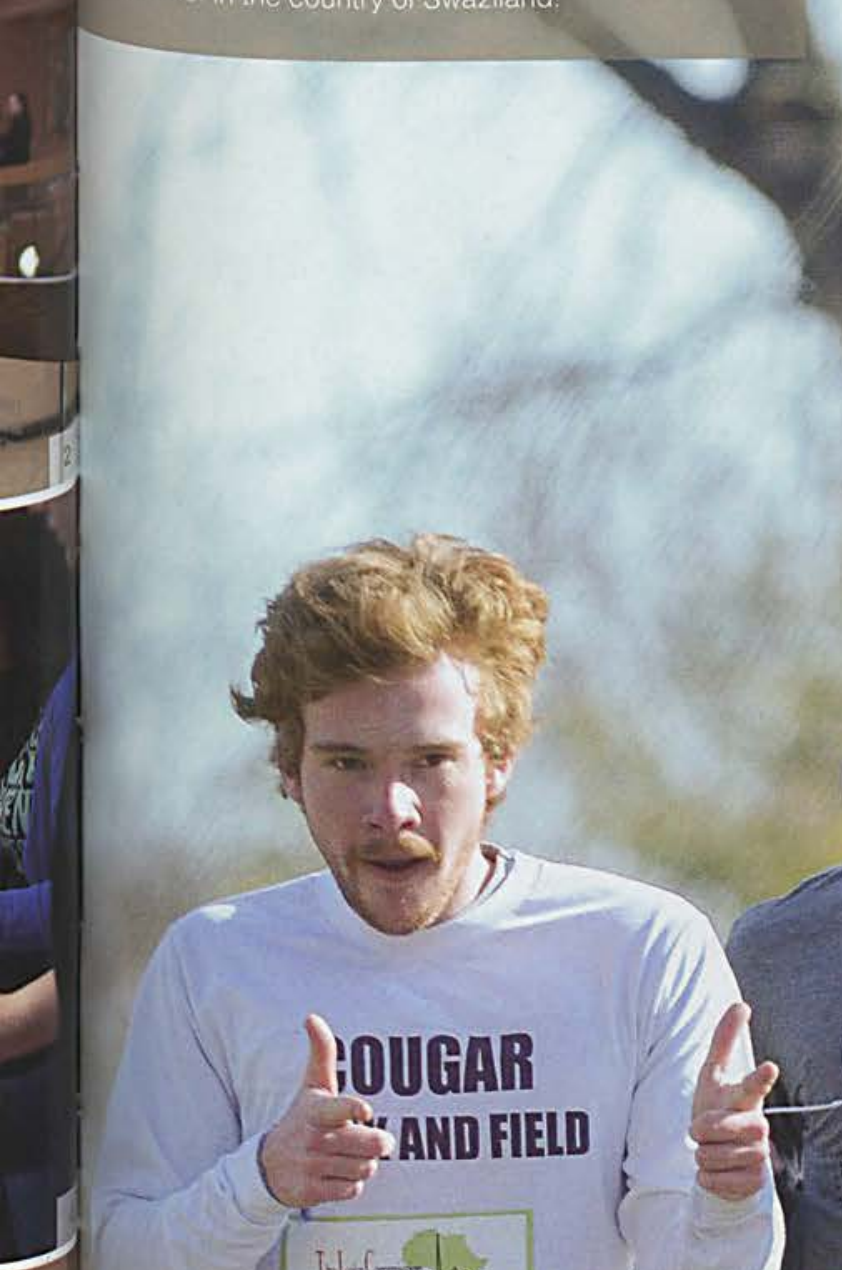

\section{ADS relief in the country of Swaziland}

Lougar
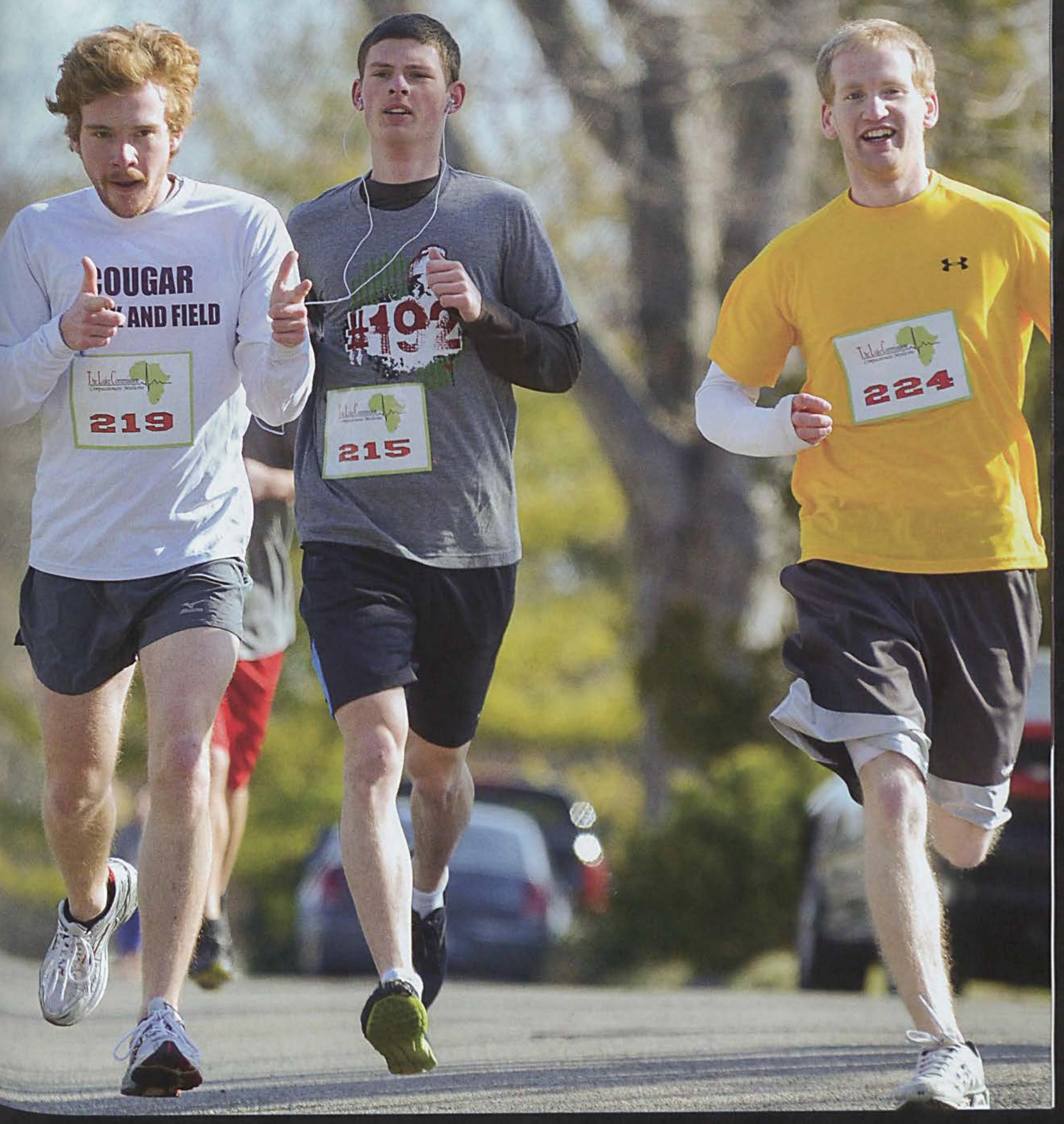

$\frac{1}{1}$

$\rightarrow$ 


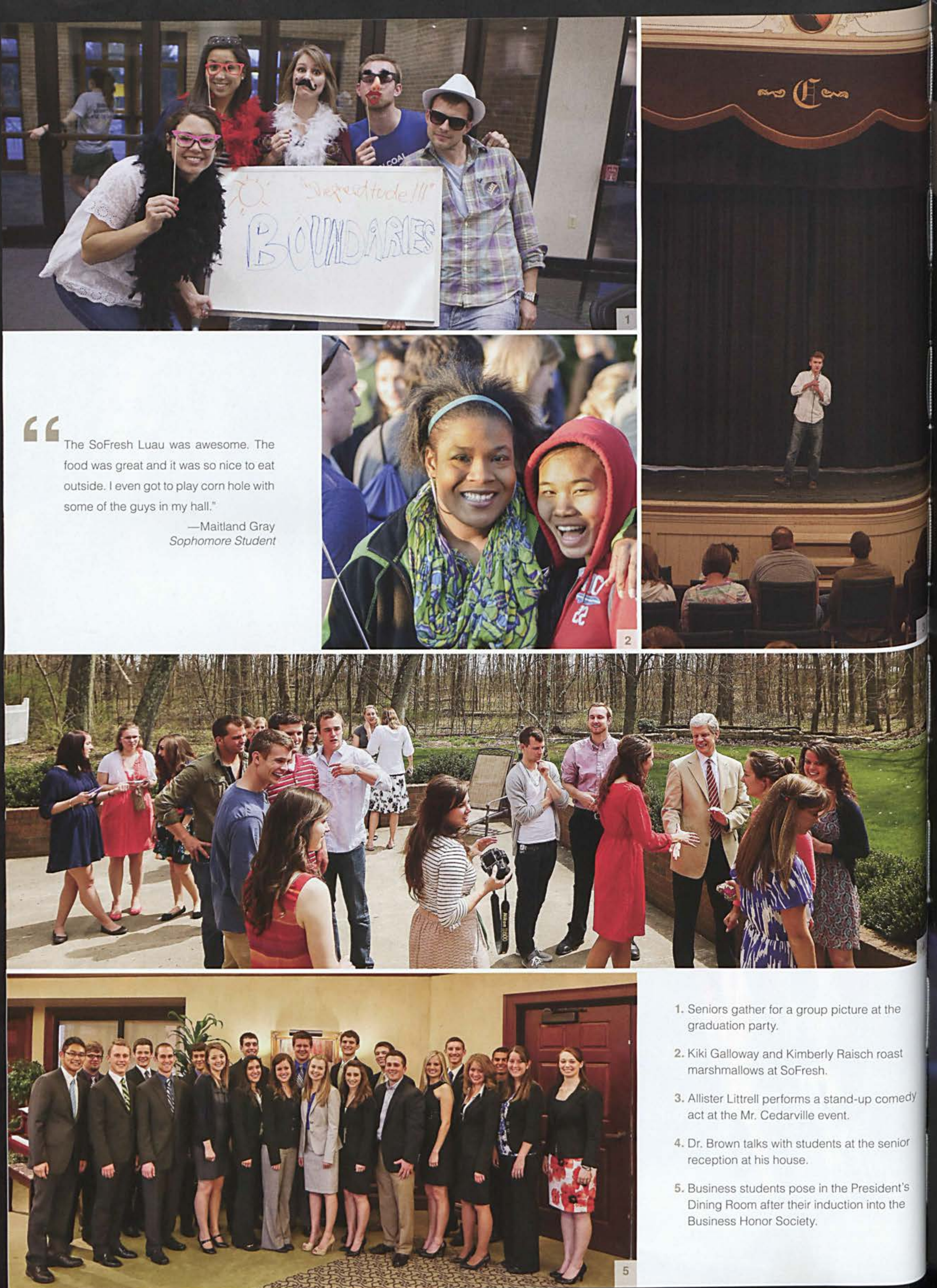




\section{SPRING EVENTS}

Eddie Liggitt performs a set for the CMC

concert in the Jeremiah Chapel.

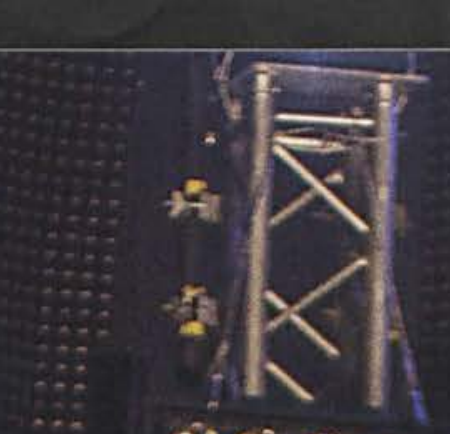

169

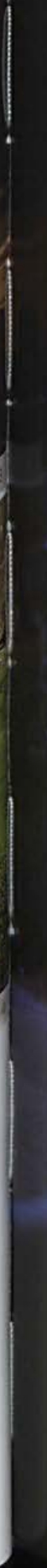

-

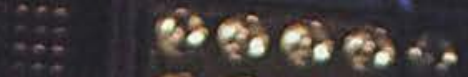

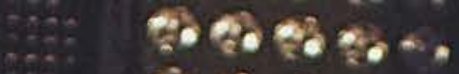

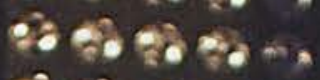

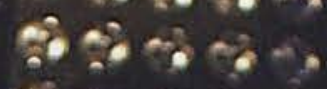$$
a+x+2,0
$$$$
\text { at } 2000
$$
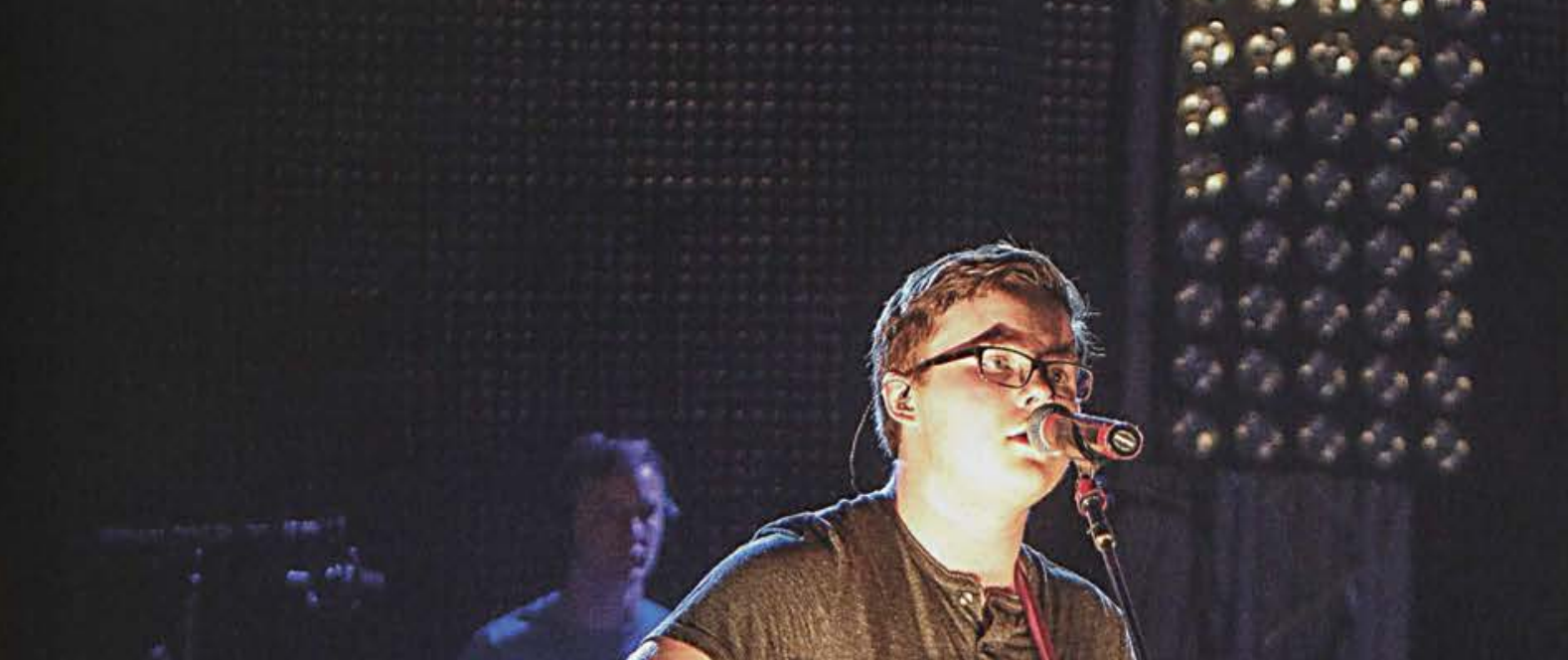

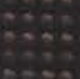
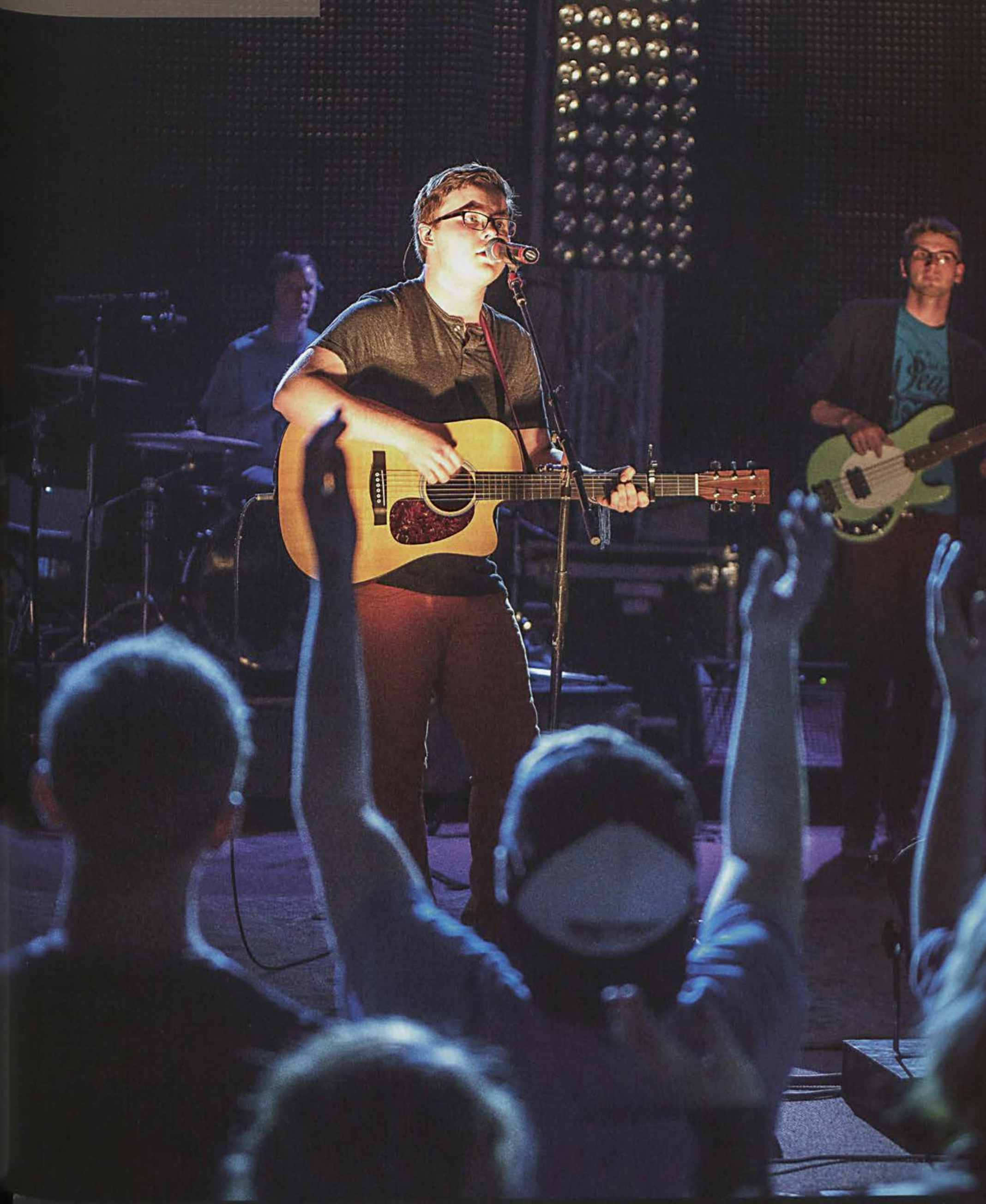

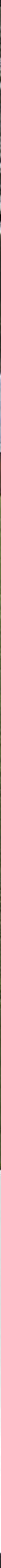


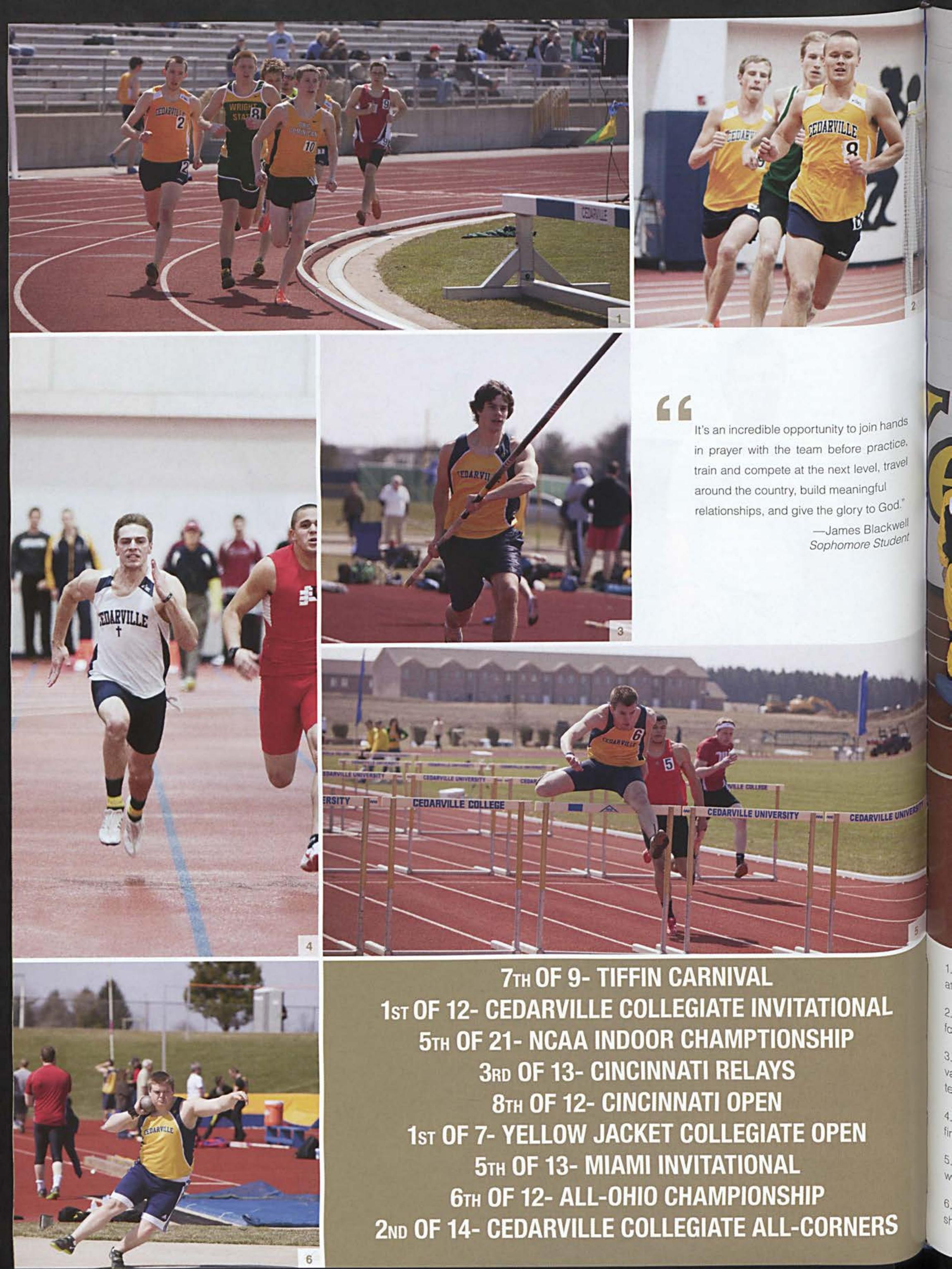




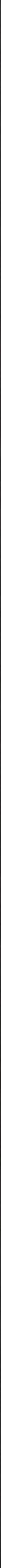




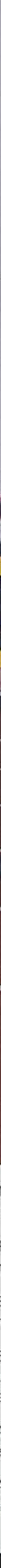





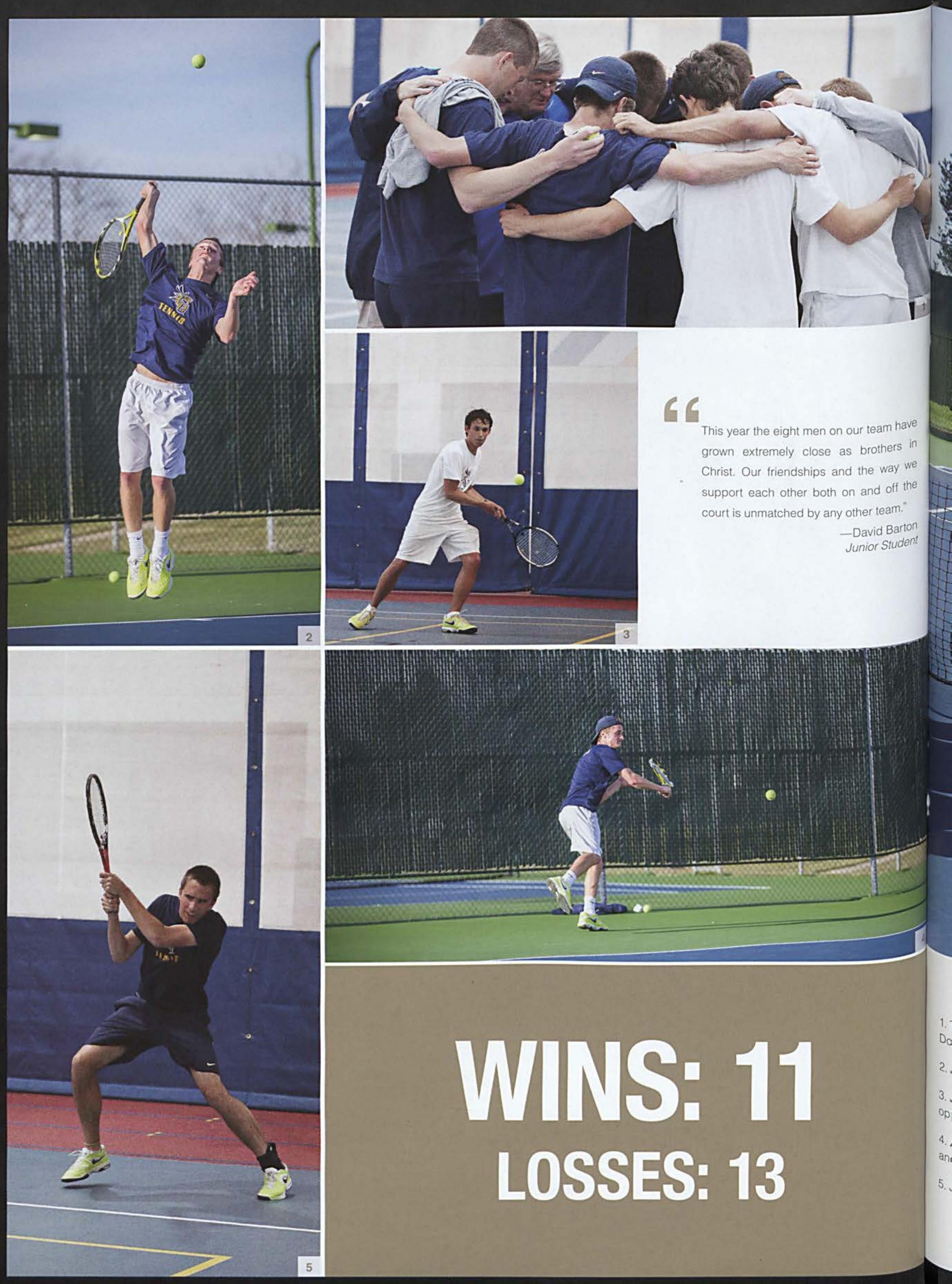




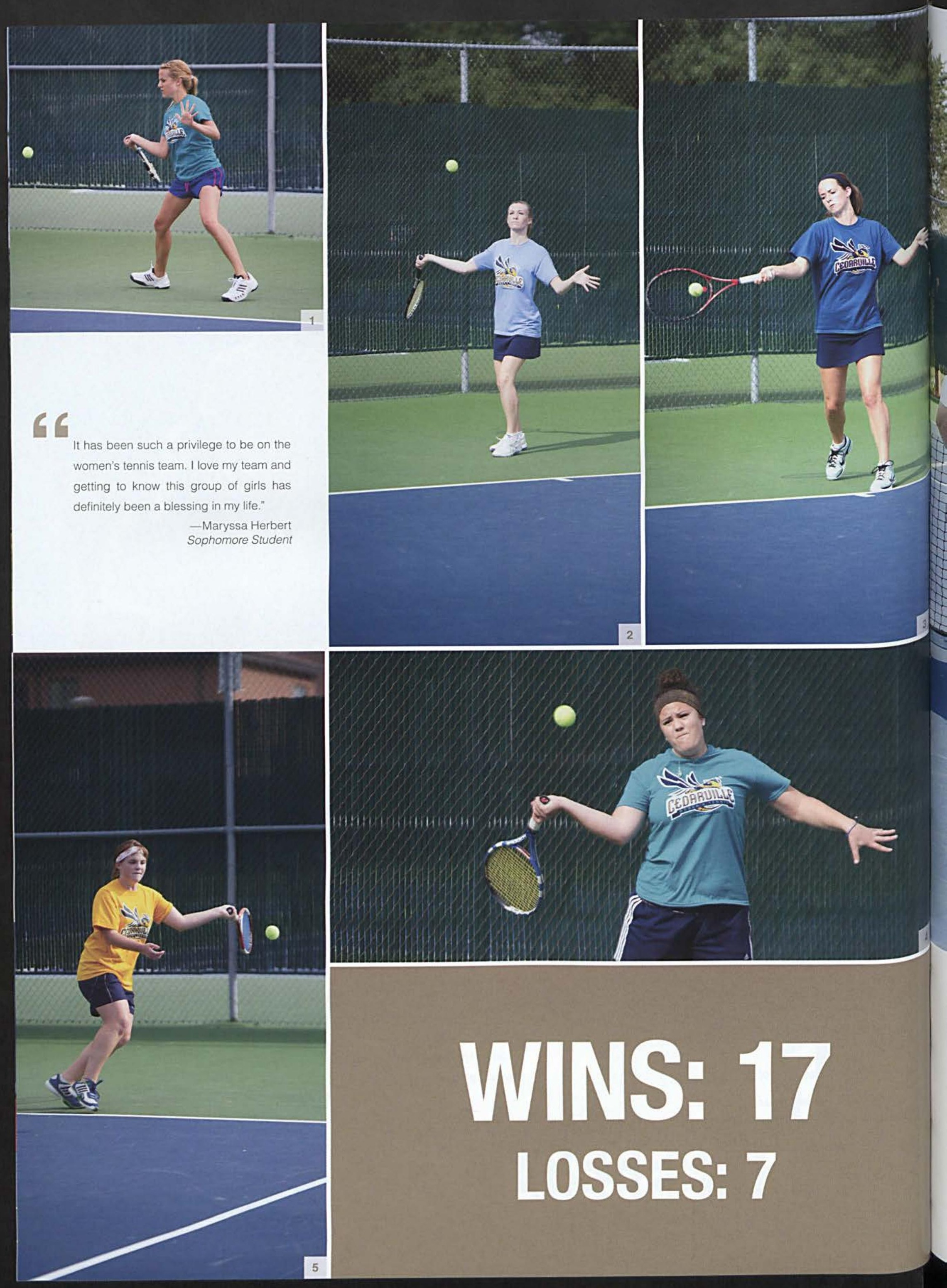


G.

- 2 centions

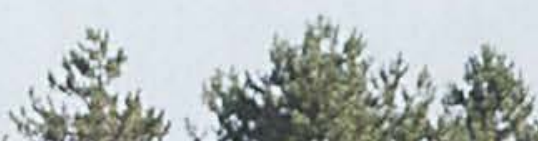

i.

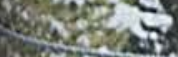

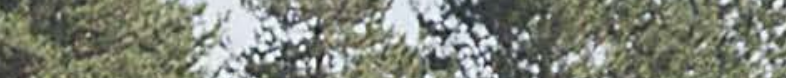

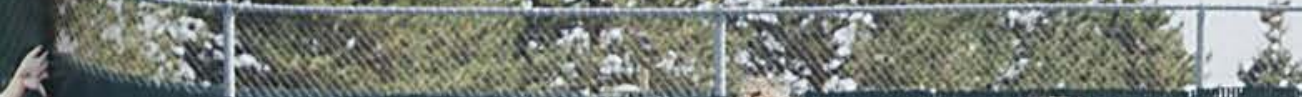

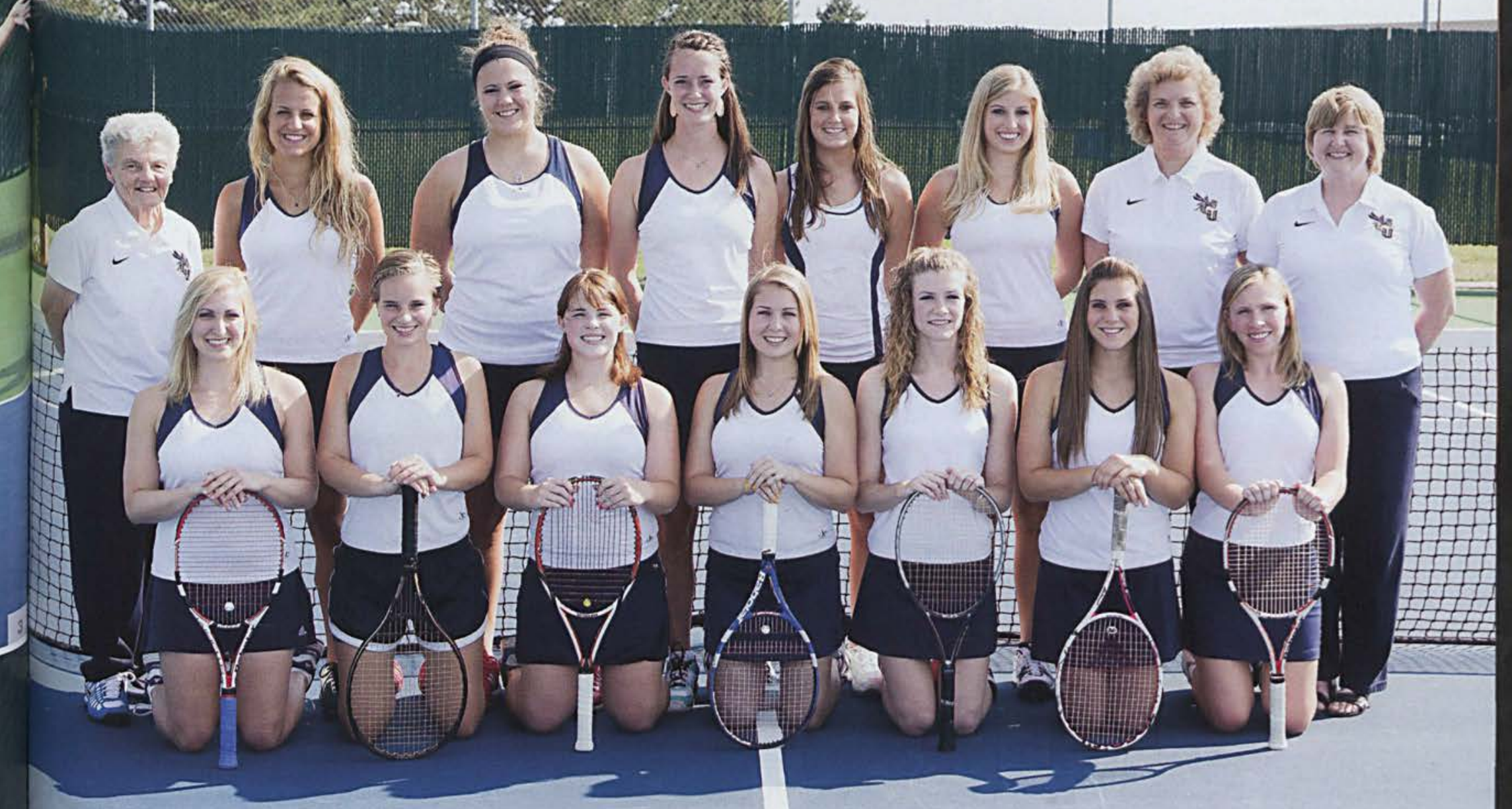

Sophomore Lily Schuler, who plays \#1 in the

team line-up, goes head to head against the

Opponent's best player.

2. Lyndsay Stine swings at the ball, striving to

keep up her record of 6-1.

3. Maryssa Herbert returns the ball in a

doubles match

4. Senior Joy Dice returns a serve.

5. Senior Melissa Stoltzfus rushes to return

the ball.

(kneeling left-to-right) Brooke Johnson, Melissa Stoltzfus, Colleen Browning. Chelsea Diamond, Lindsay Stine, Jessie Finfrock, Emma Rahn. (standing) Head Coach Dr. Dee Morris, Lily Schuler, Joy Dice, Maryssa Herbert, Courtney Heydt, Emily Paul, Associate Head Coach Dr. Pam Johnson, Team Chaplain Becky Kuhn 


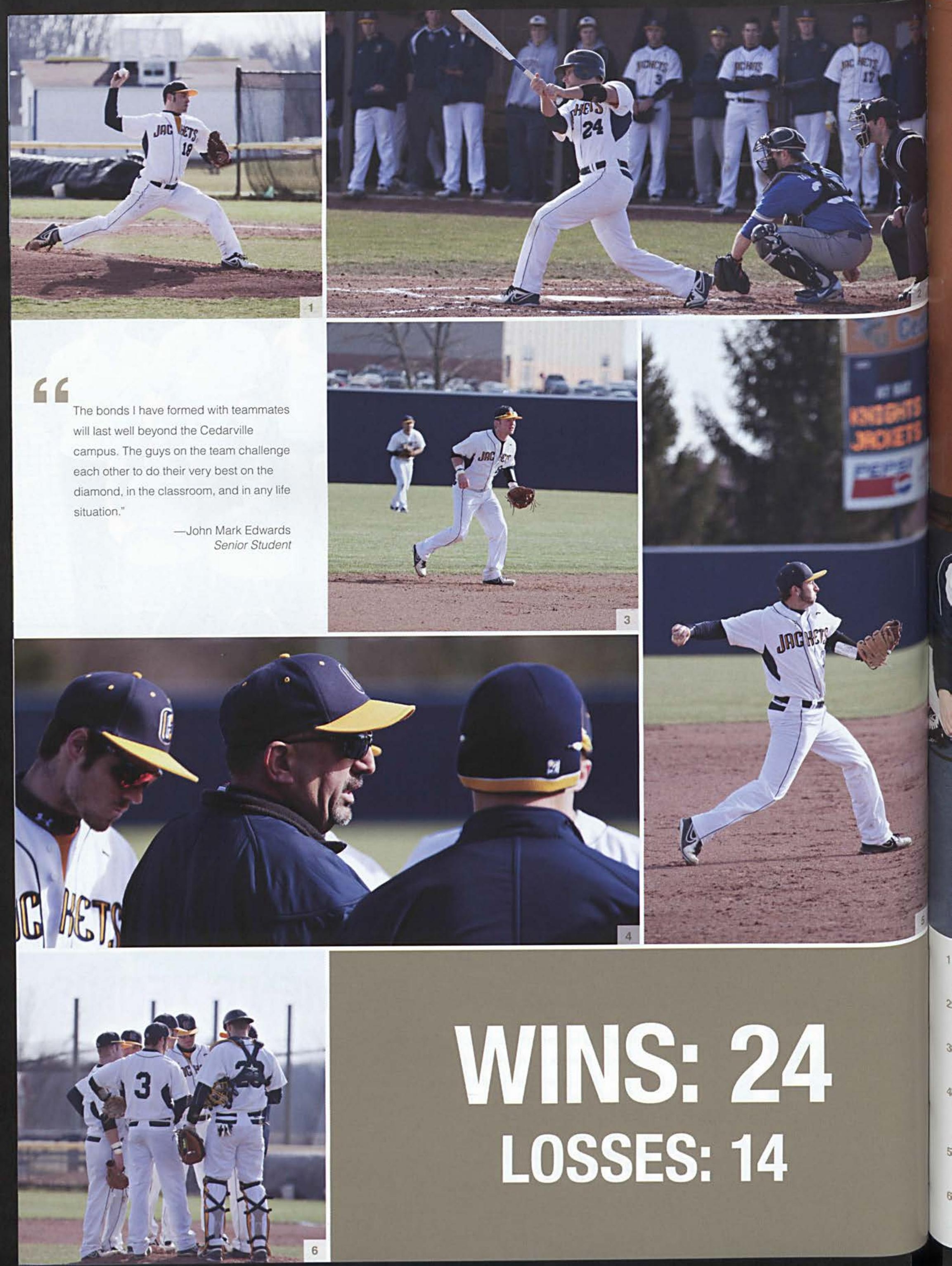




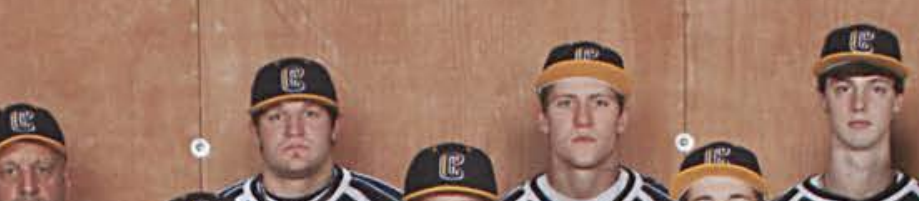
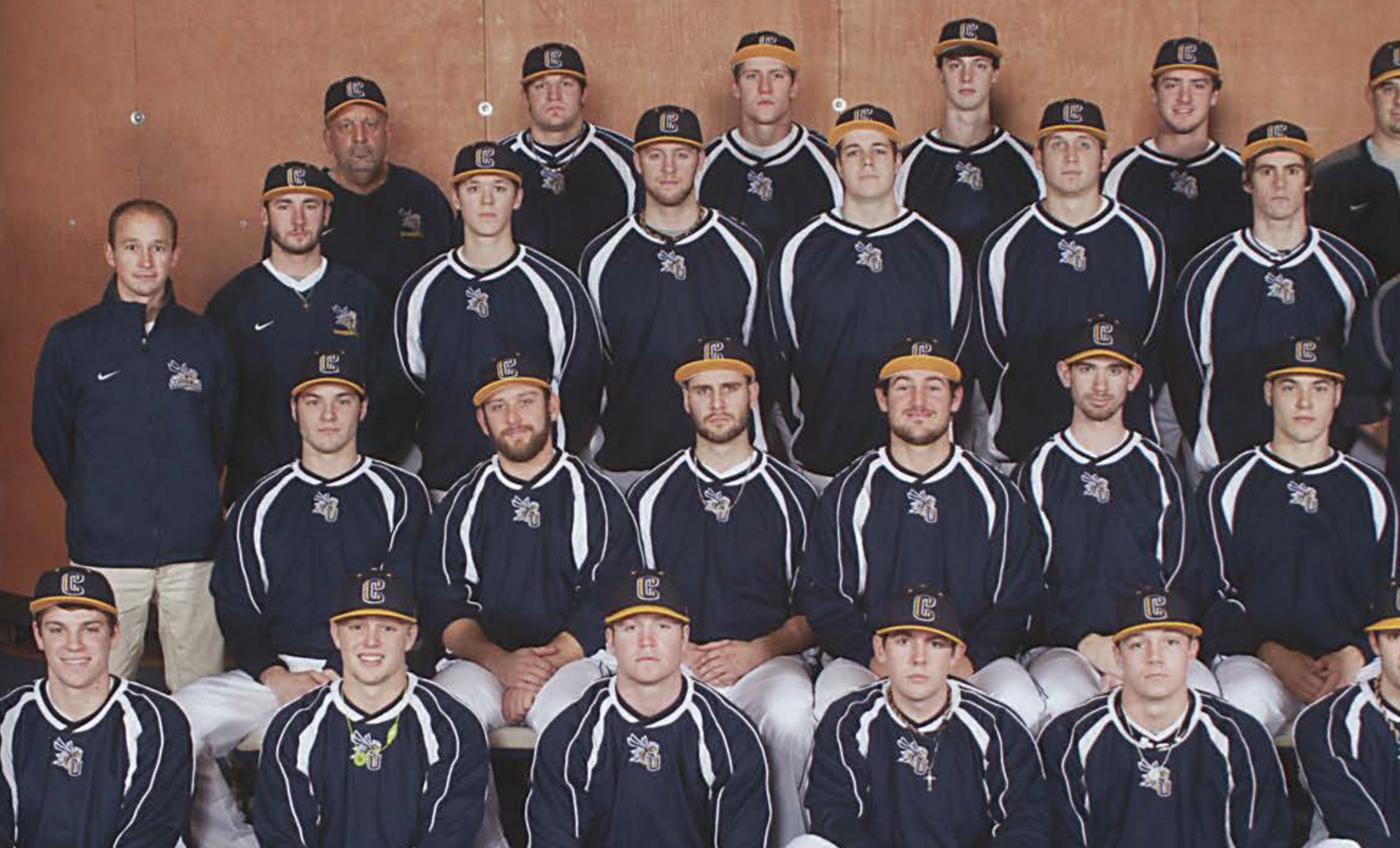

(is) is
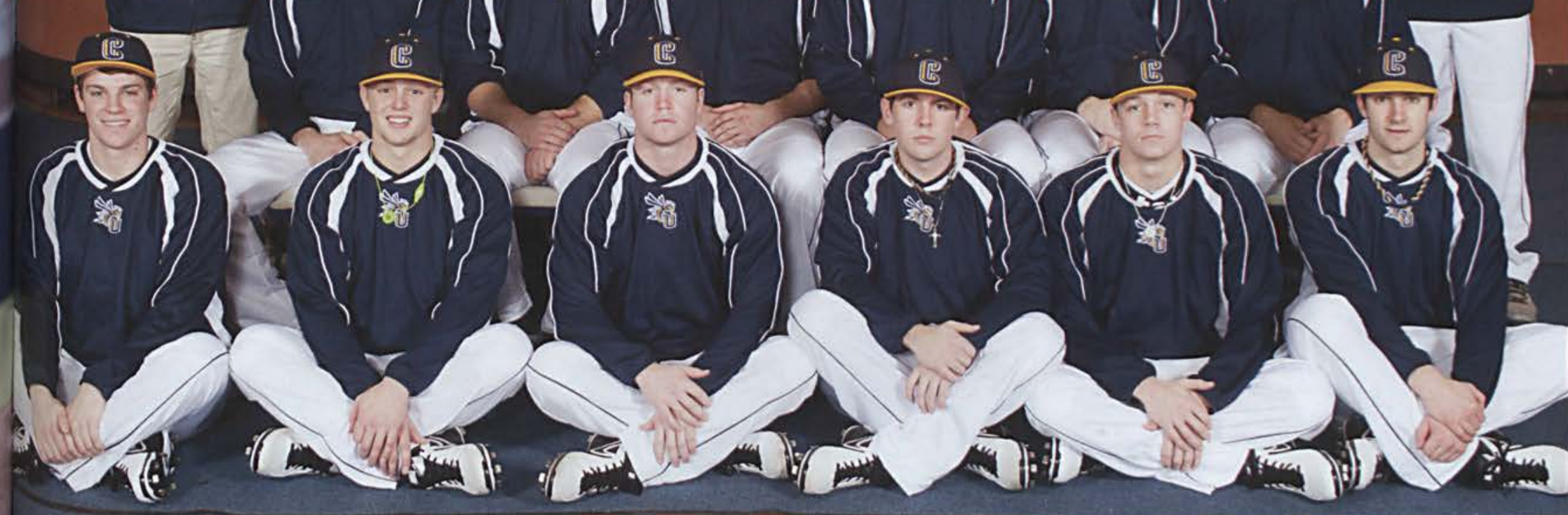

1. Junior Jacket David Ledbetter pitches a

fastball, hoping for a strikeout.

. The Cedarville team looks on as Chris Ward hits a pop fly into left field.

3. Playing shortstop, Sam Summerlin looks to Snag the ball after a hit.

4. Michael Manes, Dan Larkin, and Coach Tyler Rost discuss their offensive strategy for the next inning.

5. Tyler Hurt throws the ball to first base, trying to get an Urbana player out

6. The Cedarville team on the field gathers together for a mound visit to strategize their game plan.

\section{BASEBALL}

(front row left-to-right) David Bancroft, Tyler Hurt, Sam Summerlin, Zach Huskey, Jordan Adams, Garrett Baker. (second row) Ryan Ledbetter, Chris Ward, Rob Nesteroff, Logan Kasabian, John Mark Edwards, David Ledbetter. (third row) Assistant Athletic Trainer Kurt Beachy, Cam McWilliams, David Lenhardt, Chris Fox, Peter Martin, Jordan Ammon, Dan Larkin, Assistant Coach Tyler Rost. (back row) Head Coach Mike Manes, Steve Cardwell, Harrison Martin, Sean Larkin, Steve Kneeland, Assistant Coach Ben Galbreath. 


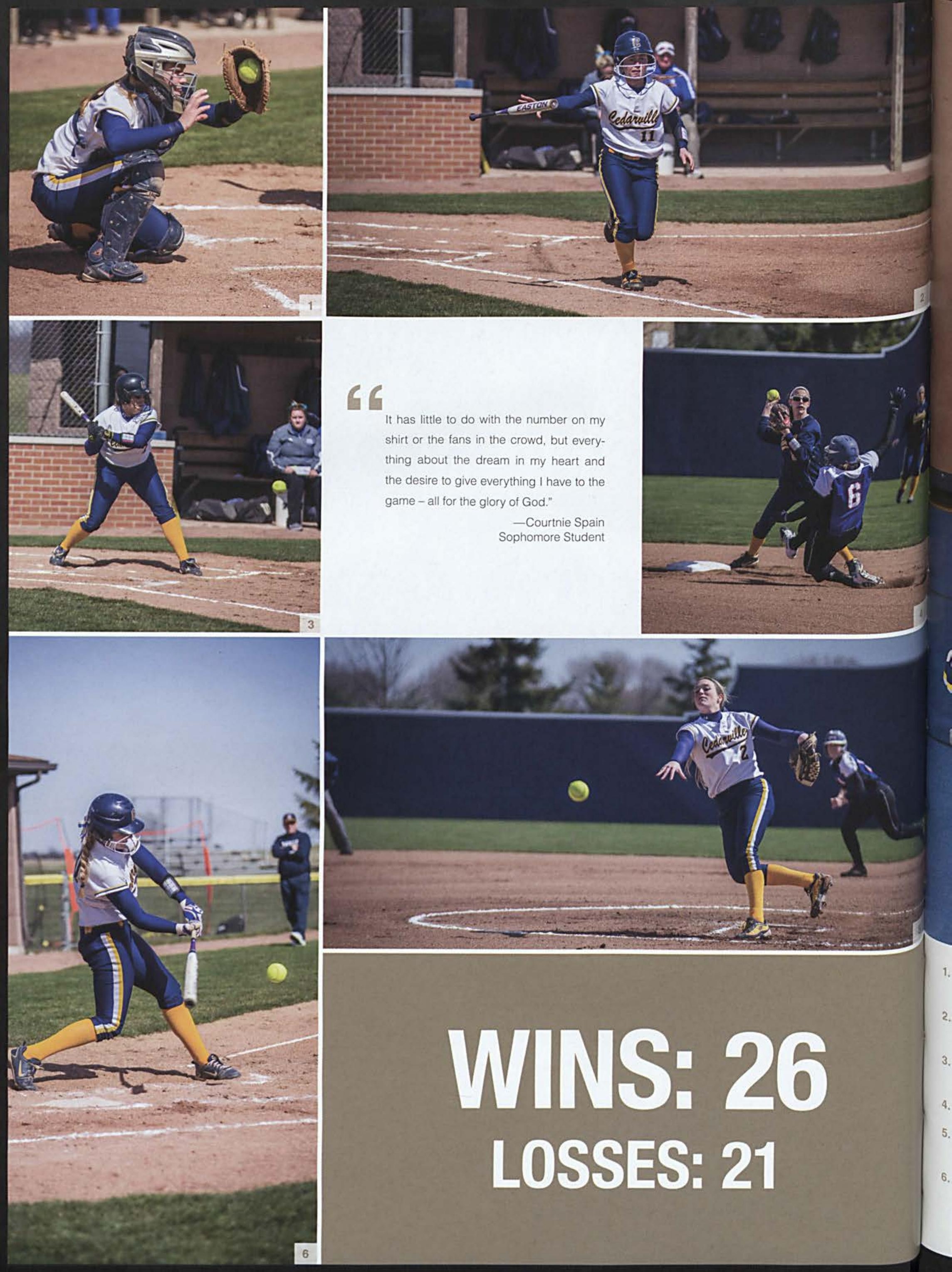




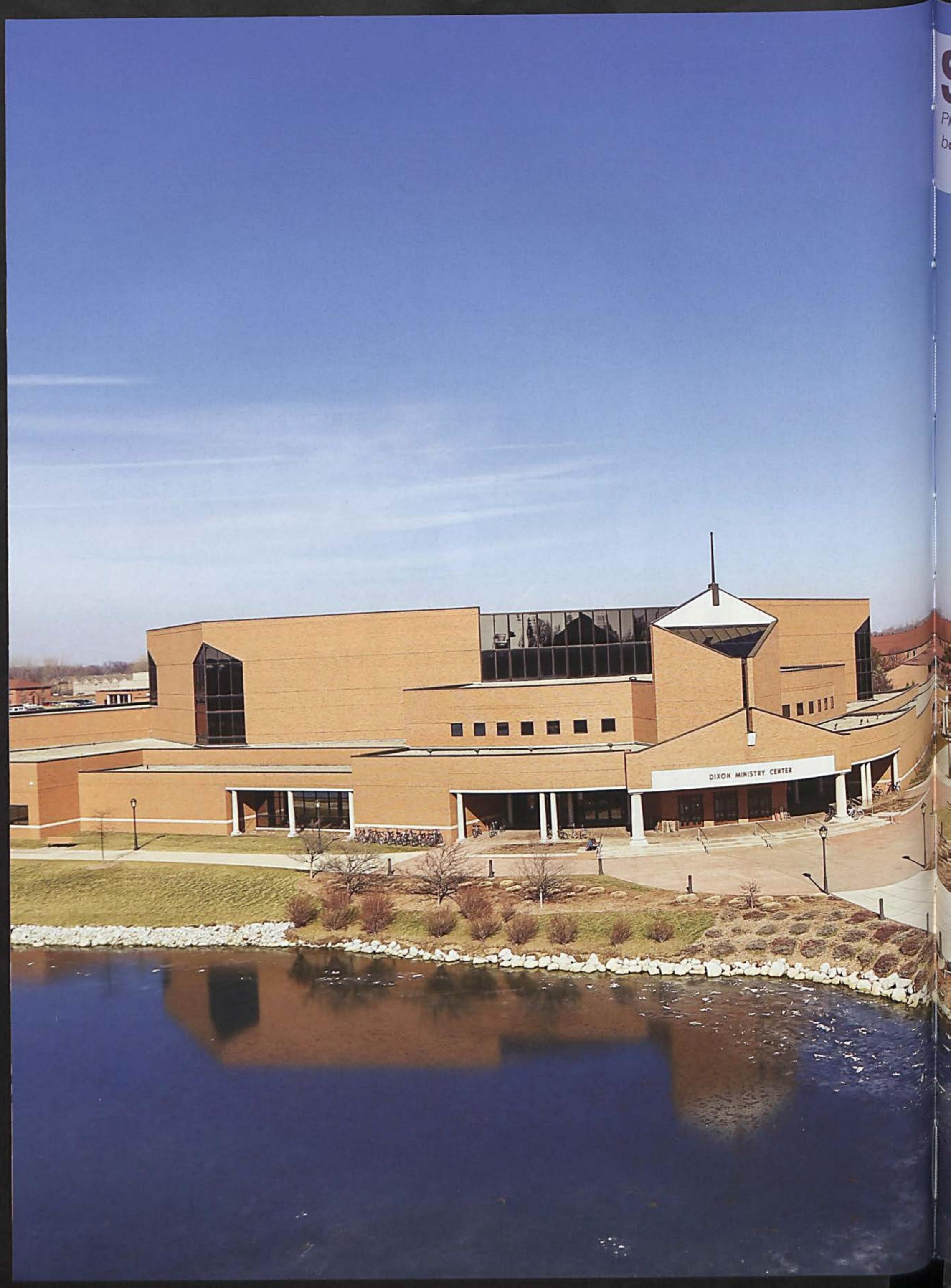




\section{SENIORS}

Philippians 3:13-14 "Brothers, I do not consider that I have made it my own. But one thing I do: forgetting what lies behind, and straining forward to what lies ahead, I press on toward the goal of the upward call of God in Christ Jesus."

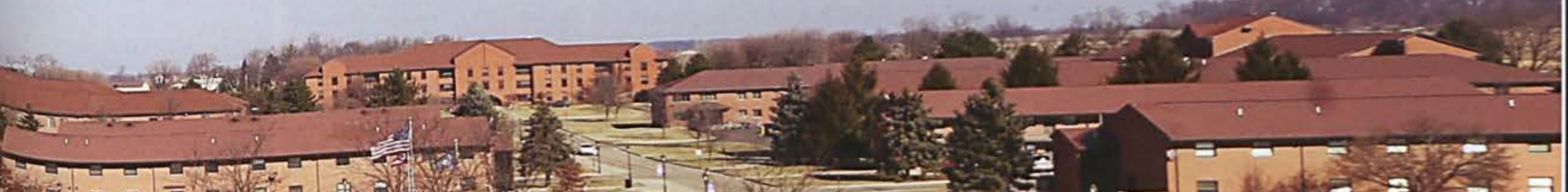

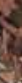
$\sqrt{2}$

Lis:-

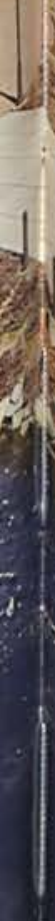

thing ne

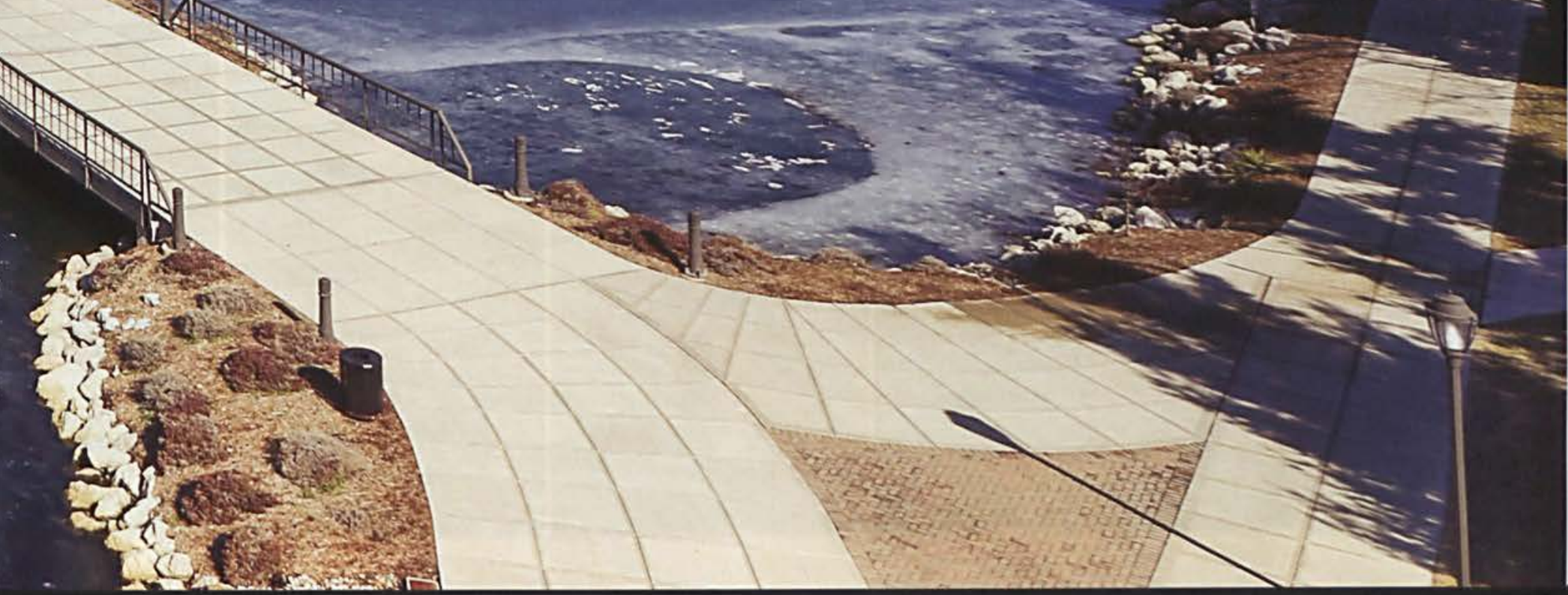




\section{SENIORS}

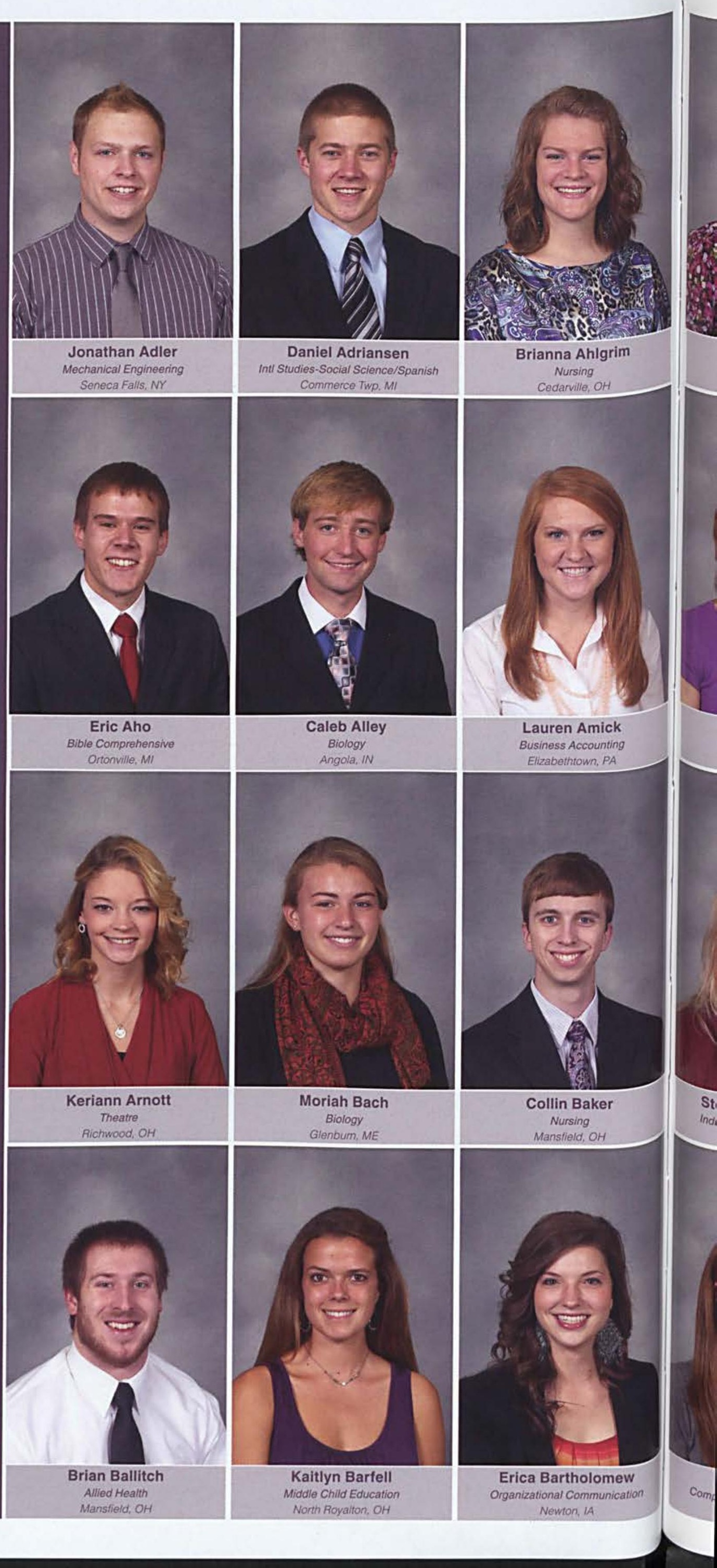





\section{SENIORS}

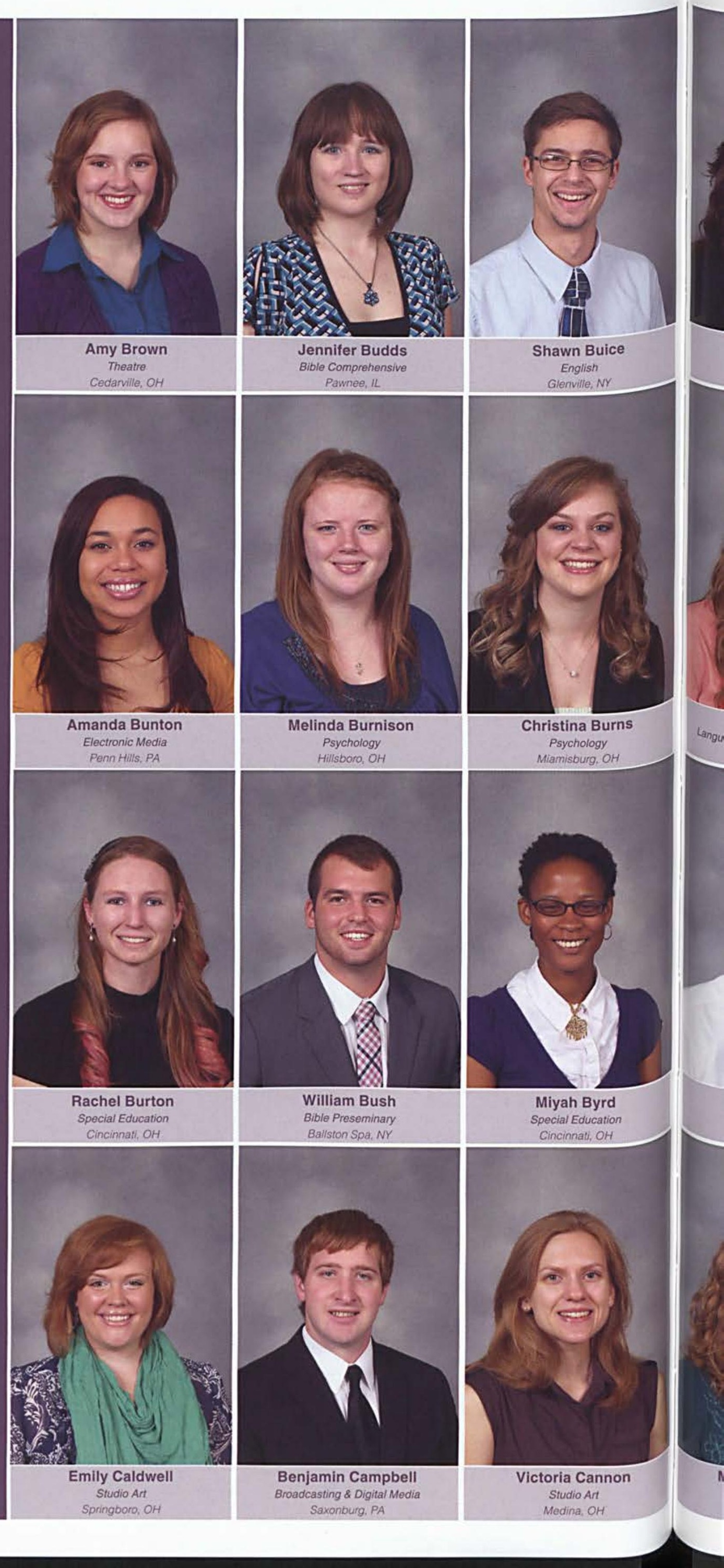




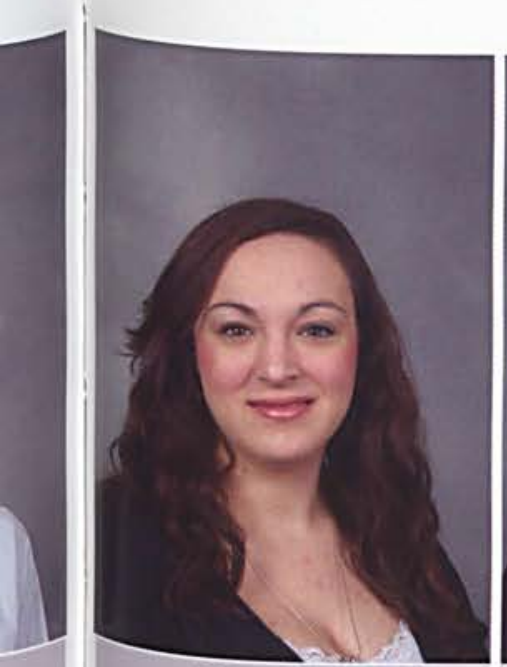

Chloe Carey

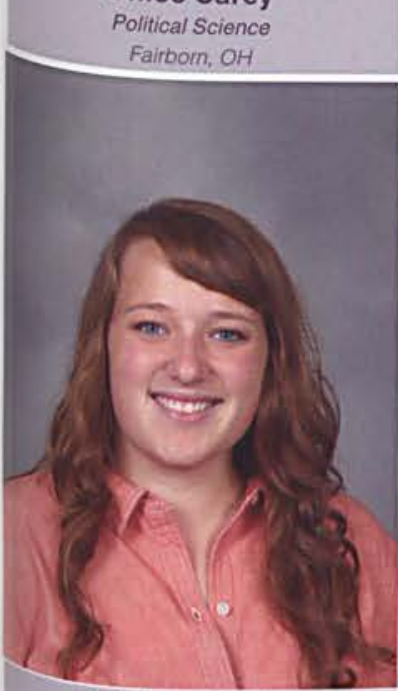

Katelynn Cassel Language Arts Education-Integrated

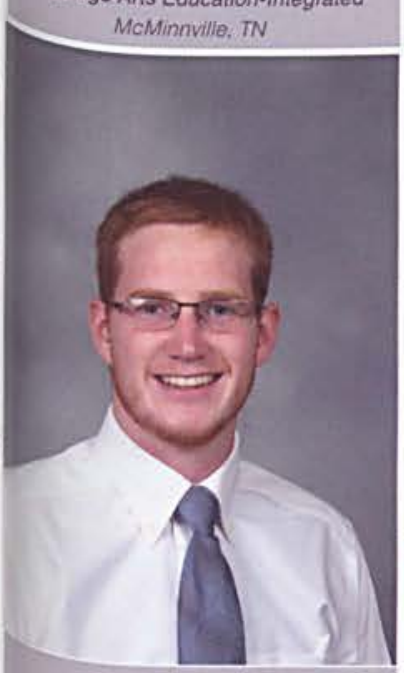

Nicholas Clark

History
Rochester. NY

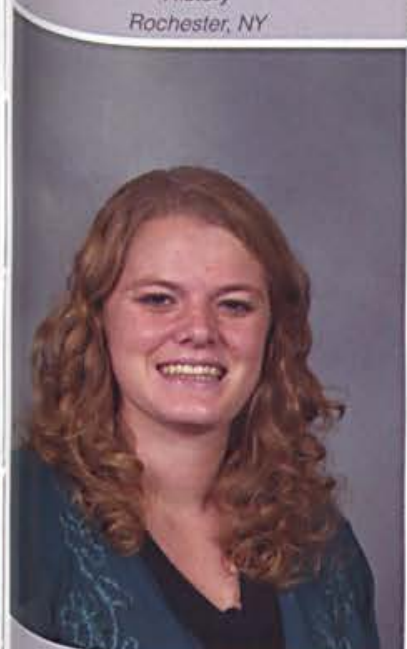

Megan Coffman TPC/Spanish

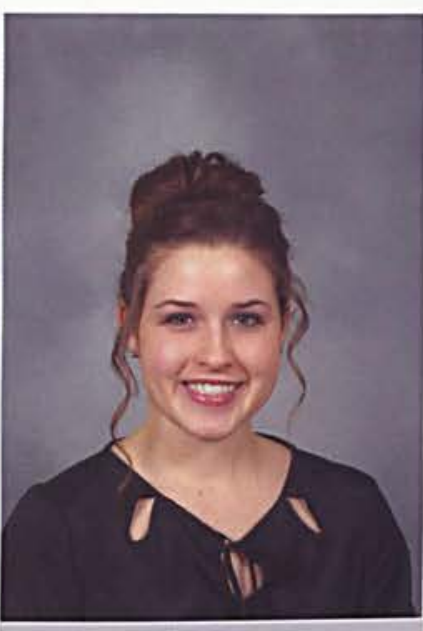

Abigail Carpenter

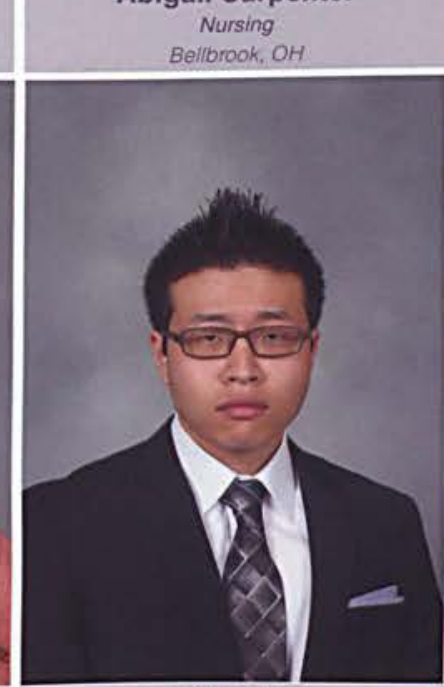

Eunho Cha

Mechanical Engineering

Federal Way, WA

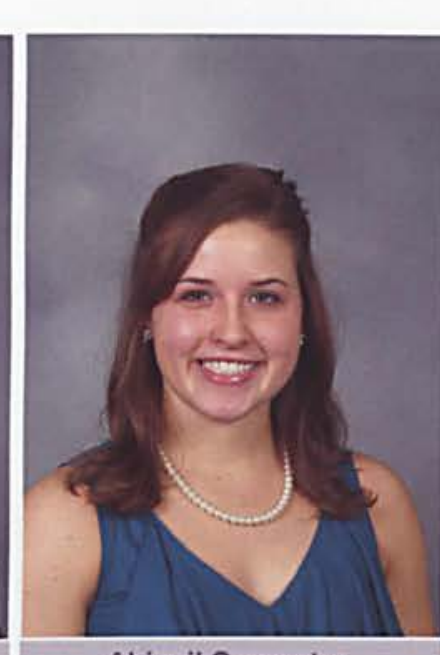

Abigail Carpenter

Nellorsook. $\mathrm{OH}$
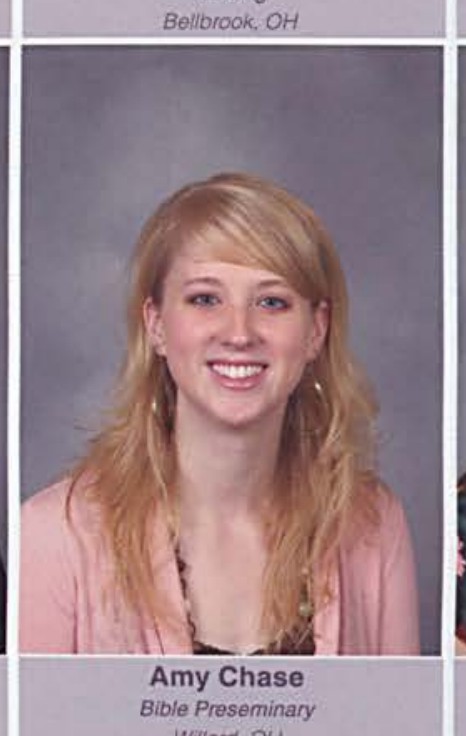

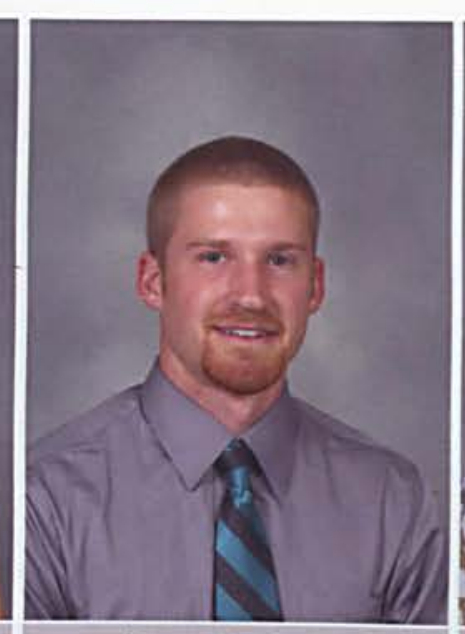

Alexander Carter

Allied Health
Presque Isie, ME

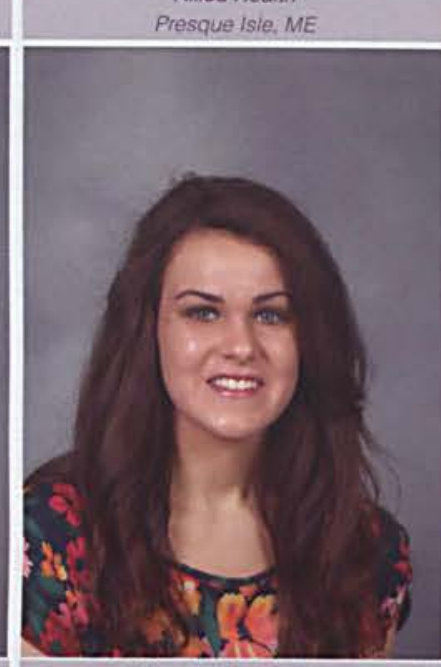

Julie Christiansen

Intemational Studies - Missiology

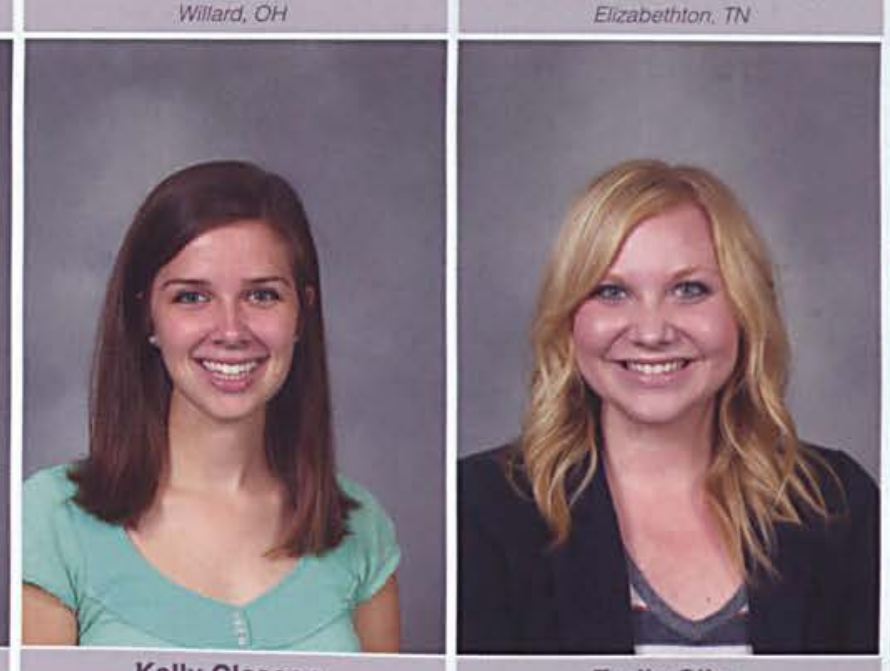

Kelly Classen

Bible Comprehensive

Bible Comprehensive

Brighton, MI
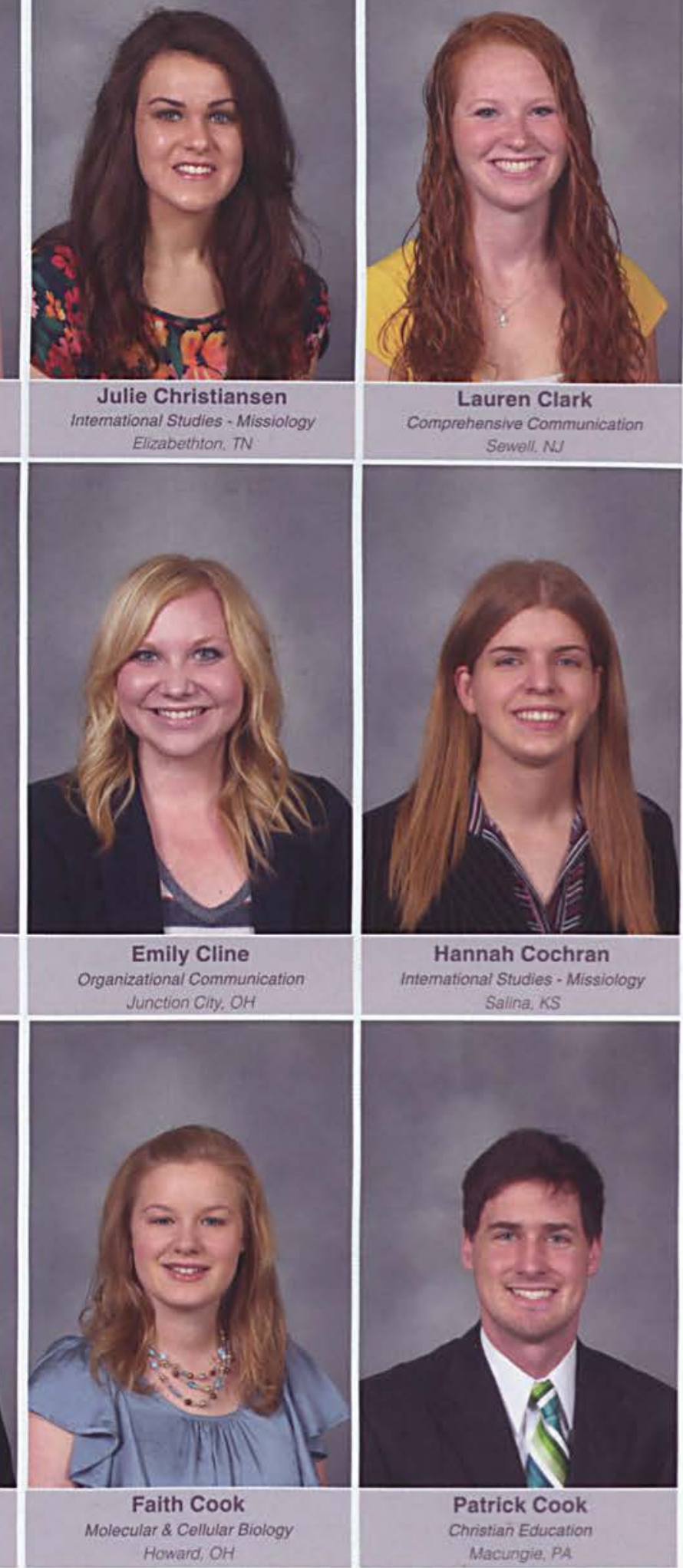

Lauren Clark

Comprehensive Communication

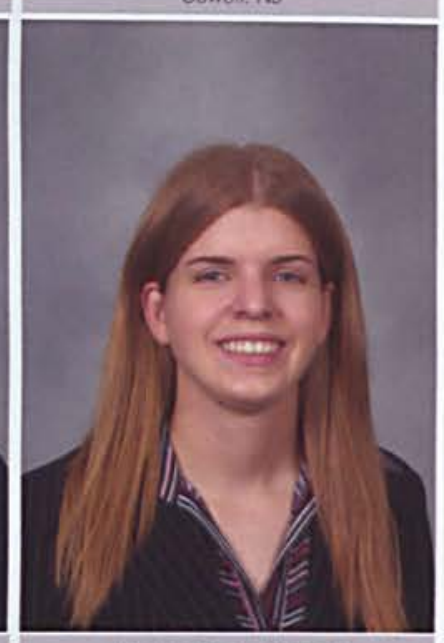

Hannah Cochran Intemational Studies - Missiology

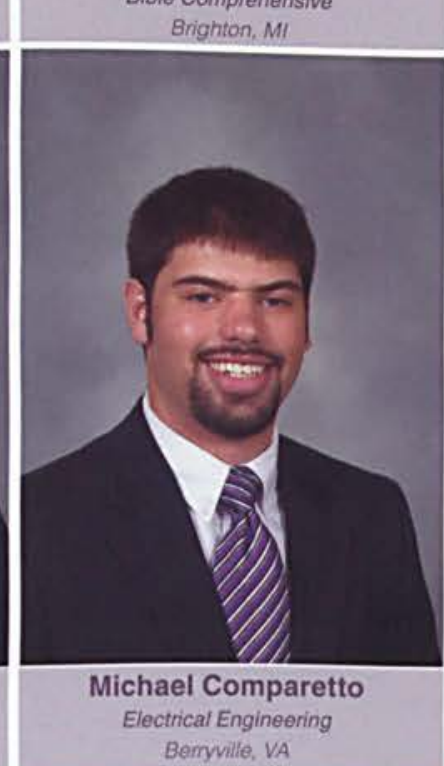




\section{SENIORS}

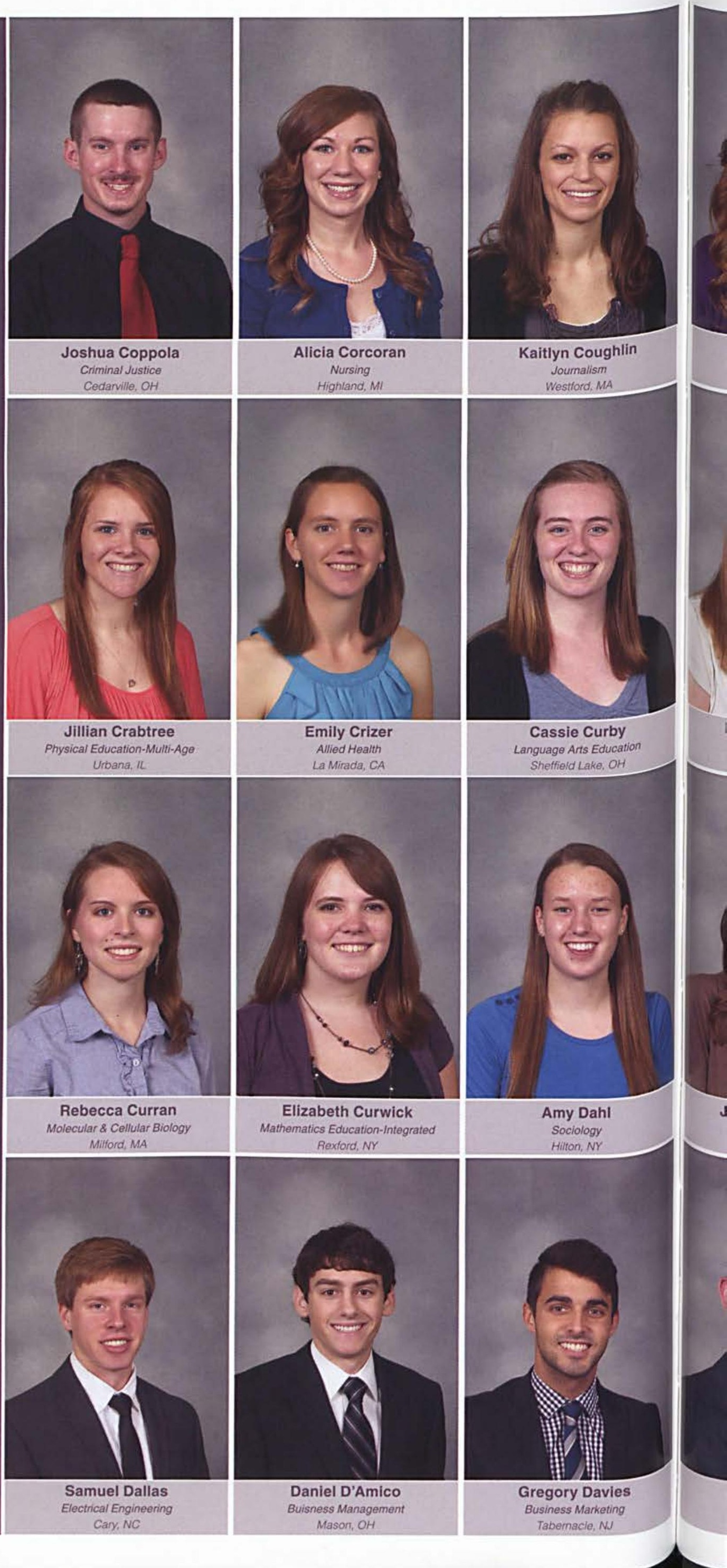




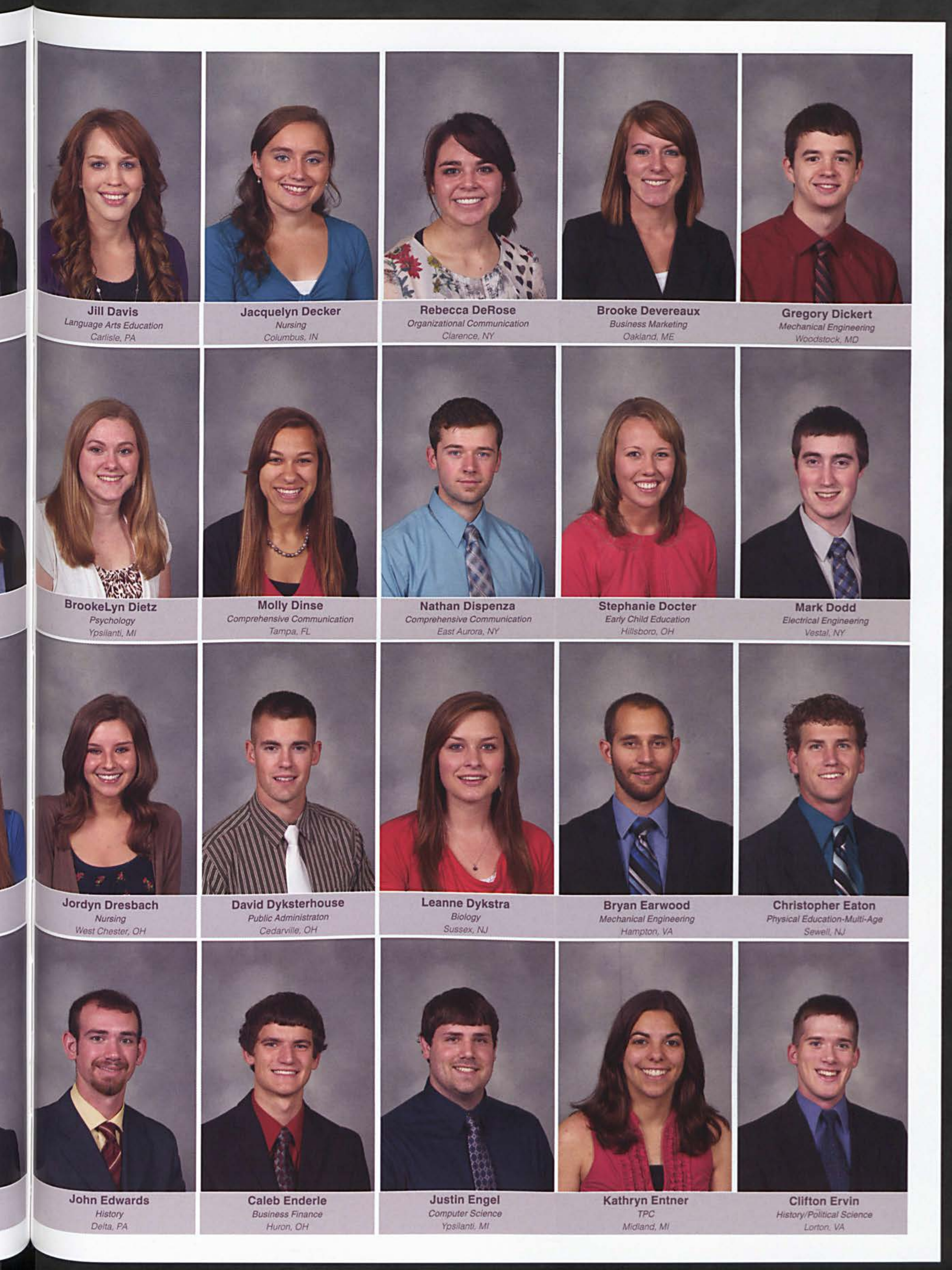




\section{SENIORS}

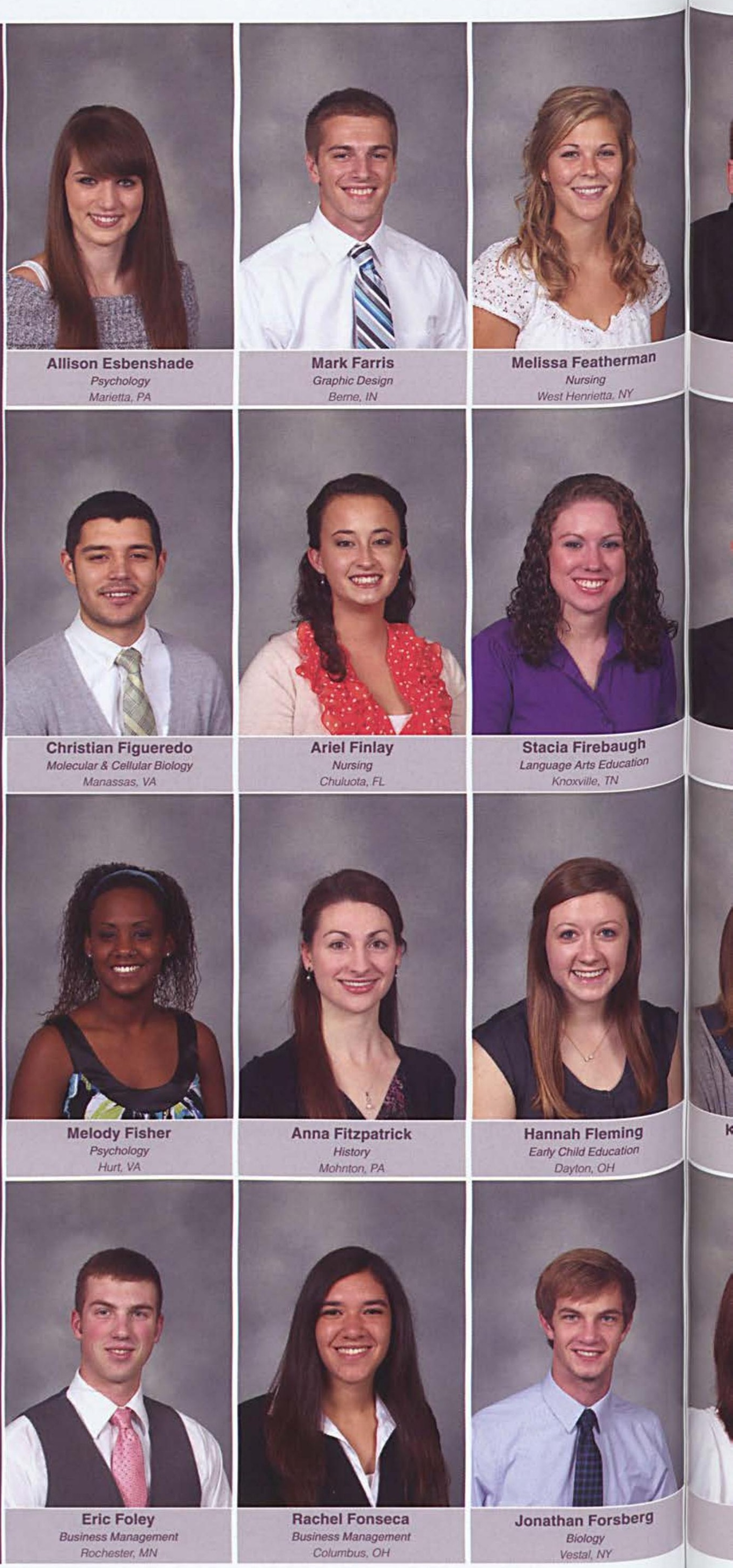




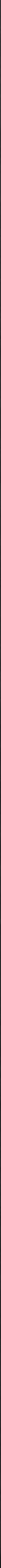




\section{SENIORS}

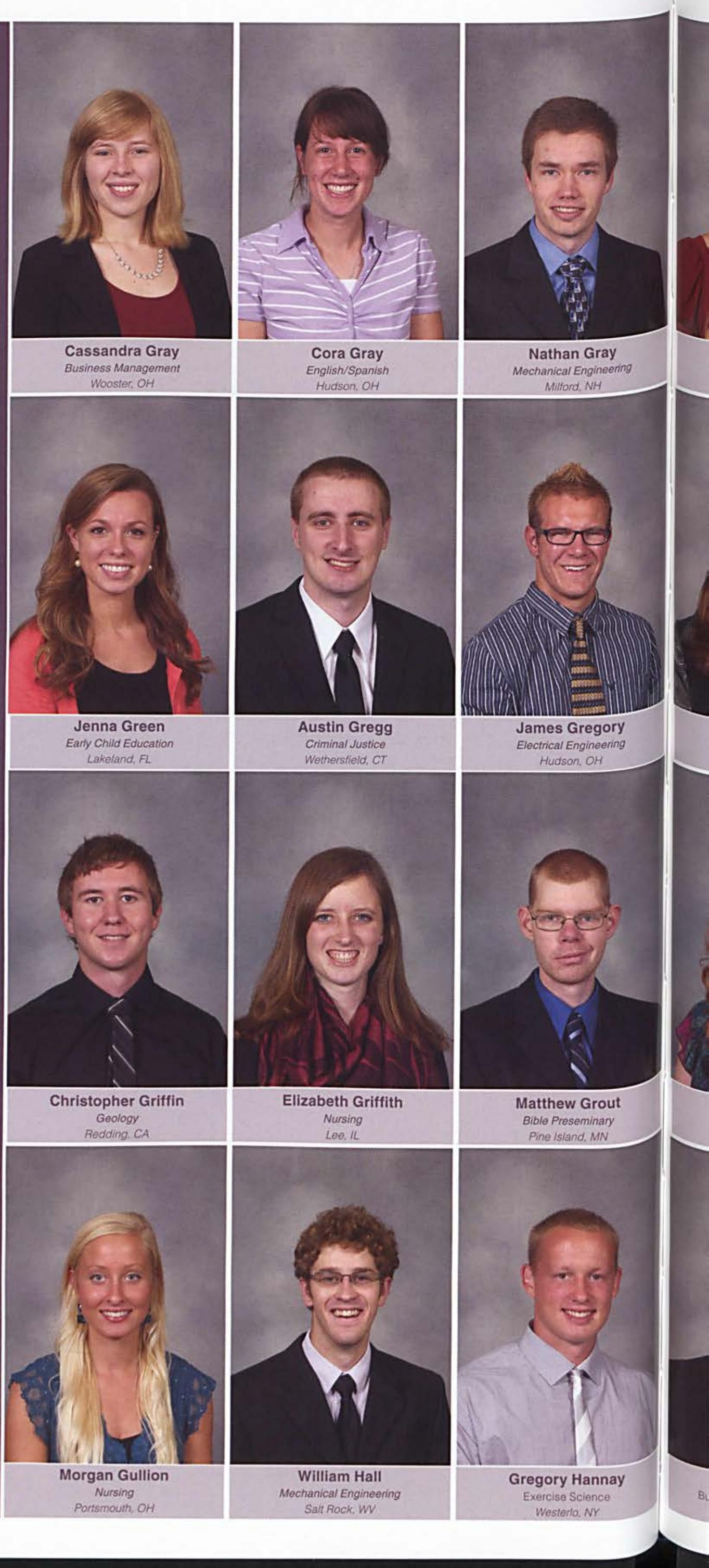



SENIORS

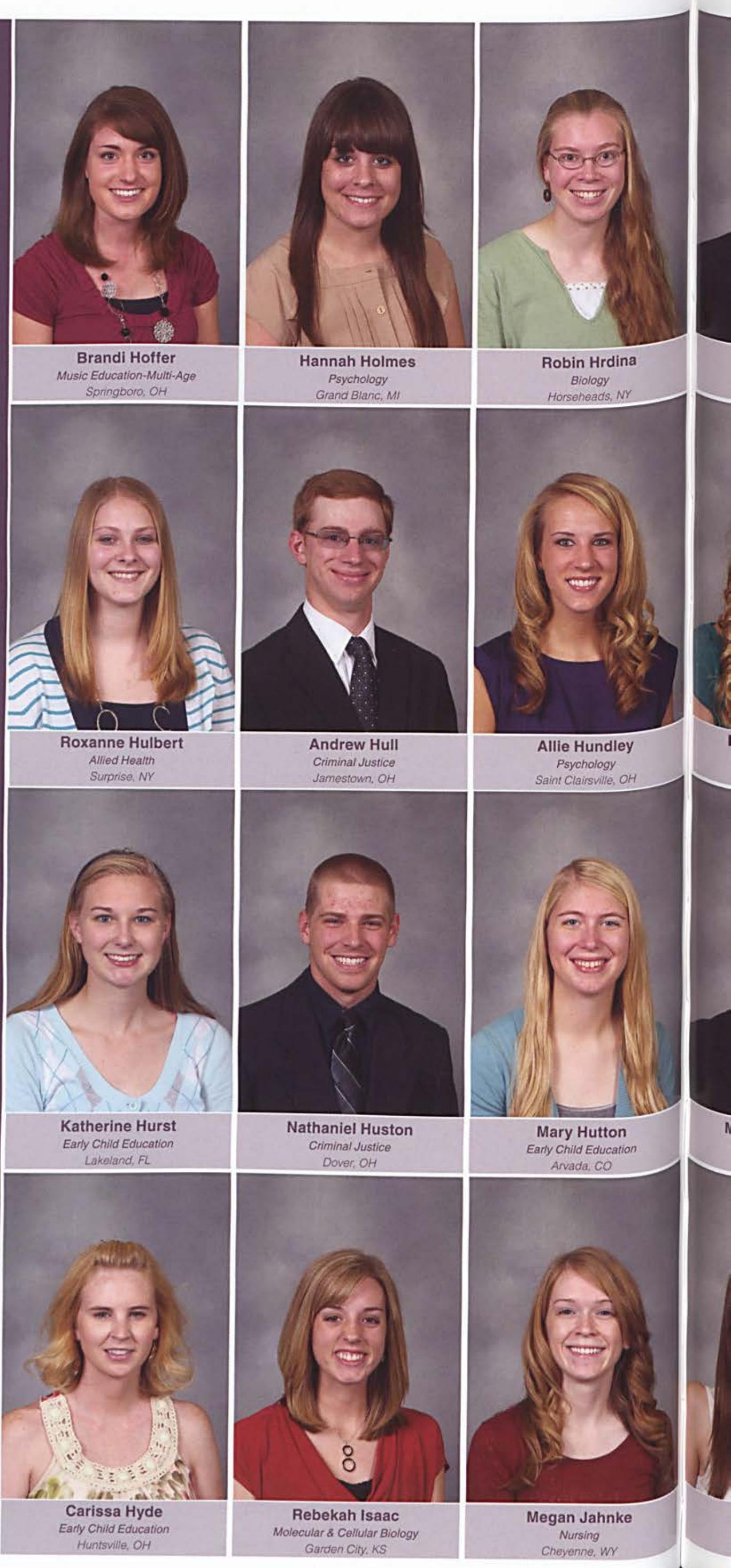




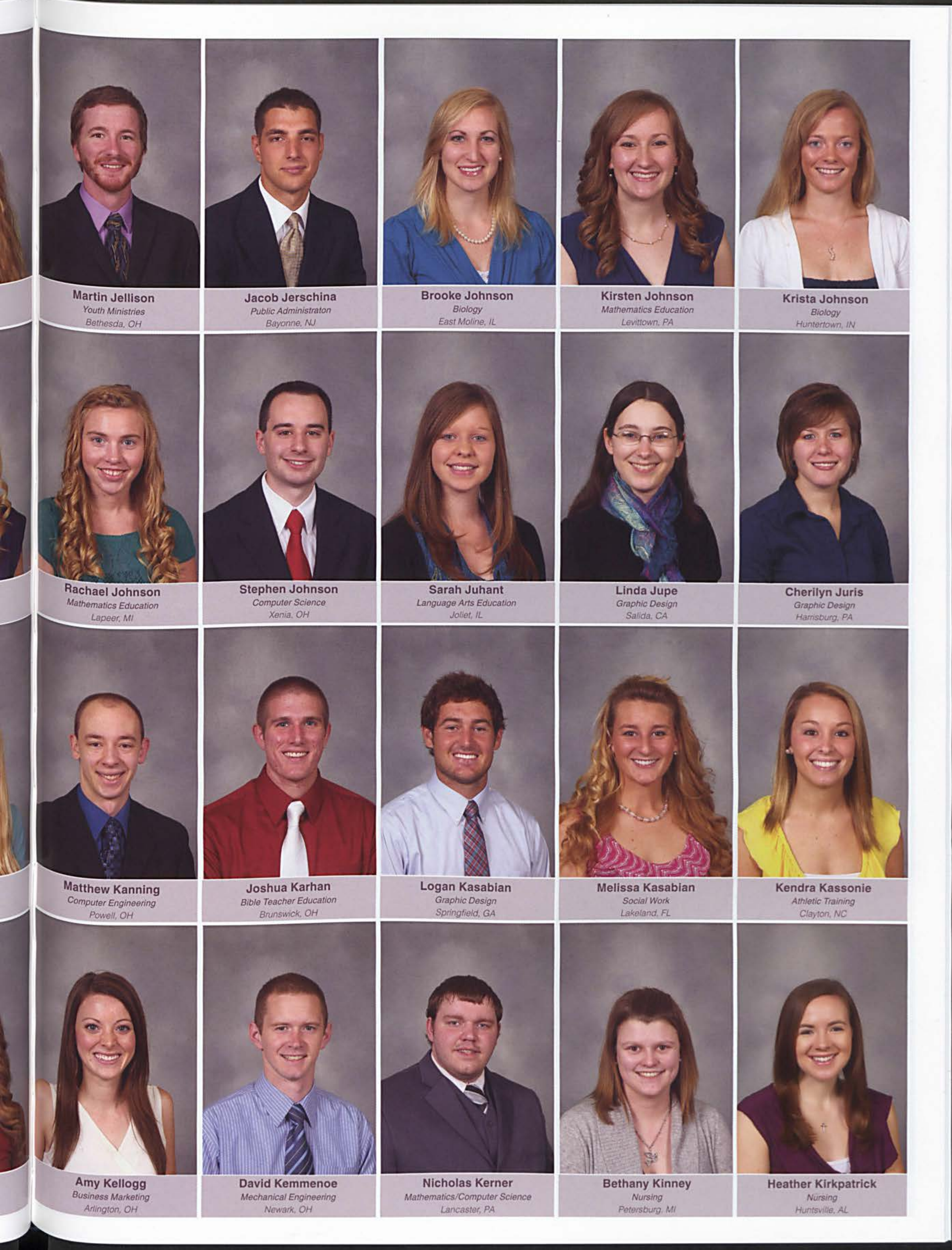




\section{SENIORS}

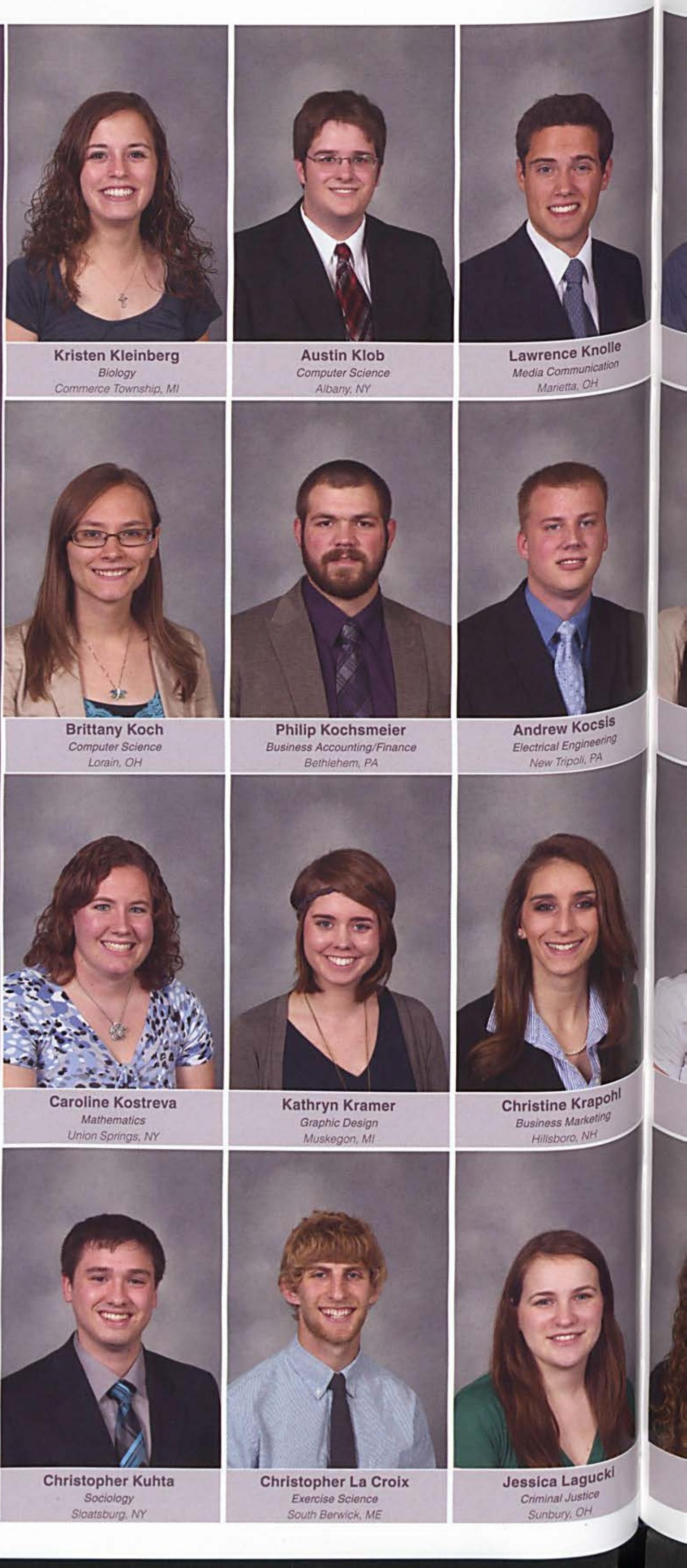




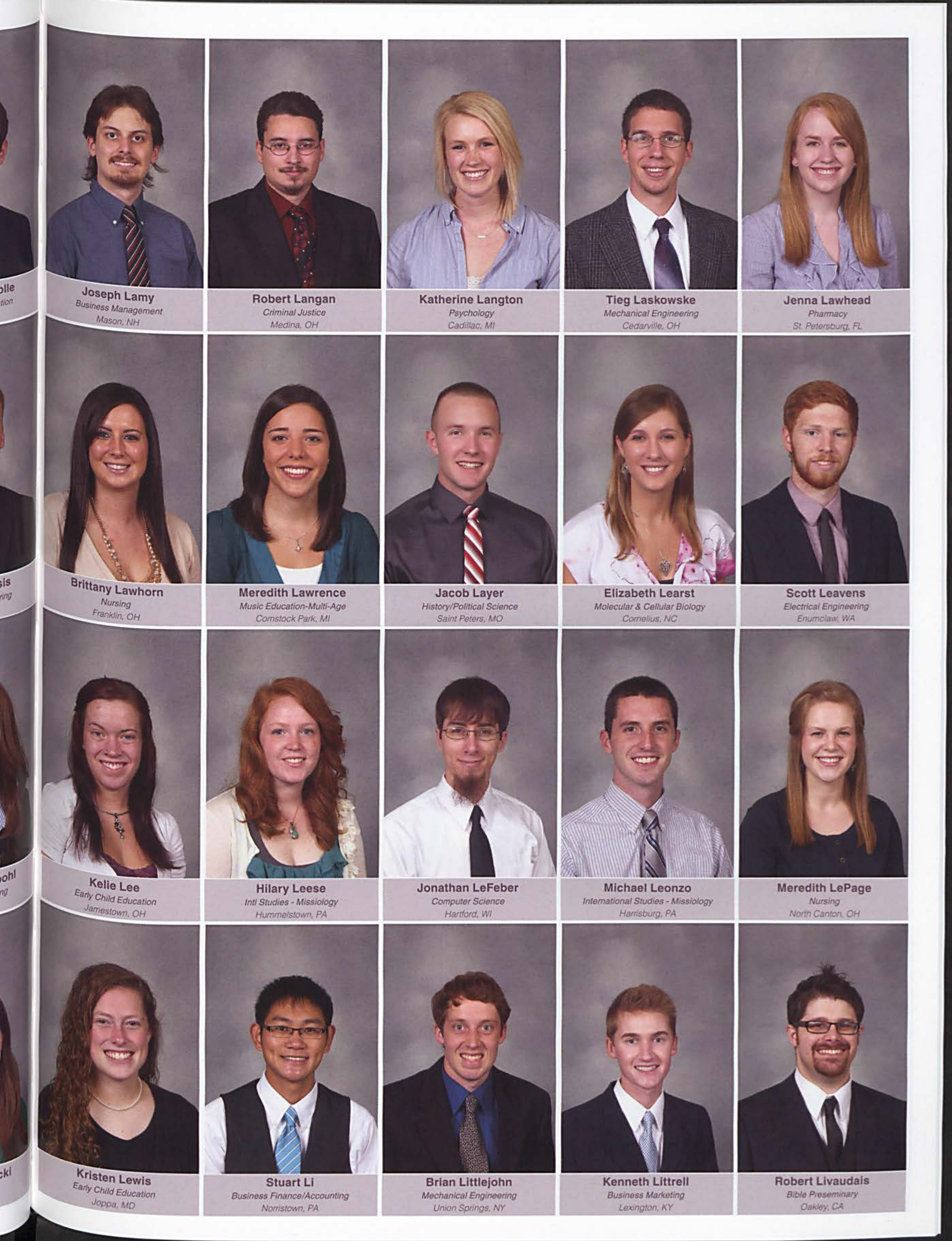




\section{SENIORS}

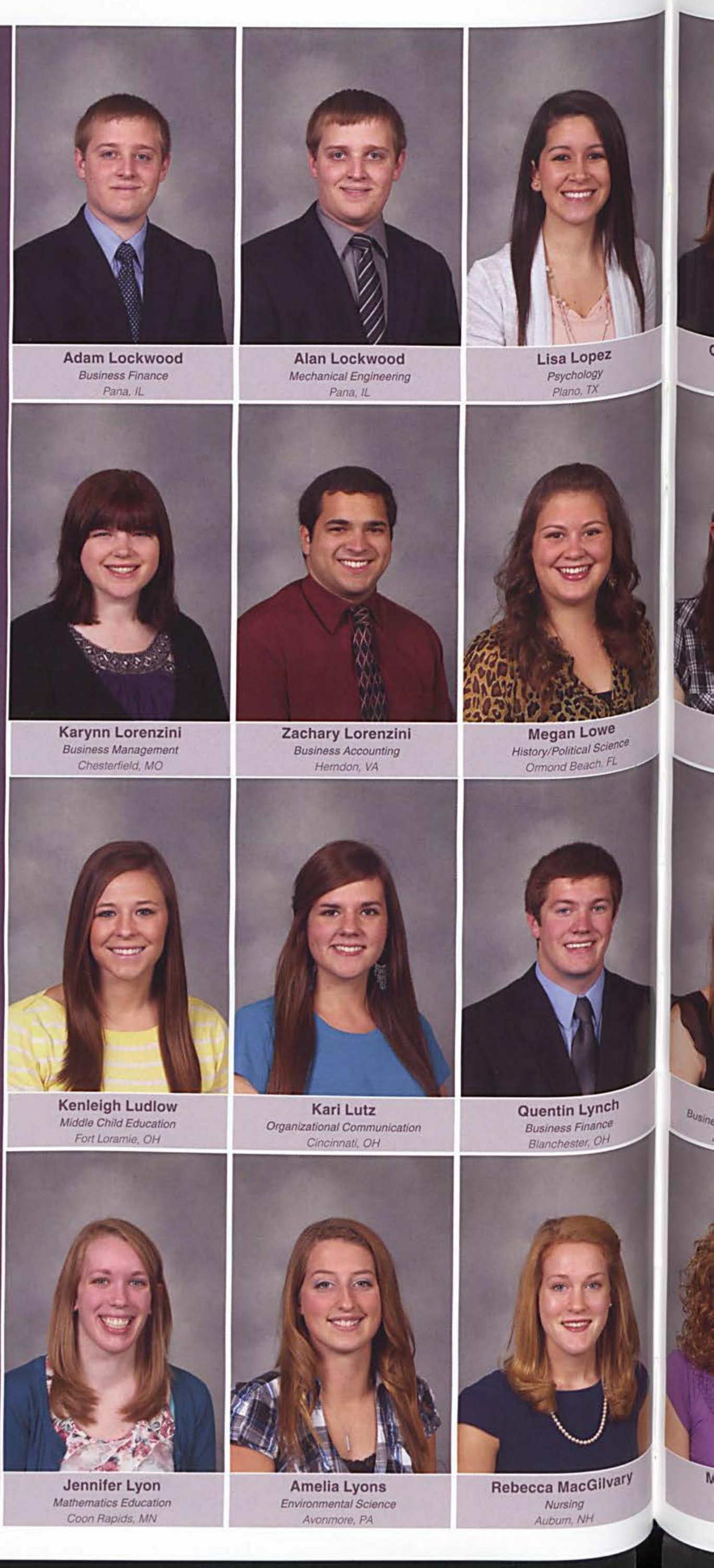




\section{SENIORS}

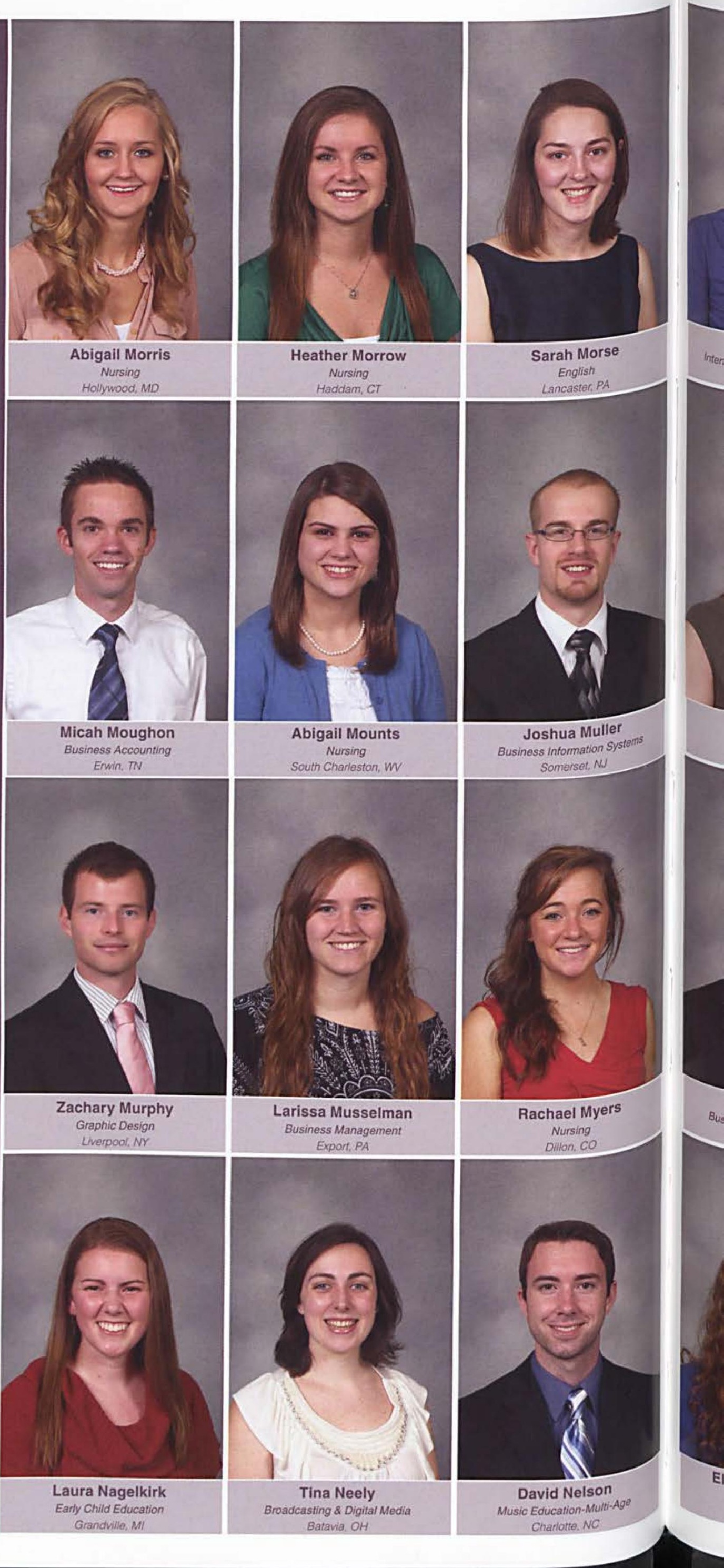




\section{SENIORS}

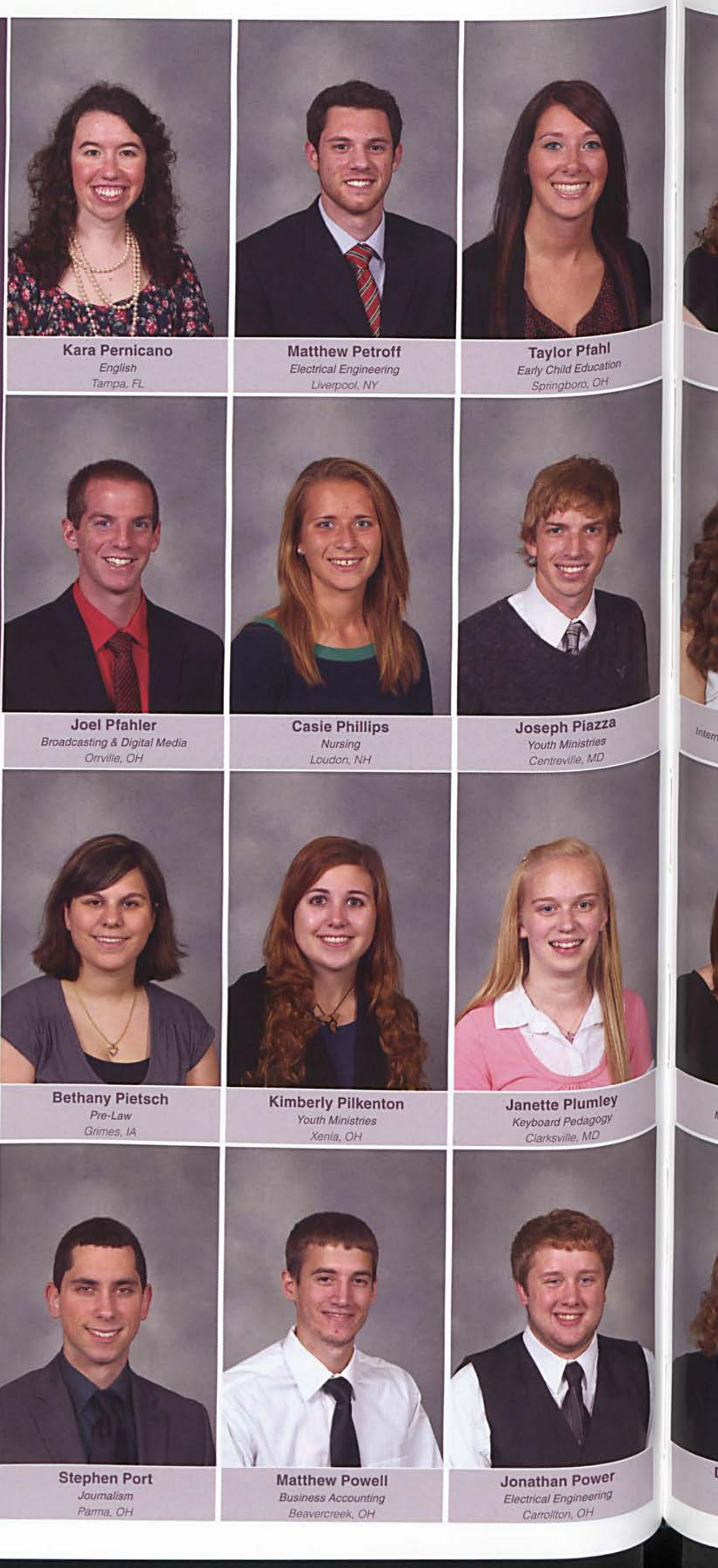




\section{SENIORS}

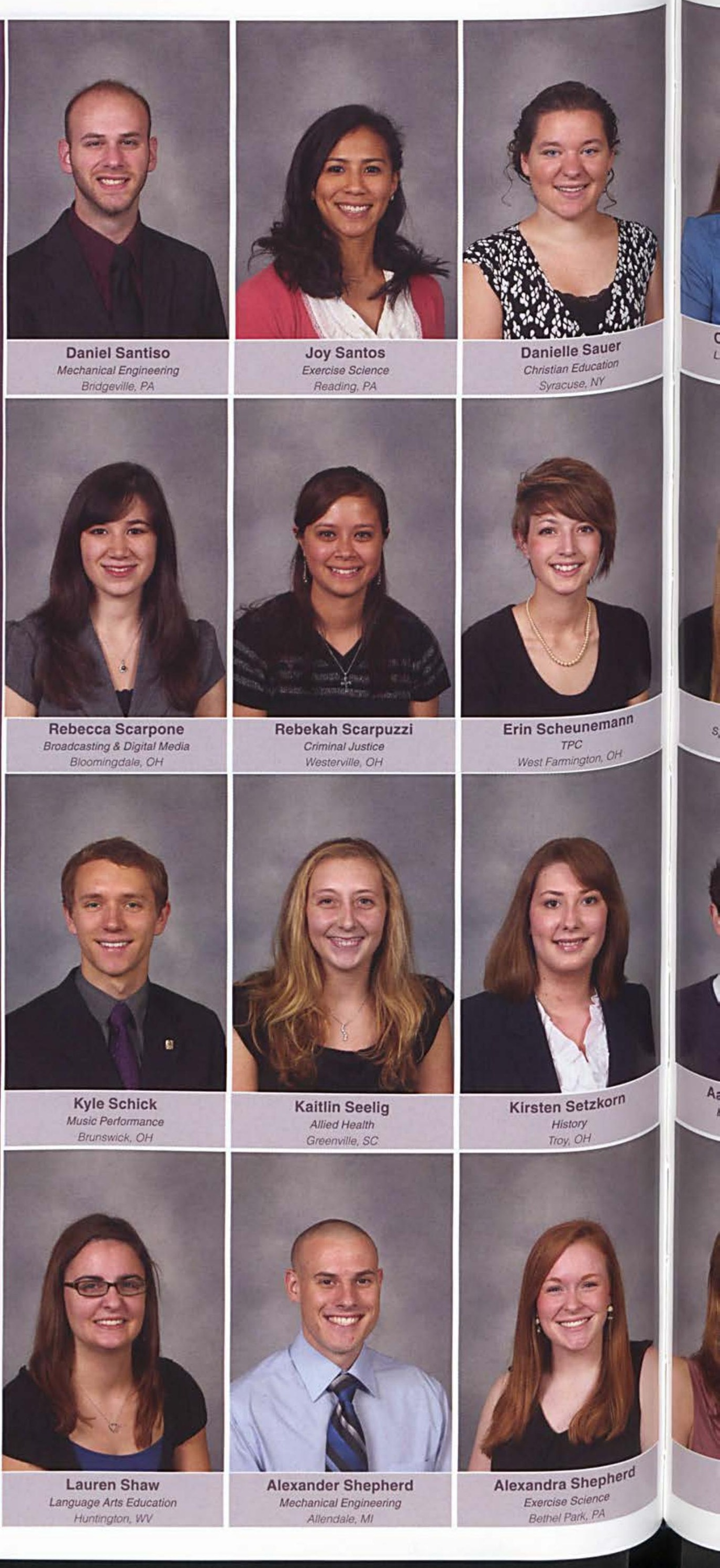



SENIORS

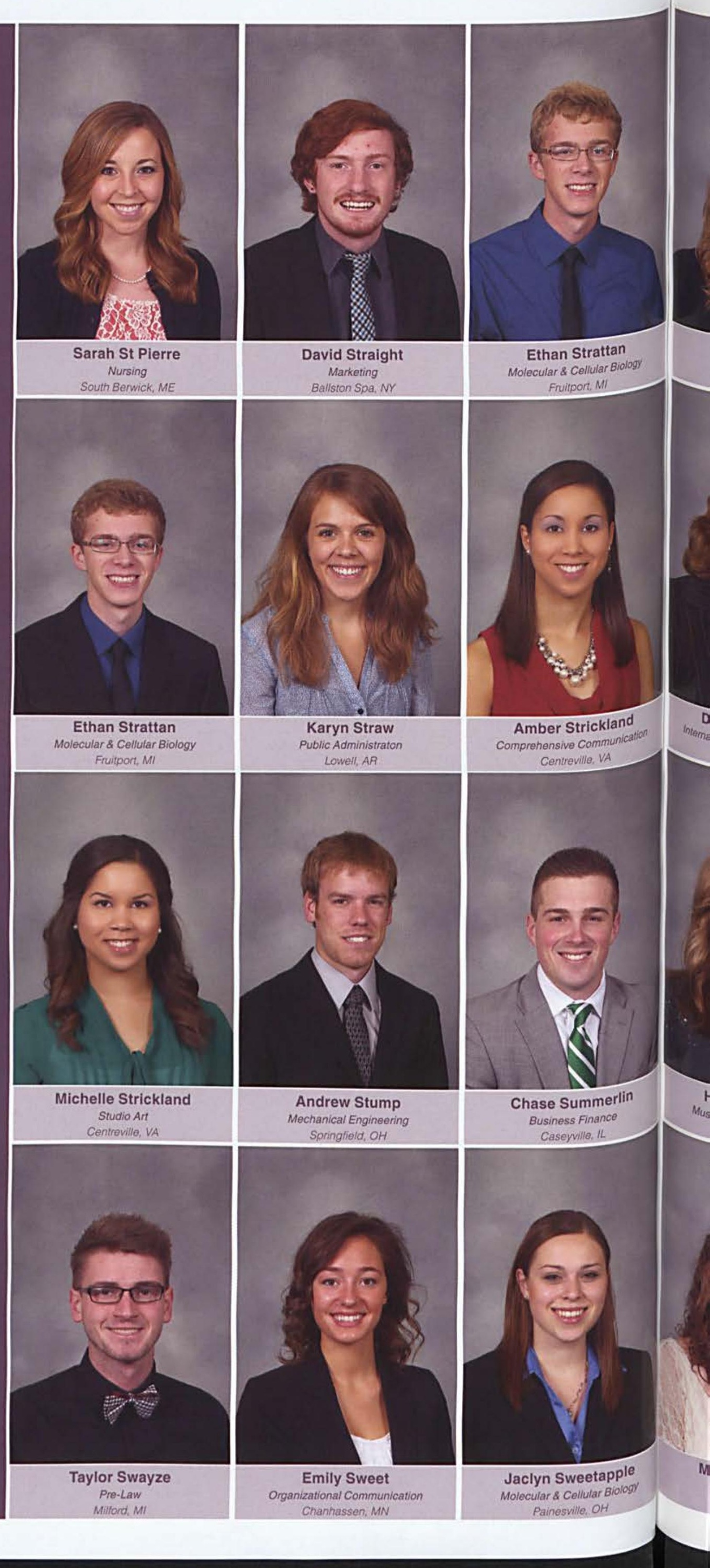




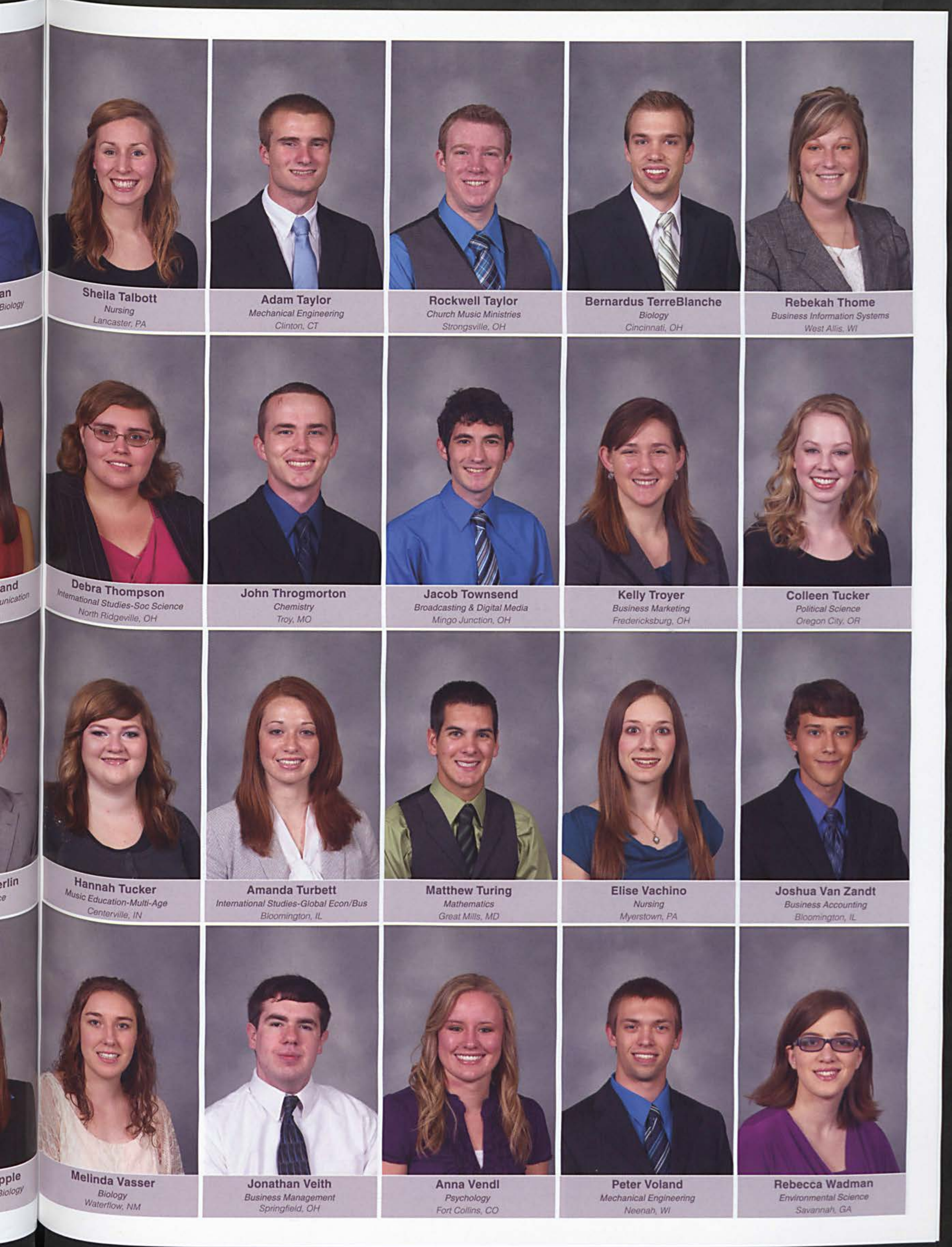




\section{SENIORS}

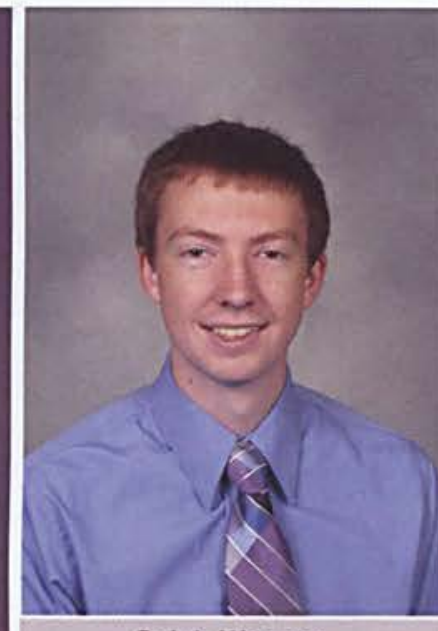

Caleb Wagner

Mechanical Engineering

Shippensburg, PA

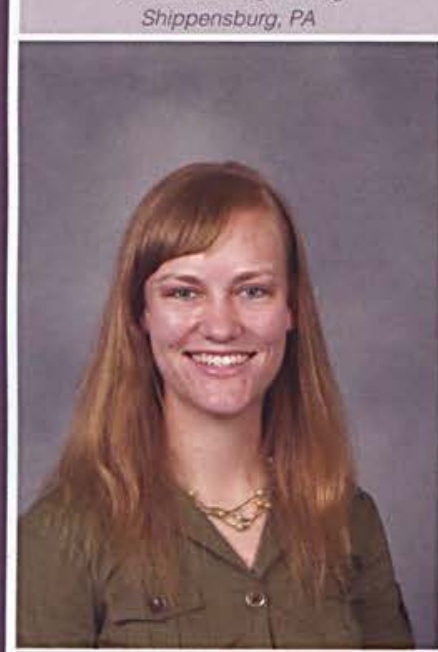

Rebekah Wallin

Criminal Justice

Torrance, CA

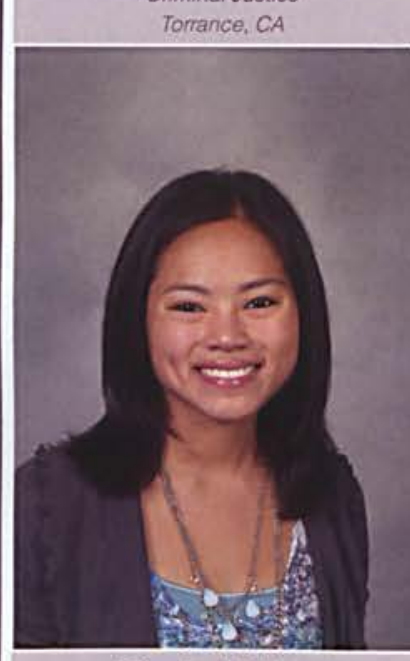

Elisabeth Ware

Nursing

Memimack, $\mathrm{NH}$

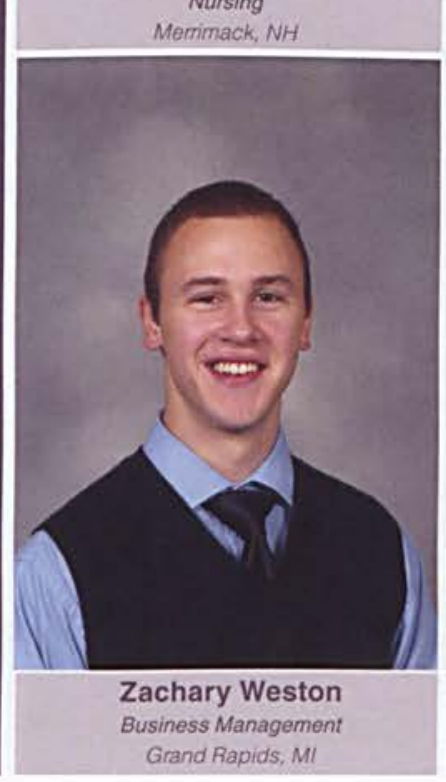

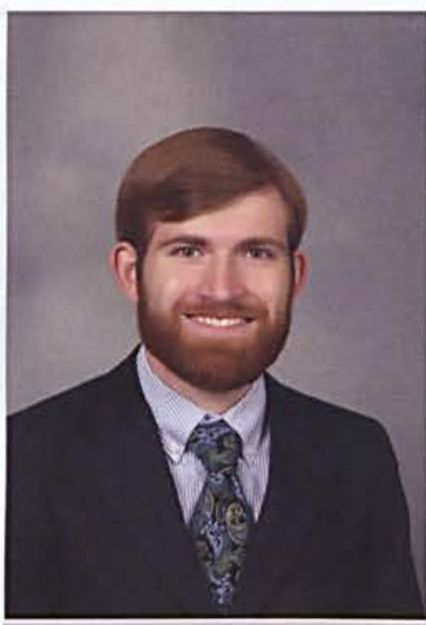

Ethan Walker

International Studies-Social Science Springfield, $\mathrm{OH}$

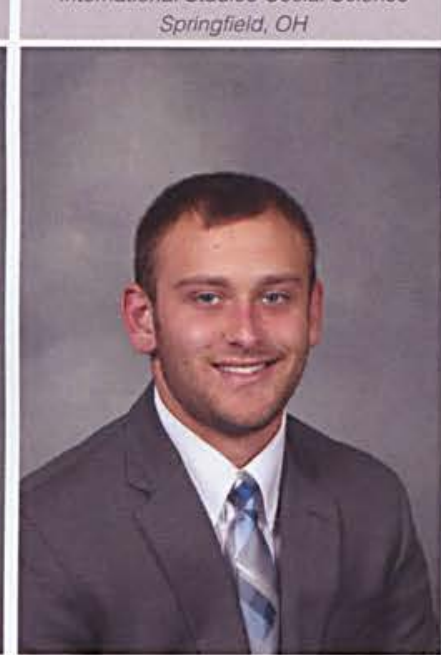

Christopher Ward

Business Finance

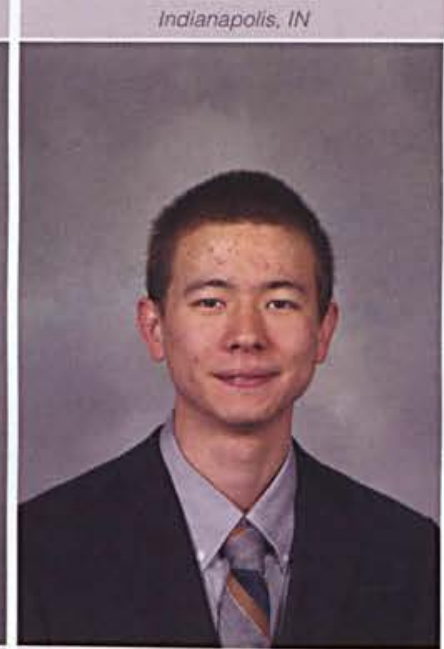

Erkai Watson

Mechanical Engineering

Cedarville, $\mathrm{OH}$

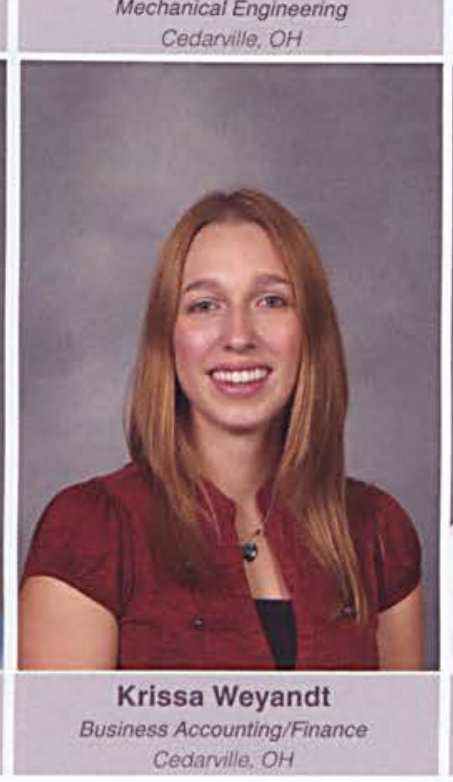

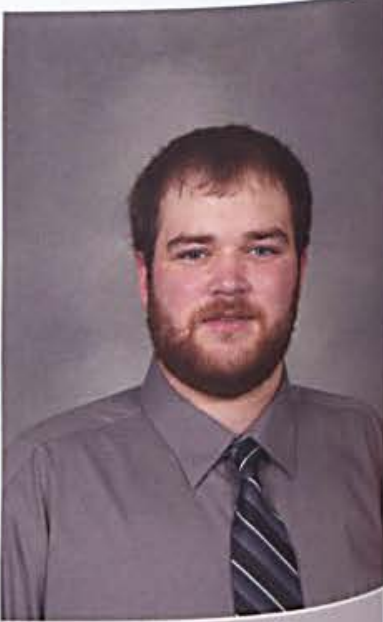

Joseph Wallin

Mechanical Engineering Mars Hili, NC

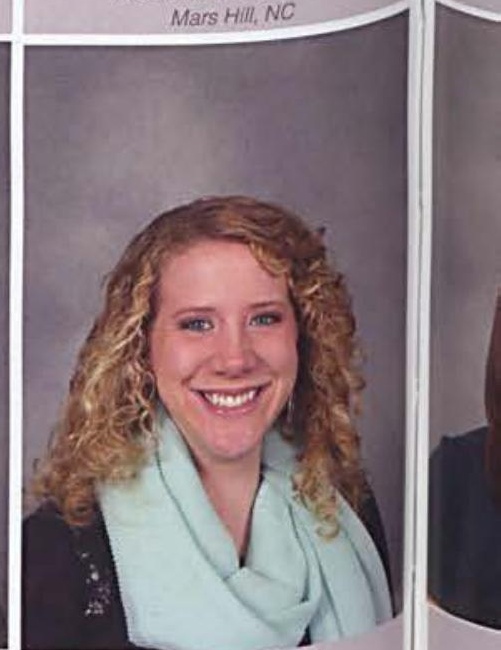

Jennifer Ward
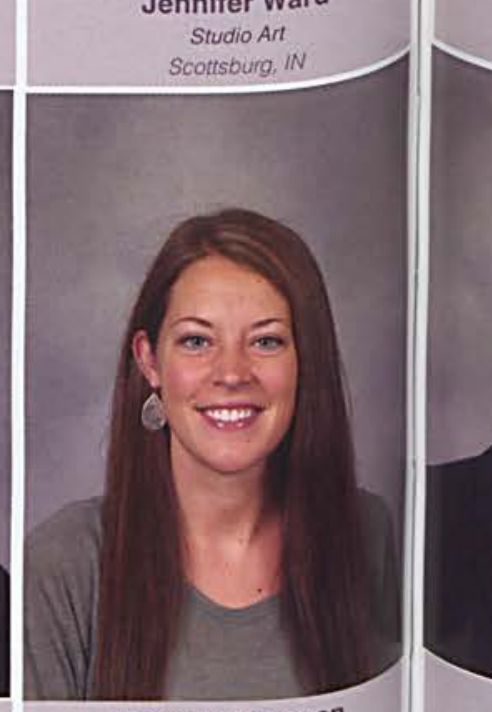

Hannah Wegman Intermational Studies-Social S Harrison, $\mathrm{OH}$

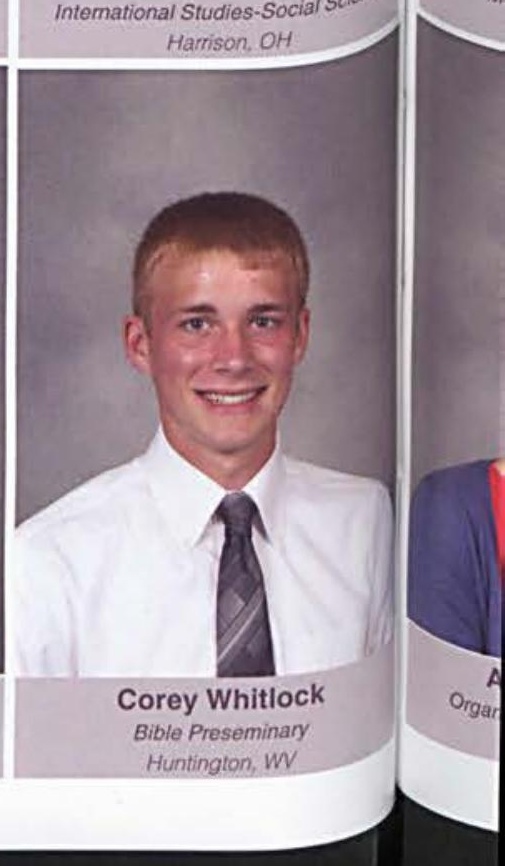





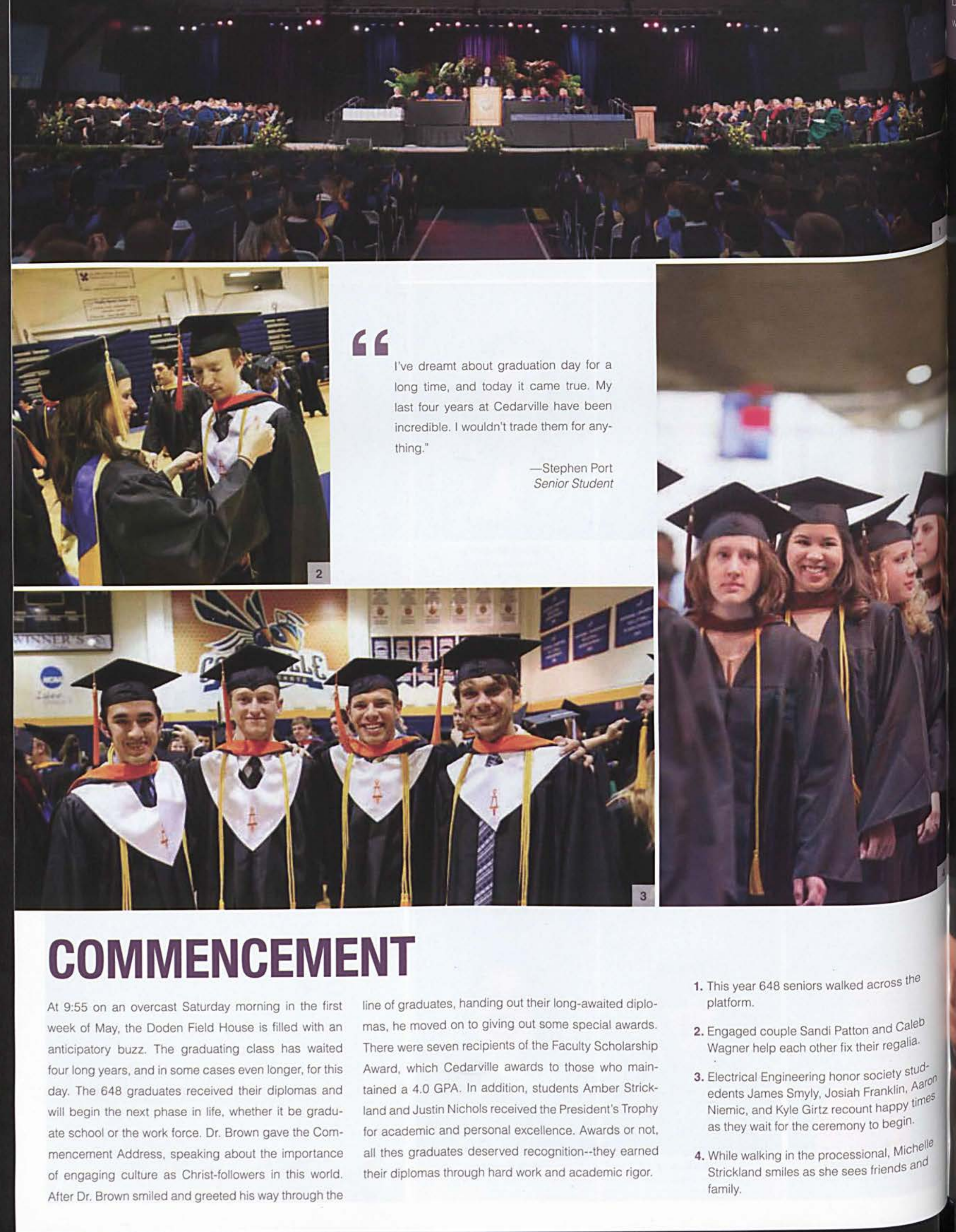




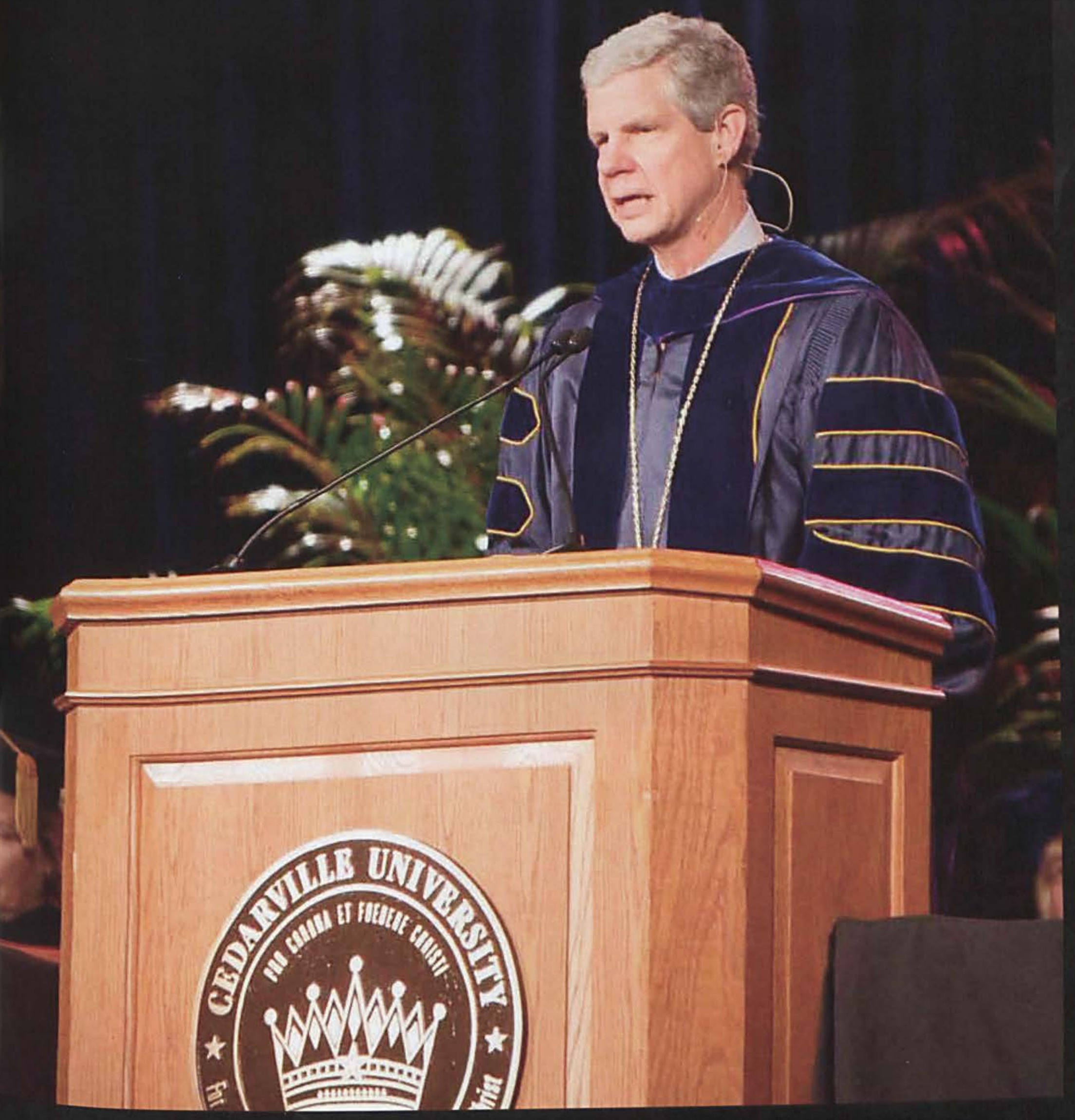




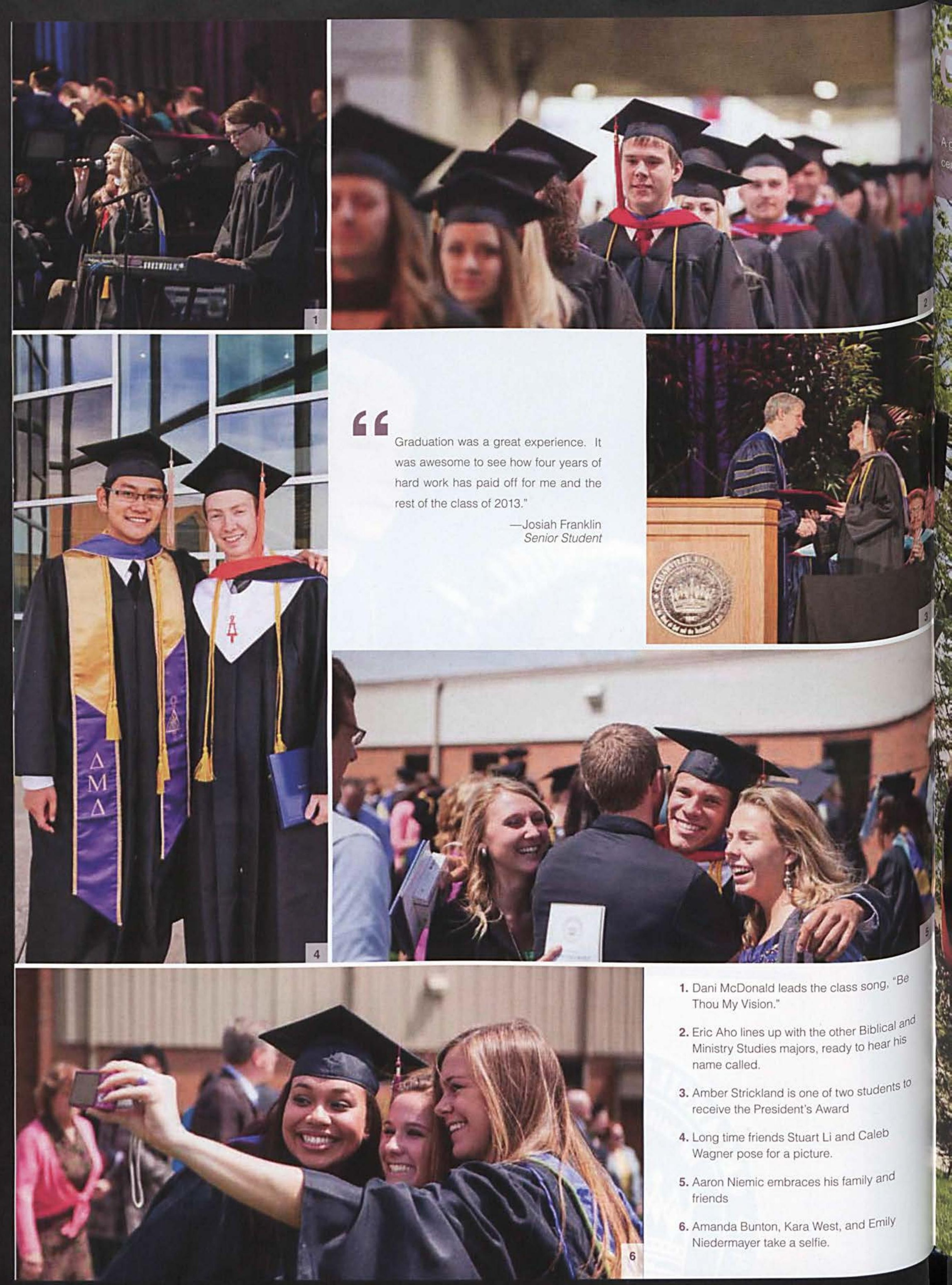




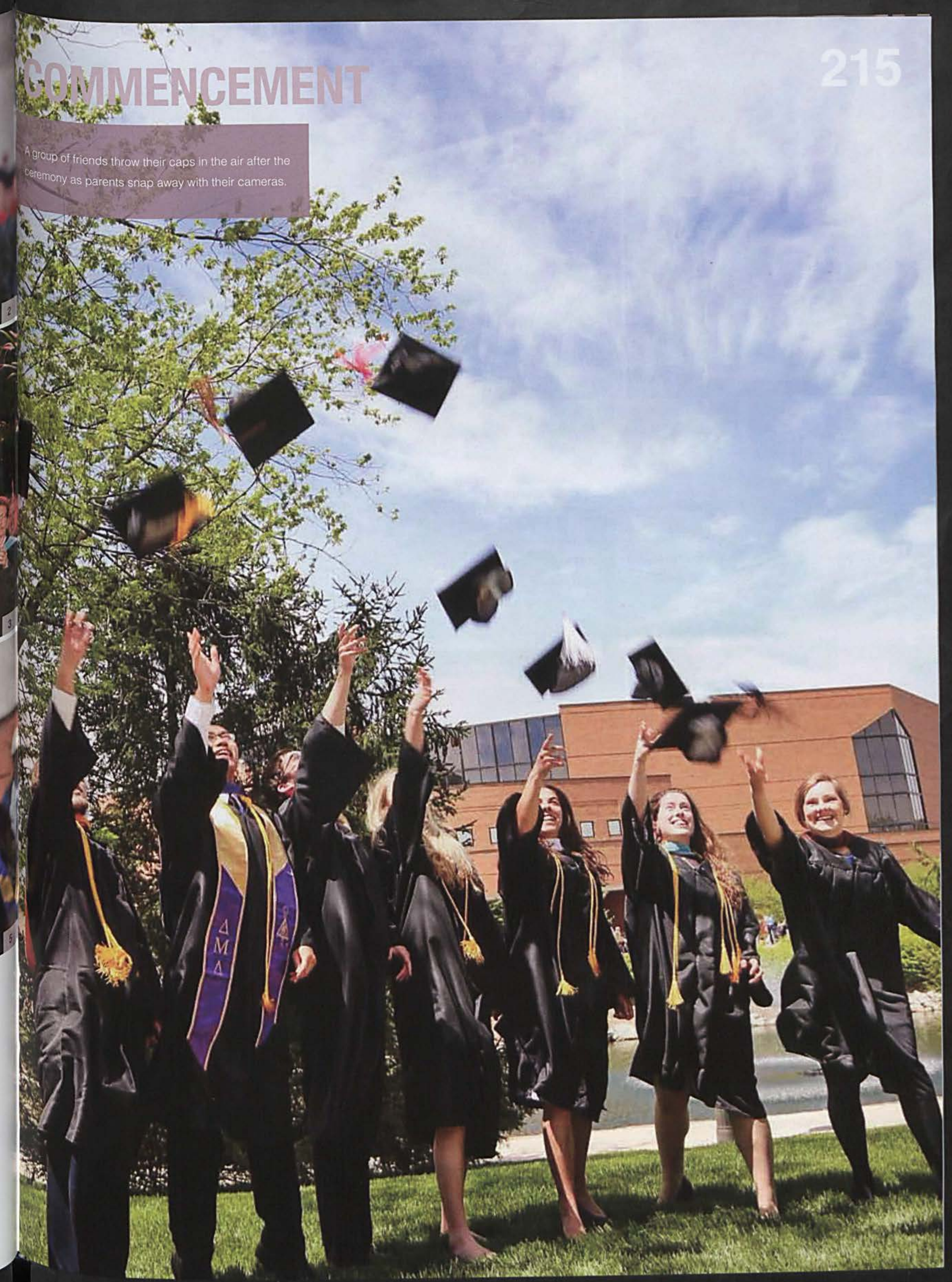




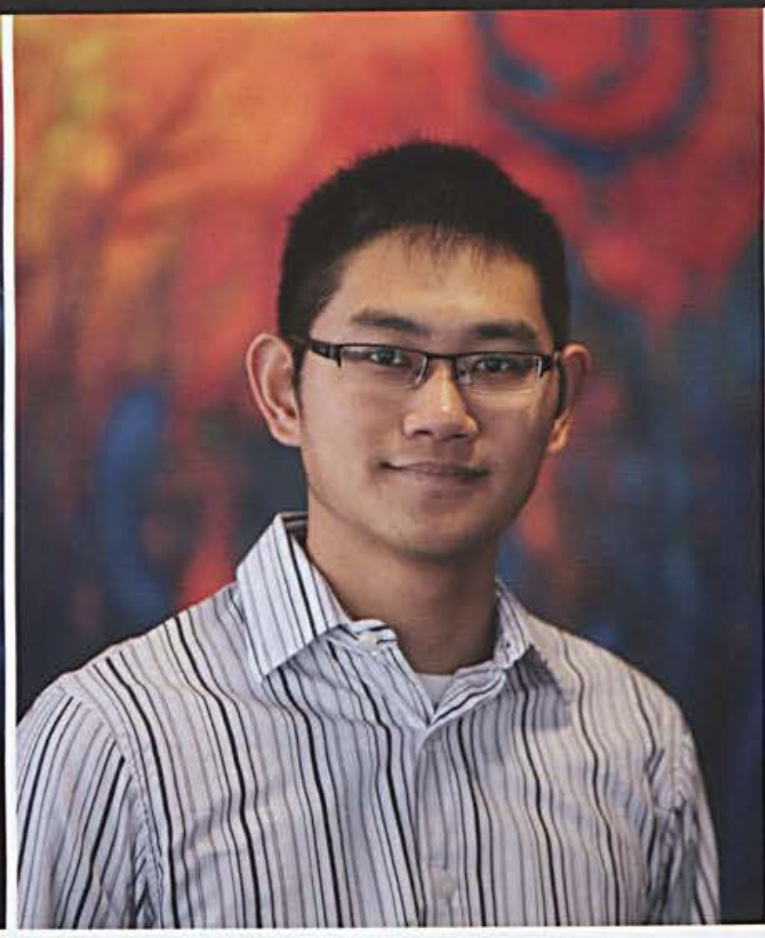

Cynthia Messer, Faculty Advisor

Cyndi is an associate professor of English and has been the faculty advisor for yearbook for thirteen years. She teaches composition, literature, grammar, and methods courses. She is the program coordinator for the AYALA major and enjoys working with future English teachers. She also enjoys spending time with her three children and husband of eighteen years.

\section{Stuart Li, Editor in Chief}

Stuart is a Senior Accounting Finance major from Philadelphia, PA. He has been working with photography on Miracle Yearbook for two years. This year he took on the challenge of being Editorin-Chief. Stuart will be going to Nicaragua as a business consultant in the education sector after graduation.

www.facebook.com/stuartandphotography
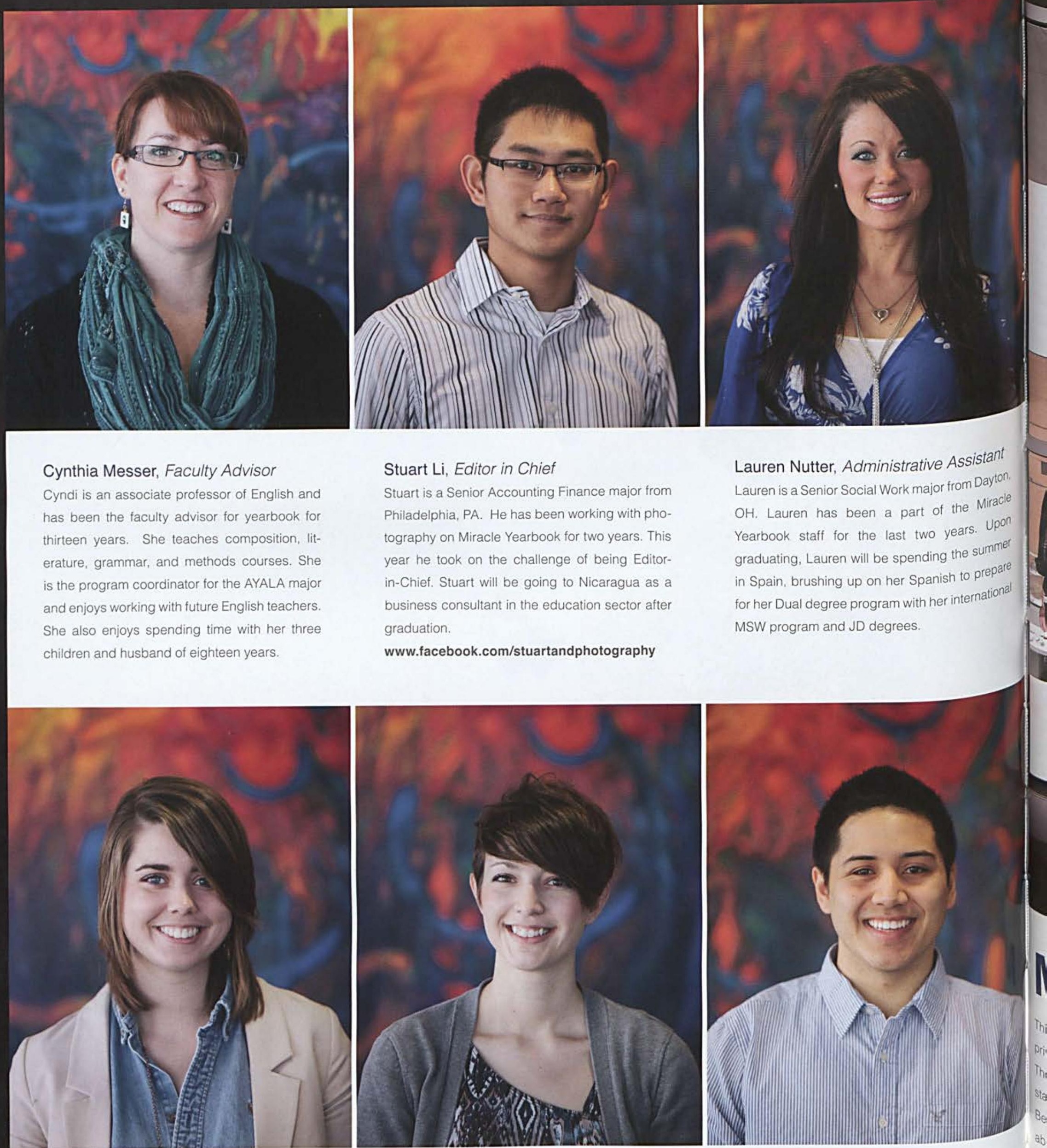

Lauren Nutter, Administrative Assistant Lauren is a Senior Social Work major from Dayton. $\mathrm{OH}$. Lauren has been a part of the Miracle Yearbook staff for the last two years. Upon graduating, Lauren will be spending the summer in Spain, brushing up on her Spanish to prepare for her Dual degree program with her international MSW program and JD degrees.

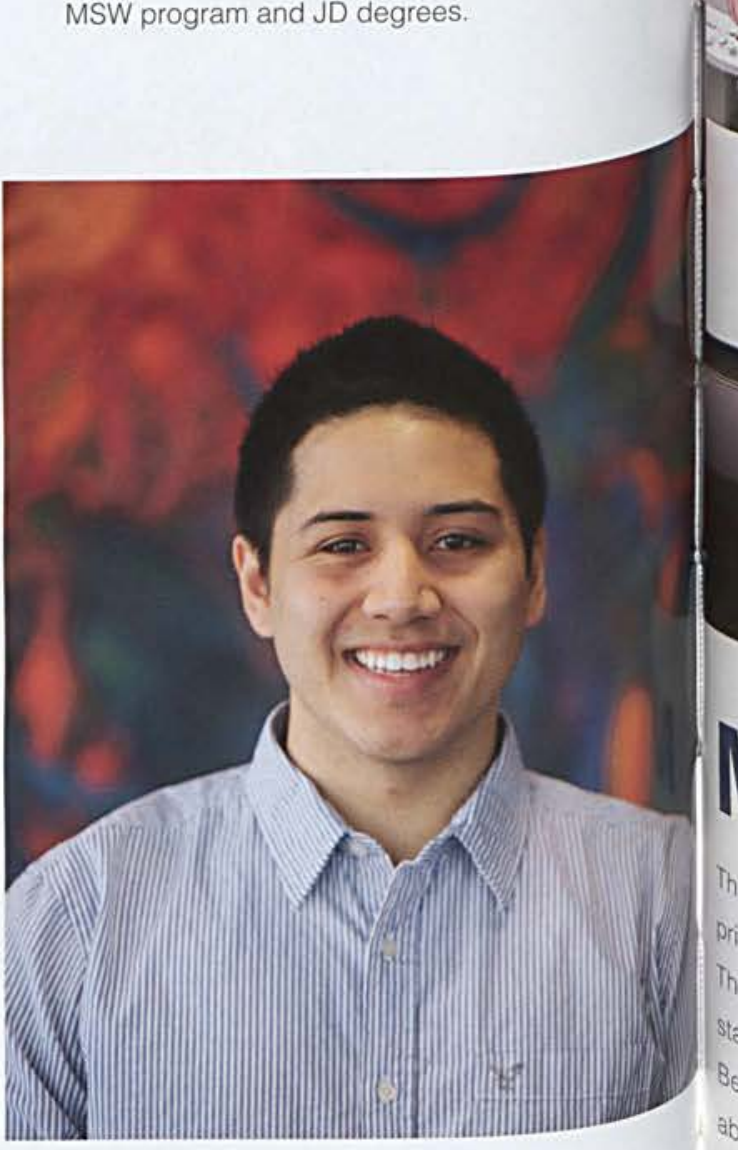

Kathryn Kramer, Design Editor

Erin Scheunemann, Copy Editor

Erin is a senior technical and professional communication major. This was her first year as copyeditor, and she enjoyed writing the stories as well as getting to know the other staff members throughout the year. After graduation, she plans to work as a graphic or marketing designer.
Alex Xayathone, Photography Editor Alex is a sophomore international studies major from South Charleston, $\mathrm{OH}$. He has been a part of Miracle for the past two years and will con tinue his same position next year. He would like to thank his photographers for working so hard the throughout the year.

www.flickr.com/axayathone 

A

Acker, Elizabeth.

Adams, Jordan

Adams, Kalina.

Adams, Mitchell.

Adel, Tehseen.

Adler, Jonathan...

Adriansen, Daniel

Ahlgrim, Brianna

Aho, Eric.

Akhnoukh, Dalia.

Alderink, Jessica

Alexander, Christian.

Alexander, Katherine.

Alexander, Nicole.

Alford, Aaron.

Allen, Jonathan.

Alley, Caleb.

Ames, Robert

Amick, Lauren

Amling Malia.

Ammon, Jordan

Ancona, Alexis.

Anderson, Arne

Anderson, Calvin.

Anderson, Claire.

Anderson, Kelli.

Anderson, Madelyn...

Anderson, Megan.

Anderson, Sarah.

Andrews, Daniel.

Andrews, Rebekah.

Ansiel, Heidi

Ansiel, Hillary.

Antoun Jacob.

Antrican, Derek

Arbogast, Shannon.

Archambault, Alexandra 31,66,175

Armstrong, Samuel.......... 104, 106, 148

Arnott, Keriann.

Arslain, Monica

66. 183

Atkins, Jamie

Auch, Ashley.

Austin, Joseph

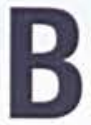

Bach, Moriah.

Baglio, Anthony

Bailey, Jerry. 66,133

Bailey, Morgan

Boker. Adam

.66

Baker, Collin

Baker, Garrett.

Baker, Jocelyn.

Baker, Jonnah.

Ballitch, Brian.

Banachowski, Jasmin.

Bancroft, David.

Banz, David.

Barber, Daniel.

Barfell, Kaitlyn.

Barkas, Julianna

Barker, Heather

Barkley, Alyssa.

Barkoviak, Aleska

Barlow, Jonathan.

Barnes, Brianne.

Barnett, Adrienne

Barnett, Witness

Barnhart, Steven

Baron, Daniel.

Baroni, Alexis

Bartholomew, Erica.

Barton, David.

Barulich, Anna

Batts, Christopher

Baver, Matthew

Baumann, Amelia.

Baumann, Clara.

Baumer, Jeanetta.

Beach, Brittany.

Beach, Michael.

Bearden, Ransom.

Becker, Meghan

Beckler, Joshua.

Becton, Austin.

Behrke, John

Bell, Amanda

Bellanti, Jordan..

Belschner, Matthew

Belton, Rebecca

Benitez, Olivia

Benjamin Carlson.

Bennett, Alysia..

Bennett, Elizabeth.

Bennett, Gregory.

Bennett, Joel.

Bercaw, Alissa

Bergsma, Emilie.

Bernecker, Mary.

Bernstein, Megan.

Berry, Reena..

Bertolino, Nicole.

Bewley, Mary Beth.

Bewley, Molly.

Bewsey, Amber

Biddinger, Ryan.

Biddinger, Stefan.

Bieniek, David.

Biggs, Kyle..

Bigler, Alison

Bisbee, Lauren.
186 Bissonette, Sydney

.181 Blackburn, Hayley.

66

148

186

31,175

66. 181

148

148

186

148

.16

27

66

66
.25

25

108
66

148

148

.66

7., 186

176, 177

148

8,20

108

66

.187

90,187

. .108

148

66

108

187

67

67

8

08

133

133
187
67

67
188

49

187

67

108

67

108

187

148

187

108

67
187

87

108

04, 106

104,106

67

67

27.108

. .148

Blackwell, James

Blair, Meredith..

Blanchard, Christa.

Blocksom, Irene...

Bode, Leah Rachel.

Boehning, Brianna.

Bok, Jihan.

Bollman, Jeremiah.

Bond, Kathleen

Bond, Nathaniel...

Bonifas, Jonathan

Bonnevie, Abigail.

Bouws, Todd

Boward, Alicia.

Boward, Victoria

Bowker, Erin...

Bowser, William.

Boyd, Marie

Bradshaw, Deanne

Bragg, Josiah

Brammer, Joy,

Brant, Micah.

Brett, Chelsea

Brewer, Megan.

Brewer, Rebekah

Bricker, Kailey

Brittan, Michael.

Brittenham, Kaysie.

Broad, Emily.

Broberg, Kenneth

Brock, Bethany

Brooker, Hannah.

Brooker, Matthew

Brown, Amy..

Brown, Anna

Brown, Austin.

Brown, Hannah

Brown Mackenzie

Browning, Colleen.

Brubaker, Sergei

Bruckner, Meghan

Brummel, Julia

Bruning, Madison.

Buck, Megan...

Budds, Jennifer.

Buettell, Stephen.

Buice, Nicole

Buice, Shawn

Bull, Rachel.

Bullis, Anna

Bullis, Emalyn.

Bullis, Luke...

Bundenthal, Landon.

Bundy, Andrew.

Bunton, Amanda.

Burkholder, Lindsay.

Burkholder, Mary...
Bouchard, Stephanie

Brumbaugh, Rebecca

$28,29,173,187$

.108

108

$23,129,187$

. .148

.187

67

. .148

131

187

187

. .148

175

\section{7}

188

108

.67

.67

.129

179

73

173

67

.67

67

108

148

188

. .131

108

188

183

67

144

67

173

08,118

148

Burnison, Melinda

Burns, Christina.

Burrell, Nathaniel.

Burton, Rachel 
Choi, Ruth

Cristiansen, Julie

108 Gristiansen, Kelsey

23 Oristini, Carolyn.

Orystal Renae.

8 Curch, Abraham.

- Iccarello. Anthony

Cimo, Dylan...

Clark, Kayleigh.

Cark, Nicholas

9 lark, Stephen

Coson Natalie.

Cossen, Kelly

Cossen, Kyle

-emens, Karissa

Cemons, Josiah..

Cifinger, Rachel

intord, Sebastian

Ane, Emily.

13 Cochran, Hannah

88 Coffman. Megan.

$5 \mathrm{Co}$, Adam.

88 Coman, Alyssa

Collins, Rachel.

Compagnone, Joseph.

Omparetto, Michael

88 Cook, Patrick.

9 Coley, Alex

49 Copola Joshua

6) araccio, Johnathan

181 Gocoran, Alicia.

9 ordle. Austin

orlito-Morell, Joseph

of Curtney, Megan...

Ouser, Victoria

Cor F, Brittany

ox Jerilyn.

abtree, Jillian

aig. Bayley.

aig. Chelsea..

awley, Aaron

Owley, Stephen

riswell, Emily.

Fizer, Emily...

foll. Maraus

omwell, Andrew

ouch, Toni.

ise, Bethany

Mise, Brandon

upi, Stephen

utiee, Jason.

Up, Ethan

Cuver, Emily.

Umberland Caleb

ummings, Laura.

undall, Casey

urby, Cassie

irby, Charissa

urby, Levi

ran, Rebecca.

189
.$\quad 189$
.$\quad 171$
.179

190

68

.190

.68

68

109

68

109

25. 139

.149

190

109

149

68

109

190

68

.68

69

109

90

109
Curtis, Austin

Curtis, Zachary

Curwick, Elizabeth

Custer, Amanda

Custer, Gavin..

Cutting, Andrew

Cymbalak, Lindsey

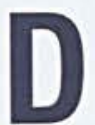

D'Amico, Daniel

D'Anna, Emily

Dahl, Amy.

Dalavai, Emilie.

Dallas, Samue

Dalton, Anna.

Dancer, Joshua.

Daniels, lan.

Daniels, Katherine

Danielson, Paul.

Dannelly, Philip.

Dark, Rachel.

Darkwah, Belinda

Darst, Casey

Darwin, Kyle.

David, Amanda

Davies, Gregory

Davis, Elizabeth.

Davis, Geoffrey.

Davis, Hannah.

Davis, Jill.

Davis, Nicole.

Davis, Samantha.

Davison, Joseph

Day, Megan...

Dayton, Heidi..

Dearing, Grayson.

Debartolo, Cecelia

Decker, Jacquelyn.

Defore, Stephanie.

DeHart, Paul

DeHart, Tabitha.

Deloye, Samuel.

Dembowski, Anna.

Deneseus, Nicole

Dennison, Joel..

DeRose, Rebecca

Detrick, Tyler

Devereaux, Brooke

DeVos, Gregory.

Devries, Ashley.

Dewhurst, Ethan

DeWitt, Brittany.

Diamond Chelsea

Dice, Joy.

Dick, Amy.

Dickert, Gregory
69

109

190

149

149

69

69

\section{(Din}

Dombrowski, David

Dossett, Elizabeth...

Douglas, Amy.

Douglas, Lydia.

Downer, Susanna.

190

Dremann, Sarah

Dresbach, Jordyn

Driggs, Janae...

Drum, Carissa.

Dubie, Jacob.

Duncan, William.

Dyksterhouse, David

Dykstra, Leanne.

Dyson, Jessica.

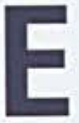

Earl, Jonathan.

Earwood, Bryan.

Ebersole, Stephen.

Eckberg, Justin...

Eckley, Andrew.

Edmonds, Corbin

Edwards, John...

Edwards, Nathan.

Eissler, Lauren

Elbert, Kellie.

Eldridge, Stephen

Eliopulos, Alexander

Ellis, Stephen.

Elwell, Hannah

Enderle, Caleb..

Engel, Justin...

English, Grant

Entner, Abigail

Entner, Kathryn.

Erickson, Brian.

Ericson Cole.

Eriksen, Michelle

Ervin, Clifton.

Erwin, Jenna.

Esbenshade, Alexander

20,23

Esbenshade, Allison.

Eustace, Victoria..

.179

Evanoff, Jessica.

Evanoff, Julia.

.110 Evans, Jennifer...

191

Ewart, Elizabeth.

.110

13,69

-.....191

22, 191

.191

149

34, 110

149

. .191

.69

.149

. .110

.149

.110

.69

191

.110

110

3 9 91 9

70

Fakhoury, Matthew 90,149

Farleman, Laura $\quad 110,174,175$

Farrell, Ryan.

150

Farris, Mark

.173, 192

Faulds. Christine 24, 25,110

Fawcett, Beniamin $\quad 70$

Faxon, Kylie. 25

Featherman. Melissa $\quad 192$

Febrizio, Luke $\quad 70$

Feltner, David $\quad 150$

Field Kathryn _ $\quad 70$

Figueredo, Christian $\quad 87,89,192$

Filcik, John_ 18, 177

Fillmore, Caitlin _ $\quad 70$

Finfrock, Jessica _. $\quad 70,179$

Finke, Anna $\quad 70$

Finlay, Ariel_ $\quad 192$

Finter, Rebecca $\quad 110$

Firebaugh. Stacia _. 192

Firmin Sarah $\quad 150$

Fisher, Melody 192 
Funk, Brian.

Funtik, John.

Funtik, Kyle.

Furrow, Leah

\section{G}

Gabel, Amanda

.110

Gahman, Brandon

Gale, Amie

Gall, Christina.

.. .193

Gallardo, Jonathan

Galloway, Keyana

Gambrell, Carl...

Gardiner, Shannah.

Gardner. Amber.

Gardner, Michael.

Gardner, Scott.

Garlick, Kimberly.

Garneau, Morgan

Garrett, Anna

Gates, Anna

Gates, Jordan.

Gatlin, Zachary.

Gauger, Natalie.

Geib, Carrie...

Gelwicks, Roger.

Gendron, Hannah.

Gendron, Rebecca.

Gentry, Kelsey.

George. Christine.

Gerber, Jared.

Gerber, Joella

Geringer, Keely...

Getchell, Emily

Getchell, Paige

Gill, Lauren...

Gillispie, Leah.

Gilmour, Connor.

Giraud, Nicole

Girtz, Kayla.

Girtz, Kyle.

Glandon, Megan

Glick, Jordan

Godbold, Eun Hyea.

Gonzalez, Alexander

Good, Kortney.

Goodliffe, Diane

Goodling Mitchel

Gordon, Deborah.

Gorog, Carolyn...

Gorrell, Rebecca

Grafton, Sharon.

Grahn, Daniel.

$. . .124,150$

23.71
Grant, Brian

Grapes, Madison

Gray, Cassandra.

Gray, Cora...

Gray, Maitland..

Gray, Nathan...

Graydon, Kristi.

Grayton, Katie

Grear, Danielle.

Greely, Myles.

Green, Bethany

Green, David

Green, Jenna

Greenbank, Gabriel.

Greene, Kyle

Greene, Margaret

Greenwood, Jonathan.

Gregg, Austin

Gregory, Carly.

Gregory, James

Gresh, Alexis.

Griesel, Carly

Griffin, Christopher

Griffith, Elizabeth

Grinalds, John

Grout, Matthew.

Gruber, David

Gualtieri, Daniel...

Guffey, Katherine

Guidone, David

Gullion, Morgan.

Gummel, Jonathan

Gunter, Katherine

Gustafson, Ryan

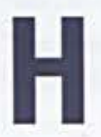

Haas, Shelby.

Haines, Lauren

Hale, Cori...

Hale, Jameson

Hall, William

Hallisy, Shannon

Halwes, Sarah.

Hammond, Emily

Hanavan, Kale.

Hanbury, Nathan.

Hands, Lisa.

Hankla, Rachel...

Hannay, Gregory.

Hanzel, Carlyn...

Harbeck, Sarah.

Hardman, Rebecca

Harkness, Aaron.

Harlos, Allison.

Harper, Shelby.
.129

...7

Harris, Hannah

Harris, Zachary

Hart, Madison

Hartman, Emily

Hartman, Steven

Hartung, David.

Hartzler, Melissa

Harvey, Claire

Harvey, Olivia

Haselton, Annelise

Haskins, Connor.

Havard, Katherine.

Hawkins, Brooke

Hayden, Josiah.

Hayes, Amanda

Hayes, Brittany.

Hayes, Christian.

Hayes, Kristen...

Haywood, David

Heathcote, George.

Hecker, Brian.

Hedges, Tessa

Hefner, Jennifer.

Heiden, Jonathan

Heiden, Molly

Hellwig, Caitlynn

Hellwig, Deric.

Helmbrecht, Megan

Helton, Tara

Henderson, Andrew

Henley, Susan.

Henry, Allison.

Her, Jonathan...

Herald, Cassidy

Herbert, Maryssa

Herman, Anna

Herrera, Miguel

Herrera, Rachel.

Hershberger, Danae

Herting, Andrew

Heuzey, Stephen

Heydt, Courtney

Hickinbotham, Lia

Hickman, Daniel.

Hicks, Courtney.

Hicks, Elizabeth.

8. 194

Hill, Colin.

Hills, Nathaniel

Hinckley, Samuel

Hines, Hannah.

Hines, Suzanne

Hinks, Robert

Hinman, Kathryn

Hise, Jessica.

Hodecker, Julia

Hoekman, Helen

Hof, Marina.

Hoffer, Brandi.

...111, 138

Hogan, Melisso

Holloway, Danielle

Holloway, lan.
195

23

158

150

....71

71

47, 111

...71

150

71

.71

71,175

151

71

195

.151

195

71

195

72

129, 195

.195

72

195

8, 111

...25, 195

...72. 120

.. .195

133

...II

151

195

140

195

179

.111

195

5. 195

72

195

195

179

72

129

2, 134

151

195

8

72

151

195

95

72

72

195

. .111

196

196

151

151

72

Holloway, Seth.

Holmes, Hannah

Holsclaw, Jared

Honeck, Melissa

Hoober, Eric.

Hooper, Grant.

Hopkins, Cassandra

Hoppe, Jeremy

Horne, William.

Hotchkiss, Bethany.

Hou, Freeman...

Houston, Rebekah

Howder, Benjamin.

Howland, Britney.

Howland, Caleb.

Howsden, Lauren

Hrdina, Robin

Huddle, Kelsey.

Huffman, Alexandra

Hughes, Laura...

Hughes, Nathanie

Hughes, Rebecca.

Huhta, Kendra.

Hulbert, Roxanne

Hull, Andrew.

Hull, Bethany

Hull, Chandler

Hull, Timothy

Hulsey, Lauren.

Hundley, Allie

Hunt, Sara.

Hunter, Cathryn

Hurley, Elise..

Hurst, Katherine.

Hurt, Tyler.

Hurton, Mary

Hurtubise, Anna

Huschilt, Joanna

Huskey, Zachary

Huston, Nathaniel

Hutchings, Josiah.

Hutchison, Jennifer...

Hutton, Mary...

Huynh, Samuel.

Huyser, Stephanie

Hyde, Carissa.

Hykes, Peter.

Imhof, Jacob. 


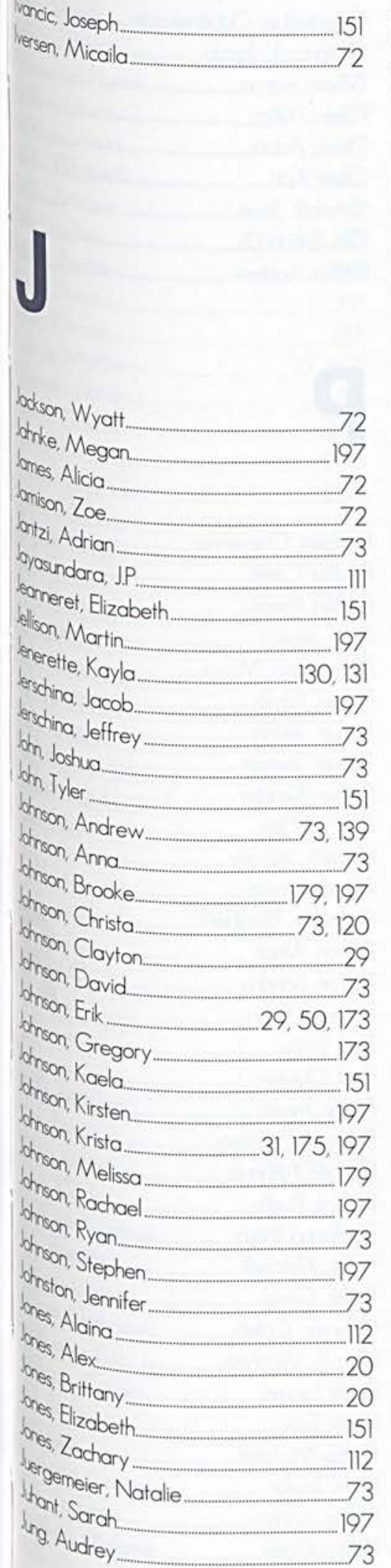

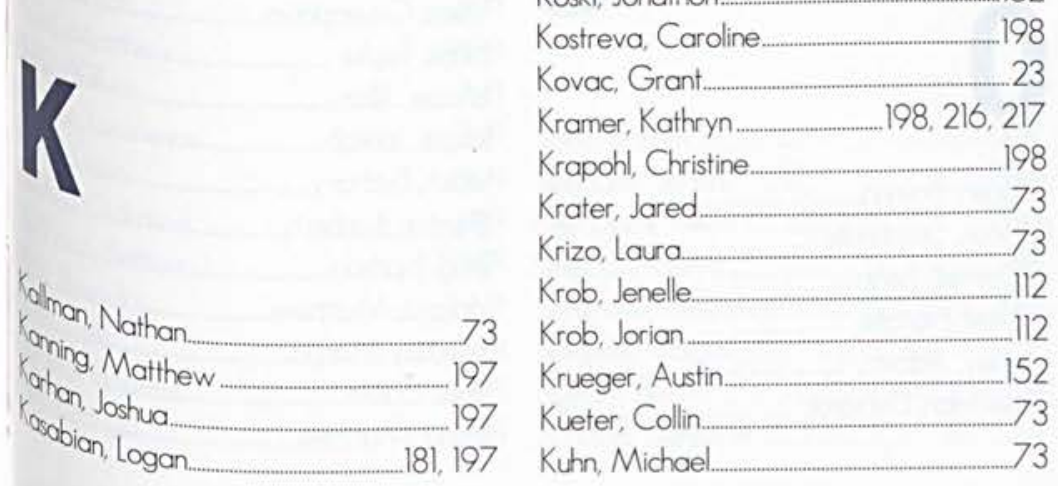

....73, 122

Kassonie, Kendra

Kearney, Jonathan.

. .112

Kee, Kevin.

Kellogg, Amy

Kelly, Ashlynn

Kelly, Caleb.

Kemmenoe, David _.......... 197

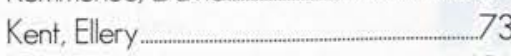

Kerner, Nicholas........______.... 198

Kester, Angelica.

Keur, Jonathan

Keys, Kristen.

Kidd, Kathleen.

Kilgore, Nathan

Kim, Myeongseop.

King, Brett.

King Colton.

King, Dillon

Kinnett, Rachel.

Kinney, Bethany

Kinsinger, Ella

Kirk, Valerie..

Kirkpatrick, Heather

Kirschbaum, Joshua.

Kisha, Michelle.

Kistler, Ericka.

Kitchen, Hayley.

Kitzmiller, Chase.

Klatt, Cierra

Kleckner, Ayana.

Kleinberg, Kristen

Klinger, Neil.

$\mathrm{Klob}$, Austin.

Klodnicki, Laura.

Kneeland, Stephen.

Knepel, Jennifer.

Knepper, Karis...

Knolle, Lawrence.

Knowland, John.

Knowland, Meredith

Koch, Brittany

Koch, Lauren...

Kochsmeier, Philip

Kocsis, Andrew

Kohl, Daniel

Kohl, Kathryn.

Kolota, Paulene

Koski, Jonathon

.73

$112,131,175$
Kuhta, Christopher

Kuiken, Christine.

Kuo, Matthew

La Croix, Christopher.

Laaman, Kristen. $31,74,175$

Lagucki, Jessica.

Laird. Ashley.

Lamy, Joseph..

Landis, Amber

Lang, Nicholas

Lang, Steven.

Langan, Robert.

Langton, Katherine

Larkin, Daniel

Larkin, Sean

Larned, Alana

Larrabee, Allison

Larson, Peter.

Laskowske, Tieg.

Law, David ...

Lawhead, Jenna

Lawhorn, Brittany.

Lawrence, Meredith

Lawson, Rebecca

Layer, Jacob.

Learst, Elizabeth

Leavens, Scott

Leber, Emily

Lee, Ethan.

Lee, Jennifer

Lee, Jordan

Lee, Kelie.

Lee, Matthew

Leese, Hilary.

LeFeber, Jonathan

Lehman, Michayla

Leigh, Jason

LeMaster, Samuel

Lenarski, Amanda

Lenhardt, David..

Leonzo, John

Leonzo, Michael.

LePage, Meredith

Levergood, Rebecca.

Levesque, Adam.

Levinsky, Kimberly

Lewis, Kristen

Lewis, Thomas.

Li, Stuart

Liggett, Morgan

Liggitt, Edward

Lightbody, Jack

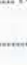

$164,199,214,216,217$

164,169

8.74, 145
.199

199

74

74

199

74

199

74

74

152

199

199

.181

2, 181

.112

74

.112

199

4.74

199

199

199

.112

199

199

199

.152

23

.112

4. 122

.199

.112

199

.152

74,171

.173

. .152

.181

8, 129

199

199

74

177

.183

199

52

\section{4}

\section{Mart}

Martin Andrew $\quad 133$

Lingaas, Jessica. $\quad 74$

Lingenfelter, Seth. $\quad 74$

Link, Alexander _ $\quad 74$

Linkous, Kayla _ 131

Linstad, Callie..__ 112

Linville, Tani... 74

Little, James _ $\quad 74$

Littlejohn, Brian _ $\quad 200$

Littrell, Kenneth..__ 200

Livaudais, Robert...._. 200

Lively, Mary _ $\quad 112$

LiVigni, Kathryn... $\quad 74$

Lockwood, Adam $\quad 200$

Lockwood, Alan _. 200

Loeffler, Sarah _ $\quad 152$

Logan, Alison____ 31, 74, 175

Logsdon, Katherine ..._. 152

Long. Stephanie $\quad 152$

Lopez, Lisa _____ 200

Lorenzini, Jessica _ 74

Lorenzini, Karynn $\quad 200$

Lorenzini, Zachary _ $\quad 200$

Lowe, Megan... $\quad 200$

Lowrance, Rachel ___ $\quad 152$

Lowry, Megan _... $\quad 74$

Luce, Benjamin _ $\quad 152$

Luce Christie 74

Luce, Jordan____ 18, 112

Ludlow, Kenleigh___ 131, 200

Luginbill, Jaye Anna ___ $\quad 74$

Lusk, Joshua _ 173

Luther. Andrew _ $\quad 112$

Lutz, Kari. 200

Lyman, Caleb__ $\quad 138$

Lynch, Quentin _ 200

Lyon, Jennifer _ 2OI

Lyons, Amelia ___ 201

Lyons, Hannah $\quad 25.74$

MacKendrick, Elizabeth ____ $\quad 112$

MacPhail-Fausey, Alexander__ 12

Maillefer, Matthew. $\quad 74$

Makoski, Sarah _ $\quad 152$

Malincon, Andrew. $\quad 112$

Manthei, Alexa $\quad 74$

Marks, Kayla ___ 112, 133

Marot, Caleb____ 152

Marquardt, Christa _ 201

Marquez, Daniel..___ 113

Marshall, Elizabeth.____ 113

Martella. Alexandria $\quad 113$

- 
Martin, Harrison

Martin, Laura..

Martin, Melissa

Martin, Peter

Martinson, Andrea

Mary, Kyle.

Mason, Gabriel

Mason, Ronald.

Mast, Austin.

Mater, Karlee.

Mathew, Susanna.

Mathews, Emily

Mathews, Faith-Hannah

Mathis, Alyssa.

Mattes, Gina

Mattison, Christopher

Mattison, Rebecca

Mattke, Travis.

Matyszczak, Matthew.

Mauk, Logan.

Maxwell, Caleb.

Mayne, William.

McAnallen, Laura

McCain, Angela.

McClain, Jonathan

McClellan, Holly,

McClenahan, Ivy.

McConkey, Jennifer

McCown, Stephen

McCoy, Jessica

McCrone, Kati.

McCurdy, Grant

McDonald, Danielle

McDonald, Jonathan

MoDunn, Andrew

McGee, Lindsay.

McGillick, Brendan

McKenzie, Frank

McKenzie, Kaitlin.

McKevitt, Dylan.

McKinley, Jessica

McKinley, Rachel.

Mclaverty, Elizabeth.

Mcleod, Alicia.

Mclysaght, Caitlin.

McMahan, Mackenzie

McMichael, Paul.

MoNeil, Michelle.

McQuarry, Alison.

McReynolds, Suzanne

McRory, Ivey.

McTaggart, Joy

McWilliams, Cameron

Meacham, Brooke.

Mead, Michelle.

Meakem, Katherine

Meeker, Rachel

Melendez, Thalia

Menendez, April

Merical, Haley
181 Merrick, Hannah

201 Mervine, Alexia

Meyer, Curtis.

Michael, Carol.

Michaud, Beau.

Miesse, Brittney

Miller, Amanda.

Miller, Bethany.

Miller, Brandon

Miller, Chasity

Miller, Jacob.

75

201

173

75

173

. .113

152,201

$113,140,217$

76,201

Miller. Mark

0,177

Miller, Mary.

Miller, Rebecca

152

Miller, Rebecca

.152

.152

Miller, Rebekah

Minich, Phillip.

Minnick, Ronald.

Miranda, Julio.

Mitchell, Rebekah

Mittelo, Aaron.

Moats, Sarah.

Mody, Andria

Moeller, David.

Moline, Meredith

Monroe, Michael

Montross, Ryan

Moon, Abigail

Moore, Alyssa

Moore, Ashley.

Moore, Brittnee

Moore, Ellen...

Moore, Garrett

Moore, Justin...

Moore, Maria

Moore, Tabitha.

Moreno, Jessica

Morley, Joseph

Morris, Abigail.

Morris, Anne.

Morris, Caleb

Morris, Daniel

31, 113, 175

.75

Morris, Stephen

Morrone, Casey

Morrow, Heather

Morrow, Kelsey

Morse, Sarah.

Morton, Tyler

Moss, Isaac

Mottinger, Kyle.

Moughon, Micah

Moulden, Mami

Mounts, Abigail

Mueller, Victoria

.152

.113

76

152

76

76

\section{0}

76

76

76

52

201

202

76

76

76

\section{2}

152

76

\section{2}

76

39

39

16

$$
\text { N }
$$

$$
\text { Nikk }
$$

$$
\text { Niss }
$$

Niss

No

$\mathrm{Noris}$

2 No

6 N

Murrell, Isaac

Muschott, Brett

Myers, Emily.

Myers, Rachael.

Myers, Victoria.

Mytren, Christopher

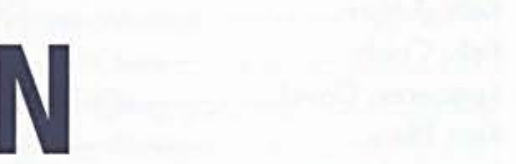

Nafziger, Jacob.

Nagelkirk, Laura.

$.170,171$

Neidig. Natalie _._._............................. 76

Nelson, Casey $\quad 76$

Nelson, David ____._._._._._. 203 
e, Jessica

ley, Janette

dexter, Hunter

sean, Laura.

mento, Anna

ore, Elizabeth.

olin Brittany.

Stephen

ter, Andrew

ter. Anna

Wh Grace.

ands. Matthew

wazki, Sarah.

well, Chelsea.

vell, Matthew

wer, Jonathan

"ers. Sean.

Wers, Timothy

m. Jacob.

ce. Caroline

Q Micah.

e. Stephanie

itchard, Katrina.

or, Joseph.

oh Kelley.

inam, Neola

les, Elijah.

D)

ogle, Rebecca

on. Emma

isch, Kimberly.

andk. Curtis

andolph, Florence.

o. Vineeta

quet, Johanna.

quet, Nathaniel

amussen, Eric.

roach. Carole

ay. Breanna

ay. Ruth.

aymond, Courtney

edfeld Melanie

-dic, Bryan

od, Matthew

oed, Trevor

seder, Lawrence

sep, Jason

eves, Garrison

ichard, Brittani

id Brenda

elly, Kyle

eineke, Marcus

eis, Rodrigo.

. 212.217

.139

77

139

77. 167

77

153

205

205

153

78

78

78

78

205

78

.114

78

31, 175, 205

153

205

179

$78,133,168$

.23

205

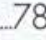

. .114

78

205

.175

205$$
. .114
$$

114
78

$31,114,175$

129

205

78

78

129

78

205

51

8. 158

.129

78
Reisenweaver, Amy

Reisenweaver, Anna

Reisner, Rebekkah

Renner, Derek

Revenaugh, Grace

Rhodes, Robert

Ricci, Marguerite.

Rich, Rebecca..

Richmond, Elijah

Richmond, Jane.

Ries, Joseph.

Rigby, Daniel

Riggleman, Erik

Riley, Scott..

Ripley, Adam.

Ripperger, Kyle.

Rivera, Matthew.

Rizzardi, Kristina.

Roberts, Hadassah

Roberts, Kathryn.

Roberts, Rachel.

Roberts, Tabitha.

Robertson, Andrew

Robertson, Janna

Robinson, Angyla

Robinson, Daniel.

Robinson, Lauryn.

Robinson, Whitney.

Rockwell, Robert

Rogers, Sara

Rogers, Stephanie.

Ross, David.

Ross, Nathaniel.

Rosso, Daniel.

Rotroff, Mark

Rowland, Luke.

Ruch, Christine.

Ruhlman, Melissa

Ruhlmann, Michaela.

Ruiz-Bueno, Amy

Ruman, Deanna

Russell, Austin

Russell, Brian

Russell, Jade

Ryan, Raegan

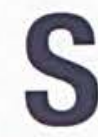

Sabo, Kristen.

Sahadak, Jerica

Sakaguchi, Kevin

Salisbury, Emma

Salzmann, Beniamin.

Samples, Abigail.

Samuel, Benita.

Sanders, Benjamin.
79

.. .153 Sanders, Lindsay

8, 205

78

78

78

78

205

205

78

78

78

23

78

205

.153

205

78

153

78

.114

153

.114

. .114

205

78

13

.. .131

78

25

27

.153

78

.153

205

138

79

114

44

144

114

205

79

205

79

129, 131

Sanderson, Ryan.

Santana, Ashley.

Santiso, Daniel.

Santoro, Justin.

Santos, Joy

Saver, Danielle.

Savard, Sara...

Sawyers, Brenna

Scanlon, Hannah

Scarcella, Timothy.

Scarpone, Rebecca

Scarpuzzi, Rebekah

Schaefer, Allison

Schaffter, Kelly

Scheunemann, Erin

Scheurer, Emily

Schick, Annelise.

Schick, Kyle.

Schindler, Stephen

Schlabach, Timothy

Schloneger, Rachel

Schmid, Anna.

Schneider, Brittni

Schnepp, Rebecca

Schoch, Maxwell.

Schoenwald, Matthew

Schroeder, Erich.

Schroeder, Niklaus

Schroll, Laura.

Schuler, Lilian...

Schumacher, Emily

Schutt, Caleb.

Schutte, Rachel.

Schweinitz, Angela

Scott, Connor

Scott, Erin...

Scott, Jeremiah.

Scott, Richard.

Seabold, Paul

Seals. Amanda.

Sears, Emily

Seegers, Caitlyn.

Seelig, Kaitlin.

Senerius, Kimberly

Sensky, Cassandra

Sergeant, Troy.

Setzkorn, Kirsten

Seybold, Rahel.

Seymour, Benjamin.

Shamburger, Timothy

Shannon, Muriel.

Sharp, Joanne.

Shaw Oieda Gloria

Shaw Oieda, Myriam.

Shaw, Lauren..

Sheldon, Andrea.

Shenk, MoKenzie.

Shepherd Alexander

Shepherd, Alexandra

114

79

79,173

79

206

23

$.175,206$

206

25

.79

.114

.153

158,206

206

.153

. .114

$140,206,216,217$

.100

.114

206

23

.153

.79

.175

.153

. .153

79

.153

.114 
Southworth, Aaron

207

Spain, Courtnie $114,182,183$

Spann, Jennifer

.183

Sparks, Brianna

Spears, Brandon.

Spencer, Andrew

Spiliotis, Danielle

Spivey, Samuel

Spranger, Amy.

Sprotberry, Jeffrey

Spudie, Erika.

80

.17

80

.131

80

.114

80

St Pierre, Sarah

183. 207

Stackhouse, Wesley _..._...................... 133

Stambach, Hailey ___ 207

Standridge, Lydia ....................... 80

Stanley, Jessica _... $\quad 154$

Stannard Grant _...__ $\quad 80$

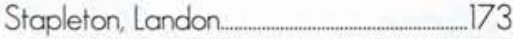

Stark, Evan _. $\quad 8,145$

Steckel, Jonathan _............. 207

Stecker, Andrew....................... 154

Steele, Joshua _..... 207

Steele, Rachel ..._... $\quad 207$

Stein, Alicyn _....................... 154

Steinhoff, Daniel _.____ $\quad 154$

Stephens, Taylor..._. $\quad 80$

Sterling, Kellee

Sternberg, Madison

Stevens, Elizabeth.

Stevenson Maggie

Steward, Scott 154

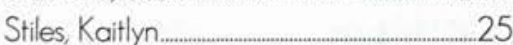

Stine, Lyndsay _...__._._._. _.....

Stitzinger, Abigail..._____._. 207

Stobbe, Lillian _....................................

Stockdale, Kevin ................................ 208

Stoker, Andrew..._._. 208

Stoltenberg, Elaina.

Stoltzfus, Melissa

179,208

Stonkus, John.

Storkel, Sarah

Storm, Victoria

Stover, Katelyn.

Straight, Alexander

Straight, David.

Straight, Sarah.

Strattan, Ethan.

Straw, Karyn..

Strawser, Payton

Strayer, Hope

Strayer, Melody

Strickland, Amber ..........34, 208, 212, 214

Strickland Michelle__. 208,212

Stump, Andrew _... $\quad 208$

Sturgeon, Cameron _..._........... 154

Sturm, Kaitlyn _..._......................... 155

Summerlin, Chase.

126, 208

Summerlin, Samuel

Sunden, Kerith..

Sutherland, Abel.
Suver, Hayden

Swanson, Danielle

Swartley, Daniel.

Swayze, Taylor.

Swedorski, Caleb

Sweet, Emily

Sweetapple, Jadyn.

Switzer, Tori

Symington, Alisha.

5

Talbott, Sheila

209

Tang, Caleb

$.91,138,140$

Tang, Hawkins

Tarr, Jessica

Tavierne, Tyler

Taylor, Adam

Taylor, Joshua

Taylor, Rebecca

Taylor, Rockwell

Teague, Courtney

Teixeira, Bethany.

TerreBlanche, Bernardus

Terrell, Meghan.

Tesmer, Jacob

Thacker, Cassandra.

Thayer, Forrest.

Thome, Rebekah.

Thompson, Ashley

Thompson, Benjamin.

Thompson, Debra.

Throgmorton, John.

Thurman, Aerial.

Thurman, Ryan.

Thut, Katelyn

Ticker, Carolyn

Toburen, Abigail

Tomlinson, Abigail.

Tomlinson, Luke

Torres, Alyson

Toth, Michelle.

Towne, Ruth

.160

81

115

209

81

Van

Van Der Wal, Elizabeth ...

Van Dyck, Carissa ...._... $\quad 25$

Van Dyke, Jonathan ....__..... 29, 173

Van Etten, Caytlin ........................ 115

Van Pelt, Jonathan _.................... 155

Van Zandt, Joshua ............... 177, 209

Vance, Laura _........ 155

Vander Plas, Taylor _......... 82, 131

VanderHart, Claire _..._... $\quad 155$

VanDyke, Caleb _._.............. 82

VanLith, Natalie ............................. 82

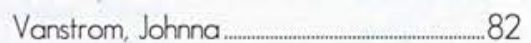

Vasser, Melinda $\quad 209$

Vaughn, Heather ............................ 82

Vazquez, Cristina …__...… $\quad 82$

Veith, Jonathan .......................... 209

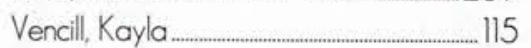

Vendl, Anna....

Venman, Caleb.

210

Venturini, Tara _............... 14, 139, 155

133,155

Vermilion, Mason

173

Vidal, Briana

.82

Vogt, Jerome _ $\quad 82$

Voland, Peter

210

Traeger, Robert...__ 52

158,209

Treece, Jordan ................................. 115

Treide, Alexander......... 104, 106

Trent, Avery _.. $\quad 81$

Trigg, Emily _..._..............................

Troyer, Kelly _...__. $\quad 209$

Troyer, Megan _..._._._._._._._. 115

Truninger, Derek _._._._. $\quad 81,171$

Tse, Nathan _..._............................

Tucker, Colleen _ $\quad 209$

Tucker, David _ $\quad . \quad 81$

Tucker, Hannah _ 209

\section{W}

Wadman, Henry.

Wadman, Rachel

.. .82

Wadman, Rebecca

Wagner, Caleb..
Wagner, Hannah.

Wah, Andrew...

Walczyk, Brian.

Walker, Ethan...

Walker, Fred.

Wall, Ryan.

Wallace, Hannah.

Wallace, Samuel.

Wallace, Stephanie

Waller, Timothy

Wallin, Joseph..

Wallin, Rebekah.

Walsh, Erin...

Walter, Jacob.

Walter, Megan

Ward, Chelsae.

Ward Christopher.

Ward, Jennifer.

Ward, Jessica

Ward, Laurel...

Warder, Amiah

Ware, Elisabeth

Wasserstein, Joel.

Waters, Thomas.

Watson, Clay ..

Watson, Erkai

Weaver, Luke

Weaver, Zachary

Wegman, Hannah

Weir, Kelsey.

Weiss, Vivian

West, Chloe.

West, Kara.

West, Morgan

Weston, Zachary

Weyandt, Maggie.

Wheatley, Joanna.

White, Andrew.

White, Paige.

Whiteside, Alexander

Whitfleld, Marybeth.

Whitlock, Corey.

Whitney, Darcy.

Widder-Varhegyi, David.

Wilbers, Joshua.

Wilcox, Rachel.

Wilkinson, Benjamin

Willeford, Megan.

Willenbrink, Joshua

Williams, Aaron.

Williams, Allison.

Williams, Bradley,

Williams, Brandon

Williams, Cody.

Williams, Dalton

Williams, Emily..

Williams, Lauren

Williams, Rachel.

Williamson, Christopher.

Willis, Tyler 


\section{X}

Wynalda, David

Wynalda, Mark 183

on, Kelsey

son. Michelle

on. Sarah.

feld Paul.

ton. Benjamin

ters, Donielle.

rs, Kelsey

rs, Zachary

nan, Ellen

er. Heather.

iewski, Kadie.

Ait, Kristina...

ack. Alison

g. Abigail

od Michael

dard, Katie

oodfeld, David

ods, Micheal.

amald, Katherine

isham, Ashley

thington, Neal.

ight, Jenna

right, Joshua

right, Kyle.

ight, Natalie

int, Nathan

ght. Nathan

Qionghui

derlich, Hannah
211

Xayathone, Alexander

$126,216,217$

Xu. Linhan

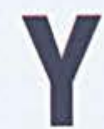

Yake, Tess

Yang, Ziwen

Yates, Seth

Yeh, Simon

Yoder, Caitlin

Yoder, David

Yorgey, Marissa

Yost, Seth

Young, Shelby

Young, Zachary

Youngquist, Natalie.

Yuchimiuk, Larisa

Yutzy, Kara
211

83

Zaharek, Kristyna 155

Zambello, Lauren.

Zavodney, Anna. 83

Zavodney, Julie. $122,162,211$

Zehel, Tiffany

Zehr, Olivia

Zeiter, Rachel.

Zender, Jordan

Ziegler, Grace

Ziegler, Jacoby

Zonca, Ethan.

Zuch, Jordan.

Zuidema, Emma

Zwiebel, Heidi

Zwirkoski, Sarah

.115

.144

83

.115

. .115

155

25

211

.83

83

21

83 83

$5,155,173$

23,155

.. .155

183

211

211

83

27. 211

\section{COLOPHON}

\section{BLISHER}

2013 Miracle Yearbook was published by Jostens, was printed at their plant located in Clarksville, he Jostens representative was Eric Howard, and ant coordinator was Crystal Gault.

\section{OVR}

cover was designed by Kathryn Kramer. The

Was taken by Stuart $\mathrm{Li}$. It is a custom litho cover Ss cover with gloss lamination).

\section{ER STOCK}

aper for this book is a $80 \#$ gloss finish on all

\section{COLOR}

The 2013 Miracle design theme was created by Kathryn Kramer.

\section{COVER}

The 2013 Miracle theme was formulated by the editors and faculty advisor with a photo book theme.

\section{PRODUCTION}

The 2013 Miracle Yearbook was produced on Macintosh computers. Layouts were constructed using Adobe InDesign CS4 and CS5. Copy was written in Microsoft Word and was imported into Adobe inDesign.

\section{TYPOGRAPHY}

The main font used in the 2013 Miracle Yearbook is Helvetica Neue with various versions including Light, Bold, and Bold Condesned.

\section{PHOTOGRAPHY}

The majority of the photos were taken by the photography staff. Student submissions were also accepted. All team photos and several others were taken by Scott Huck, Cedarville University photographer. was used for class portraits

\section{INQUIRIES}

The Miracle Yearbook office may be contacted for additional information about this book's production at miracle@cedarville.edu or (937) 766-4995. 

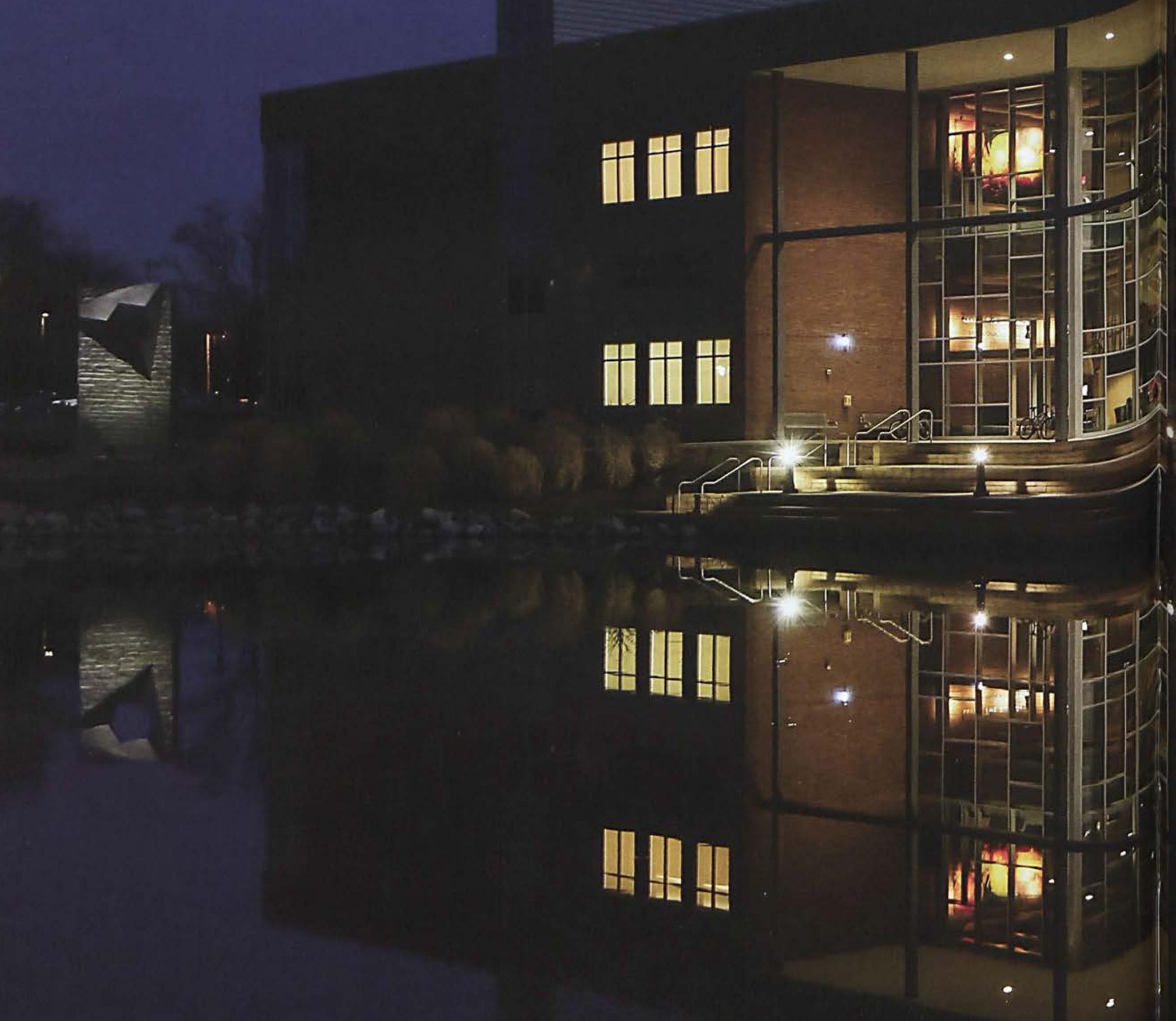


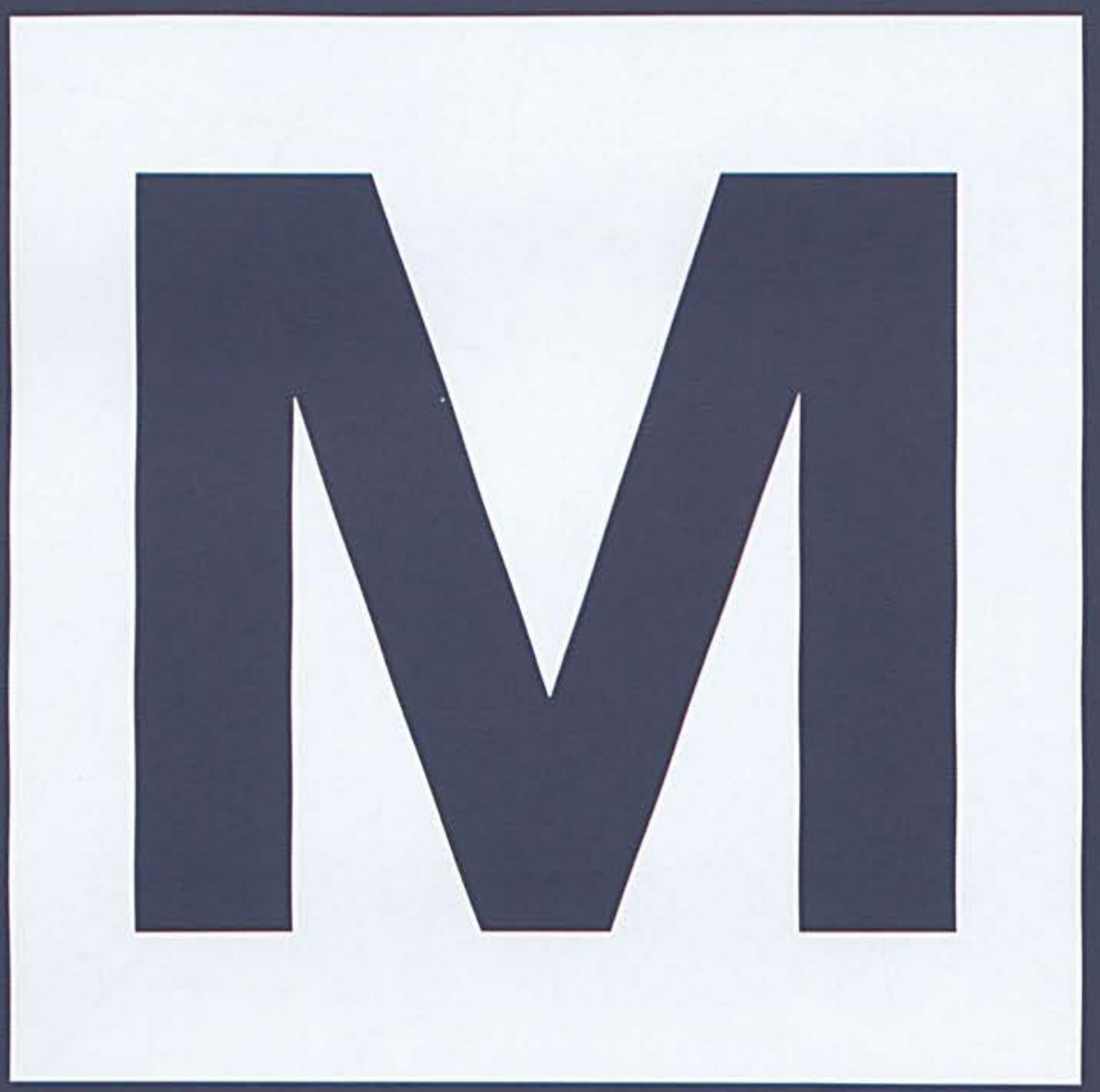





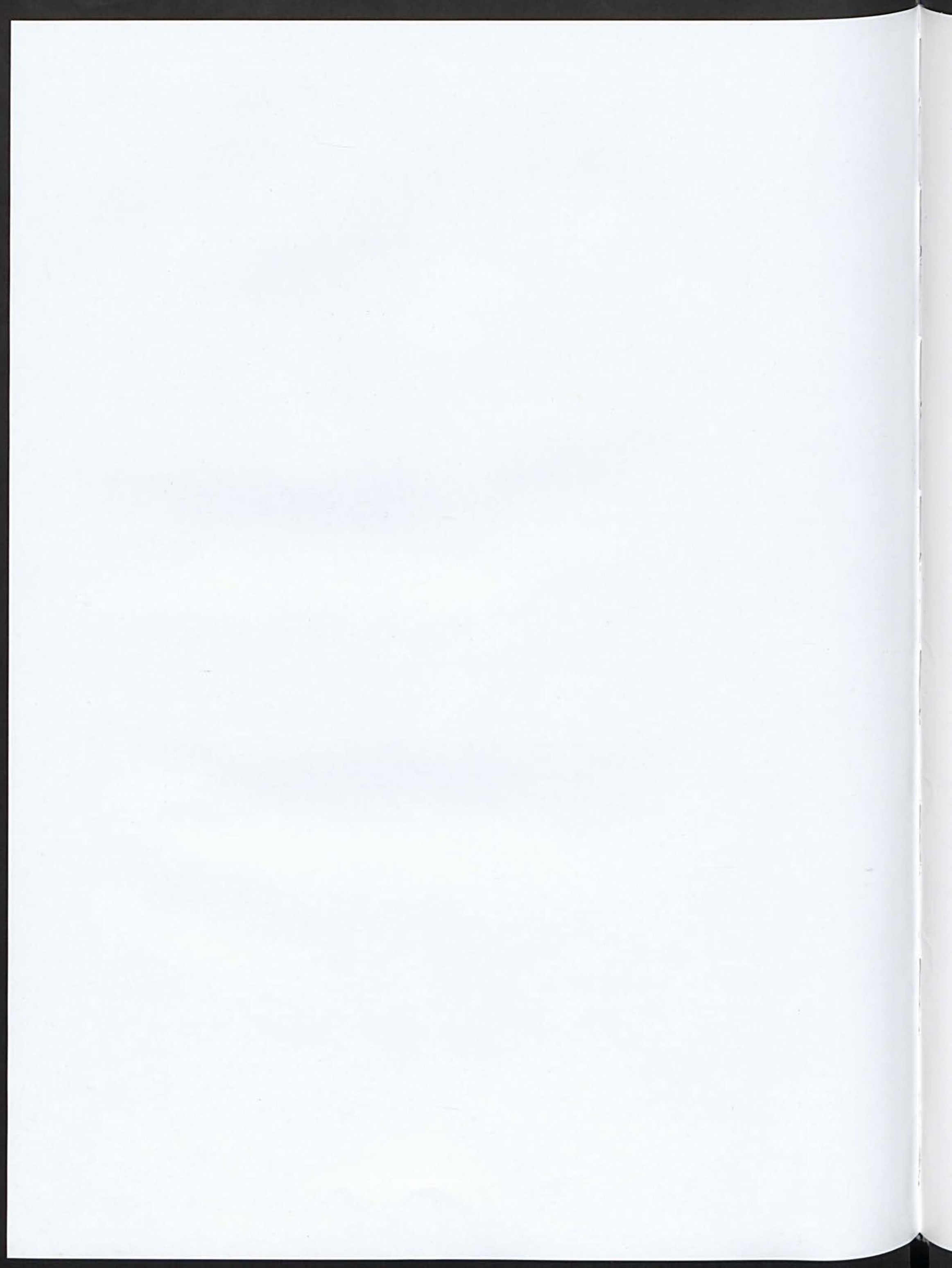





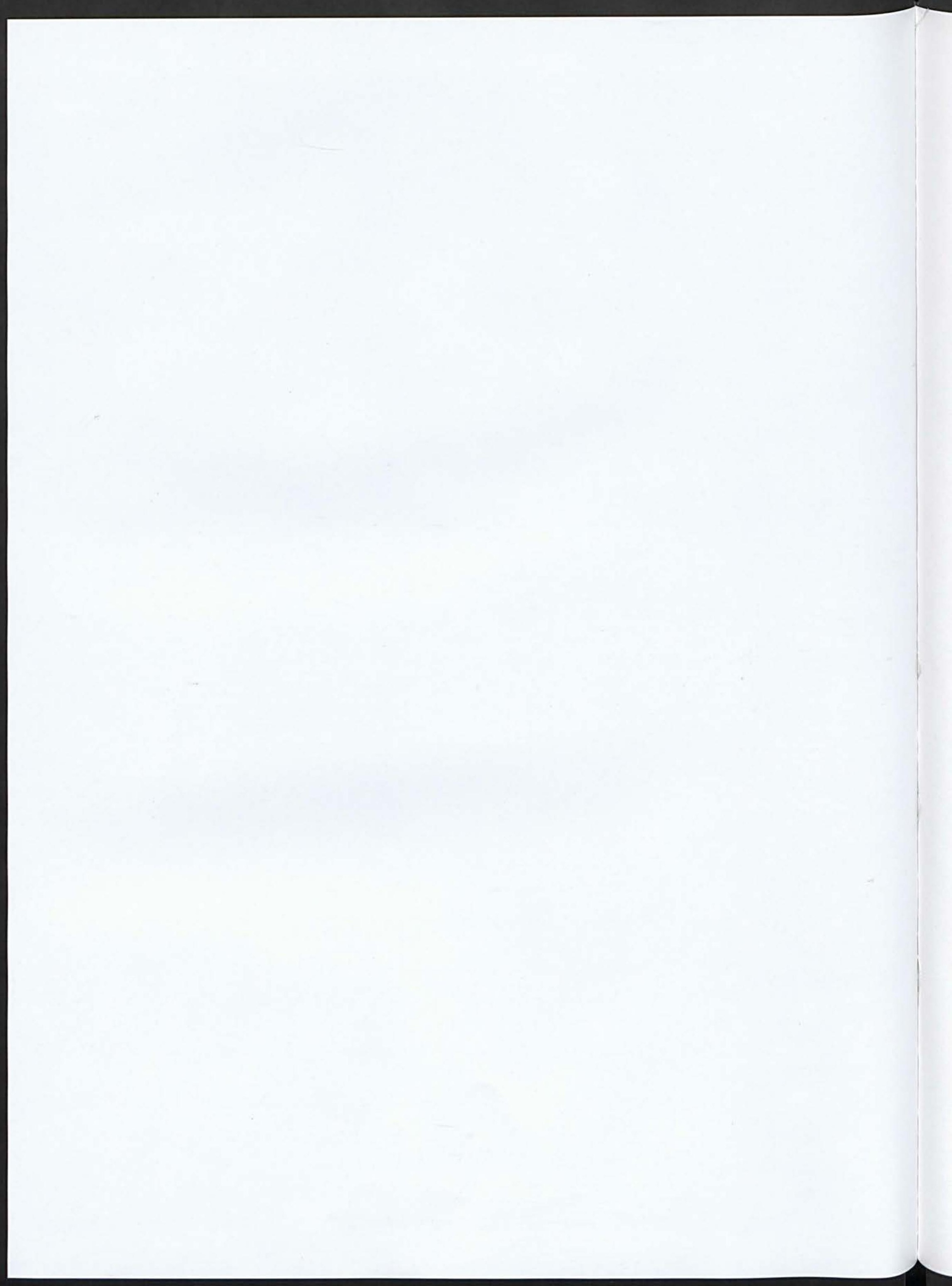





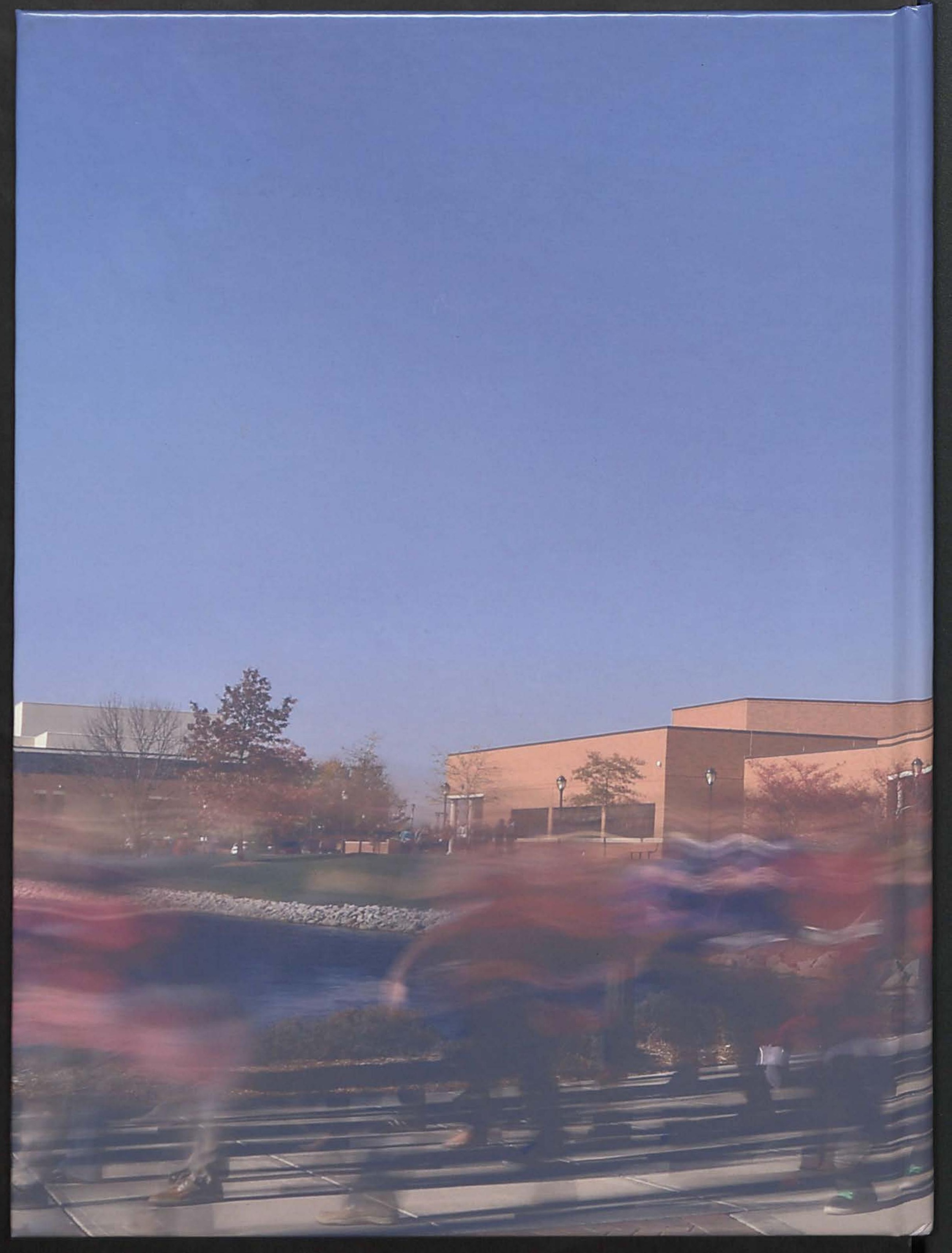

